Fall 1987

\title{
1987 Miracle Yearbook
}

\section{Cedarville College}

Follow this and additional works at: https://digitalcommons.cedarville.edu/yearbooks

Part of the Higher Education Commons, Organizational Communication Commons, and the Public Relations and Advertising Commons

\section{Recommended Citation}

Cedarville College, "1987 Miracle Yearbook" (1987). Yearbooks. 10.

https://digitalcommons.cedarville.edu/yearbooks/10

This Book is brought to you for free and open access by DigitalCommons@Cedarville, a service of the Centennial Library. It has been accepted for inclusion in Yearbooks by an authorized administrator of DigitalCommons@Cedarville. For more information, please contact digitalcommons@cedarville.edu. 


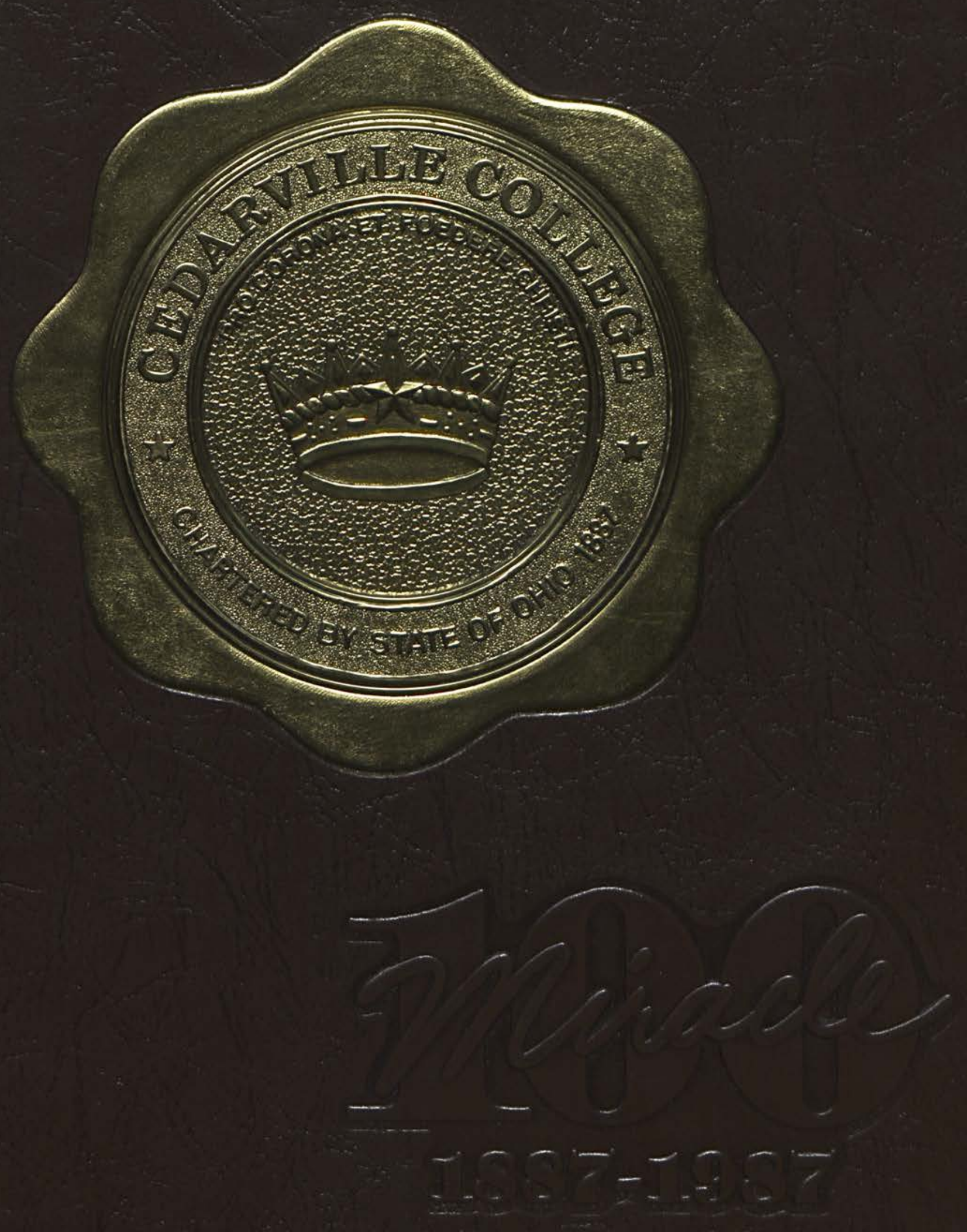






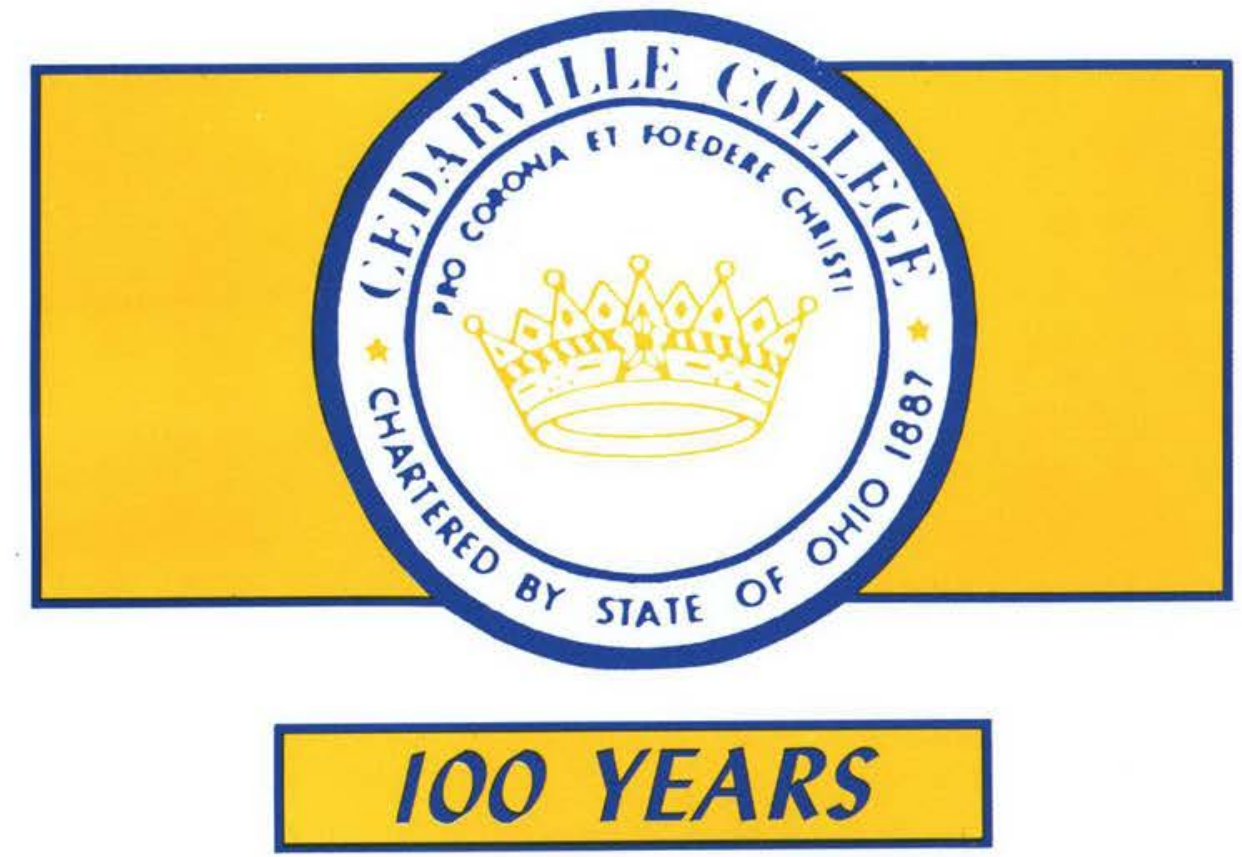

"For The Word of God..."
and Testimony of Christ"

\section{THE I987 MIRACLE}

\section{Cedarville College}

Cedarville, Ohio Volume \#34

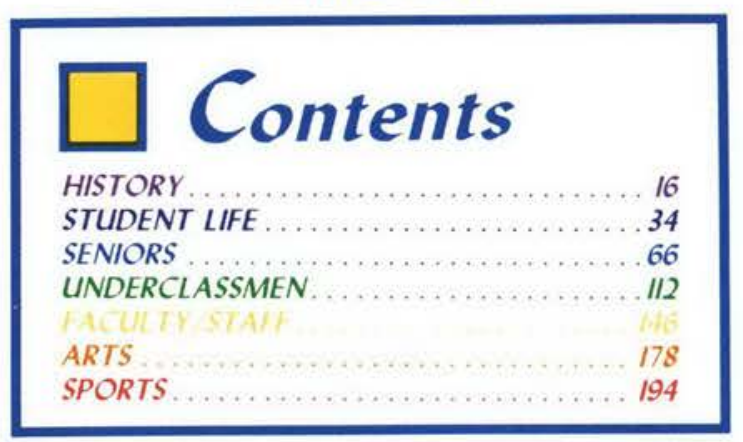



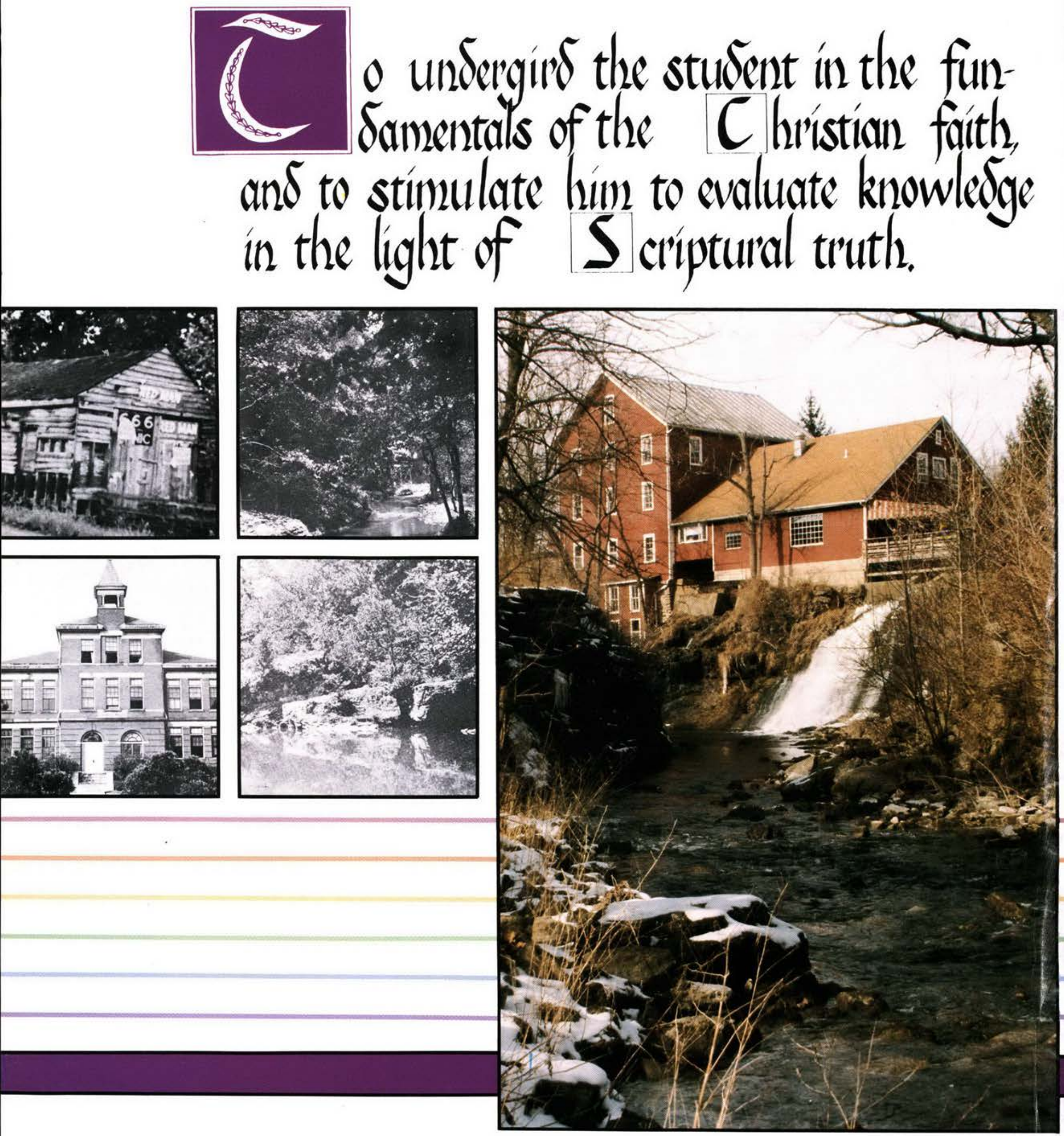


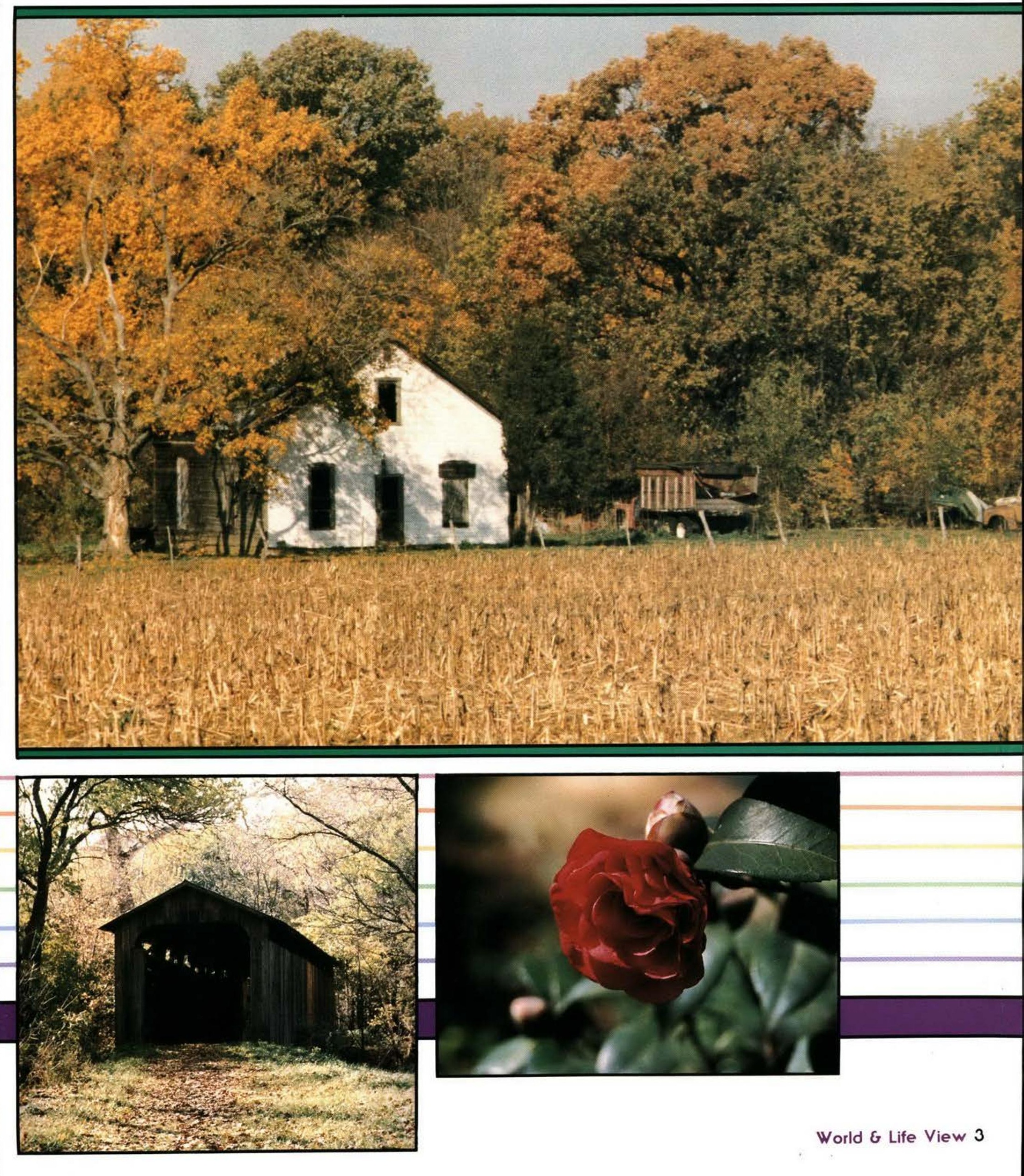




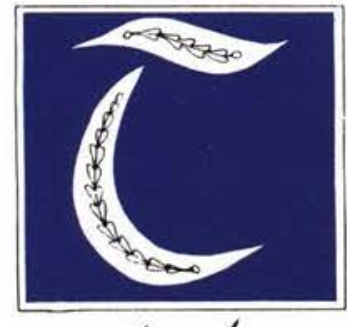

- encourage growth in Christian character in each student, and to help the student accept his responsibility in faithfull Christion service.
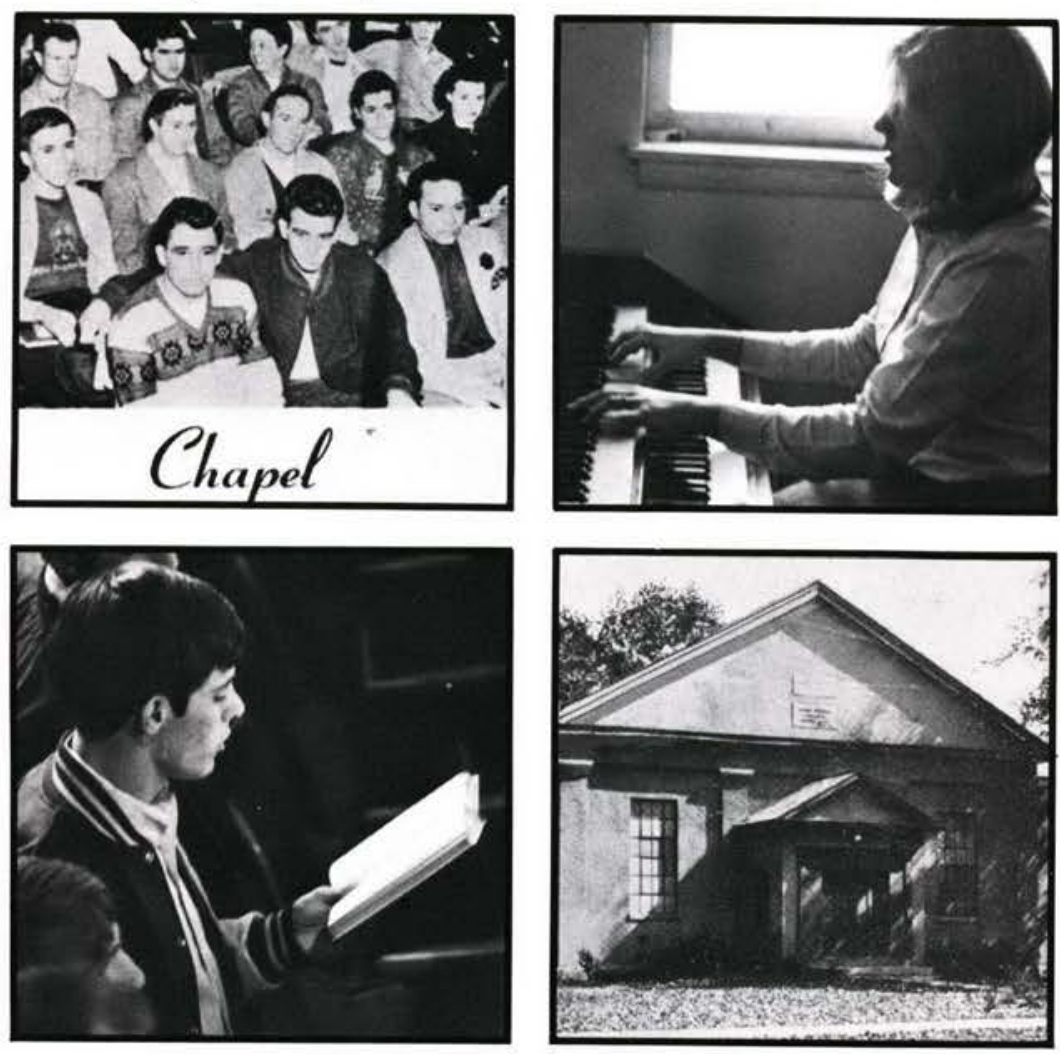

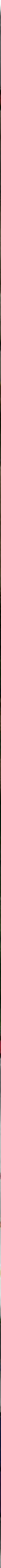

Christian Service 5 


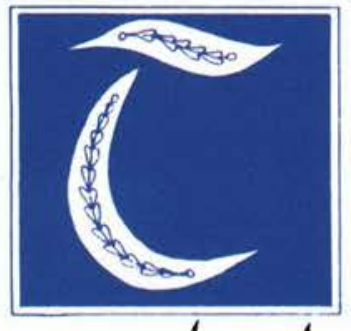

- increase the stubent's awareness of the worls of iseas and events which are influencing our contemporary culture, and to prepare the student to knowledgeably participate in our society.
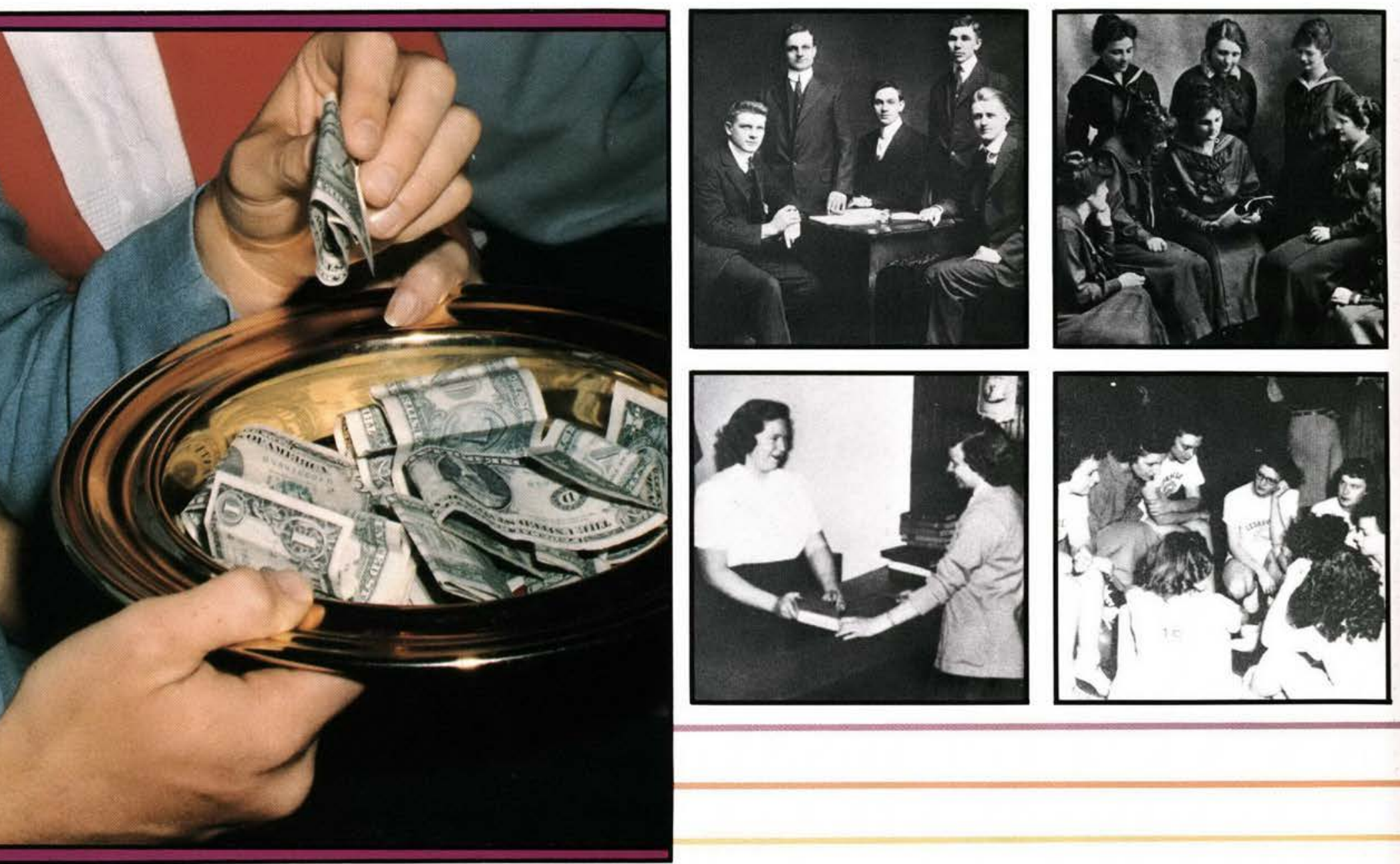

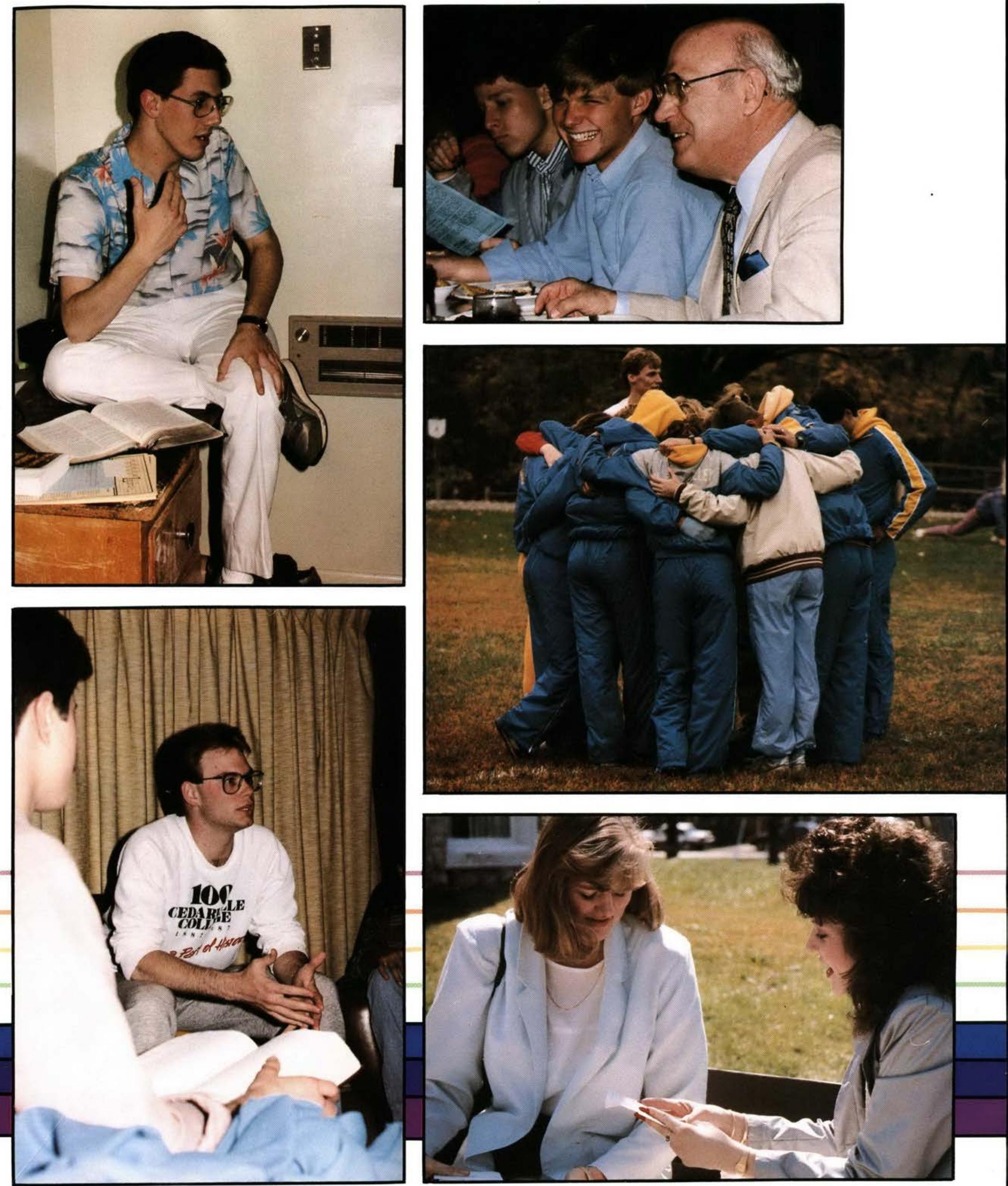

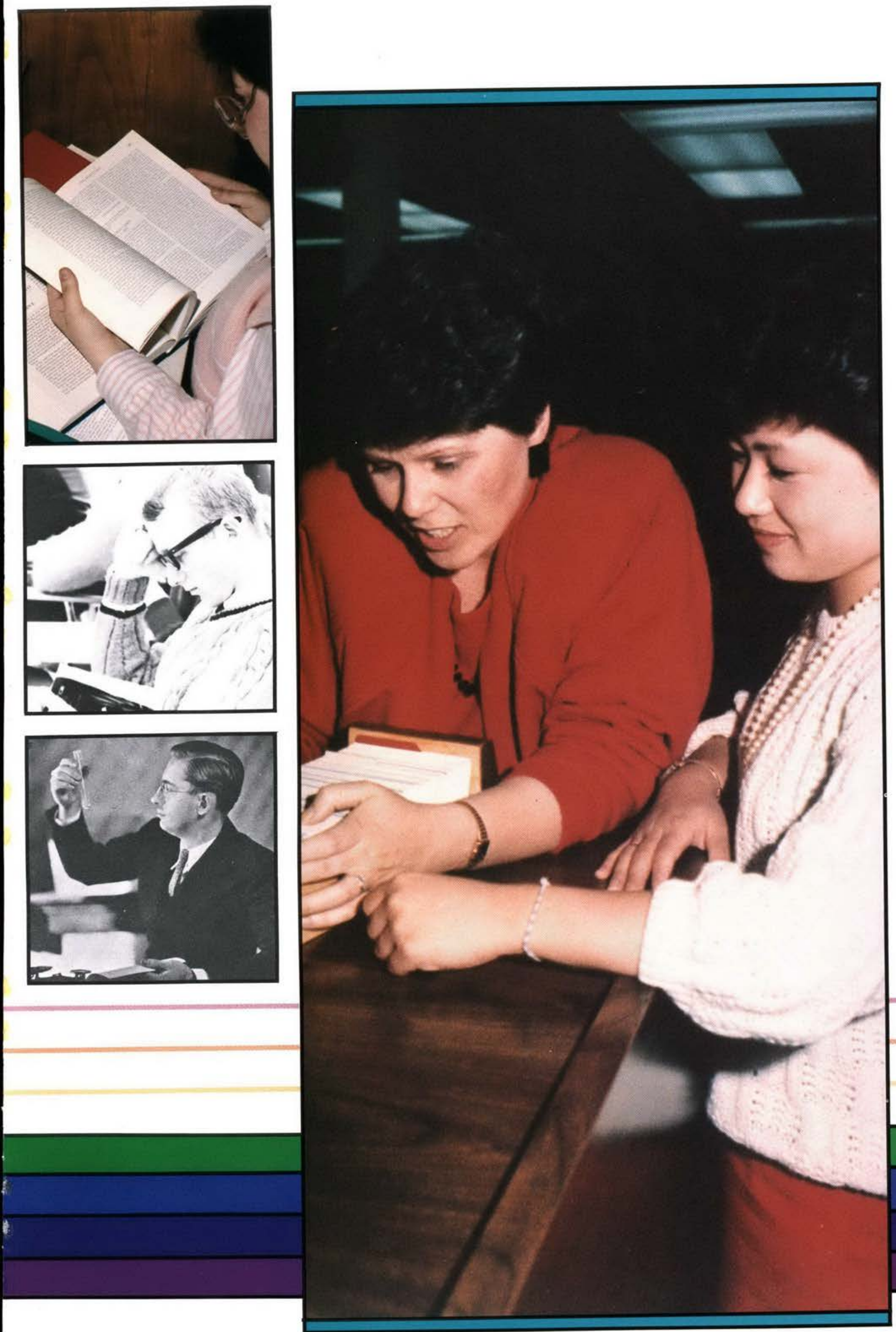

Critical Thinking 9 


\section{o provise sufficient opportunity for students to practice the skills of communication.}

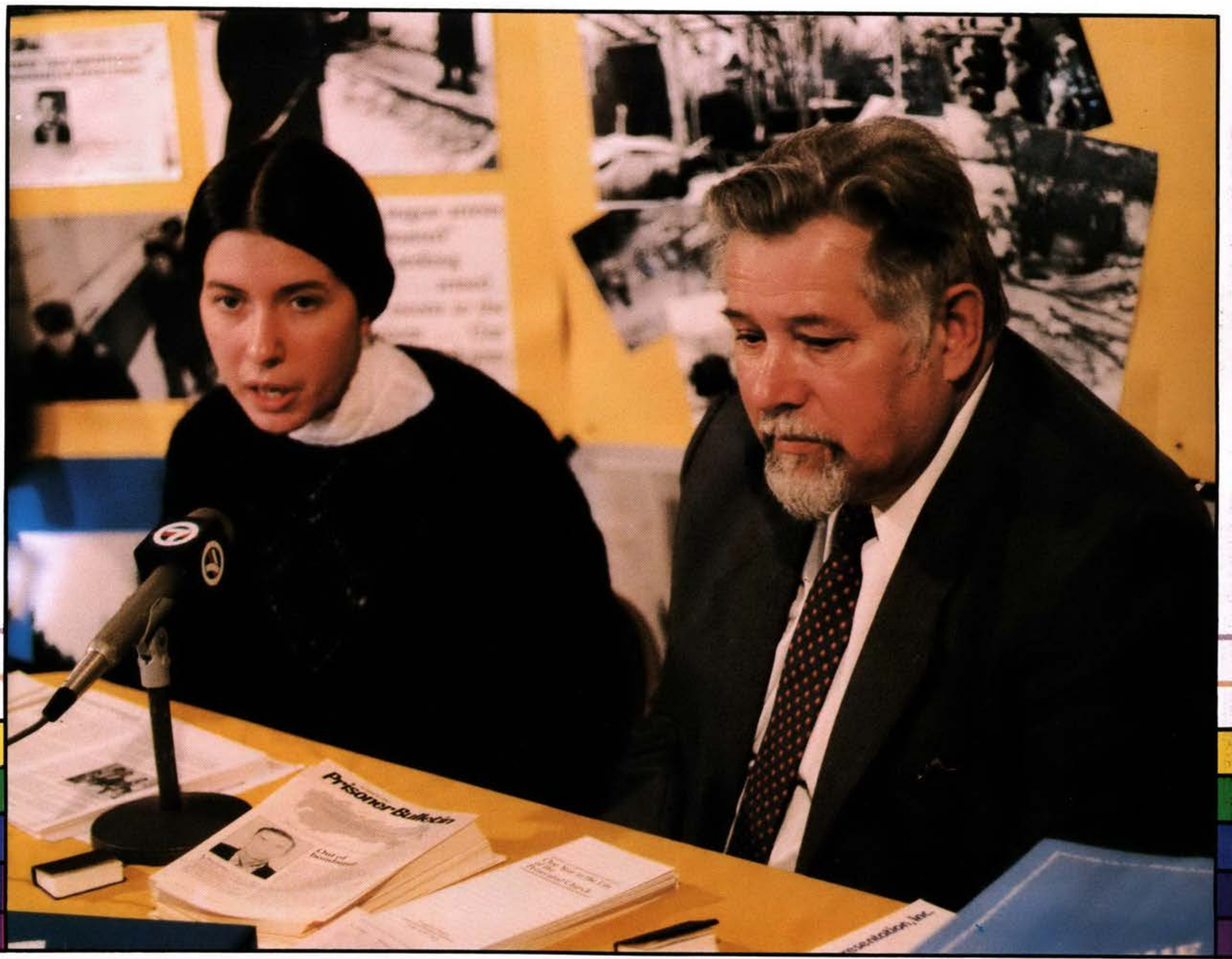



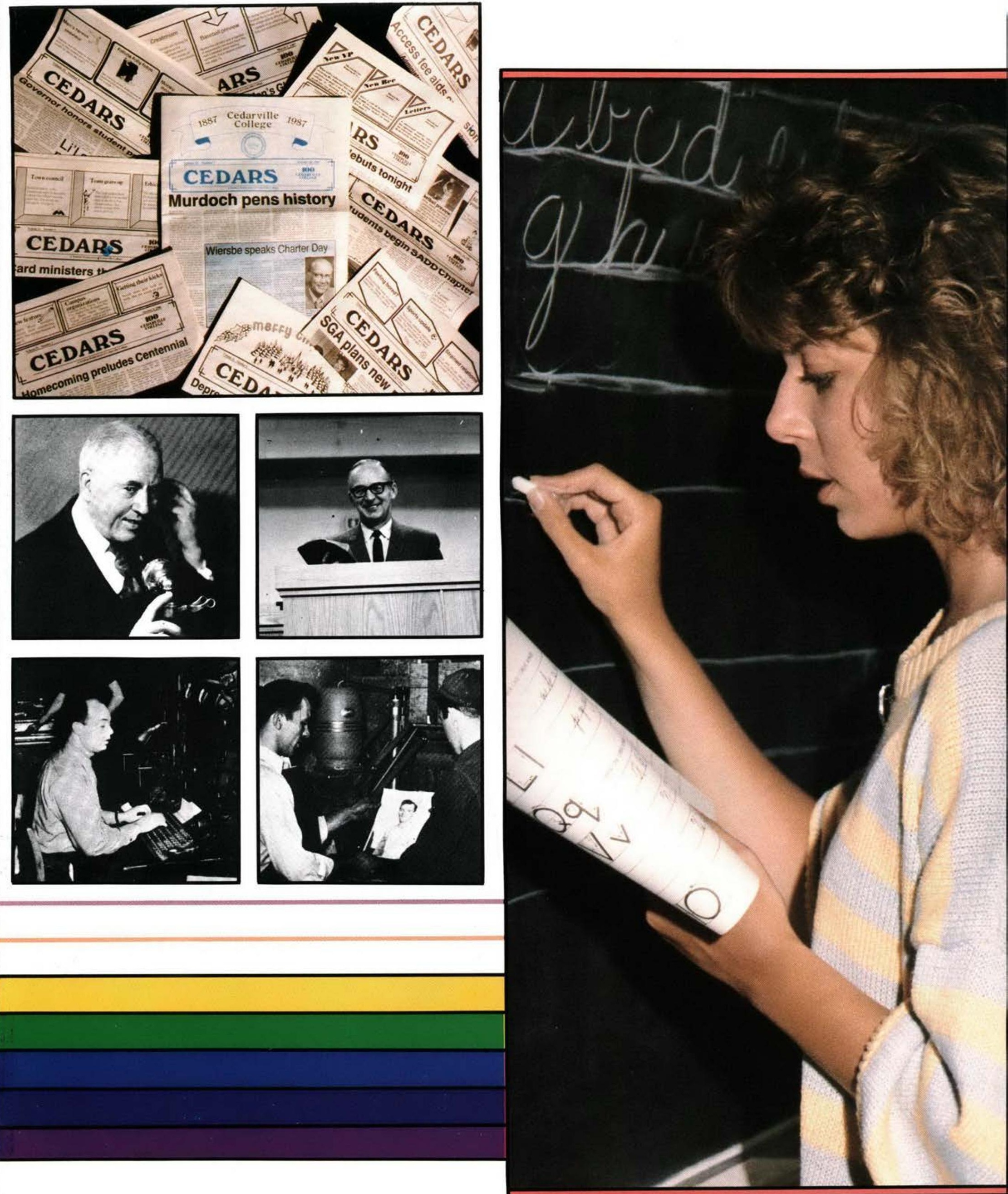


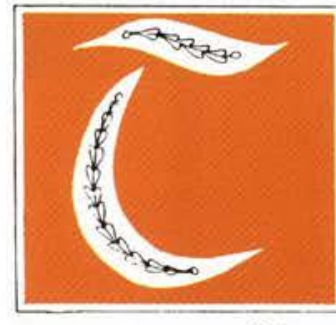

- offer opportunities for academic specialization and preparation for graduate stusy, and to assist the student in selecting and preparing for a vocation.

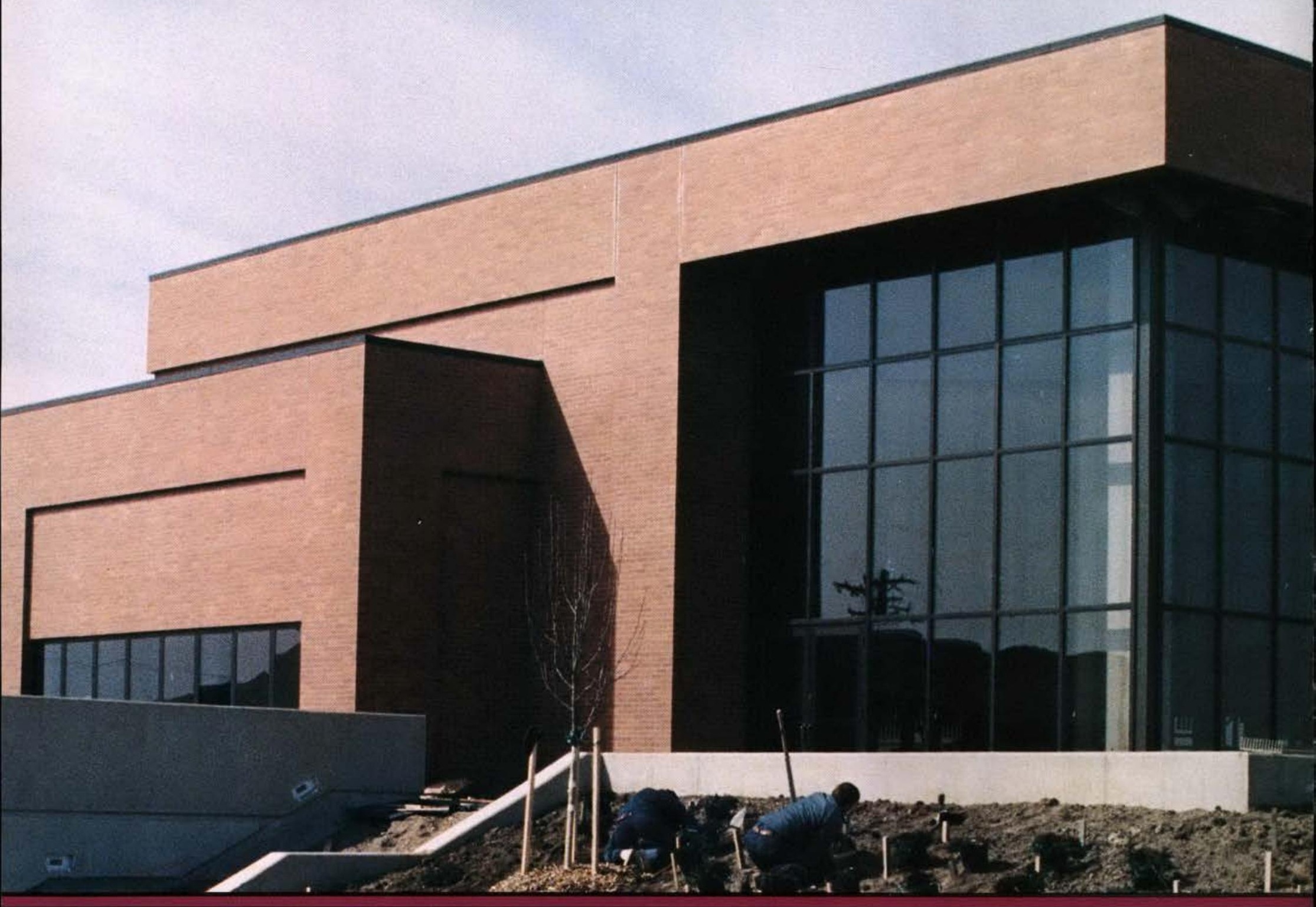



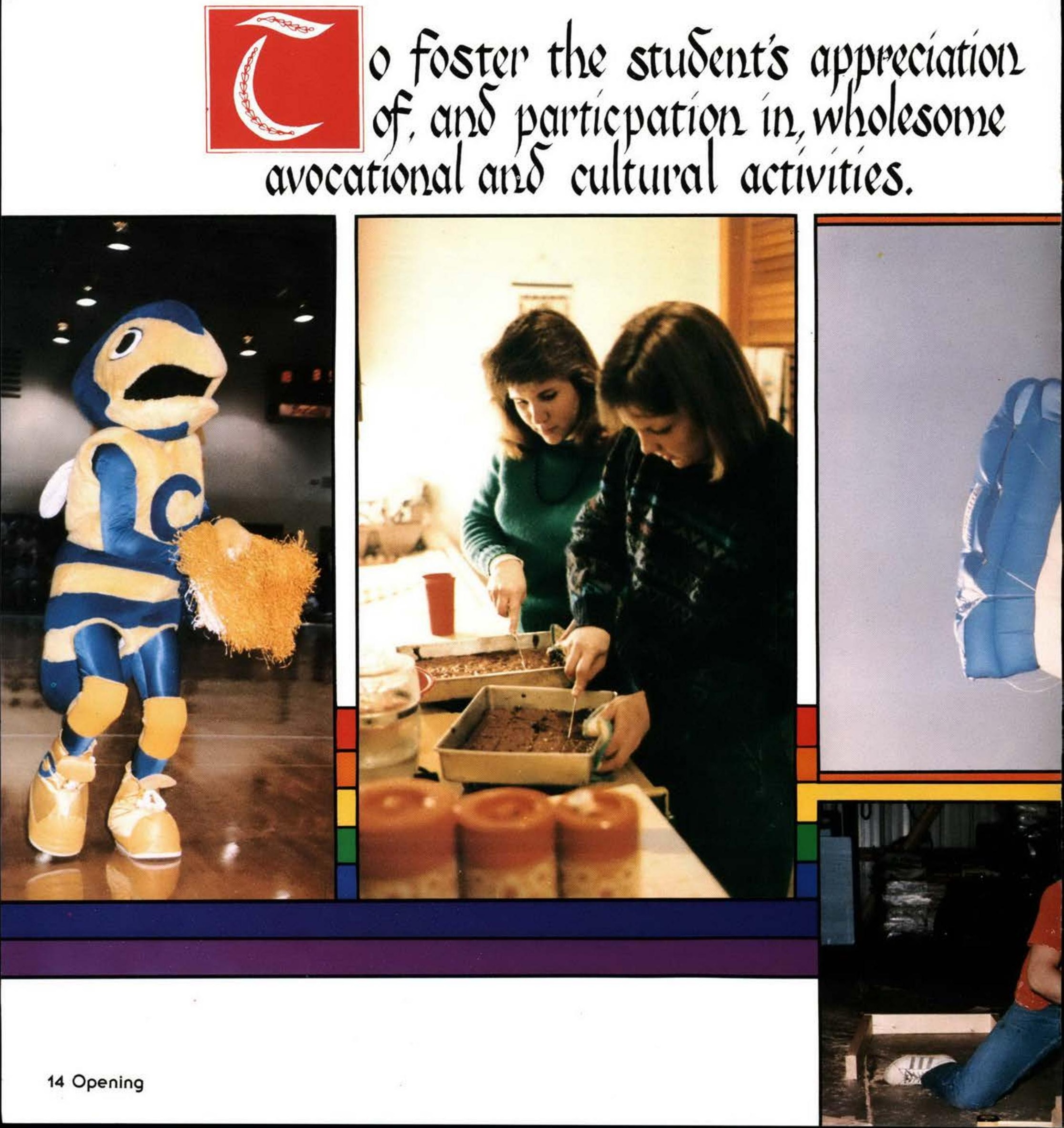

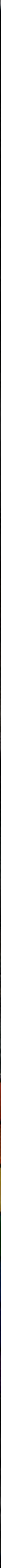


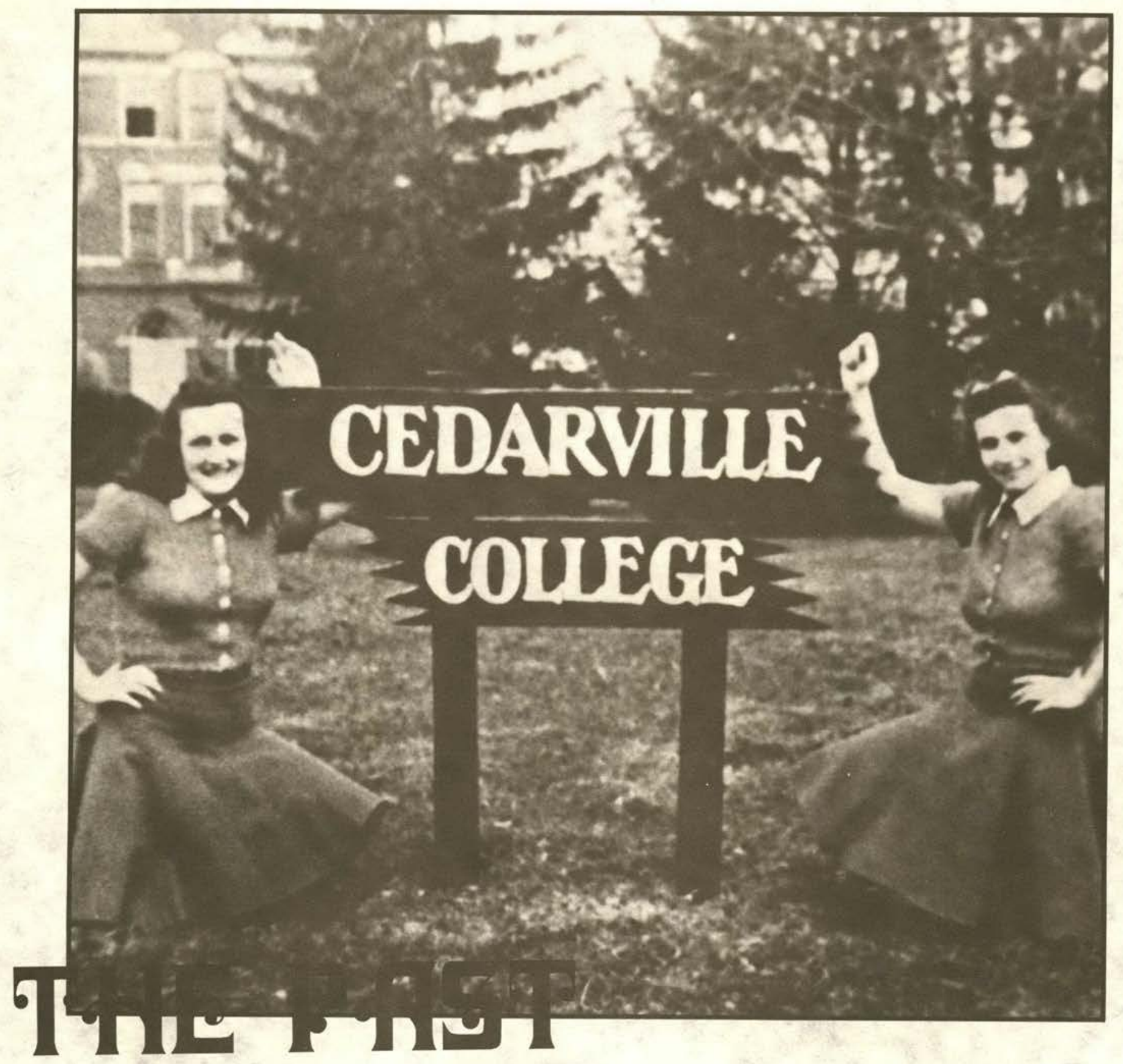




\section{THE FOUNDATION}

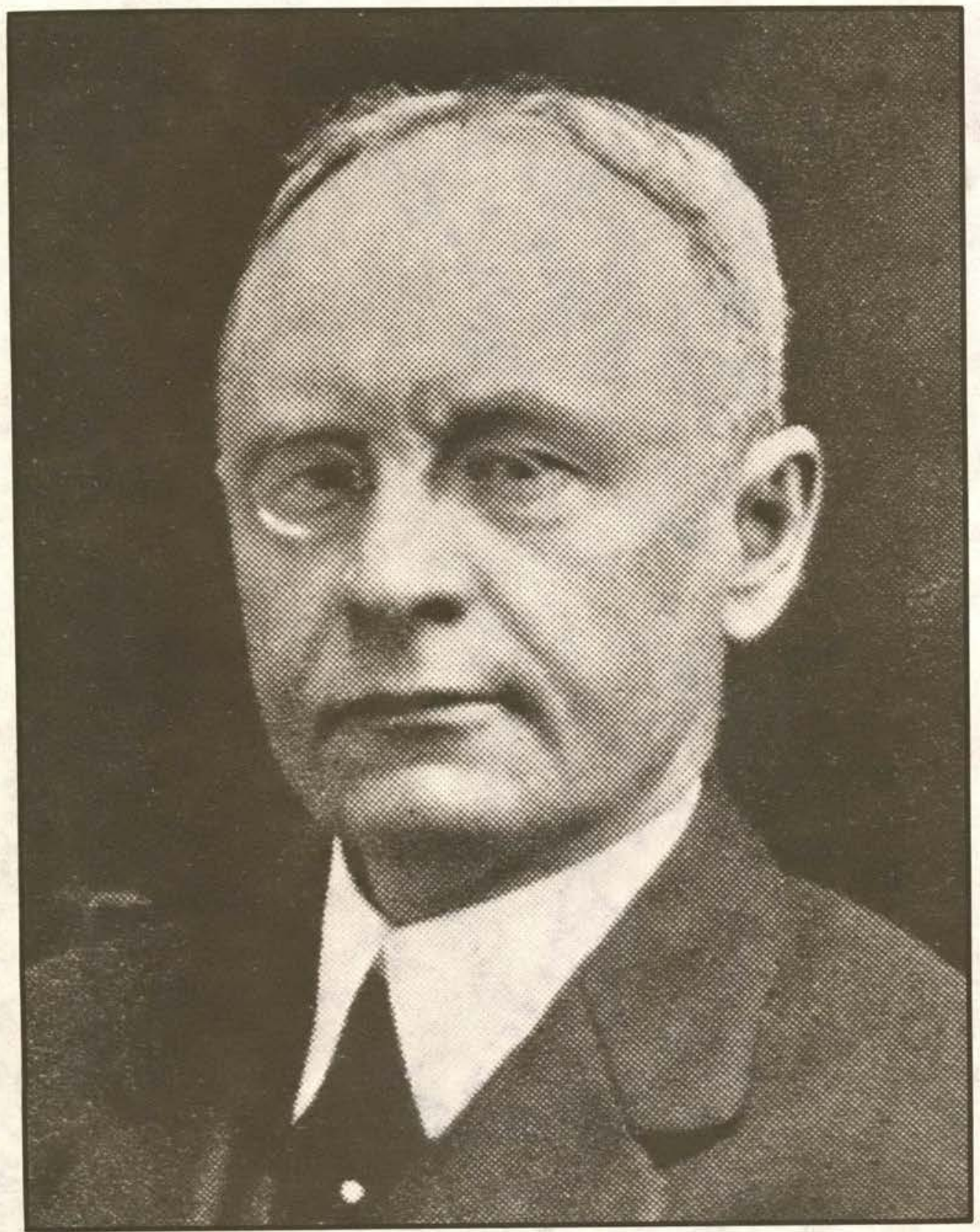

The foundation of Cedarville College's history was formed by the college's first president, Dr. David McKinney. He was a welleducared and articulare man who had complered his undergraduate program at the University of Pennyslvania before artending the Reformed Presbyterian Seminary in Philadelphia.

During his 21 years as president, McKinney, while communting to Cedarville twice a week, rerained his pastorare of the Plum Street Reformed Presbyterian Church in Cincinnati. His stable leadership and administrarive abilities greatly contributed to the college's formative years, although his primary commirment was to the pastorate.

Mckinney spent most of his term away from the college campus, bur he accomplished many admirable goals: three new buildings were added, including a new li-

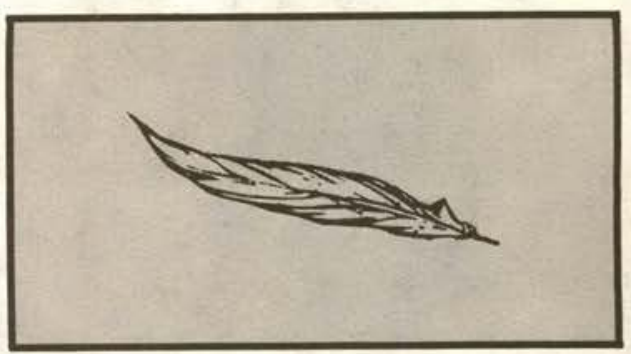

David McKinney, 1st President (1897-1915)
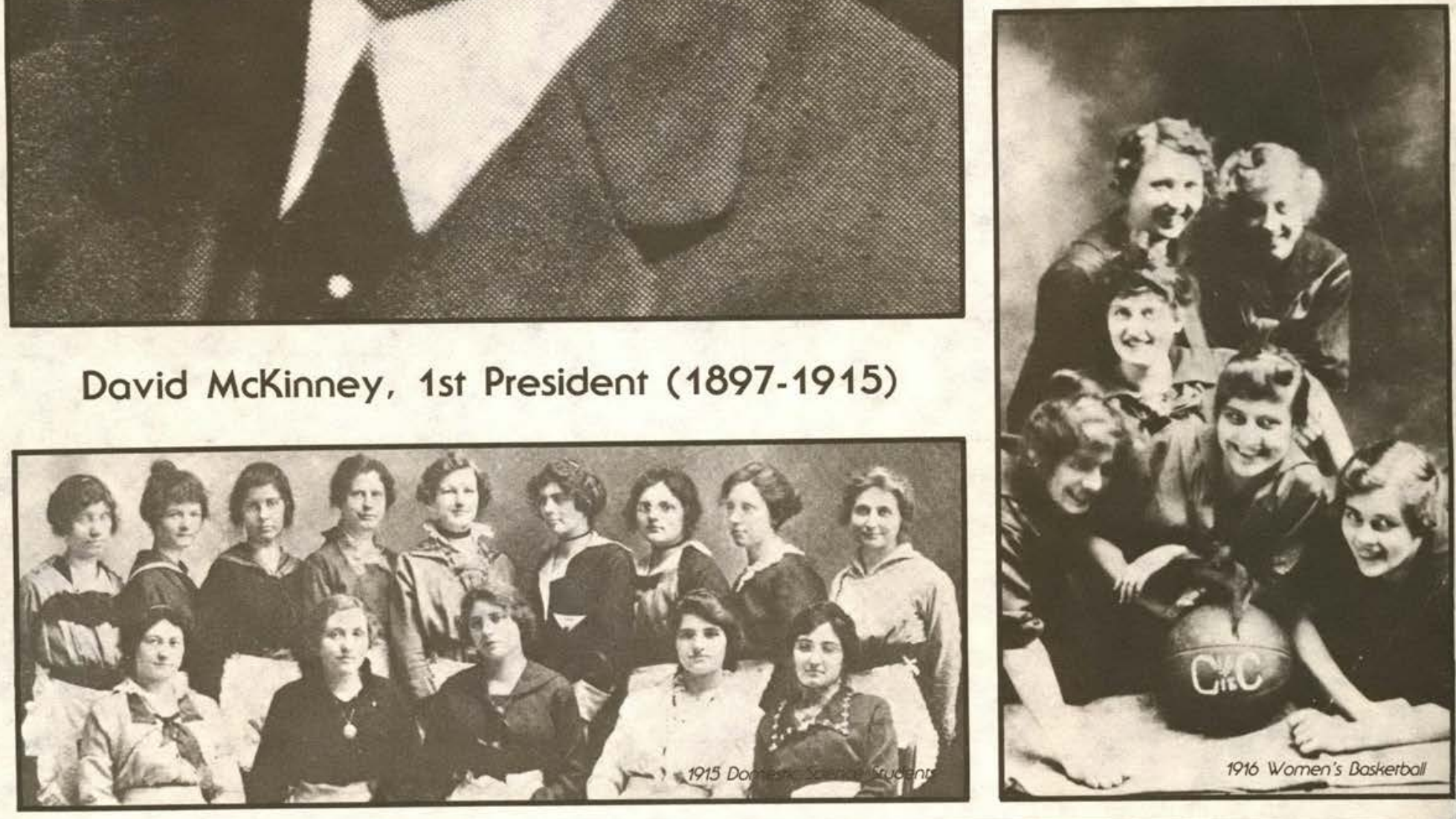


\section{Cedanitur,}

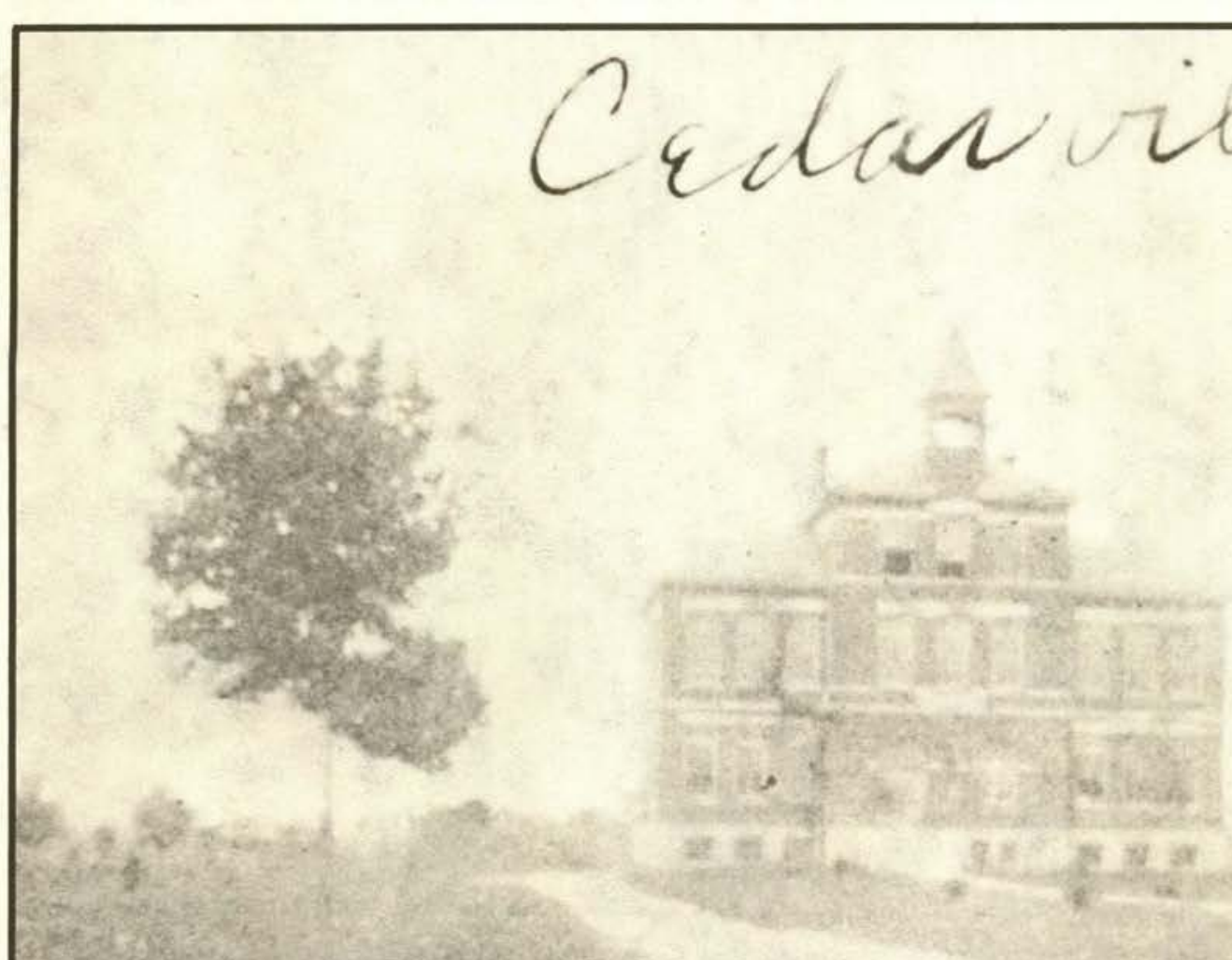

An Early Sho
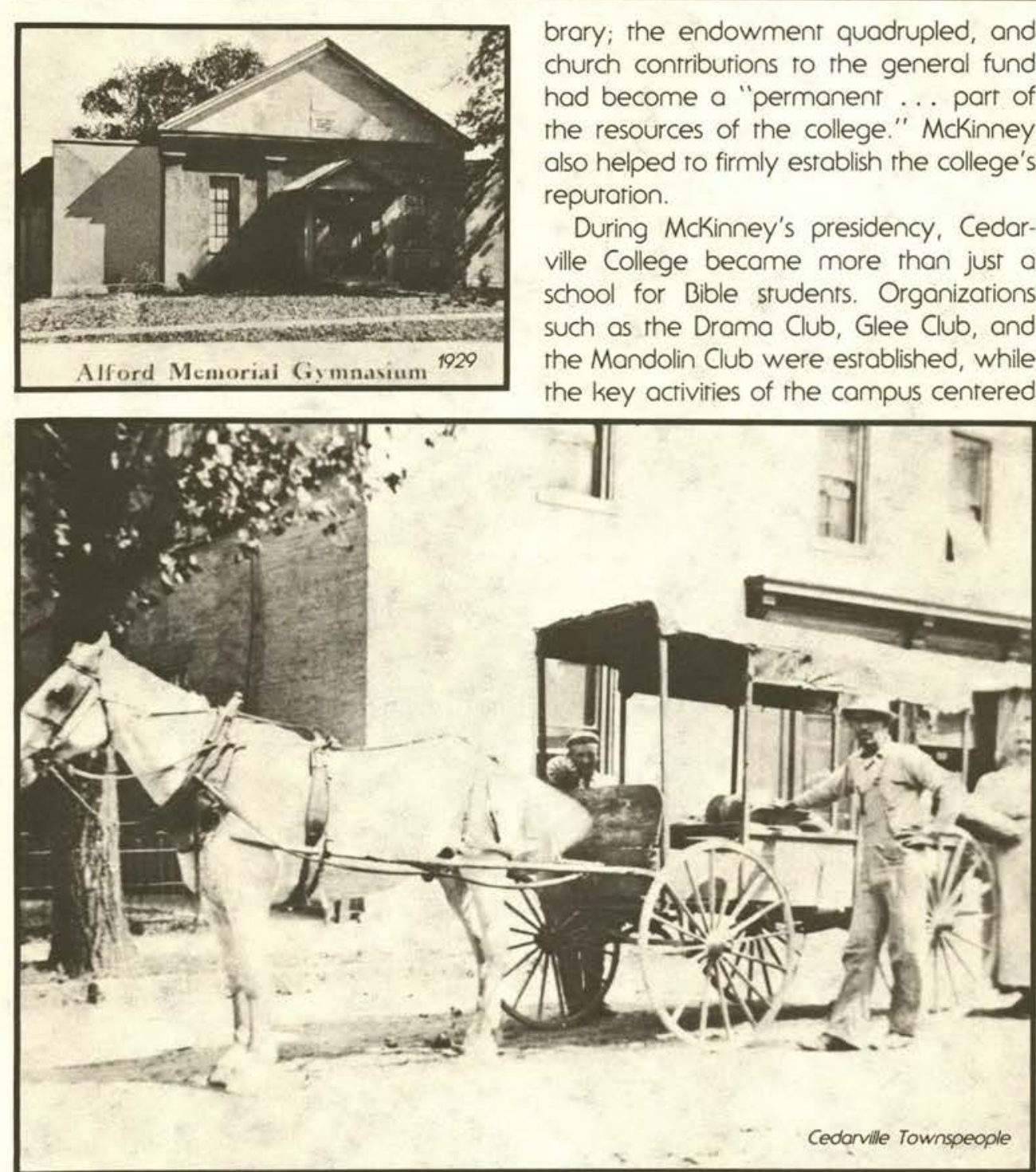

brary; the endowment quadrupled, and church contributions to the general fund had become a "permanent ... part of the resources of the college." Mckinney also helped to firmly establish the college's repuration.

During McKinney's presidency, Cedarville College became more than just a school for Bible students. Organizarions such as the Drama Club, Glee Club, and the Mandolin Club were established, while the key activities of the campus centered

in the literary organizarions known as the Philadelphian and Philosophic Societies

In addition to academic programs, the college also enjoyed a vigorous arhleric program. Sports for men included baseball and foorball, and baskerball soon followed. Adding this sport allowed women to compere os well.

As the college grew, both Mckinney and the trustees recognized the need for a resident administrator, so Mckinney resigned from the presidency.
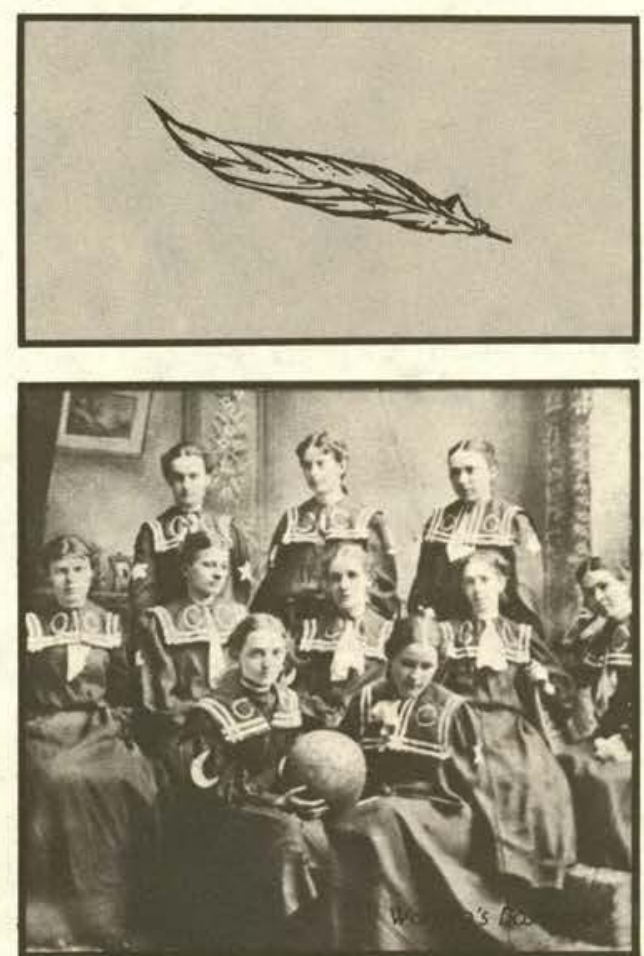
As the college prepared for its 40th anniversary, in 1934, Dr. David Mckinney was invited to be the commencement speaker. The 74-year old Mckinney accepted the invitation; however, his sudden dearh less than a month before graduation prevented him from fulfilling that commirment. Mckinney's place ar commence ment was taken by his son, Rev. D.A. Mckinney, who pastored the Oakland Presbyterian Church in Springfield, Ohio.

Dr. David Mckinney was a grear man of God who devored many years of service to Cedarville College and the cause of Christ. His influential leadership provided the college with a strong foothold for its furure success.

by Kristin Webber
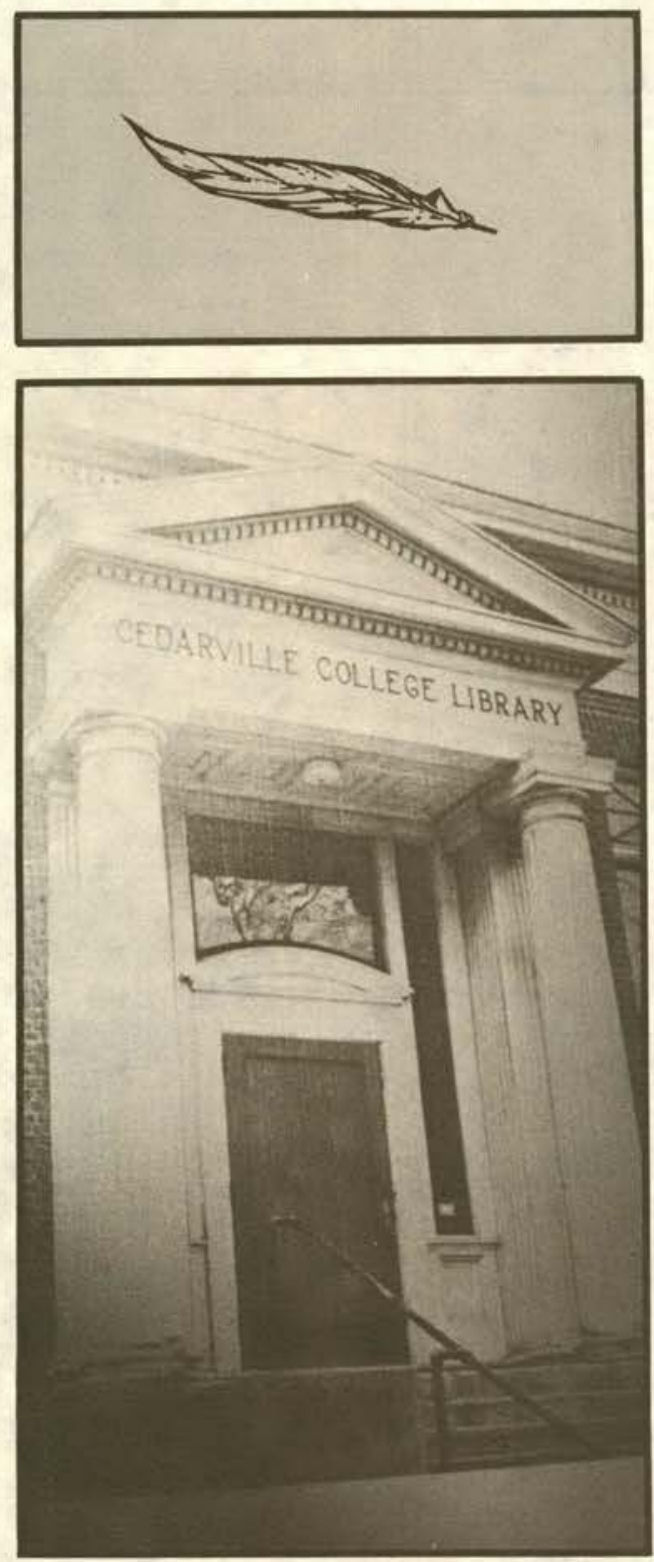
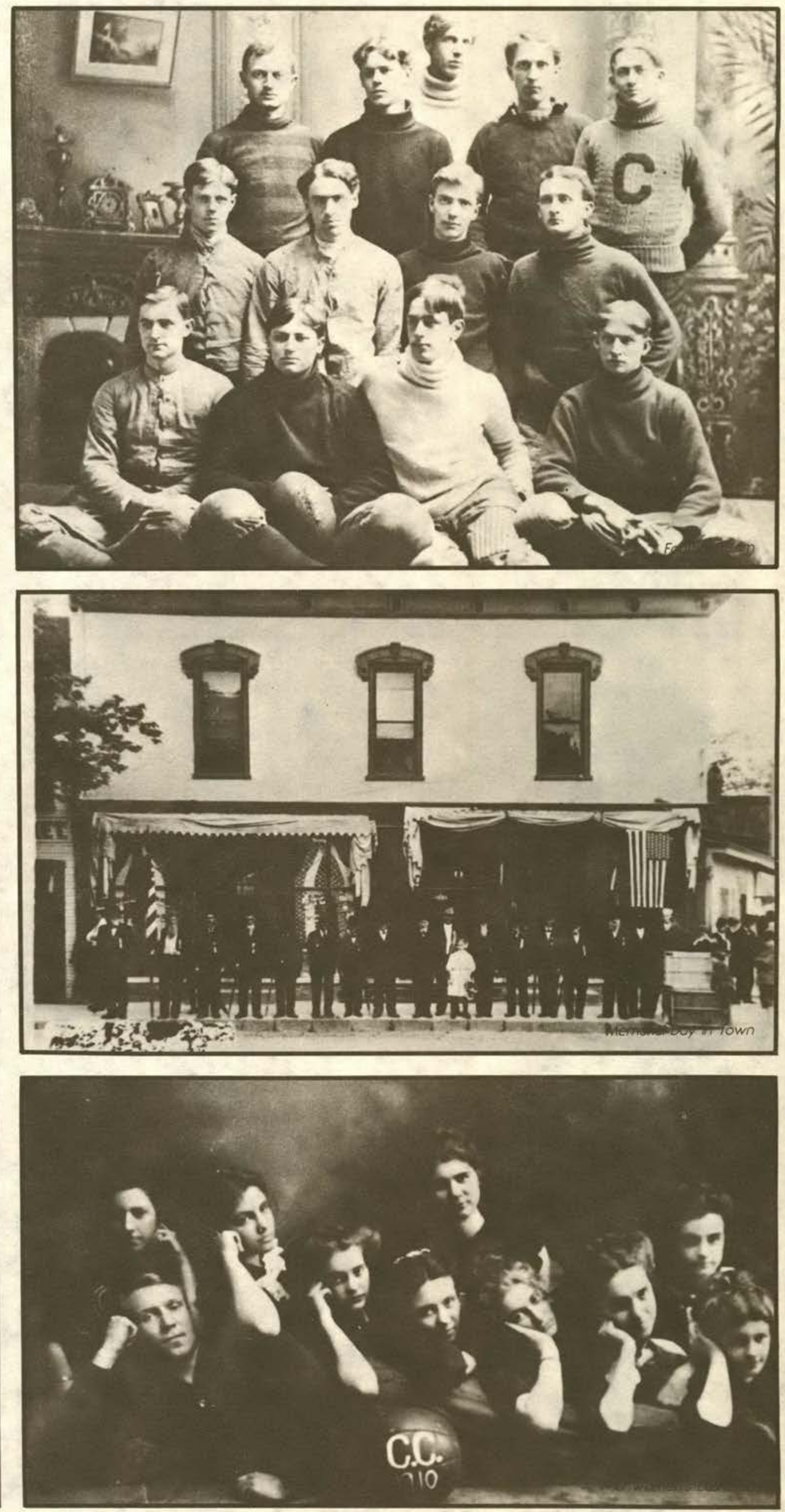


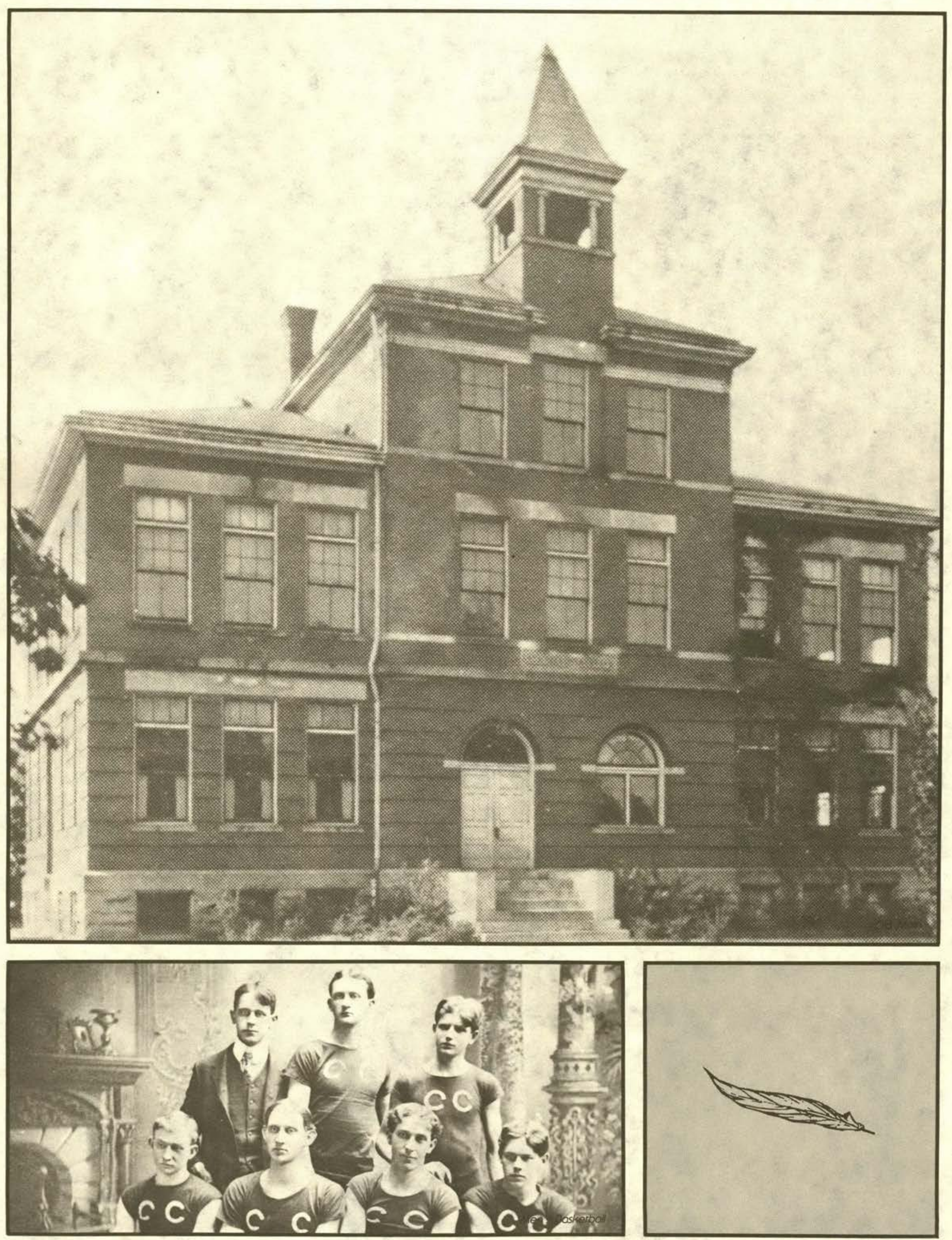

History 21 


\section{THE MCCHESNEY DAYS}

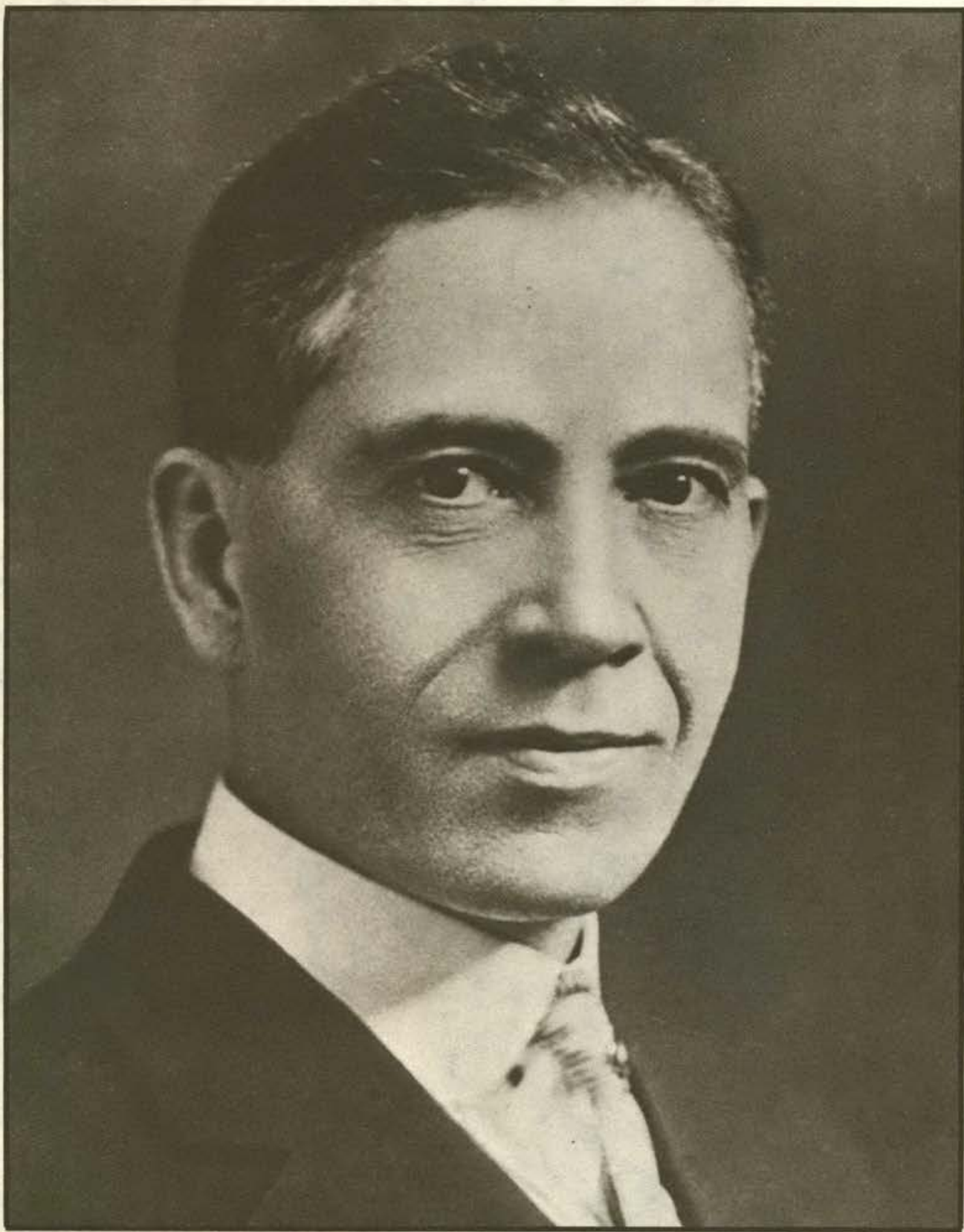

\section{Wilbert McChesney, 2nd President (1915-1940)}

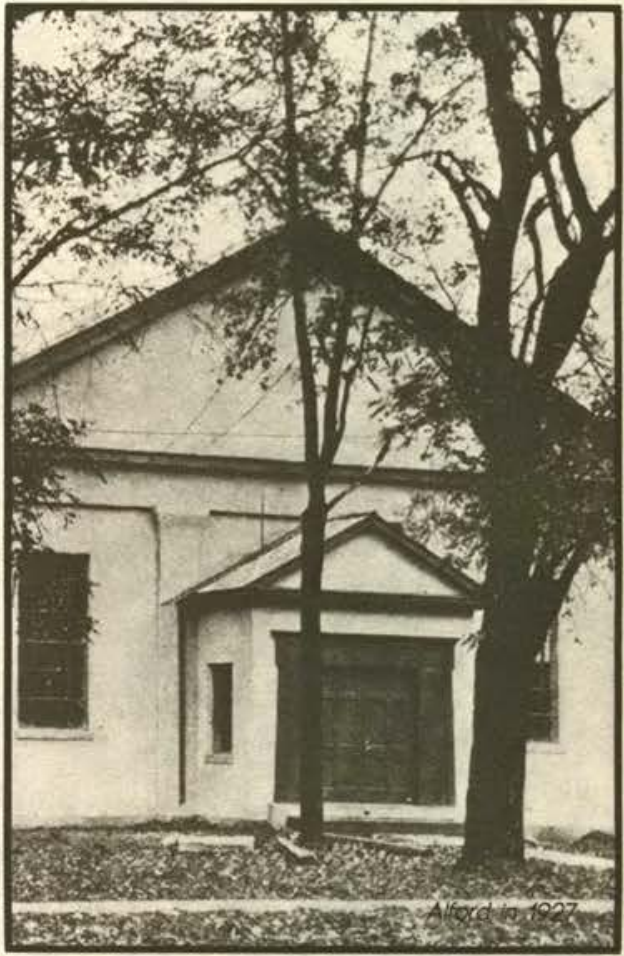

When David Mckinney resigned in 1915 Reverend Wilbert Renwick McChesney was unanimously elected the second president of Cedarville College. Teaching for 21 years, McChesney had been the first faculty member hired by the college in 1884 . He was an ordained minister and skilled pulpit orator but had spent his entire career in the classroom reaching subjects such as Greek and systemaric theology. Devoting his life to the college, he also served as Vice President in 1909 and Dean in 1913.

One of the most serious problems McChesney faced when beginning his presidency was the college's lack of resources, parricularly financial. In his inaugural address, he promised a major fundraising drive. However, as the Unired Stares became more and more alarmed abour
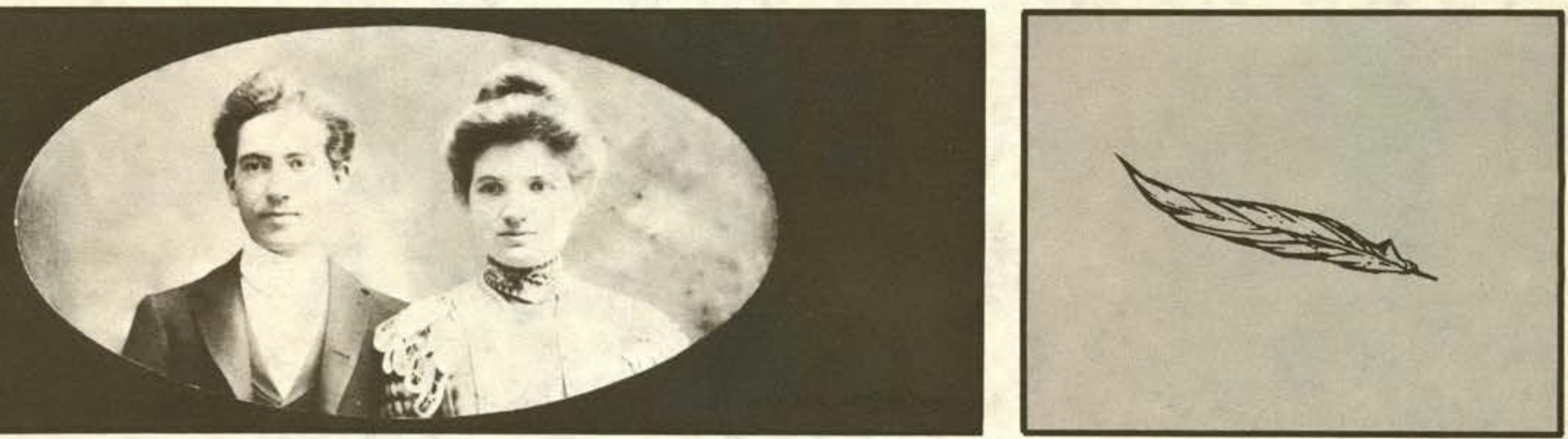


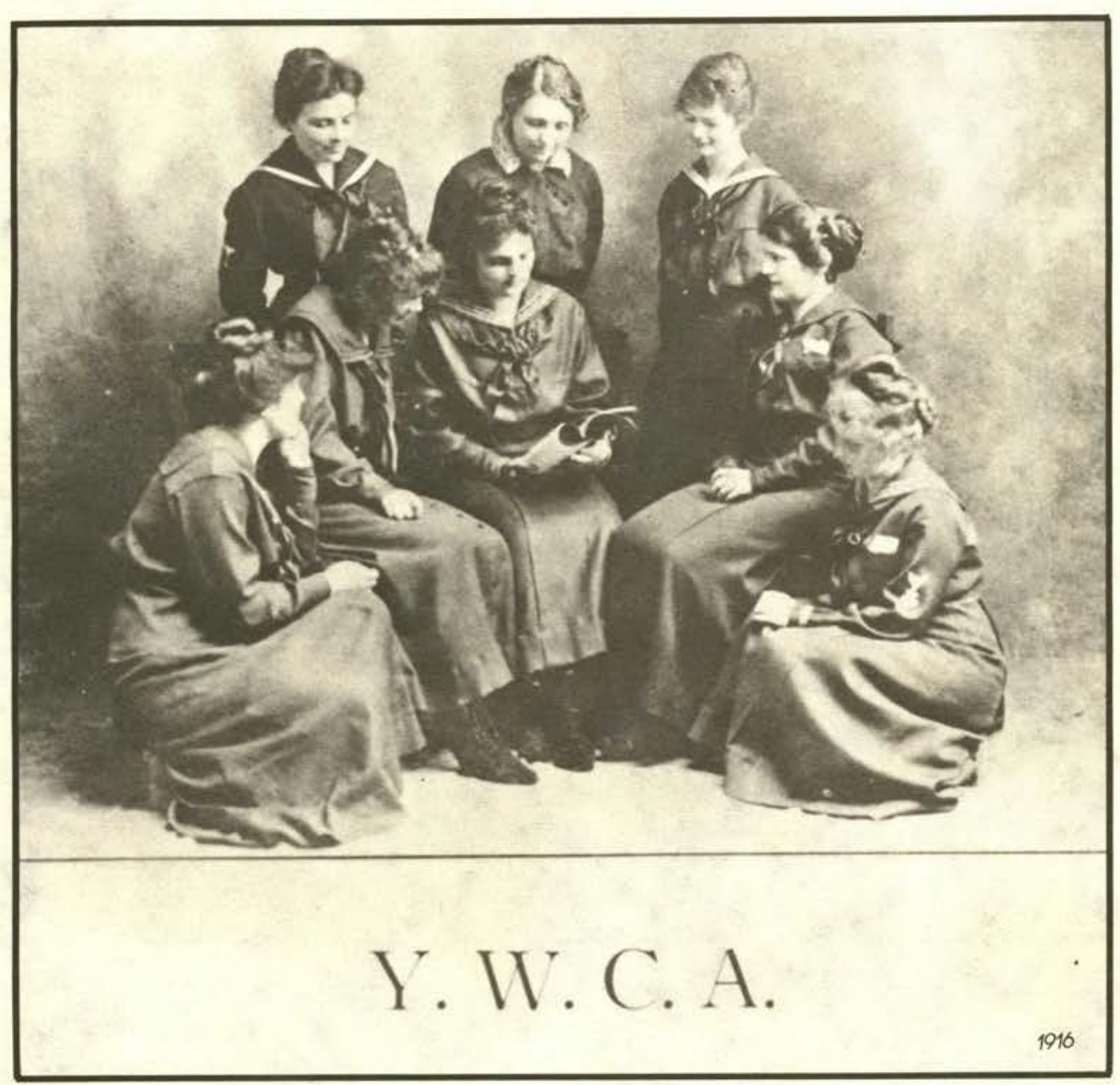

entering World War I, McChesney decided to delay his fund-raising efforts.

After the war, the college's enrollment began to climb; by 1920, 116 students strained the campus' limited facilities, by 1921, 135 students were enrolled. That year, McChesney asked the Board of Trustees for an endowment and building program. Announced by the Cedarville Herald, he launched a successful $\$ 200,000$ campaign for the school. He appealed to the citizens of Cedarville and Greene County, threatening that the college would have to close down or move if it did not raise needed funds.

During his early years as president, McChesney continually urged the trustees to meet the state of Ohio's minimum standards. Sarisfied by the school's efforts to meet these standards, inspectors for the Ohio Stare Associarion and Department of
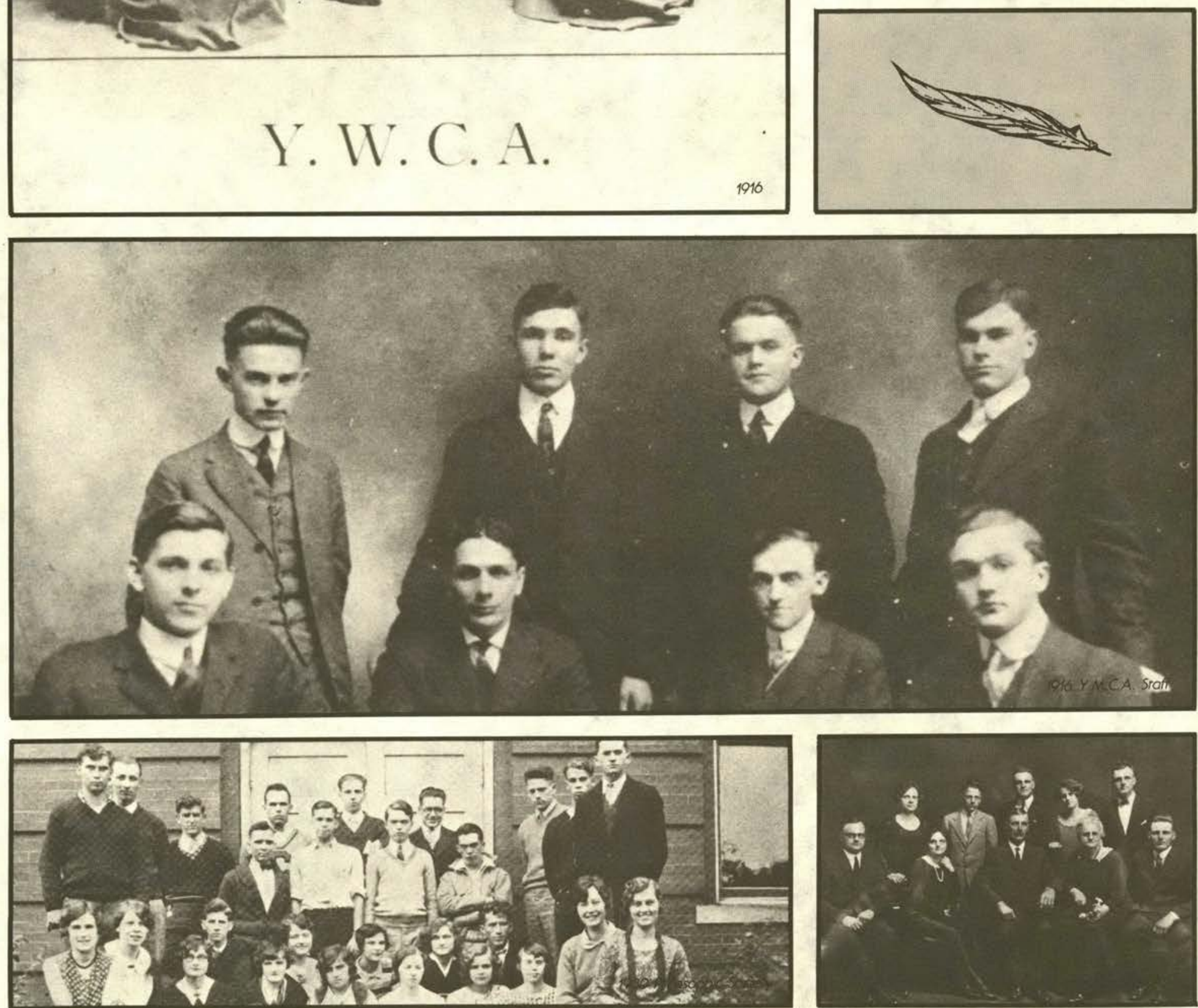
Public Instruction even called Cedarville "one of the best small colleges in the stare."

In spite of this academic success, the college's financial problems grew worse. In 1928, the General Synod of Reformed Presbyterian Churches gave up all control of Cedarville; thus, the college lost its supporting constituency. The Presbyrerian Church in the U.S.A. gradually filled the openings on the Board of Trustees, but the college was unable to win the support of that denomination in the students or donations.

In the midst of these financial problems,
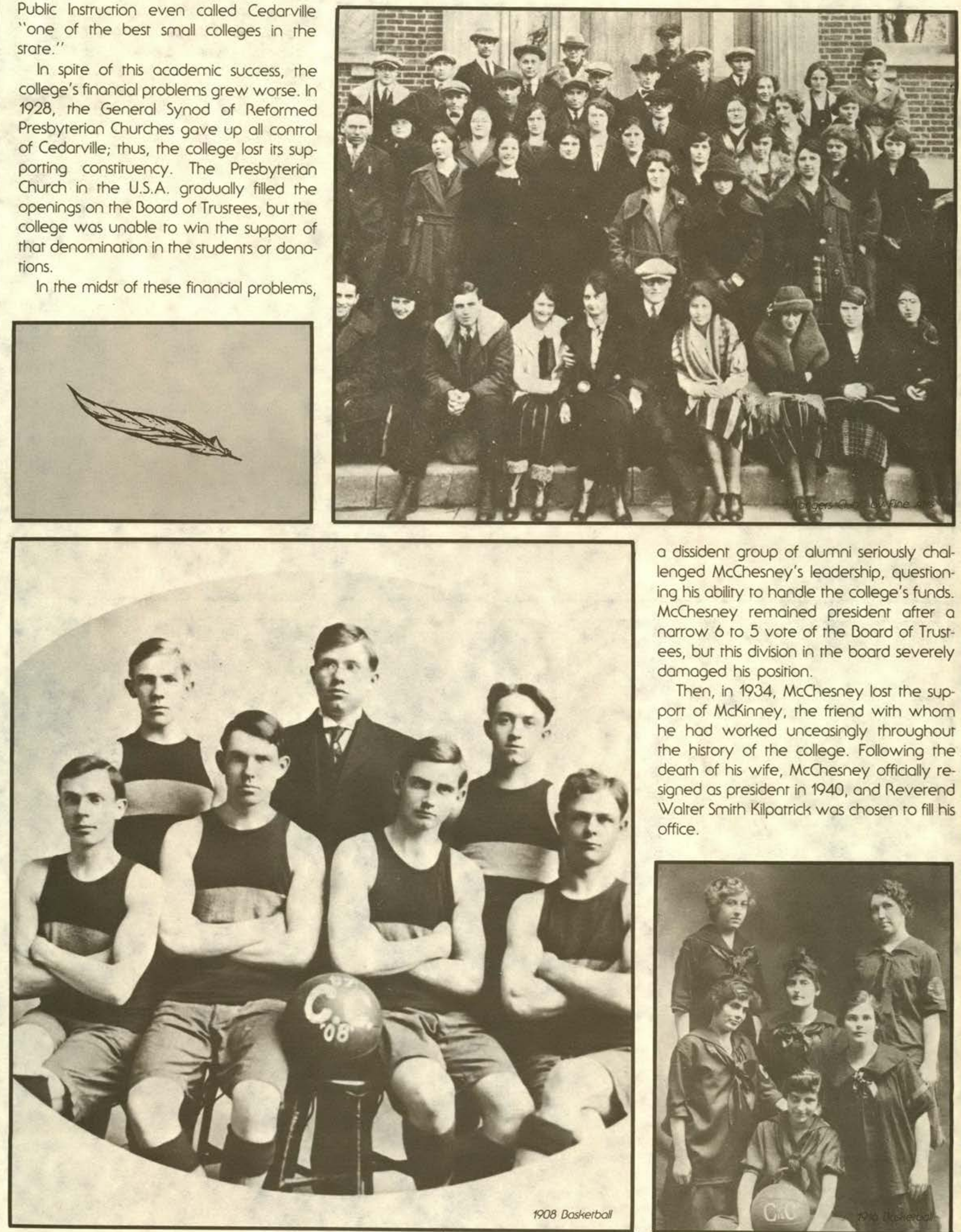

a dissident group of alumni seriously challenged McChesney's leadership, questioning his abiliry to handle the college's funds. McChesney remained president after a narrow 6 to 5 vore of the Board of Trustees, but this division in the board severely damaged his position.

Then, in 1934, McChesney lost the support of Mckinney, the friend with whom he had worked unceasingly throughour the history of the college. Following the death of his wife, McChesney officially resigned as president in 1940, and Reverend Walter Smith Kilparrick was chosen to fill his office.

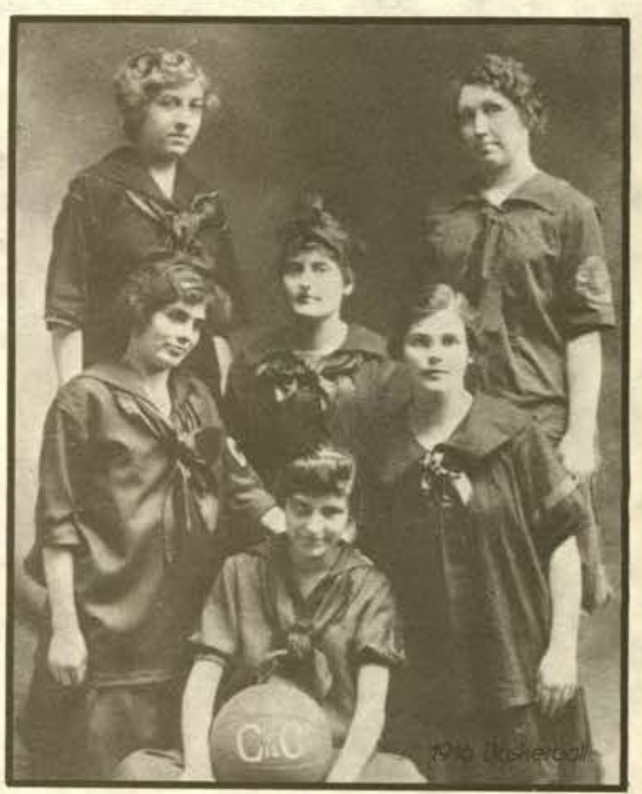




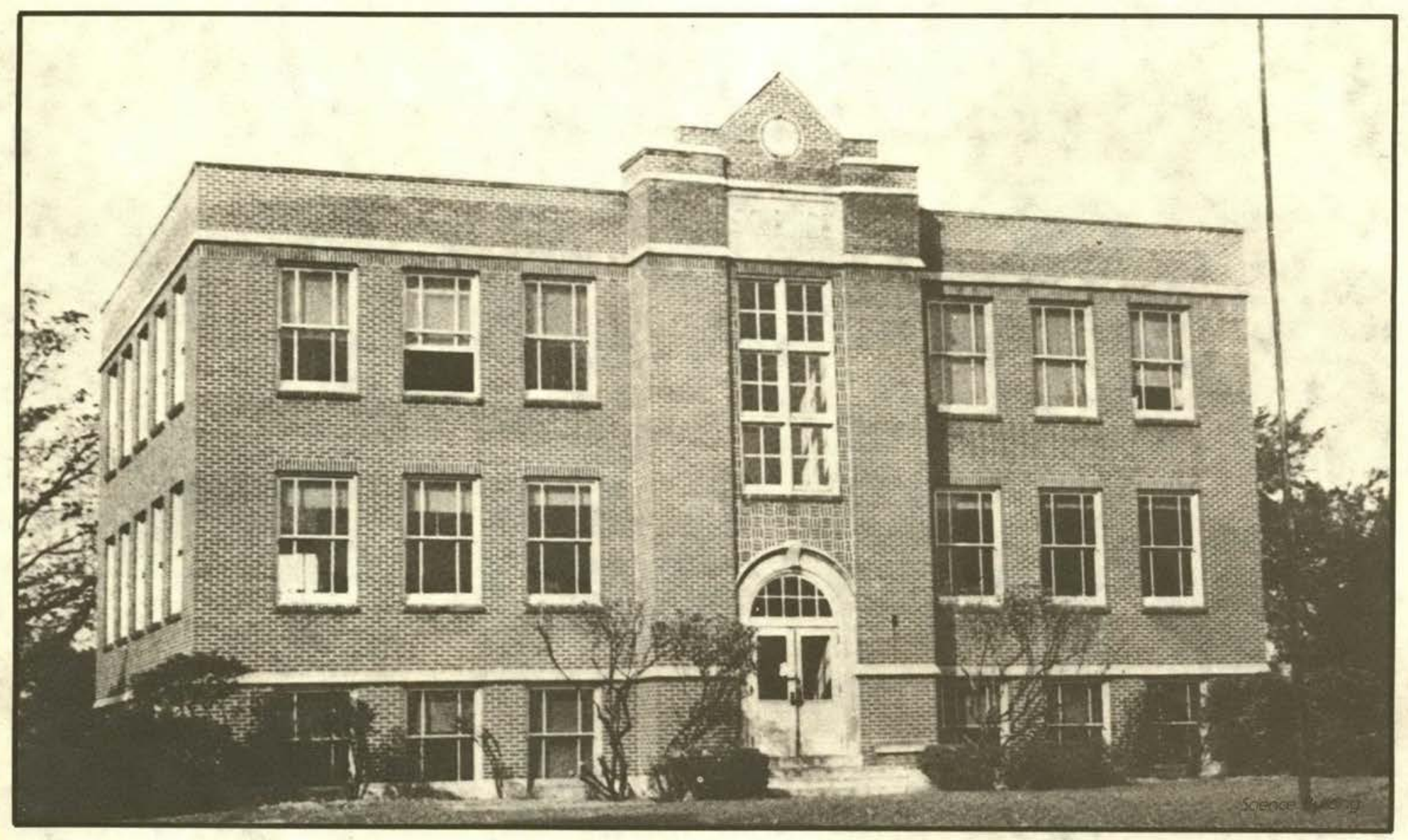

Despire his problems with the Board of Trustees, McChesney was well-respected by alumni and by the community. For instance, from 1932 until his dearh, he was elected by a wide margin to the Ohio Legislarure every term bur one. As president, he grearly enjoyed traveling to churches to preach the Gospel and raise support for the college he believed in. An edirorial in the Cedarville Herald called McChesney "a first cirizen of his community and of the religious community at large." The piece went on to express, "He lived each day what he preached and what he raught as well as what he advocared for the uplift of his fellow cirizens." Lisa Fawcert

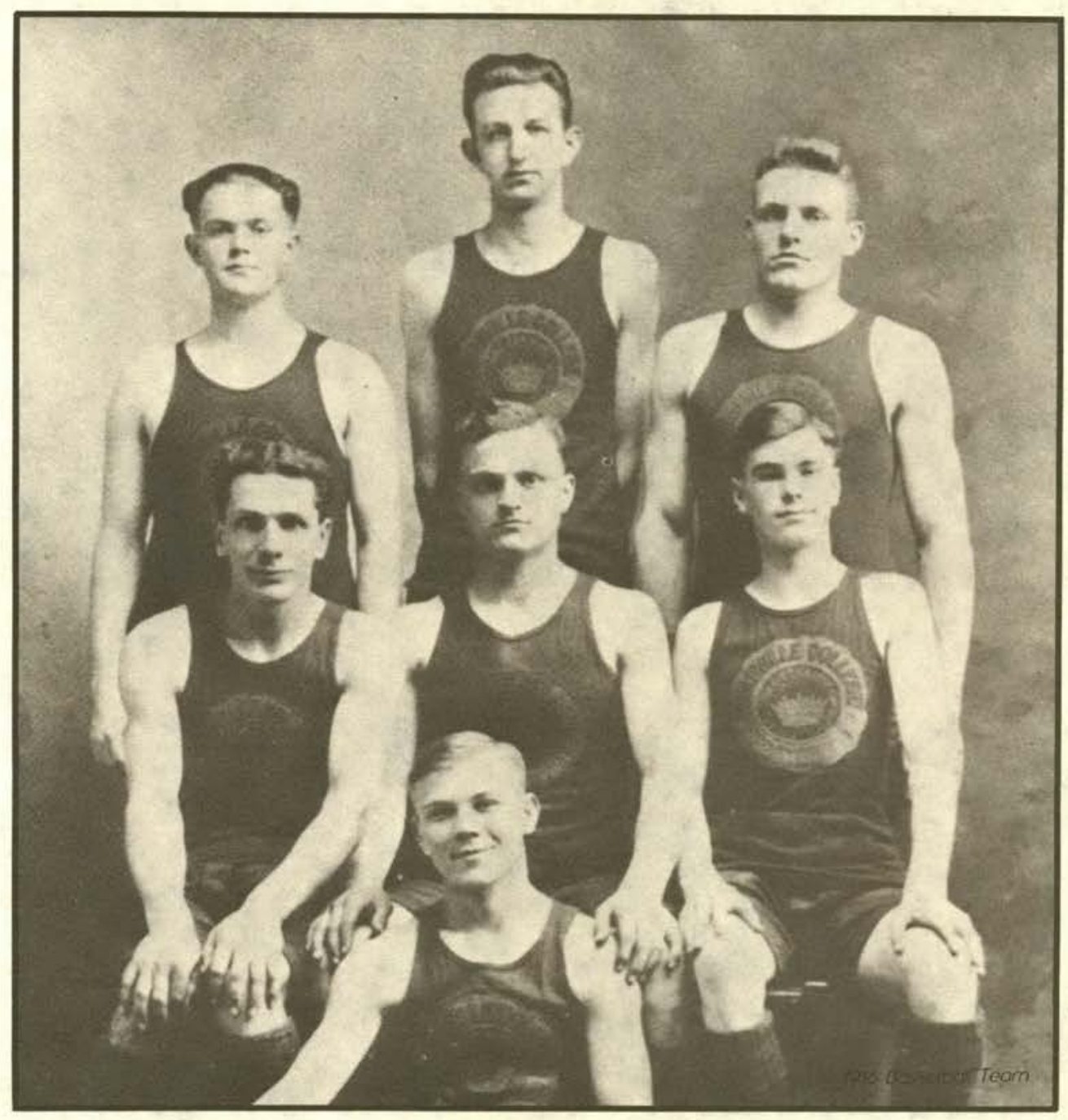




\section{A MIRACLE BEGINS}

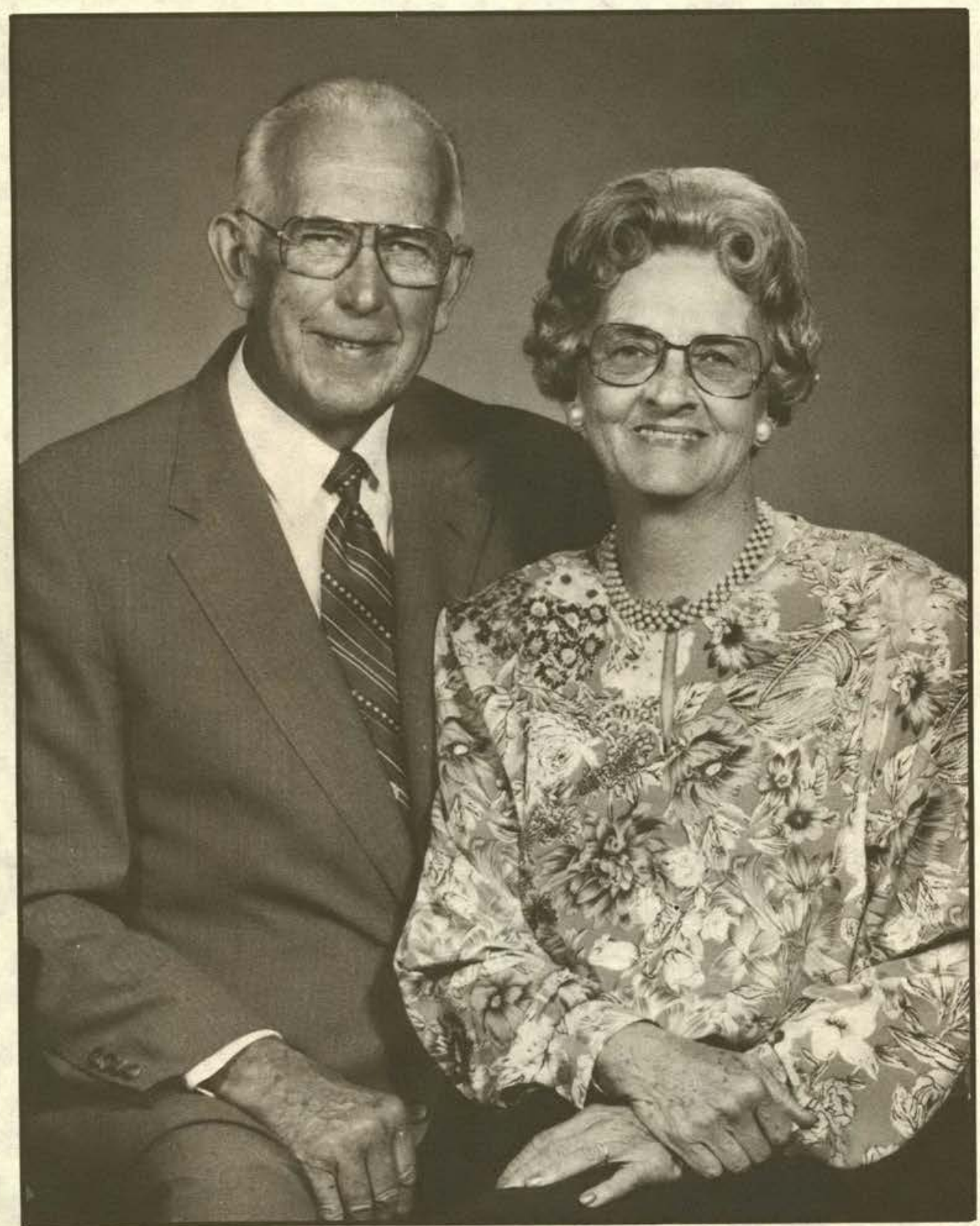

James T. Jeremiah's leadership of $\mathrm{Ce}$ darville College can be characterized in two words: faithful stewardship. Under his guidance the school has realized the porential that Jeremiah saw in 1953 when he first investigared the possibilities of merging the resources of Cleaveland's Baptist Bible Institute with the physical plant of a then ailing Cedarville College.

Jeremiah's efforts to persuade the B.B.I. irustees toward merger led to meetings with the Presbyterian trustees. Both groups agreed that the mission of Cedarville College must focus on training young people for many professions, so that every segment of sociery might have a beacon to stand, as the school morro stated, "for the Word of God and the restimony of Jesus Christ." The merger complete, borh Dr. and Mrs. Jeremiah began to invest the ralents that God had given them in the school while Jeremiah continued as a full time pastor in Dayton, Ohio.

At the starr of Jeremiah's involvement with Cedarville, his raw materials consisted

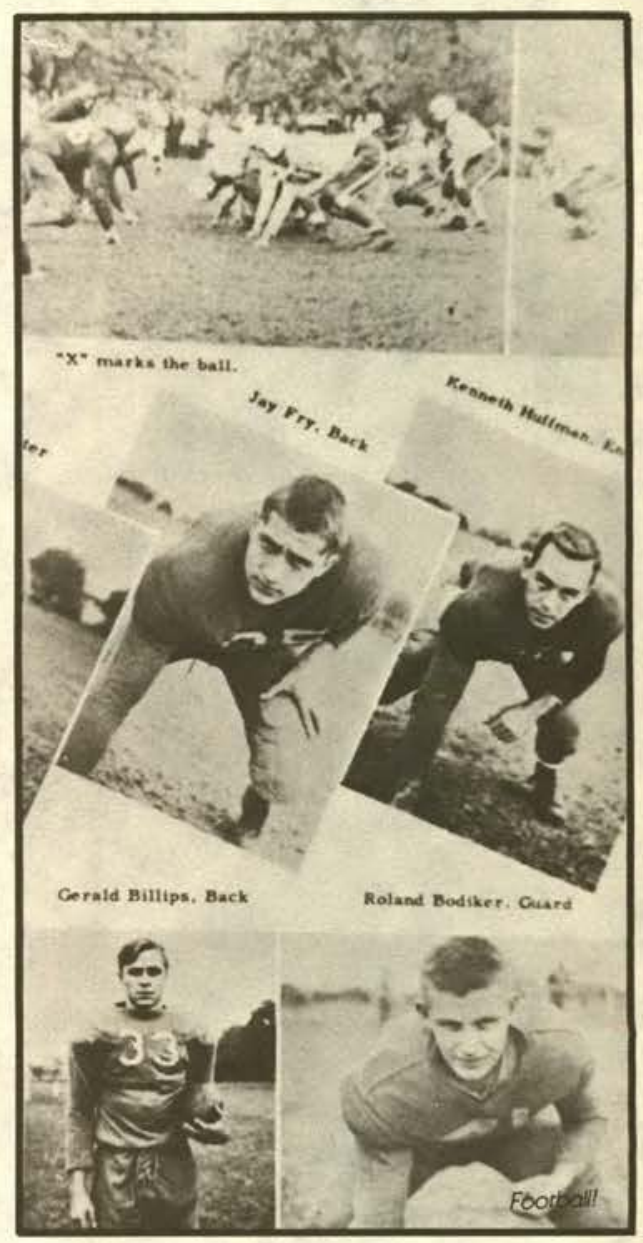



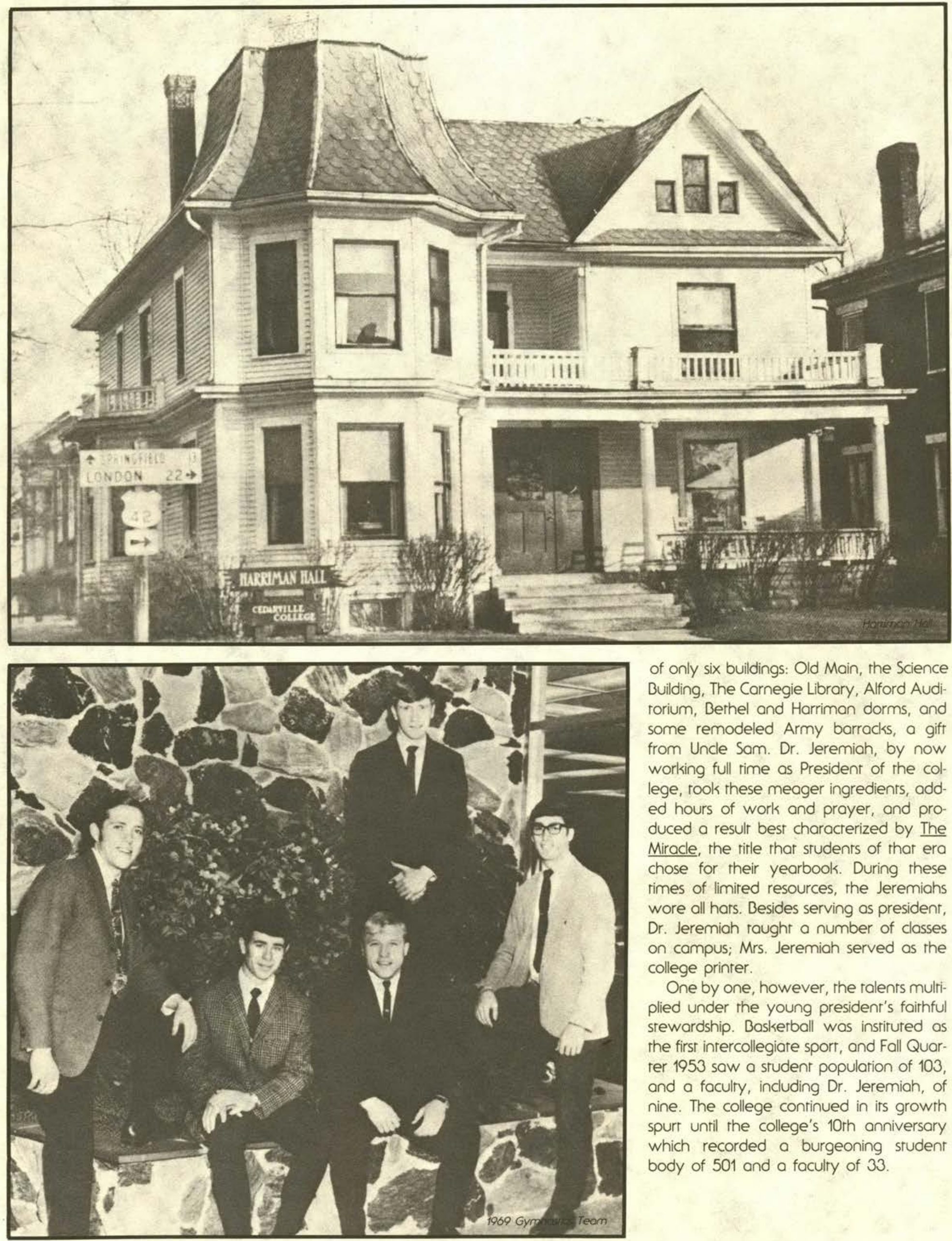

of only six buildings: Old Main, the Science Building, The Carnegie Library, Alford Audirorium, Berhel and Harriman dorms, and some remodeled Army barracks, a gift from Uncle Sam. Dr. Jeremiah, by now working full time as President of the college, rook these meager ingredients, added hours of work and prayer, and produced a result best characterized by The Miracle, the title that students of that era chose for their yearbook. During these rimes of limired resources, the Jeremiahs wore all hats. Besides serving as president, Dr. Jeremiah raught a number of classes on campus; Mrs. Jeremiah served as the college printer.

One by one, however, the talents multiplied under the young president's faithful stewardship. Baskerball was institured as the first intercollegiate sport, and Fall Quarter 1953 saw a student population of 103 , and a faculty, including $\mathrm{Dr}$. Jeremiah, of nine. The college continued in its growth spurt until the college's 10th anniversary which recorded a burgeoning student body of 501 and a faculty of 33 . 


\section{Cedarville College Library and Launge}
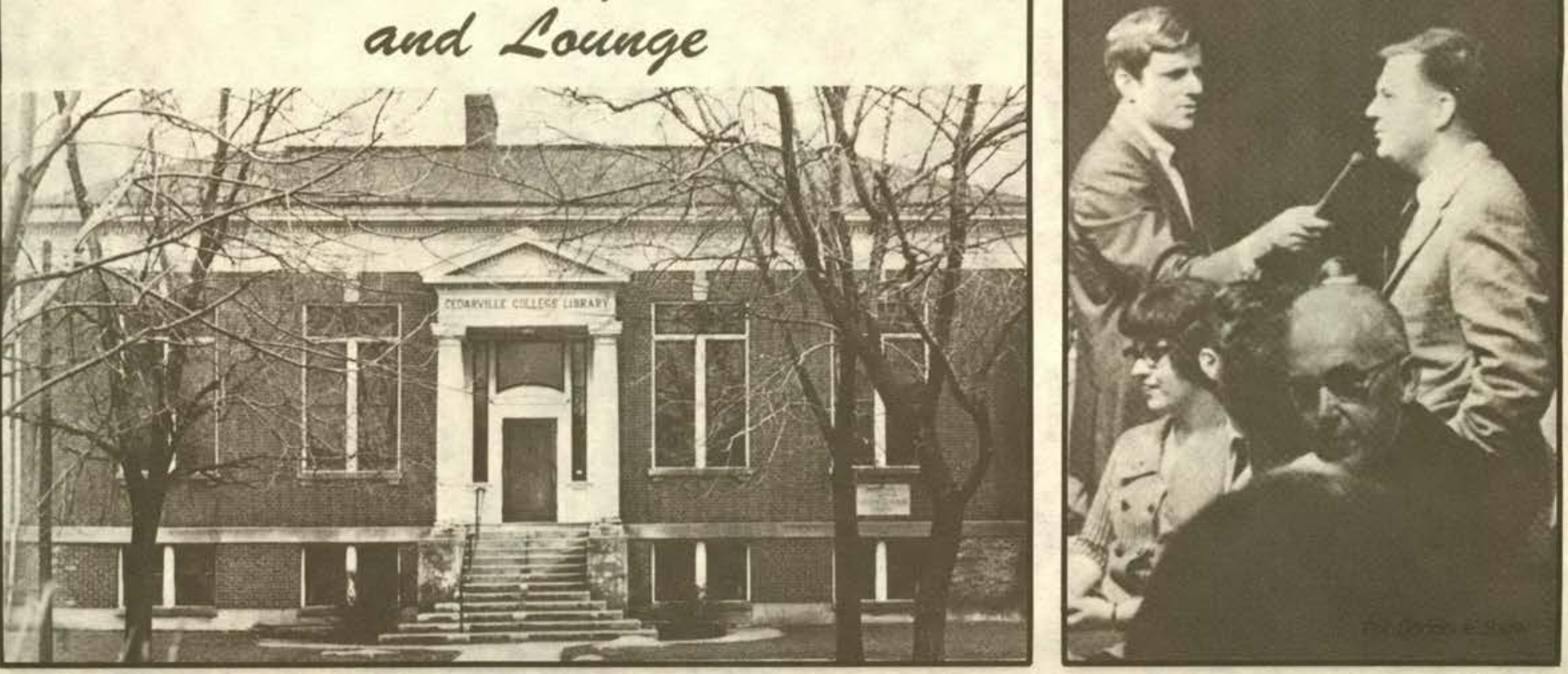

With such added growth came added responsibility as well, bur $\mathrm{Dr}$. Jeremiah proved himself yer again as a fairhful steward: he charted the expansion of the cam. pus and pur togerher an administrarive sraff capable of managing the growth needs. Dr. Clifford Johnson was hired as Academic Vice President and worked ro. ward accreditarion by cerrifying the reach. er educarion program with the stare of Ohio. Consecurive student services Deans Richard Mcintosh and Don Rickard institured financial aid services, the counseling department and the placement office. Lee Turner was hired to staff the develop. ment office, and he began the Torch mag.
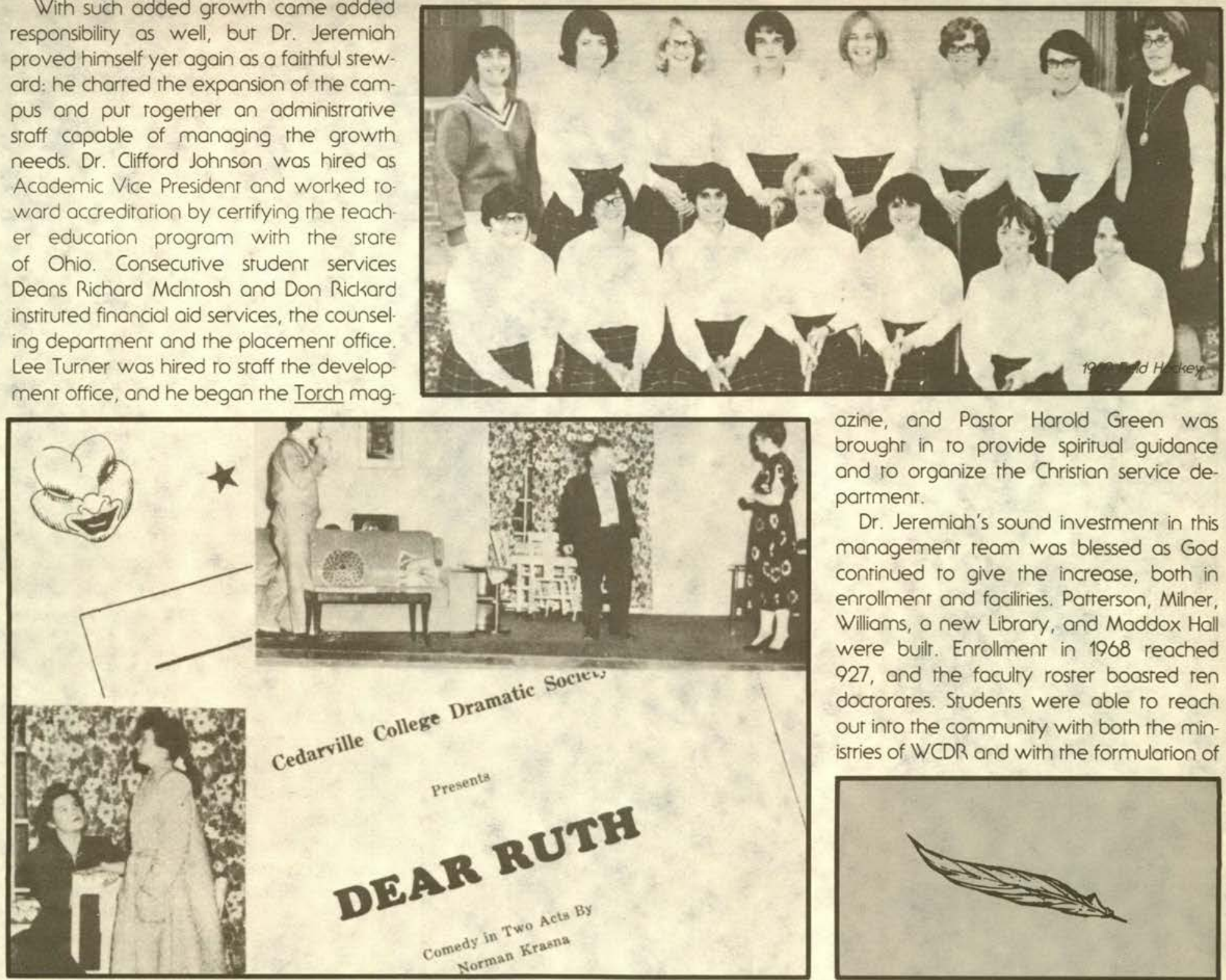

azine, and Postor Harold Green was brought in to provide spiritual guidance and to organize the Christian service department.

Dr. Jeremiah's sound investment in this management ream was blessed as God continued to give the increase, both in enrollment and facilities. Patterson, Milner, Williams, a new Library, and Maddox Hall were built. Enrollment in 1968 reached 927 , and the faculty roster boasted ten doctorates. Students were able to reach out into the community with both the ministries of WCDR and with the formulation of

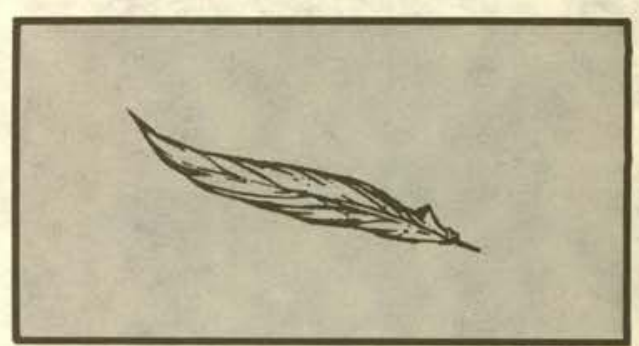



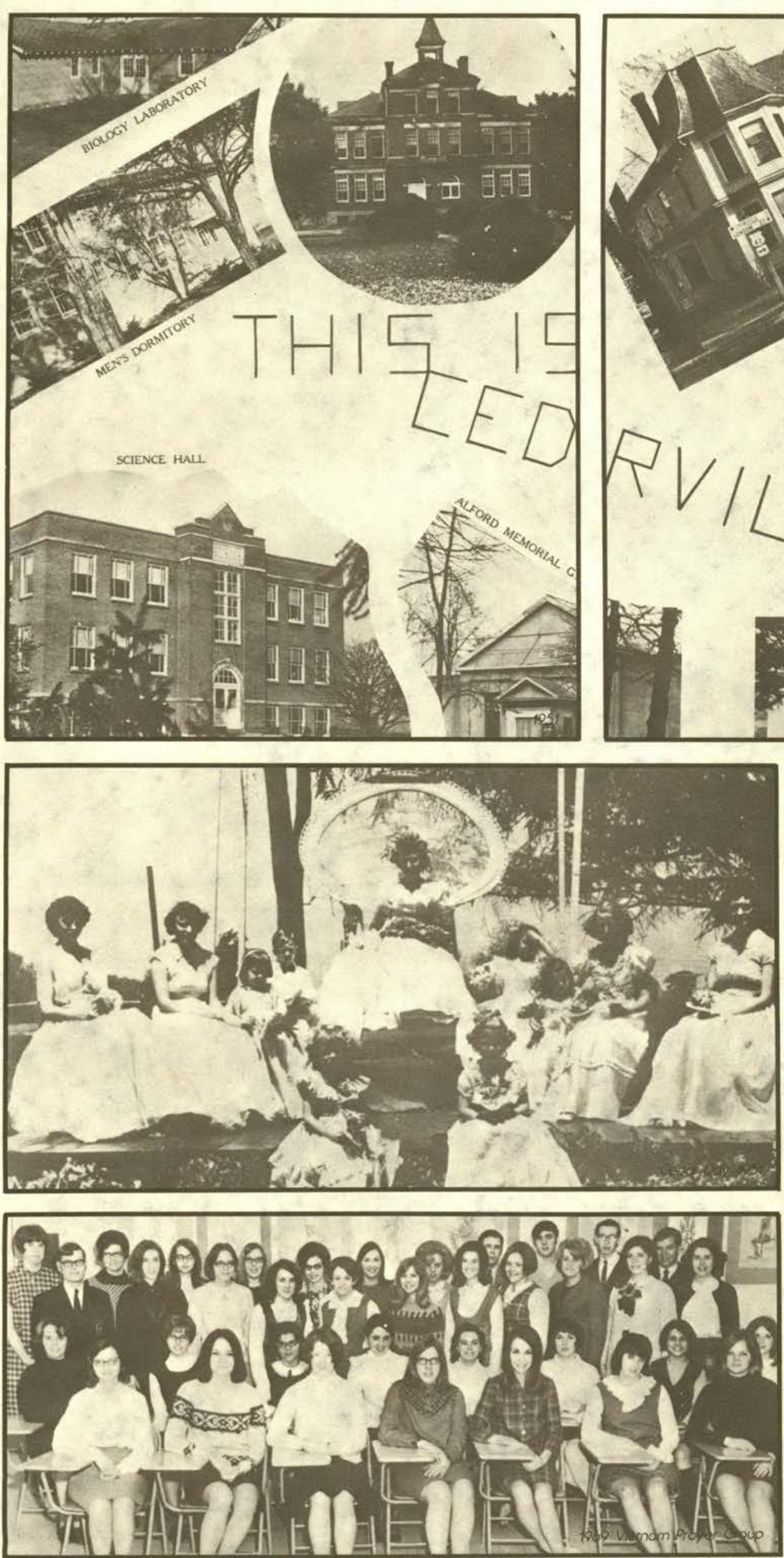

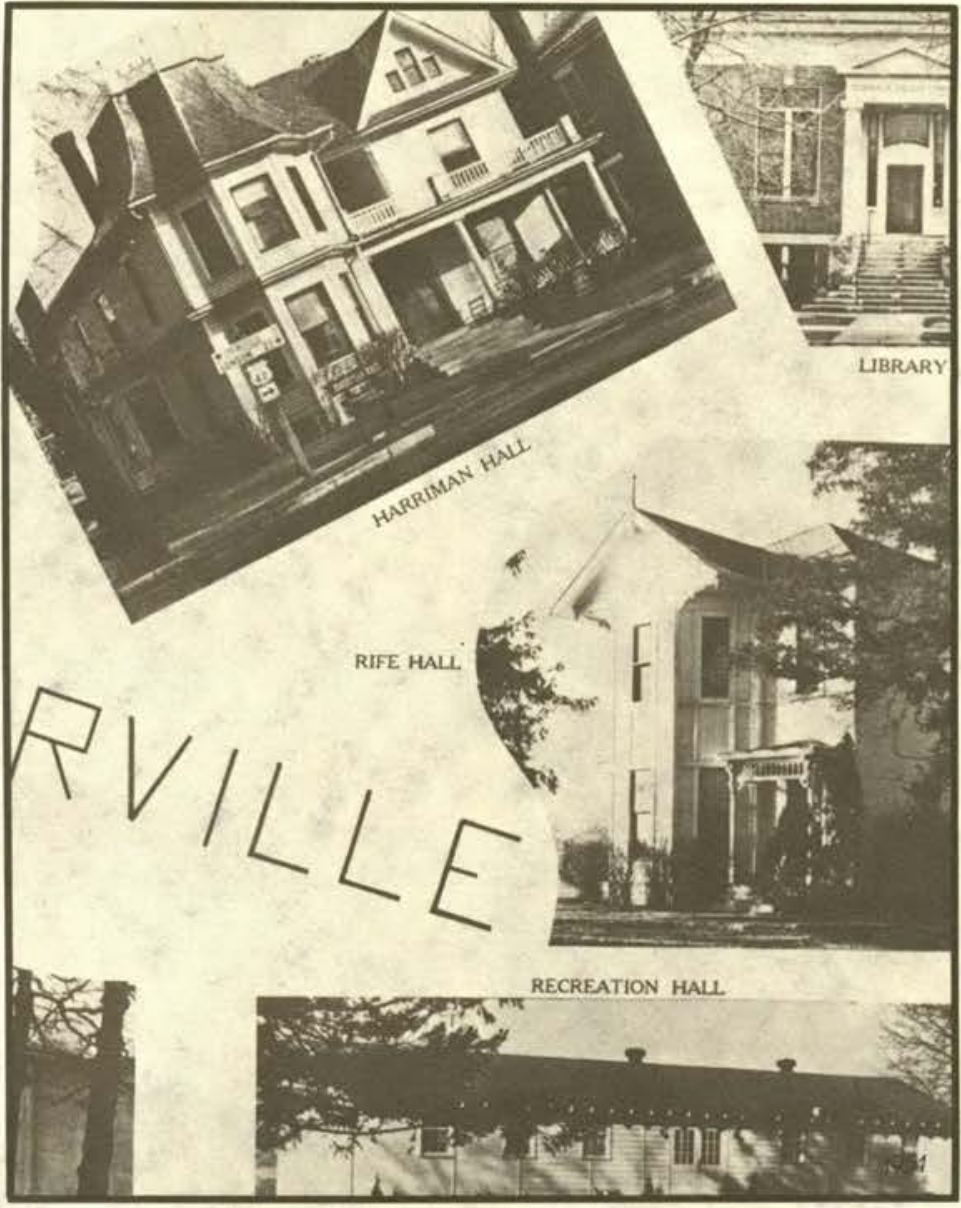

a Red Cross disaster ream on campus.

As the years passed and the college grew, Dr. Jeremiah once again showed his faithfulness as a steward of God's gifts. The college had expanded by 1976 to an enrollment of 1221, a popularion which required two chapel services. As Jeremiah surveyed the needs of the college, he realized that new facilities would have to be built, requiring a leadership full of energy and vitality. Unsure of his ability to fulfill such an awesome responsibility, Jeremiah wrore in 1978 ,

"My question is, how can I best help in the program of progress that lies before us? Frankly I am nor sure I can carry the heavy responsibiliry that is required of me

With the good of the college in mind Jeremiah decided that the best stewardship would be his resignation and the passing of respoinsibility to a younger man with more of the physical resources needed to perform the awesome job of president.

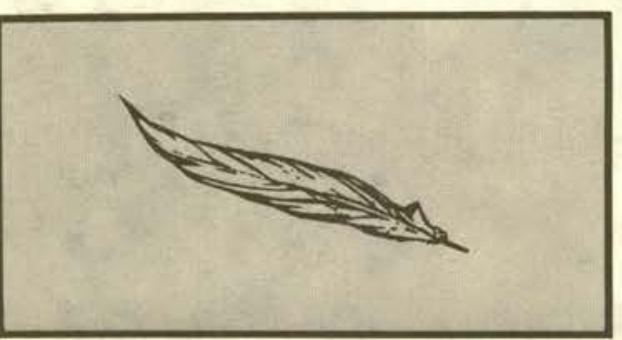




\section{A GROWING COLLEGE FAMILY}

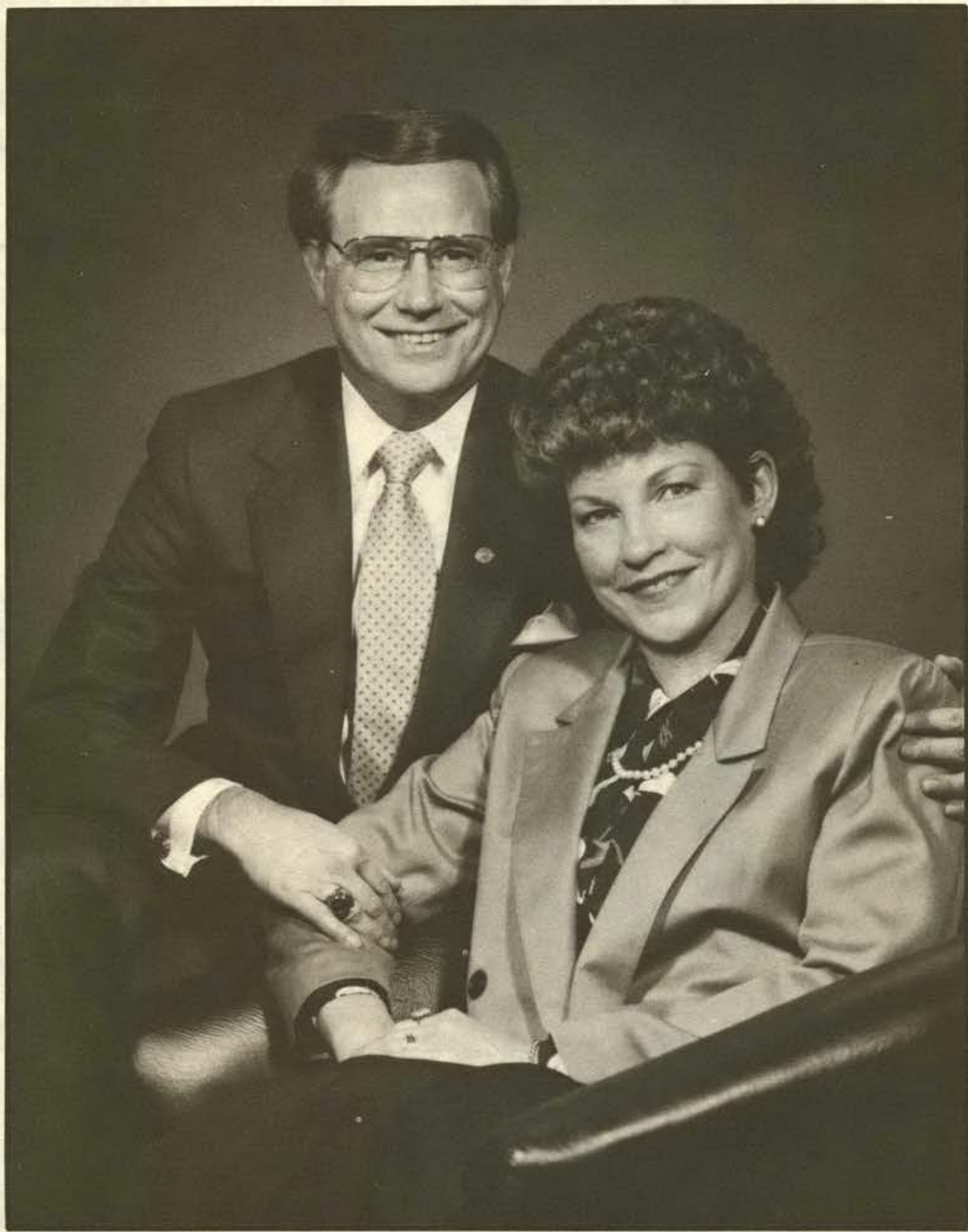

A scene very familiar to the students of Cedarville College often illuminares the Monday morning chapel service. Over 2 thousand voices obediently respond to the greetings of the man beaming broadly, exhorting earnestly from behind the massive podium which bears the college emblem. Over 2 thousand faces look toward him expectantly, the man who serves as a for more animared emblem of the current trends of Cedarville College. The faculty, staff, and student body join voices to offer up before him the chorus "Christ is All I Need," the self-adopred theme of their director behind the pulpit, President Paul H. Dixon. President Dixon has publidy pronounced chapel services, "the heartbeat of every Christian College," and the Monday morning chapel scenario appropriarely captures the pulsing essence of the Dix-

\section{Paul H. Dixon, 8th President (1978- )}
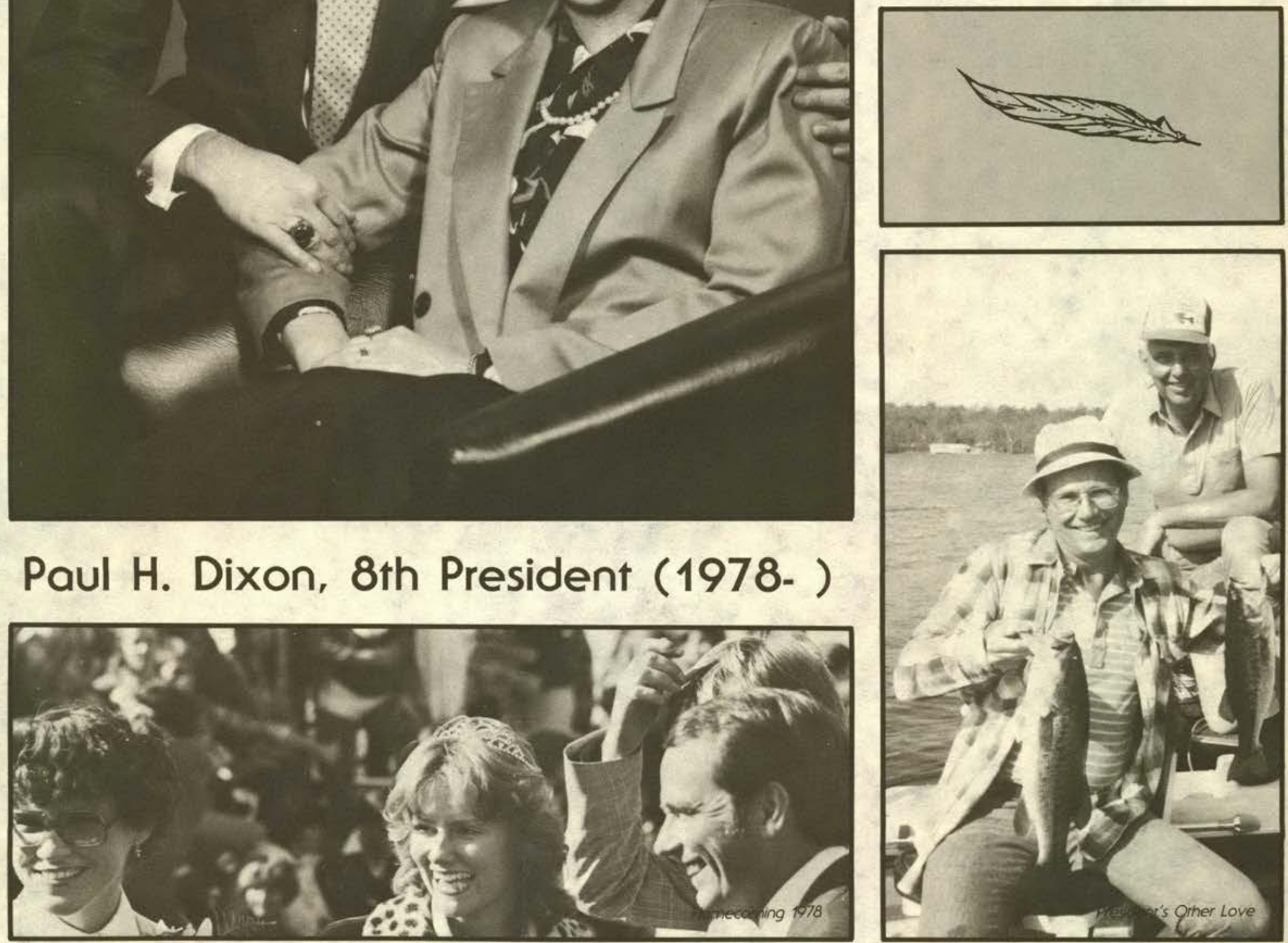


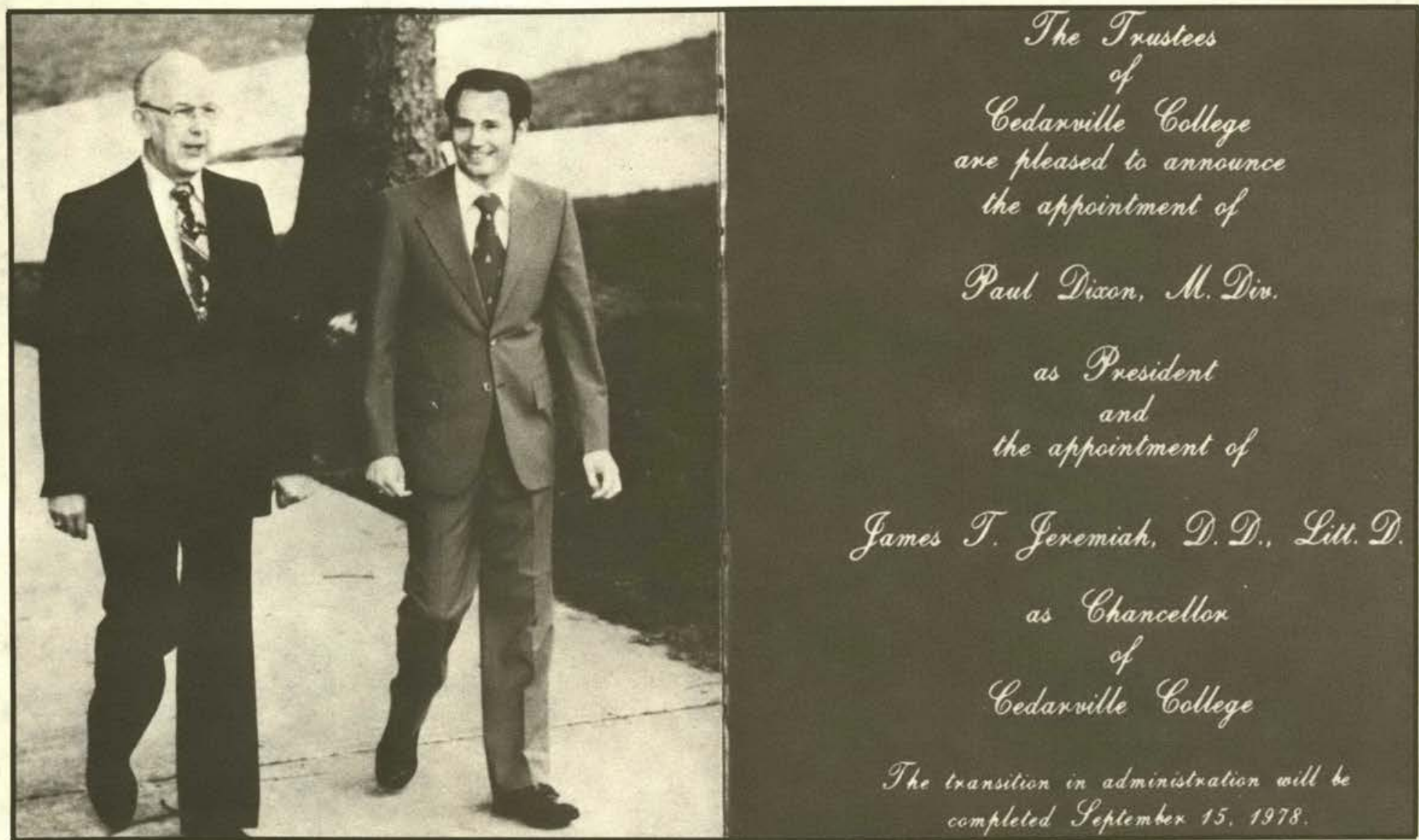

on era.

The Dixon era ar Cedarville College can be accurately termed pulsing by its very narure. Steady, fast paced and constant, the life's blood of Dixon's work ar Cedarville College flows nor only with his personal ambirion and visions for the College's potential but also from maintaining mo-
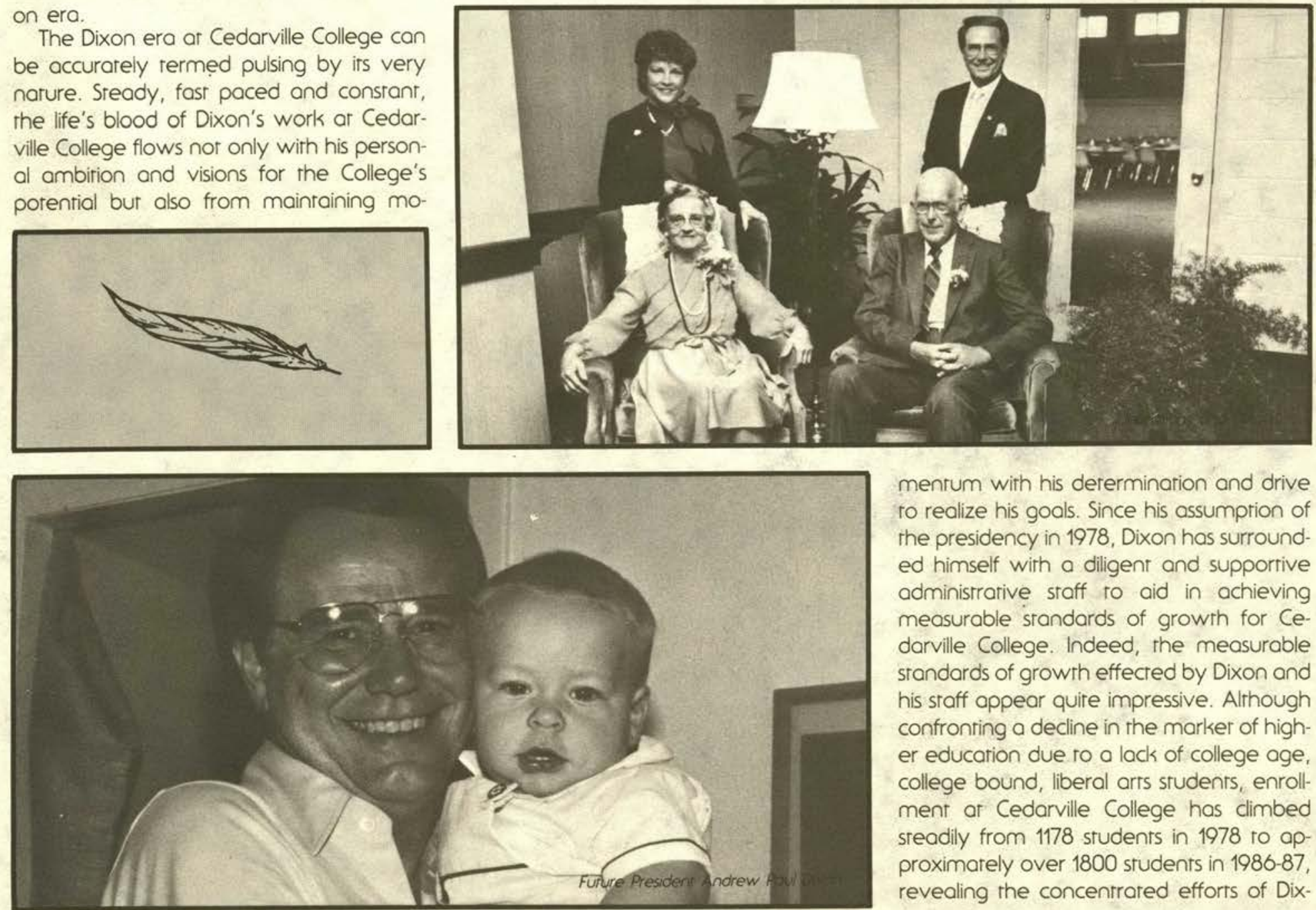

menrum with his derermination and drive to realize his goals. Since his assumprion of the presidency in 1978, Dixon has surrounded himself with a diligent and supportive administrative staff to aid in achieving measurable standards of growth for $\mathrm{Ce}$ darville College. Indeed, the measurable standards of growth effected by Dixon and his staff appear quite impressive. Although confronting a decline in the marker of higher education due to a lack of college age, college bound, liberal arrs students, enrollment at Cedarville College has climbed sreadily from 1178 students in 1978 to approximately over 1800 students in 1986-87, revealing the concentrated efforts of Dix- 

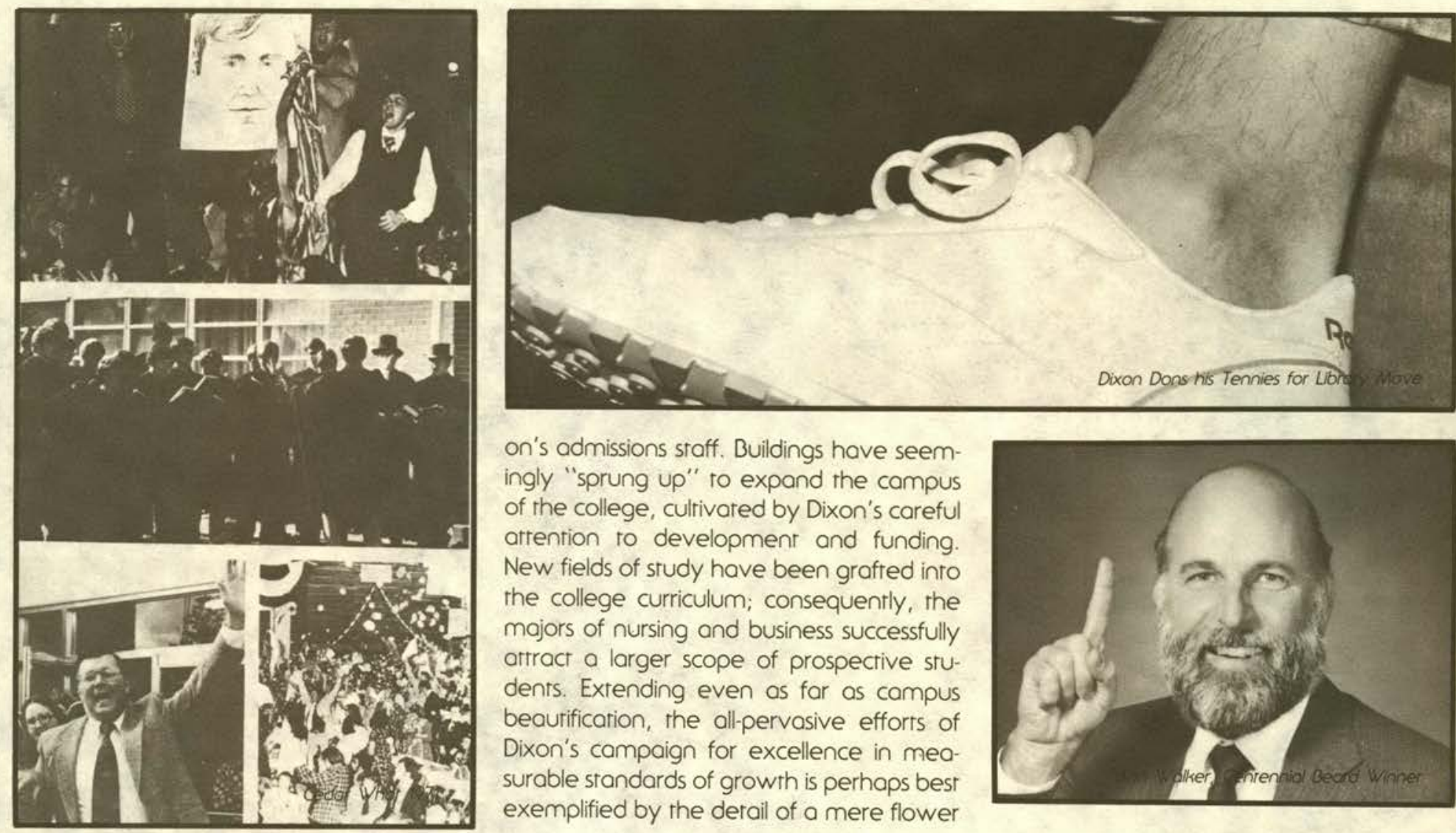

on's admissions staff. Buildings have seem. ingly "sprung up" to expand the campus of the college, cultivated by Dixon's careful attention to development and funding. New fields of study have been grafted into the college curriculum; consequently, the majors of nursing and business successfully attract a larger scope of prospective students. Extending even as far as campus beaurification, the all-pervasive efforts of Dixon's compaign for excellence in measurable standards of growth is perhaps best exemplified by the derail of a mere flower
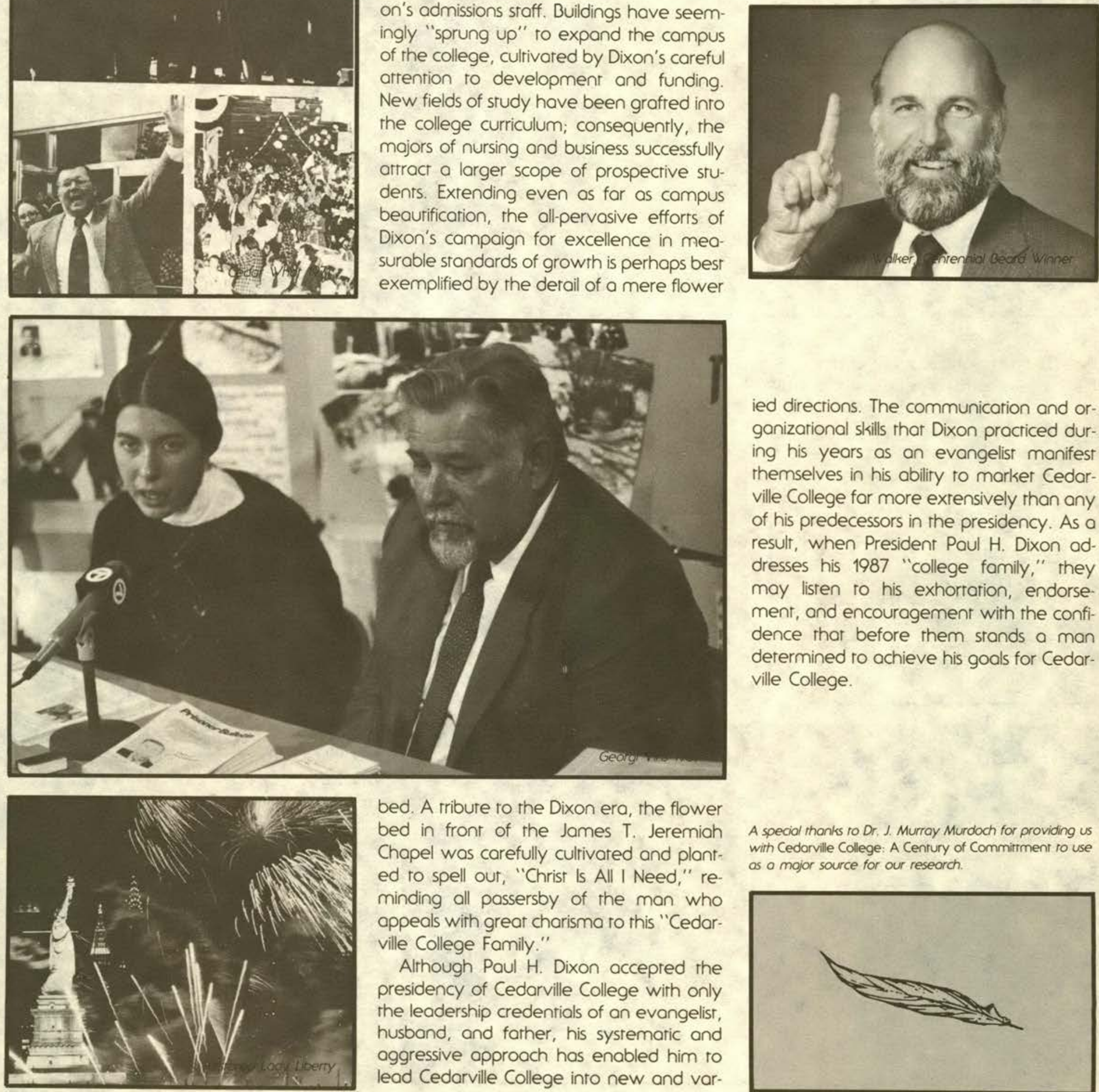

bed. A tribute to the Dixon era, the flower bed in front of the James T. Jeremiah Chapel was carefully culrivared and planted to spell our, "Christ is All I Need," reminding all passersby of the man who appeals with great charisma to this "Cedarville College Family.

Although Paul H. Dixon accepred the presidency of Cedarville College with only the leadership credentials of an evangelist, husband, and farher, his systemaric and aggressive approach has enabled him to lead Cedarville College into new and var-

ied directions. The communication and organizarional skills that Dixon practiced during his years as an evangelist manifest themselves in his ability to marker Cedarville College far more exrensively than any of his predecessors in the presidency. As a result, when President Poul $\mathrm{H}$. Dixon addresses his 1987 "college family," they may listen to his exhortation, endorsement, and encouragement with the confidence that before them stands a man determined to achieve his goals for Cedarville College.

A special thanks to Dr. J. Murray Murdoch for providing us with Cedarville College: A Cenrury of Commirment ro use as a mojor source for our research.

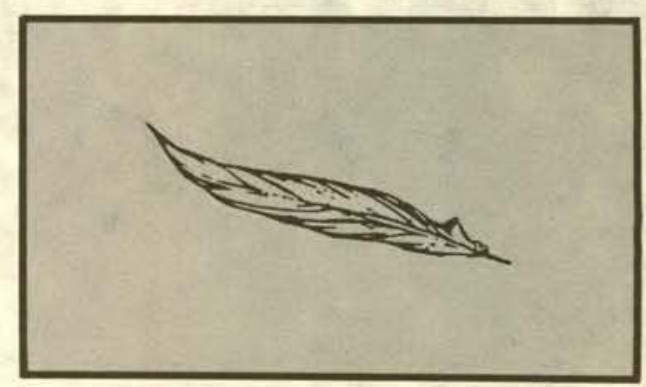




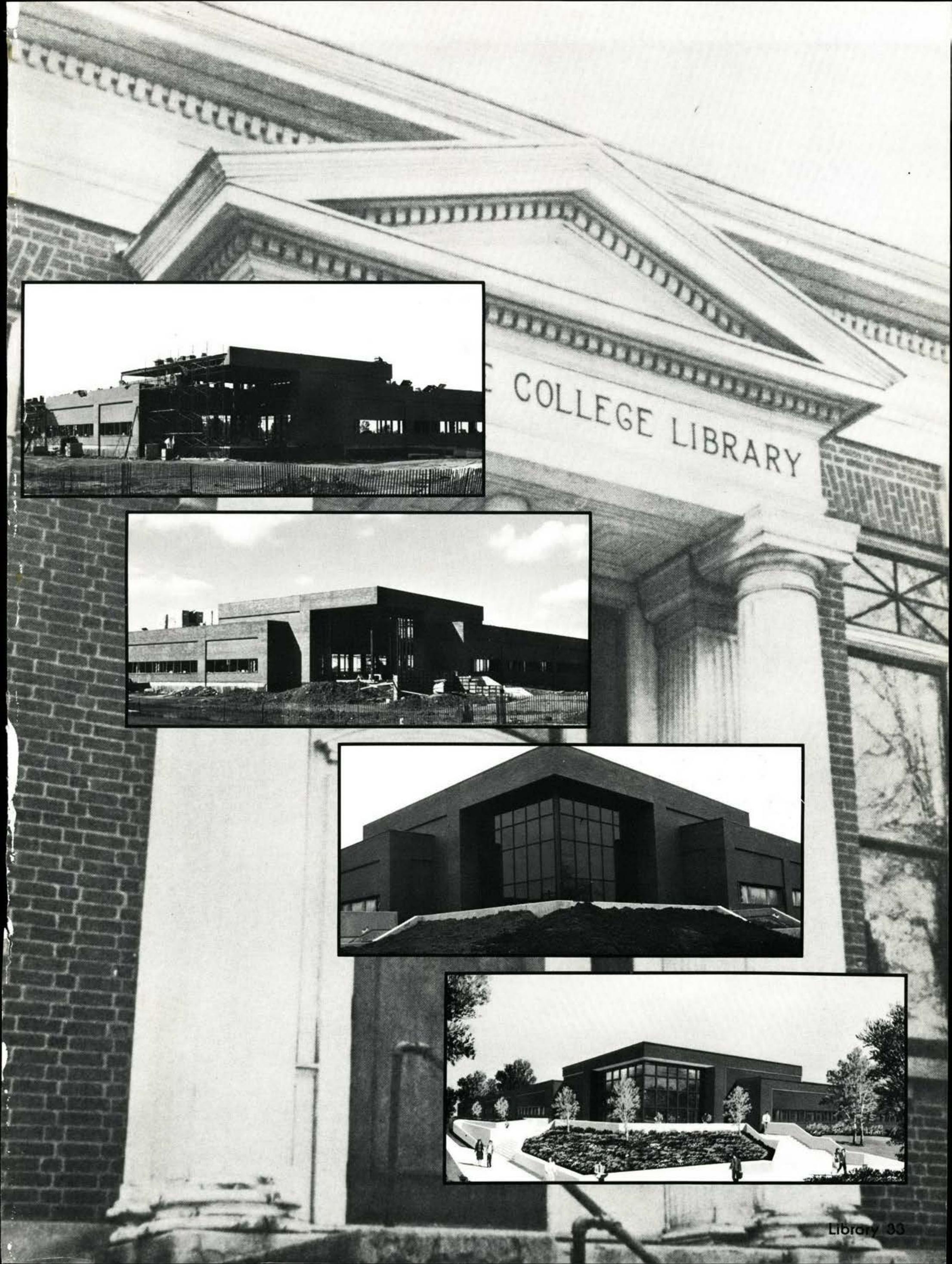



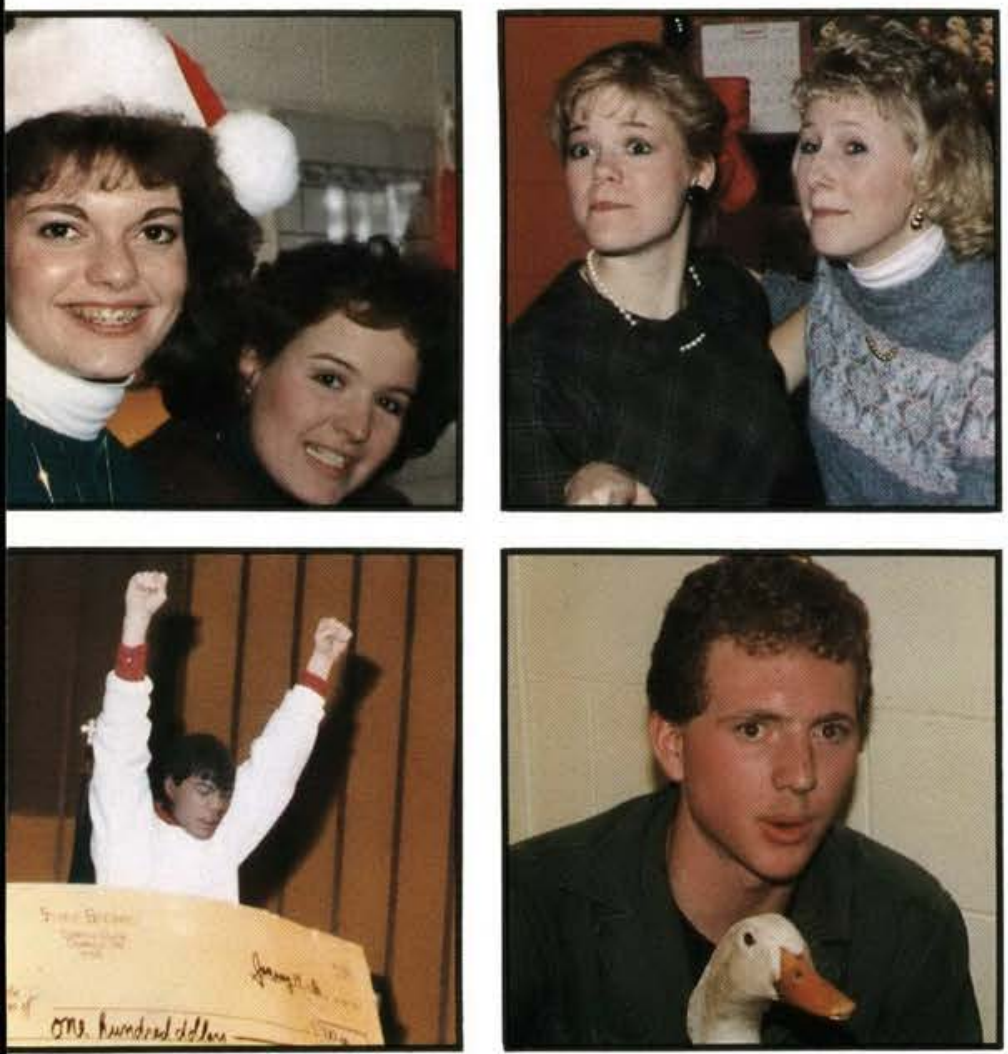

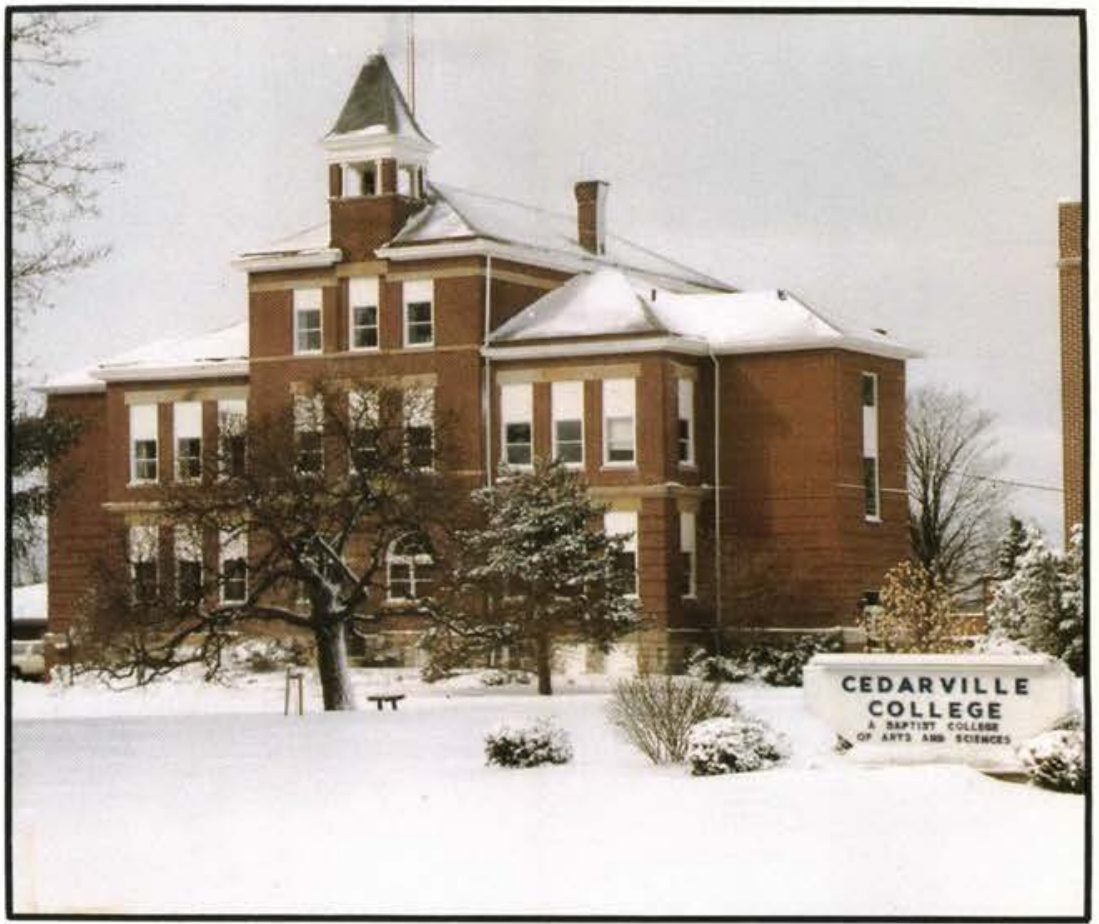

\section{Student Life}

"The more things change, the more they stay the same," even at Cedarville. College students in general may have changed a great deal since 1887, but in the lirtle burg of Cedarville College, life continues along its traditional four-year cycle much as it did a century ago.

The freshmen are srill trying to hide their insecurities; and the upper-classmen, despite regulations to the contrary, continue their generally mild-mannered initiotions. Classes are cut or used for sleeping; days are spent sunbarhing and socializing; and nights are wiled away at all-night study sessions, popcorn parties, water fights, or Late Night With David Letrerman.

Oh, to look at pictures it may seem like student life has changed immensely since 1887, but undernearh the change in artire and hair styles, the Cedarville College student has remained much the same.

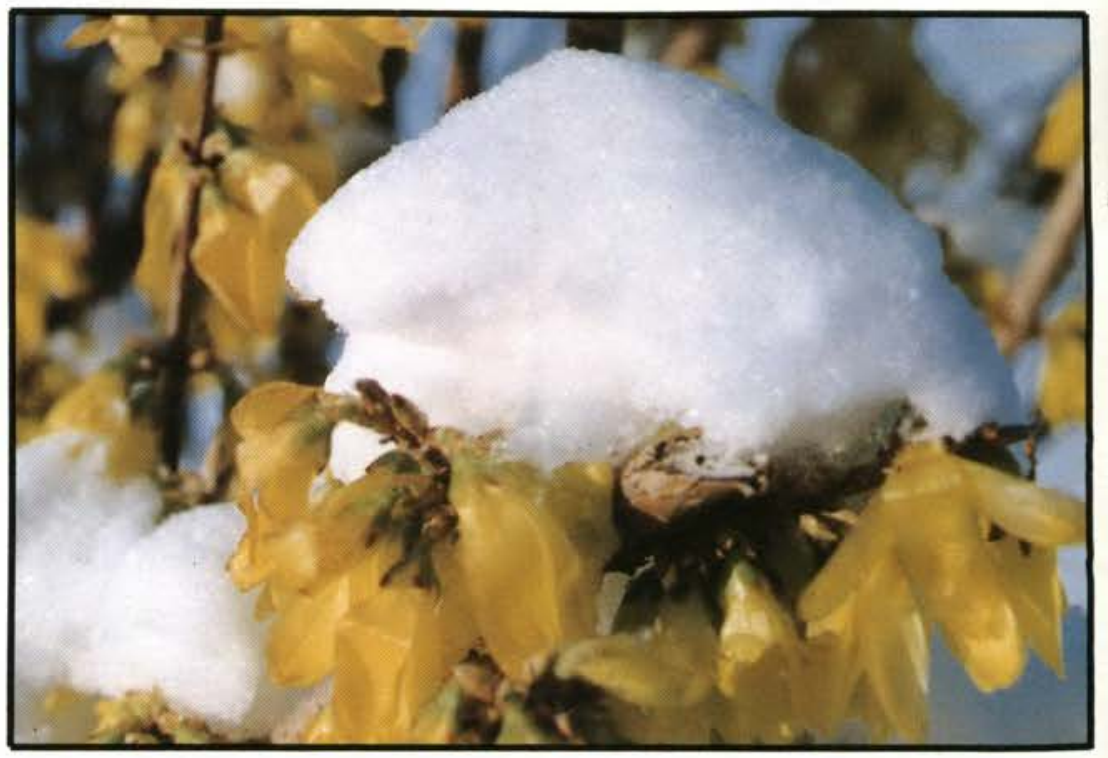




\section{1 \\ Che ${ }^{C}$ Cedrus}

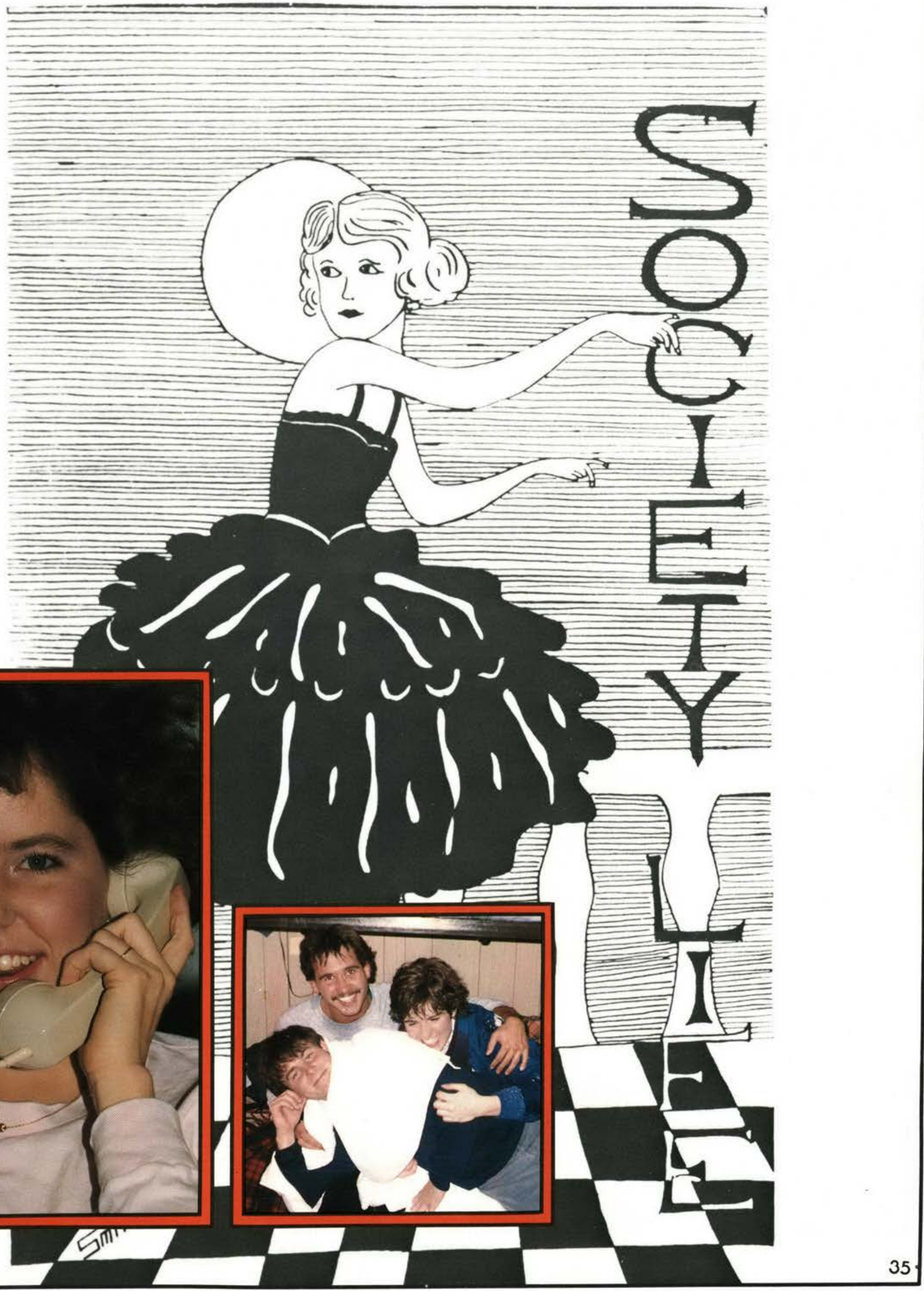




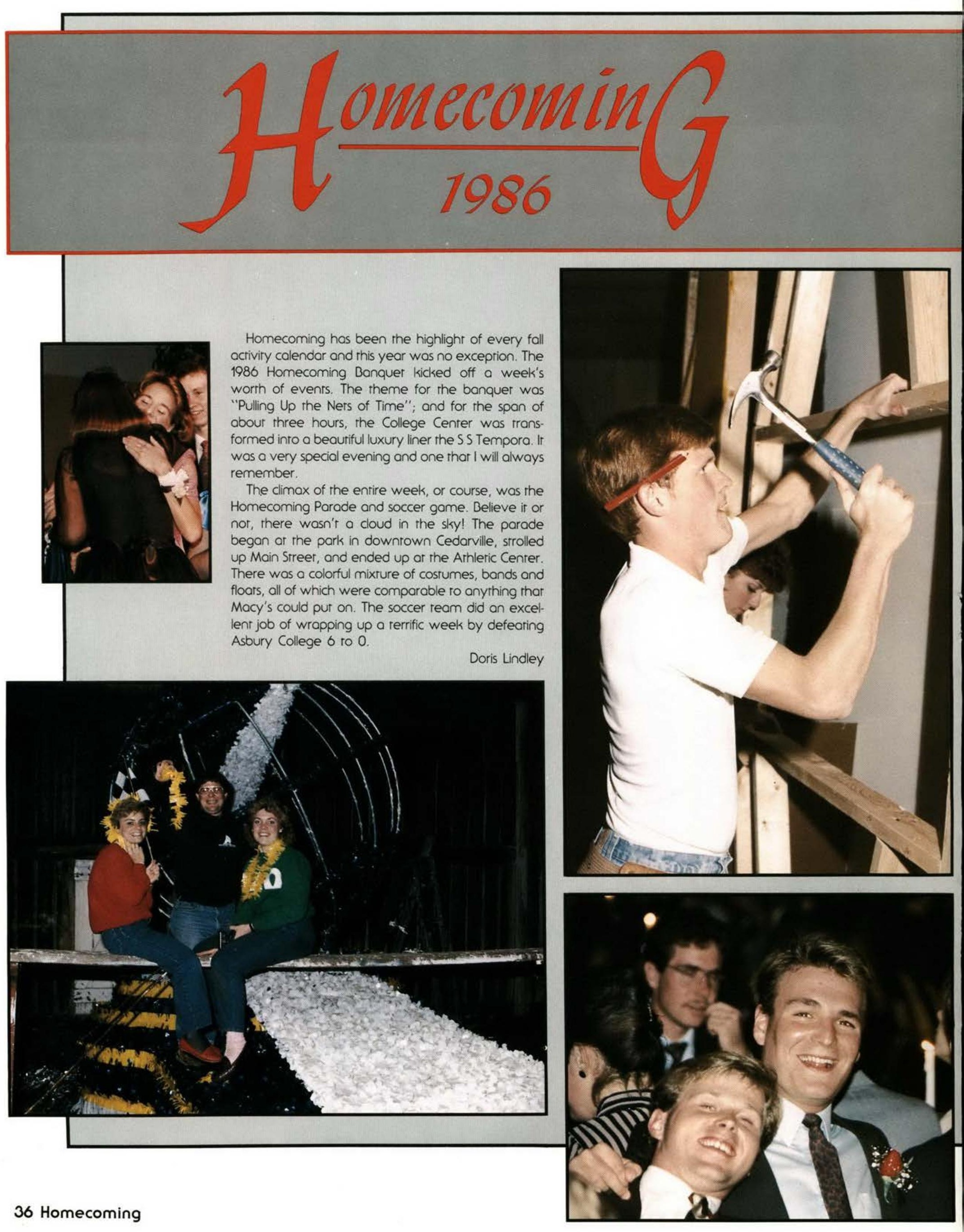



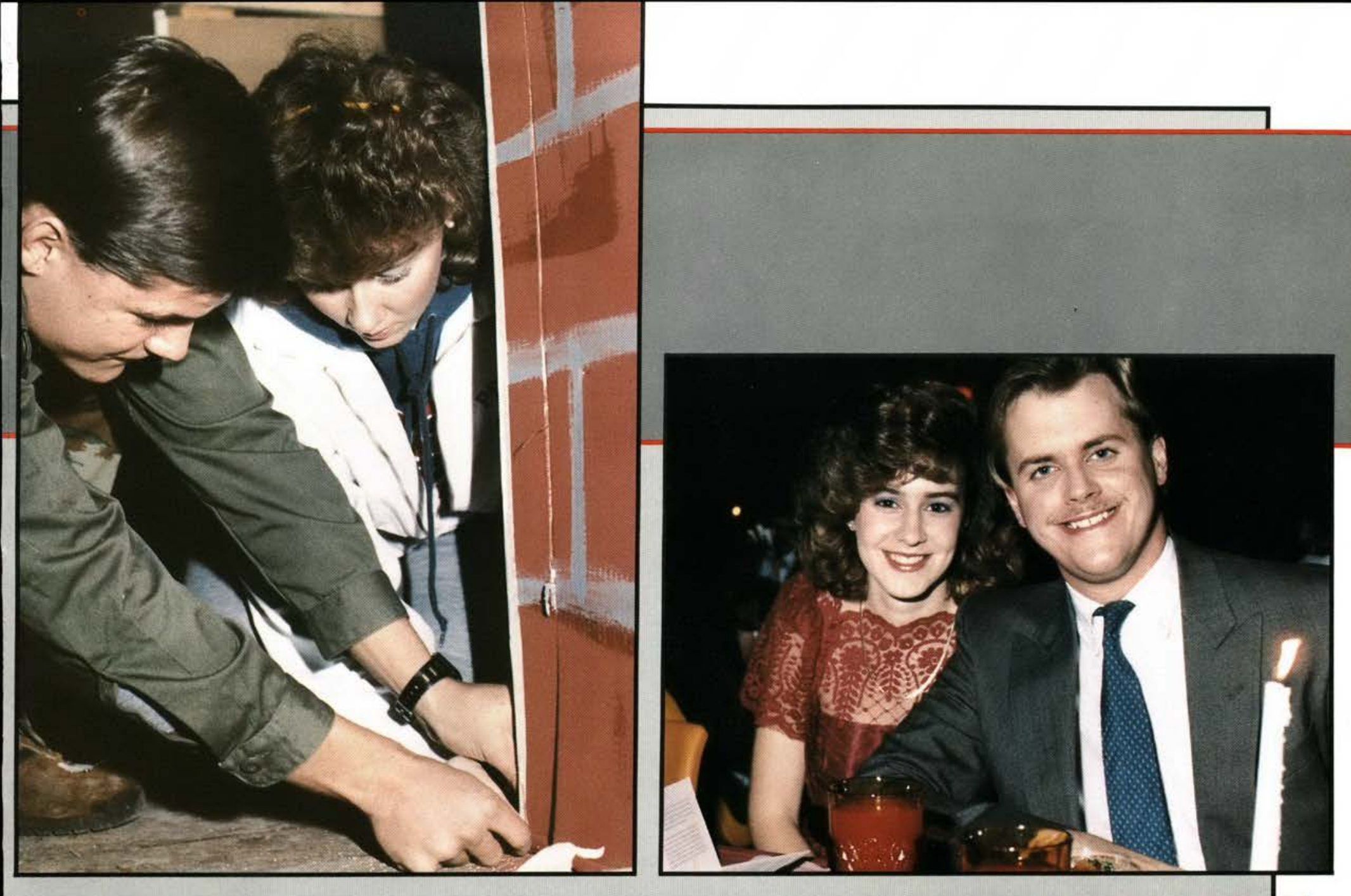

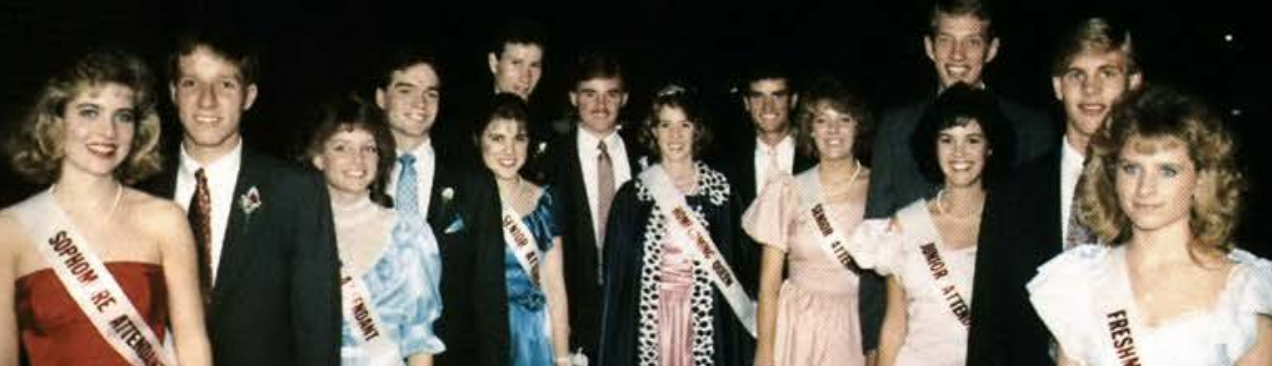

1 신
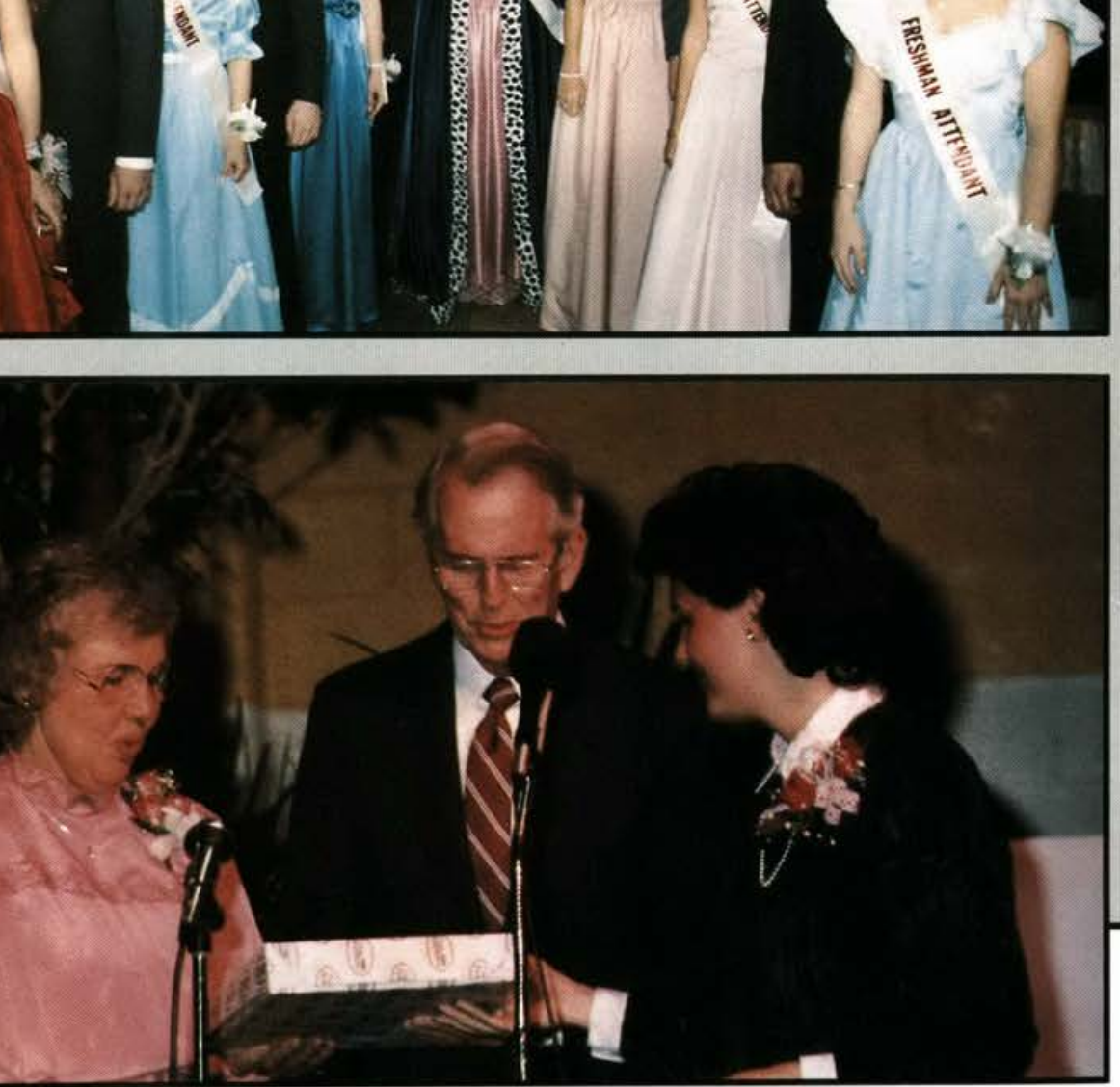

Homecoming 37 


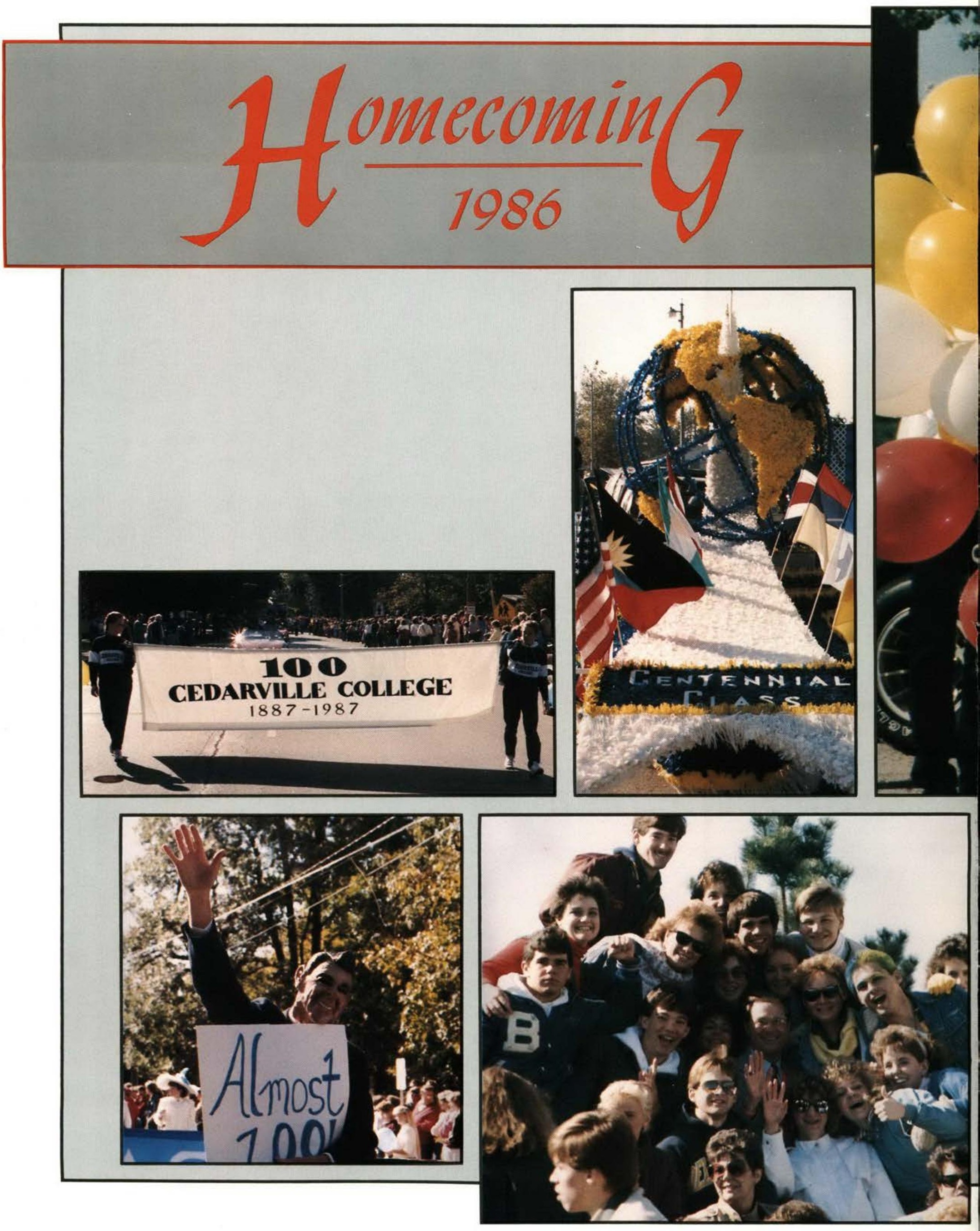




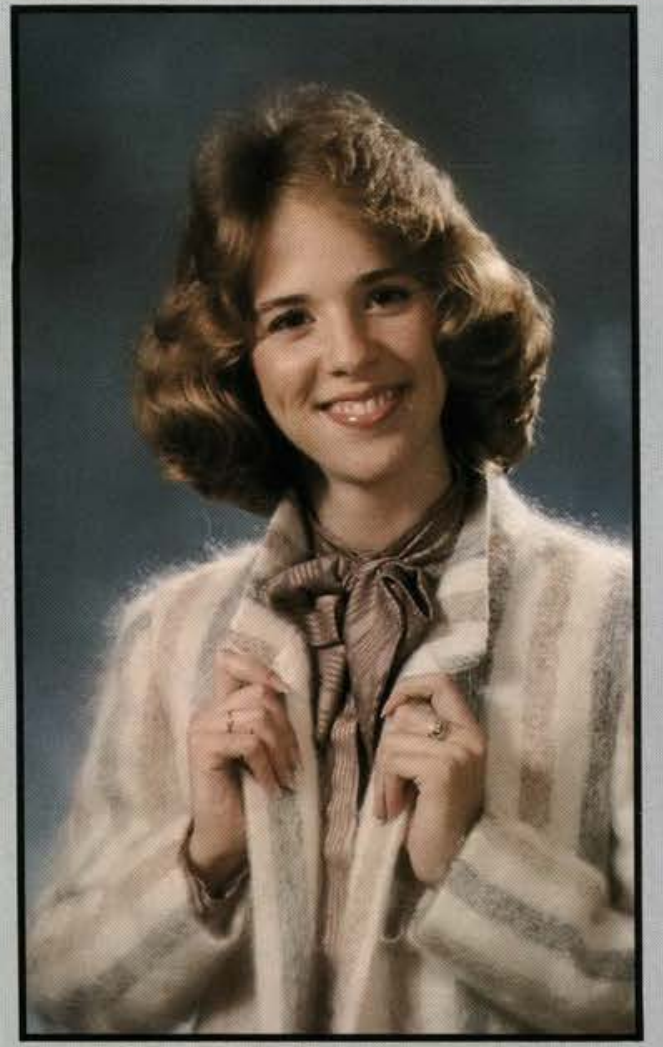

\section{Doris Lindley}

Our 1986 Homecoming Queen wos a 21 year old business adminis: rration mojor from Balimore, Maryland. A previous Homecoming attendant, Doris was actively involved on campus as a member of $A D O$, PiDelto, and KEA
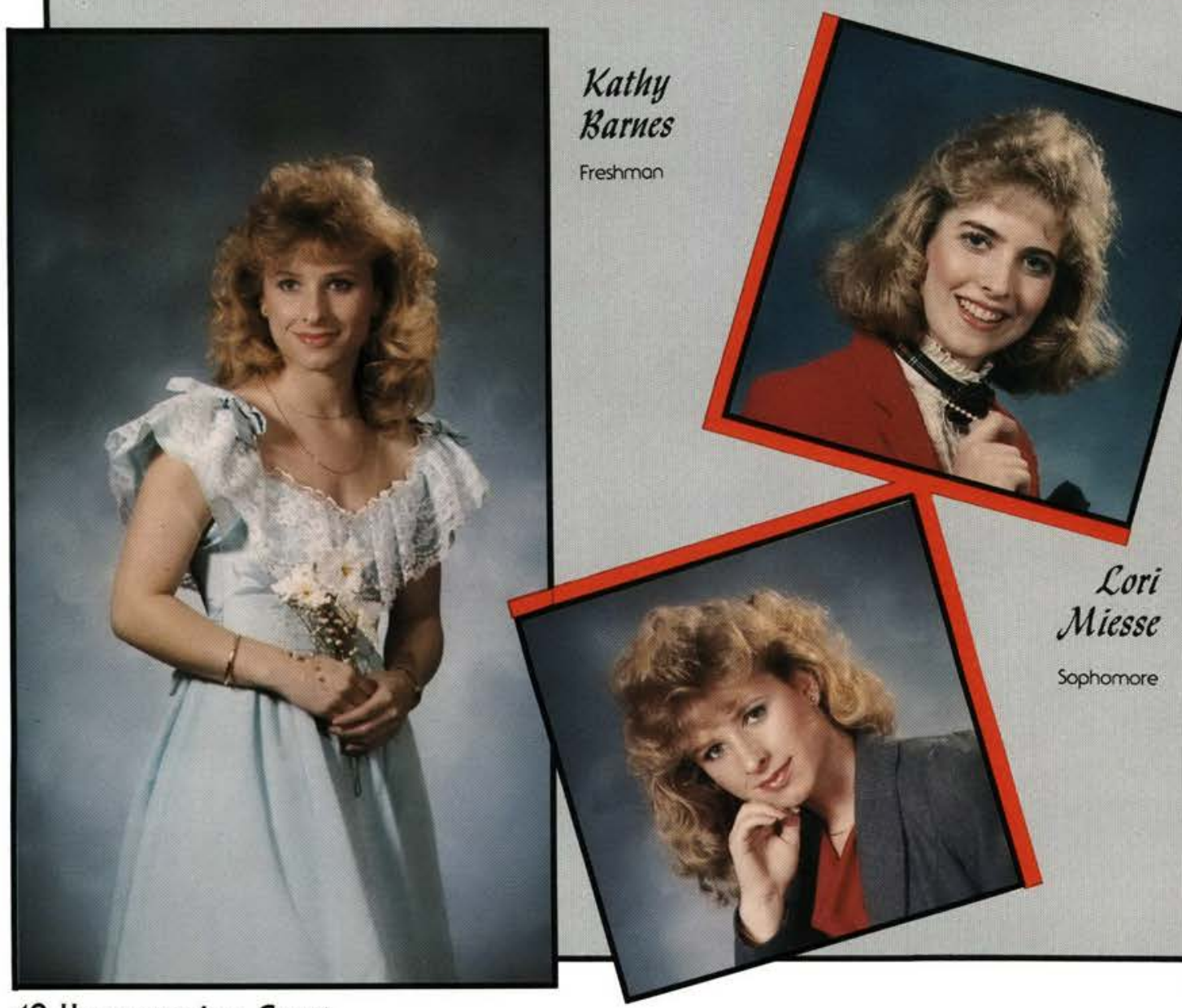


\section{CHARTER D}
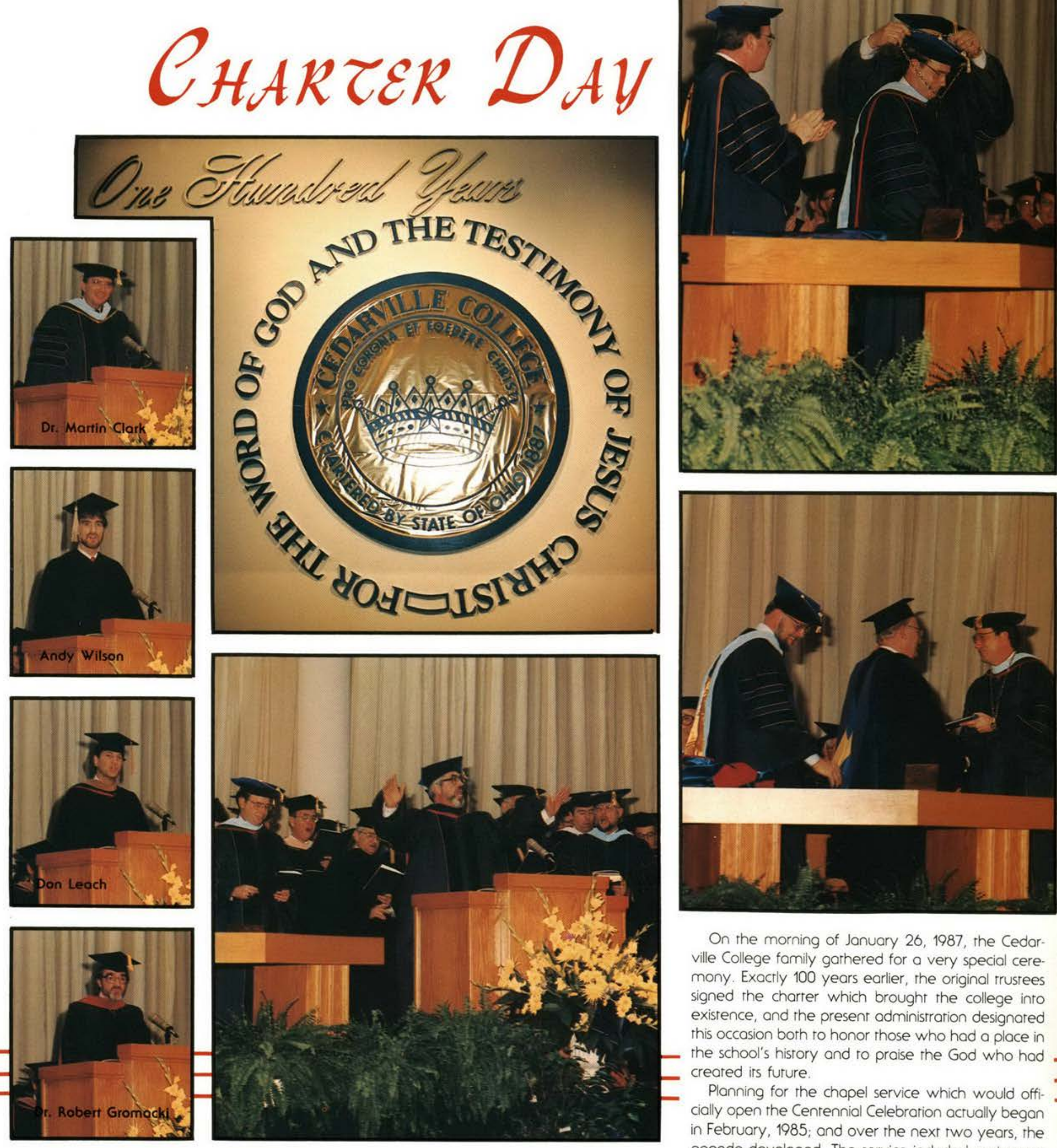

On the morning of January 26,1987 , the Cedarville College family garhered for a very special cere. mony. Exactly 100 years earlier, the original trustees signed the charter which brought the college into existence, and the present administration designated this occasion both to honor those who had a place in the school's history and to praise the God who had created irs furure.

Planning for the chapel service which would officially open the Centennial Celebrarion actually began in February, 1985; and over the next rwo years, the agenda developed. The service included an invocarion by Chancellor James T. Jeremiah, a cenrennial address by Warren W. Wiersbe, and a presidential challenge by President Paul H. Dixon. Inrerspersed with special music and congregational singing, the opening ceremony provided a beauriful and fitring introduction to the year ahead. 

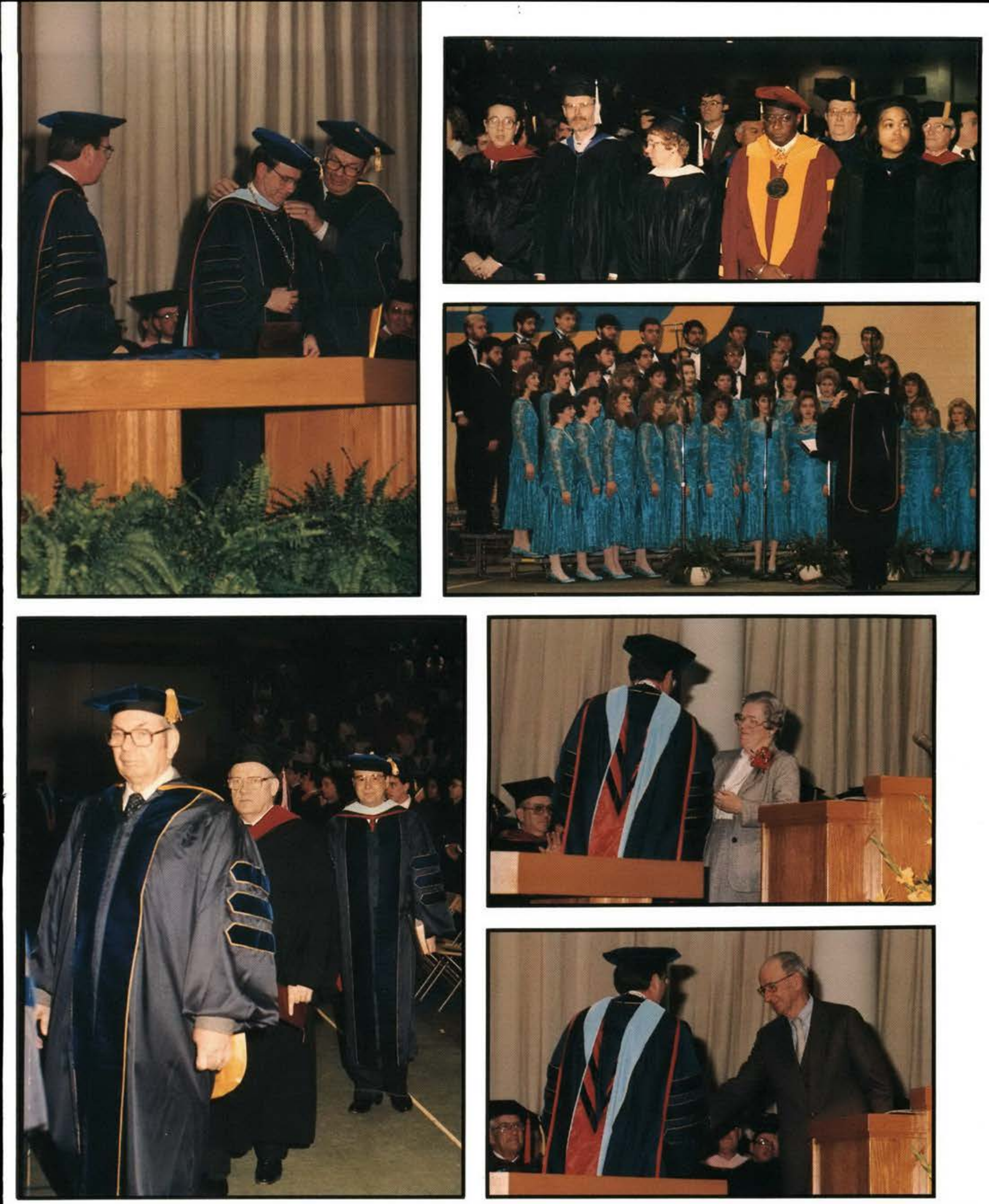

\section{Proverbs 23:23}

"Buy the truth, and sell it not ..." 

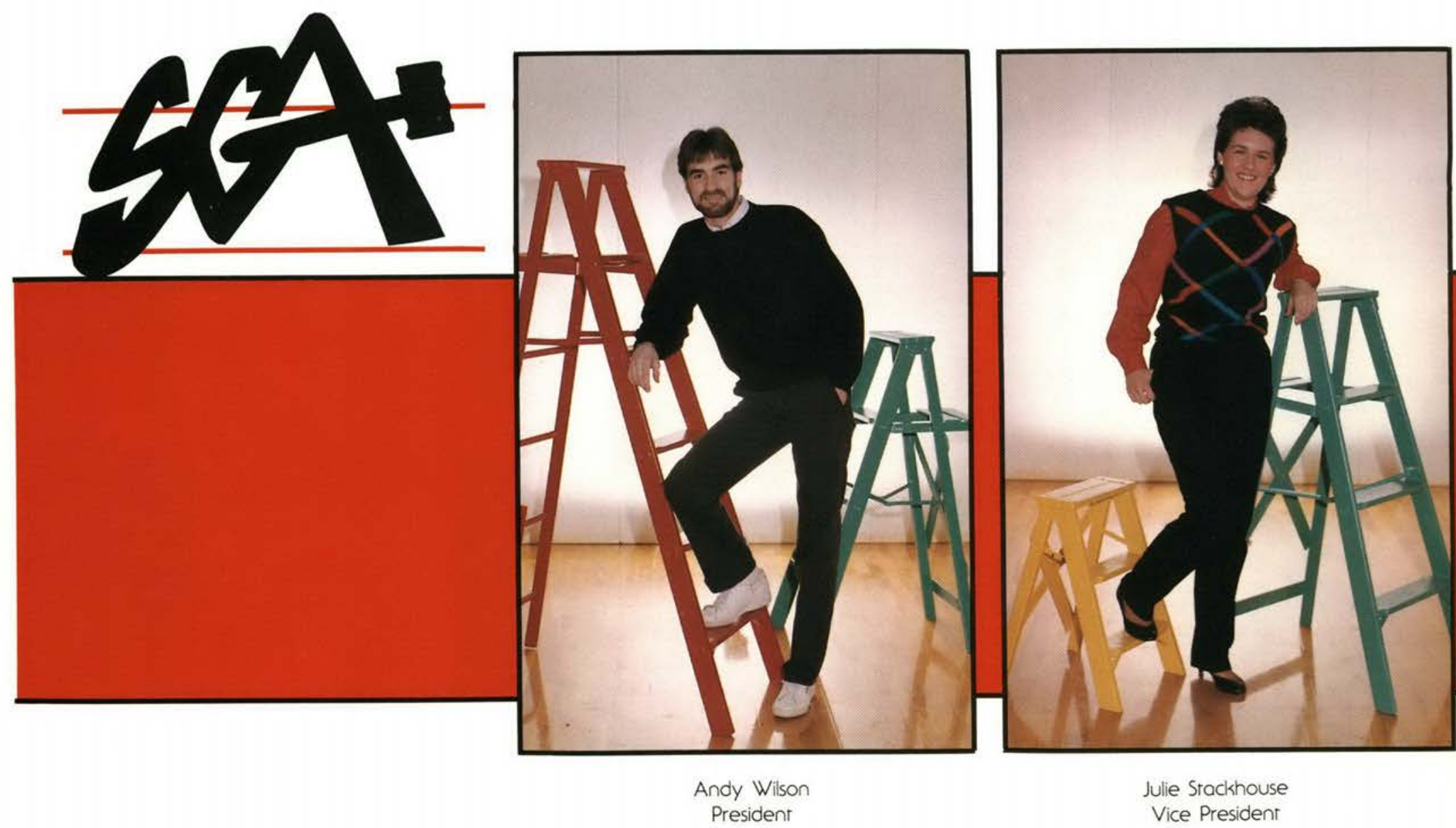

Julie Stackhouse

Vice President

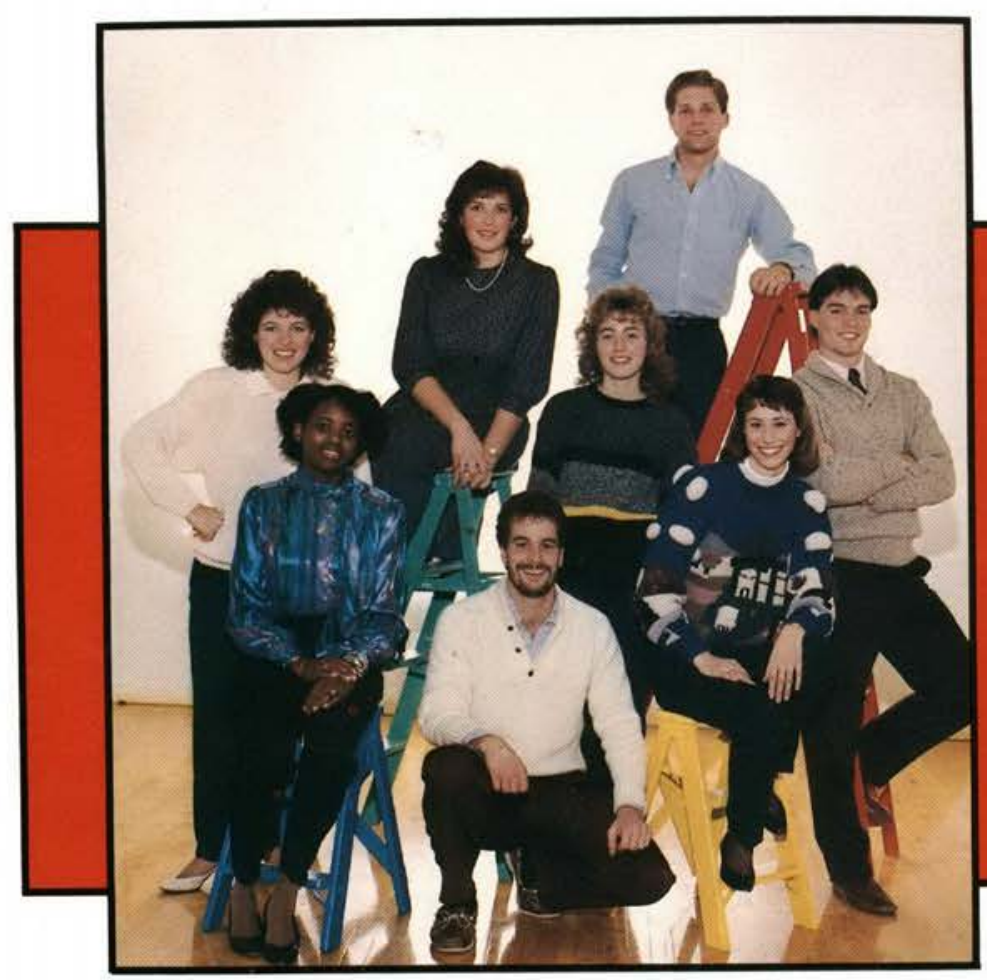

Senior Officers

Clancy Cruise Sreve Campbell Danielle Kizer Charles Jackson

Yvonne Facey Tracy McCoy Karhi Harris Lindo Lons

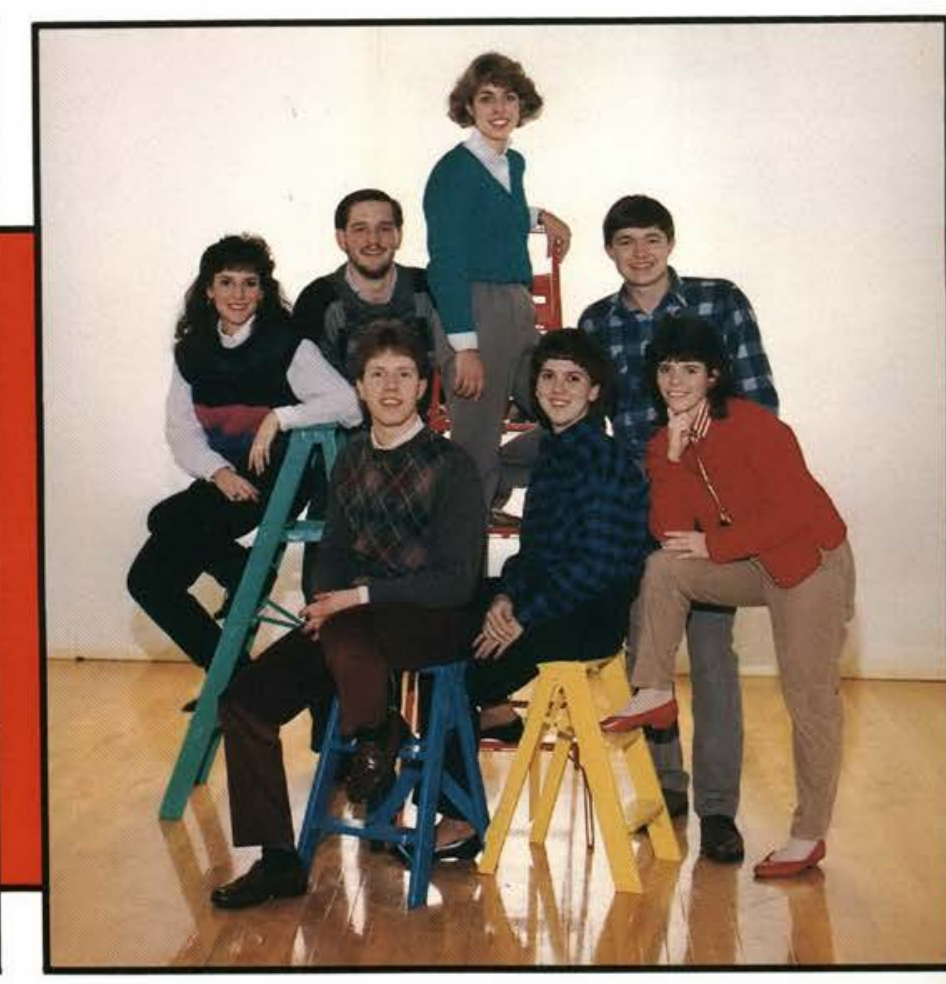

Junior Officers:

Tim Beoch Kristina Hoddleman Erin Mulberry Kendra King
Christine Friedkin Van Holloway Missy Hall 


\section{Qlma chater}

De come with eager bearts to trace

The silver which we know is there

And bidden wealth benond compare.

We ask that we who have Jtis call.

who know that gesus paid it all, chary own a deeper love toward men

for whom Ghrist died and rose again.

Jur paths have led to Gedarville,

Where we seek to do our osaviors will.

ape must impart the living word.

And rescue those who have not heard.

Ret us who have been amply fed

Erovide a dying world with bread.

O. let us be God's bumble tool,

That He man use us and our school. 

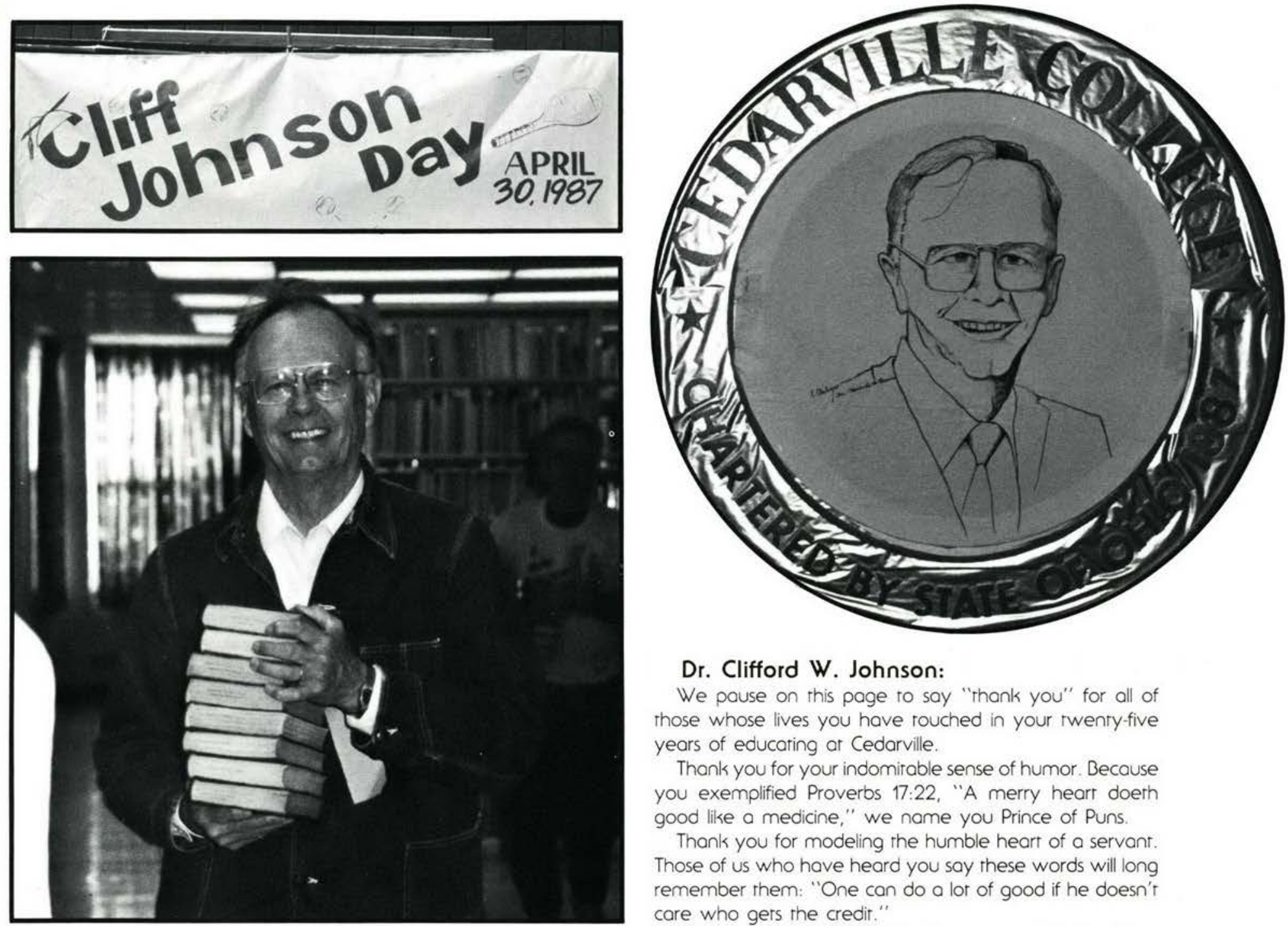

Dr. Clifford W. Johnson:

We pause on this page to say "thank you" for all of those whose lives you have rouched in your twenty-five years of educating ar Cedarville.

Thank you for your indomitable sense of humor. Because you exemplified Proverbs 17:22, "A merry hearr doerh good like a medicine," we name you Prince of Puns.

Thank you for modeling the humble heart of a servant. Those of us who have heard you say these words will long remember them: "One can do a lor of good if he doesn't care who gets the credit.
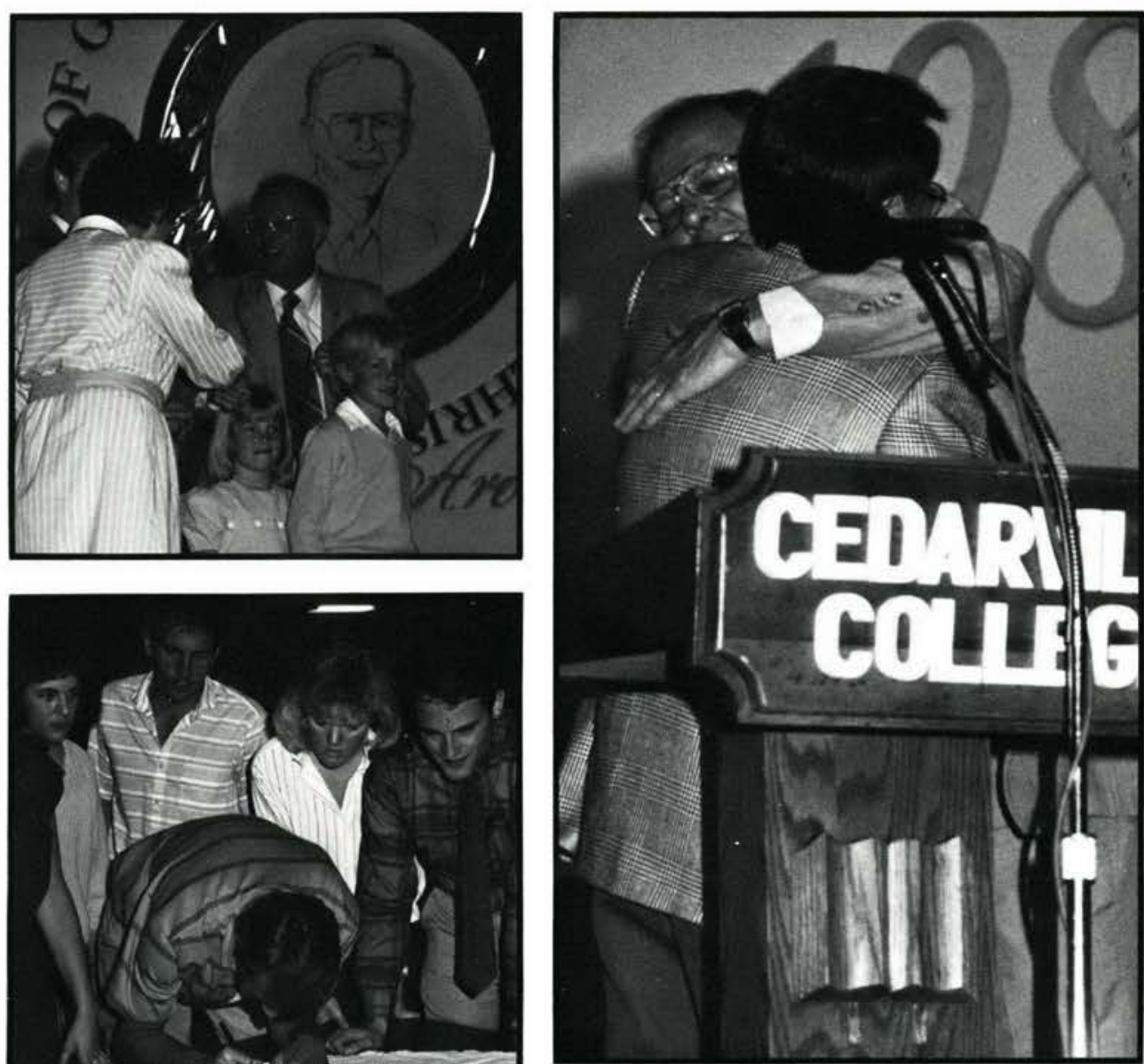

Thank you, especially, for allow. ing us the freedom to find the crearive limirs of our abilities. Your confident enthusiosm in our ralents opened vistas of achievement otherwise undreamed. We hope you will long stay with us, enriching our lives, spurring us on to berrer things. God Bless You.

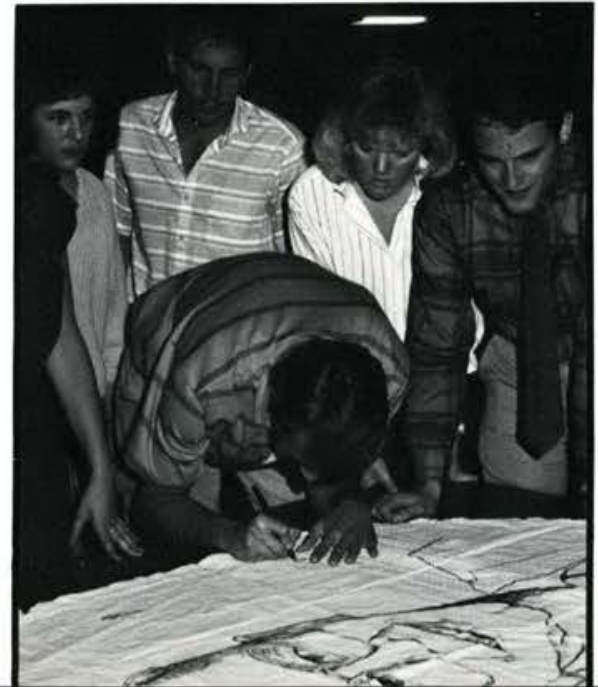




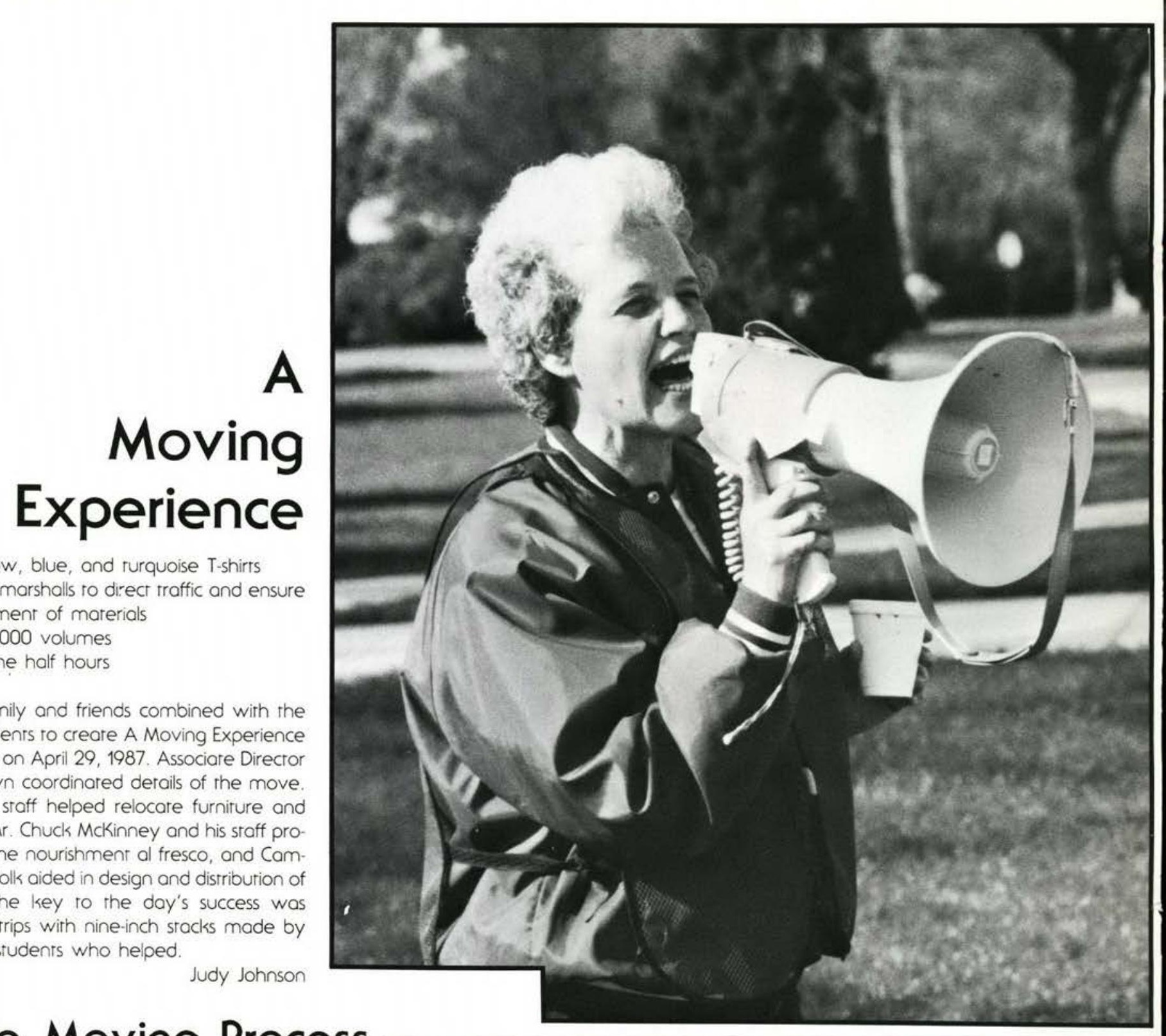

- 1500 yellow, blue, and rurquoise T-shirts

- Nearly 40 marshalls to direct traffic and ensure proper placement of marerials

- Over 100,000 volumes

- Six and one half hours

College family and friends combined with the above ingredients to creare A Moving Experience for the library on April 29, 1987. Associare Director Stephen Brown coordinared derails of the move. Mainrenance staff helped relocare furnirure and equipment. Mr. Chuck McKinney and his staff provided noonrime nourishment al fresco, and Campus Acrivities folk aided in design and distribution of T-shirts. But the key to the day's success was innumerable trips with nine-inch stacks made by each of the students who helped.

The Moving Process
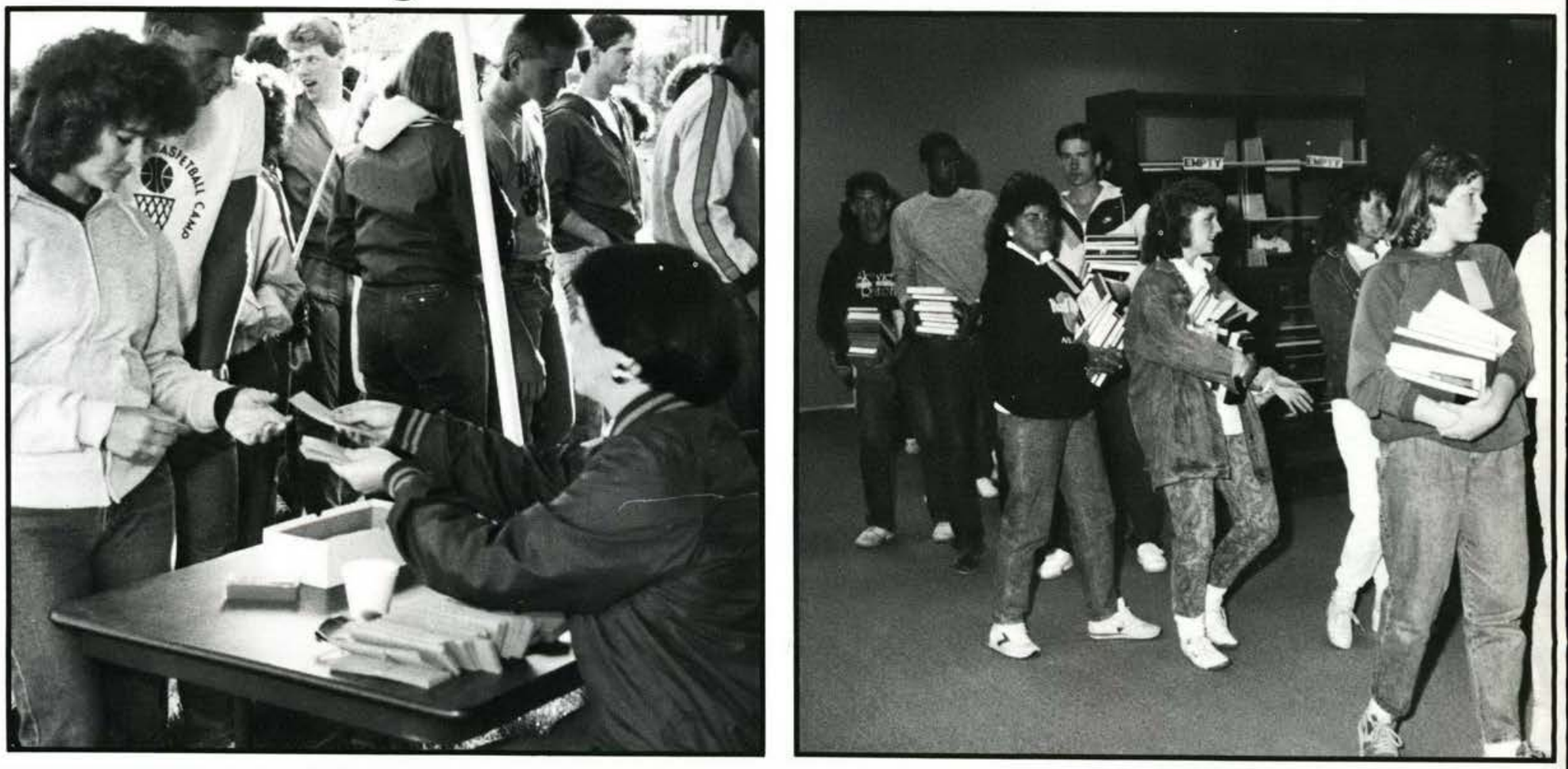


\section{$z^{z}$ Z

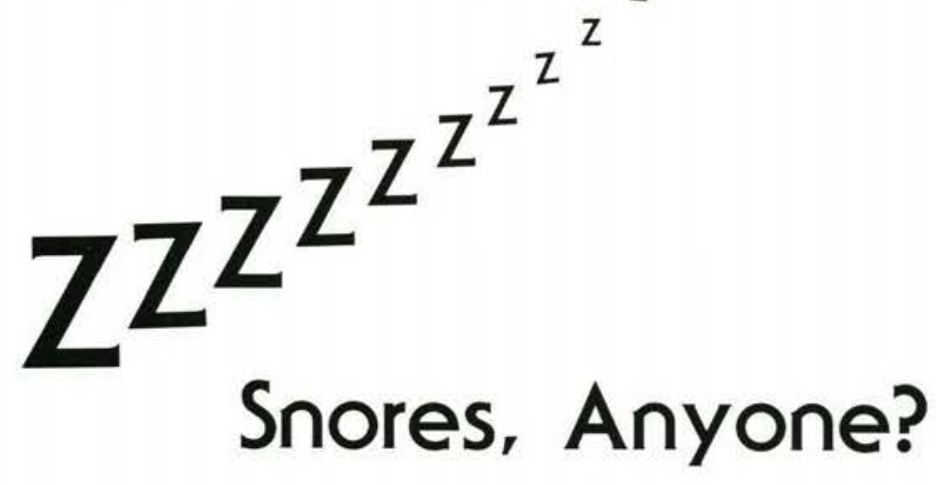

You pay $\$ 1230.00$ for a bed - usually bunked - in a scrunched room with a roommare who chews too loudly. You'd think you'd ger some quality rest, righr? Bur the night congests with papers and books and projecrs and exams, and by the time you hit the pillow if you ever do - your brain is so caffeinared that you lie rwirching contorting the visages of your stuffed animals until the day breaks. So you compensare. You sleep in subrle ways. Like 8:00 classes, wedged on a hymnal in Chapel, Physical Science, R.A. prayer meetings, sprawled on a News brief at lunch, 3:00 classes, or on the way home from a lare pass with a Melancholic.

The CC lounge is a likely spor with couches that are rather scratchy (bur it's a long way back to the dorm). If you have time for a nap, your roommare will probably type a paper. If you go to bed early, the phone will ring ar least 3 rimes. The library can be peaceful unless you get near a social butrerfly who whispers as loudly as your roommare chews.

But then your roommare claims you haven't changed your sheers for 9 weeks. And you say the only crud on those sheers is the stuffing from a murilated reddy bear. And that's the only thing resting in peace on a $\$ 1230.00$ bed.

Rurh Margraff
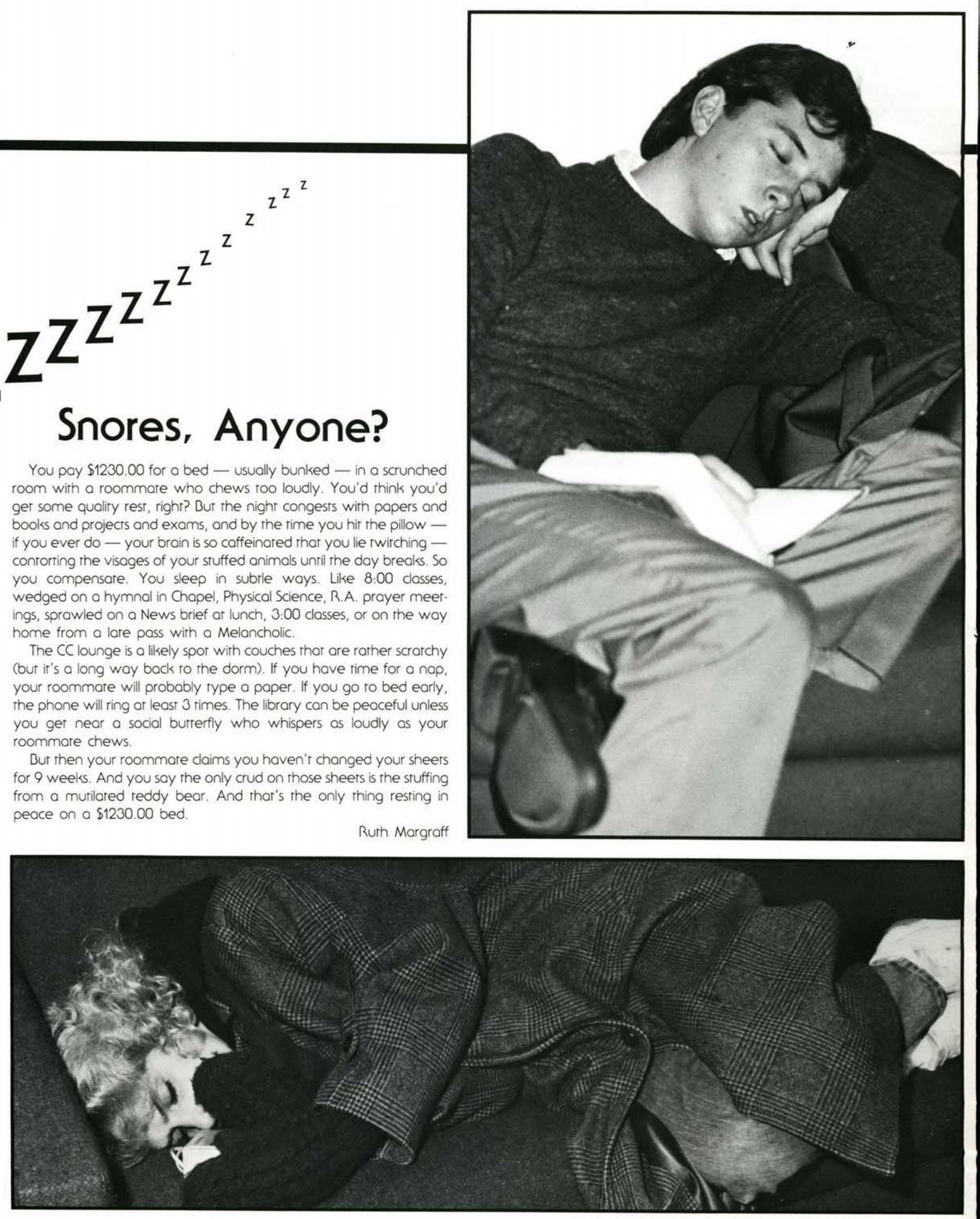


\section{AX Student Talent Show}
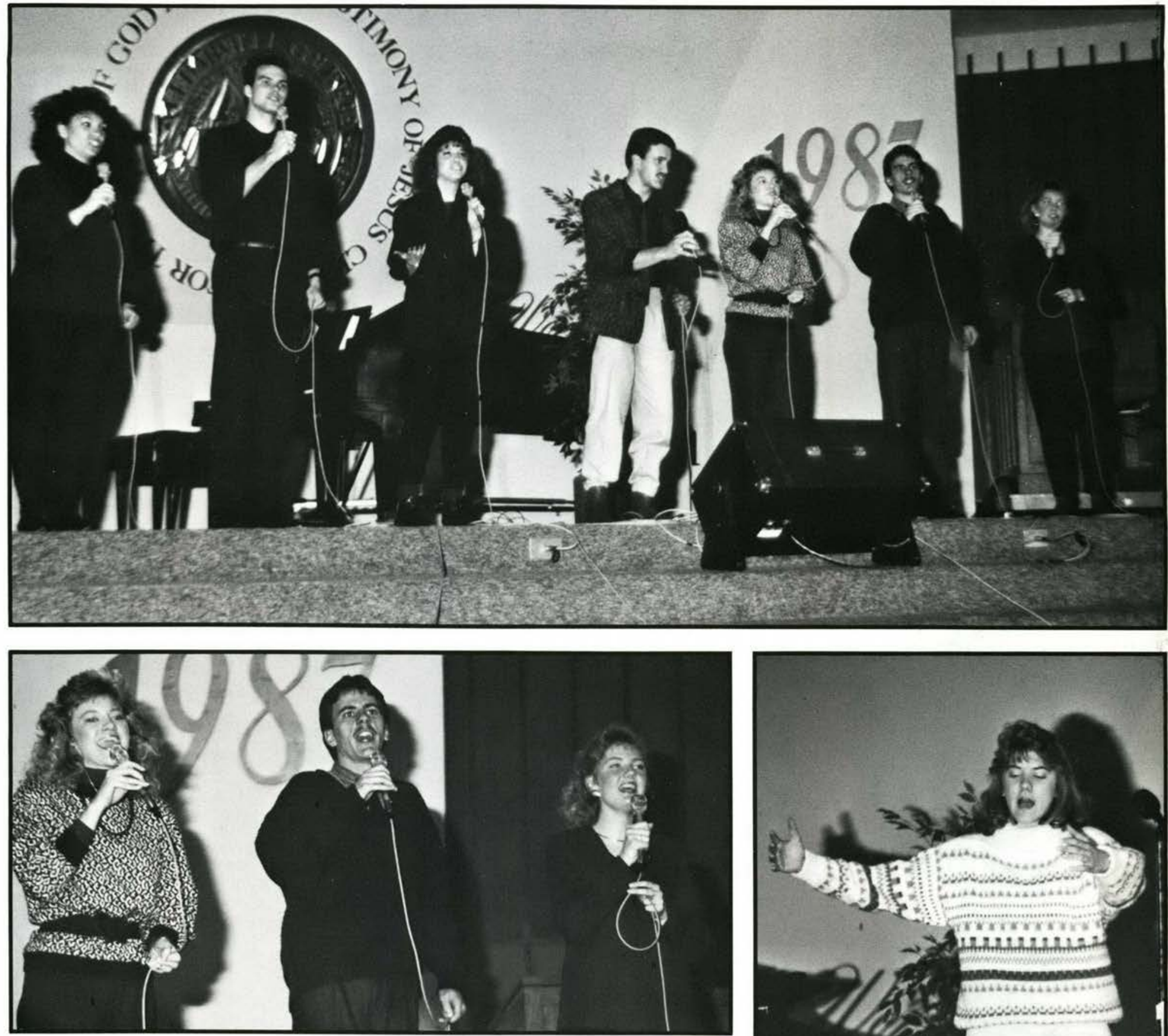

This year's Alpha Chi Talent Show once again gave us a lively balance of humorous and serious performances. With acrs ranging from dramaric readings to the music of P.D.Q. Bach and Sandi Parti, the C.C. students exemplified their crearive energies and hidden ralents in an artempt to win the coveted first prize. When the final votes were counred, the group "Destiny," consisting of Debby Rotramel, Rob Paswarer, Kim Sweer, Jeff Ves rrand, Karla Hines, Malcolm Lines and Marry Murphy, topped the list with their version of "All That You're Looking For."

Scorr Beartie
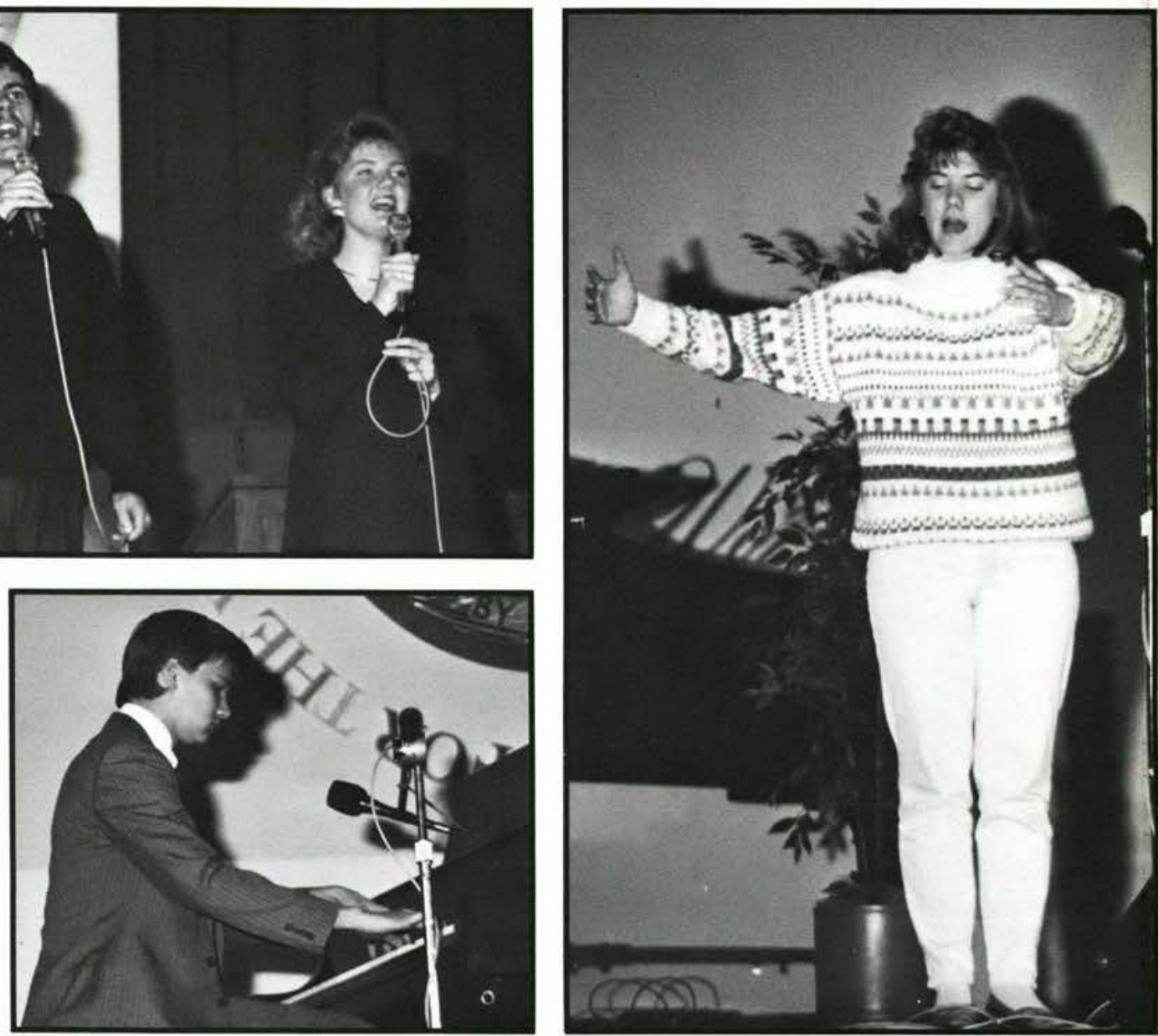


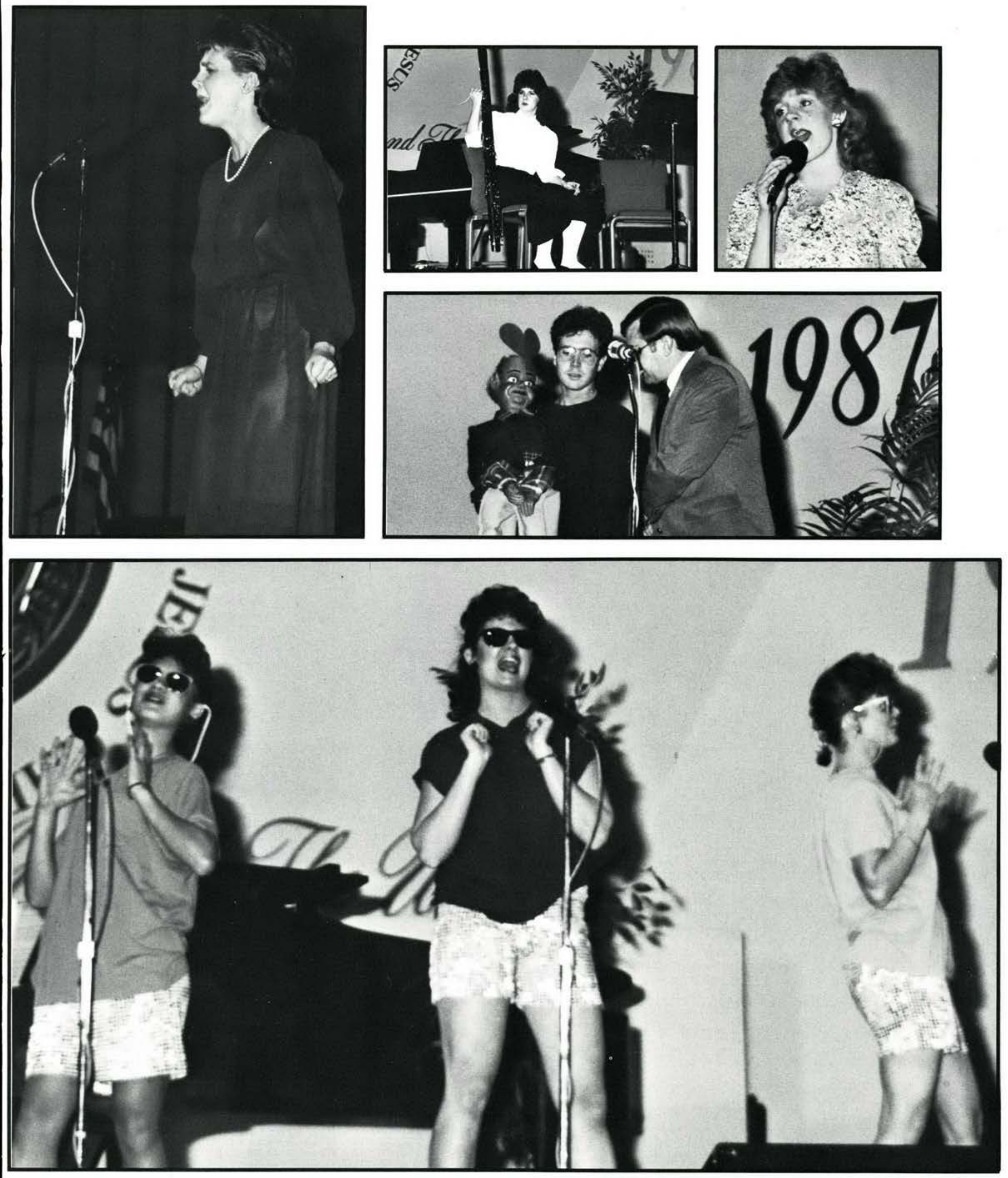



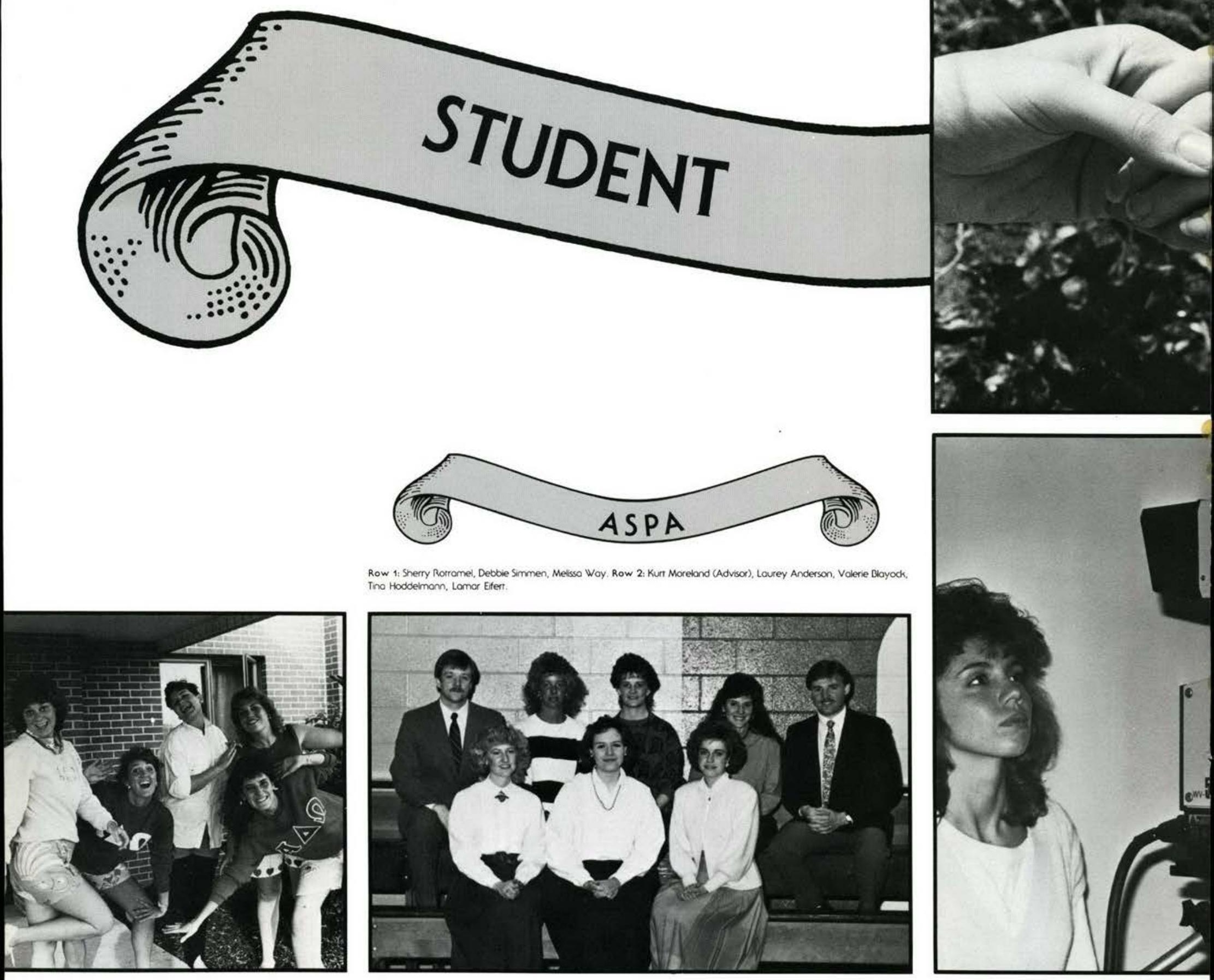

Row 1: Sherry Rorromel, Debble Simmen, Melisso Woy. Row 2: Kurt Moreland (Advisor), Lourey Anderson, Volerie Blayock, Tino Hoddelmom, Lomar Efert.
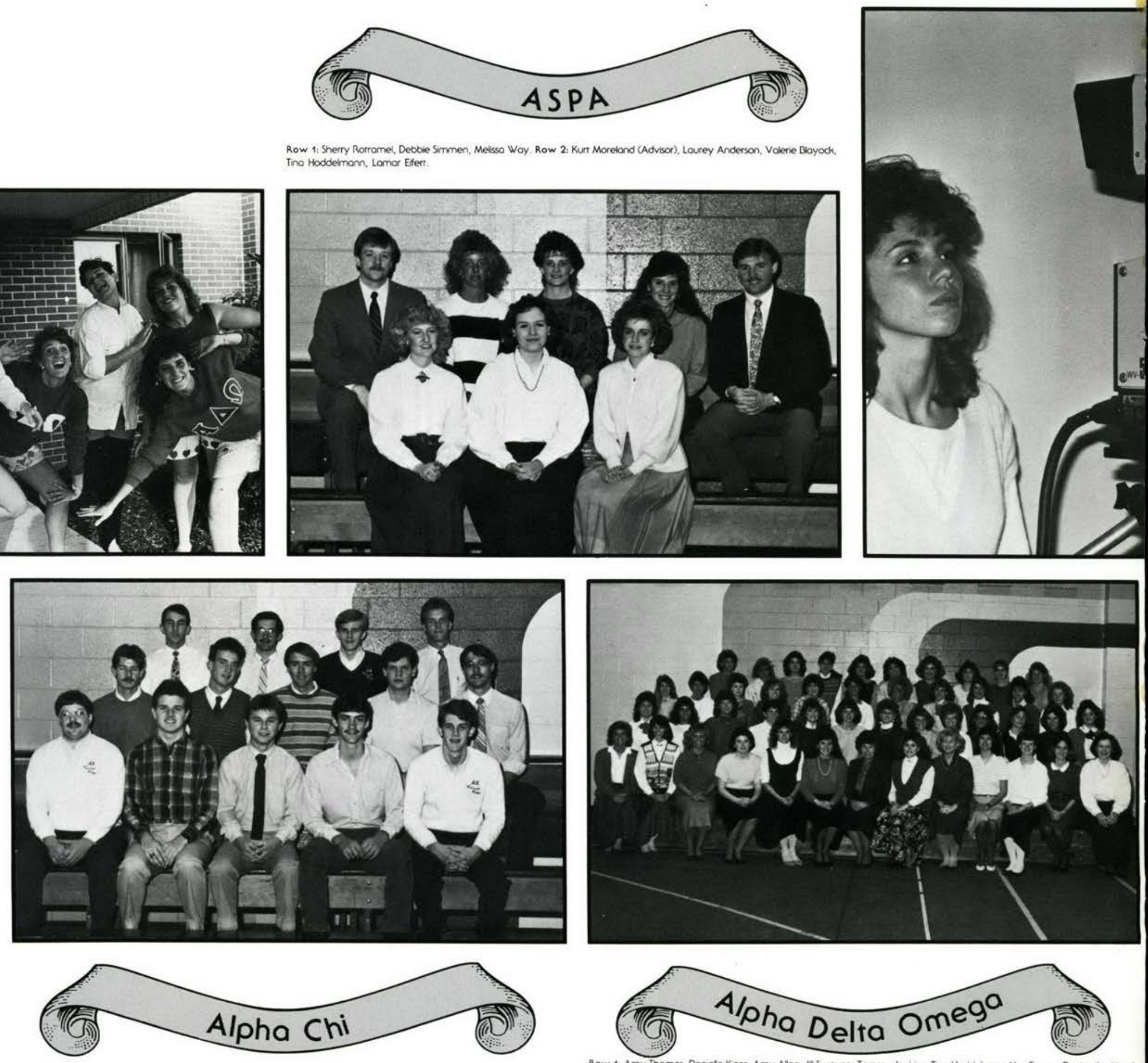

Row 1: Perry Chiorell, Oris Horrel, Jeft Cryzyk, Loren Johnson, Joel Hoskinson Row 2: Gene Evons, Jonarhan Popa, Scon

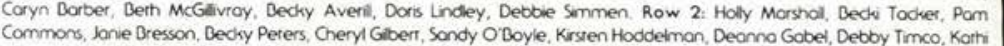

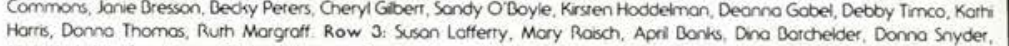
Michelle Lelah, Sue Moyer, Dridgor Vissmon, Theresa Henry, Krishne Warson, Carhy Lombord. Row 4: Korhy Reynolds, Kely

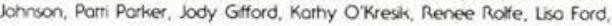



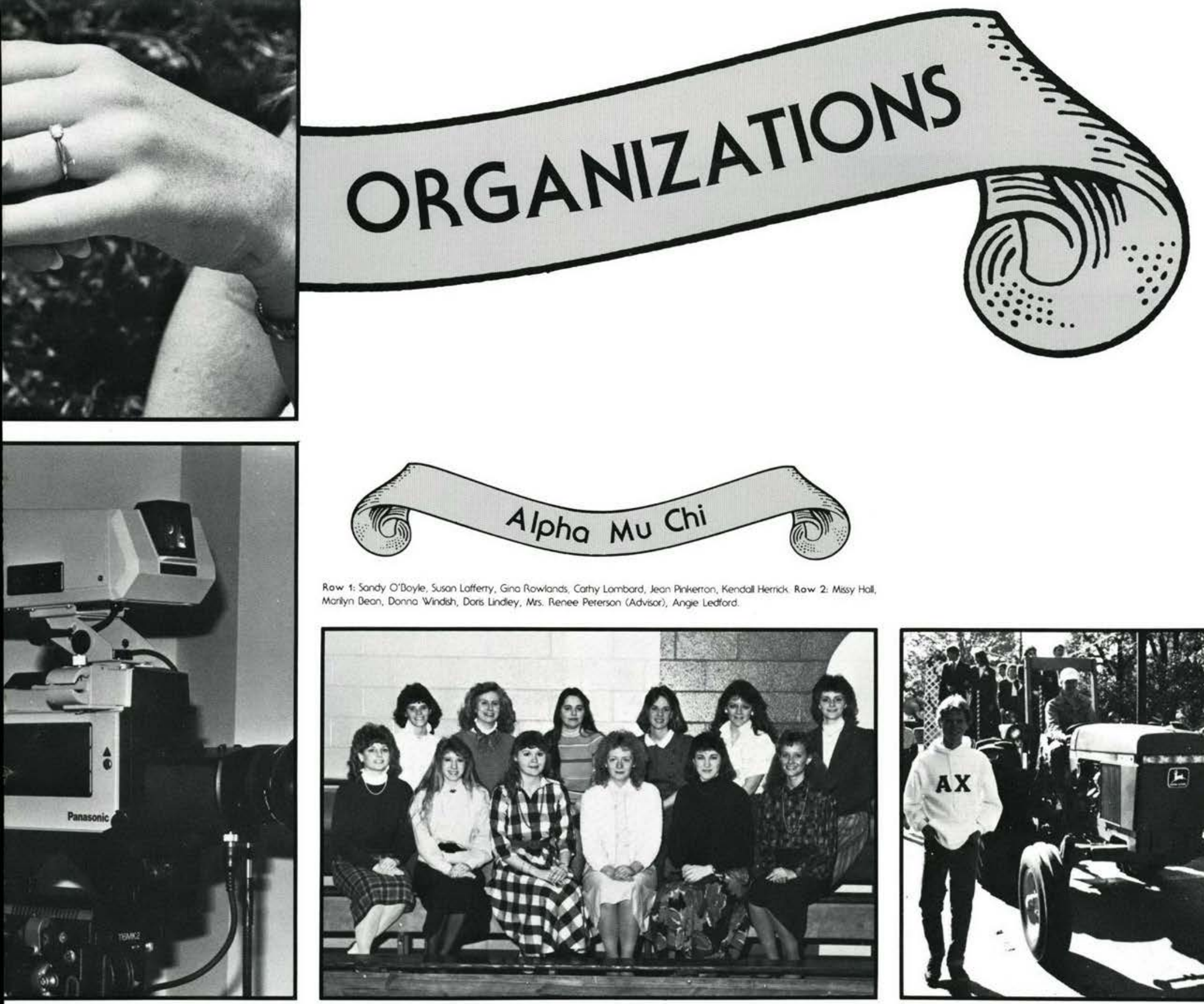

Row 1: Sondy O'Doyle, Susan Lofferty, Gino Rowlands, Carhy Lombard, Jeon Prikerton, Kendall Herrick Row 2: Missy Hall, Morlyn Dean, Donno Windish, Dors Lindey, Mrs. Renee Pererson (Advisor), Ange Ledtord
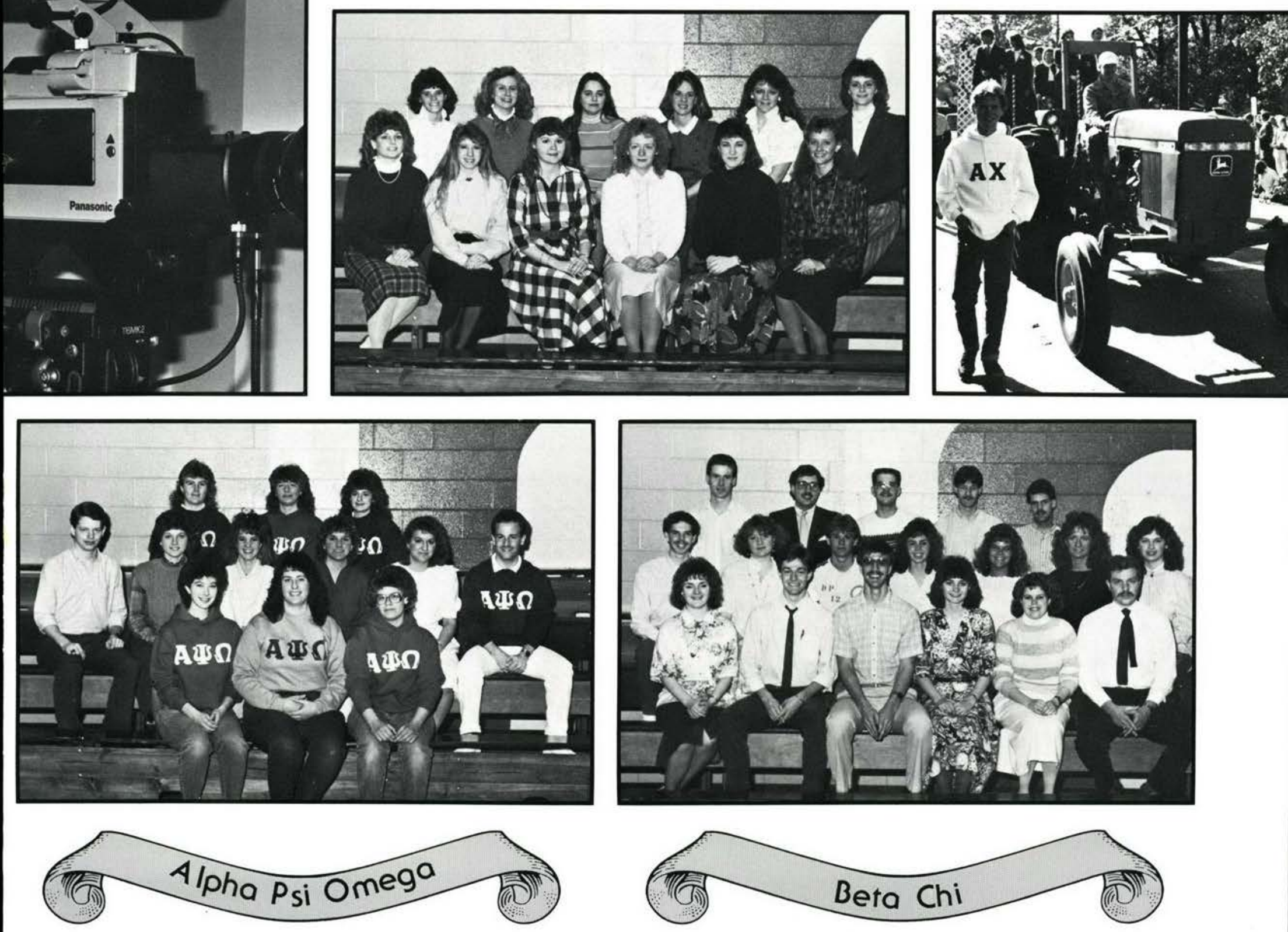


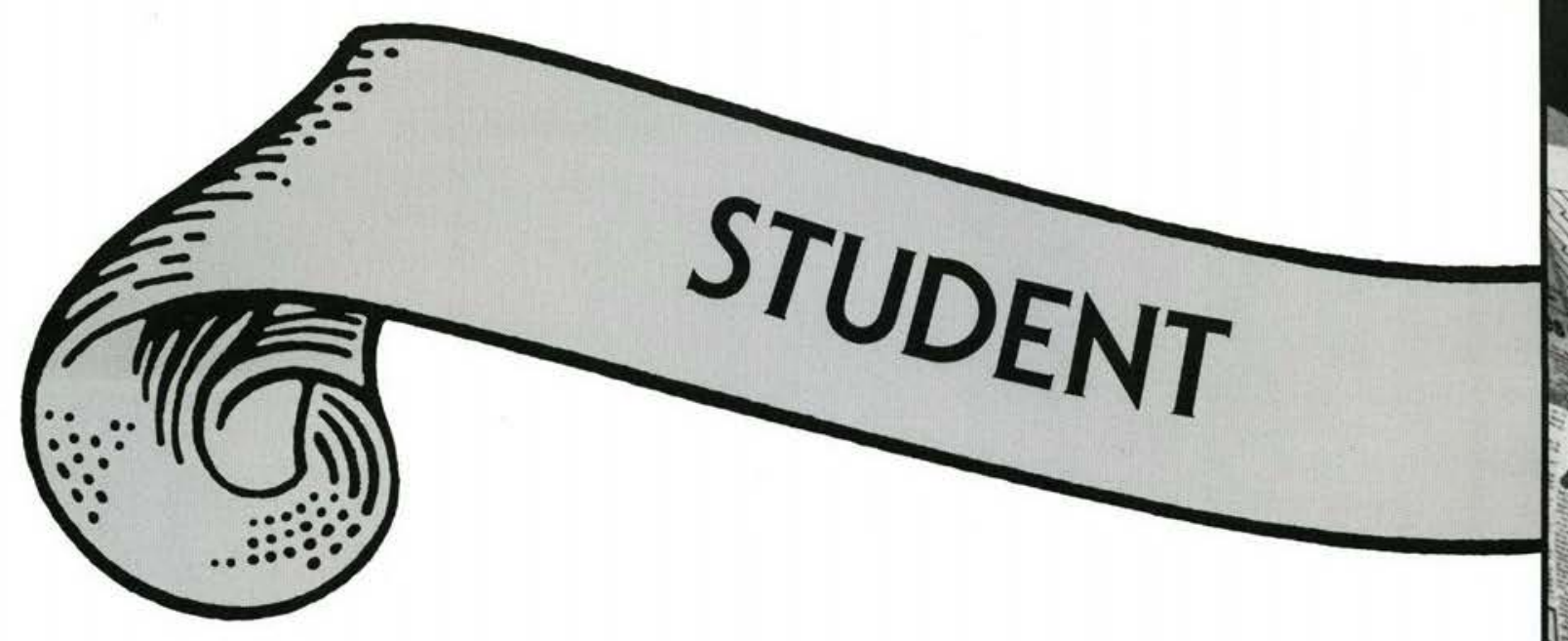

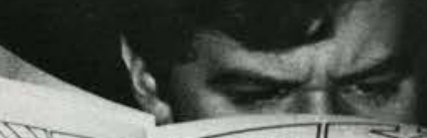
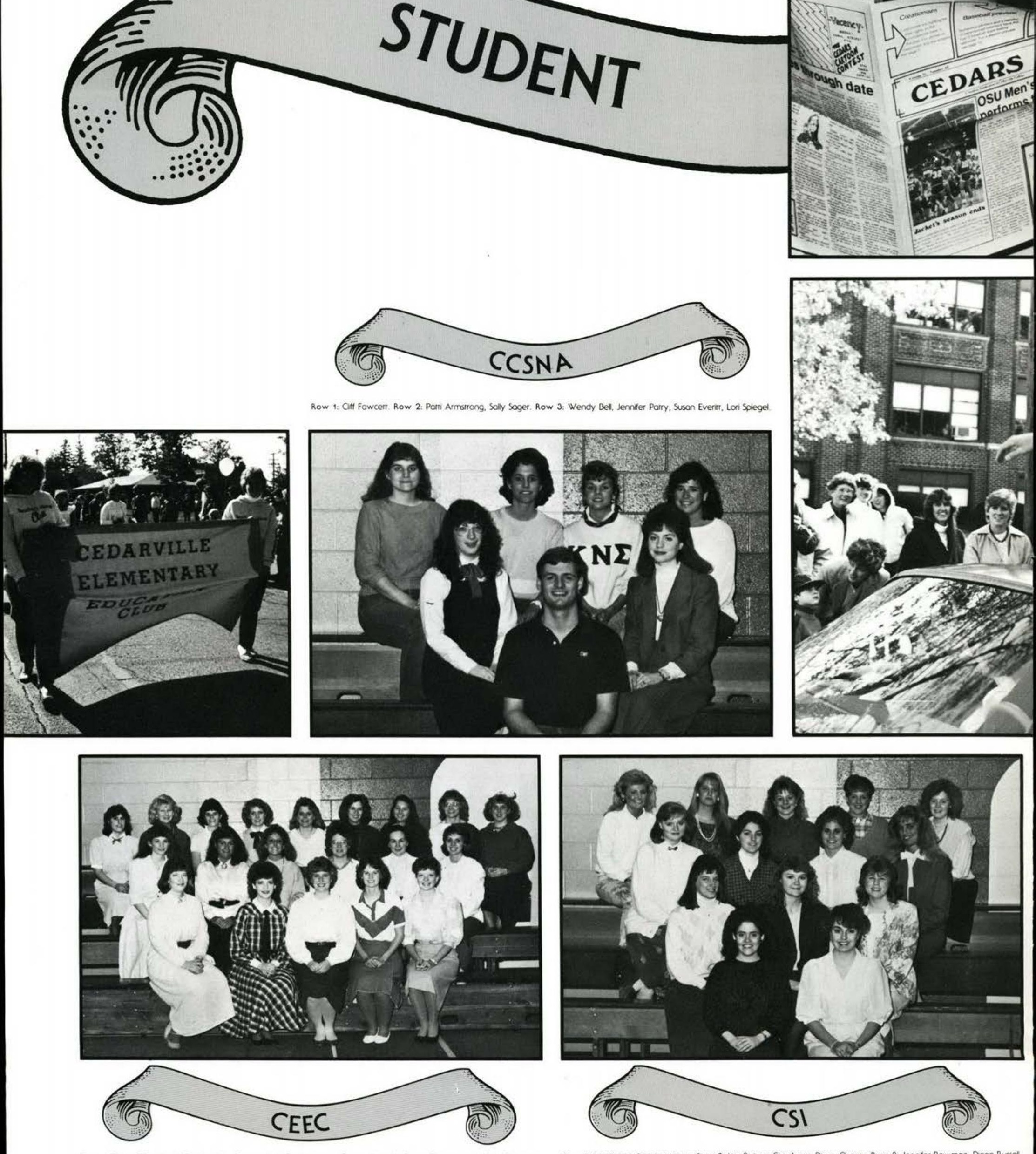

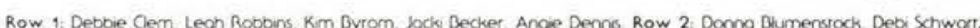

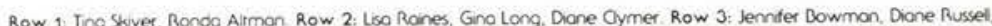
Showna Denney, Brendo Weber, Dorcos Sommers, Peggy Colter. Row 3: Desh Moon, Coryn Dorber, Missy Hol, Sandy Giber. Lso Payne, Debbie Schwarz Row 4: Goye Horden, Jule Sronehouse, Crissy Srowell, Hendi Ryan, Debbie Fogie 


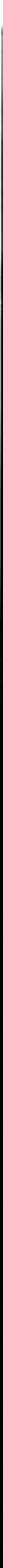



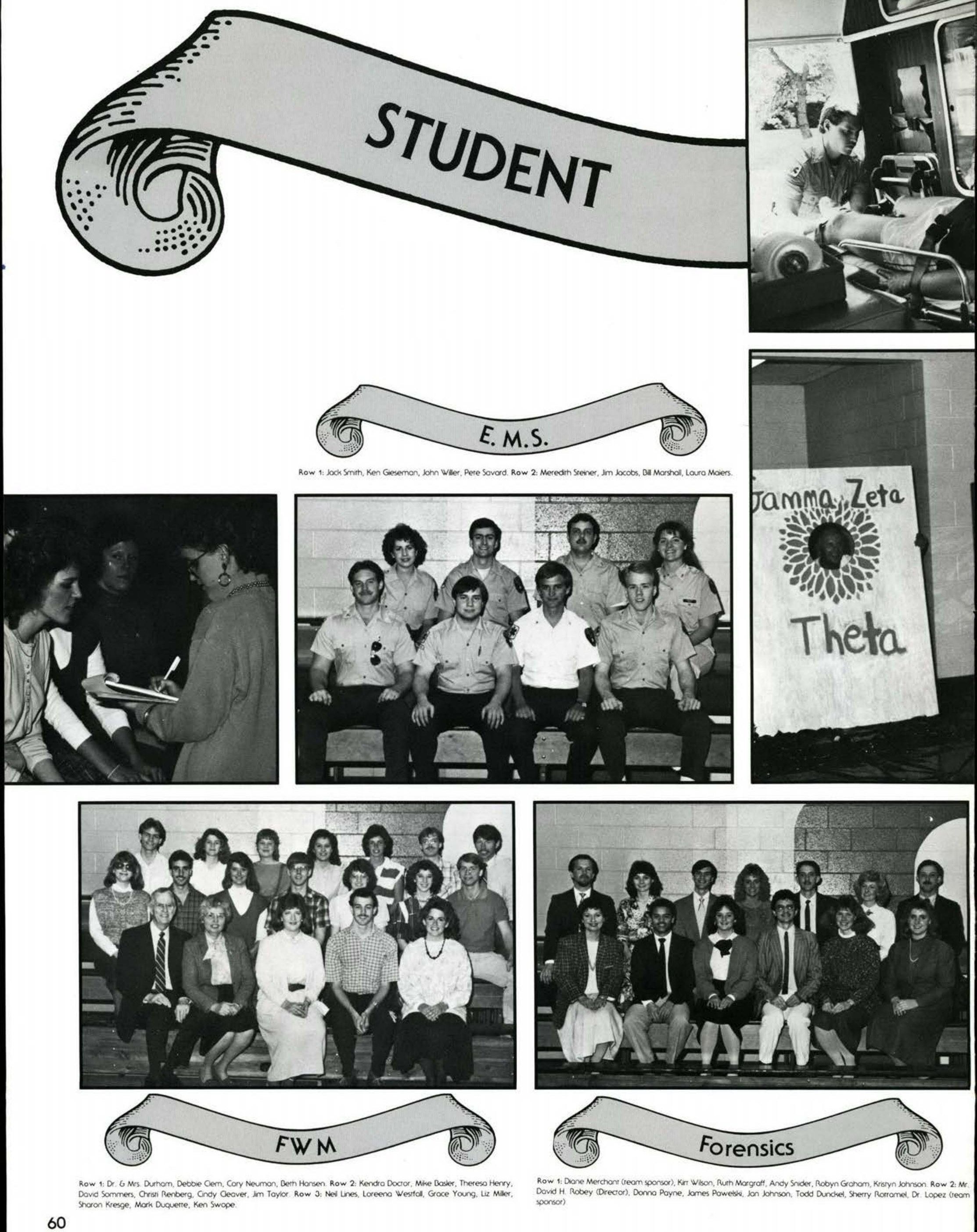

Row 1: Dr. 6 Mrs. Durnom, Deboie Cem, Cory Neumon, Berh Honsen. Row 2: Kendro Doctor, Mkke Baster, Thereso Henry, 


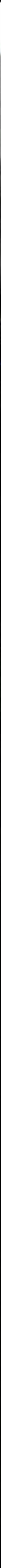



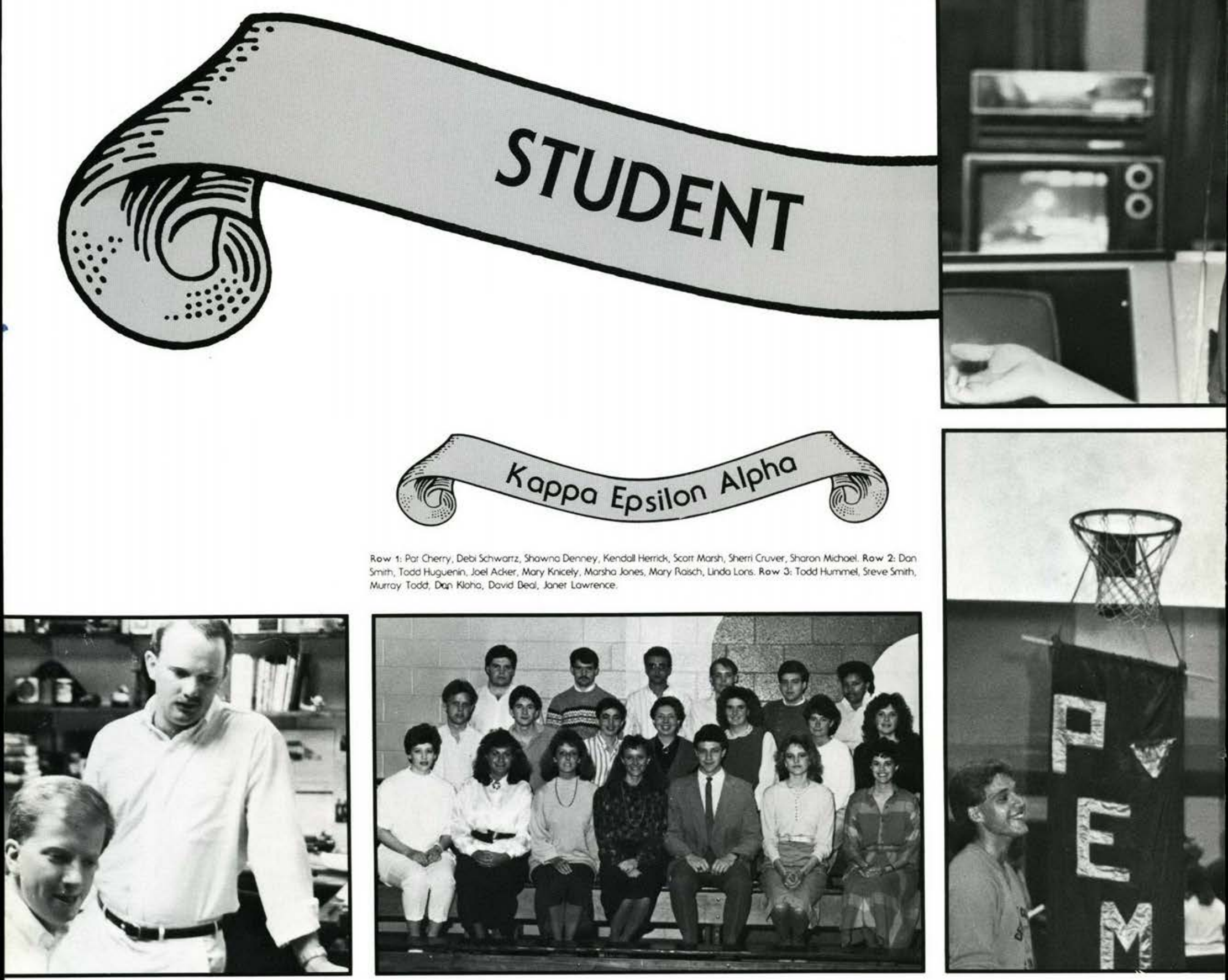

Row 1: Por Onerry, Debi Schwortz, Showno Denney, Kendall Herrick Scort Marsh, Sherri Crwer, Shoron Michoel. Row 2: Don Smith. Todd Huguenin, Joel Acker. Mary Knicely, Morsho Jones, Mary Roisch, Lindo Lons. Row 3. Todd Hummel, Steve Smith, Murroy Todot, Dan Kloho, Dovid Ded, boer Lowrence.
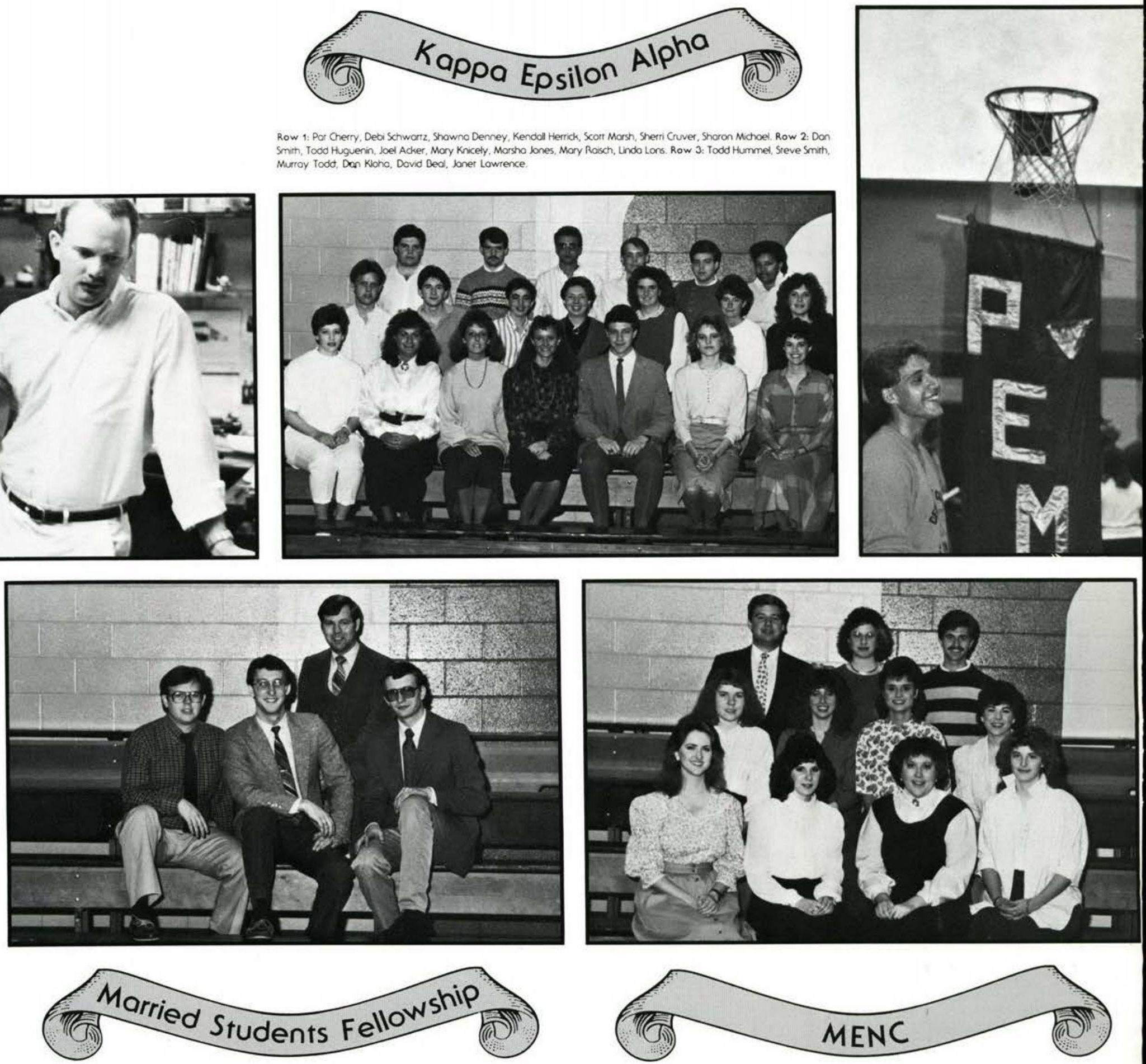


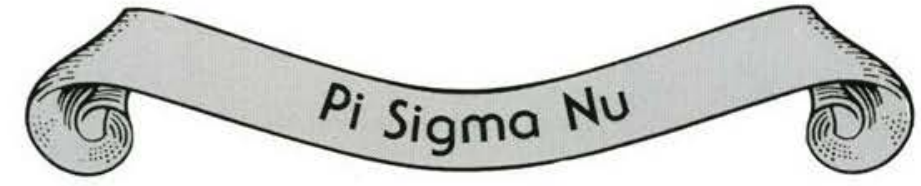

Row 1: Steve J. Schepers, Cregan Cooke. Keith Beres. Chris Link. Row 2: Tom Smatt, Rob Hicks, John Oswald. Andy froset. Row J. Derek Whalen. Tom Hill, Rick Deaman. Mark Swander. Row 4: John Angus. Rob Paswaters. Andy

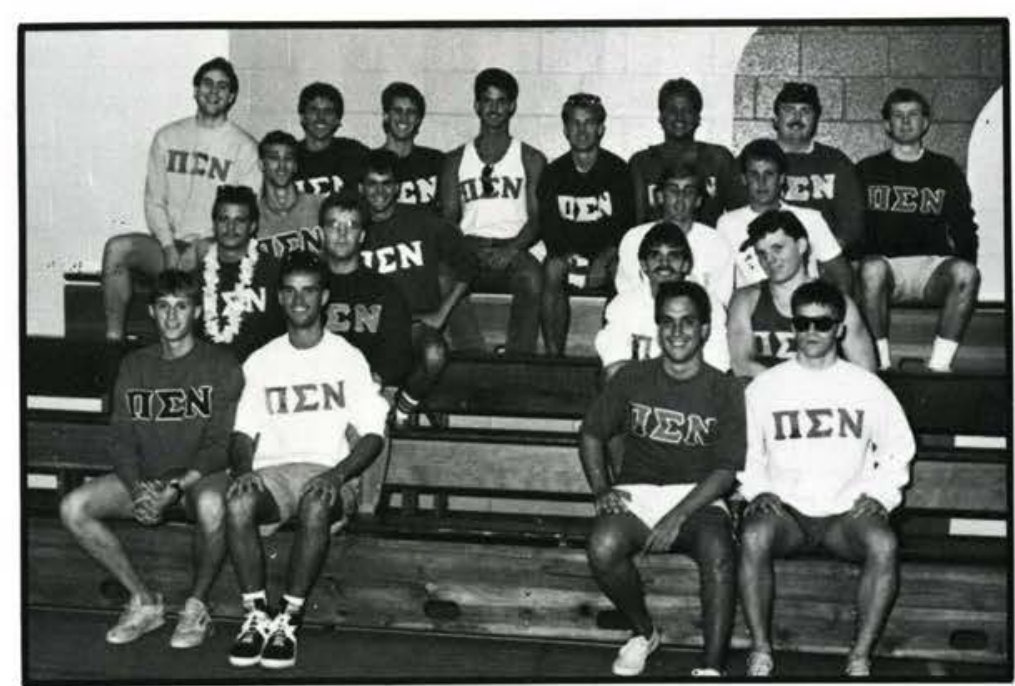
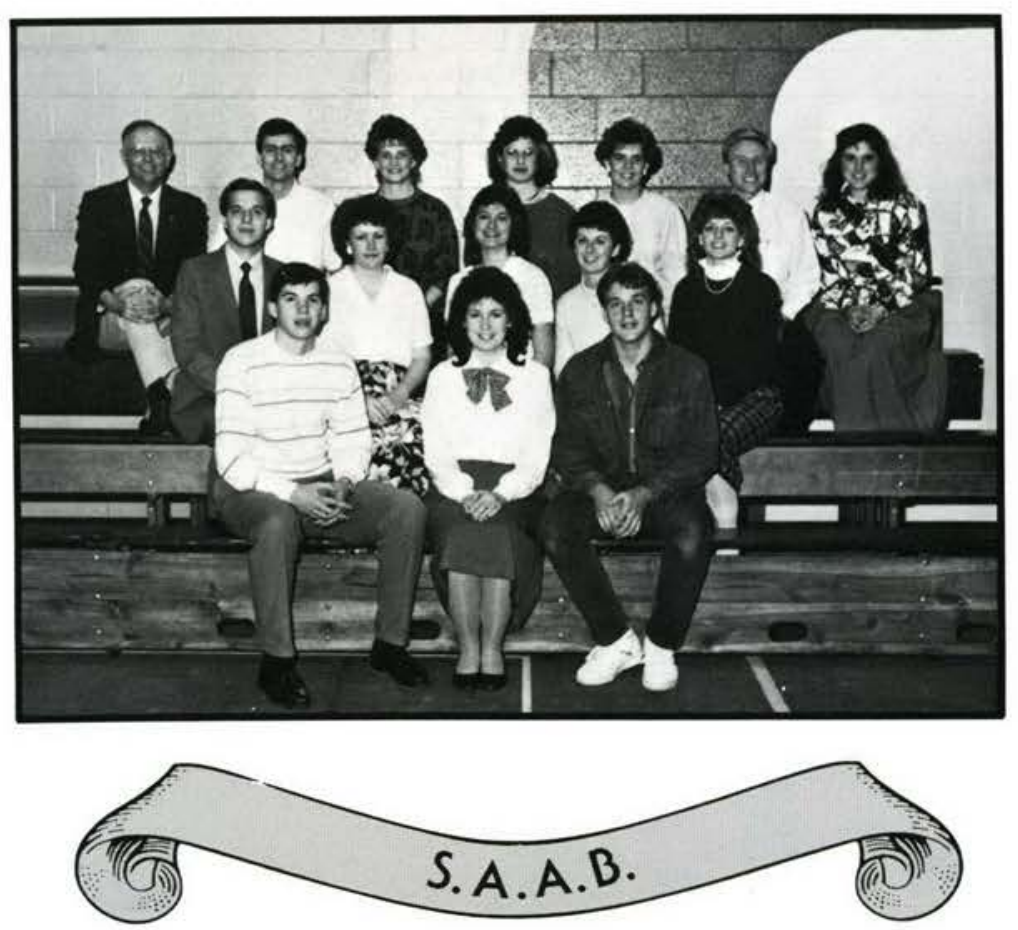

Row 1: Brion Sturgis. Holly Marshall. Mike Londis, Row 2: Eric Heimuth. Sandro Kennedy, Beth McGillivroy, Kim 

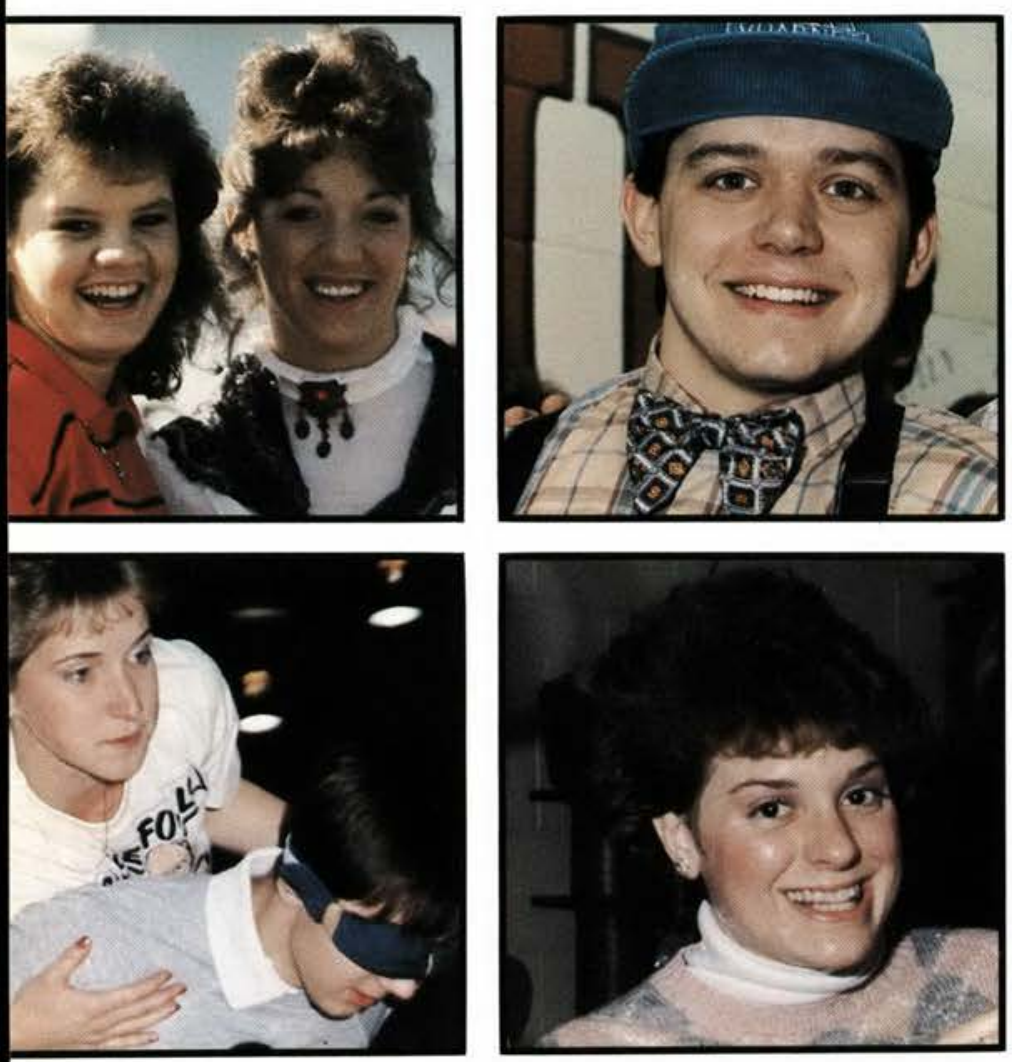
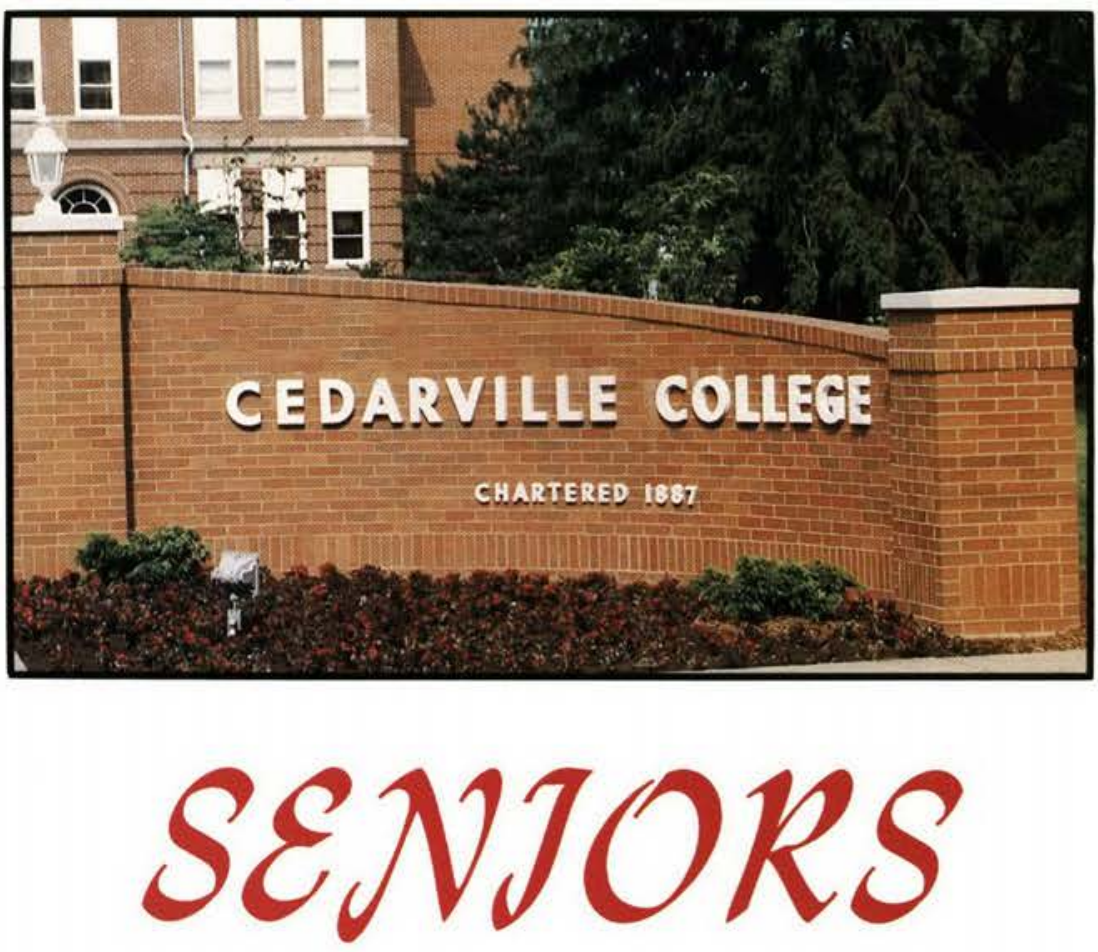

The time has arrived, and the Centennial class must pack their bags and move onto greater heights. Cedarville College has had a tremendous impact in each of our lives to broaden the spiritual and academic horizons ahead. Daily we have been encouraged and challenged to strive for Christian excellence through chapel messages, Christian ministry opportunities, and by our fellow classmates. This vast number of activities has helped our class become more unified.

We have been involved in a variety of fund raisers: letter mailing for the Alumni, donut sales, T-shirt sales, cake/balloon sales, and numerous film presentations. Our oneness has also helped us to become the victorious, undefeated, all school class-clash champs for the past four years, along with this year's 100 -mile run winners. Our class was also successful in changing the format of the survival kits. Now more parents are ordering, and the time for assembly and delivery has decreased.

It has been a memorable 4 years through all the joys and sorrows. We pray as a class that not only will our accomplishments be remembered, but also that our testimony and zeal for the Lord will influence the lives of others, for it was our desire to do everything with all our heart.

Yvonne Facey

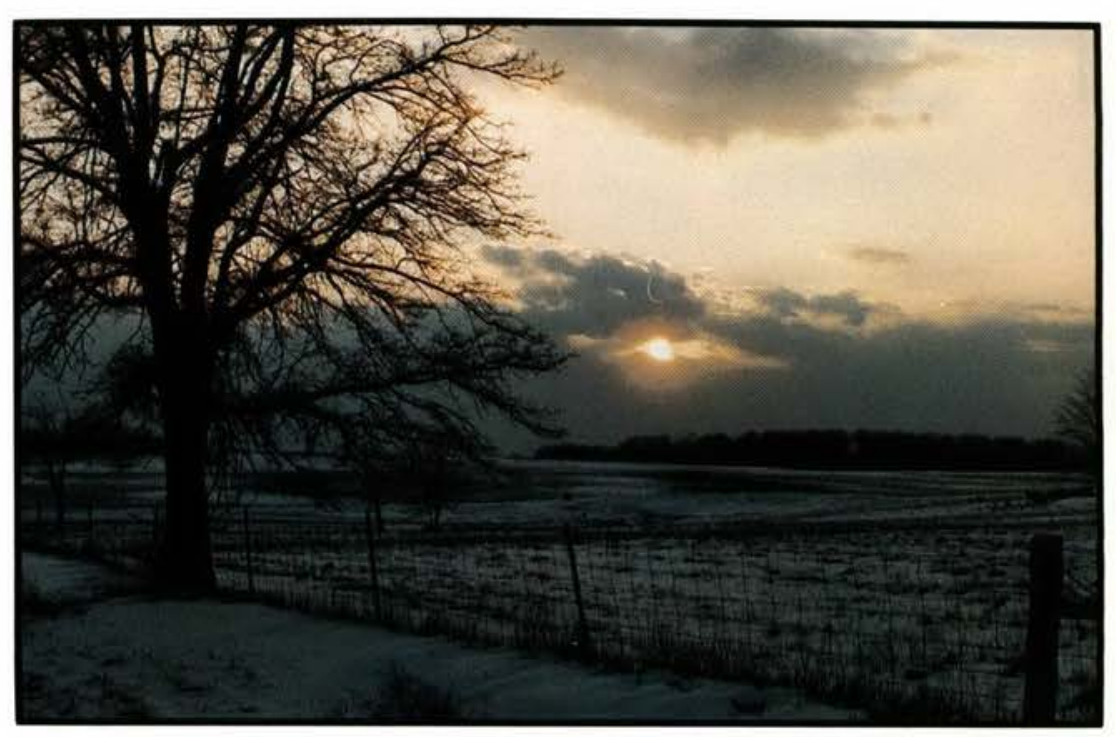




\section{9}
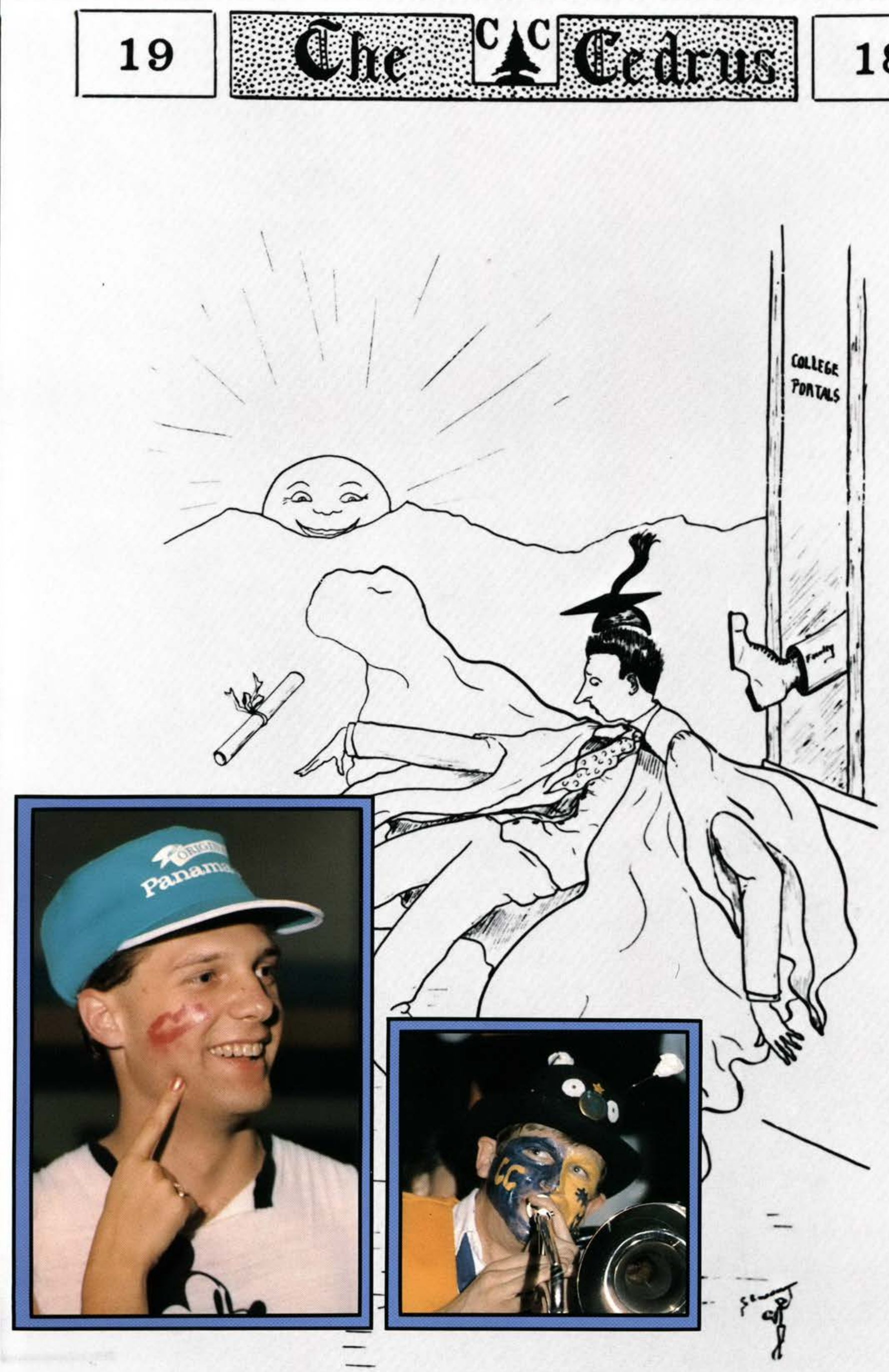


\section{A Night to Remember}

The 1987 Junior Senior banquer was most cerrainly "A Night to Renember." The special day began as many couples had heir piatures taken together on campus and then departed for the Nerhe lands Hotel in Cincinnati. Everyone enjoyed the meal and fellowstip during the first half of the evening. Then the vinging ensemble Sonlight presented a selection of several songs followed py an intermission which allowed couples to "mingle." The sentor tribure was one to remember! Coordinatad by Ruth Margraf the tribure was very likely the best that Cedarvilt had ever seen. As the banquer came to a close, so did A Nighr ro Rementper."
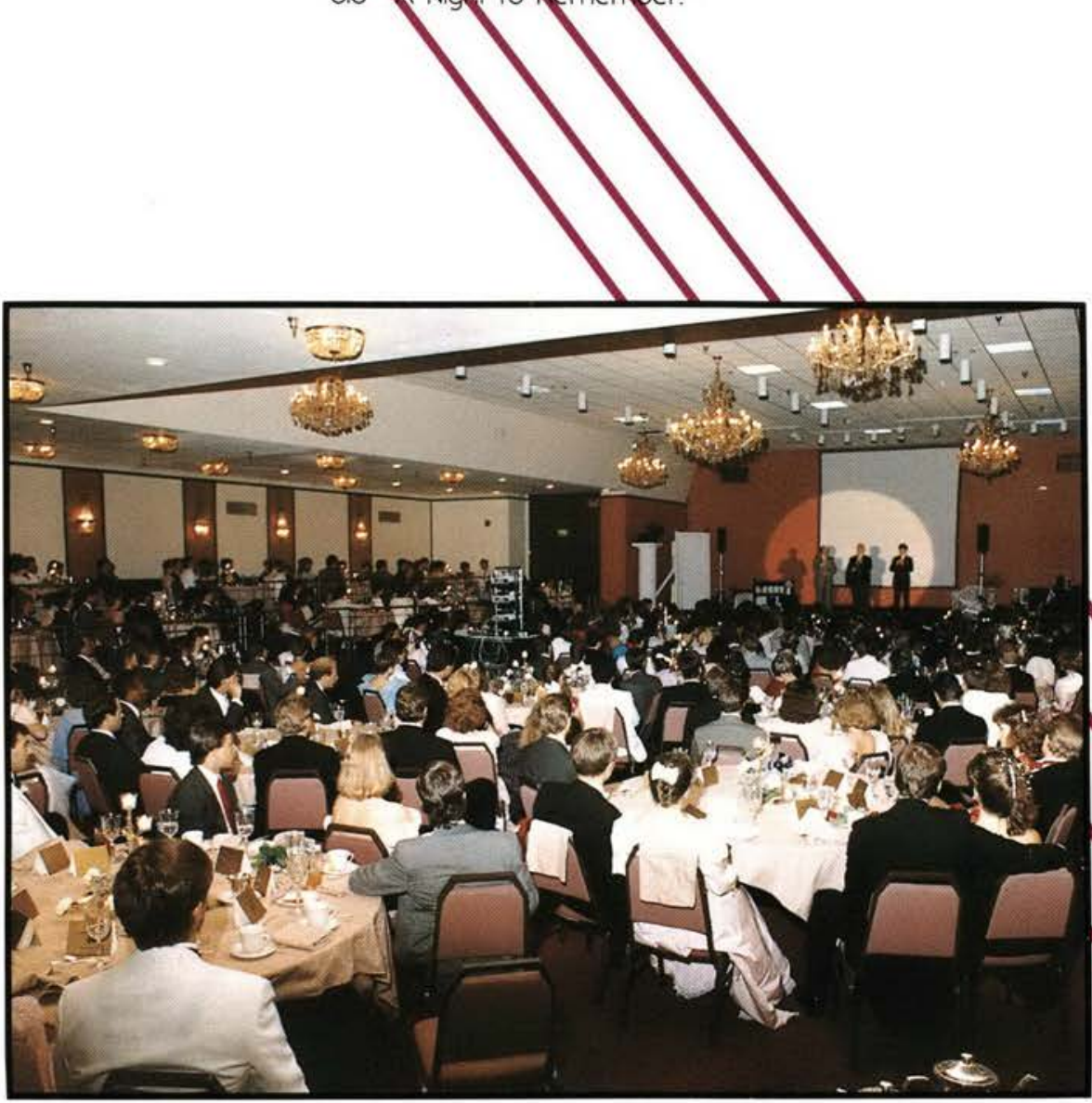
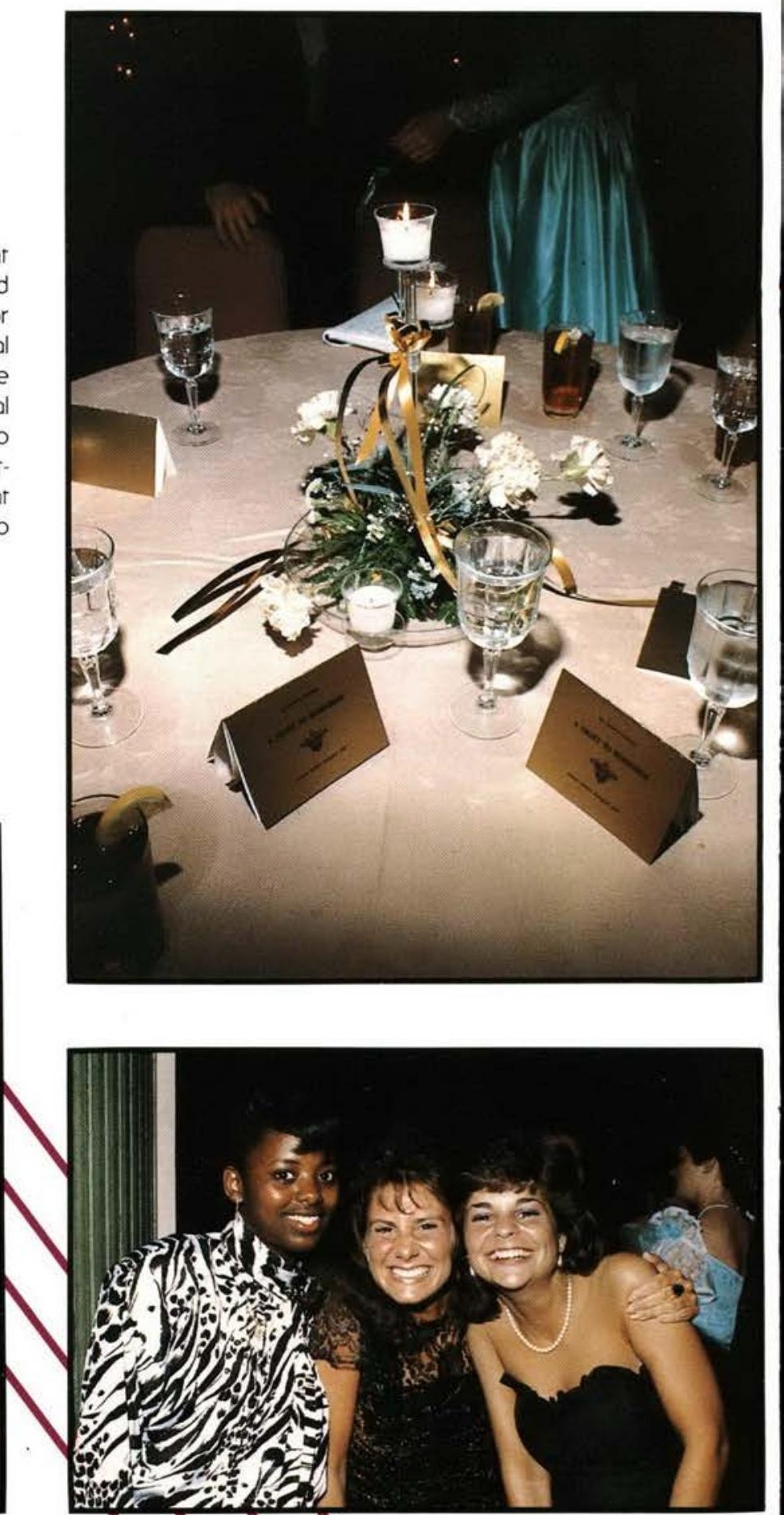
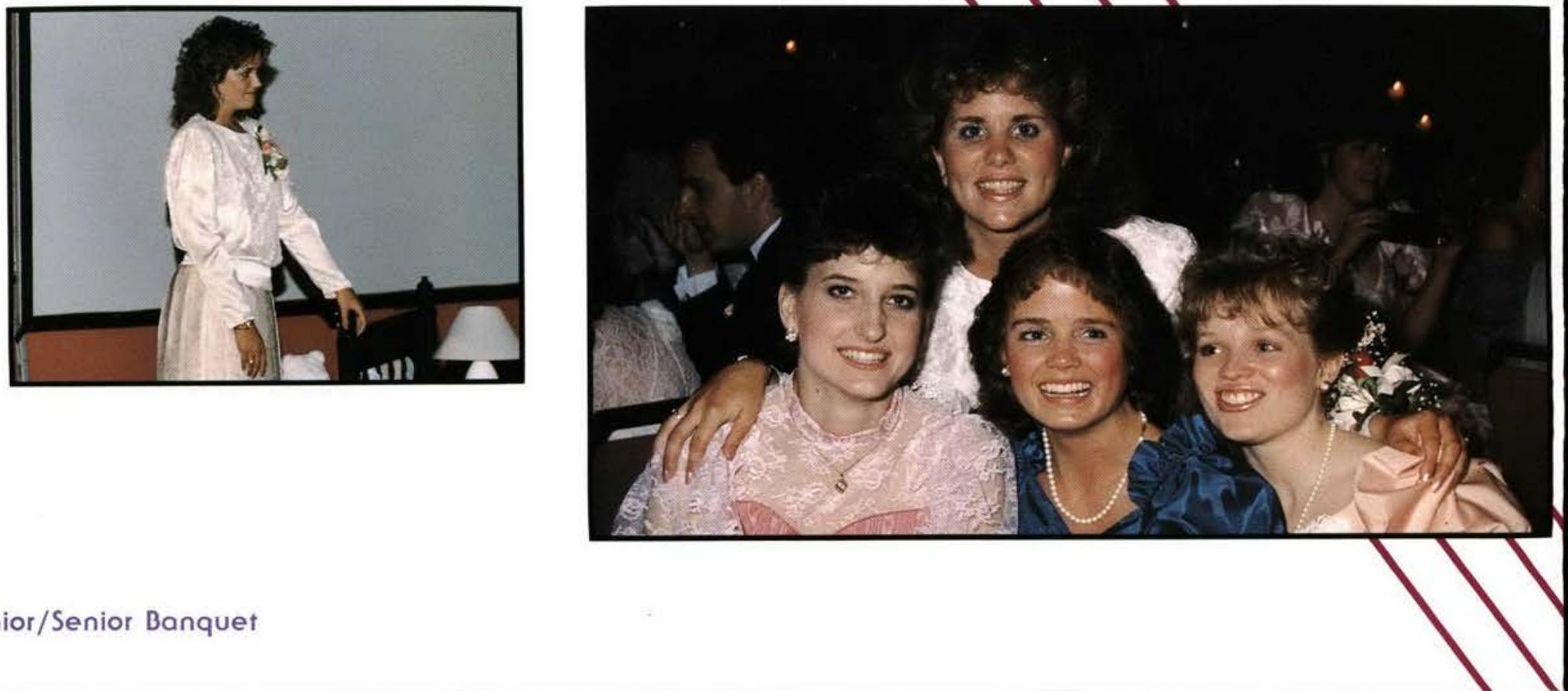



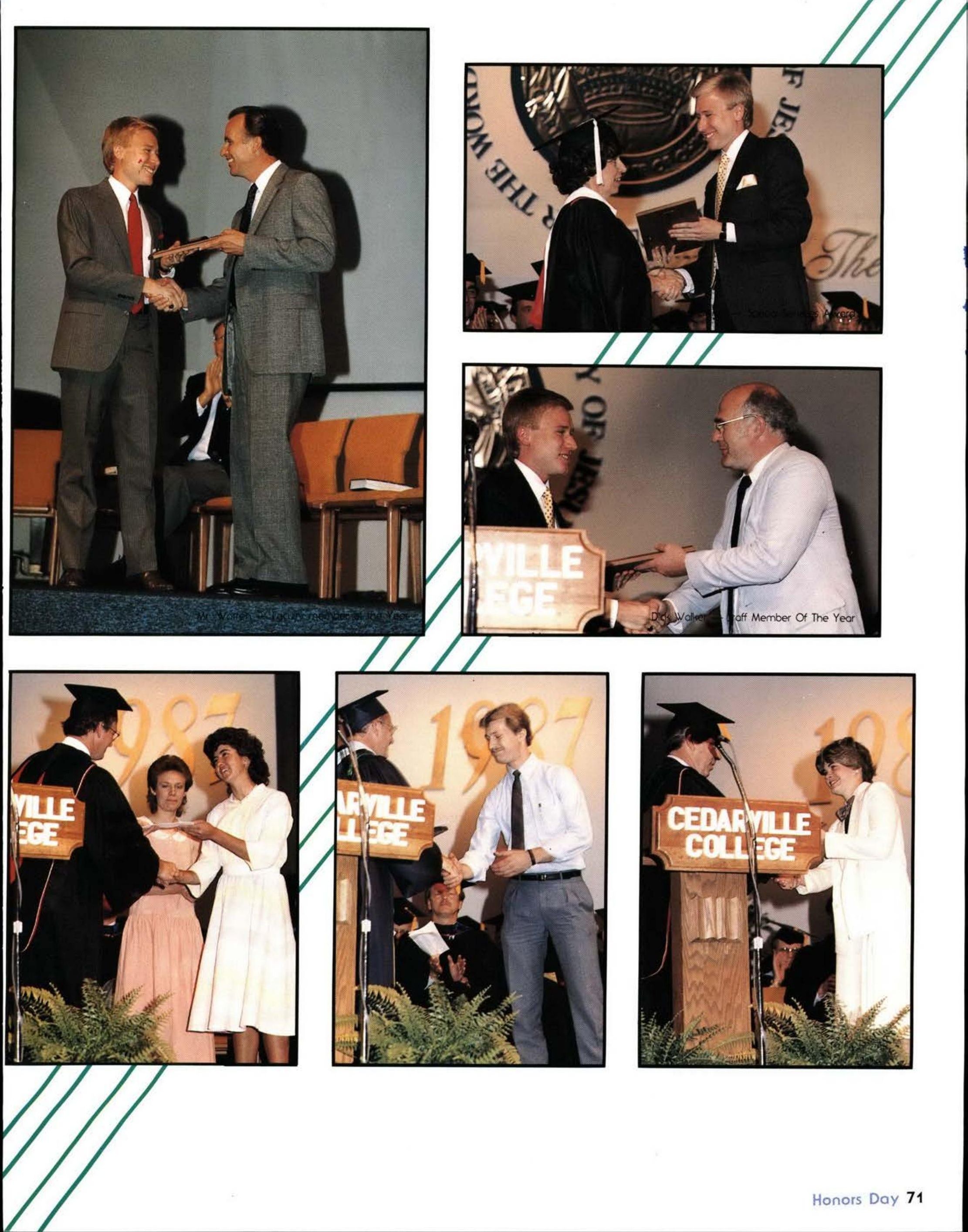




\section{Who's Who 1987}
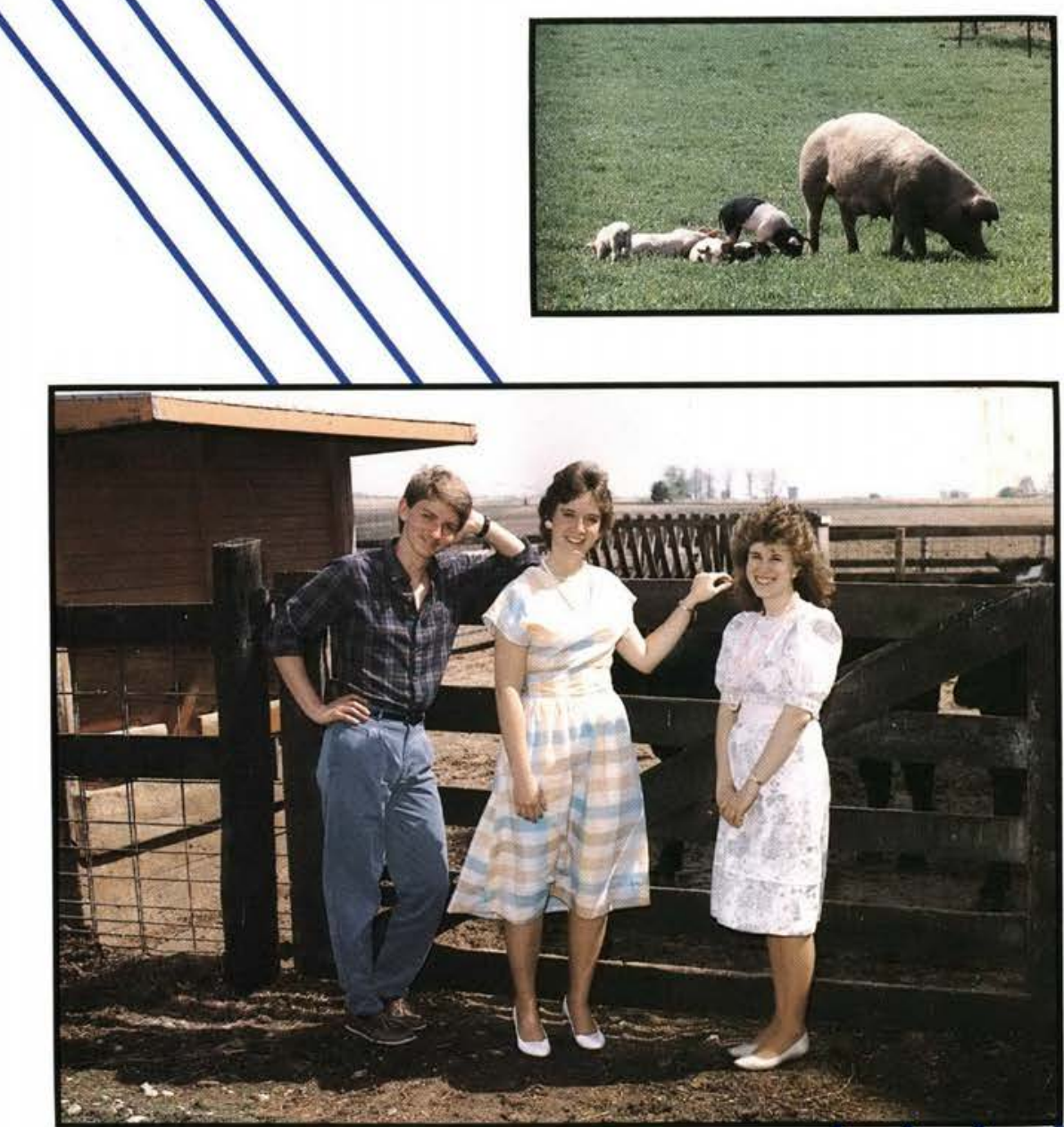

Brandon Waltz, Toni VanBeveren, Linda Shaffer

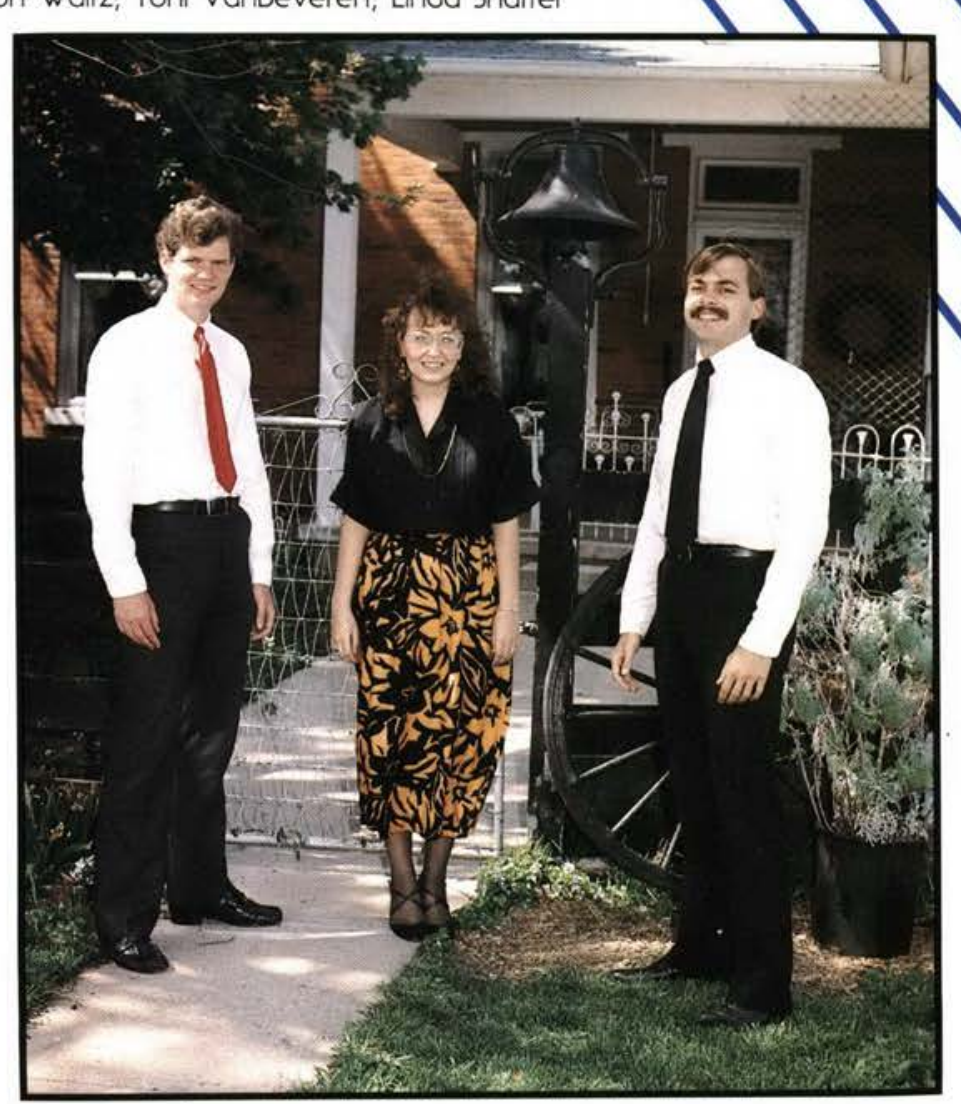

Dave Benner, Berh King, Phil Hohulin

Lisa Tyson, Laura Harrsough, Berh Hoecke 


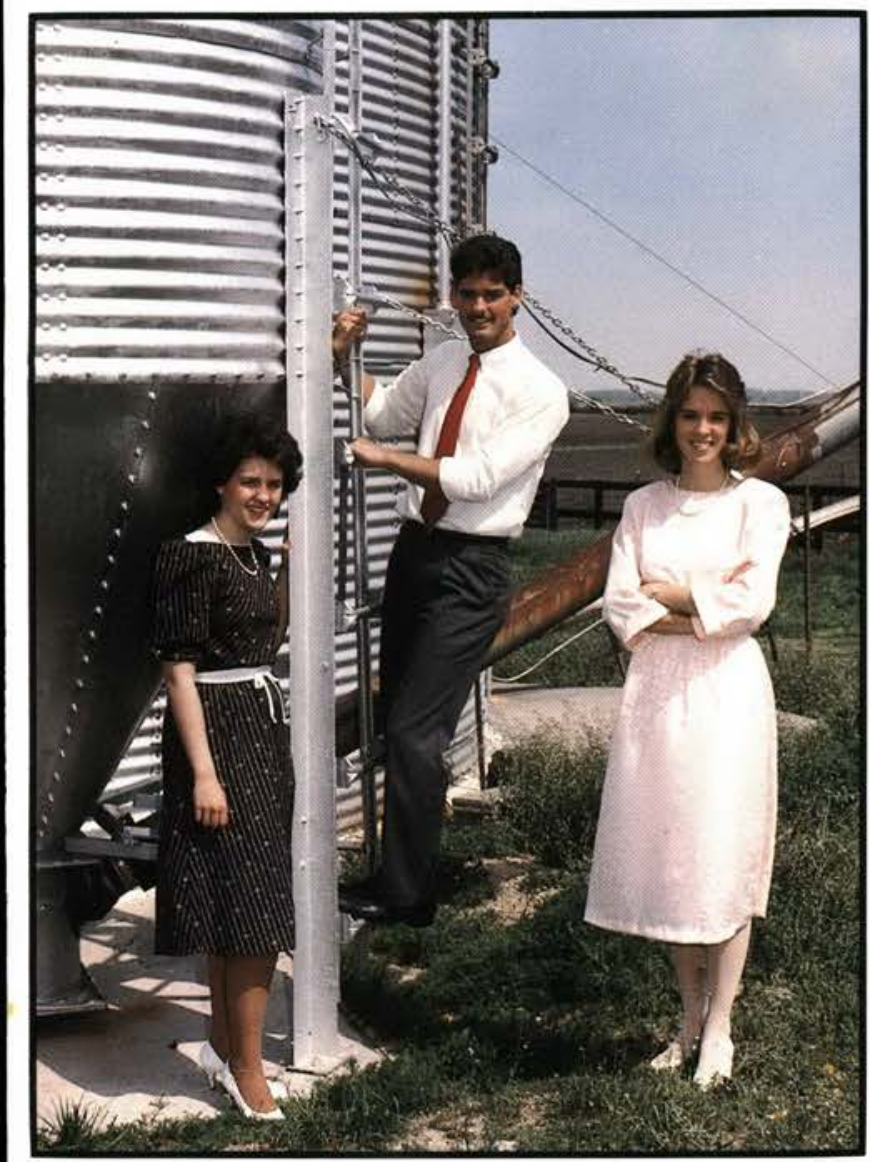

Julie Srackhouse, Rick Seidel, Doris Lindley

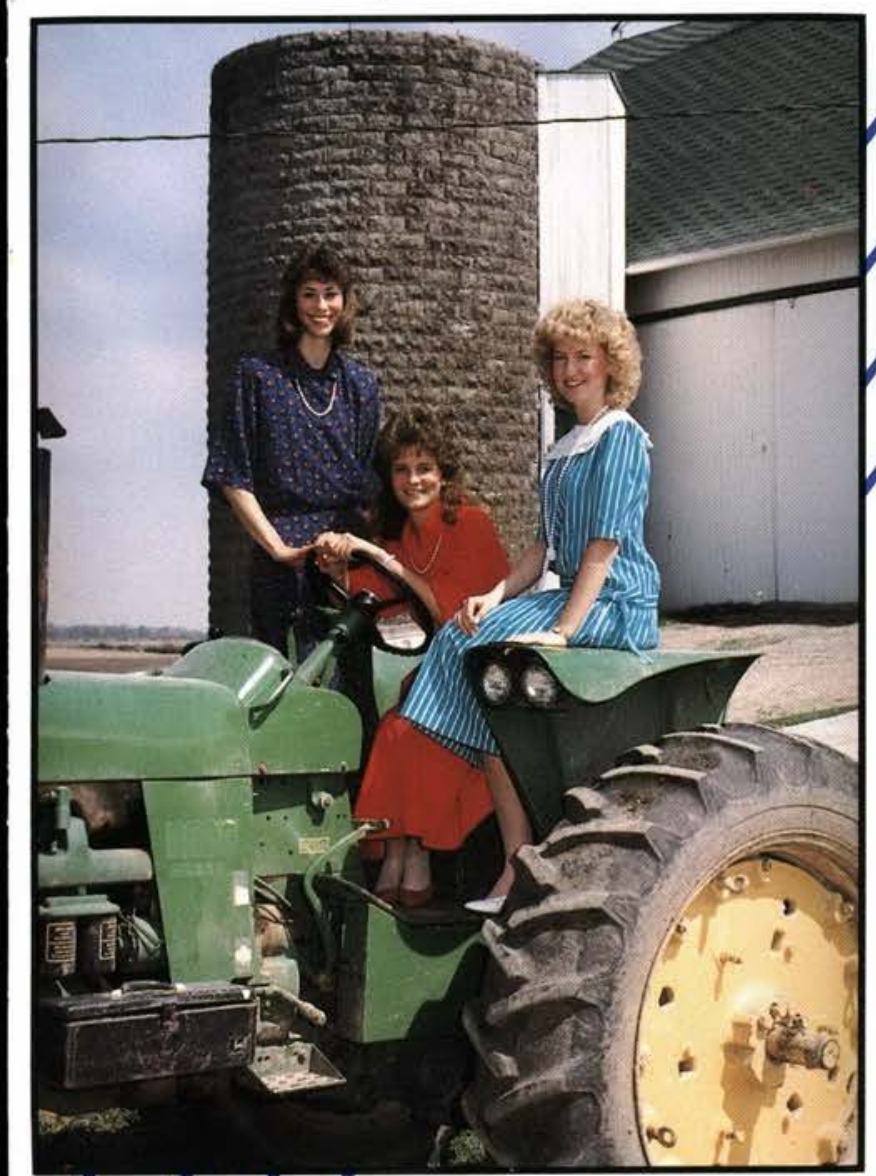

Danielle Kizer, Valerie Blaylock, Sherry Rorramel
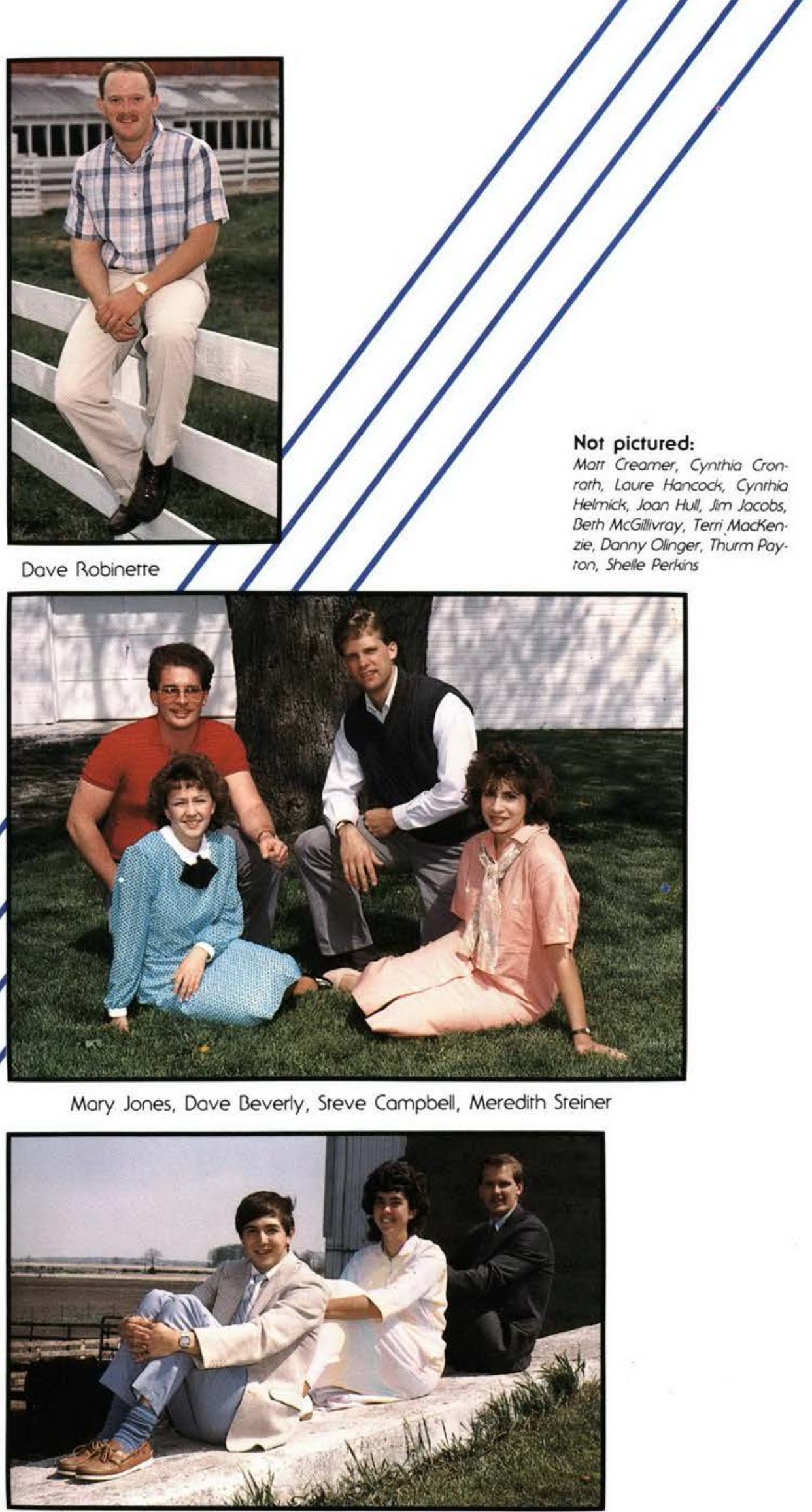

Jamie Beight, Nadine Terrill, Greg Moran 


\section{Centennial Graduation}

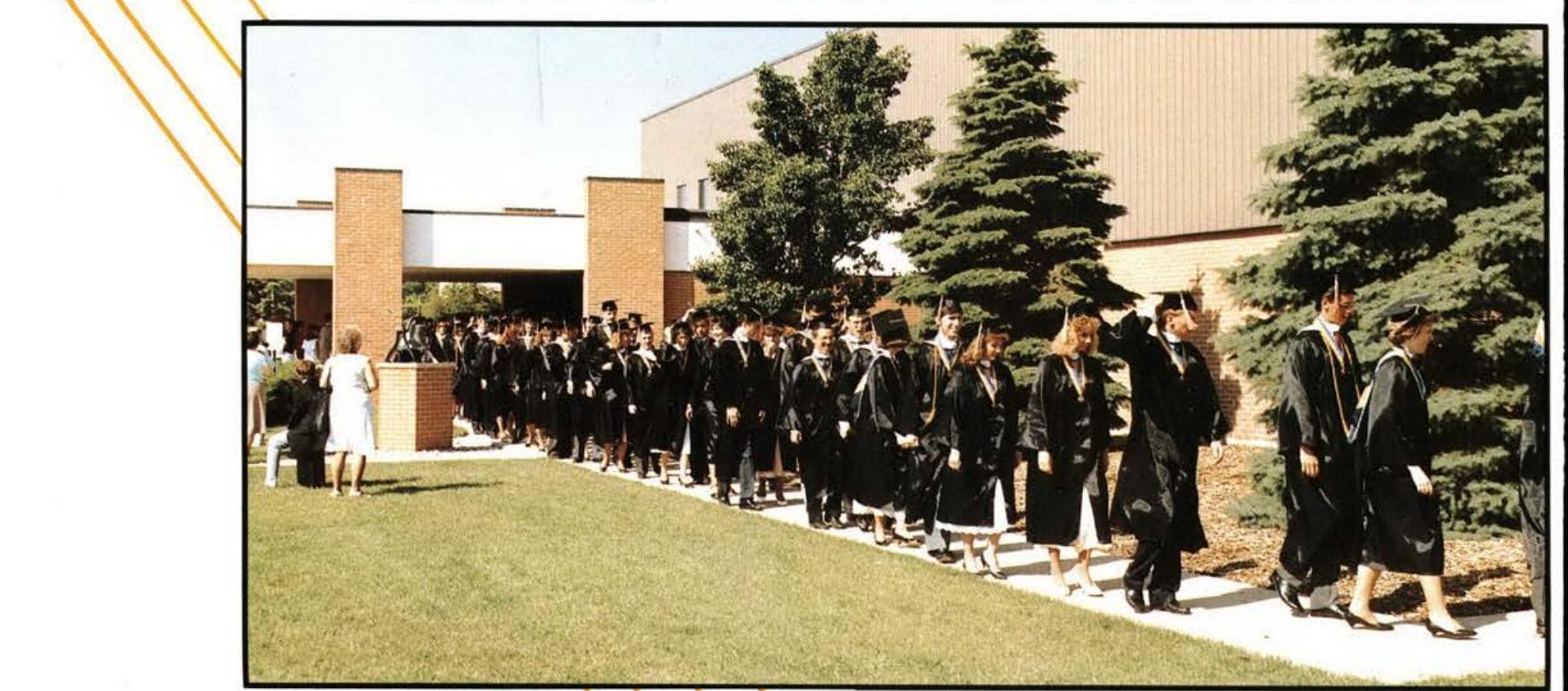

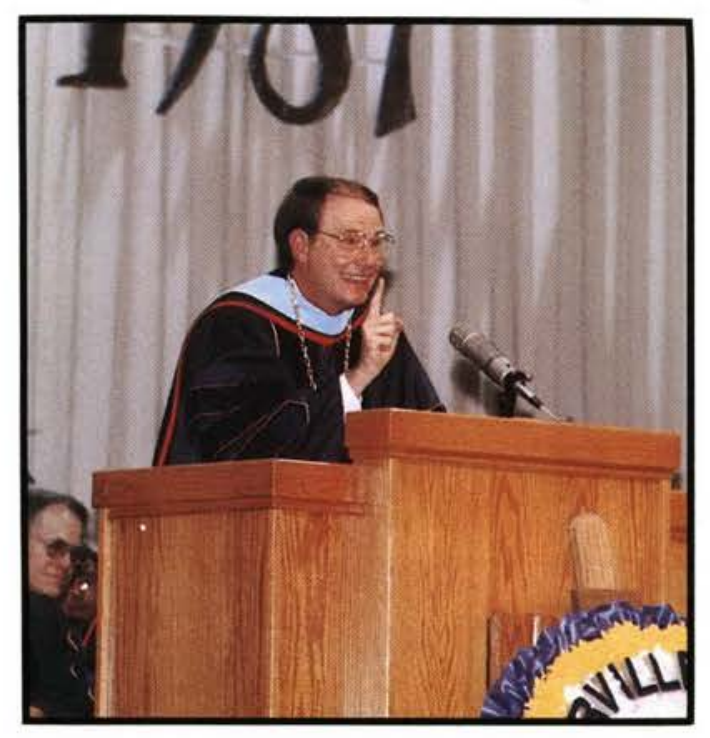

Centennial Class Verse

"For I know the plans I have for you," declares the Lord, "plans to prosper you and not to harm you, plans to give you hope and a furure." Jeremiah 29:11 NIV 

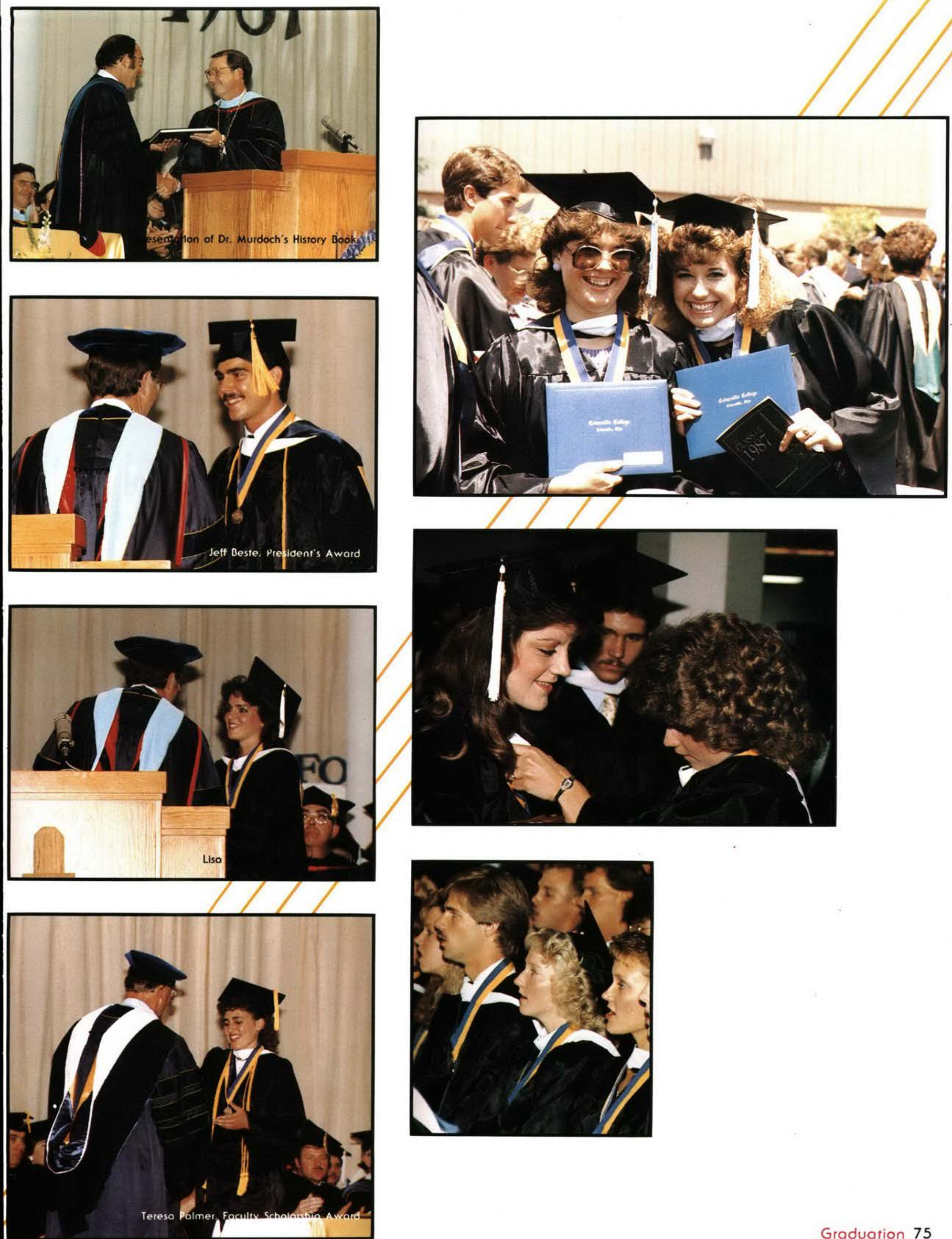


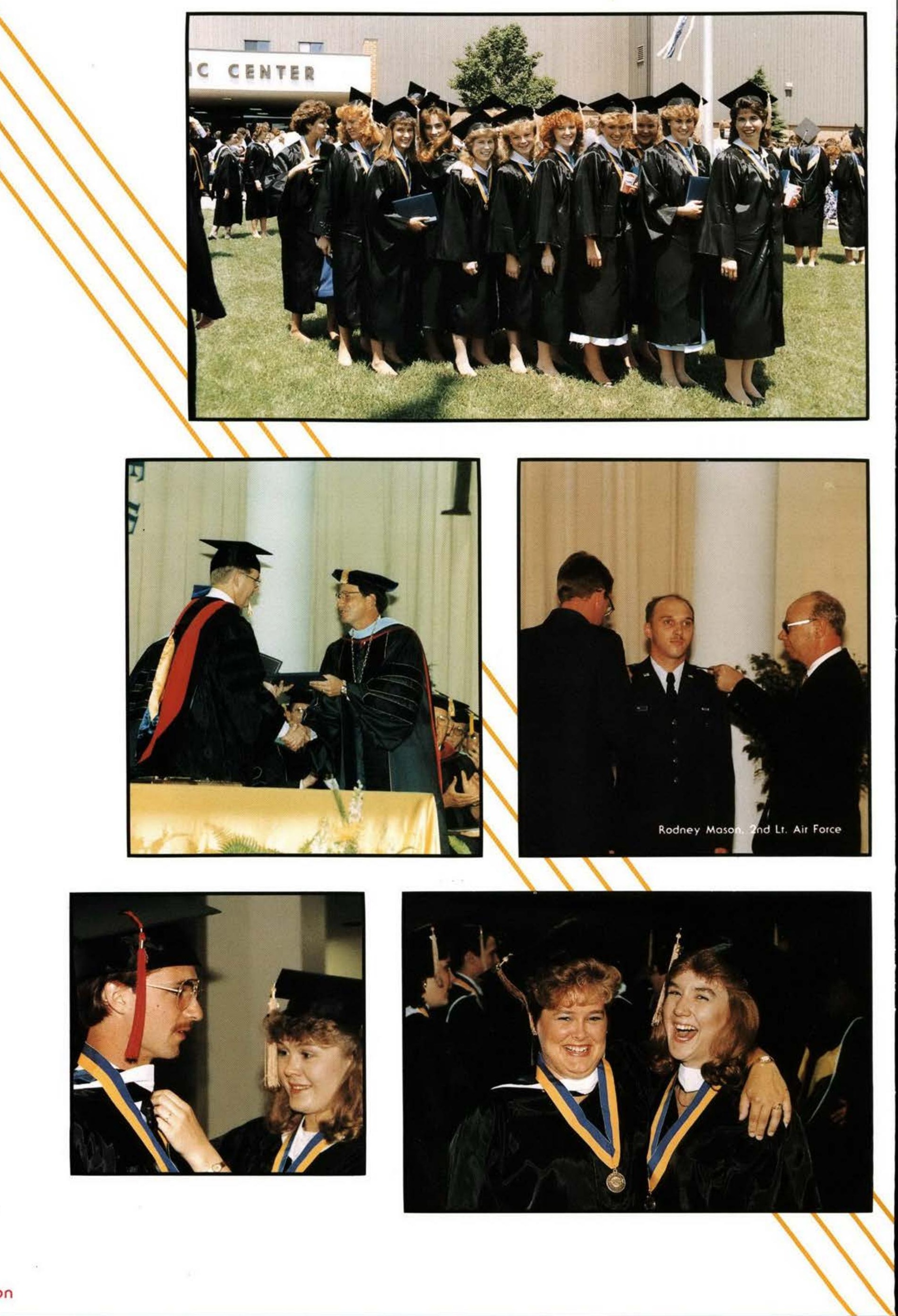




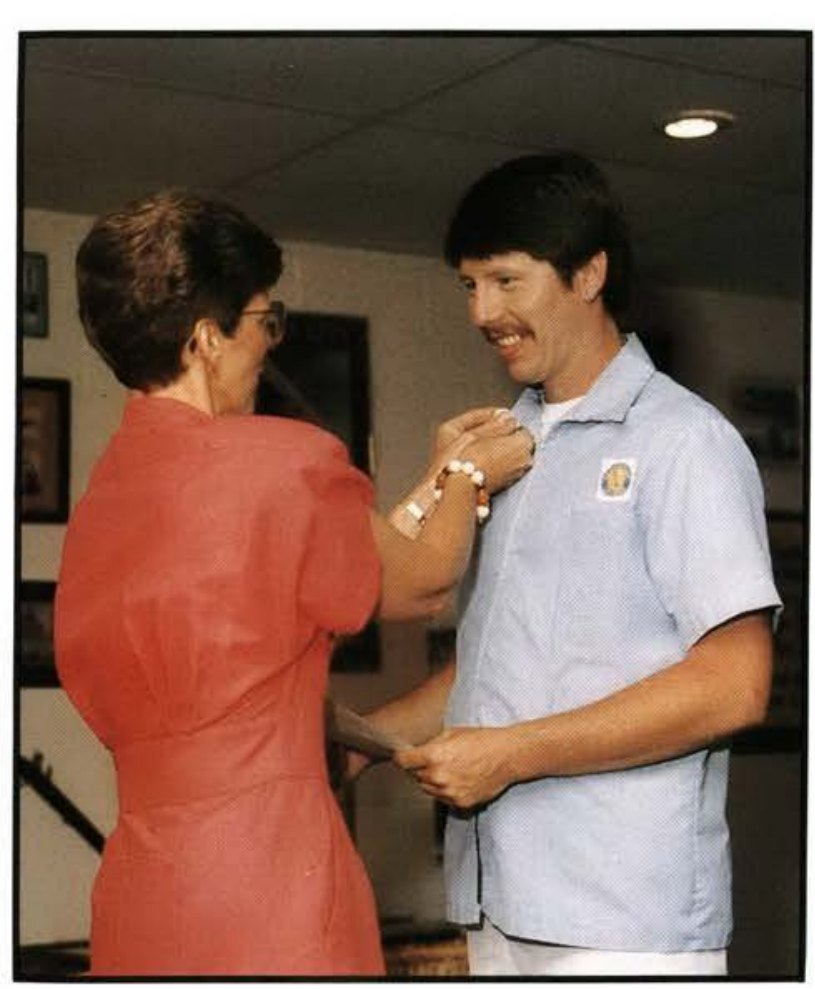

\section{Nurses' Convocation}

Friday, June 5, marked the second annual Nurses' Convocation for Cedorville College. Over 300 relarives and friends garhered in the Student Center fo honor the nursing class of 1987.

The nurses peresented their class gift of literarure for the department of nursing to Dr. Irene Alyn, chairman of the department. Dr. Alyn then gave the charge to the class. Special awards were presented to four graduares: Marla Sue Fuller, Berh J Woecke, Carherine M. Lombard and Lynda Masters Marriof. Becky Dye
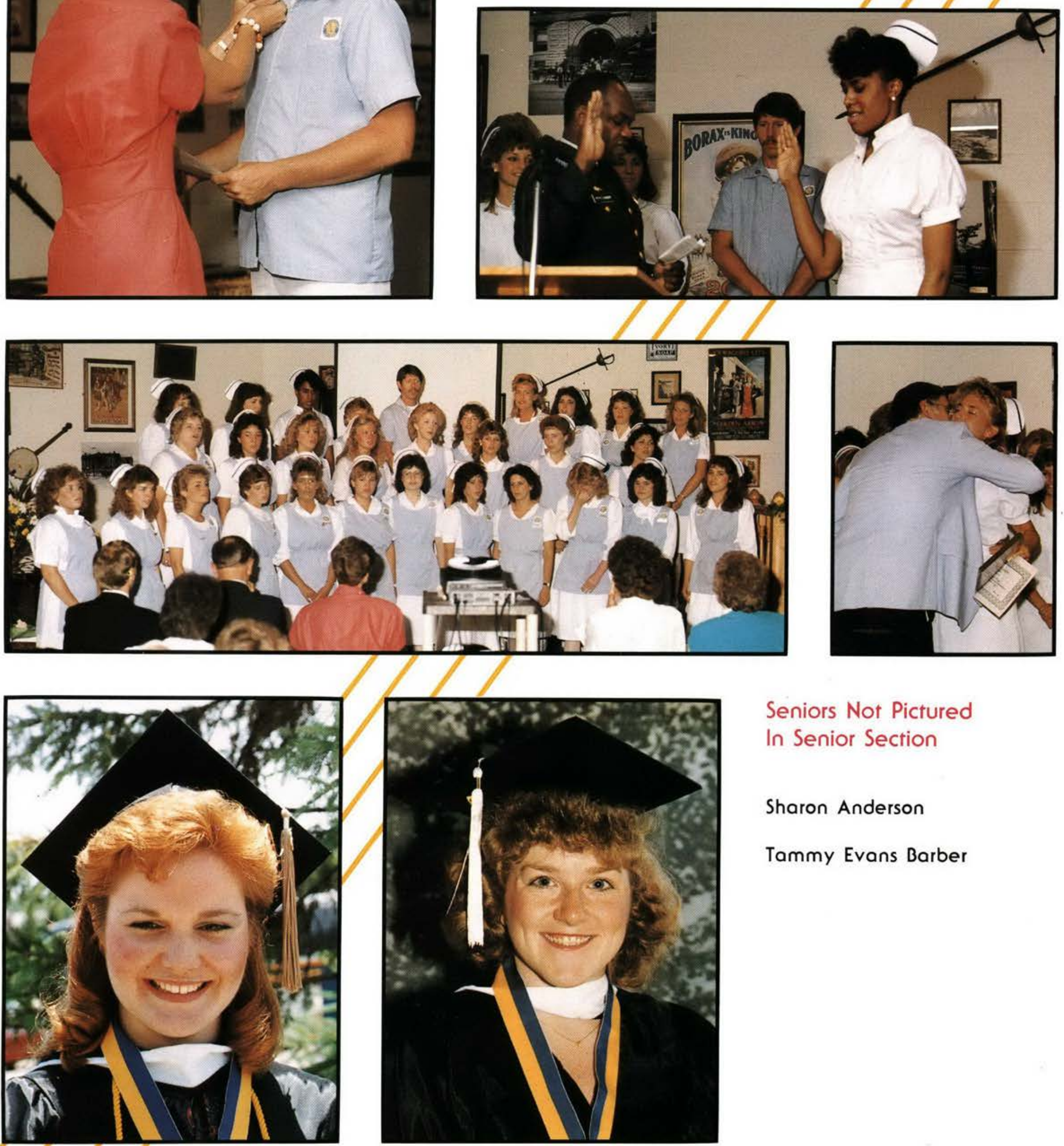

Seniors Not Pictured In Senior Section

\section{Sharon Anderson}

Tammy Evans Barber 


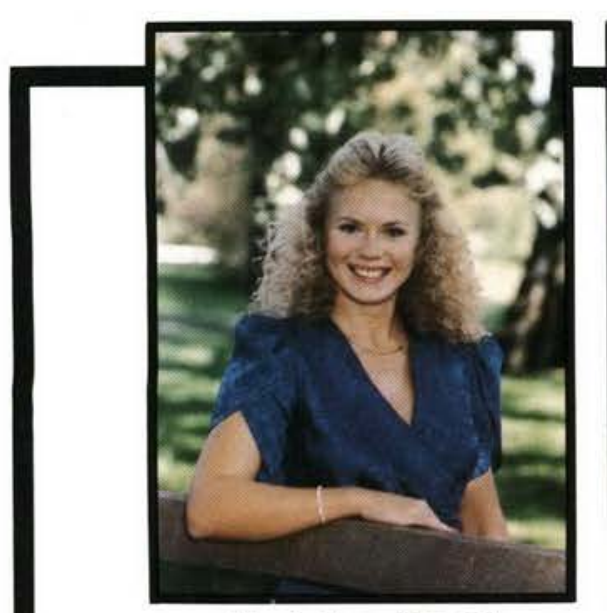

Cheri Ann Abresch

Nursing
Los Vegos, NA

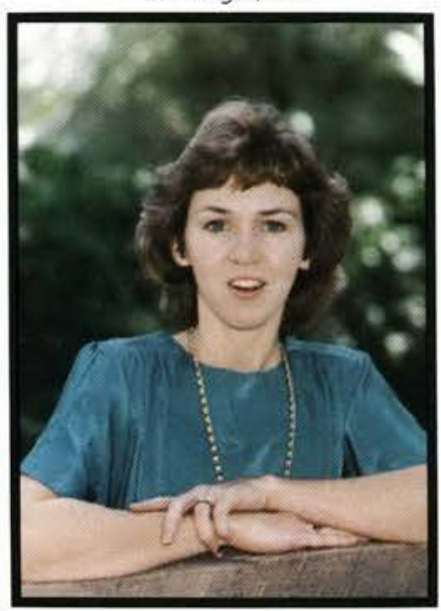

Cynthia Ray Anderson

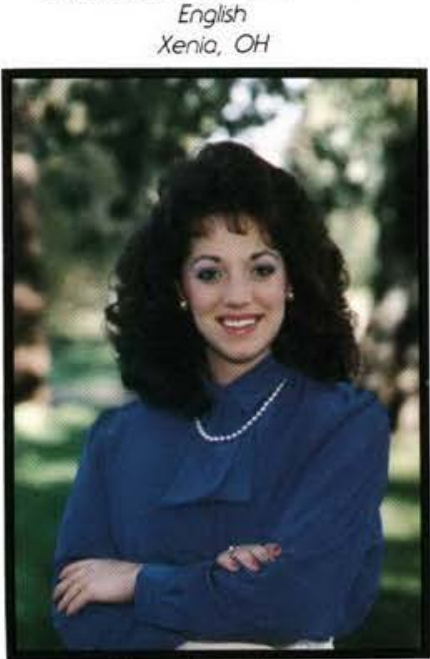

Lisa Armstrong Elementary Educarion

Rochester, MN

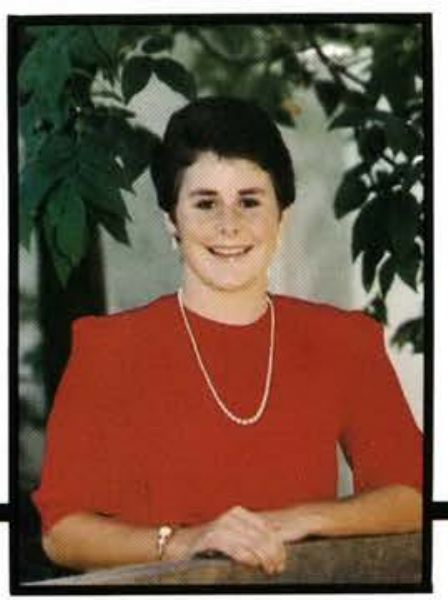

Susan Marie Baldis

Communicarion Arts

Simsbury, $C T$

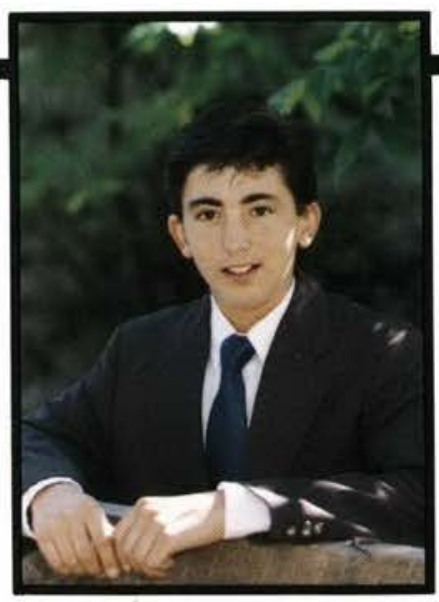

Joel David Acker

Business Administrarion

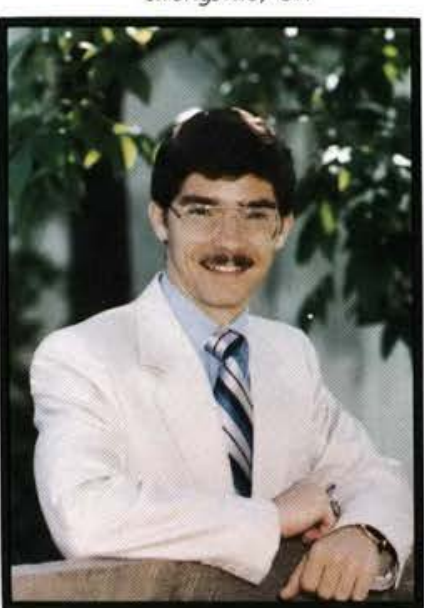

Kurt D. Anderson

Communication Arts

Soopoulo, Brozil

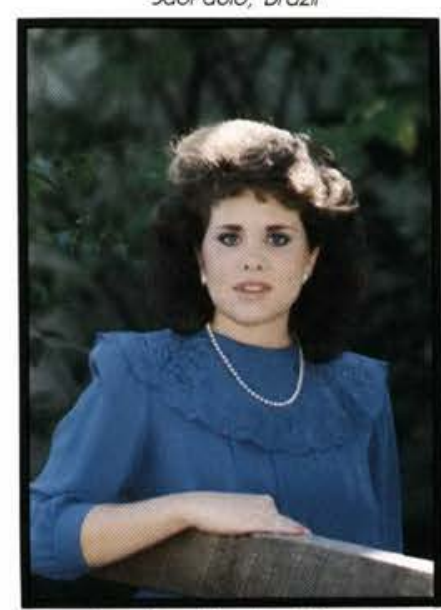

Denise A. Auckland

Nursing

Soringfield, $\mathrm{OH}$

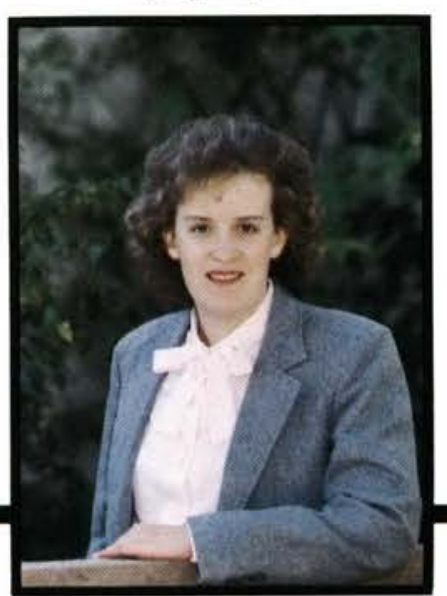

Sarah Ann Barr

Psychology

Downers Grove, IL

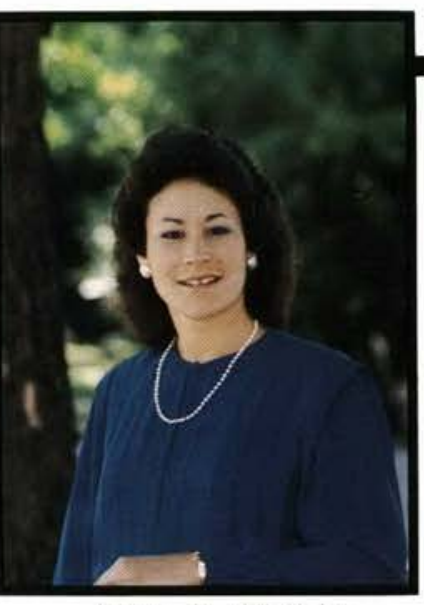

Laura D. Albright

Nursing
Grand Ledge, M

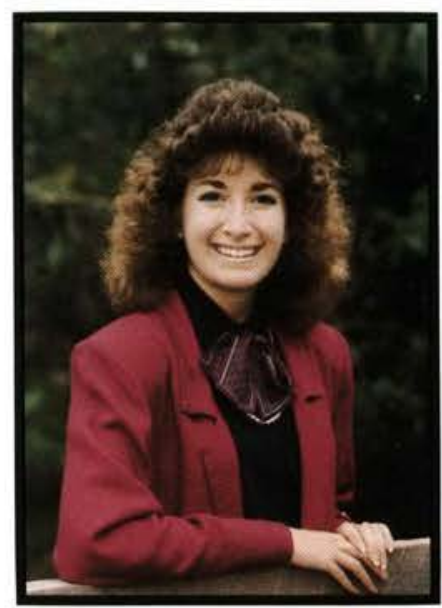

Leslie Marie Anderson

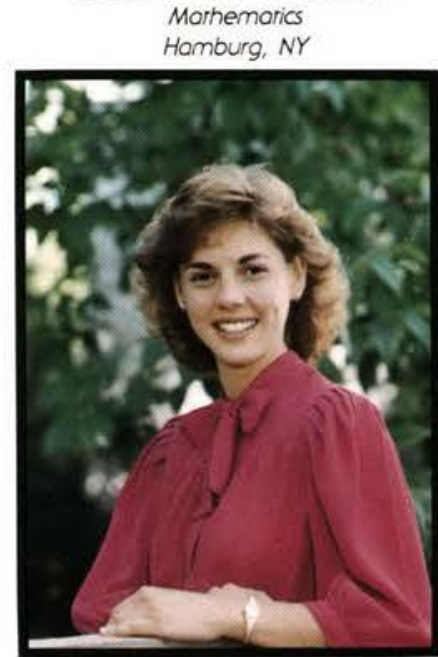

Sharon R. Augenstein Biology

Columbus, $\mathrm{OH}$

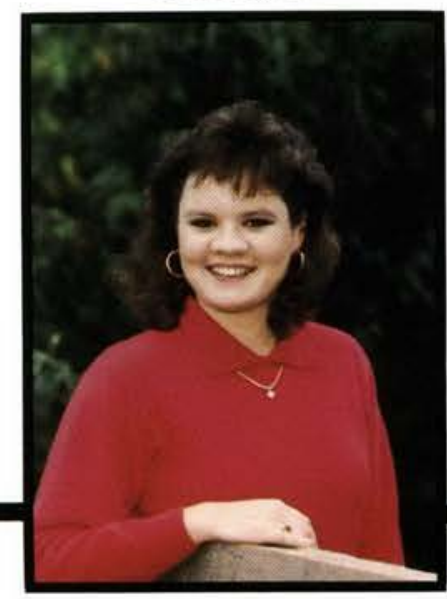

Tammy Jo Barton

Elementary Educarion

Wheoron, I

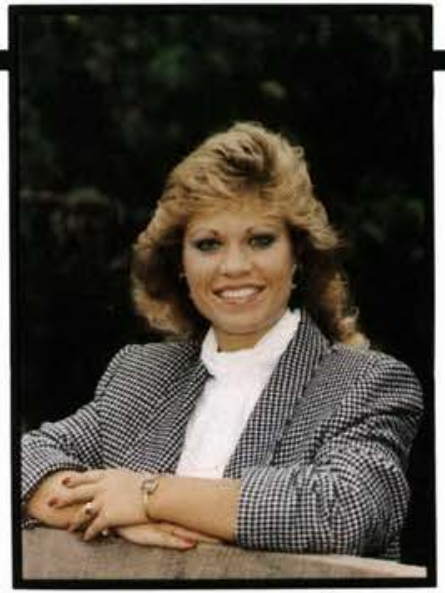

Amy Suzanne Allen

Psychology

Oxford, MI

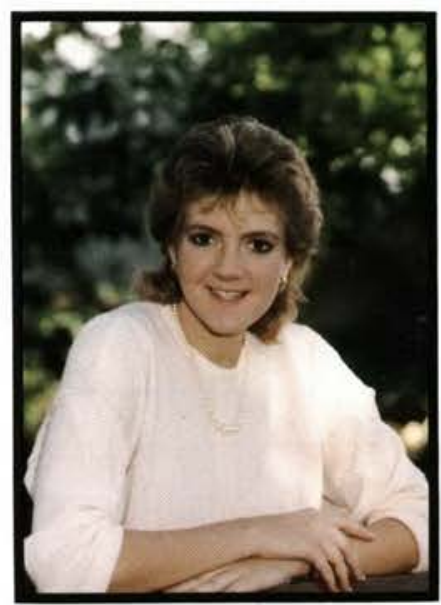

Lorelei Anderson

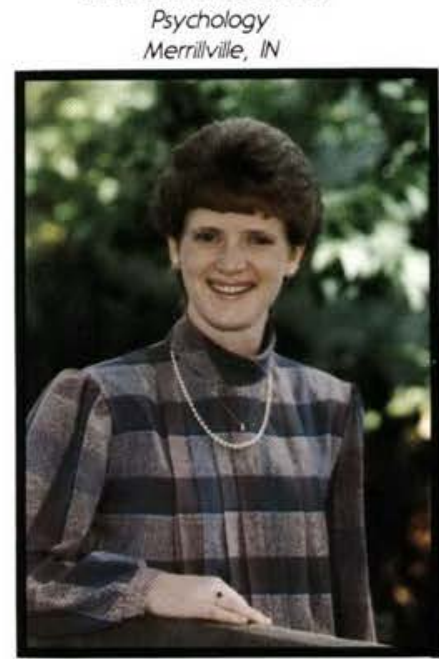

Rebecca Jeanne Averill Elementary Educarion New Hompron, IA

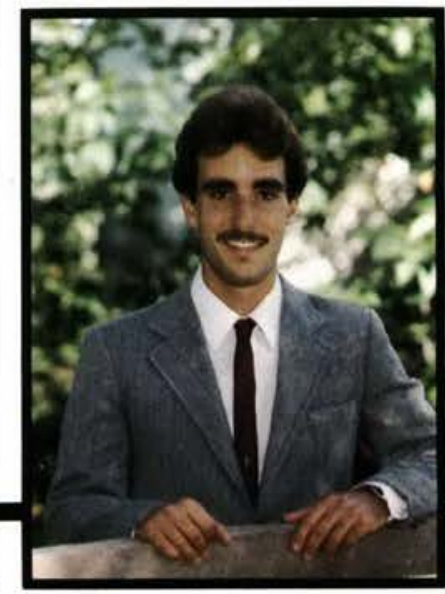

Michael Paul Basler

Business Administrorion 


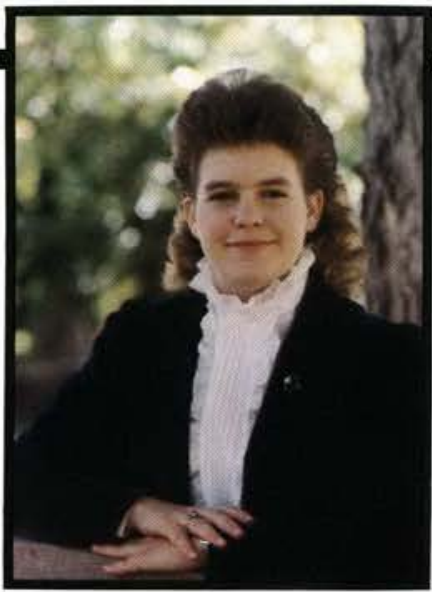

Denise M. Bass

Physical Educorio

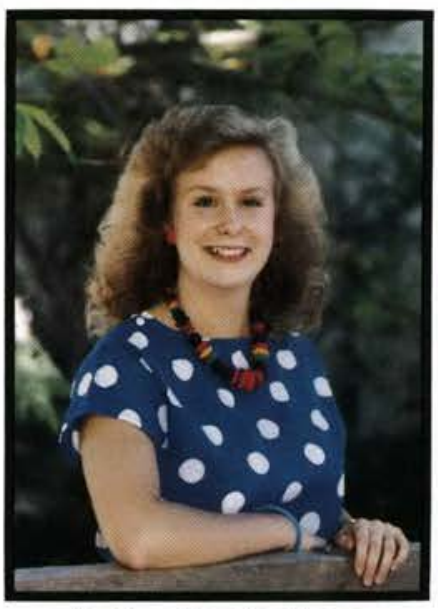

Marilyn Louise Bean

Nursing
Vest Chicago, It

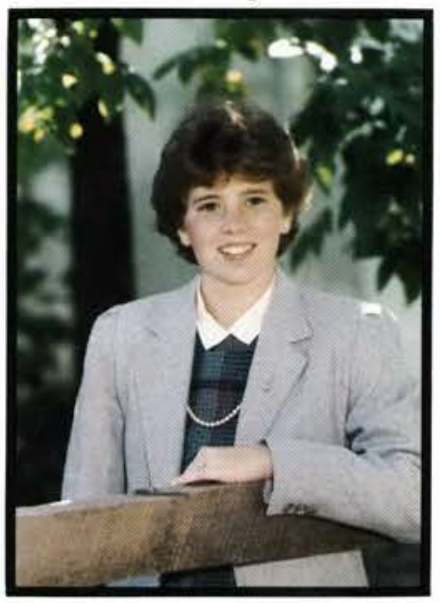

Debbi Lynn Berdy

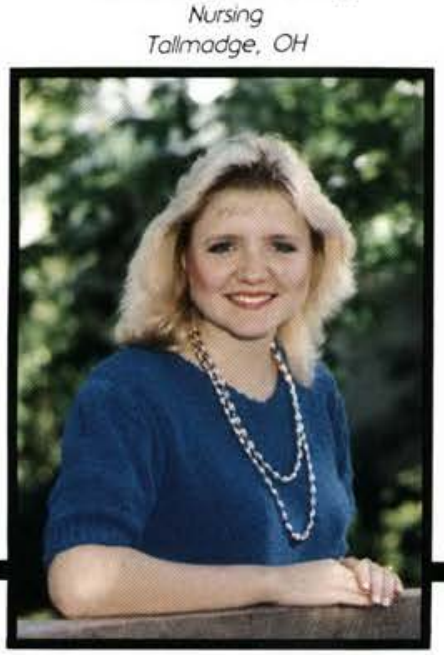

Tamra Noel Bielski

Elementary Educarion

Flint. MI

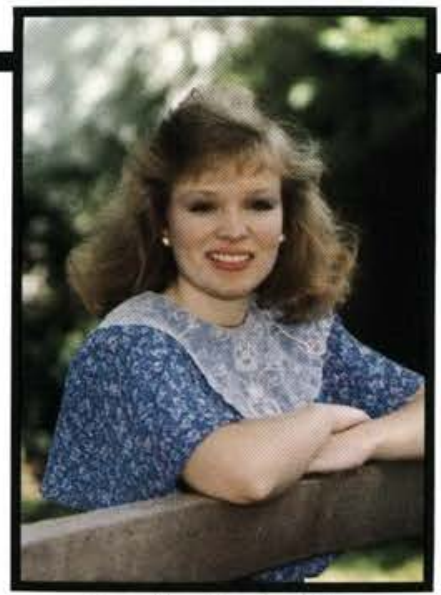

Dina M. Batchelder

English Education

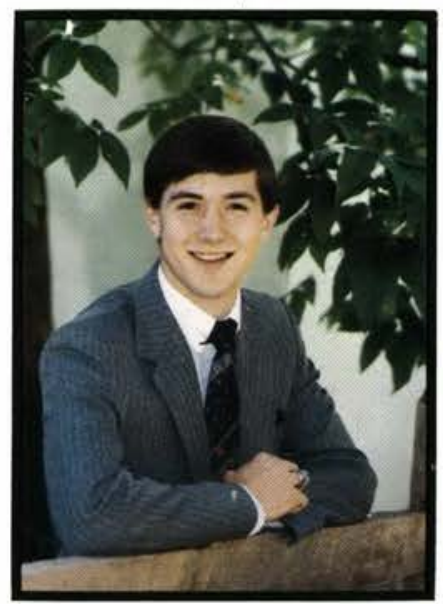

James D. Beight

Preseminary Bible Mojor

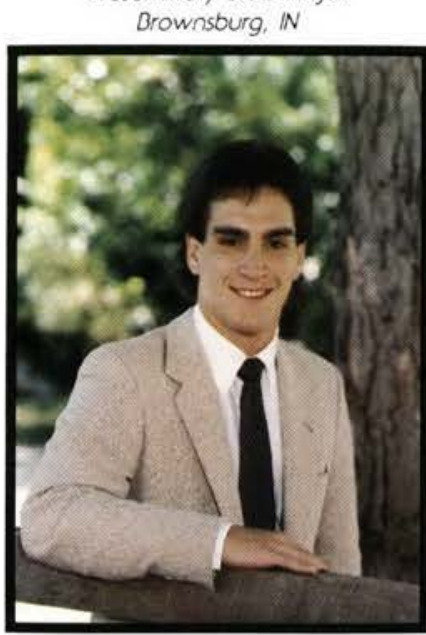

Keith A. Beres

Business Administrarion

Akron, $\mathrm{OH}$

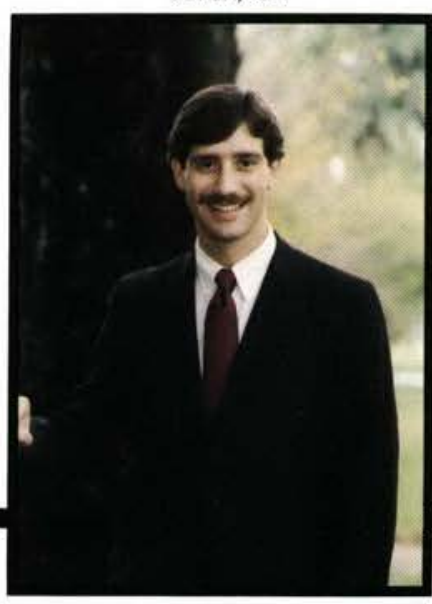

John R. Bigelow Jr.

Preseminary Bible Major

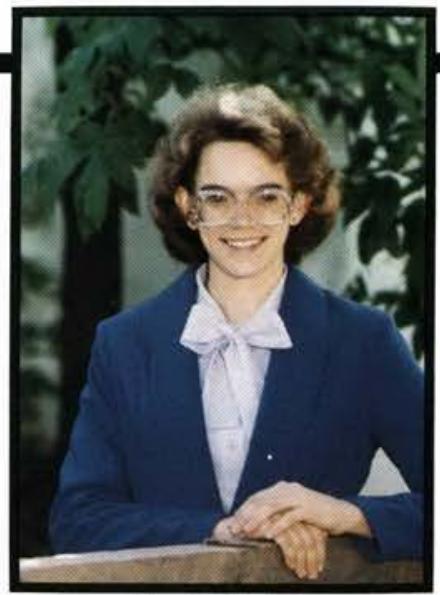

Jane Marie Baughman

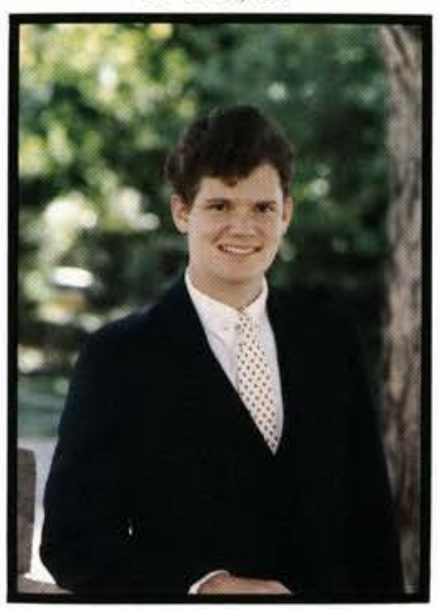

David Jay Bennett

Preseminary Bible Mojor

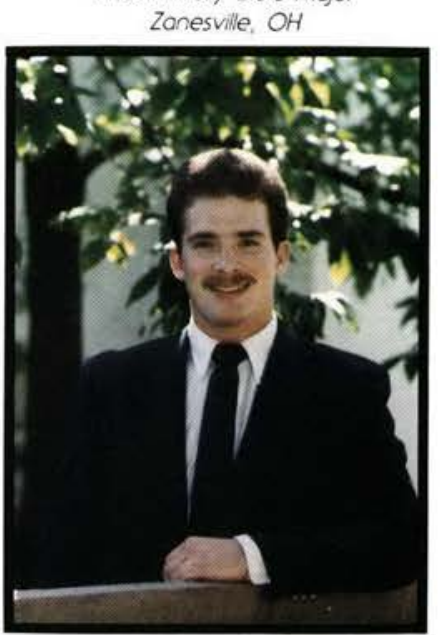

David Edward Beverly

Preseminary Bible Major

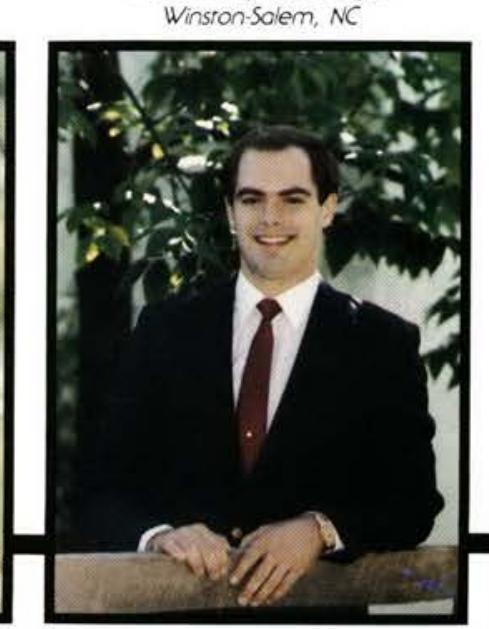

Adam Bird

Biology

New Richmond, $\mathrm{OH}$

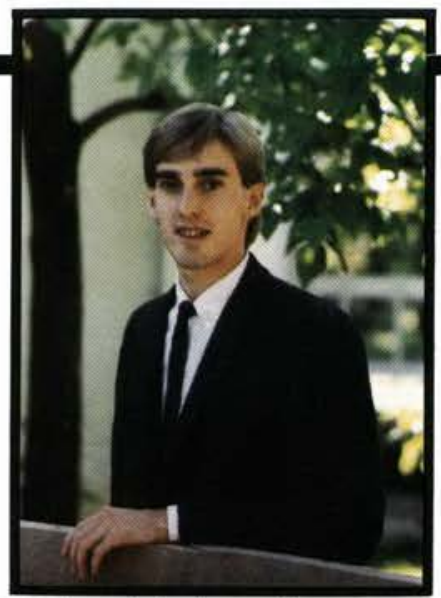

Richard Anthony Beaman

Business Administration

Springfield, ith

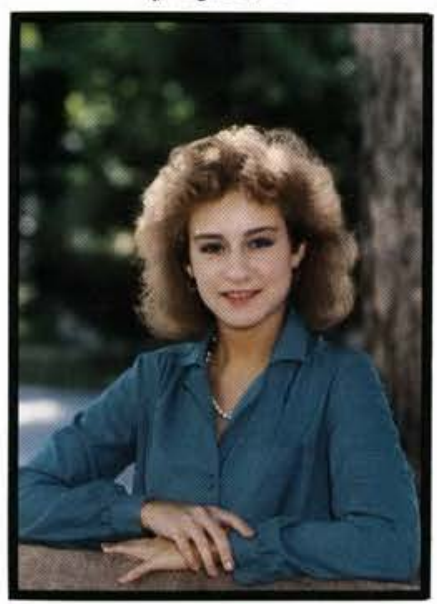

Rebekah J. Bennett

Business Administrarion

Lokewood, OH

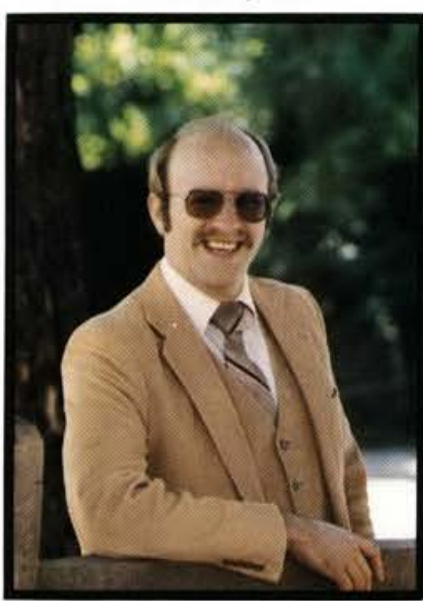

Stephen D. Bicknell

Comprehensive Bible

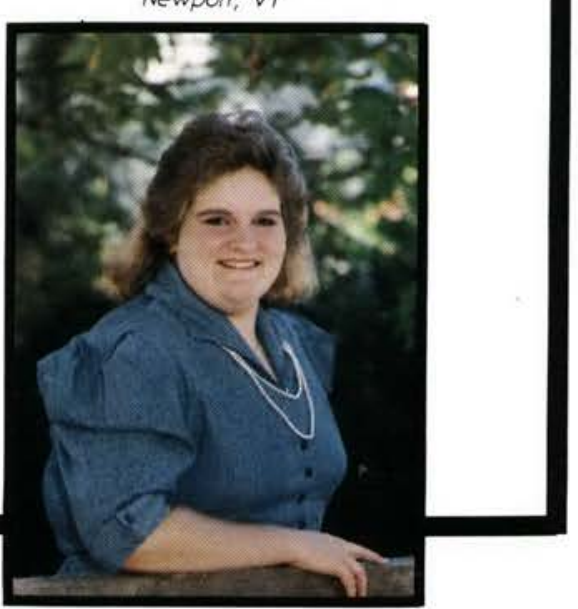

Suellen Black

Nursing 


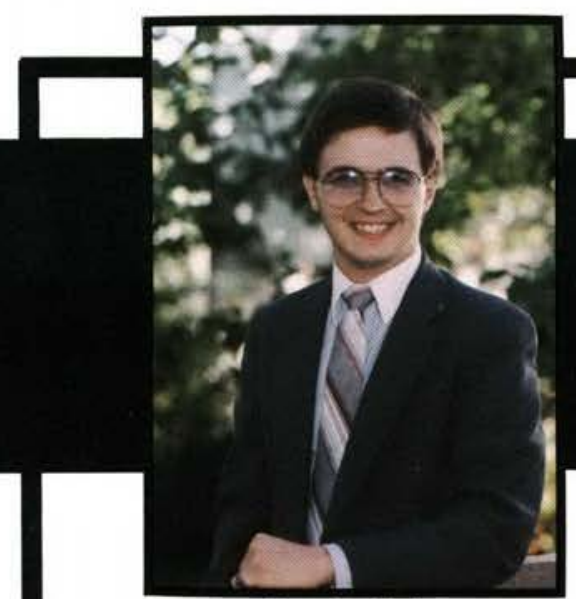

Arthur S. Blaker

Comprehensive Science

Medino, MI

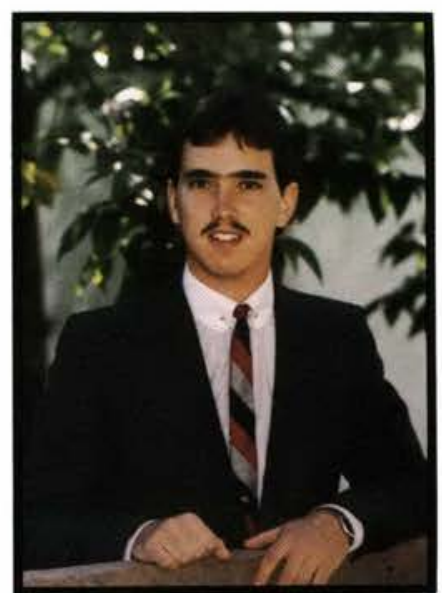

Jonathan F. Bresson

Business Administration

Milwoukee, WI

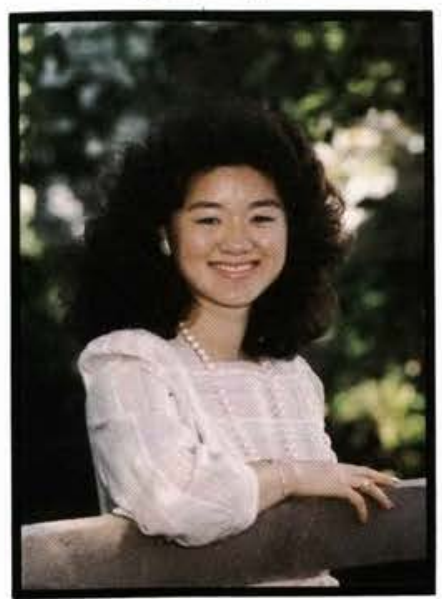

Ruth M. Brown

Elementory Educarion

Port Jervis, NY

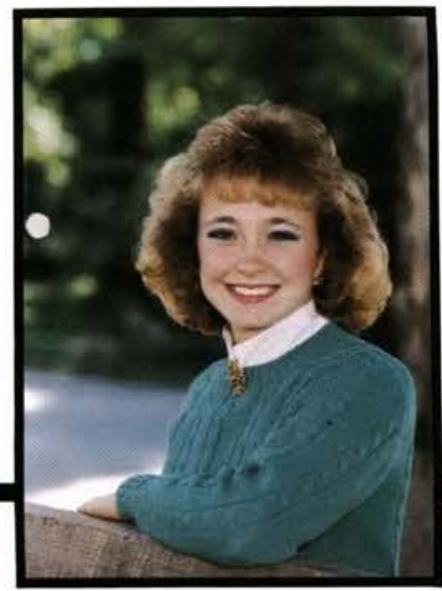

Lynne B. Calisti

Communicarion Arts

Greensburg, PA

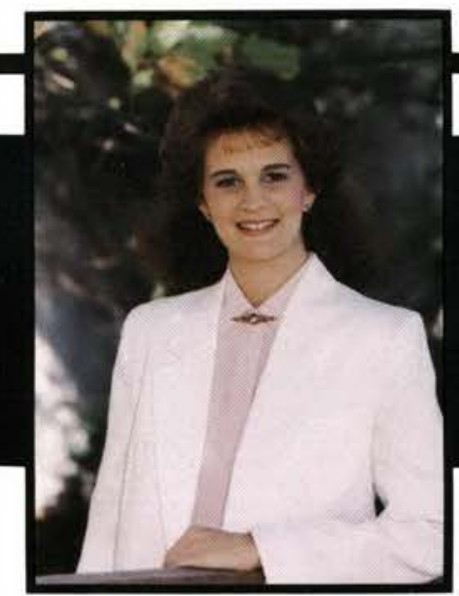

Valerie A. Blaylock

Communications Arts

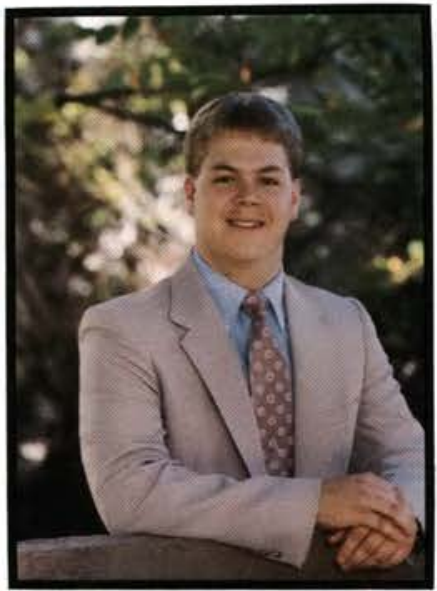

Timothy Allen Brock

Psychology

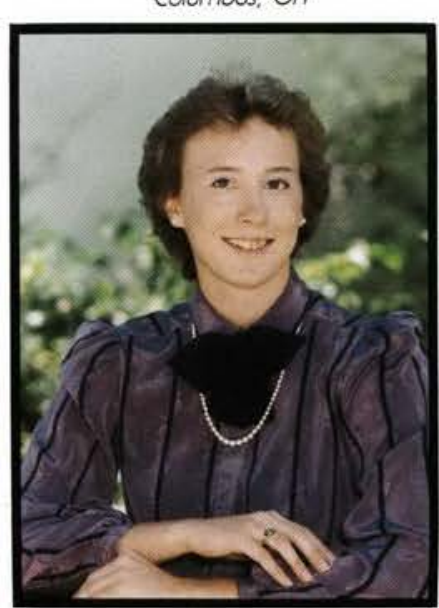

Julie Ann Butler

Comprehensive Science

Sreamboor Rock, IA

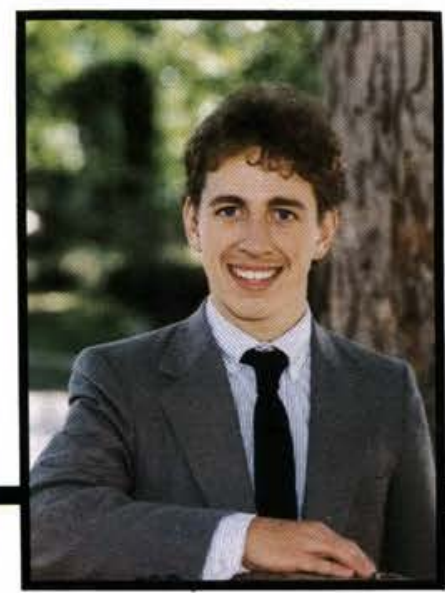

Lyle Campbell Business Administrarion

Rovenno, $\mathrm{OH}$

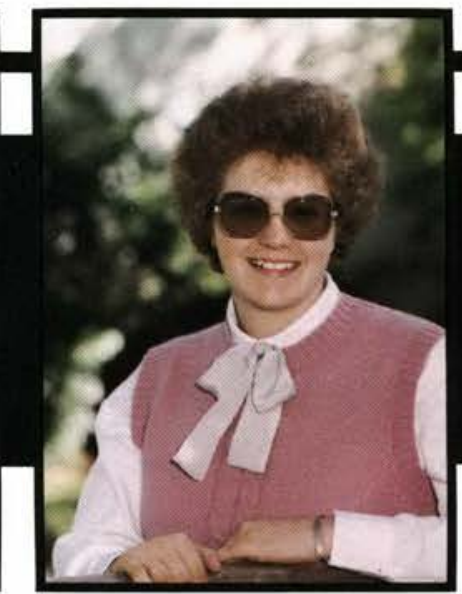

Joy C. Boersmo

Psychology

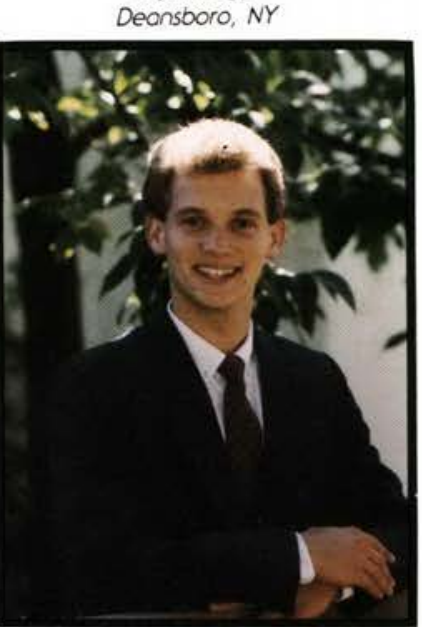

Robert Lee Broline Accounting

Sougus, CA

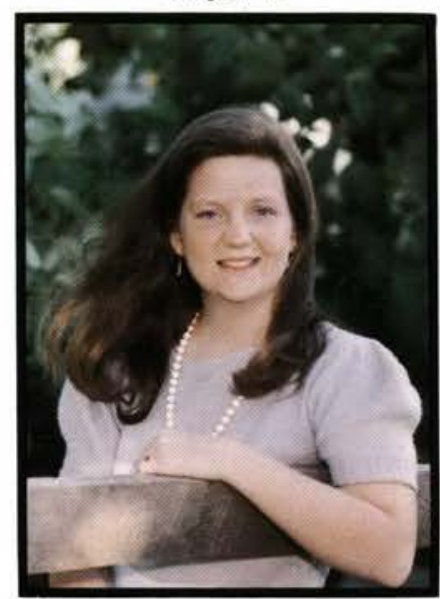

Jeanne Louise Butzlaff

Elementary Education

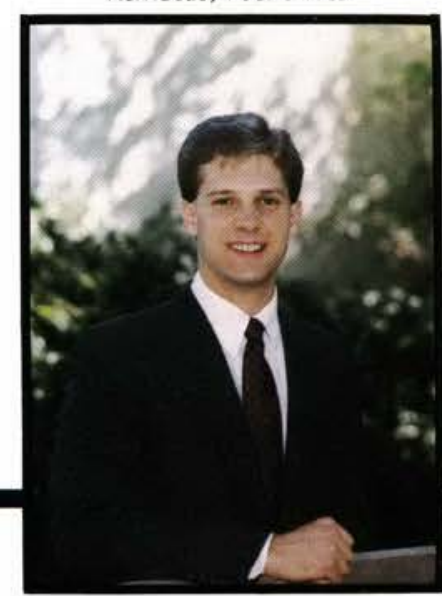

Steven Mark Campbell

Accounting

Plymouth, IN

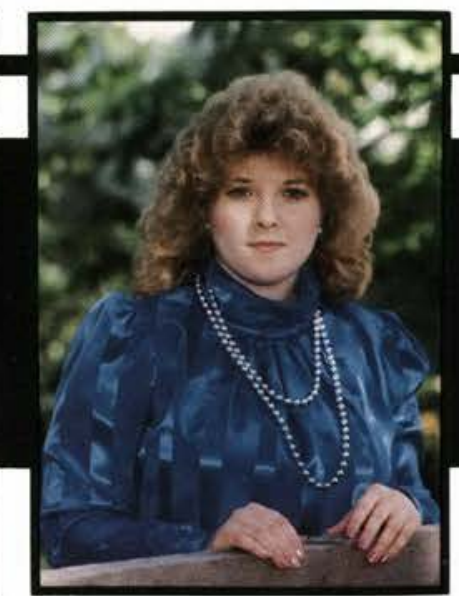

Theresa Diane Bonning Elemenrary Education

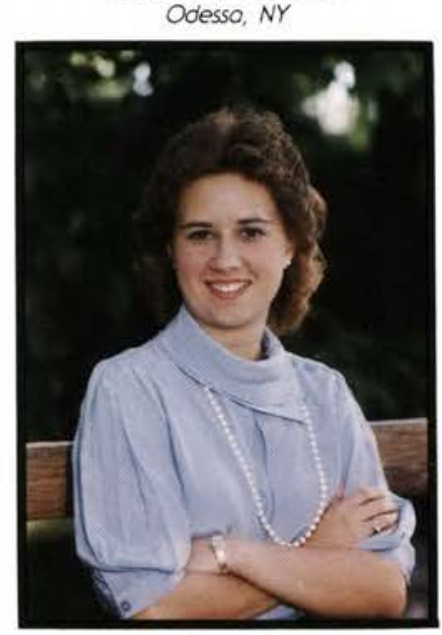

Diane Lee Brown

Comprehensive Bible

Lancoster, $\mathrm{OH}$

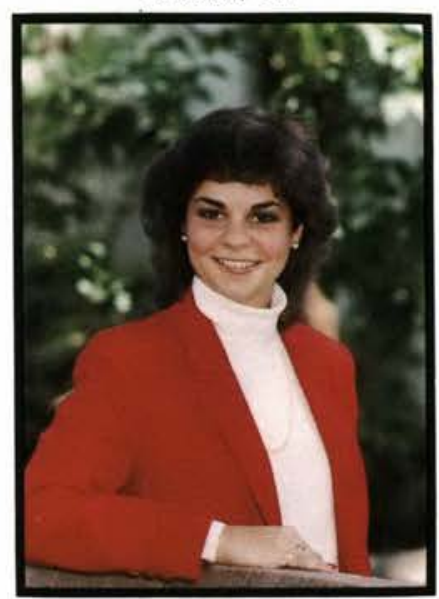

Lorrie Ann Byrd

Behovioural Science

Cenrervilie, $\mathrm{OH}$

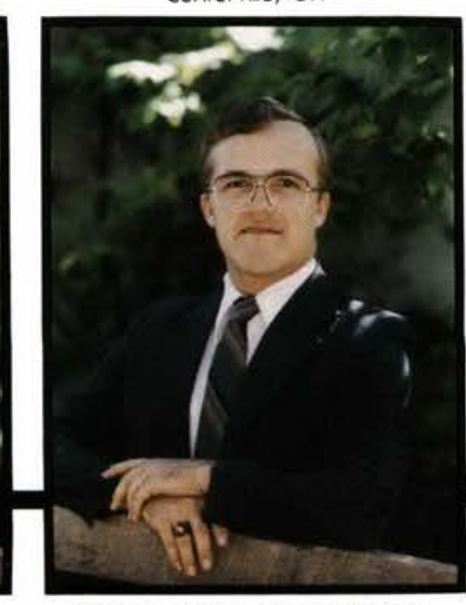

Richard Jonathan Carey

Marhemaric 


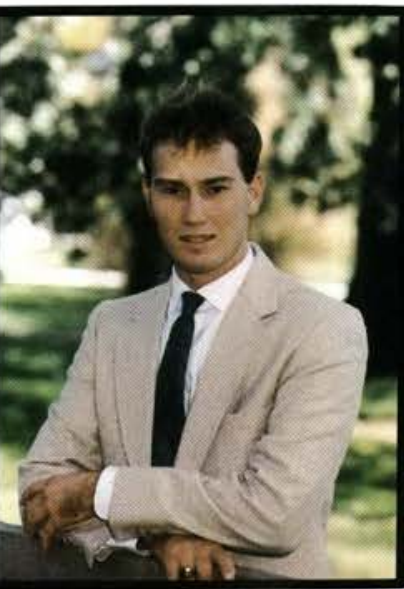

Sydney S. Caruthers Business Administrtation Ocola, FL

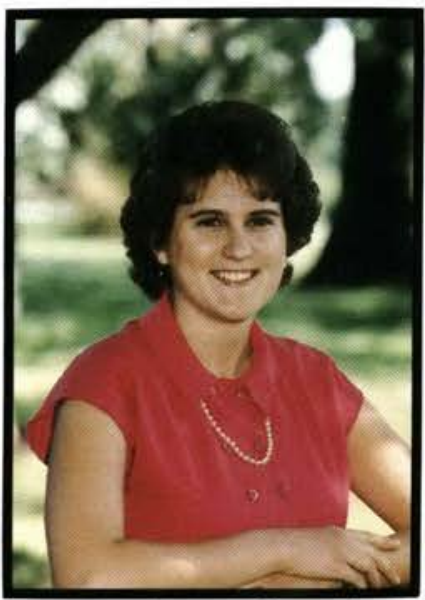

Lori Renee Castee Elemenrary Educarion Midland, M/

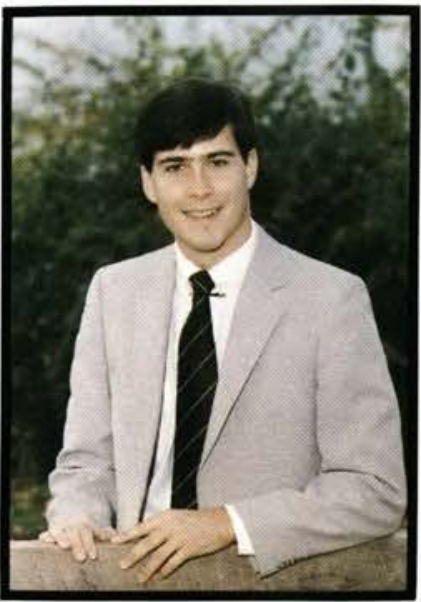

Douglas Cherry Marhemaric Srerling, IL.

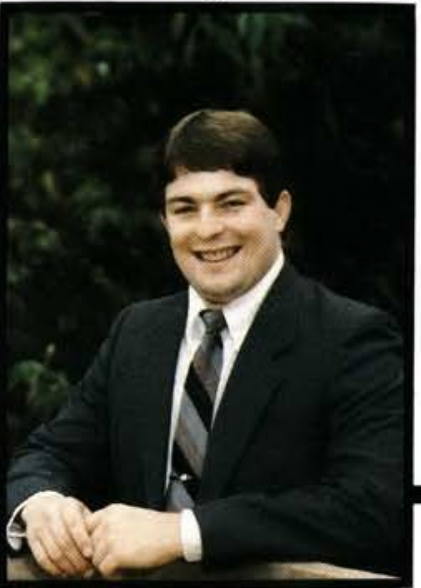

Perry Ercole Chiarelli Business Administration Lincoin Pork, M

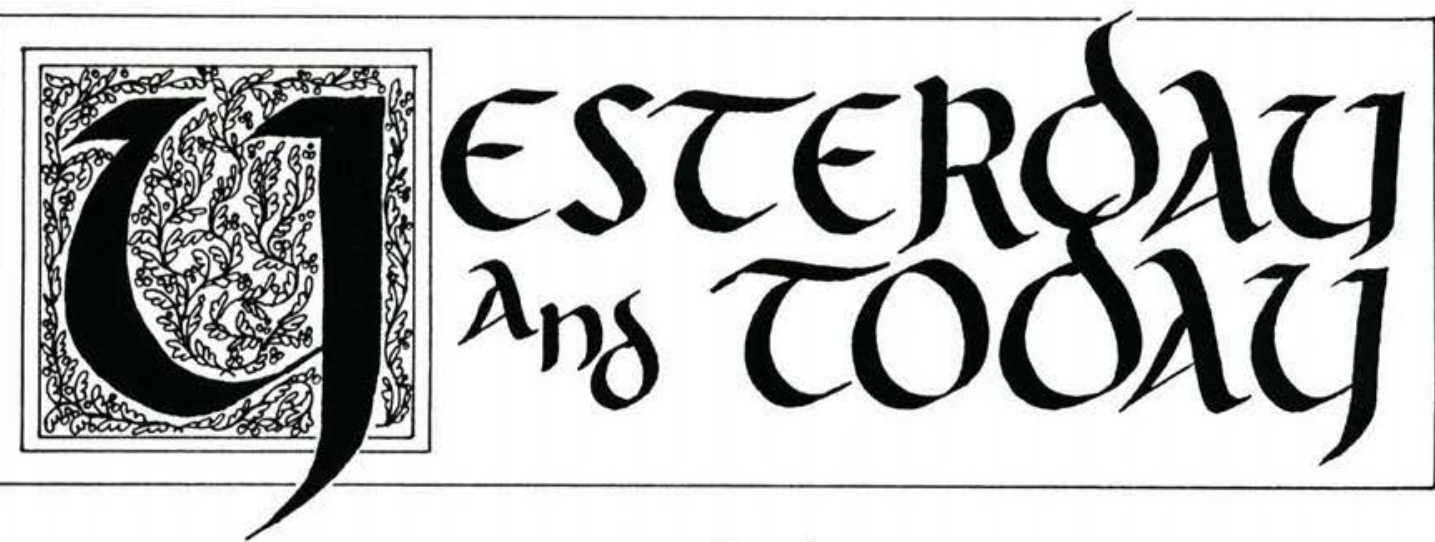

Fads \& Fashions

Quite often, trends set in the college arena carry over into national fashions, and trends across the nation always show up on college campuses. However conservative Cedarville may be considered, those trends are as prevalent here as at any other college.

In early editions of the Cedrus (predecessor to the Miracle). Cedarville students first began wearing gold-rimmed glasses, bow ties, the original tortoise-shell frame, and scarves with blouses. It's taken a few years, but those trends are finally catching on here.

Like most other schools, Cedarville has also had its fads. Before the letter jacket came along, it was a must to own a school sweater; and the college yearbook contained joke pages and "wills" from senior to underclass-

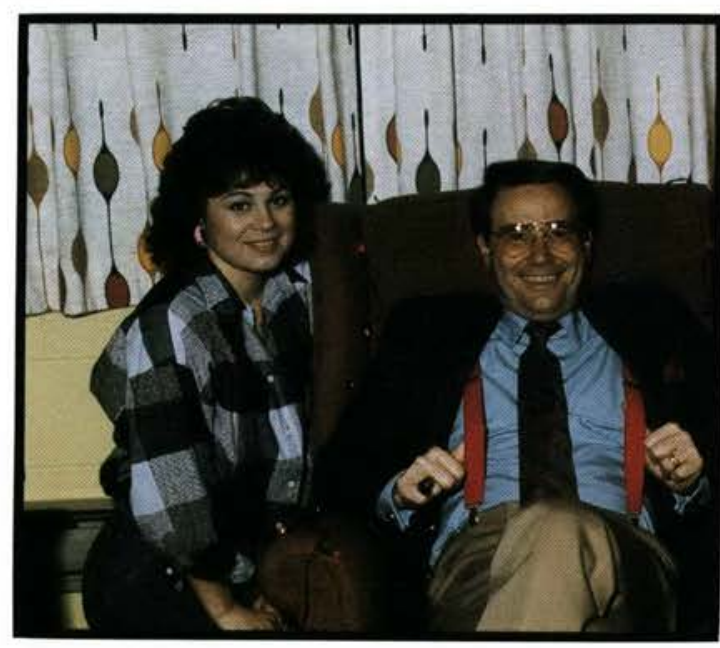
men.

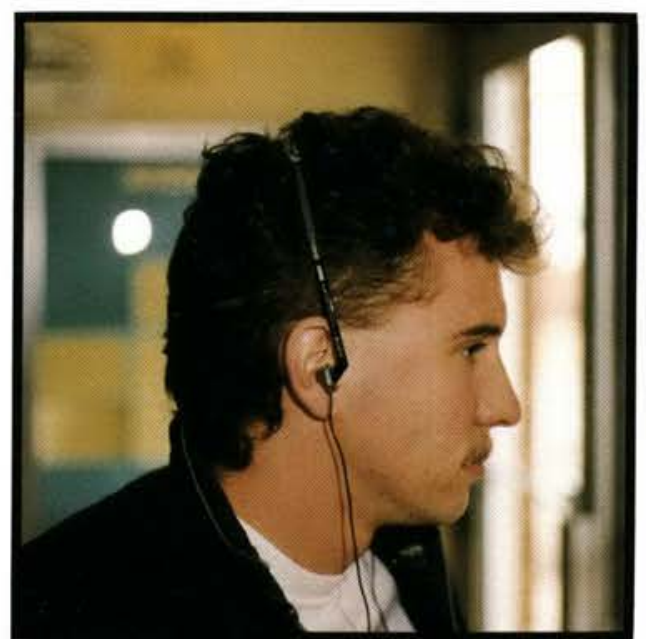

Today's Cedarville students continue to shop at thrift stores, wear big, dark coats that are at least thirty years old, and wear suspenders with baggy old pants and high top sneakers. After concerts and basketball games, they throng to $H$ G R Dairy Bar for tornadoes and gummy worms. And they, too, are affected by national trends: tanning booths, aerobics, head sets, and Levi's 501's.

Perhaps Cedarville is slightly isolated, resting at the edge of a tired little hamlet in rural Ohio. but the students have always been at the heartbeat of fashion, wherever that may be.

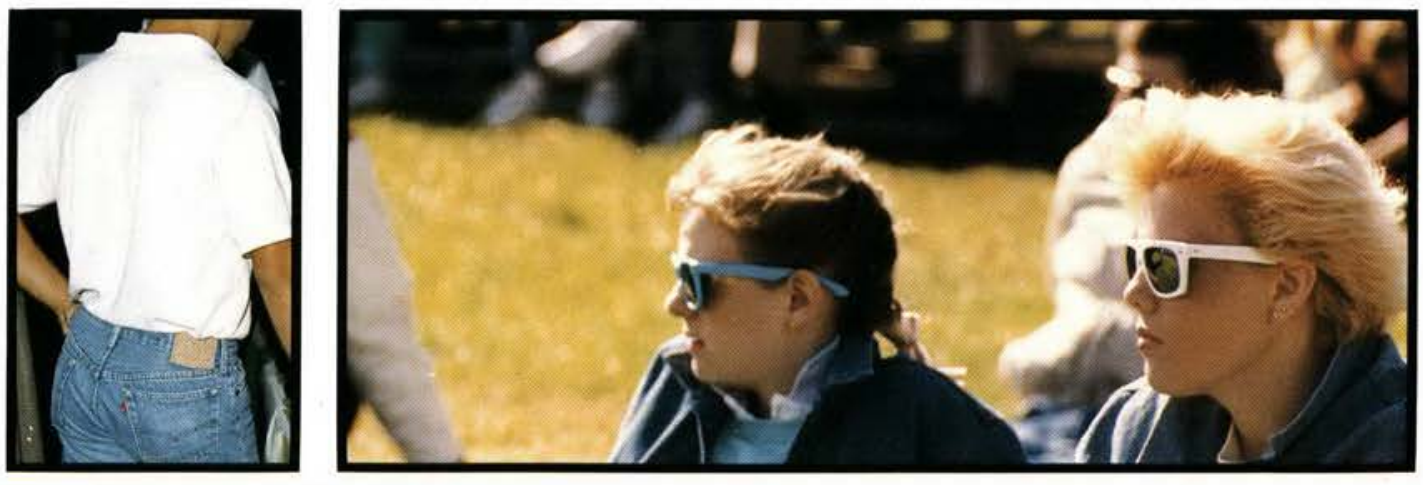




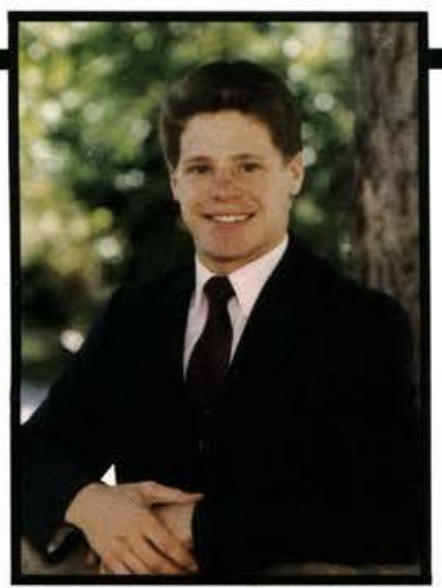

Tony Lee Childers

Preseminary Bible Mojor

West Lafayerte, $\mathbb{N}$

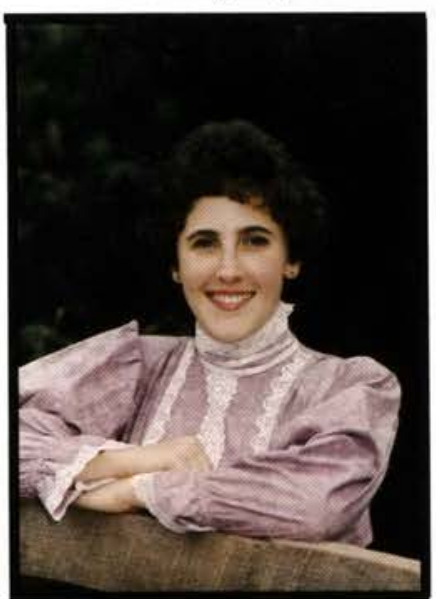

Kristine E. Cline

English Educorion

Rockbridge, $\mathrm{OH}$

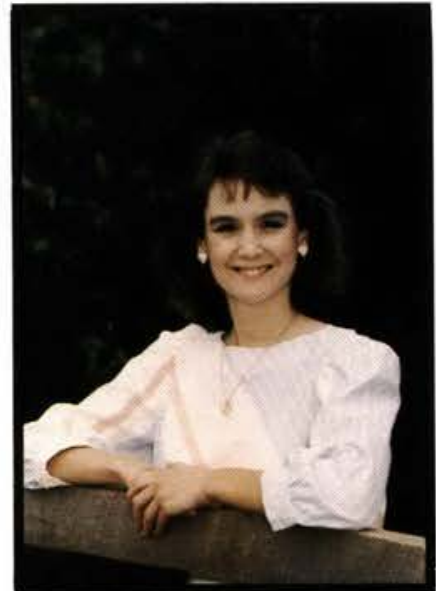

Mary E. Coons

Business Administrarion

Jeffers, $M N$

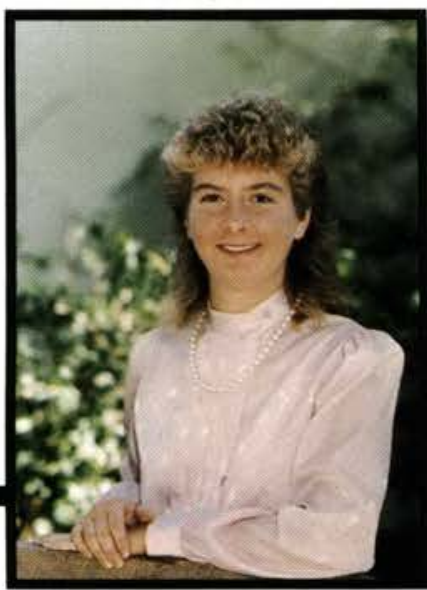

Angela K. Cremeans

Psychology

Troy, MI

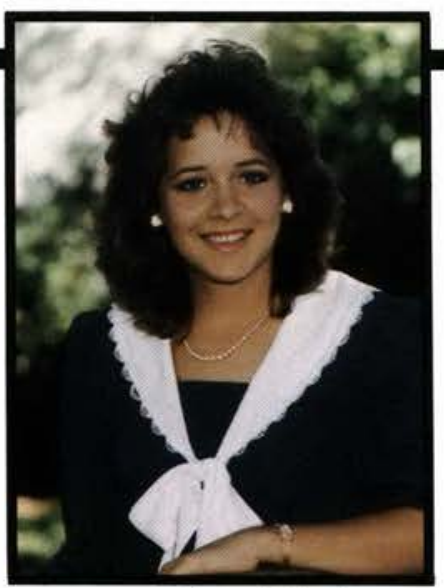

Lynn Cheryl Clark

Elemenrary Educario

Cedarvilie, $\mathrm{OH}$

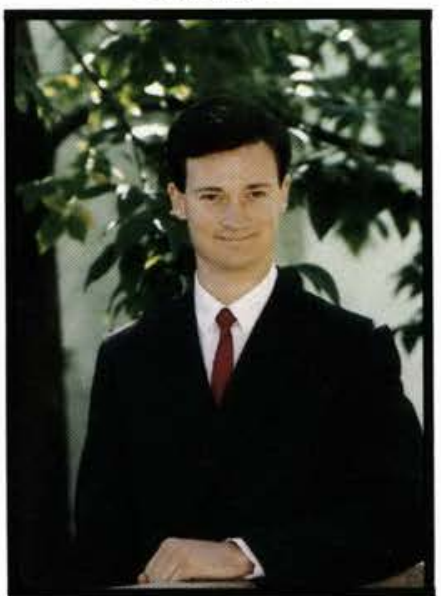

Steven David Coates

Preseminary Bible Mojor

Muskegon, M

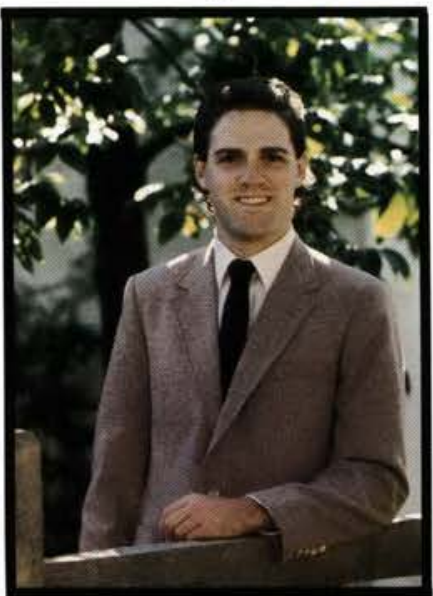

David Matthew Corry

comprehensive Bib

Springfield, $\mathrm{OH}$

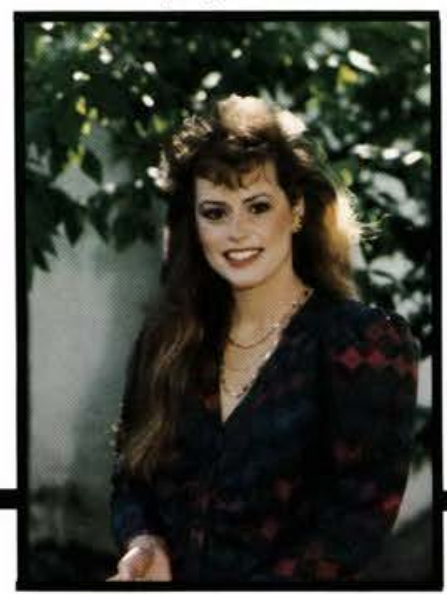

Cynthia H Cronrath

English Educarion

Worsontown, PA

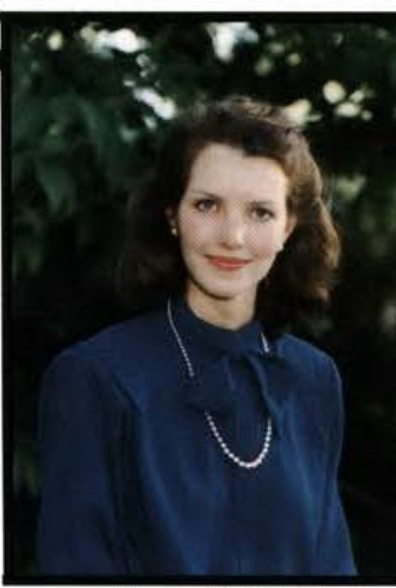

Tracy D. Clark

Business Administrotion

Columbus, $\mathrm{OH}$

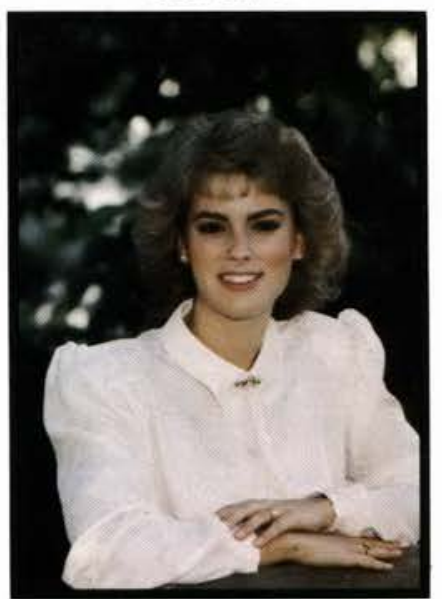

Erin Elisabeth Coffman

Elementary Educarion

Tiffin, $\mathrm{OH}$

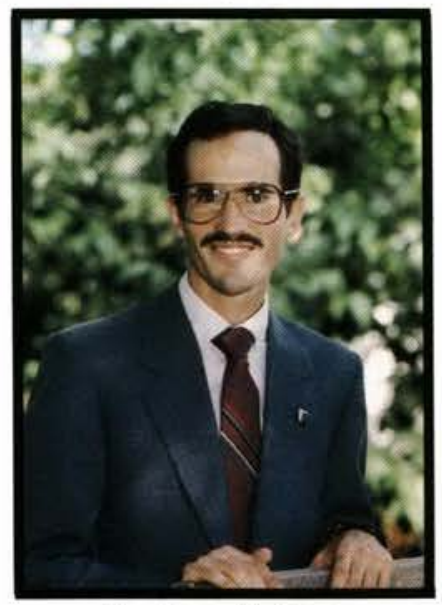

Stephen M. Cox

Physical Educarion

Soo Poulo, Brozil

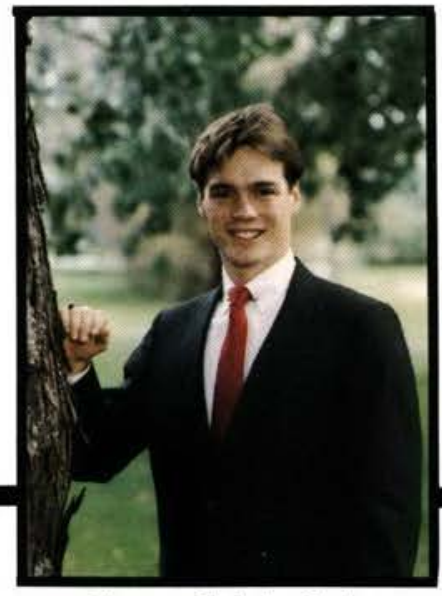

Clancy Calvin Cruise

Comprehensive Bible

Woosies, $\mathrm{OH}$

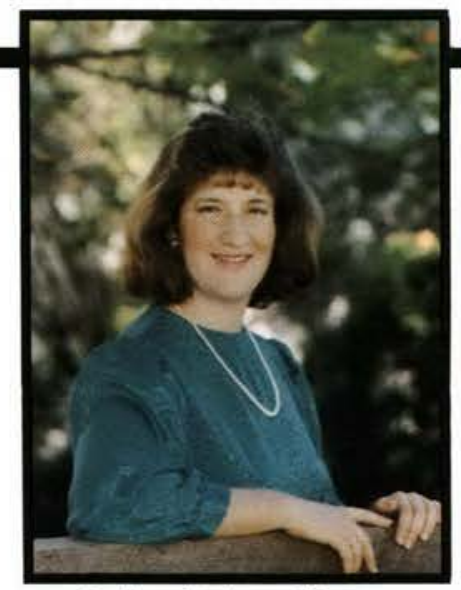

Deborah Ann Clem Elementary Educarion

Sondy, UT

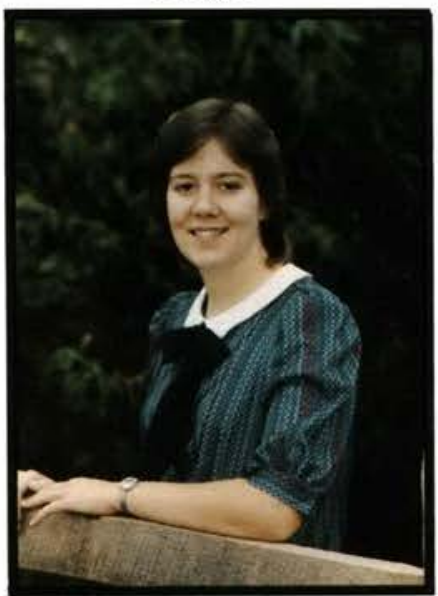

Mary Ruth Cook

Music Educorion

Mansfield, $\mathrm{OH}$

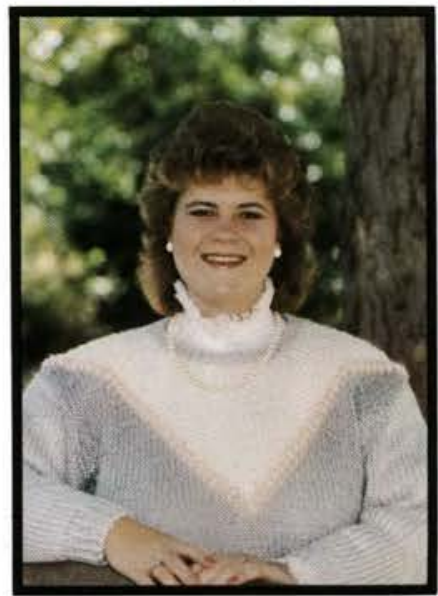

Lynn Dee Cramer

Grindsry Eoucaion

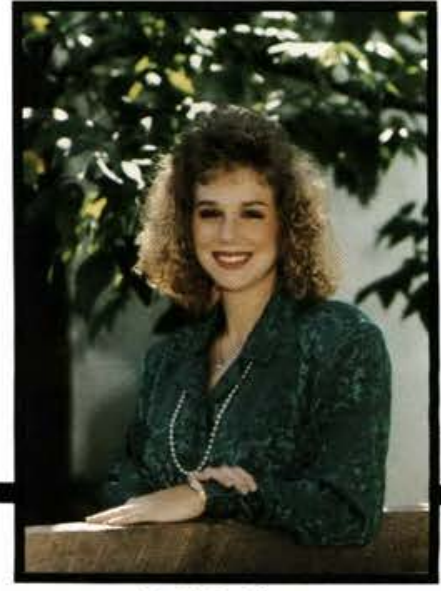

Gail Daiker

Business Administrarion Sodus, NY 


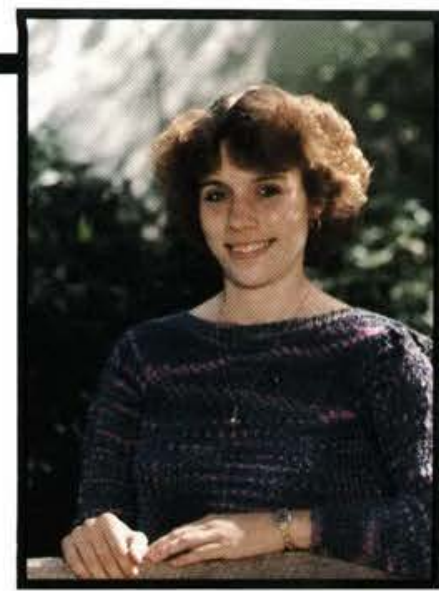

Christine F. Dail

Elementary Educorion

Ann Arbor MI

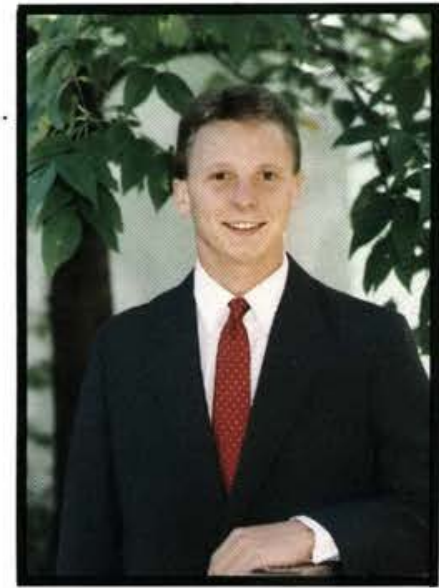

Timothy Paul Davis

Business Administration

Hoddon Heighrs, NJ

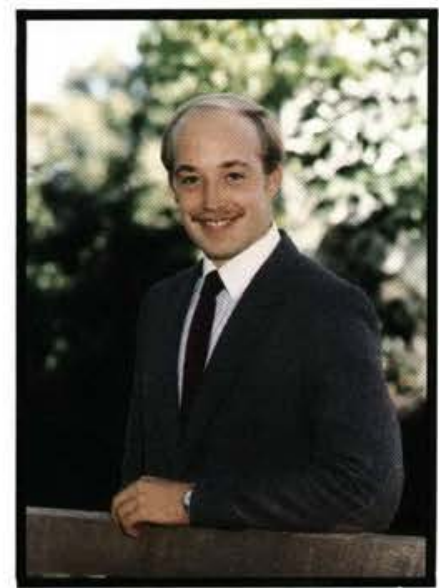

Wayne Edward Degelman

Business Administration

Unionrown, $P A$

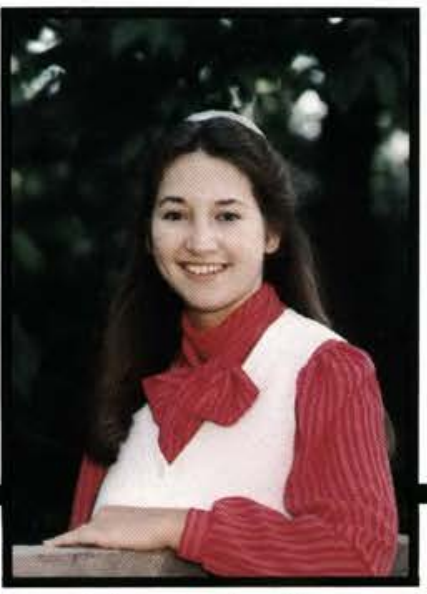

Debra Louise Diem

Elementary Educario

McAlisrerville, $P A$

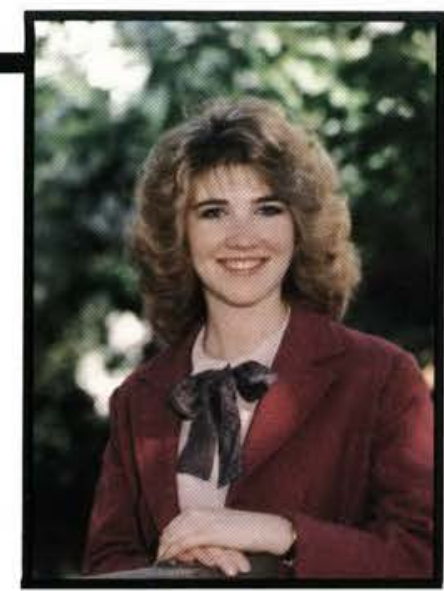

Janna L. Dalton

Comprehensive Bible

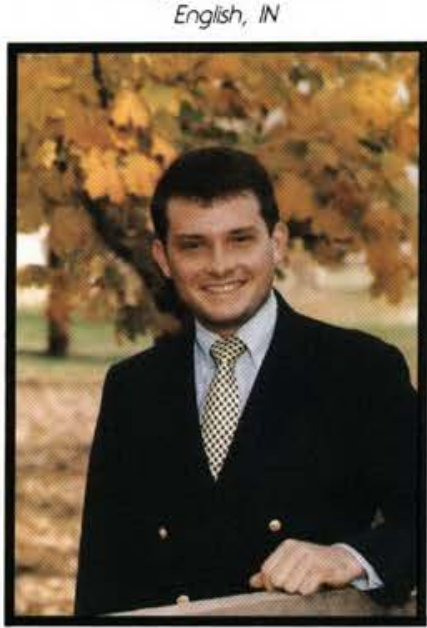

Tim Day

American Studies

Bellecenrer, $\mathrm{OH}$

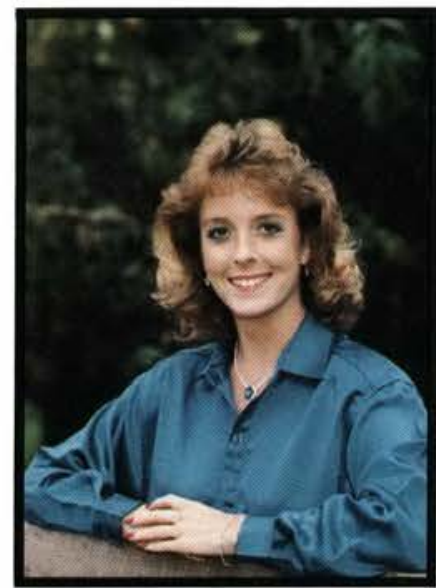

Shawna Marie Denney

Elemenrary Educarion

Hebron, KY

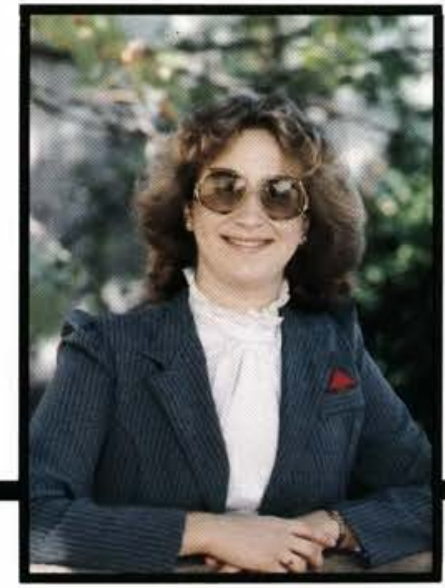

Kendra Jean Doctor

Elementary Educario

Grand Hoven, MI

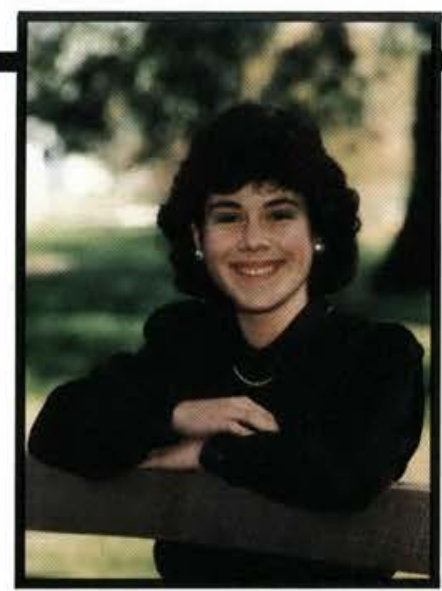

Wendy Diane Datzman communication Arts

New Orleans, LA

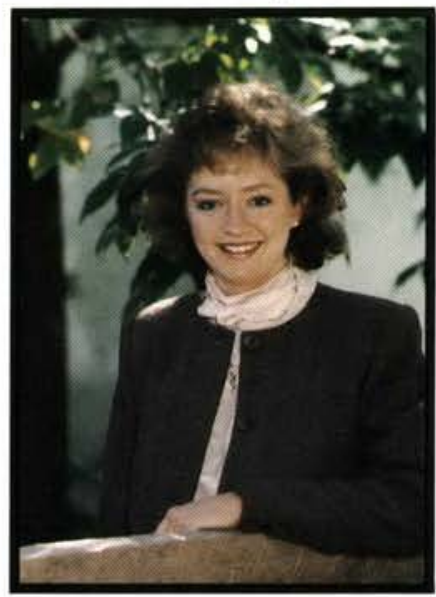

Jenny Sue Dean

Accounring

Findlay, $\mathrm{OH}$

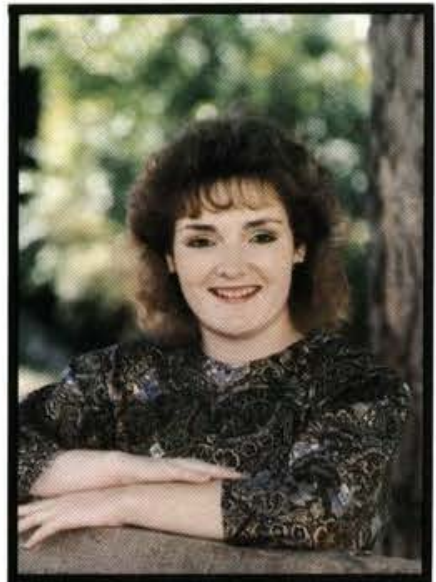

Judy K. Denning

Broodcosting

Nework, OH

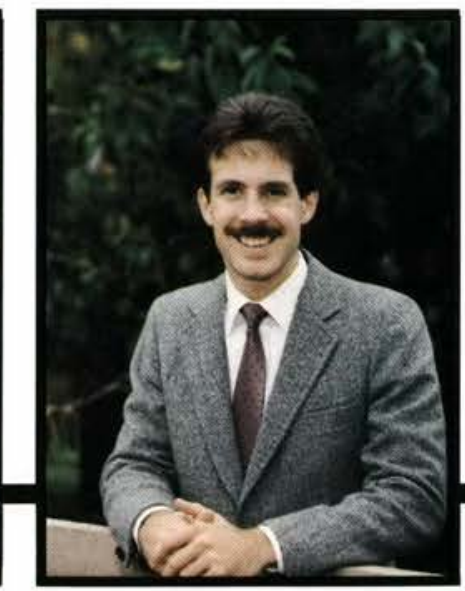

David Frank Dooley

Behovioral Science

Crowfordsville, IN

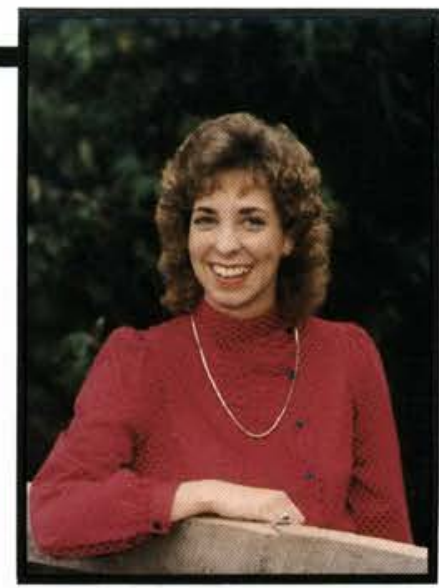

Kimberly A. Davis

English Educorio

Gombrils, MD

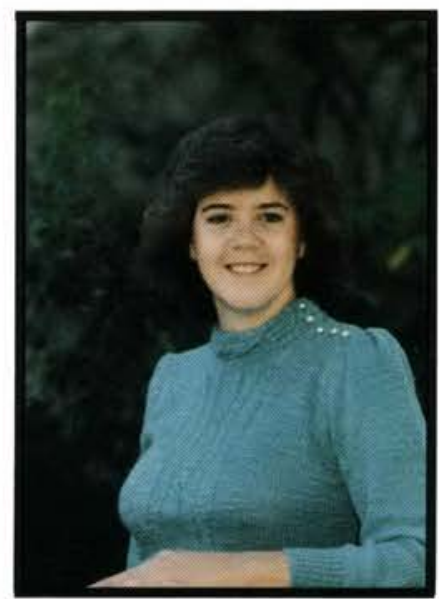

Susan Marie Dean

English Educarion

Mr. Clemens, M

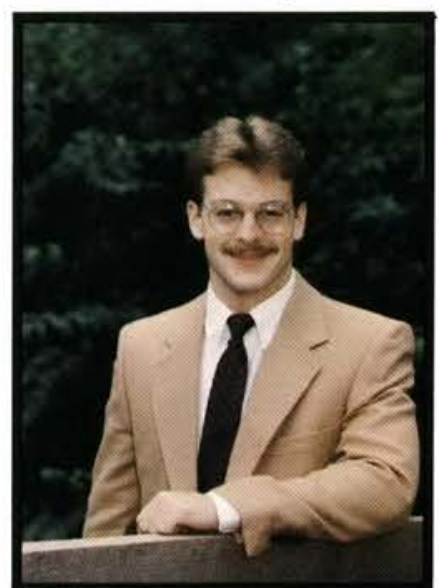

Matthew J. Dickinson

English Educorion

Willomson, $N Y$

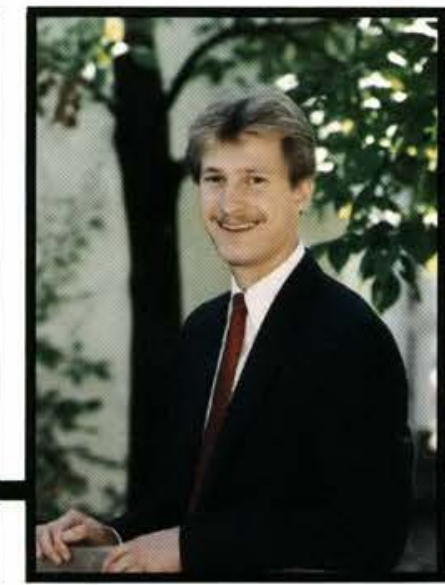

David J. Douglas

Preseminary Bible Mojor

Middlerown, OH 


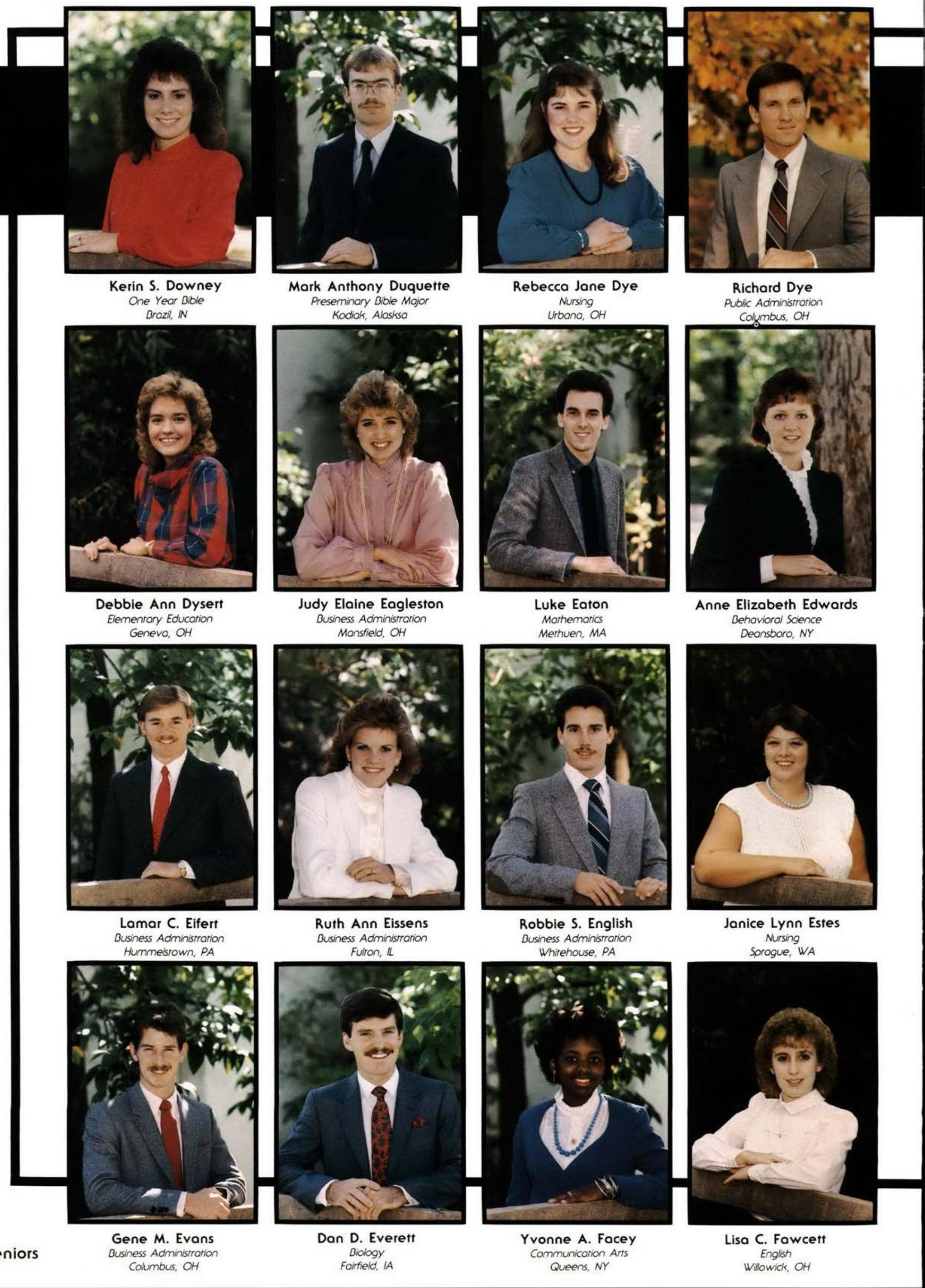




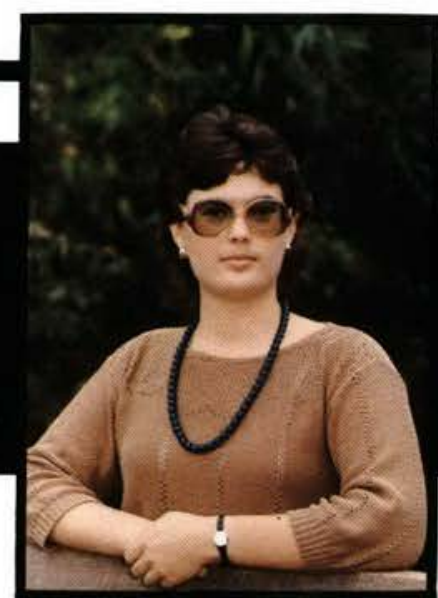

Alice L. Fenstermaker Elementary Educarion Arcodia, $\mathrm{OH}$

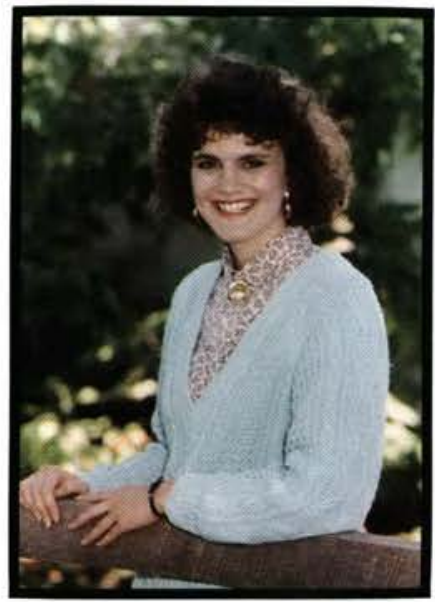

Loura Marie Fish

Elementory Educarion

Johesborough, TN

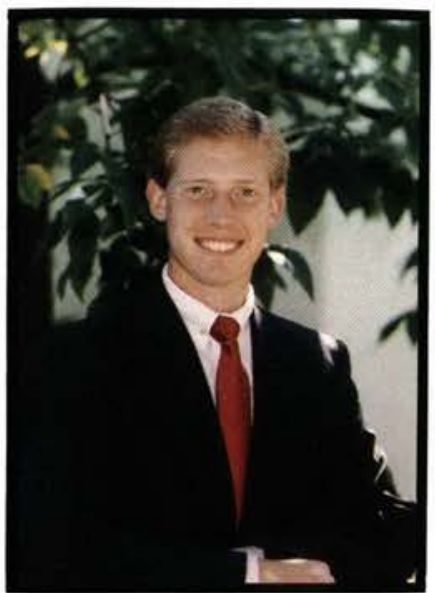

Philip R. Fogle Jr. Morhemarics

Springfield, VA

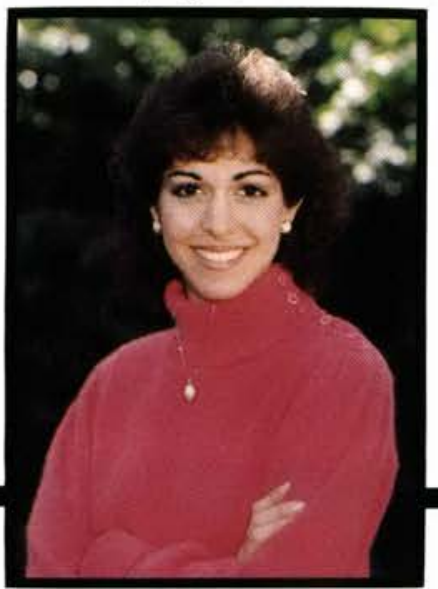

Claire Anne Fortosis

Psychology

Rockford, MI

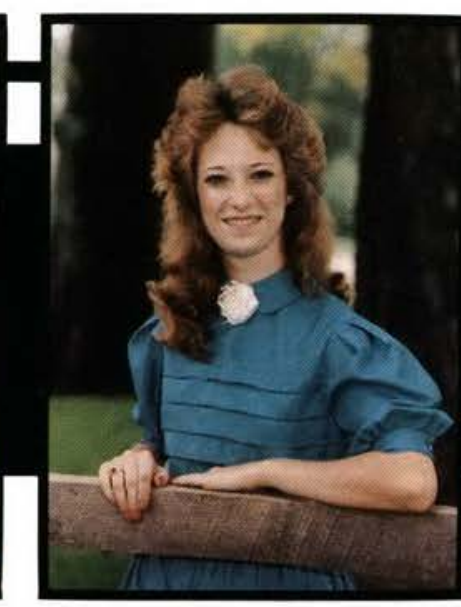

Wendi Anne Figary

Elemenrary Educarion Norwich, NY

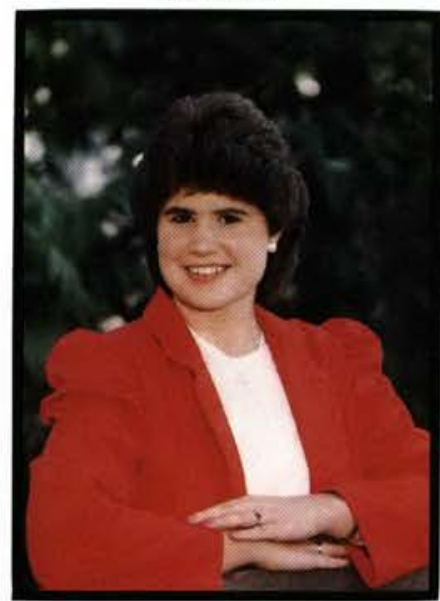

Jodie Lynn Flach Elemenrary Educarion Glodwin, Ml

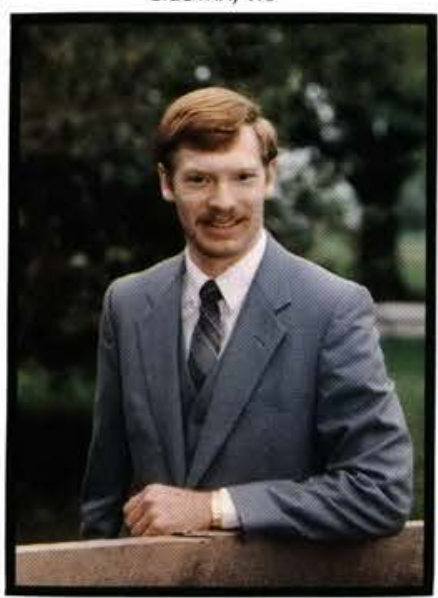

Andrew Ford

Comprehensive Bible Grove Giry, PA

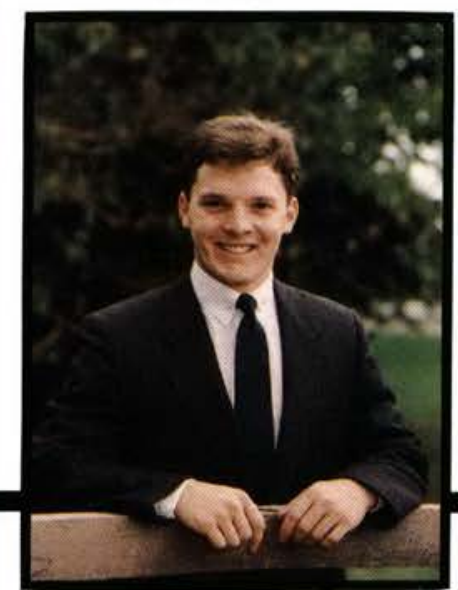

Andrew L. Fraser

Compurer informarion Systems Flint, MI

\section{ESTEROATT proboit}

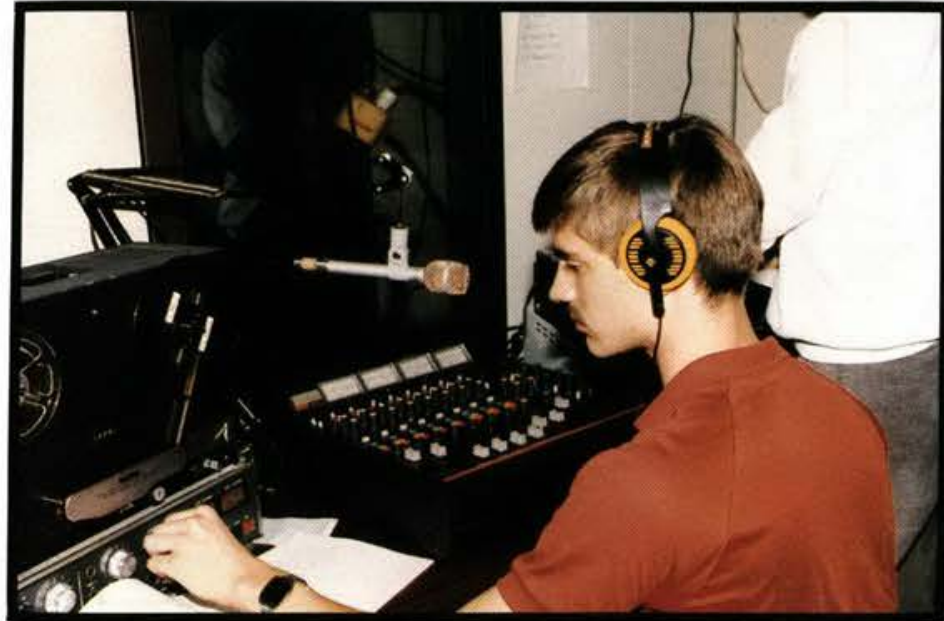

Common Majors

Long ago our beloved Cedarville College was fondly known as BBI (Baptist Bible Institute). Because of its beginning under Baptist ownership as a Bible college in those years, the students majored in some form of Biblical studies. Graduates most often became pastors, missionaries, or Christian ministry workers.

As Cedarville made the transition to a liberal arts college and began its work toward accreditation, the majors offered varied. Today the most popular major is business, with emphases in administration, computer information systems, and secretarial science. The recent accreditation of the growing nursing program has reinforced an already strong program and the second most commonly chosen major. Consistent with its heritage, Cedarville has maintained a powerful Bible department whose various em. phases incorporate the third largest major today. The education program, too, has remained popular throughout the years and expanded from its small beginning years ago to certify teachers for both Christian and public schools in nearly every field possible.

Although the majors which were most commonly declared in the early days of Cedarville College are no longer at the top of the list, Cedarville College has maintained and encouraged the tradition of excellence in each of the liberal arts divisions as well as in the original Bible department.

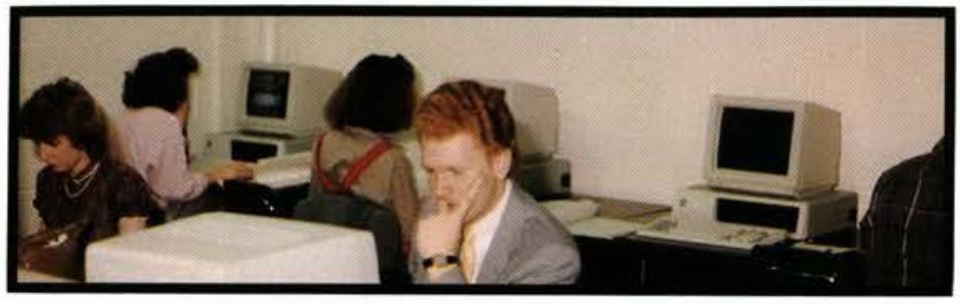




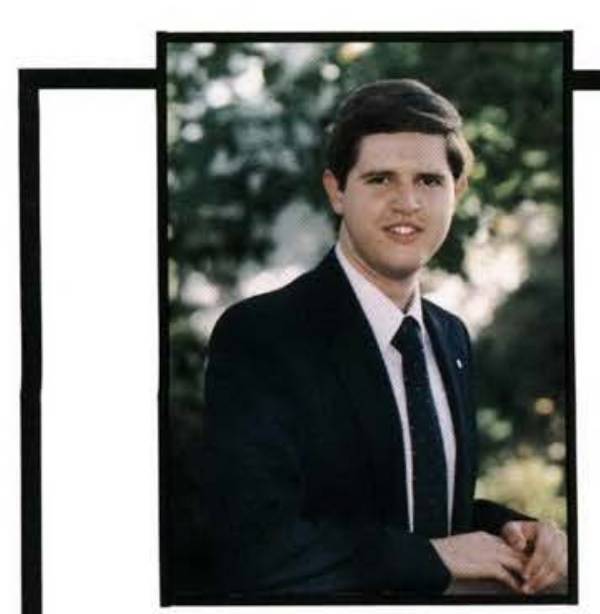

Robert A. Fread

Comprehensive Bible

Loveland, $\mathrm{OH}$

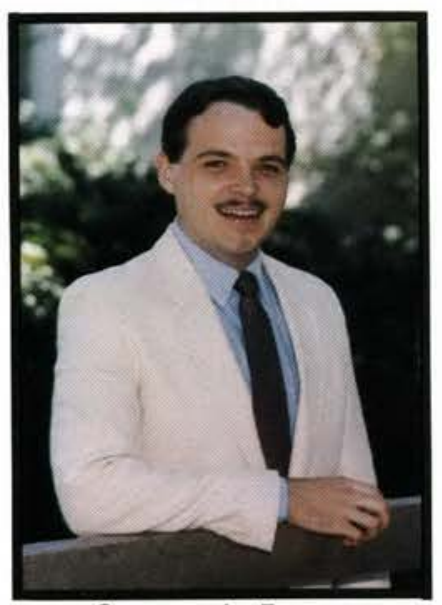

Gregory L. Frye Computer Informarion Systems

Ionia, MI

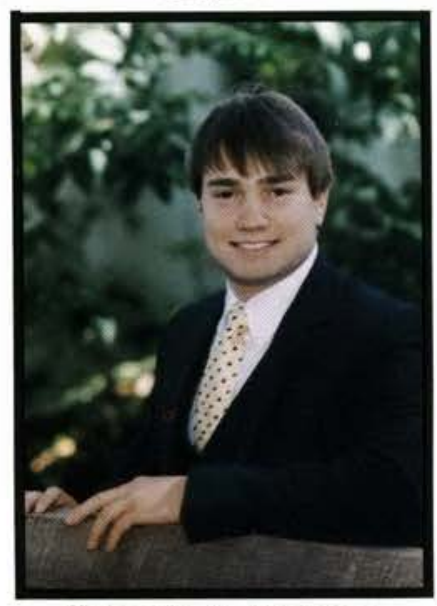

Kenneth A. Giesman Accounring

Pirrsburgh, PA

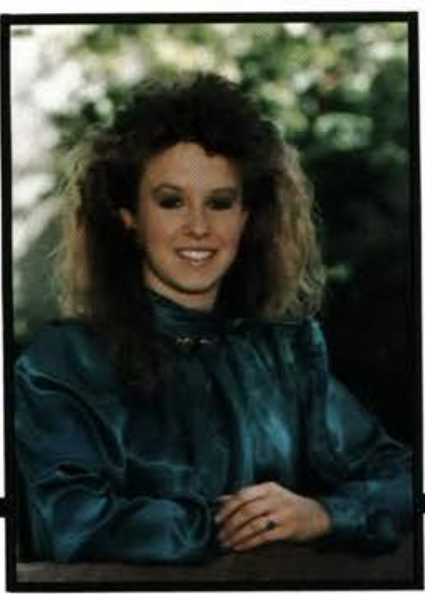

Wendy Jo Grady

86 Seniors

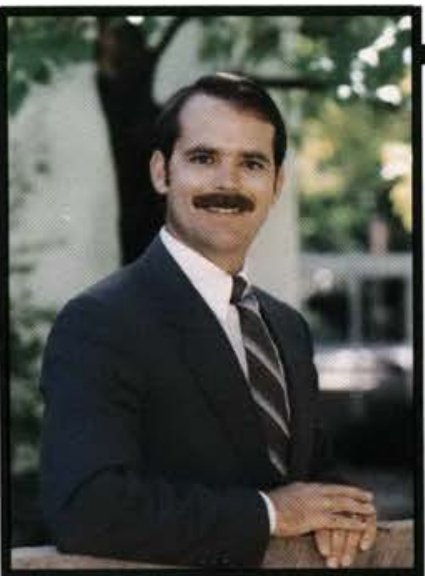

Randall G. Frederick

Business Administrorion

Poroskola, $\mathrm{OH}$

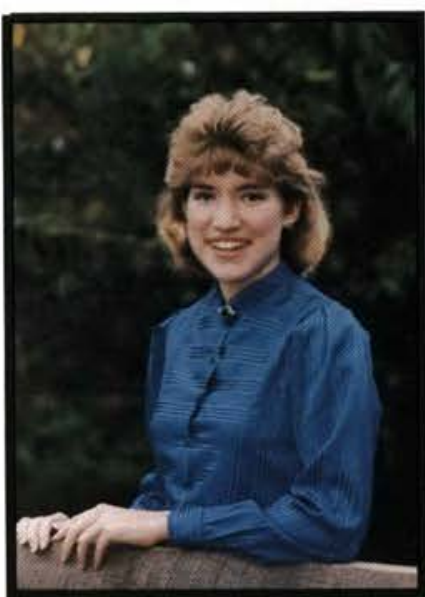

Marla Sue Fuller

Sourth Bend, IN

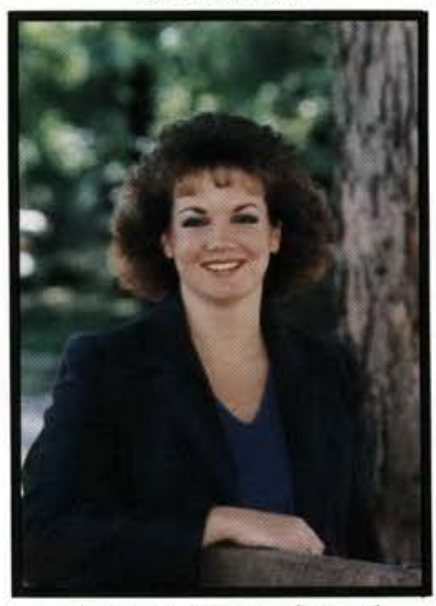

Jodean Marie Gifford Elementary Educarion

Urbano, $\mathbb{N}$

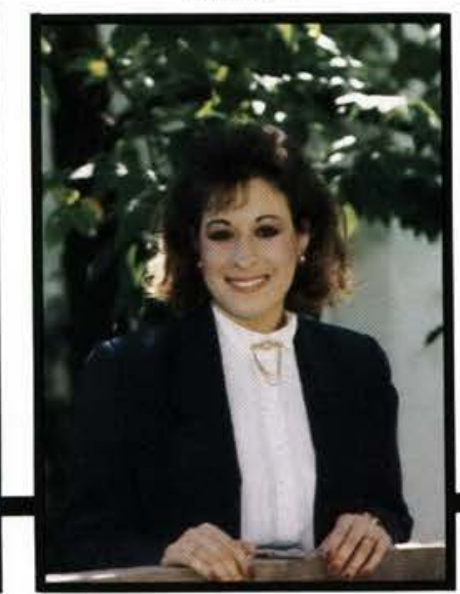

Kelly Ann Grant

Business Administrarion

North Canron, $\mathrm{OH}$

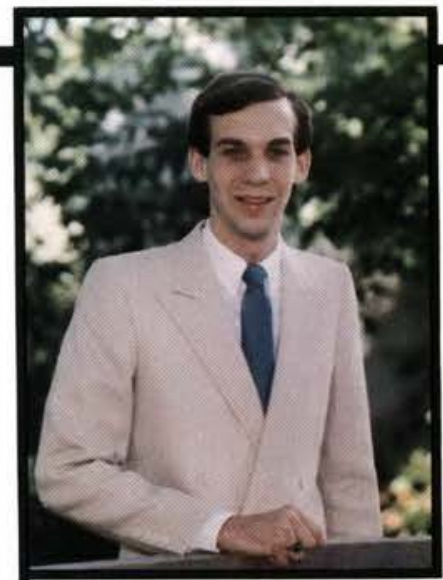

Todd T. Free

Business Administrarion

Coshocton, OH

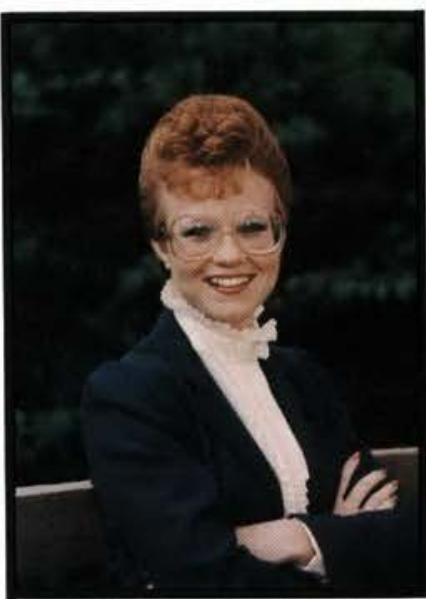

Deanna K. Gabel

Quincy, II

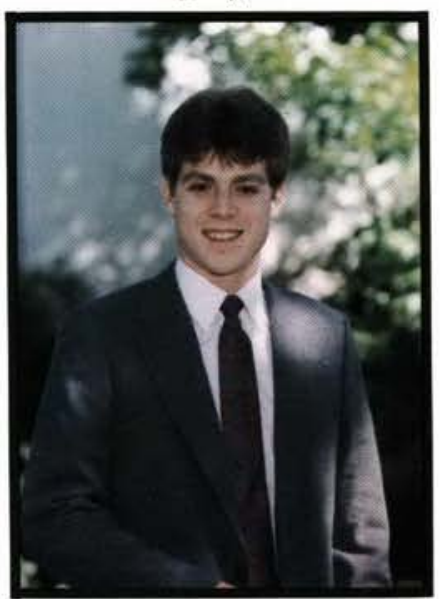

Jeffrey W. Gilbert

Broodcosting

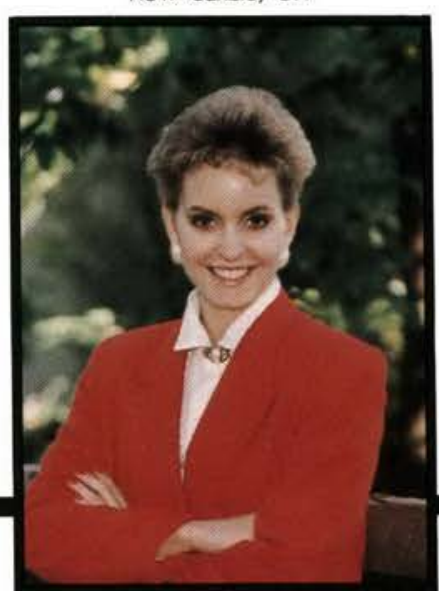

Tracy E. Grimes

English

Ankeny, IA

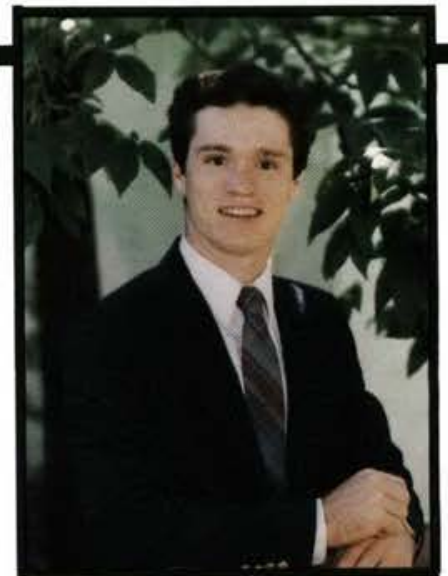

David C. Frey

Comprehensive Bible Dovison, MI

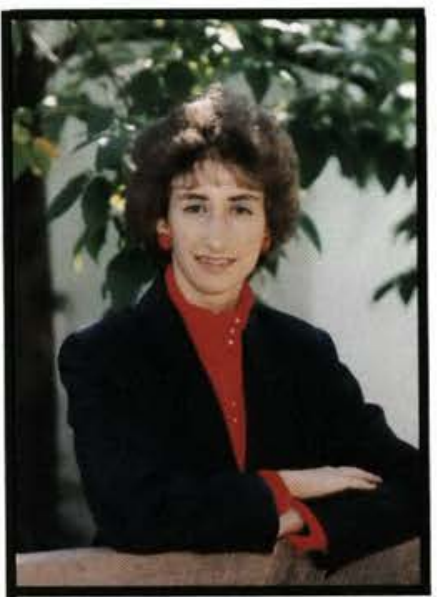

Janet Sue Garmatter

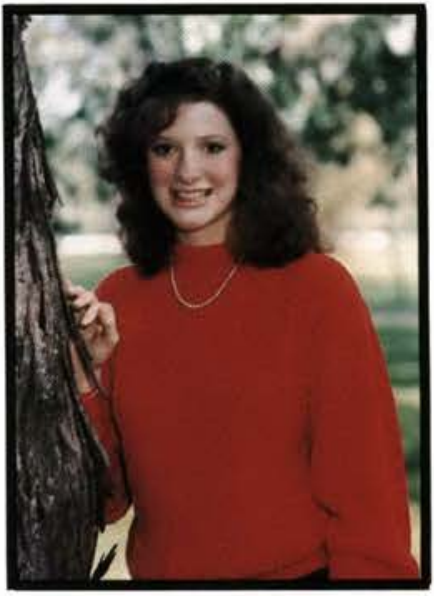

Anne L. Gordon Nursing

Willamsport, PA

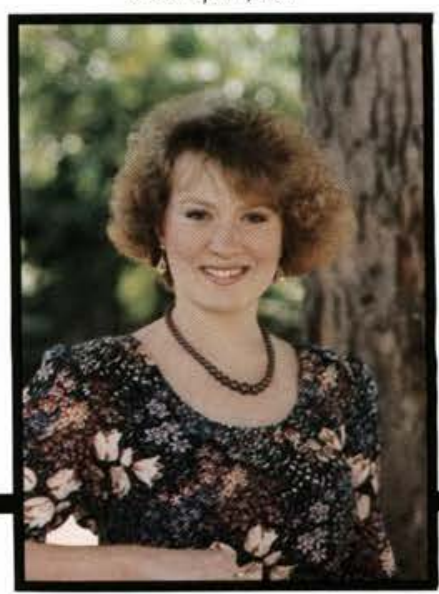

Virginia Ann Gruneisen Broadcosting

Mansfield, $\mathrm{OH}$ 


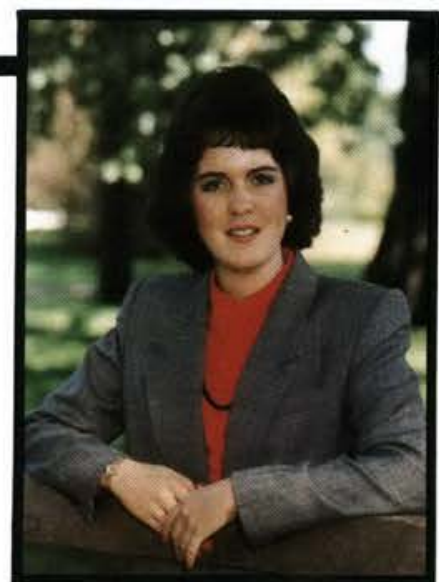

Amy Jo Guest

Business Adminissrorion

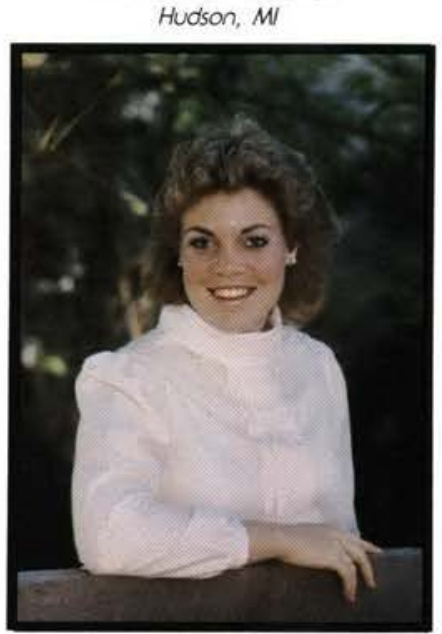

Lyn M. Hackenberry Elementary Educoriion

Henrierto, NY

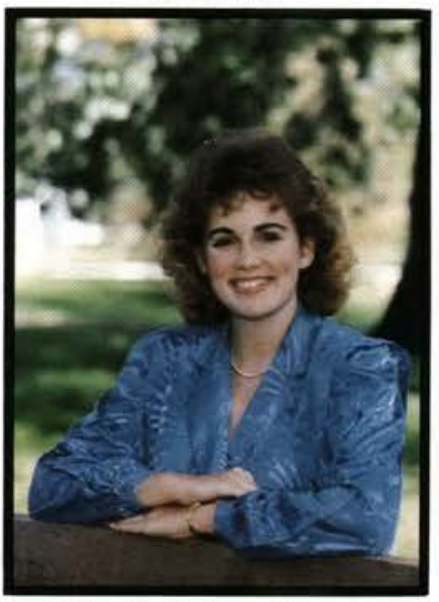

Elizabeth Joy Hansen

Nursing

Sroren Island, NY

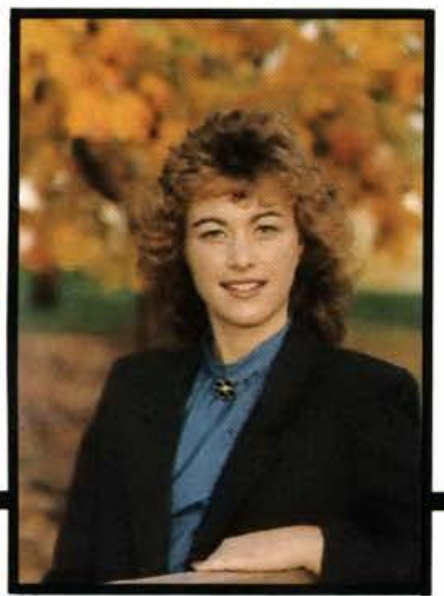

Kathi Harris

Behovioral Science

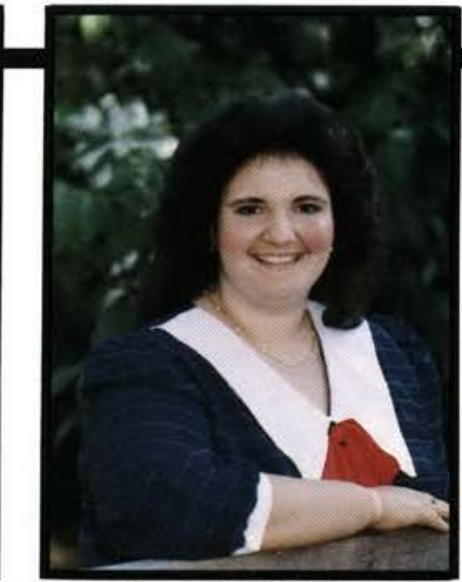

Stacy L. Gunther

Elemenrary Educarion

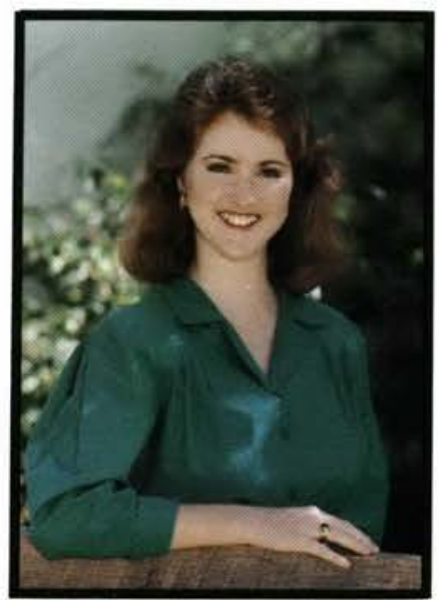

Rebecca Jo Haga

Music

Kingman, $\mathbb{N}$

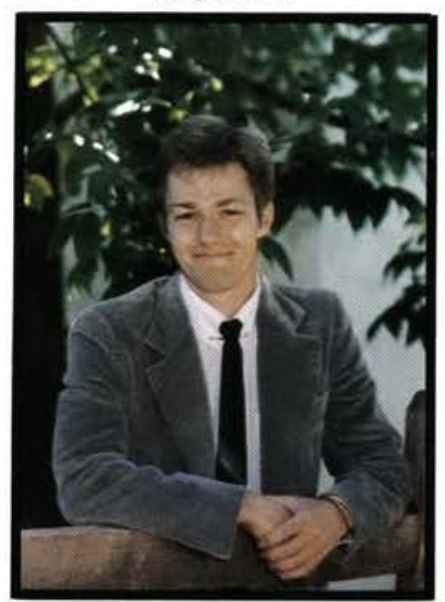

Steve J. Hanson

Broodcosting

Worerloo, IA

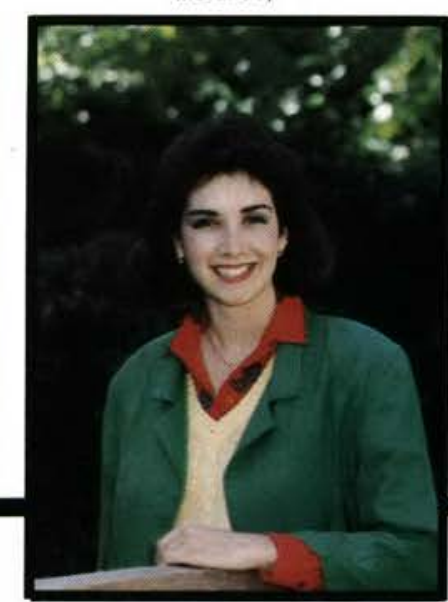

Laura A. Hartsough

Nursing

Sourh Bend, IN

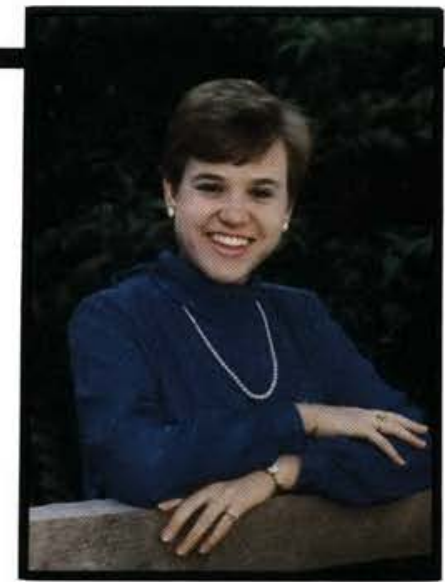

Brenda Jo Gust

Nursing

Hilron, NY

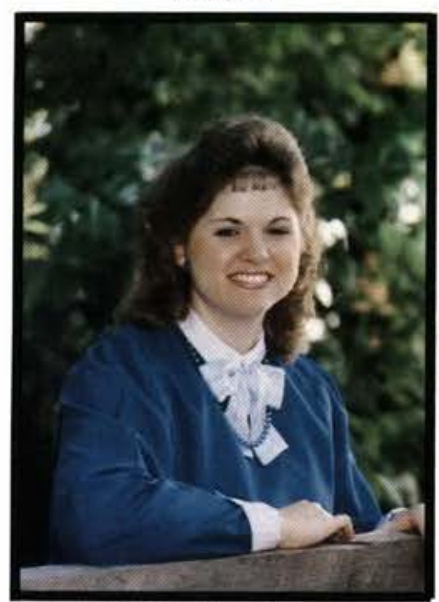

Kelly Hamilton

Elemenrary Educarion

Noshvile, MI

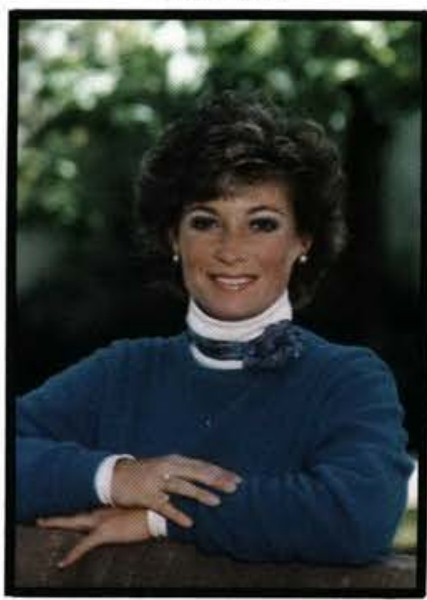

Judith E. Happe

Physical Educarion

Mocedon, NY

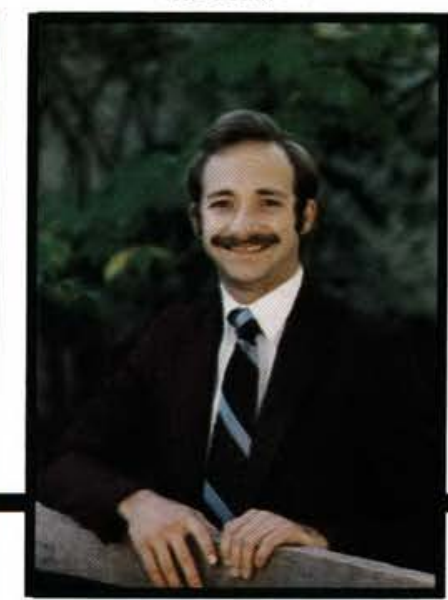

Richard G. Haywood

Biology

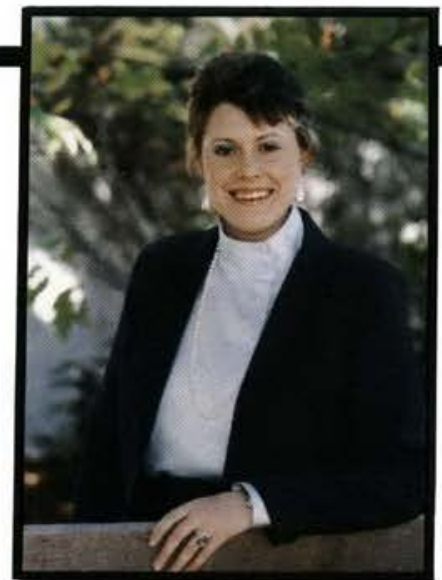

Lori R. Haberli

Psychology

Rhinelander, WI

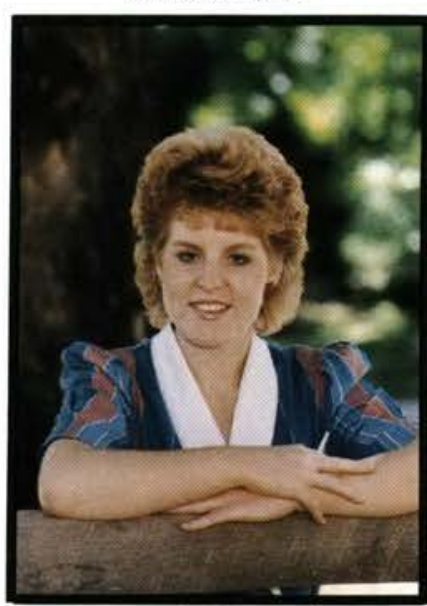

Laure S. Hancock

Political Science

Jackson, MI

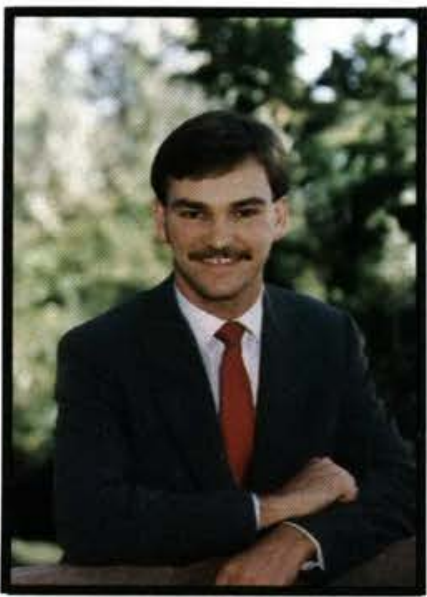

Stephen J. Harper

Psychology

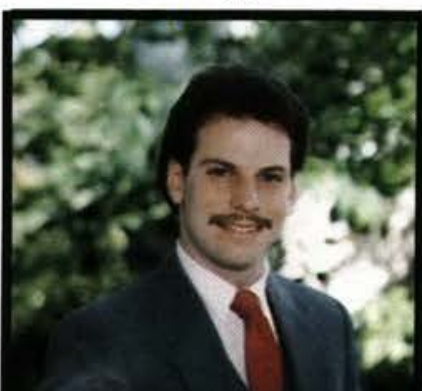

Larry Leox Heacox II

Morhemarics

Beech Grove, IN 


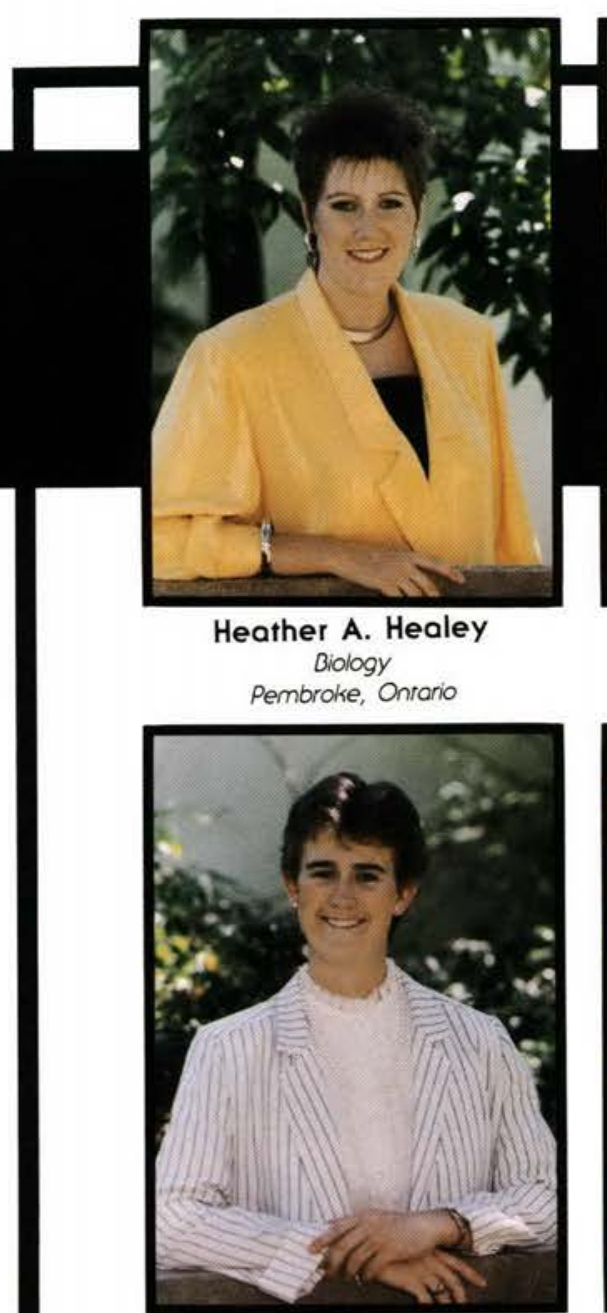

Kellie L. Helmick Compurer Informarion Systems

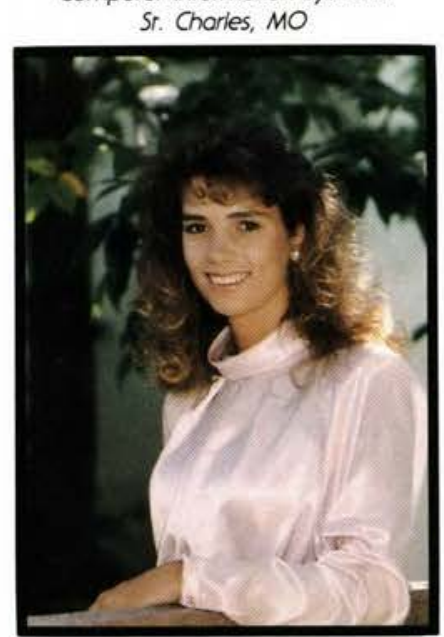

Rebecca L. Henthorn

Psychology

New Marrinsville, WV

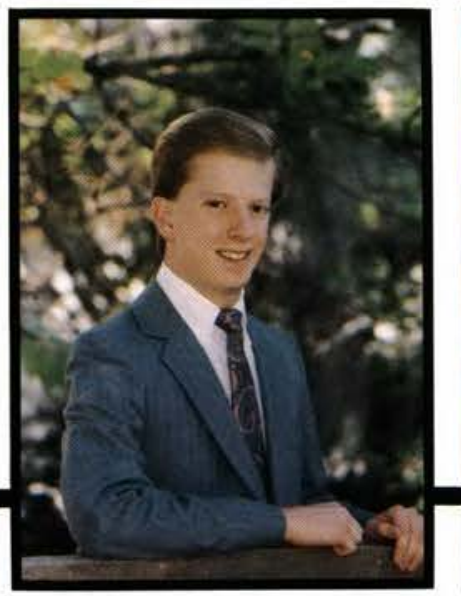

Mark Joseph Hinman

88 Seniors
9

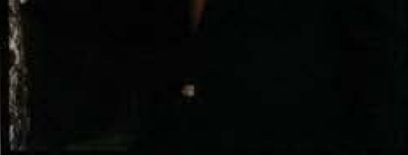

Jeffrey Lee Heffelfinger

Psychology

Troy, $\mathrm{OH}$

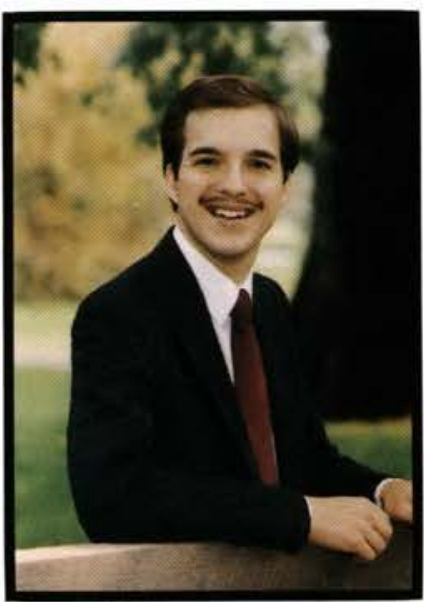

Eric D. Helmuth

Psychology, Music

Springfield, $\mathrm{OH}$

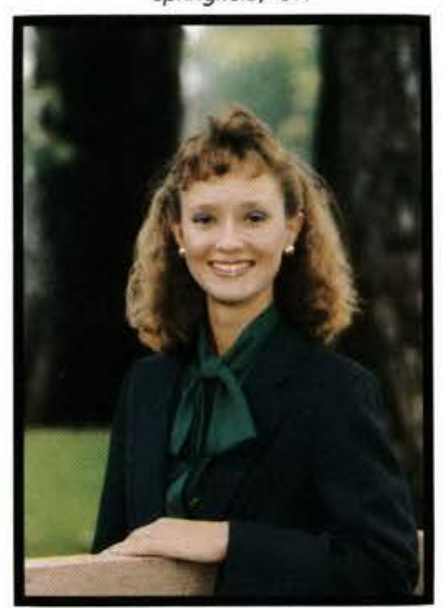

Kendall Lynn Herrick

Accounting

Morsholltown, IA

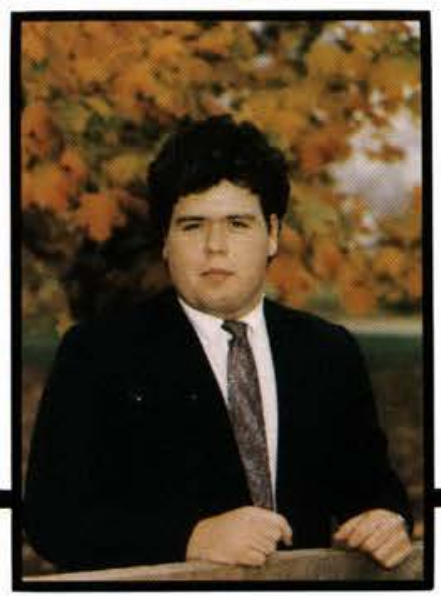

Richard Hobby

Commuinicorion Arts

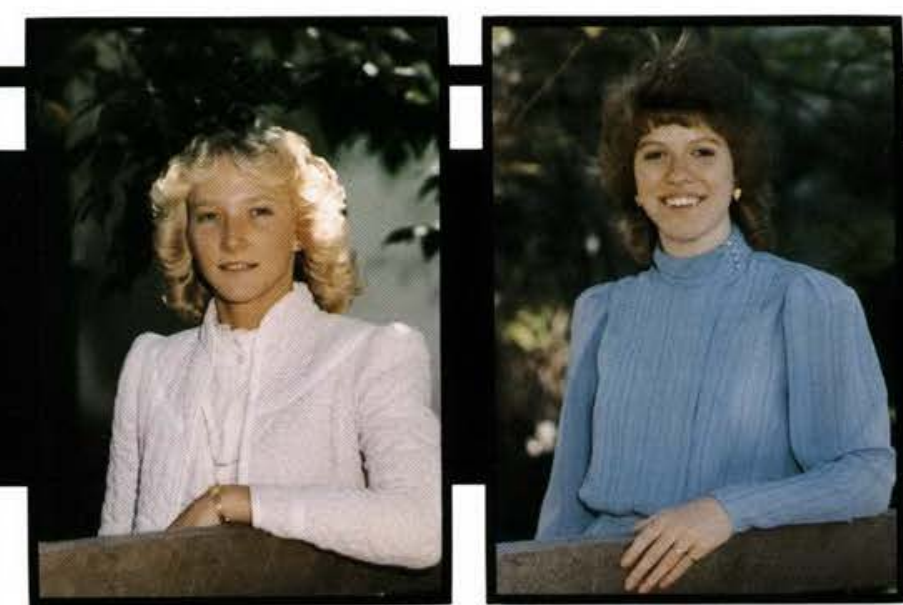

Cynthia Joan Helmick

Speech
Clayton, $\mathrm{OH}$

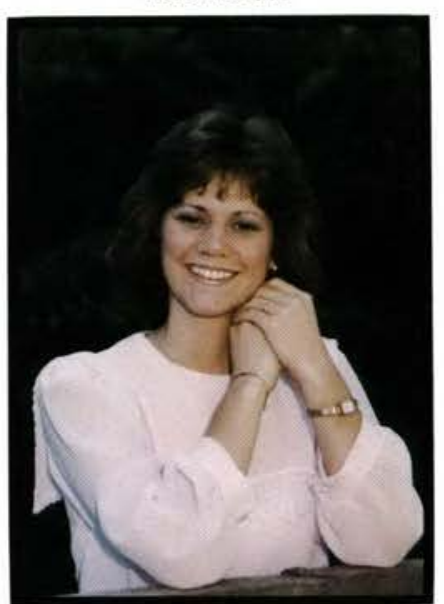

Nadeen Kay Henderson

Elemenrary Educarion

Springfield, $\mathrm{OH}$

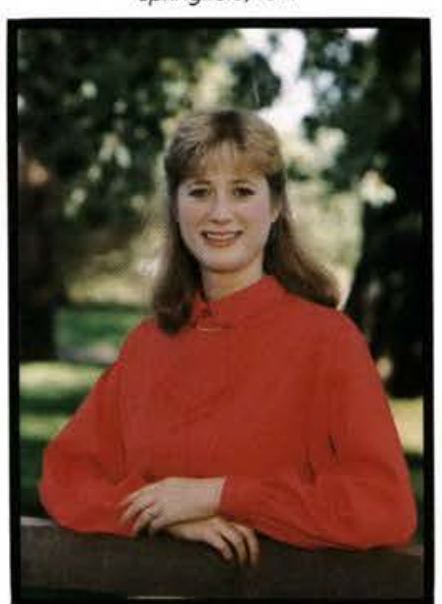

Hope Hibbard

Elementary Educarion

Woodhul, NY

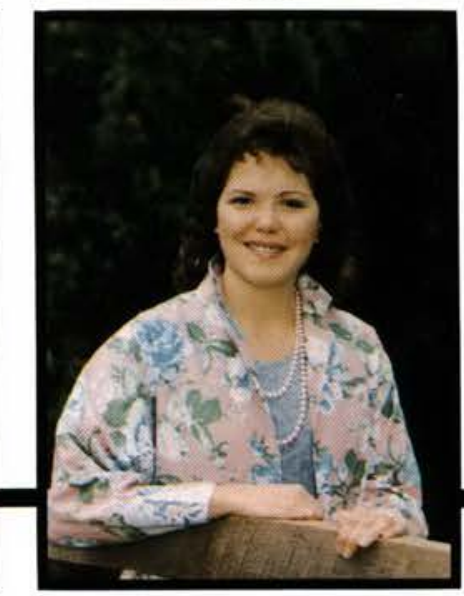

Virginia Marie Hochstettler

Nursing

Harrod, $\mathrm{OH}$

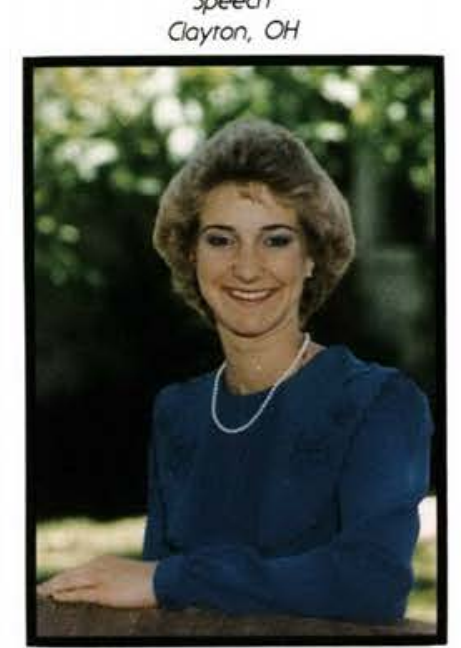

Lisa Beth Henry

Behovioral Science

Woppingers Folls, NY

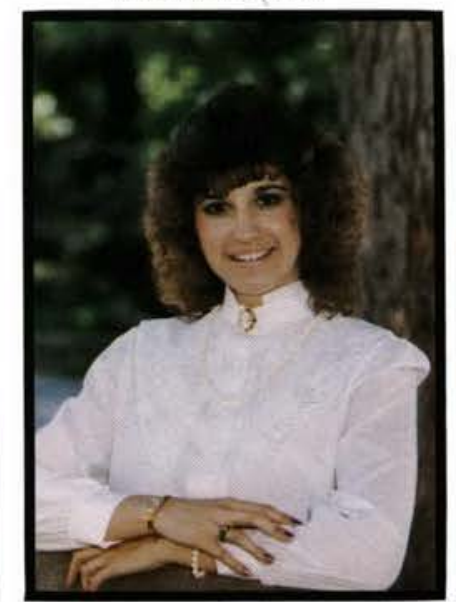

Karla Marie Hines

Psychology, Behovioral Sc Polk, $\mathrm{OH}$

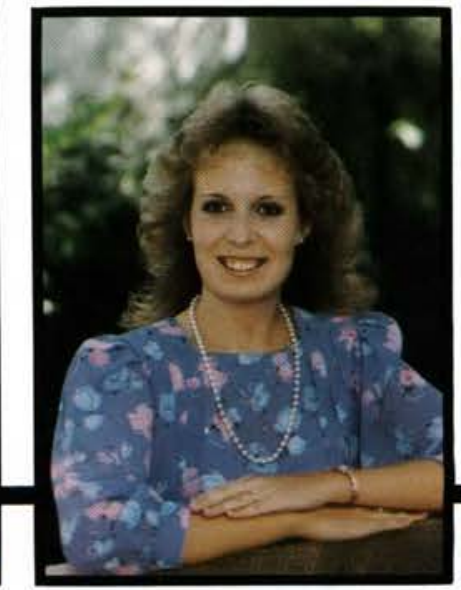

Beth J. Hoecke

Nursing

Bereo, $\mathrm{OH}$ 


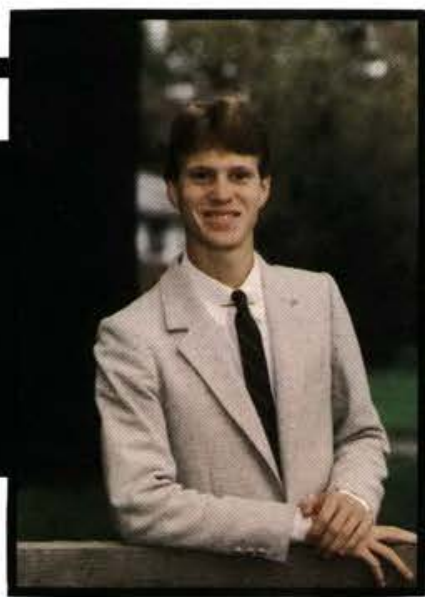

Christopher James Hoefler Comprehensive Bible Milwoukee, WI

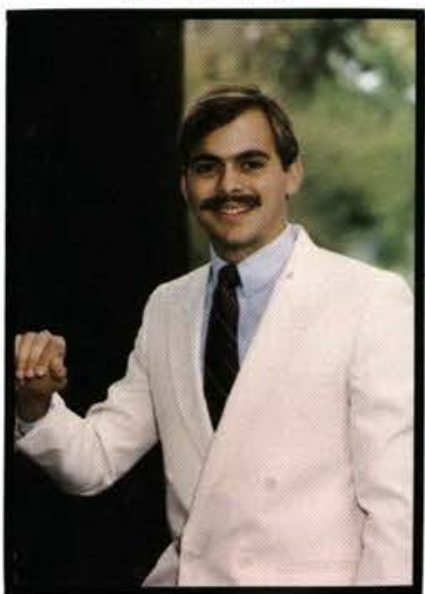

Philip J. Hohulin Preseminary Bible Major South Bend, IN

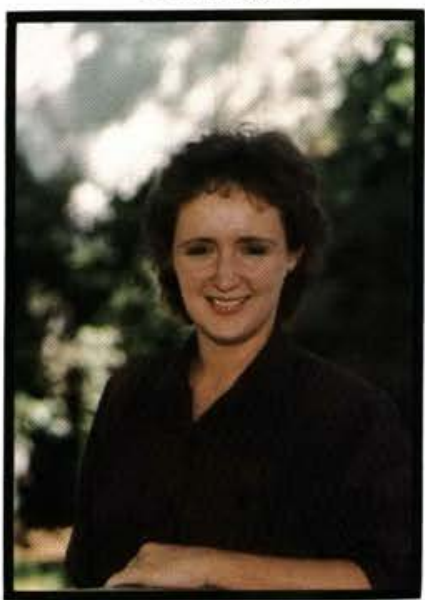

Deborah Holt Psychology Xenia, $\mathrm{OH}$

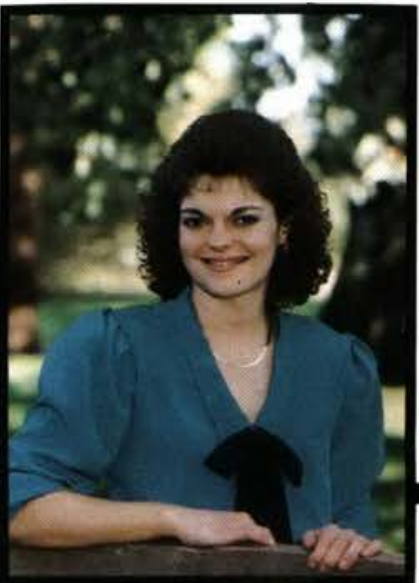

Shellie Horton

Communicarion Arts Lonsing, Mi

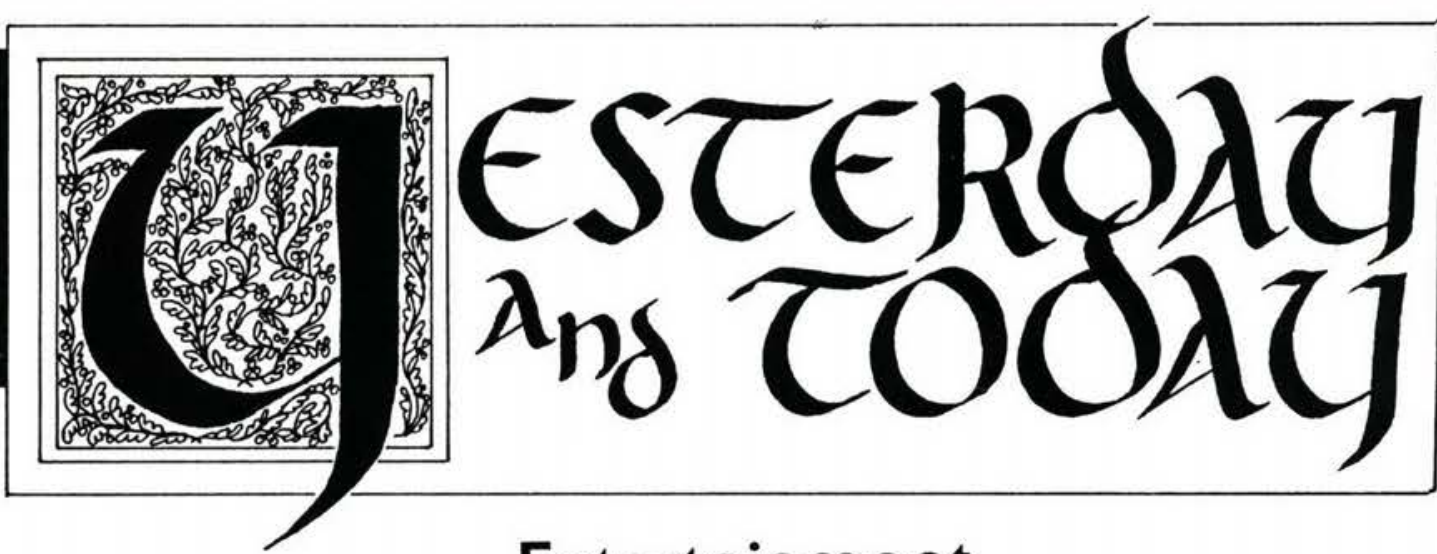

Entertainment

One of the most important reasons for attending college is to have fun. Unfortunately, unlike most large universities and colleges, Cedarville College does not have the benefit of an exciting locale. Surrounded by cornfields and cattle, college students here are hard pressed for entertainment. Throughout its $\mathbf{1 0 0}$ years, therefore, the college's students have resorted to creating their own forms of entertainment.

In the early days, life was simpler and quieter, and students were content to participate in country walks, drives, idle pranks, and freshman initiations. As the years passed, though, the activities became unique.

During the thirties, new students were welcomed to the campus with a faculty/administration tea, and they closed the year with a mock wedding, usually secrefly planned and clandestinely carried out.

The fifties brought a genuine "Social Life Committee" who planned all the events of the year to keep the students busy. Each class and organization produced its own play and presented it to the rest of the student body. The Student Wives' Fellowship continued its activities through the fifties and put on teas and banquets for the ladies.

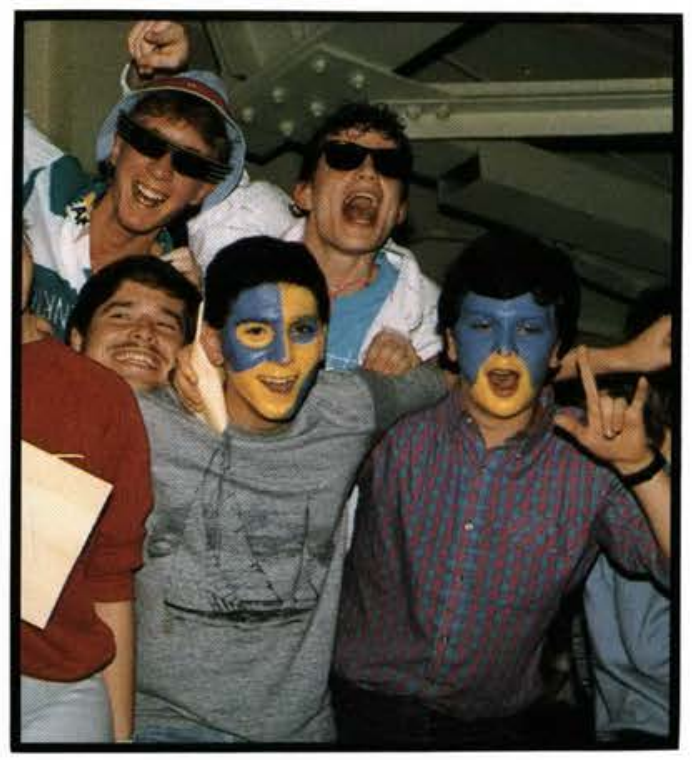

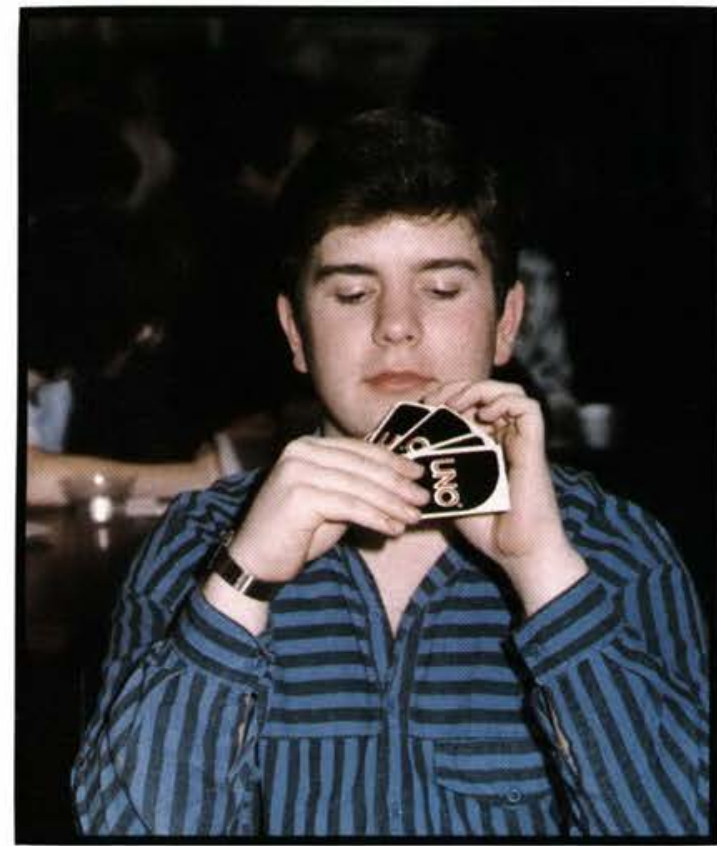

The sixties and seventies saw an increasing number of televisions on campus, which provided passive entertainment for those students who were not content with the games at the student center, sports in the gym, or other extracurricular activities.

Today, too, Cedarville students have found new forms of entertainment. The lovely Cedarville Lake provides canoeing, relaxing, and duck watching in the spring and skating and hockey in the winter. Few students miss those unbelievably unusual basketball games, complete with the Bee and pep band; and the intramural schedule includes every existing sport and some athletic activities which exist nowhere outside of this little village. "Young's runs" are a never-fail solution to almost any problem: studies, boredom, loneliness. And there is always, of course, plenty of country for a walk or bike ride.

Cedarville College students may never know what really motivated those men long ago to build a college in this "unique" setting, but its creative students somehow manage to find ways of making life fun here in the cornfields. 


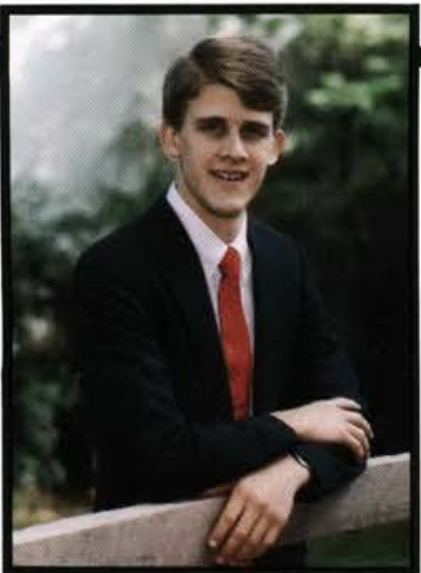

Joel M. Hoskinson

Accounting, CIS

Lewisberry, PA

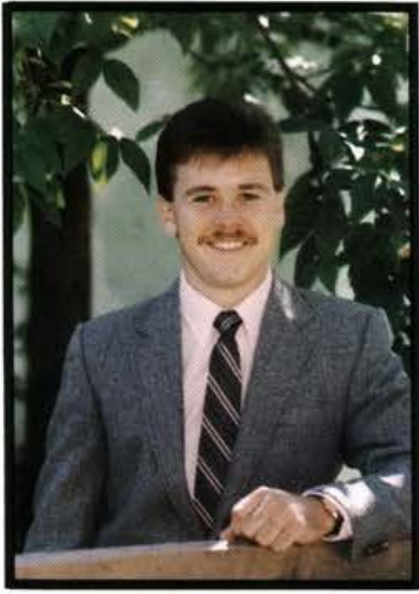

Paul R. Hughes

Business Administrotion

Pirman, NJ

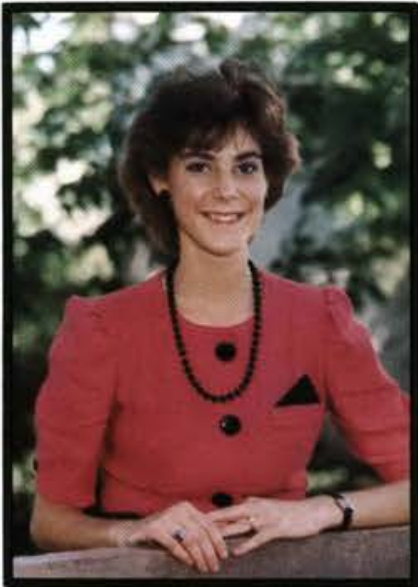

Norma Kay Hutchison

Behovioral Science

Minter, AL

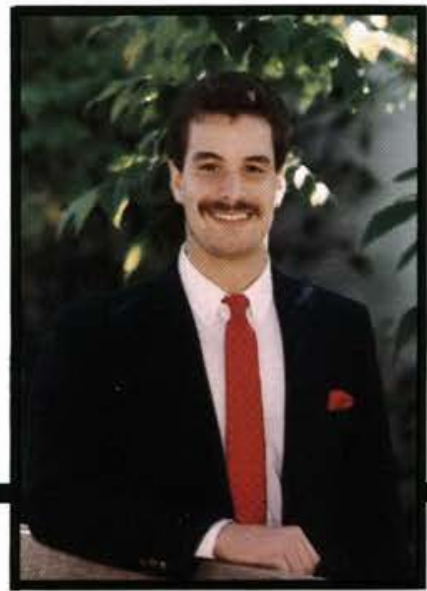

L. Charles Jackson Jr.

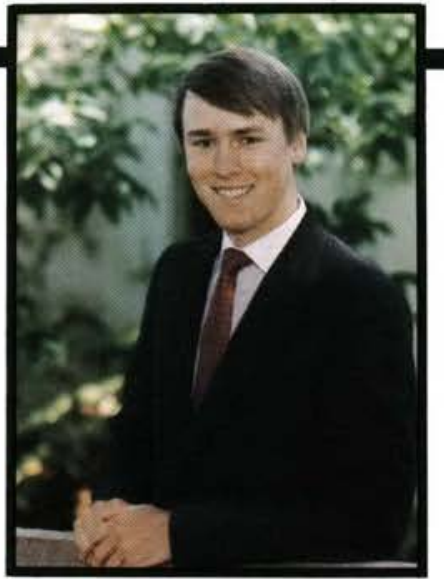

Scott J. Howder

Compurer Informarion Sysrem

Kogoshimo, Jopan

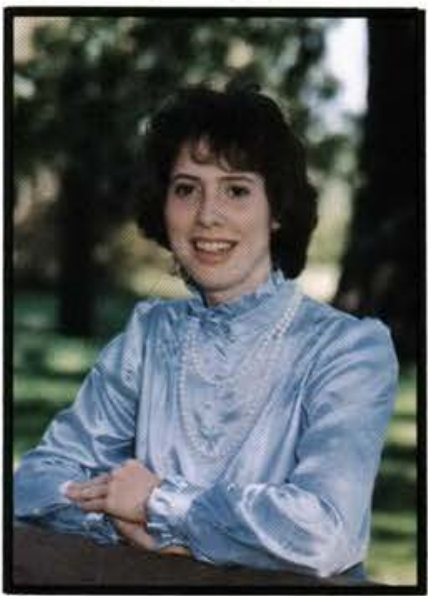

Joanie Marie Hull

Accounting

irerloken, NY

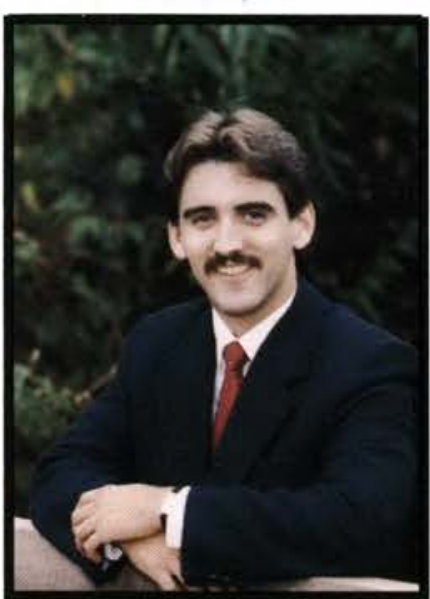

Jeffrey A. Imhoff

Accounting

Riverview, Ml

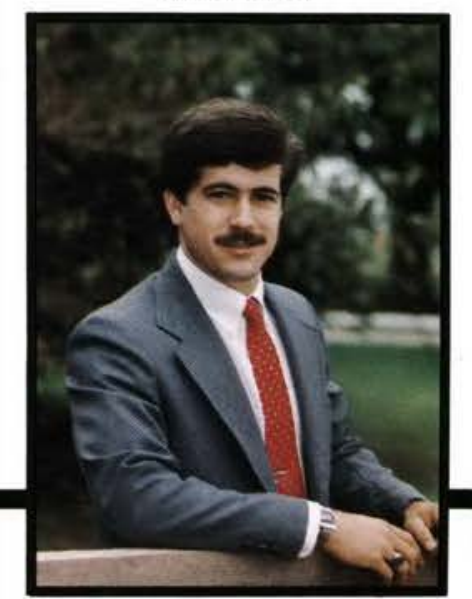

Richard D. James

Management, $C D$

Wiscosser, ME

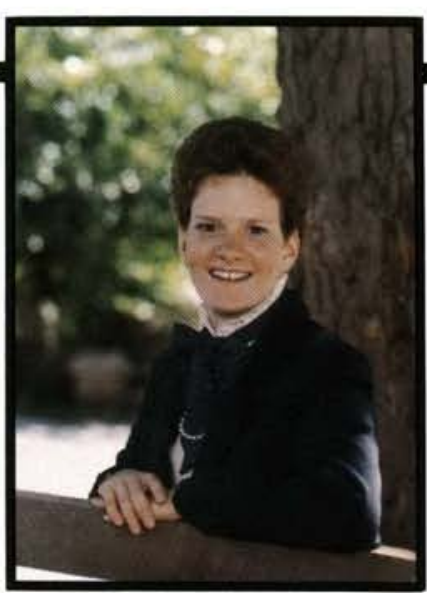

Dorothy Elaine Howdyshell Pickeringron, $\mathrm{OH}$

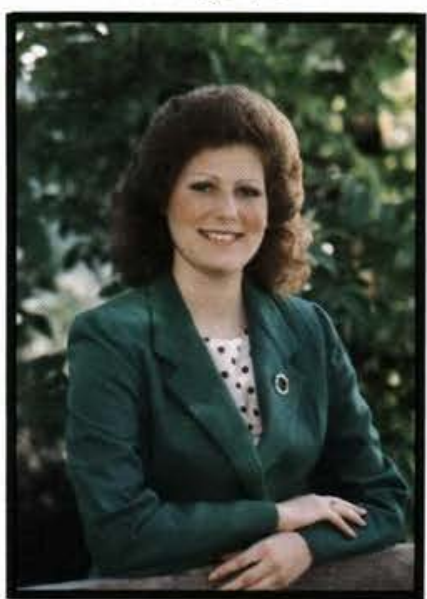

Paula M. Hunn

Behovioral Science

New Lebanon, $\mathrm{OH}$

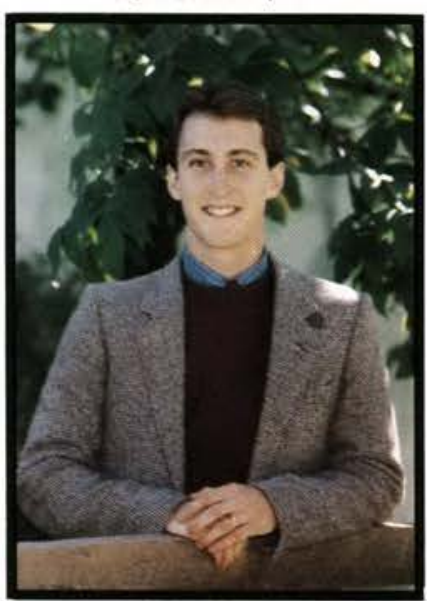

John T. Irving Jr.

English

Cedarville, $\mathrm{OH}$

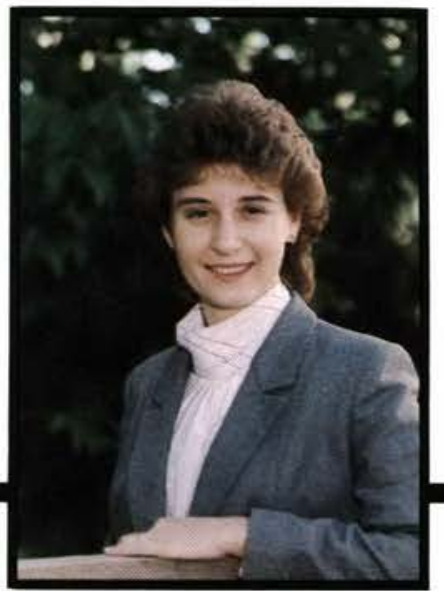

Kimberly Dawn Jenkins Business Administrorion

Lebanon, $\mathrm{OH}$

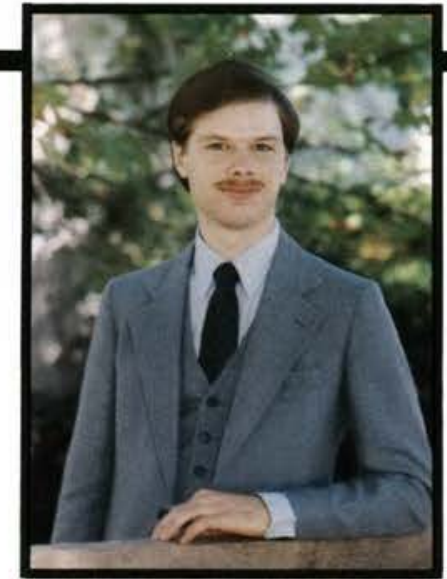

Mark David Huebscher

Marhemarics

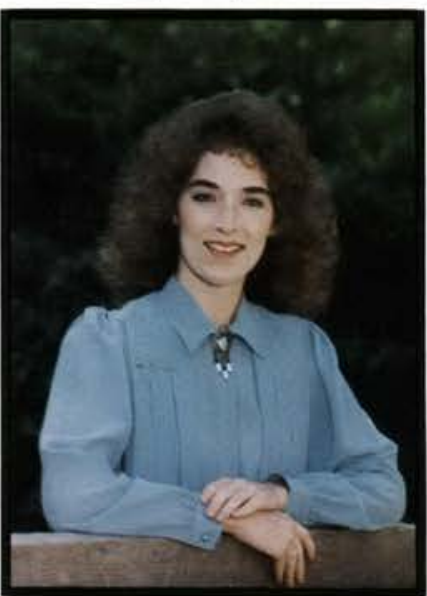

Judy M. Hunt

Nursing

Continenral, $\mathrm{OH}$

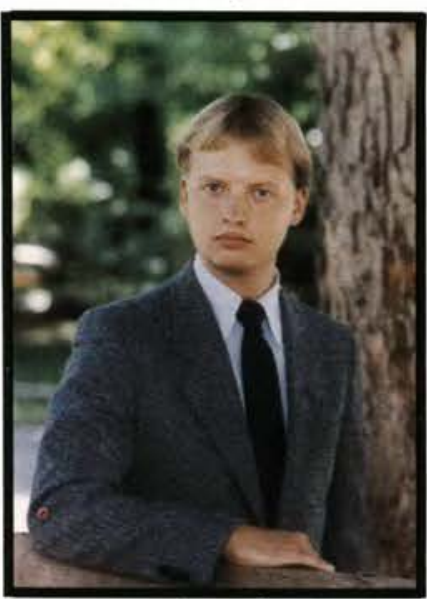

Douglas Scott Iverson

Missory

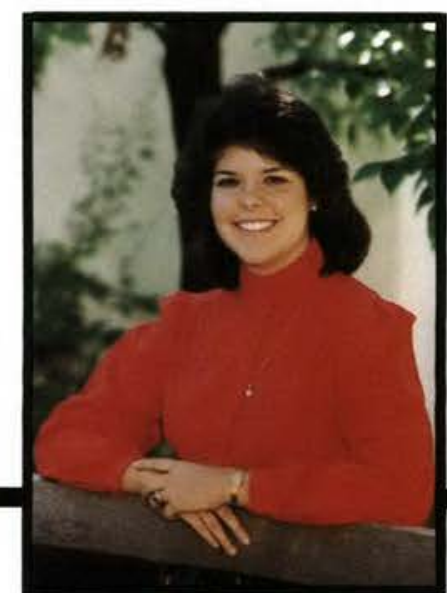

Susan L. Jessop

Elemenrary Educarion 

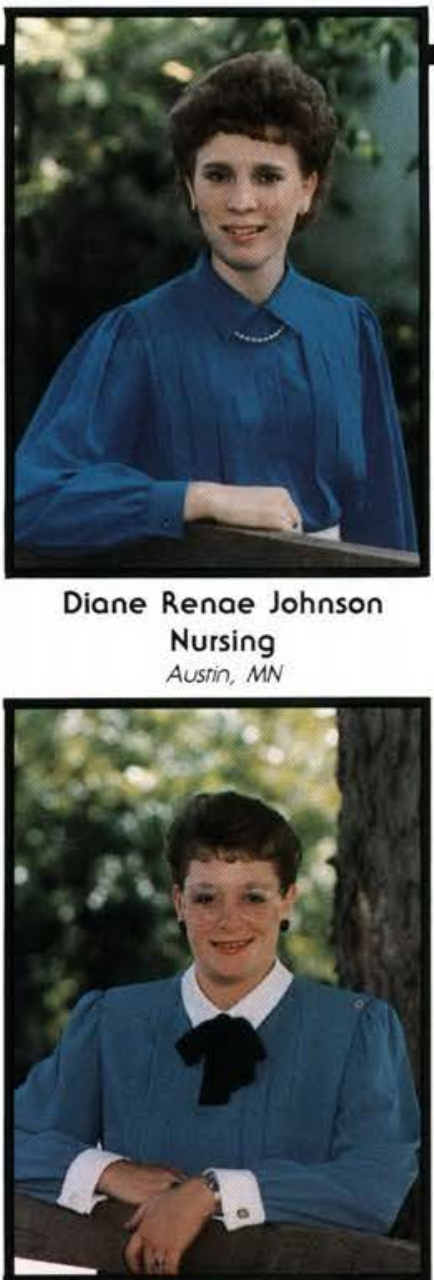

Kelly K. Jones Elementary Educarion

Union Grove, WI

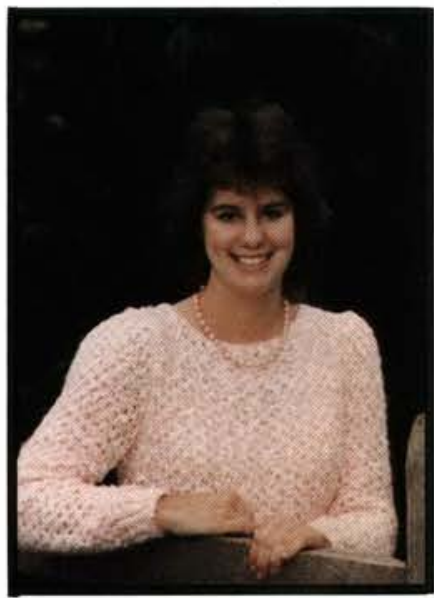

Valerie Colleen Jones English Ohiman, IL

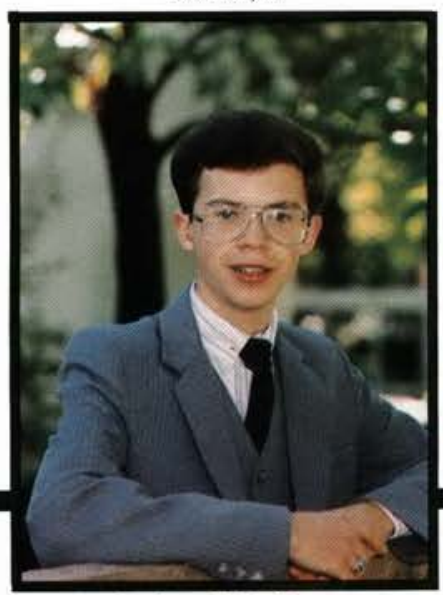

Richard T. Kester

Polirical Science

Akron, NY
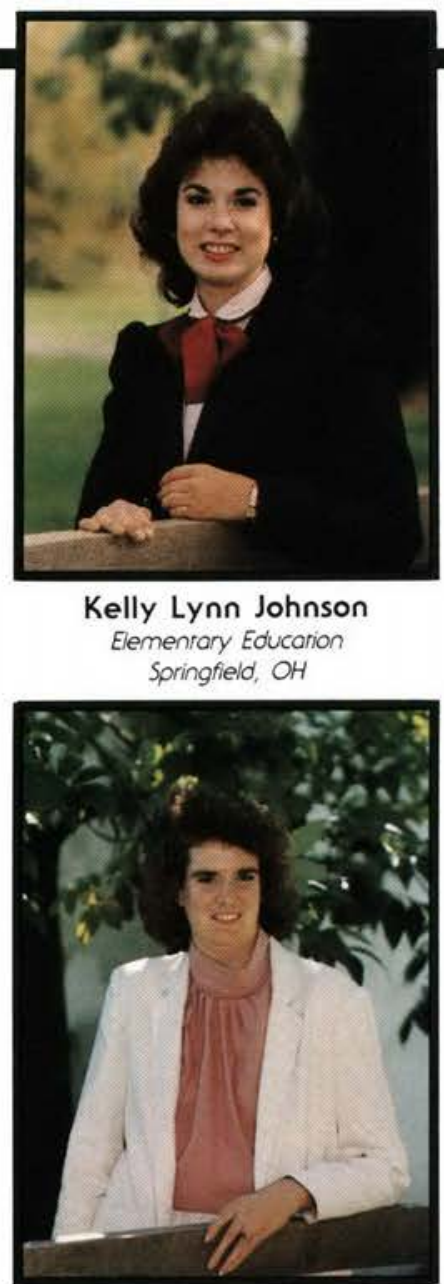

Marsha J. Jones

Accounting

Wellman, IA

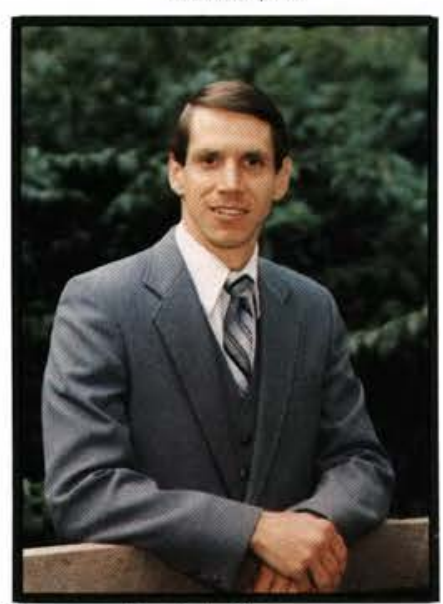

Fred E. Kanagy

Preseminary Bible Mojor

Wolford, ND

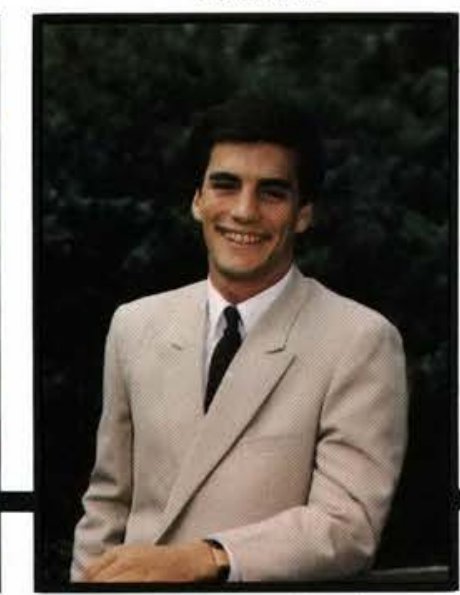

David William Ketcham Business Administrarion Wyoming, MU

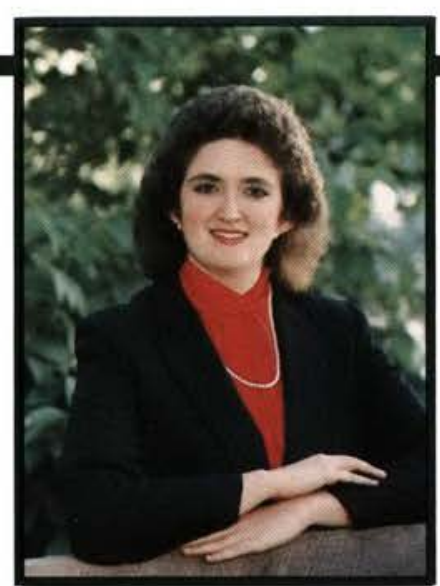

Virginia I. Johnson

Behovioral Science

Pierson, Ml

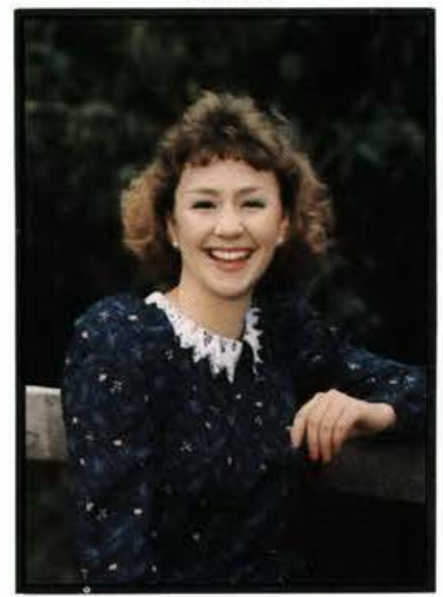

Mary C. Jones

History

Cherokee Villoge, $A R$

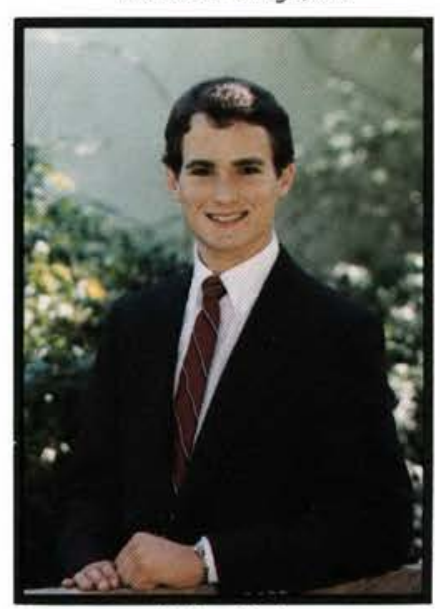

John Kejr

Business Administration

Tucson, $A Z$

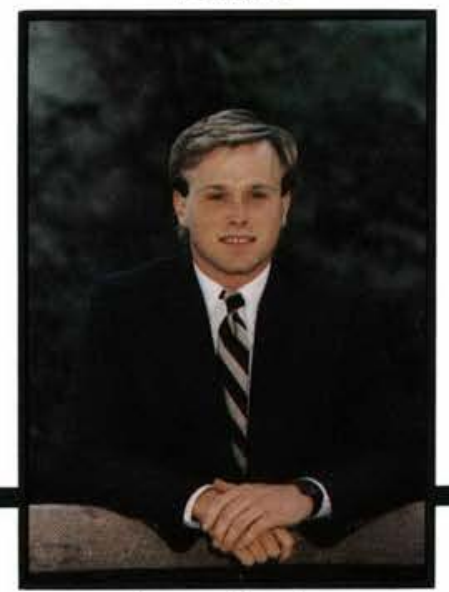

James Ketring

Business Administrotion

Woukee, IA

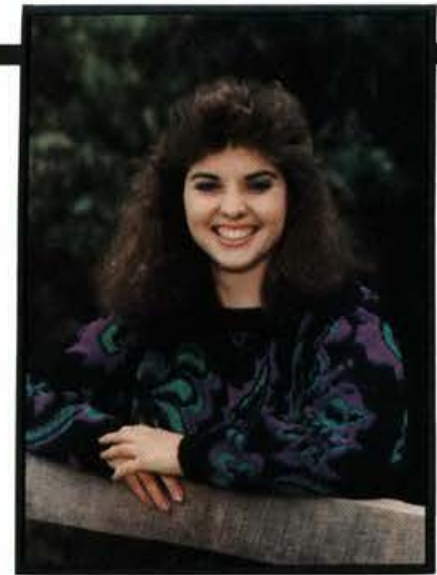

Robin L. Johnston

Elementary Educorion

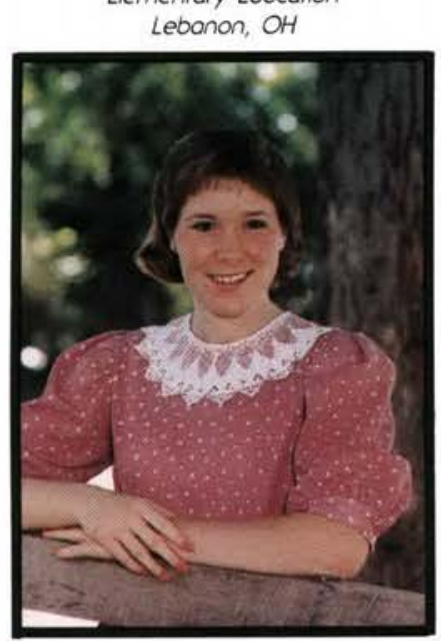

Suzanne Eve Jones Elemenrary Educarion

Rochester, NY

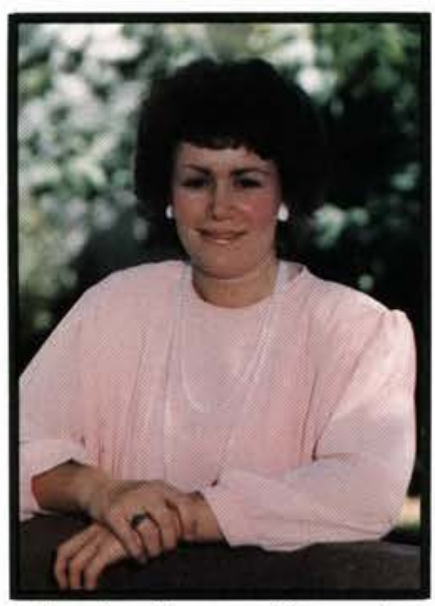

Sandra Frances Kennedy

Behovioral Science, Psychology Cambridge, Ontorio

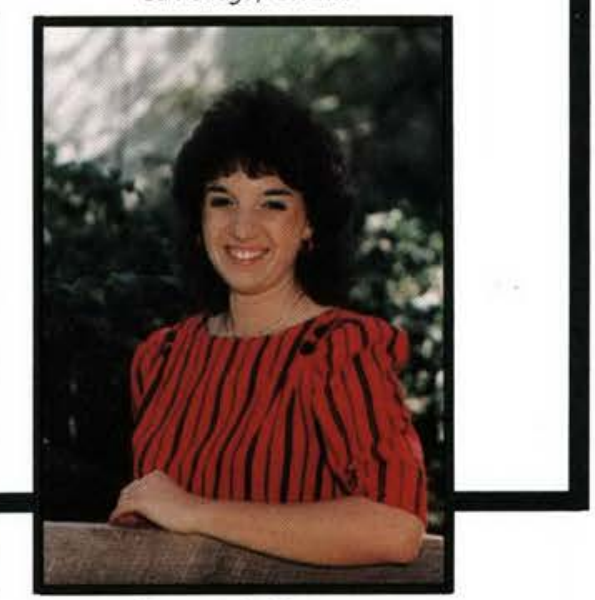

Susan J. Kiener

Nursing

Srrongsville, $\mathrm{OH}$ 


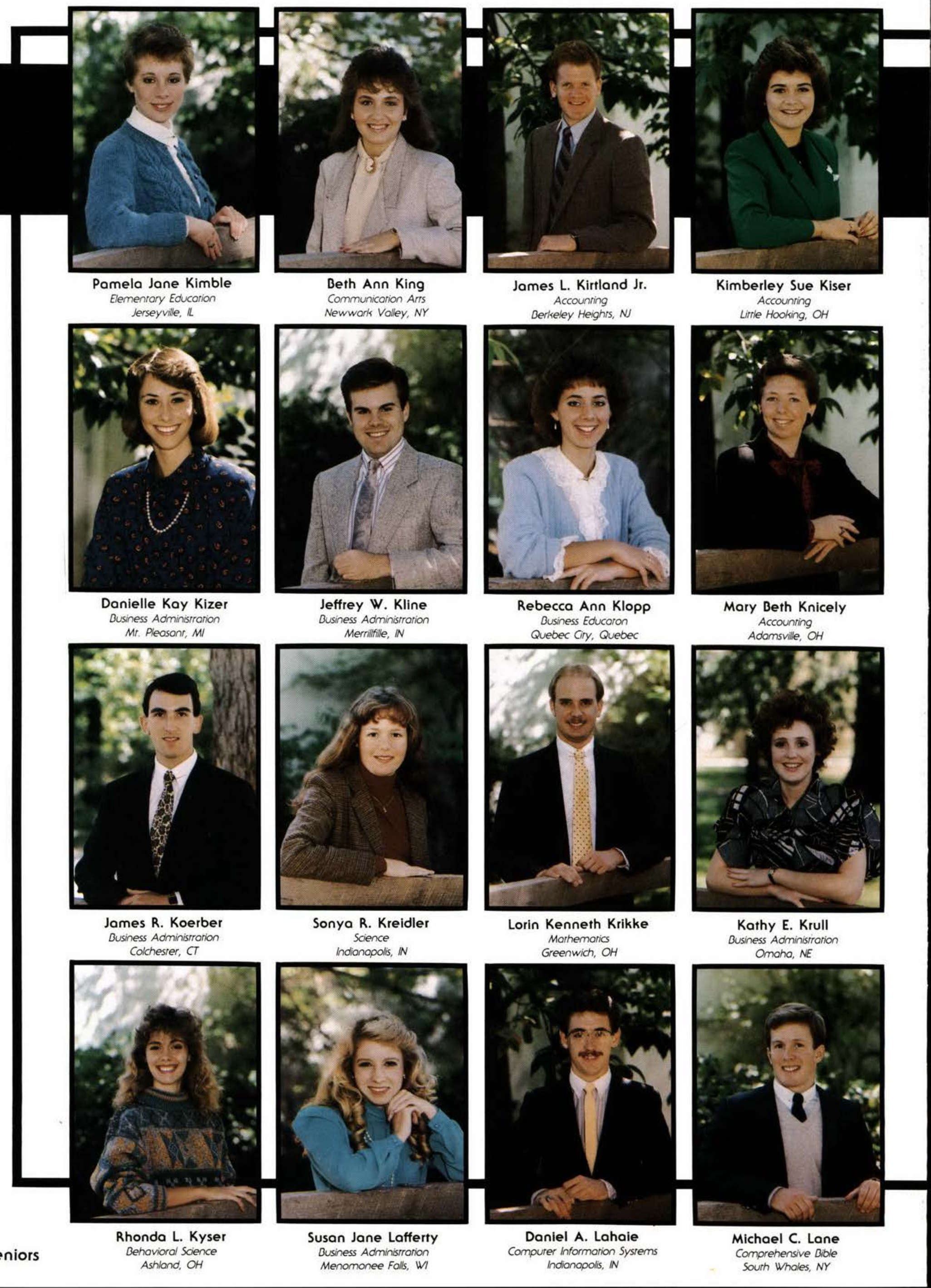




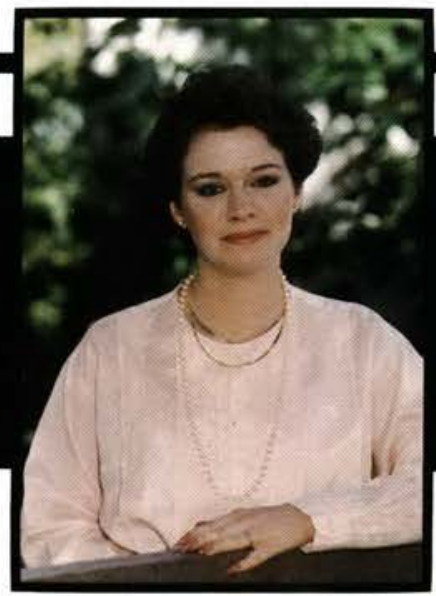

Sandra M. Lauterbach Business Administrarion Buckeysrown, MD

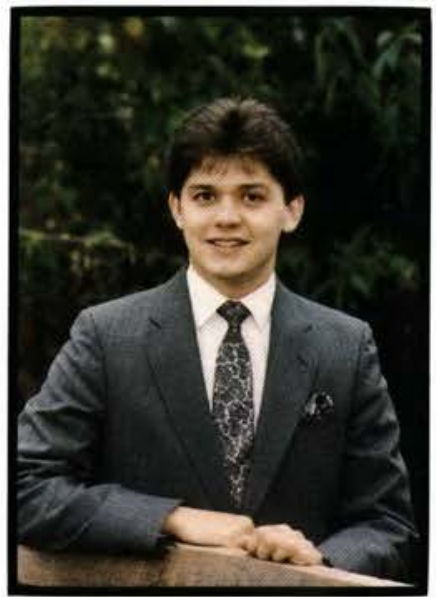

Michael David Law Music Educotion Norwich, NY

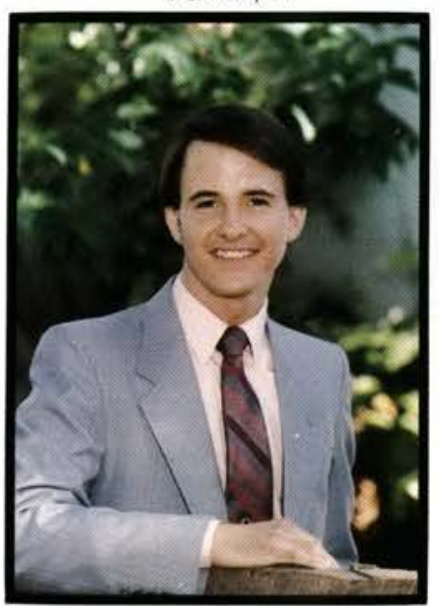

Jeffrey A. Leach Spanish Odenton, MD

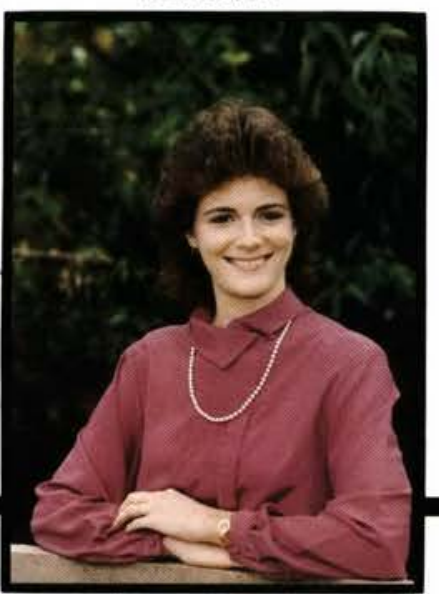

Angie Ledford

Business Administrarion Union Giry, GA
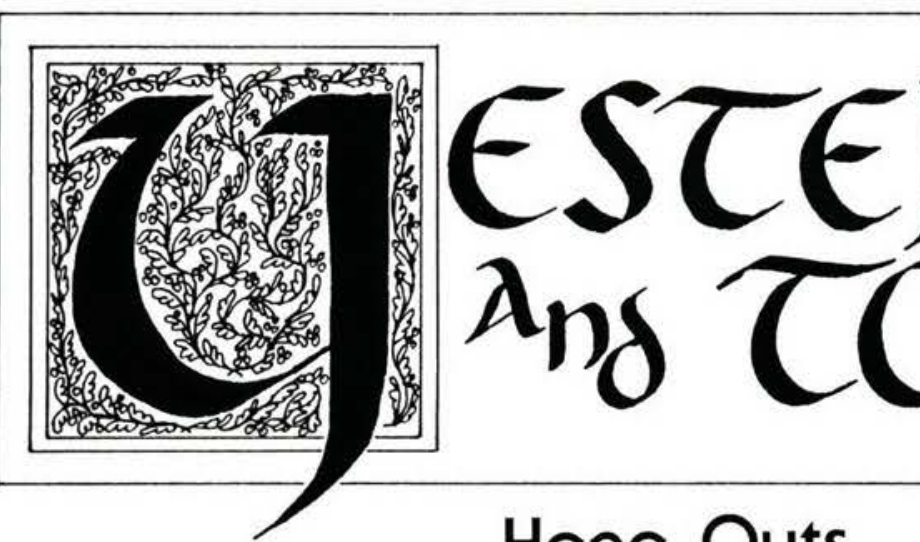
ROA $\delta \lambda$

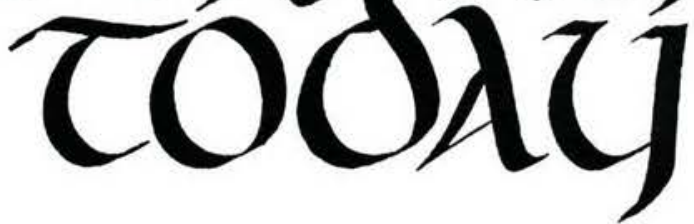

Hang Outs

The locations may have changed, but $\mathrm{Ce}$ darville students still "hang out" for the same reasons they did fifty years ago - food and fun. These pastimes are so popular that in the thirties the "Chewin' Club" (an eating organization) had to be divided into two separate clubs. When nothing else could be obtained, students resorted to country walks searching for apples.

In later years, these needs were met at a variety of "hang outs," but several are still fondly remembered, although they no longer exist. The eighties' local Cedar Junction site was once the Big Wheel, a much patronized sixties "hang out." It was that era's answer to
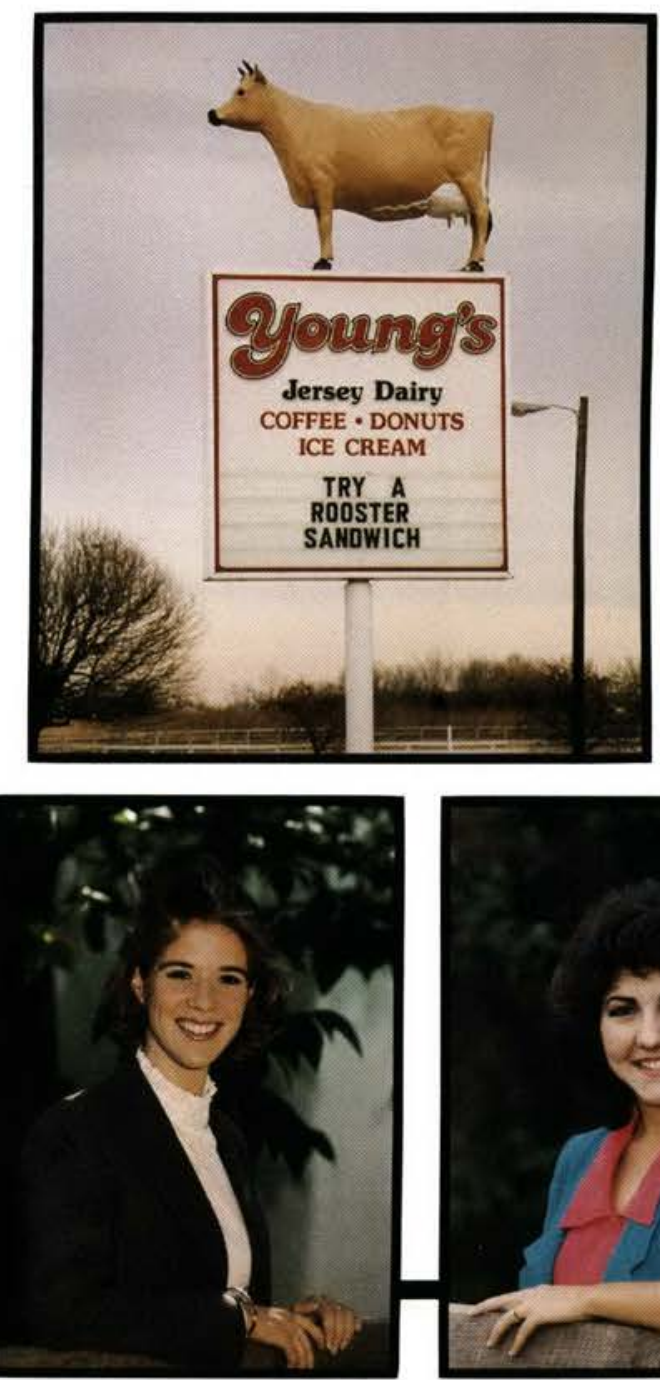

Doris Ann Lindley Business Administration Boltimore, MD
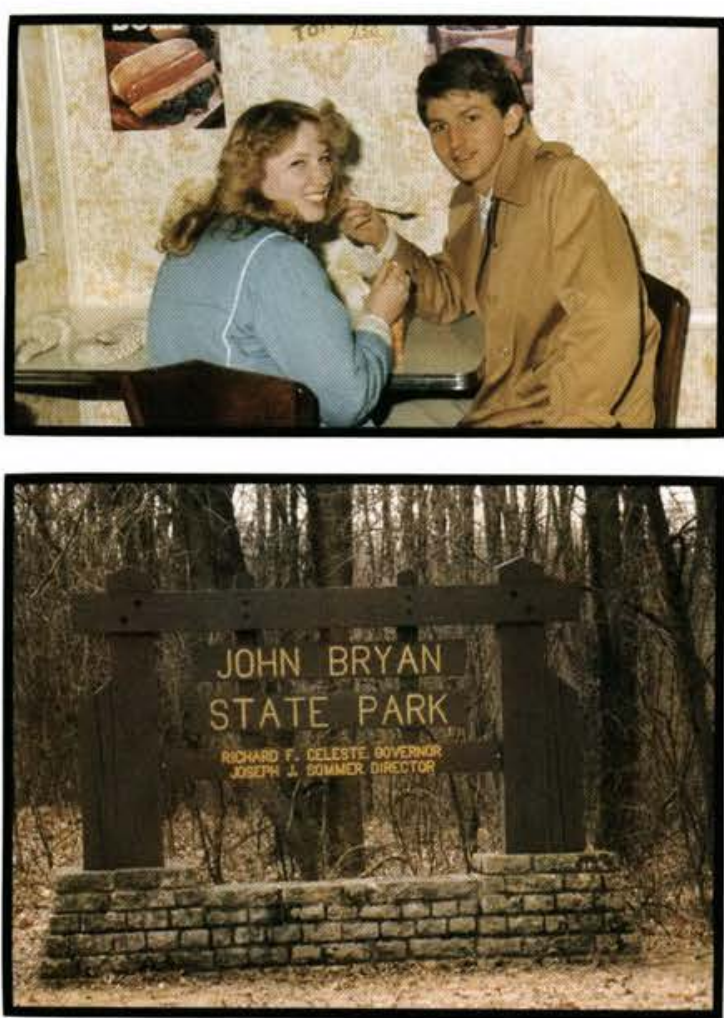

the H G R Dairy Bar. And during the blizzard in the seventies, no place was more populated than the college cafeteria; the staff served cold cuts to game-playing, snow-bound students. Today Cedarville students "hang out" at a variety of places ranging from the campus Gavelyte Deli and student center to the ever popular Young's Dairy (open 24 hours). During spring quarter, the reservoir is the "backyard" at this home away from home.

Time may have changed the locations for Cedarville student "hang outs," but it's still hard to find another group of people who are so highly motivated by the desire for anything ed. ible and an opportunity to laugh than today's CC students. 


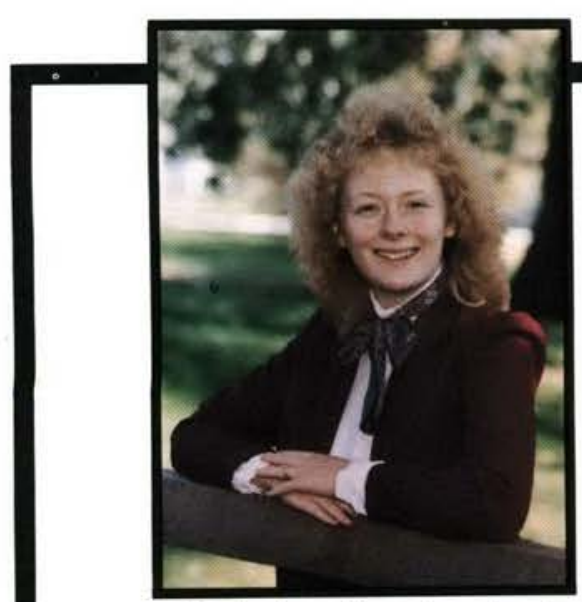

Catherine Mary Lombard

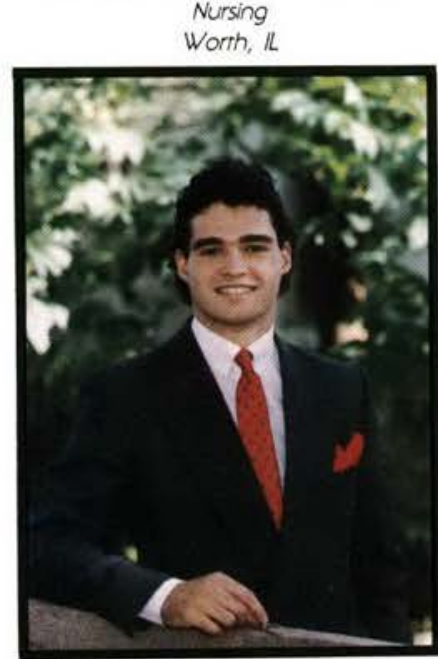

Sheldon Scott MacGillivray American Srudies

Toronto, Onrorio

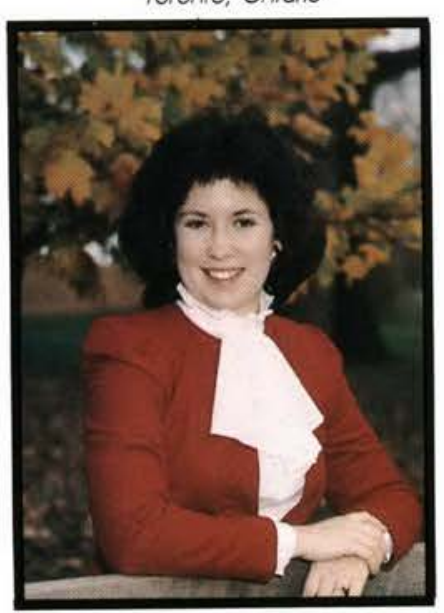

Holly S. Marshall

English Educotion

Phoenixville, PA

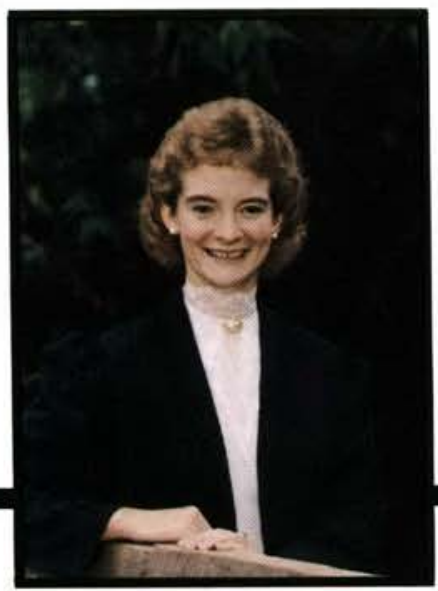

Kimberly A. Masters Physical Educarion

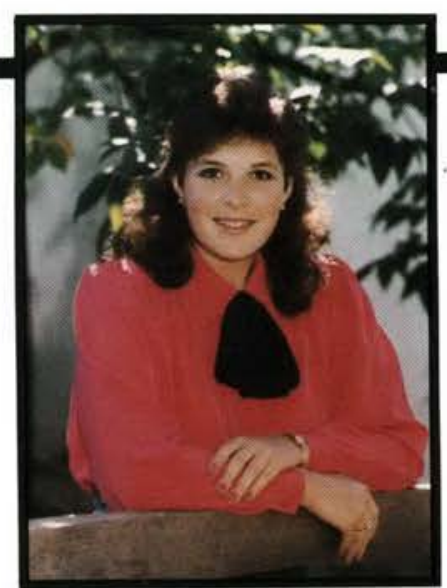

Linda A. Lons

Business Administration

Medino, $\mathrm{OH}$

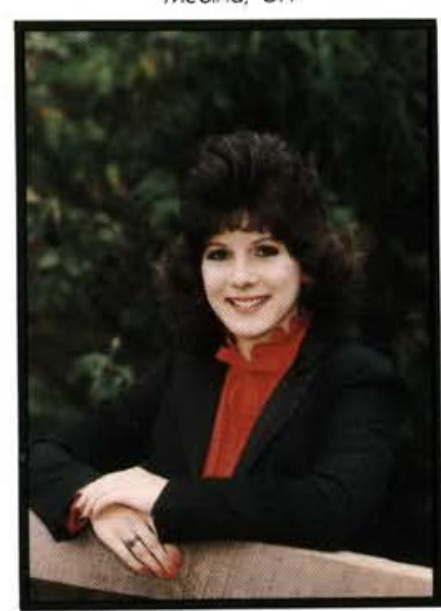

Terri Lynn Mackenzie Music Educarion

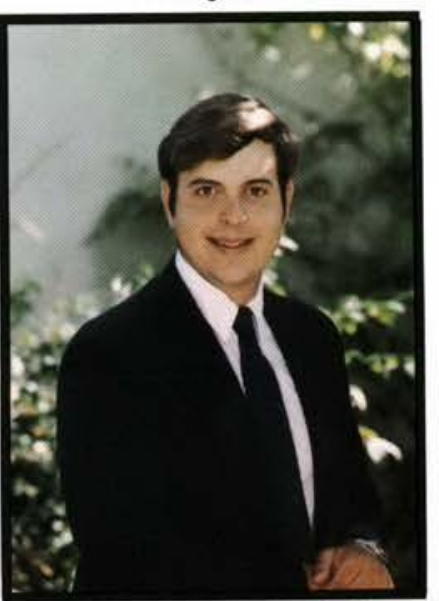

William Paul Marshal

Accounting

Schroon Lake, NY

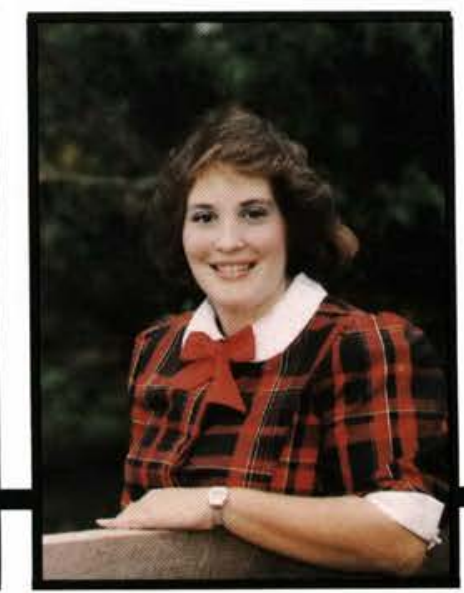

Brenda Kay Mastin

Nursing

Covingron, $K Y$

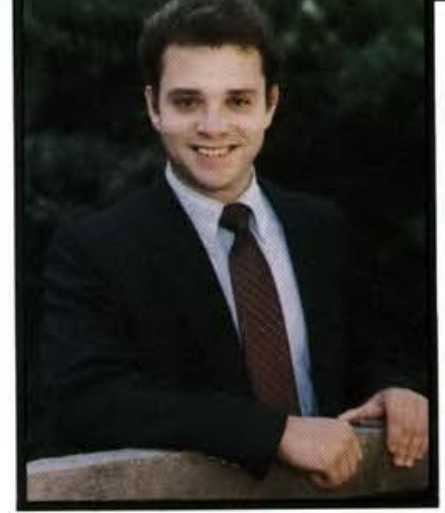

Donald H. Lough Jr.

Comprehensive Bible

Schroon Loke, NY

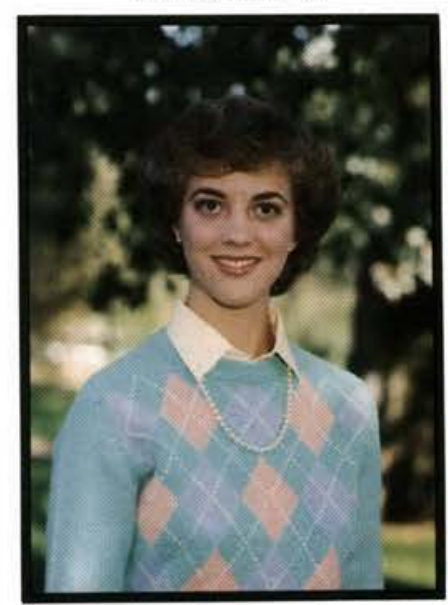

Lisa Manion

Business Administration

Plano, il

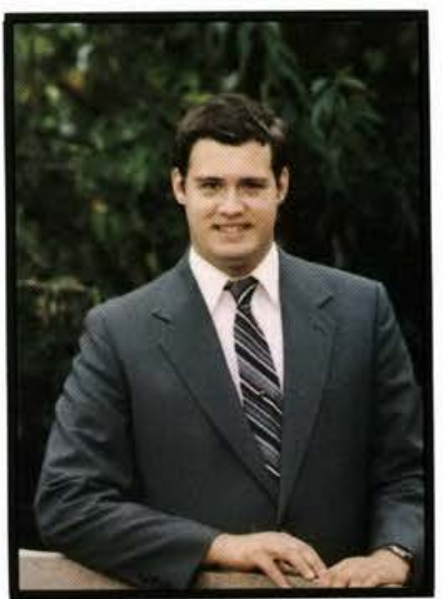

Ronald L. Martin

Comprehensive Bible

Verona, W/

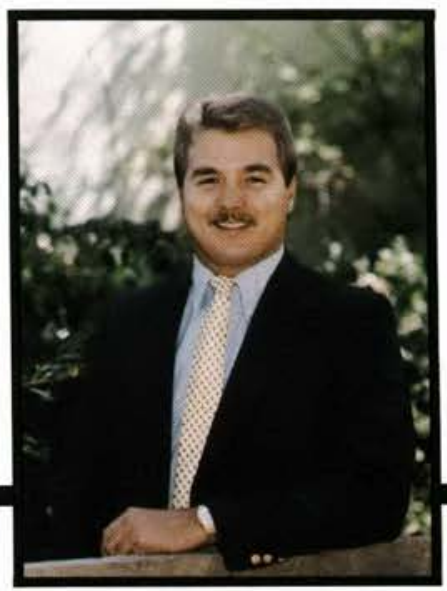

Kelly R. Mathis

Business Administrotion

Chillicorhe, $\mathrm{OH}$

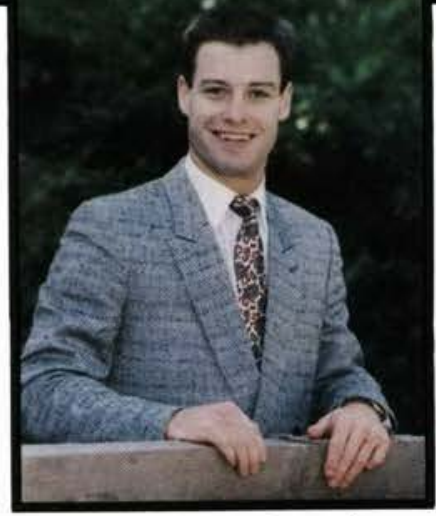

Roger Dale Luttrell

Communicorion Arts

Columbus, $\mathrm{OH}$

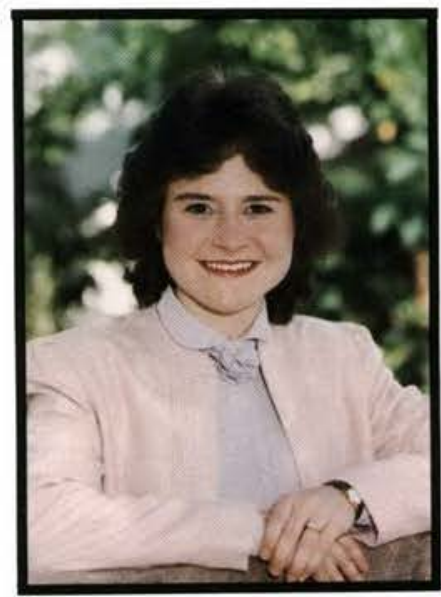

Lynda L. Marriott

Nursing

Cincinnori, $\mathrm{OH}$

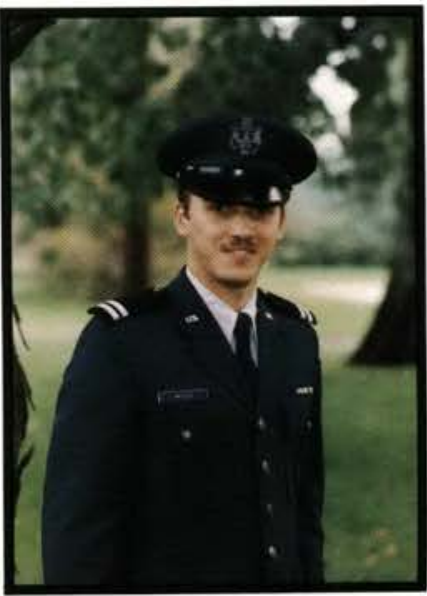

Rodney Mark Mason

Accounring
Hunringron, WV

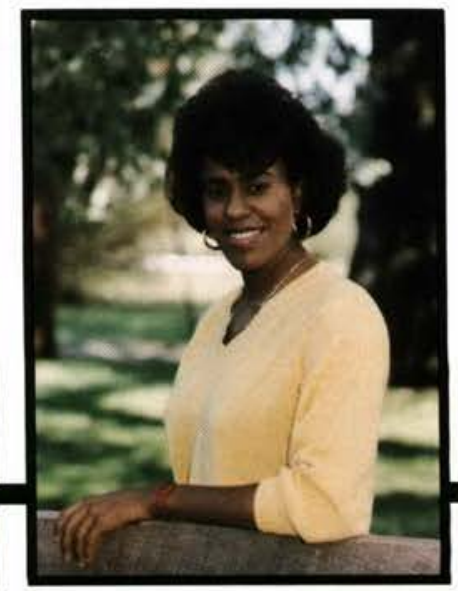

Tanya McBee

Nursing

Columbio, MD 


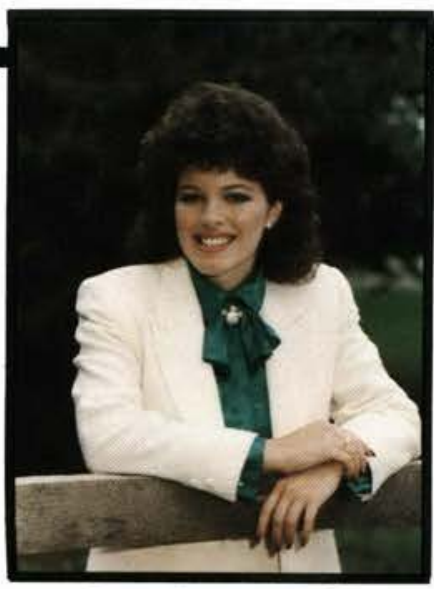

Tracy Lynne McCoy Psychology

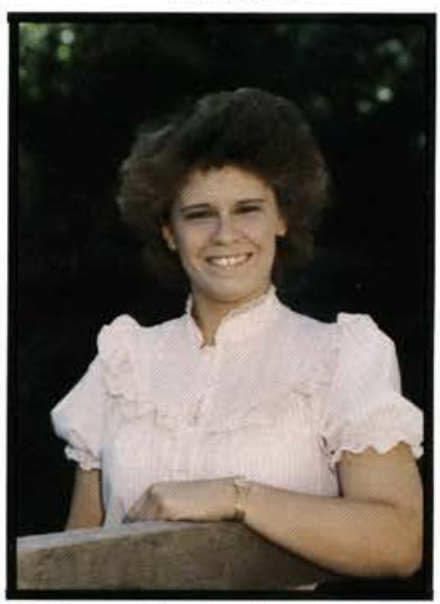

Dana Gayle Messinger Behaviord Science Tiffin, $\mathrm{OH}$

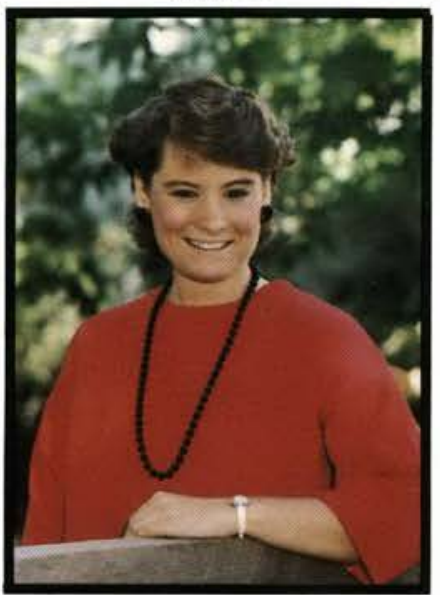

Sherrie Lee Mills

Criminal Justice

Erie, PA

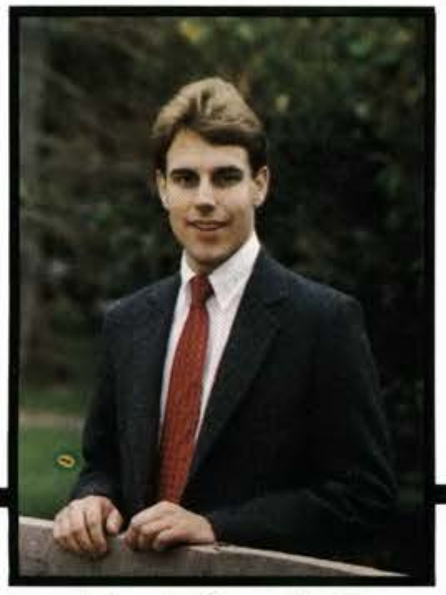

John Andrew Moore

Social Science

Hinckley, $\mathrm{OH}$

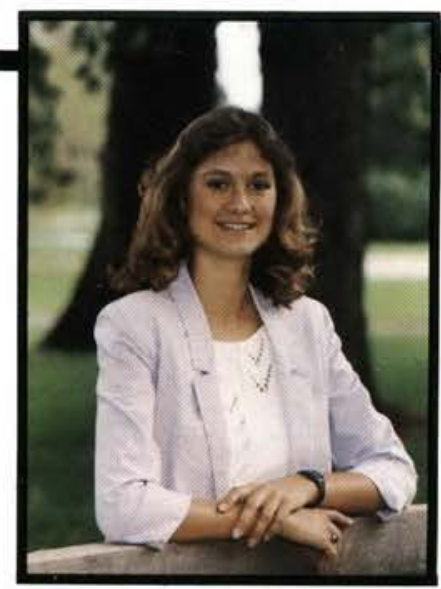

Elizabeth M. McGillivray English

Cedarville, $\mathrm{OH}$

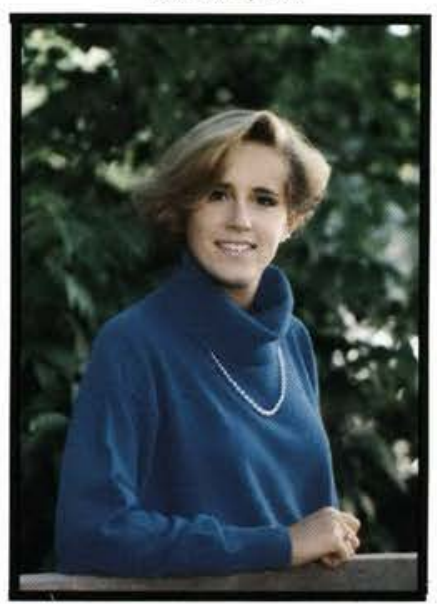

Jill Meridith Michonski

English

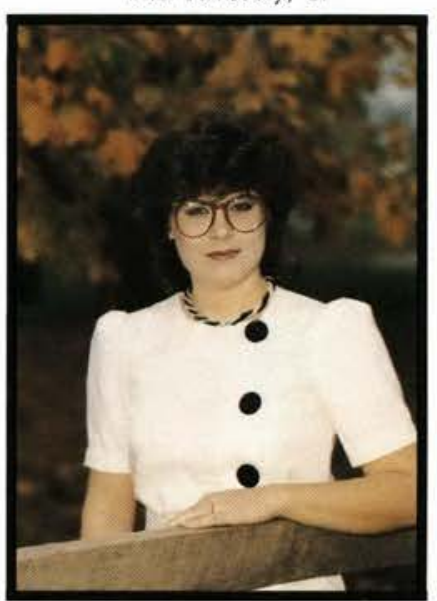

Donna Mitchell

Physical Educarion

Xenia, $\mathrm{OH}$

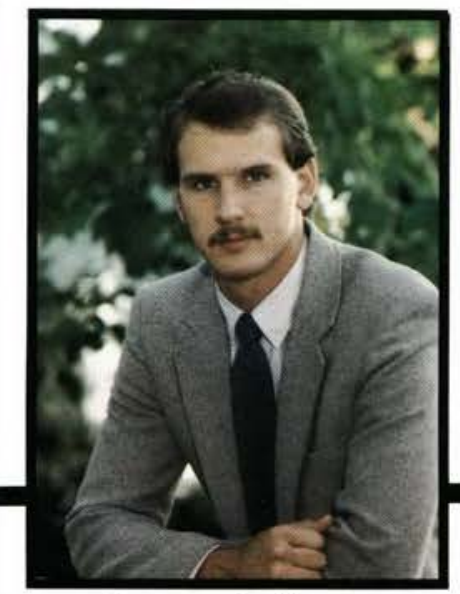

Gregory S. Moran

Accounring

Alexandrio, VA

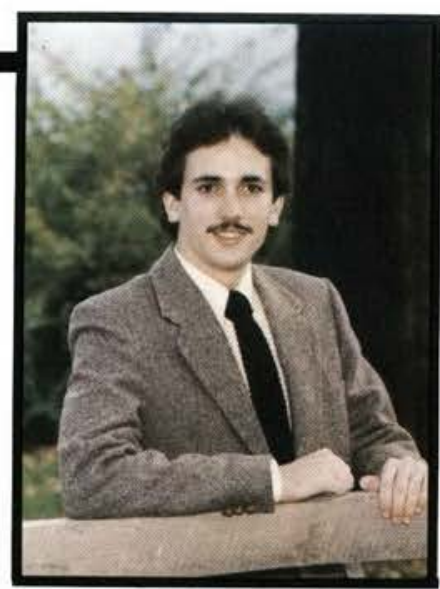

Mark Mclntyre

Behovioral science

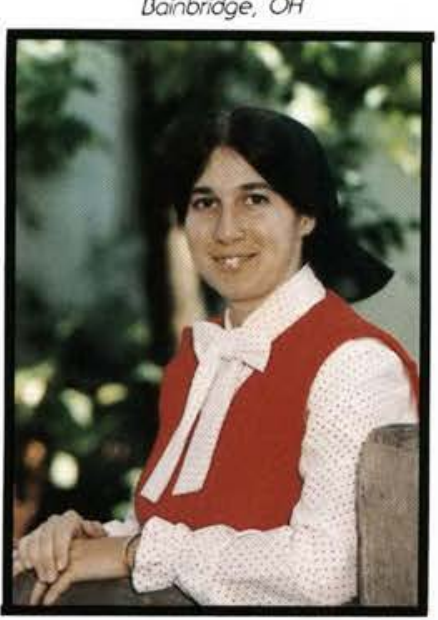

Shirley Miller

Elementary Educario

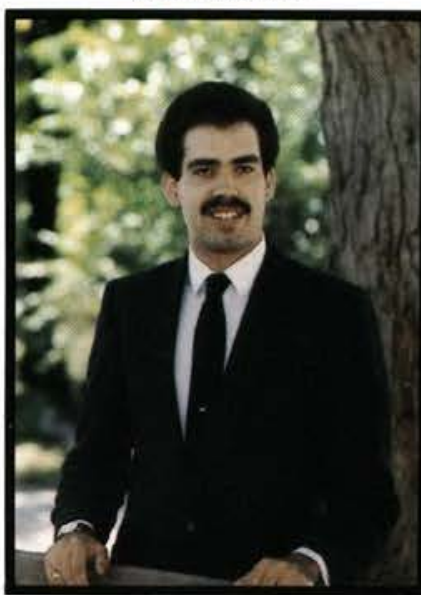

Brett Earl Montford

Broodcosting
Mr. Hope, KS

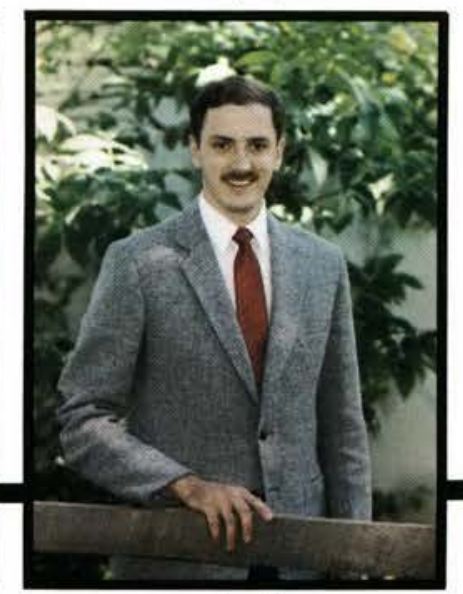

Dan L. Morris

Business Administration

Morierto, $\mathrm{OH}$

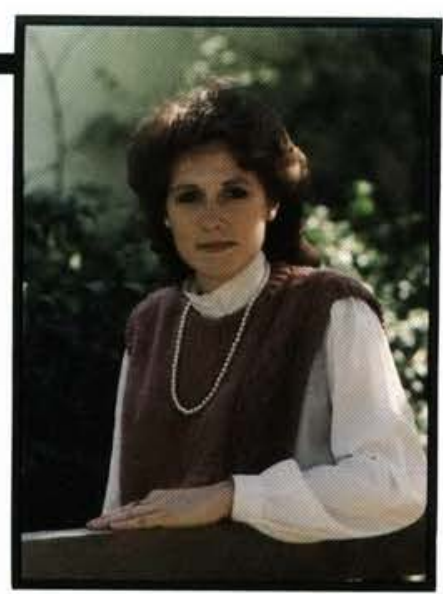

Carol Marie McKinley Elementary Educorion Codilloc, MI

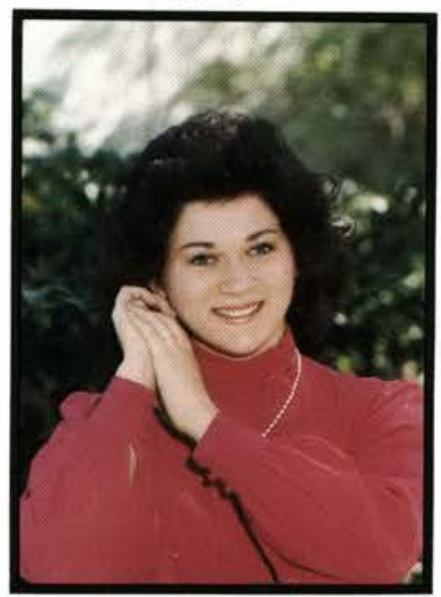

Tricia Dawn Miller Elementary Educarion Cosstoain, $\mathrm{OH}$

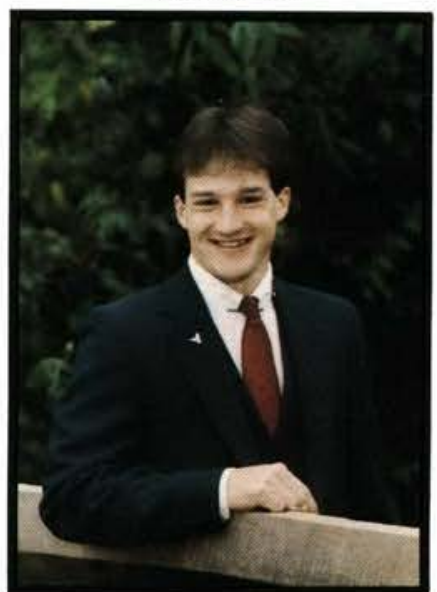

Bryan Edward Montross

Biology

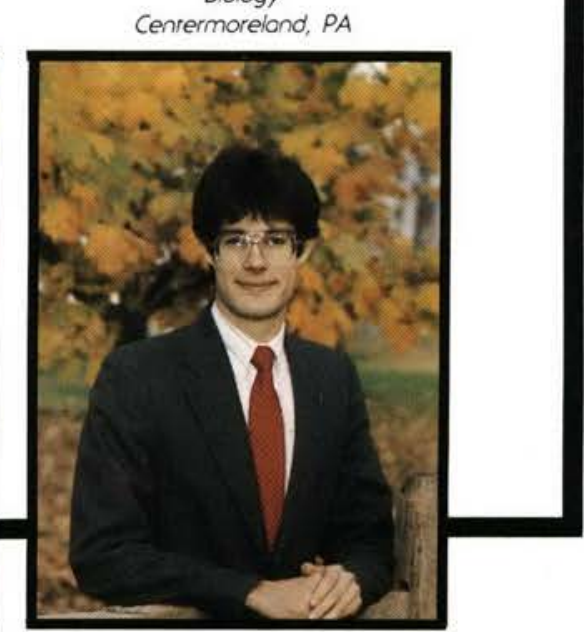

Dean Morse

Accounting 


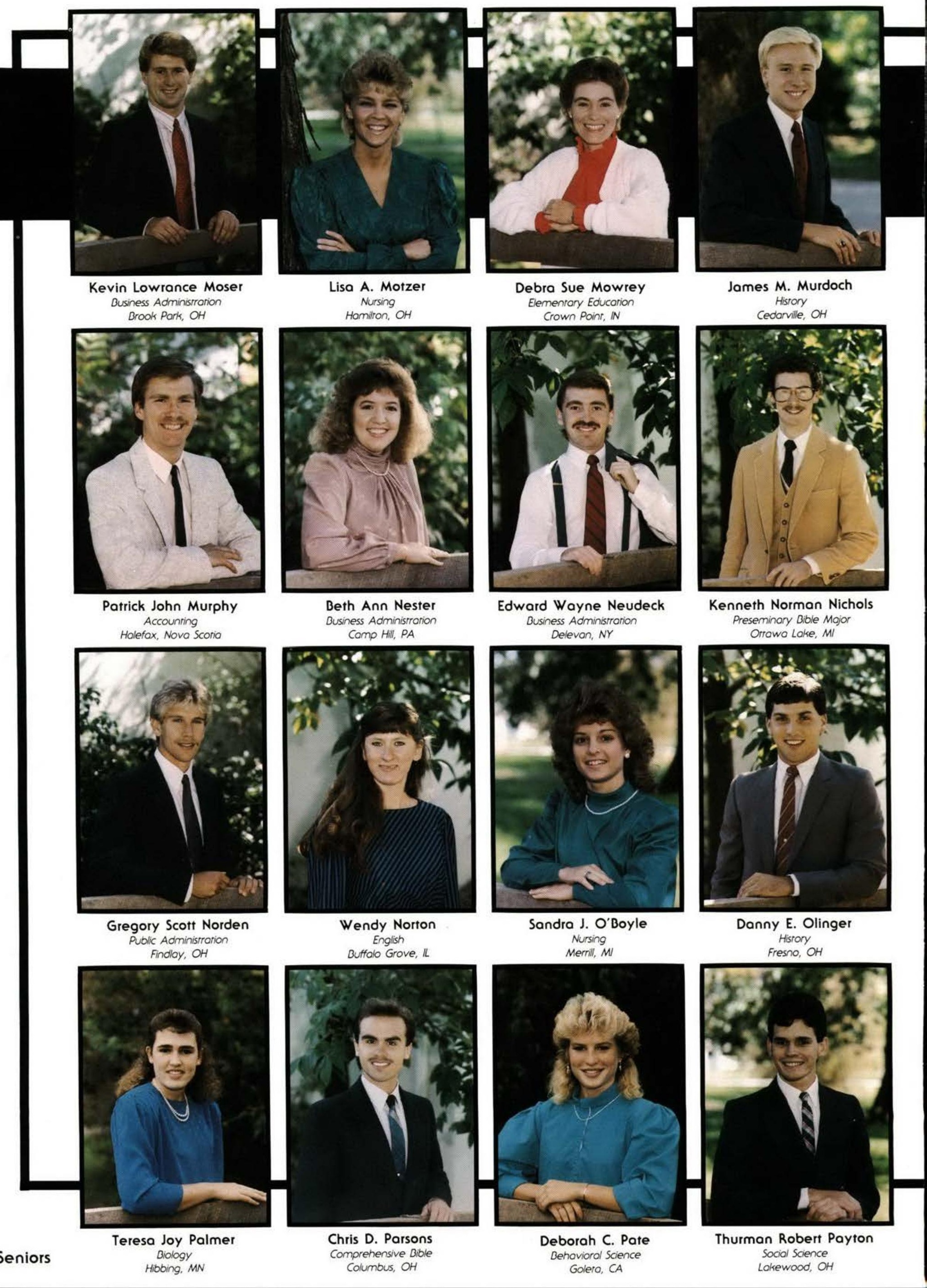




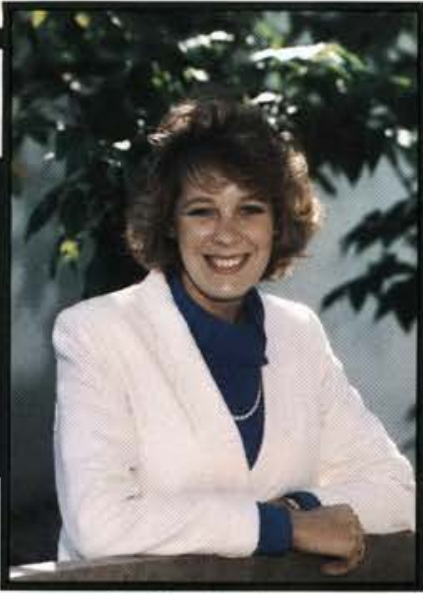

Shelle Perkins Accounting Hinckley, $\mathrm{OH}$

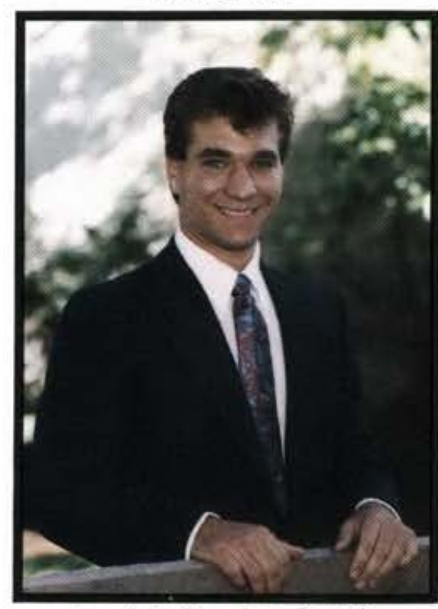

Daniel Charles Petek Business Administrarion Medina, $\mathrm{OH}$

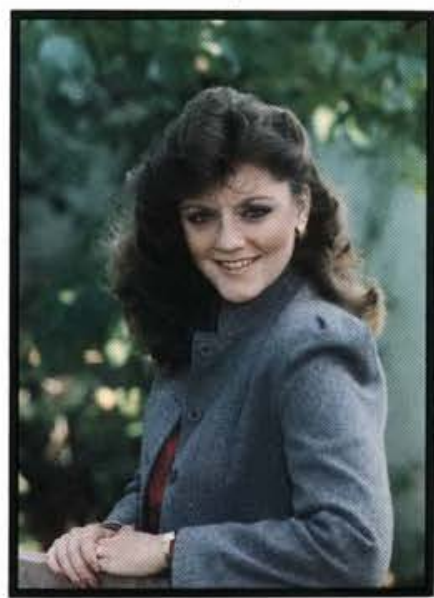

Reneé A. Peterson English Educorion Srerling, VA

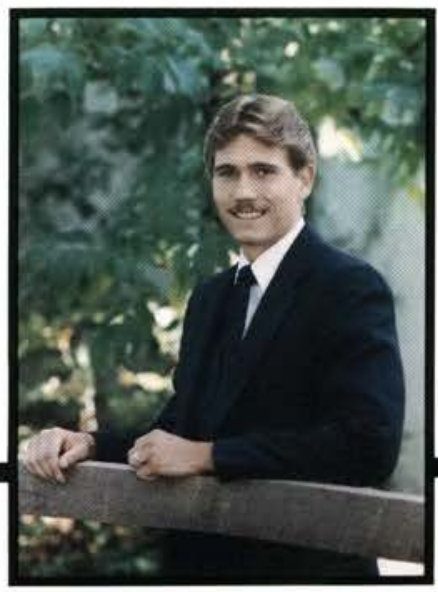

Thomas L. Peterson Business Administrorion Belmonr, NY

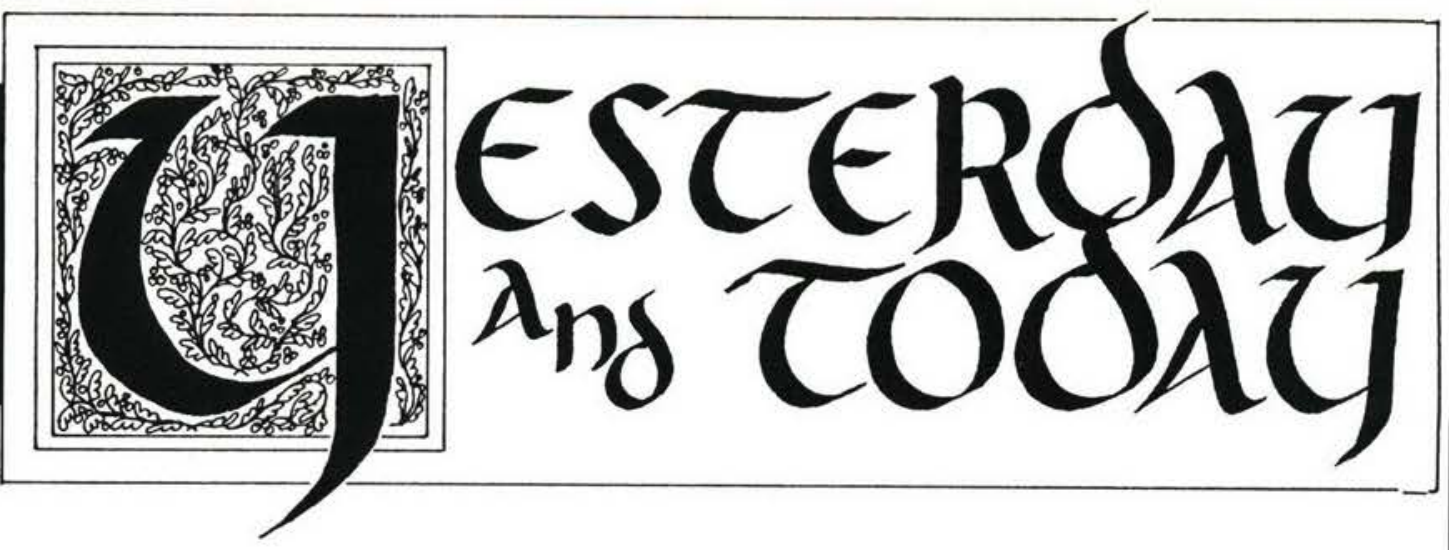

\section{Earning Money}

Simply by definition of being college students, the necessity for earning tuition and spending money creates a variety of options for the student to take. Although more Cedarville students work off-campus today than ever before, the on-campus employment opportunities haven't changed much.

Popular off-campus jobs vary from cashiers and baggers at Kennedy's in Xenia, pushing pizzas at Pizza Hut, or scooping ice cream at $\mathrm{H}$ \& R Dairy Bar to such odd jobs as church janitor, attendant at the YMCA, and mechanics and gas station attendants at area businesses. To some, those jobs during college are merely a means to earn needed cash; but for others, they are the beginning of a career.

Working on-campus, though, rarely leads to a bright future in the employee's respective field. Few students hope to spend end-

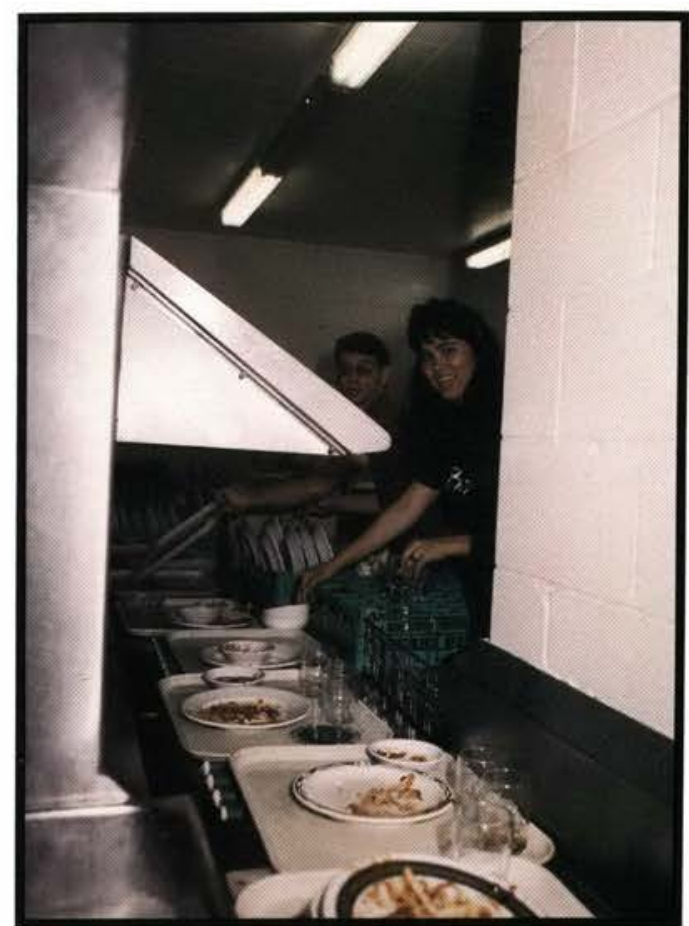

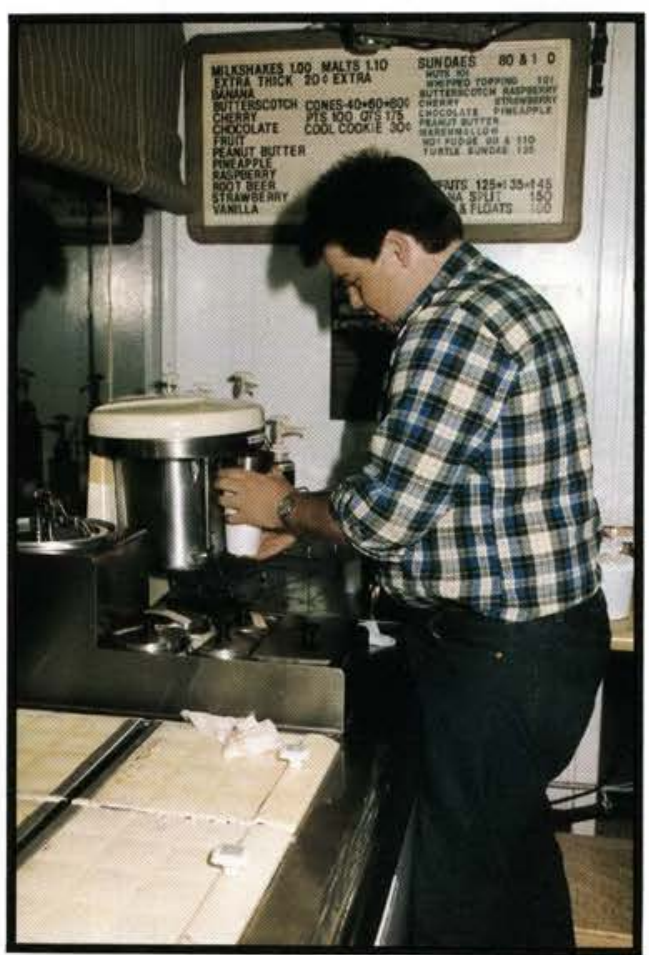

less hours working in the dish pit, cleaning rest rooms, or riding a TORO. Just as Cedarville College students years ago worked at the College Dining Club, on the housekeeping staff, or in various other campus jobs, students today find similar means to provide the funds for their days here at Cedarville. 


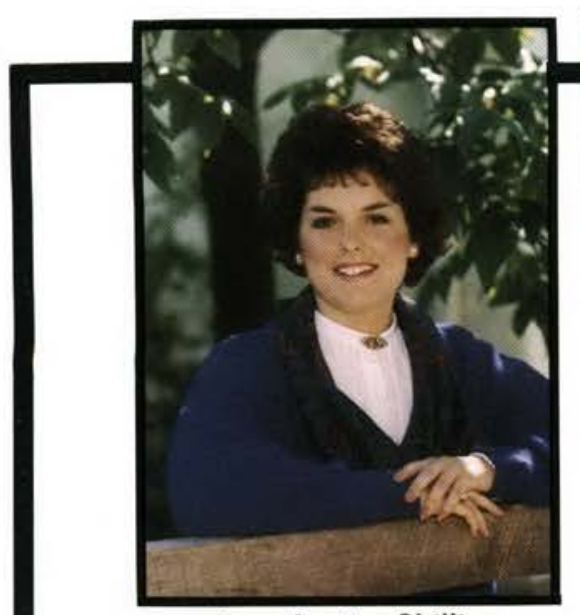

Amy Louise Phillips

Psychology

columbus, $\mathrm{OH}$

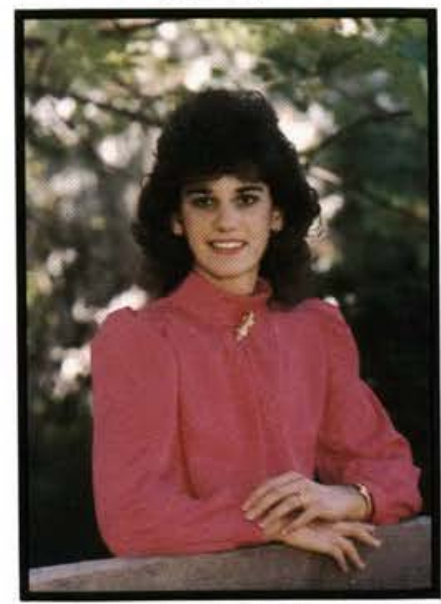

Kim E. Potts

Monirose, PA

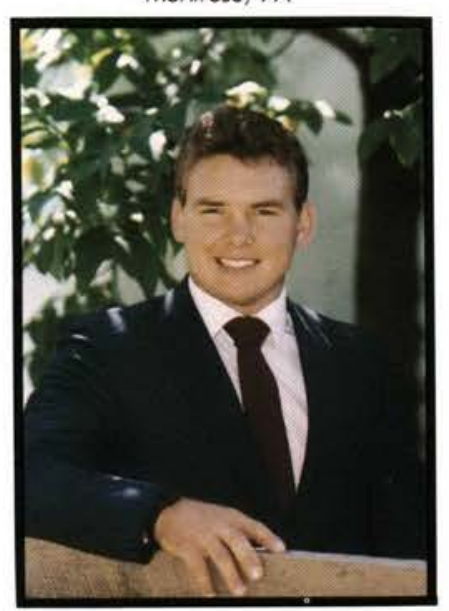

Roger Lee Randall

Political Science

Boise, 10

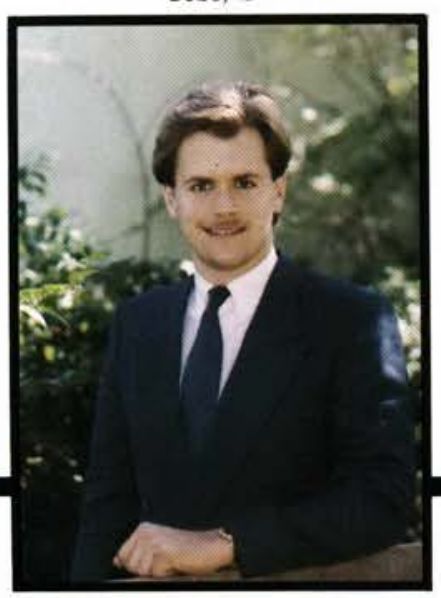

George Edwin Reede Jr.

98 Seniors

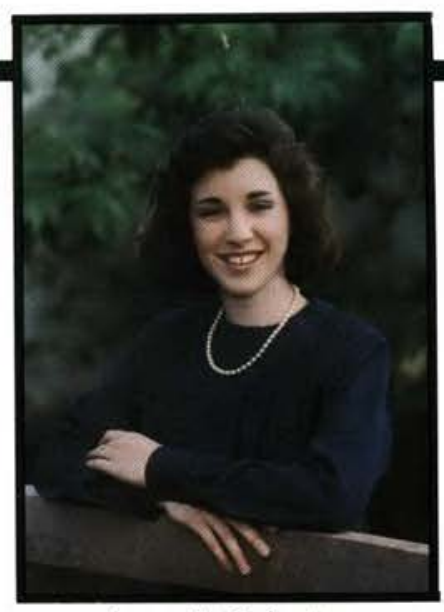

Jean E. Pinkerton

Nursing

Dugsour, VA

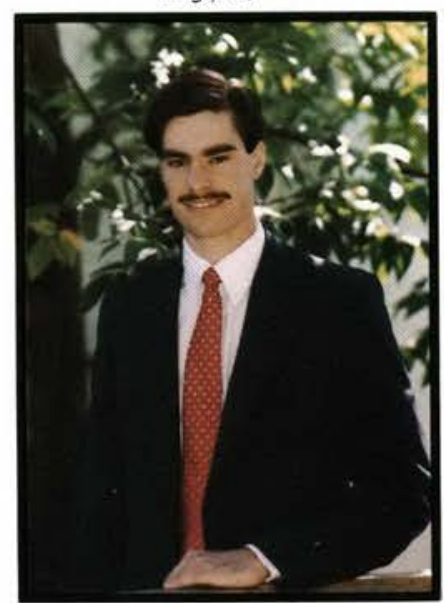

Douglas E. Pugh

Accounring

Lovisville, $\mathrm{OH}$

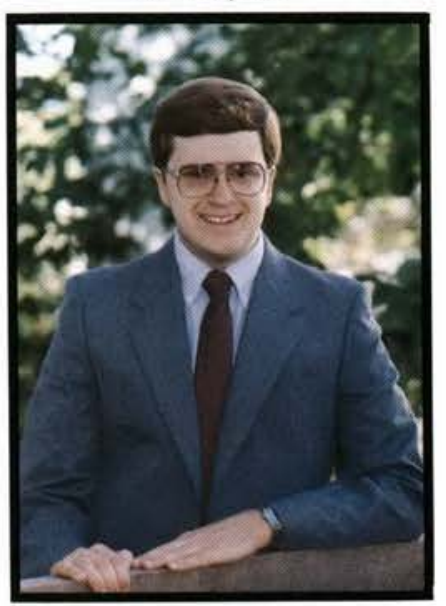

Daniel Howard Rase

Comprehensive Bible

Portsmouth, $\mathrm{OH}$

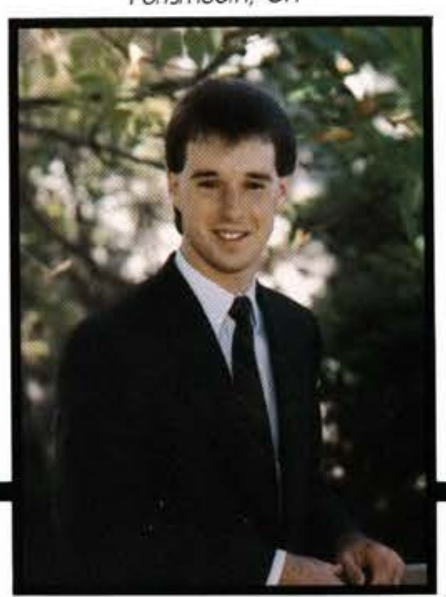

Lawrence G. Reeder

Computer Informarion Sysrems Columbus, $\mathrm{OH}$

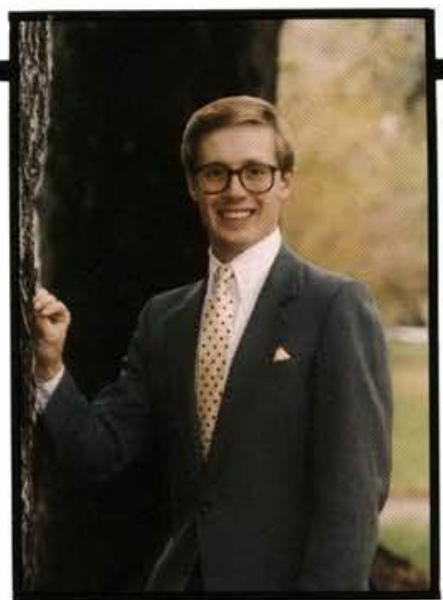

Jeffrey S. Piper

Preseminiary Bible Mojor

Kennewick, WA

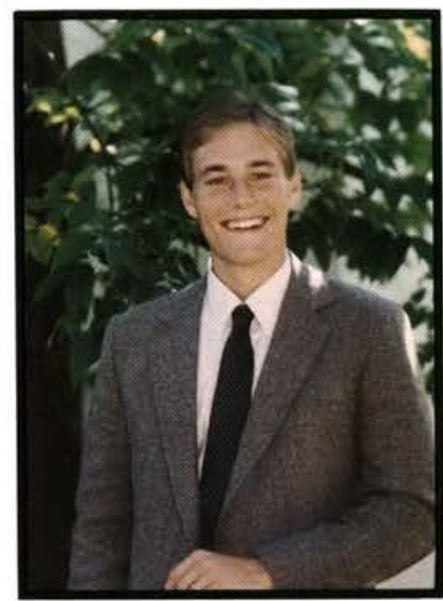

Ferdinand Henry Rajchel

Psychology

Newark, $\mathrm{OH}$

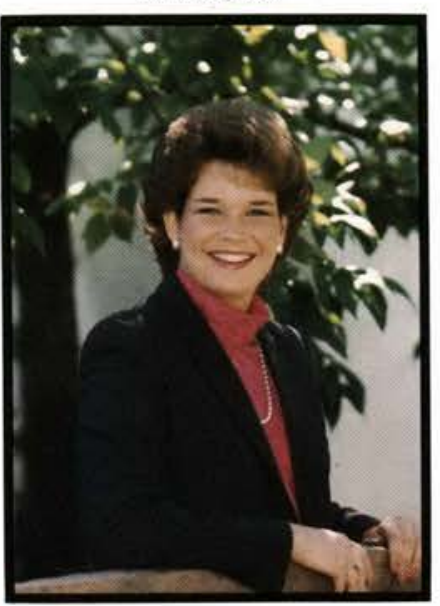

Kara Janelle Ray

Business Administrotion

Lebanon, $\mathrm{OH}$

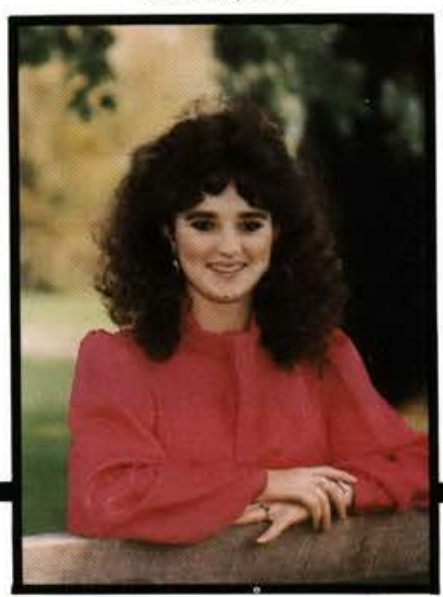

Jennifer Eileen Reeves

Accounting

Derry, PA

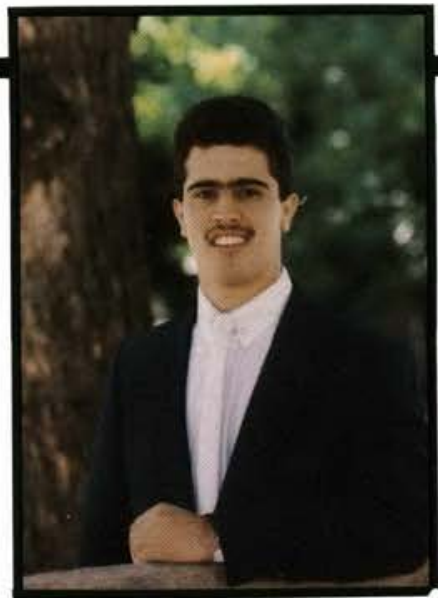

Rolando Ponce

Biology

Rio Pledros, Puerro Rico

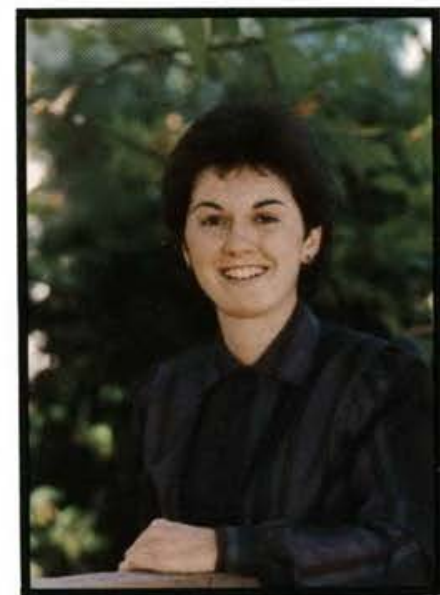

Kimberly Jean Ramsier

Physical Educarion

Wooster, $\mathrm{OH}$

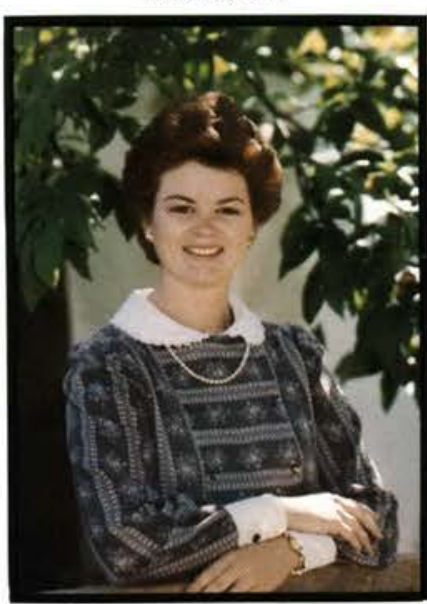

Patricia J. Reed

Elementary Educarion

St. Johnsbury, VT

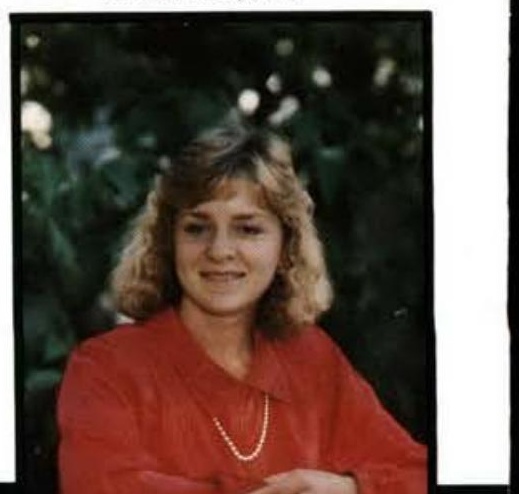

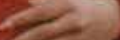

Rebecca D. Reid

Elementary Educarion

Romeo, Mi 


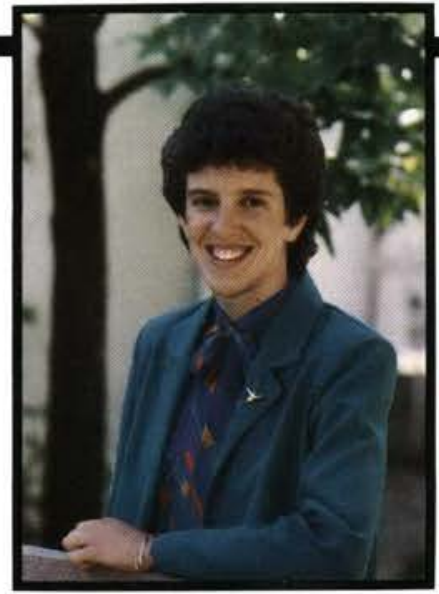

Cheryl A. Rendle Physical Educarion

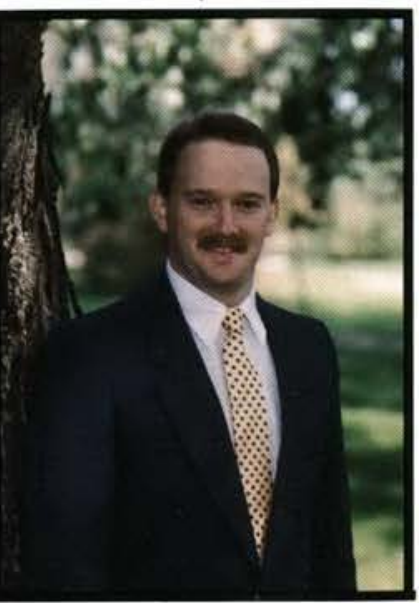

David Lee Robinette

Preseminary Bible Major

Cincinnati, $\mathrm{OH}$

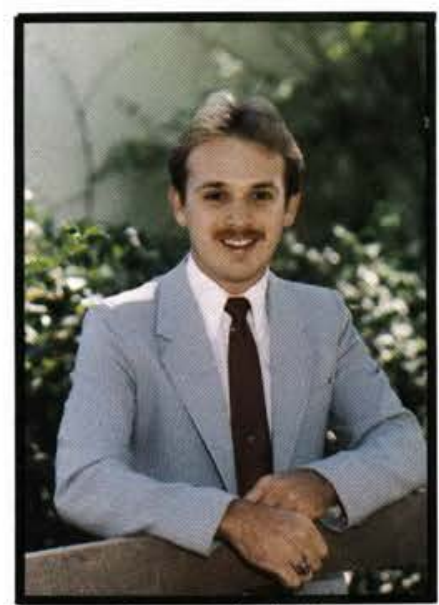

Darren L. Ruby

Business Administration

Elizoberhrown, PA

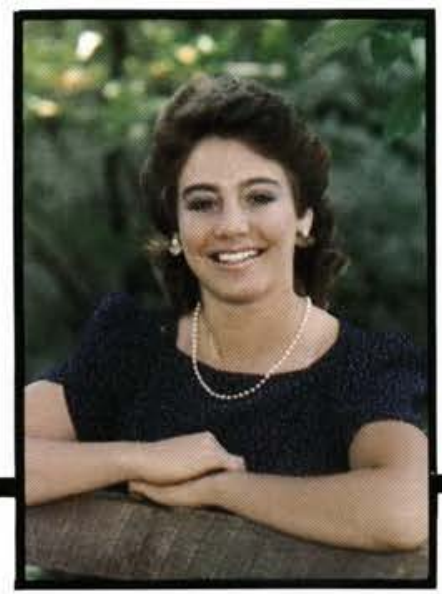

Melody Ann Schultz

Elementary Educarion

interlachen, FL

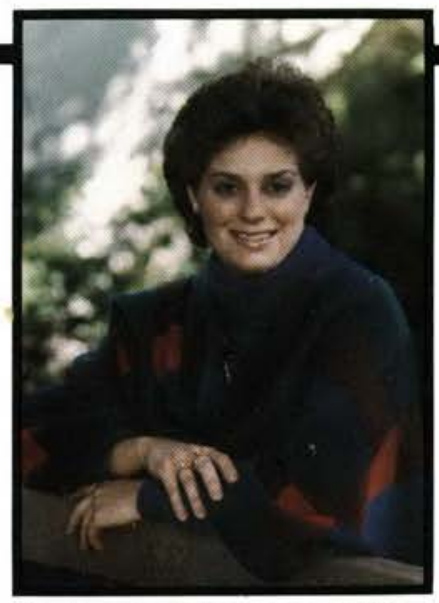

Katherine Dorris Reynolds Elementary Educarion

West Bend, WI

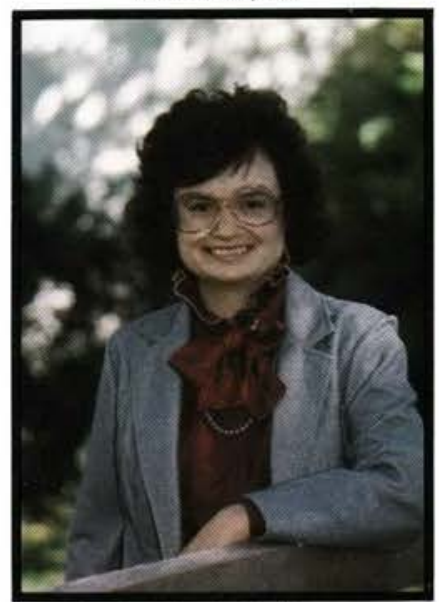

Kimberly J. Robinson

Florence, $K Y$

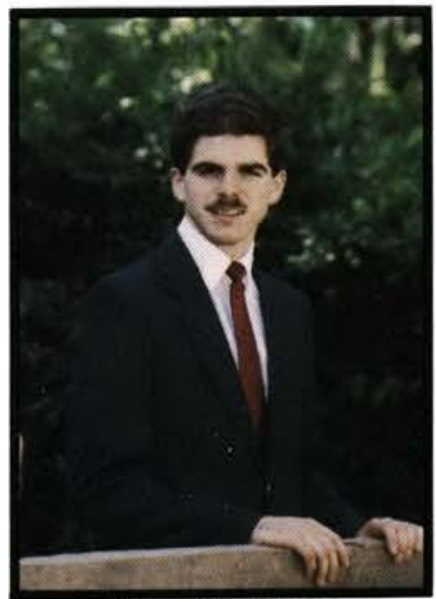

Glen W. Sallee

Business Administrorion

Ferndale, MI

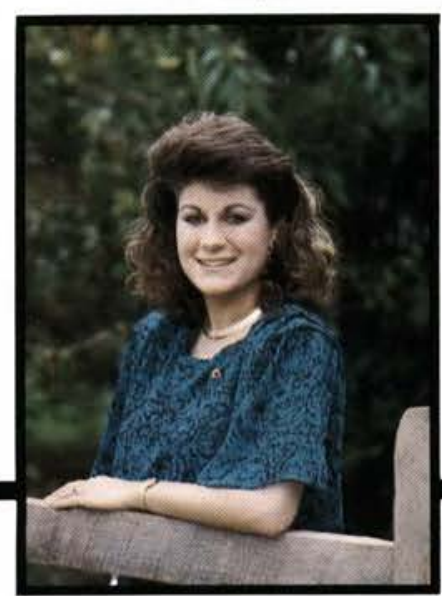

Deborah S. Schwartz

Elementary Educarion

Almond, NY

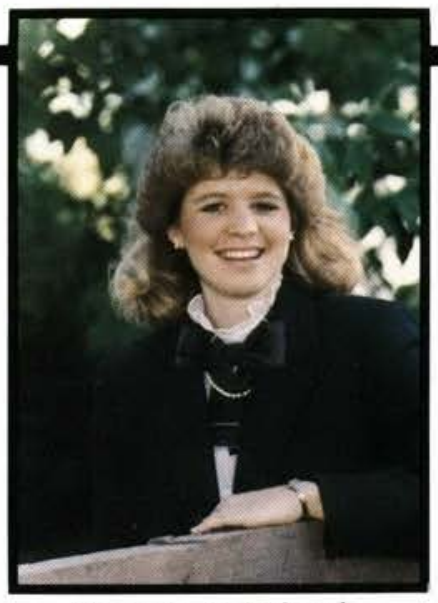

Pamela Jo Rickard

Elementary Educarion

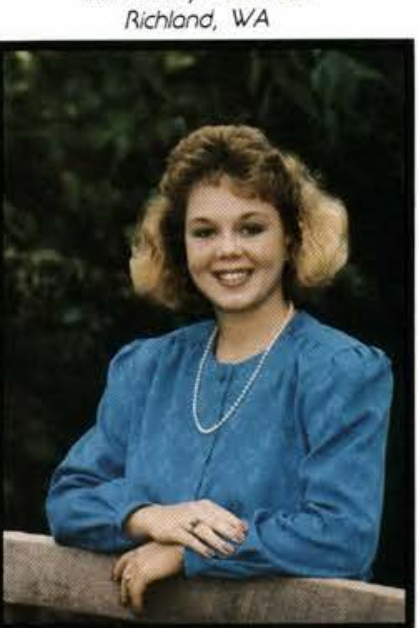

Renee Jeanne Rolfe

Elementory Educorion

Woodland, ME

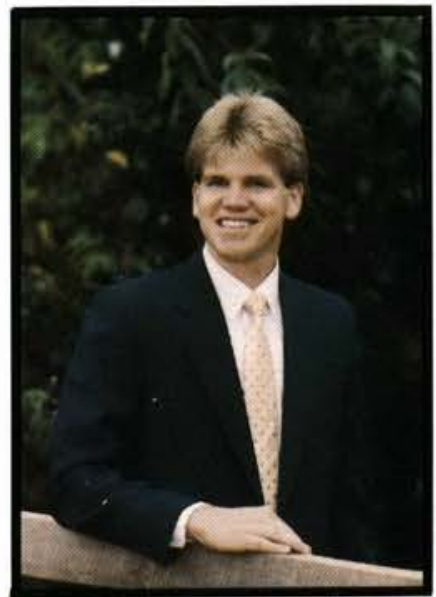

Timothy Mark Schaket Business Administrario

Cincinnari, $\mathrm{OH}$

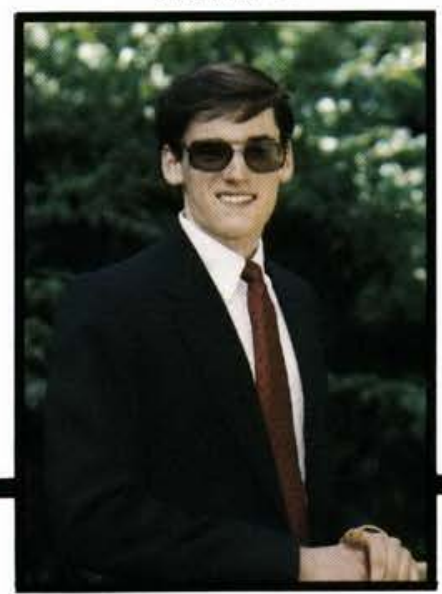

Daniel R. Searles

Accounting

Pontioc, IL

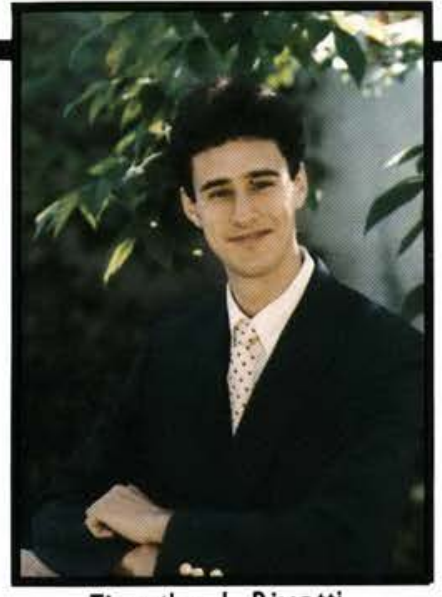

Timothy J. Rivetti

Business Administration

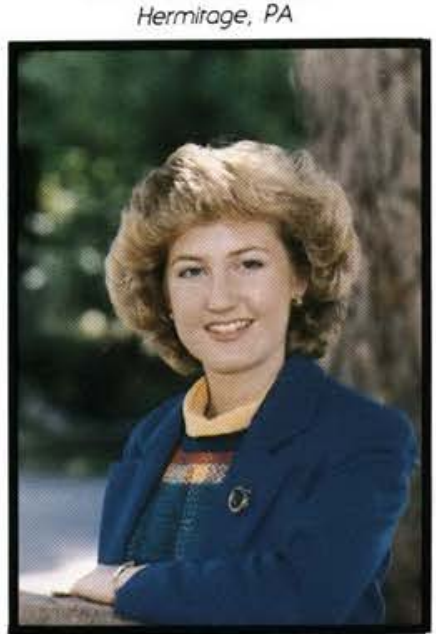

Sherry M. Rotramel

Communicarion Arts

Elkhart, IN

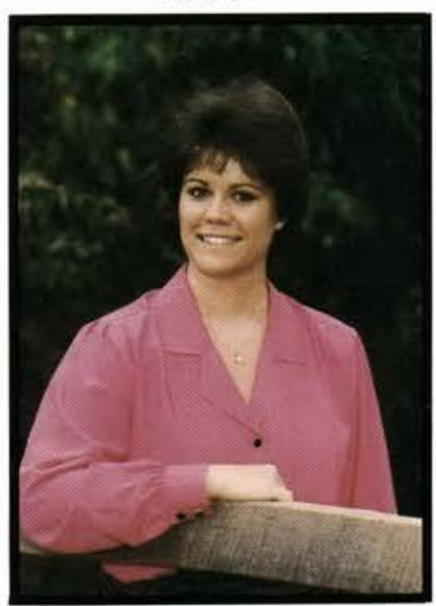

Elizabeth A. Schultz

History

Grove Ciry, OH

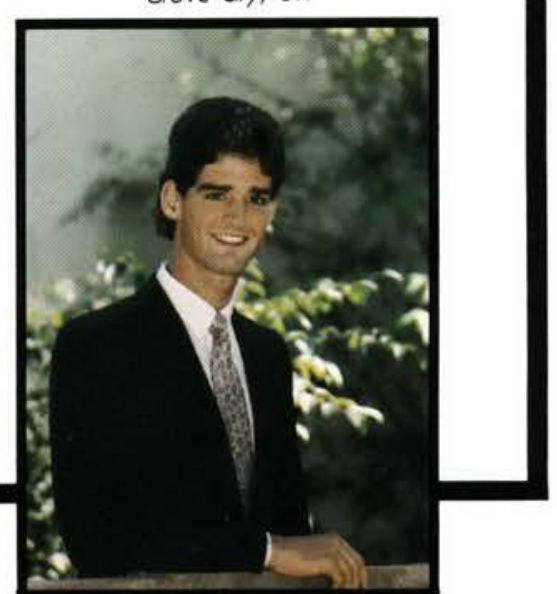

Erick J. Seidel

Preseminary Bible Mojor

Springfiled, VA 


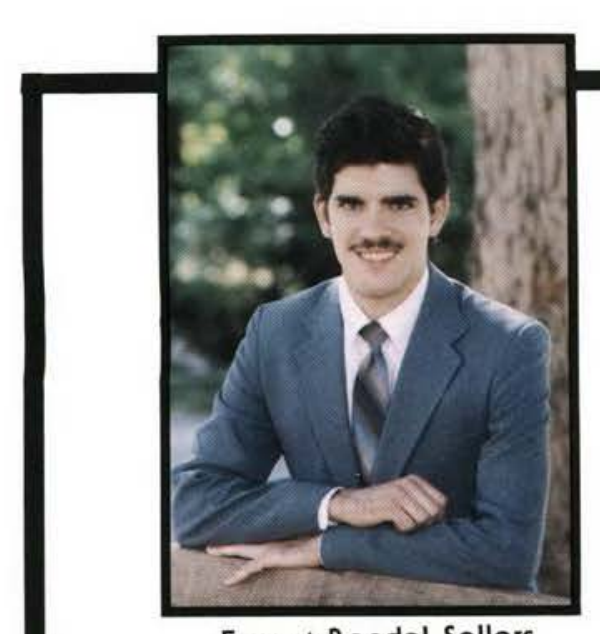

Forrest Randal Sellers

Communicorion Arts

West Chester, $\mathrm{OH}$

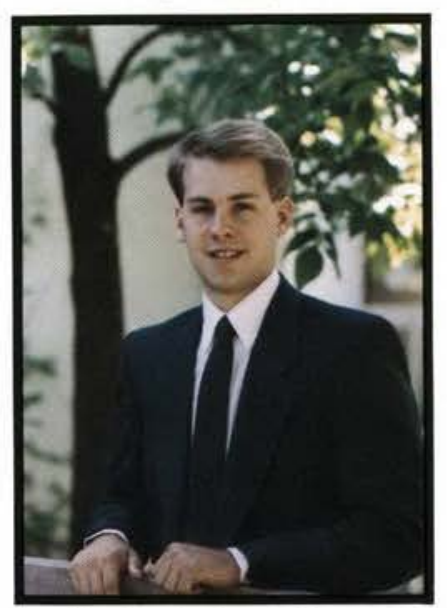

Kevin Andrew Shaw

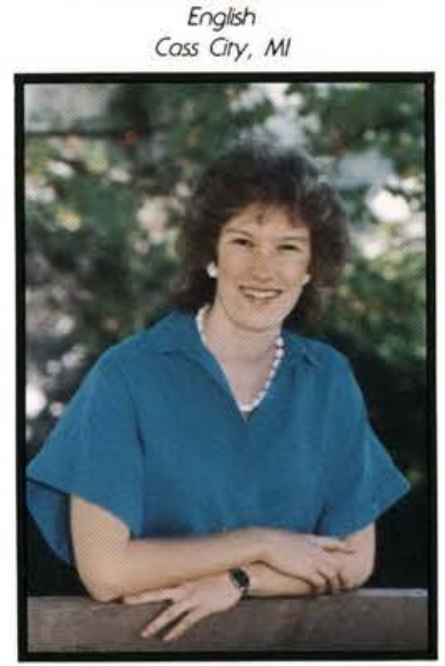

Kathryn Marie Sibert Marhemorics Educario

Martinsburg, WV

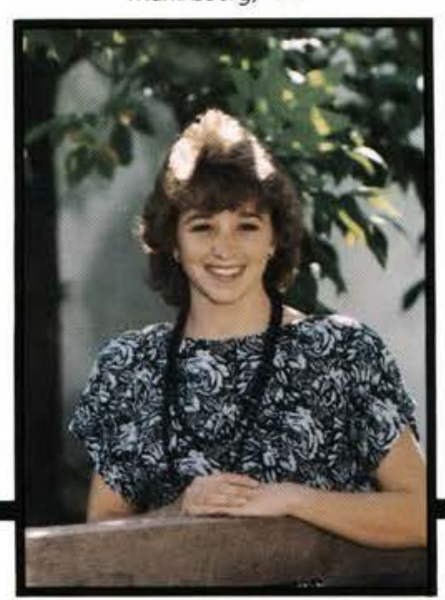

Tammy W. Sloan

Business Administration

Mr. Pleosont, IA

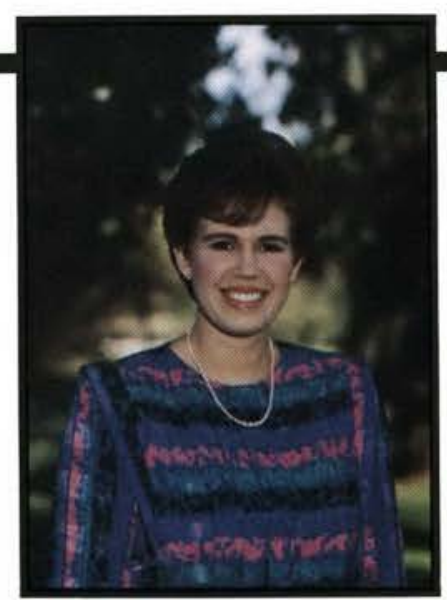

Judith Semmelroth

Elemenrary Educarion

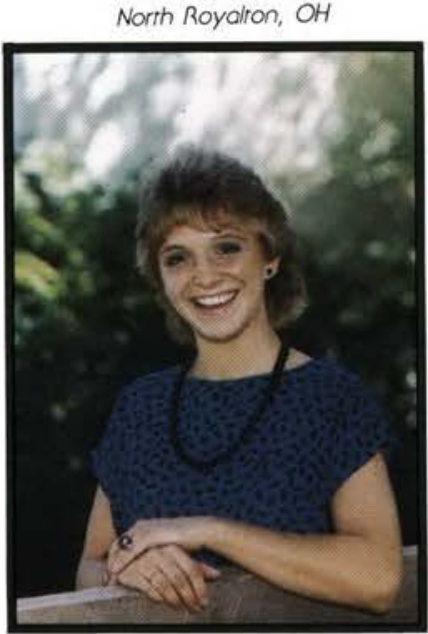

Deena Renee' Shriver

Physical Educarion

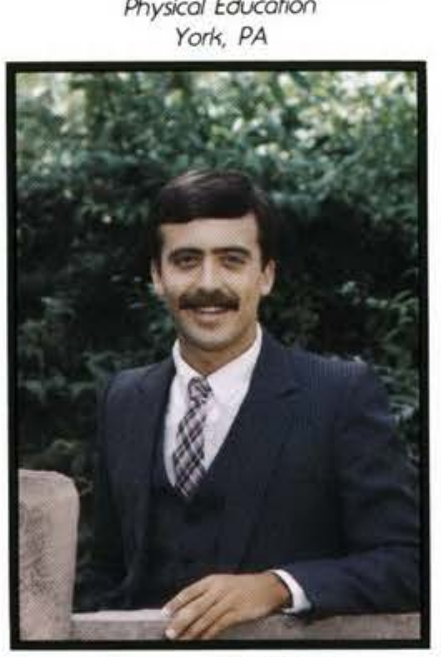

Thomas D. Simic

Bible Comprhensive

Rockville, IN

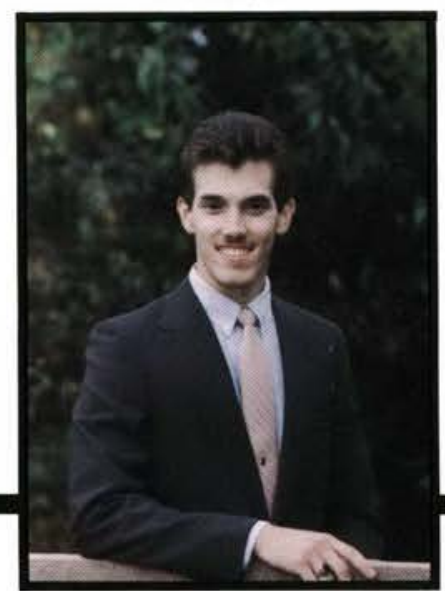

David A. Smallman

Business Administrarion

Sirongsville, $\mathrm{OH}$

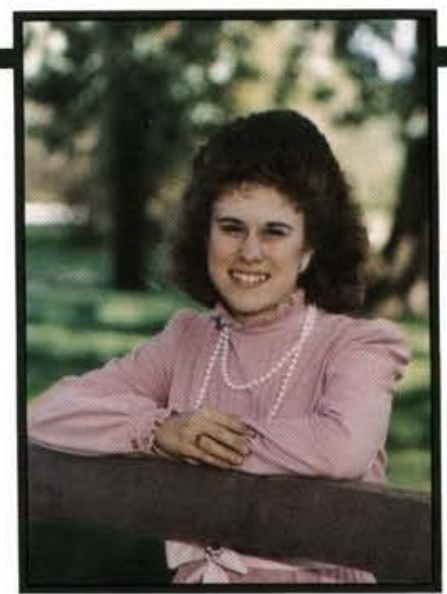

Linda L. Shaffer

English Educarion

indianapolis, IN

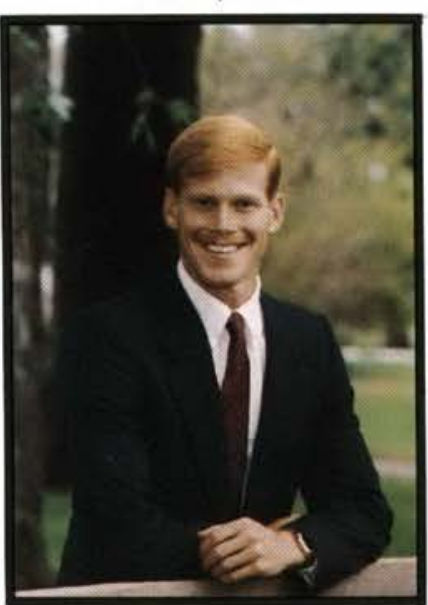

Eric L. Shrum Preseminary Bible Mojo

Dayion, $\mathrm{OH}$

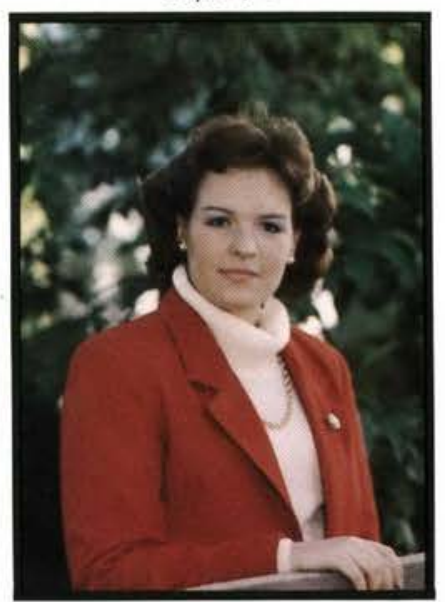

Deborah Lee Simmen Communicorion Arts Morron, IL

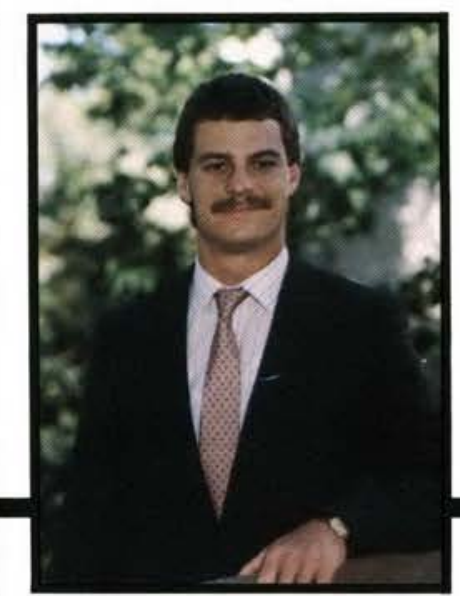

Thomas James Smart Psychology

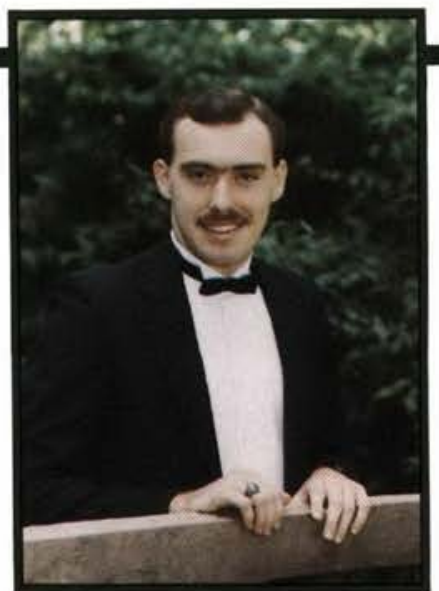

Terry B. Sharp

lorksburg, WV

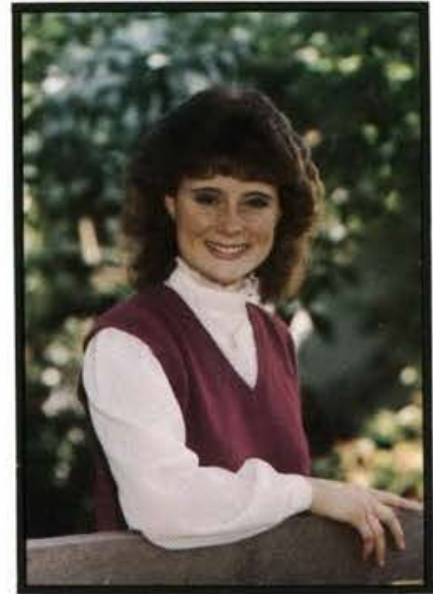

Sheri Lynn Shuler Elementary Educorio

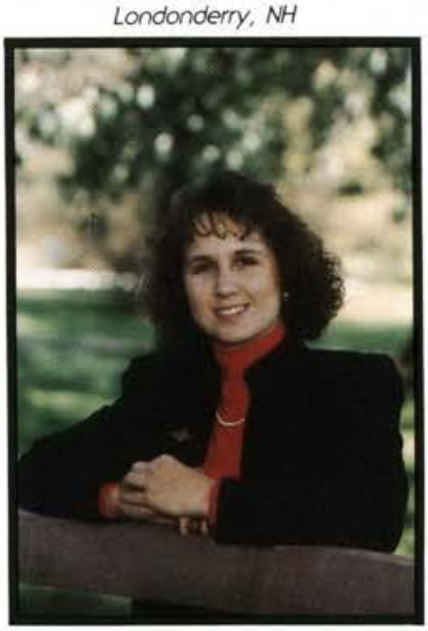

Rebecca Ellen Sinclair Communicarion Ar sheridon, M

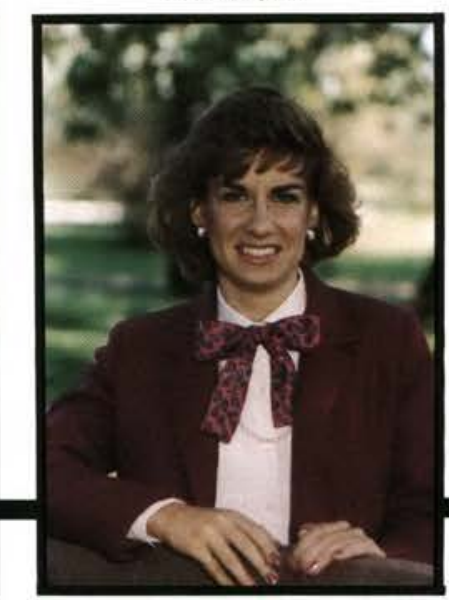

Liana Smith

Elementary Educorion

Ado, M 


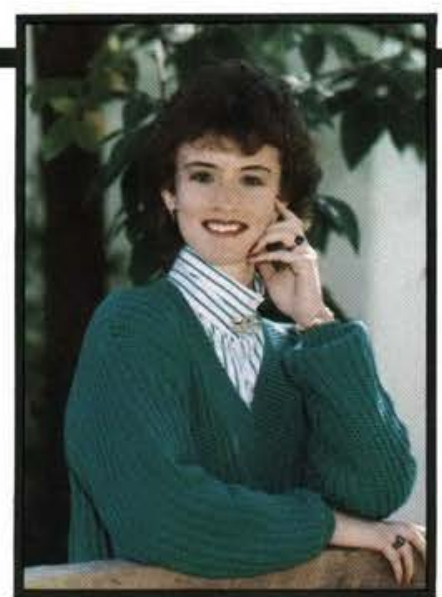

Pamela Miriam Smith

Business Administrorion

Germantown, WI

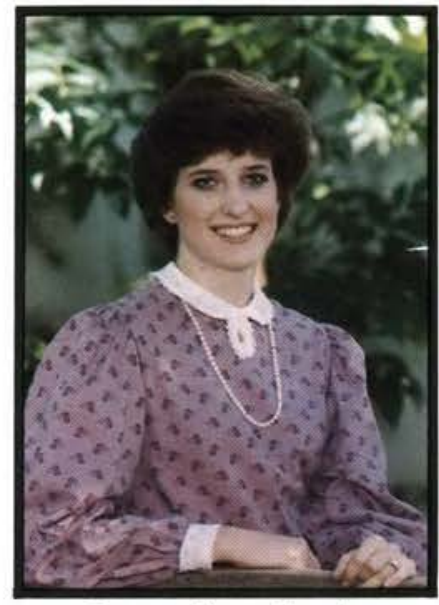

Donna Jean Snyder

Elizoberhrown, PA

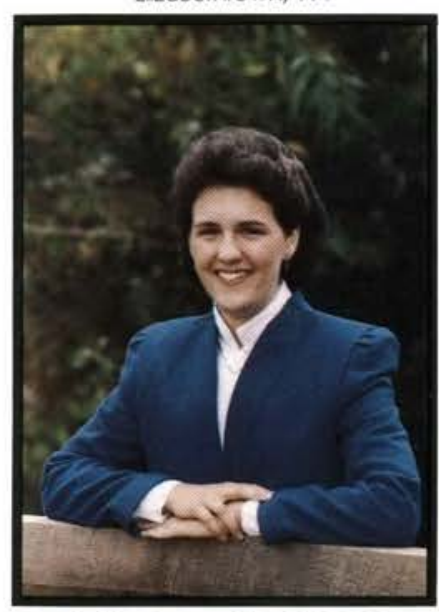

Julie Lynn Stackhouse

English

Moorestown, $N$

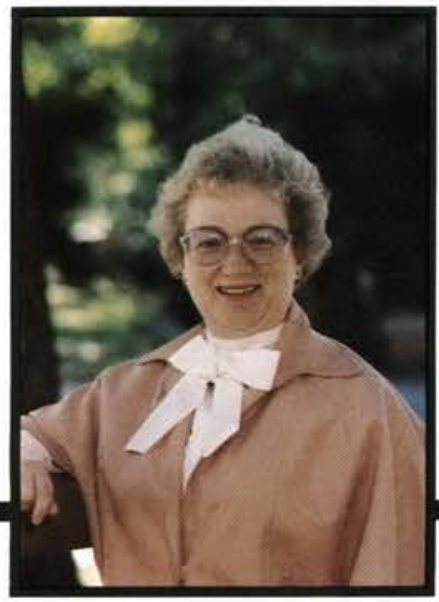

Loretta M. Strock

History

Cleveland $\mathrm{OH}$

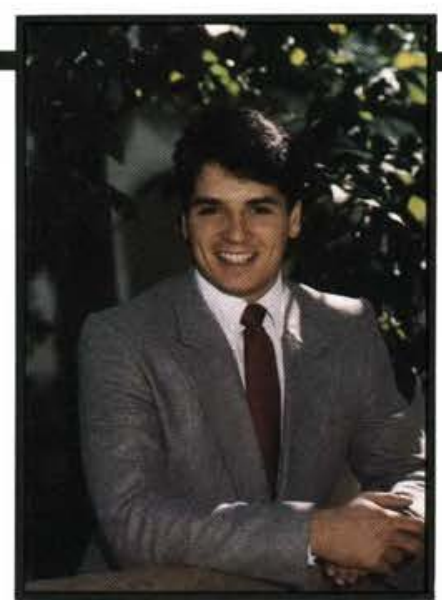

Robert J. Smith

Communication Arts

Colgare, WI

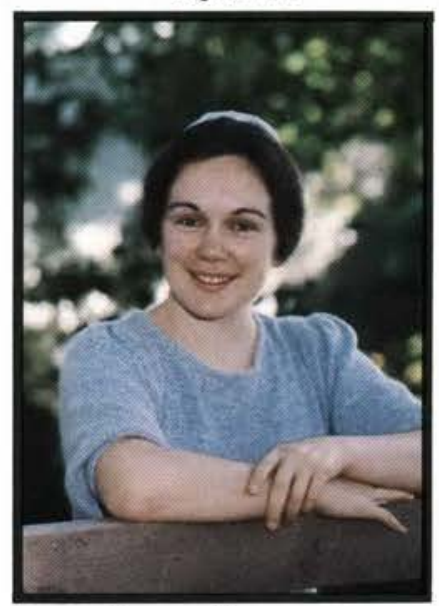

Dorcas Sommers

Elemenrary Educarion

Hartvilie, $\mathrm{OH}$

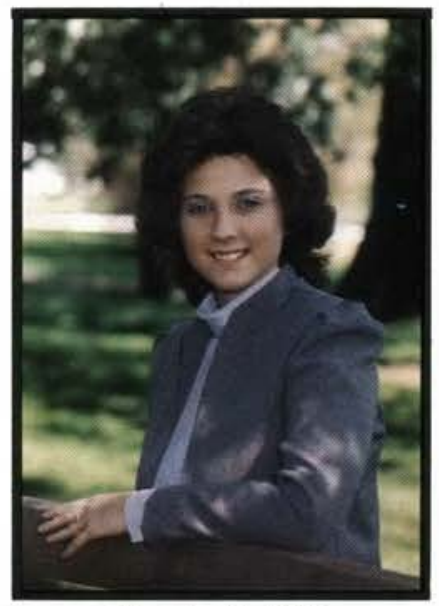

Wendy A. Stith

Behavioral Science

Milan, $\mathrm{OH}$

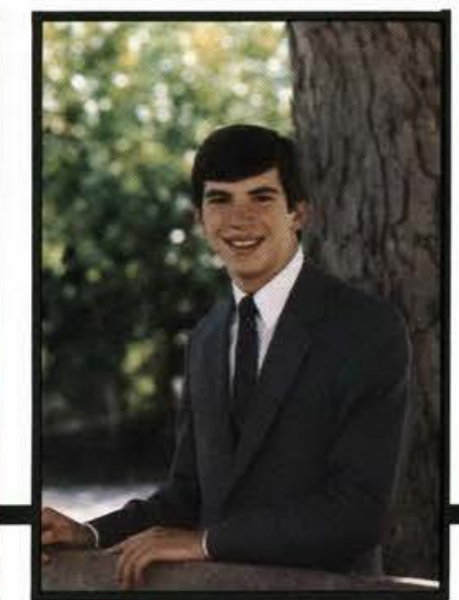

Brian Paul Sturgis

Compurer Information Systems

Foirfox, VA

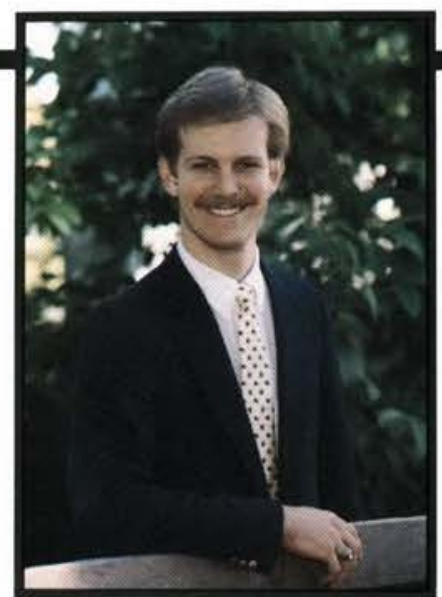

Ronald M. Smith Jr.

Marhemarics

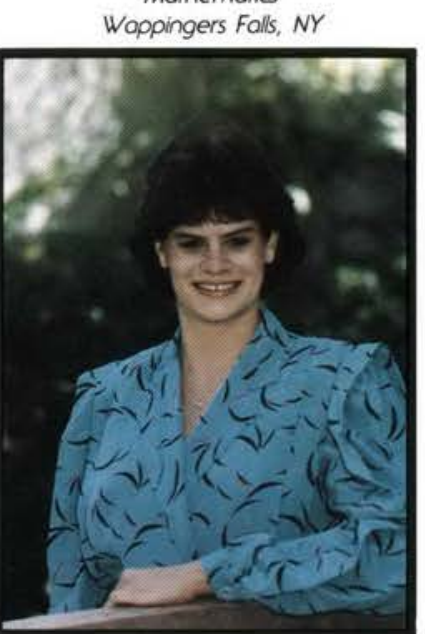

Sheila Ann Southwell

English

Hudson, MI

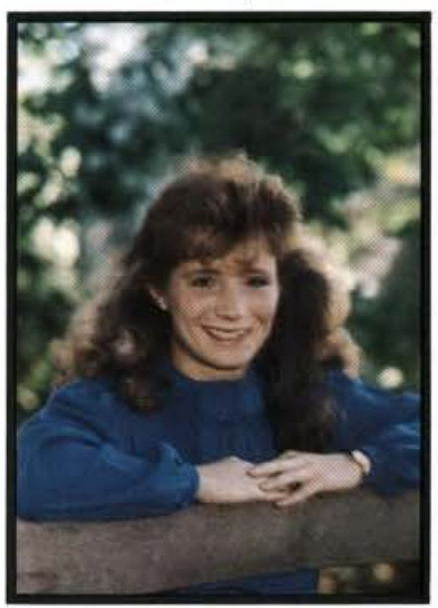

Jill K. Storer

Elementary Education

Soringfield, $\mathrm{OH}$

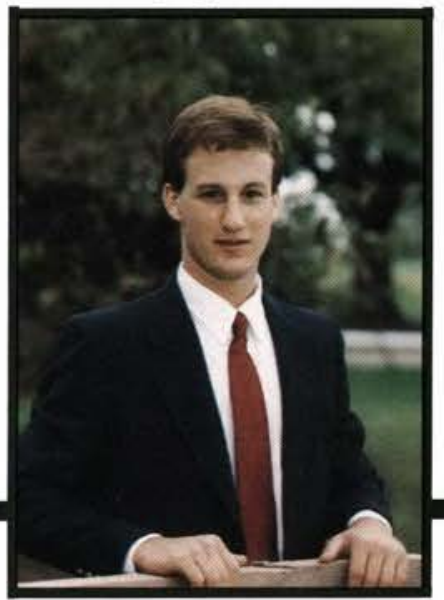

Mark Alan Swander

Business Administration

lonesville, Mi

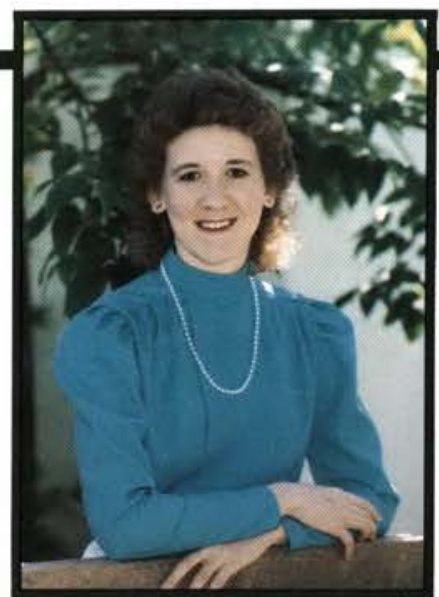

Andreo B. Snyder

Marhemorics

Romeoville, il

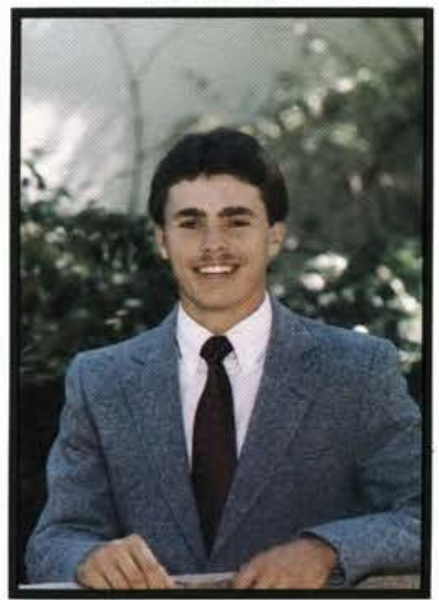

Samuel T. Springer

Business Administration

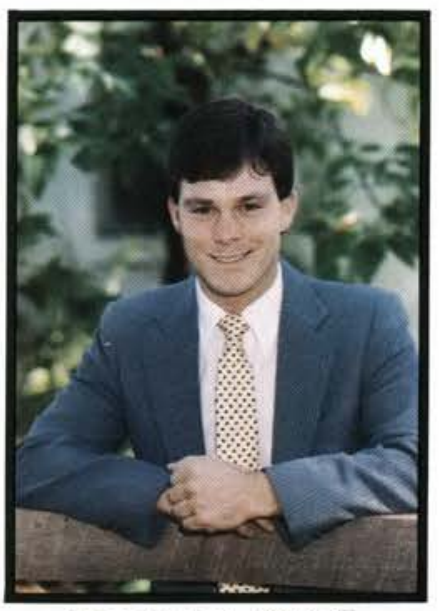

Ronald Lee Stowell

Elementary Educarion

Midland, MI

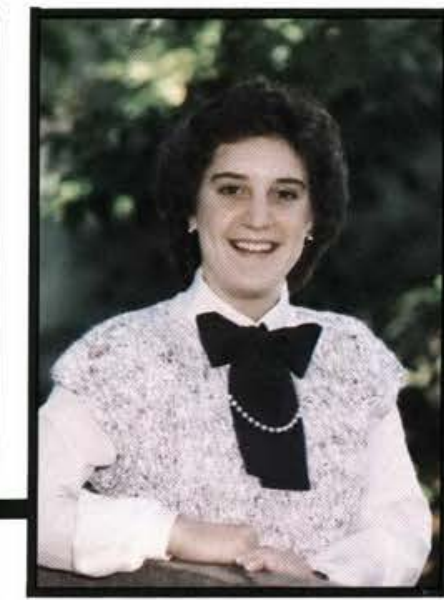

Pamela M. Swanson

Elementary Educarion

Cuyahogo Folls, $\mathrm{OH}$ 


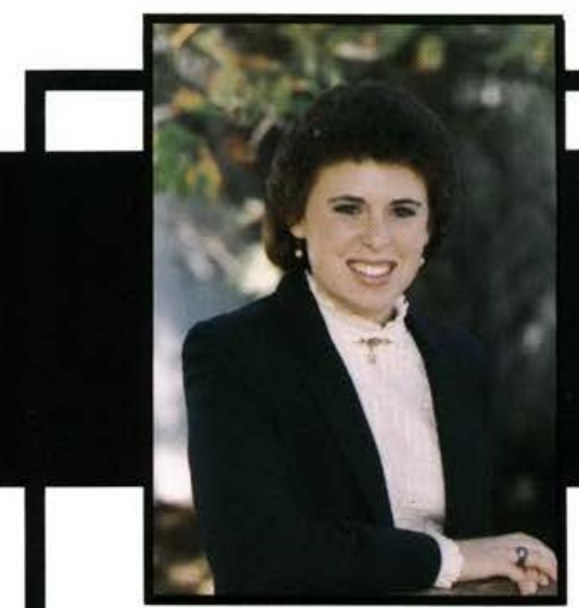

Jill A. Swayzee

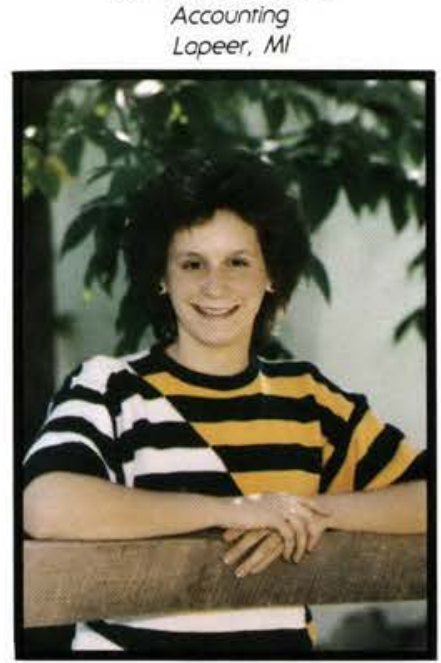

Carrie Marie Tansey Nursing

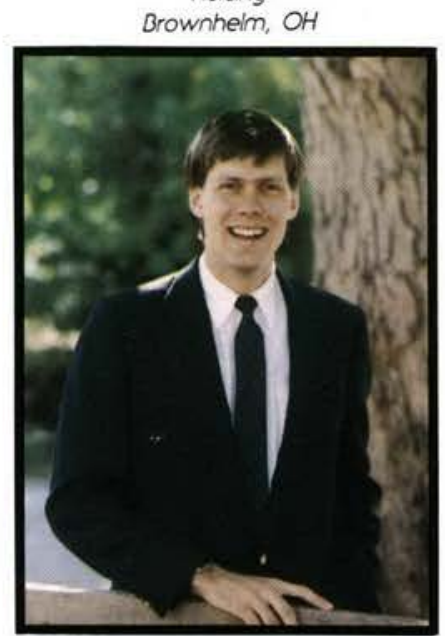

Steven Keith Terpstra Accounting Sully, IA

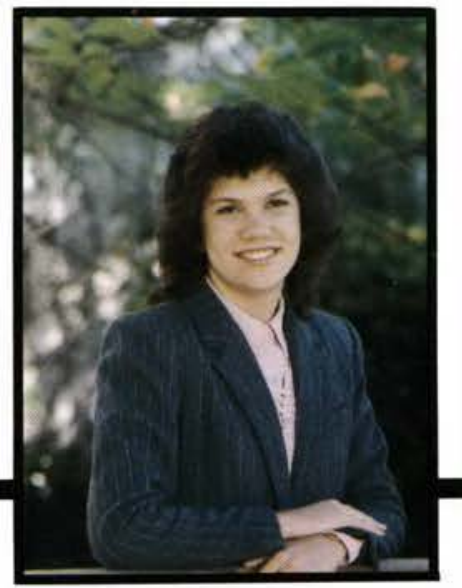

Lydia B. Thorton

102 Seniors
Compurer Informarion Sysrems Moson, $\mathrm{OH}$

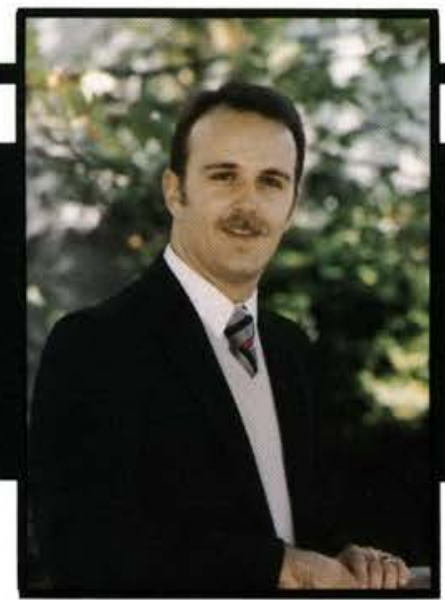

Mark Frederick Sweitzer

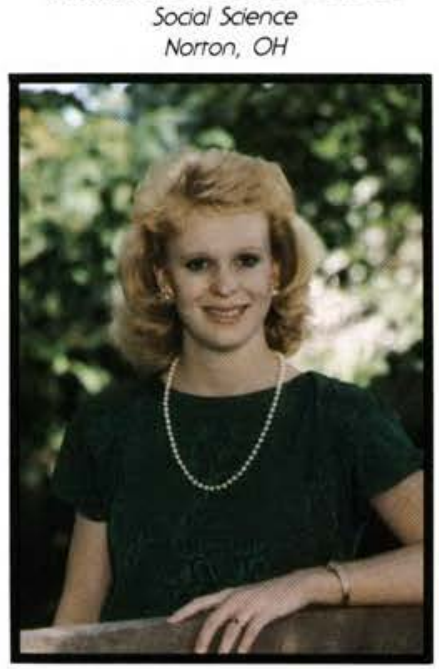

Shelley Anne Tarke

Psychology

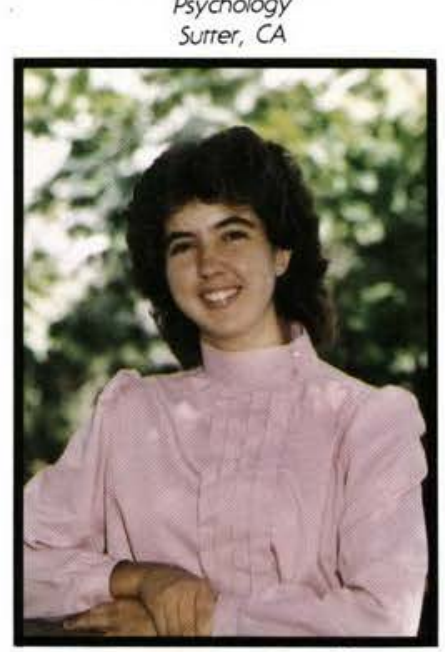

Nadine Lin Terrill

Elementary Educarion

Xenia, $\mathrm{OH}$

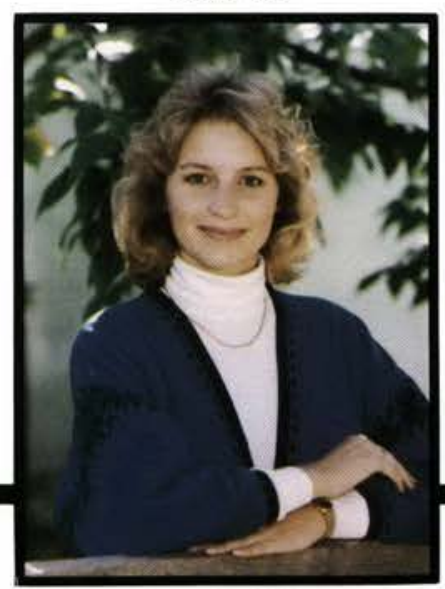

Doris I. Tober

Spanish

Grand Ropids, M

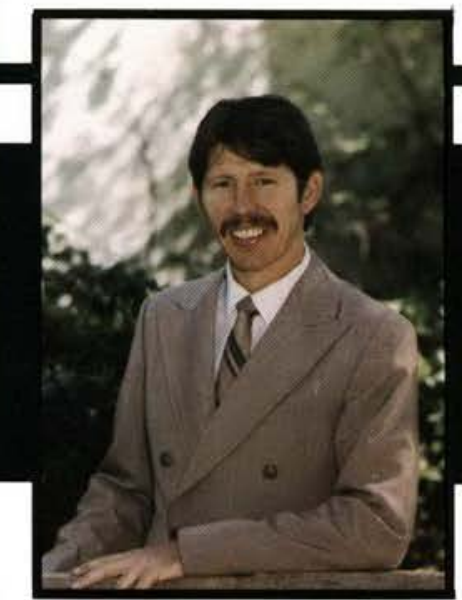

Kenneth E. Swope

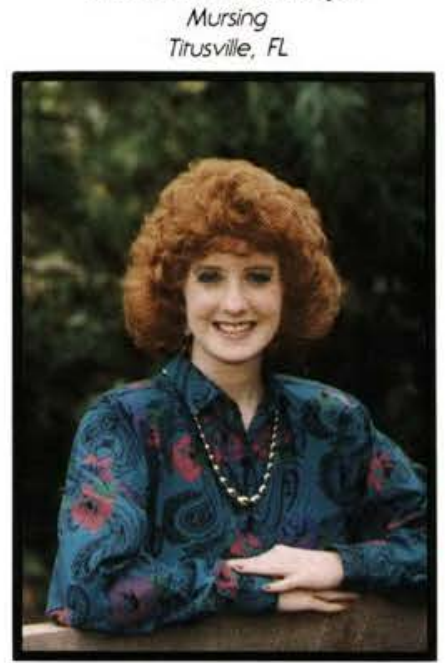

Allison Kay Taylor

Business Administrarion

Edgewood, $K Y$

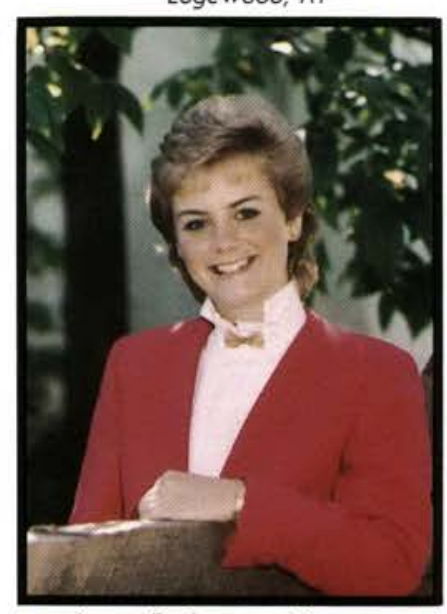

Amy Rebecca Thomas

Kertering, OH

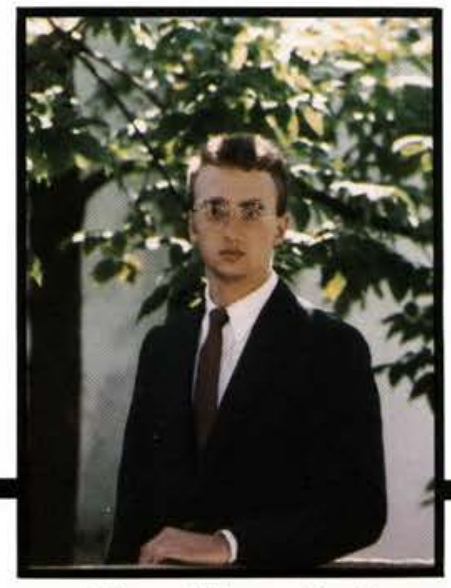

Edward Murry Todd

Accounting

Ploinview, MN

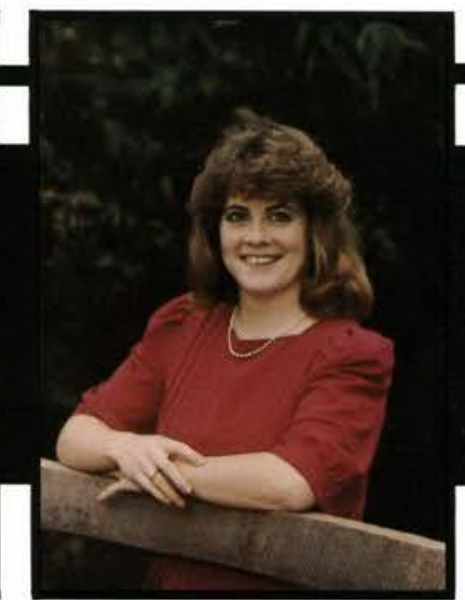

Deborah Ann Synnnott

Elementary Education

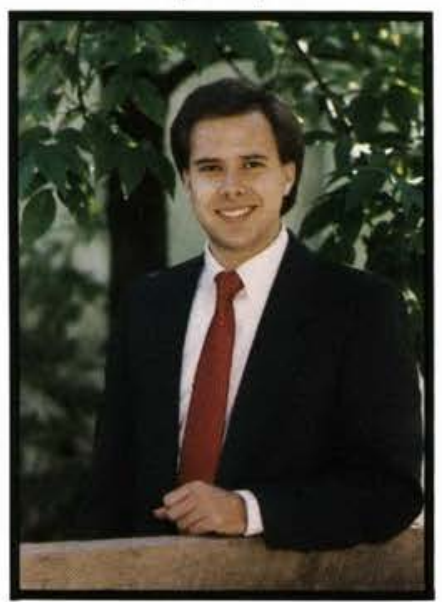

Walter L. Taylor

Public Administration

Cedarville, $\mathrm{OH}$

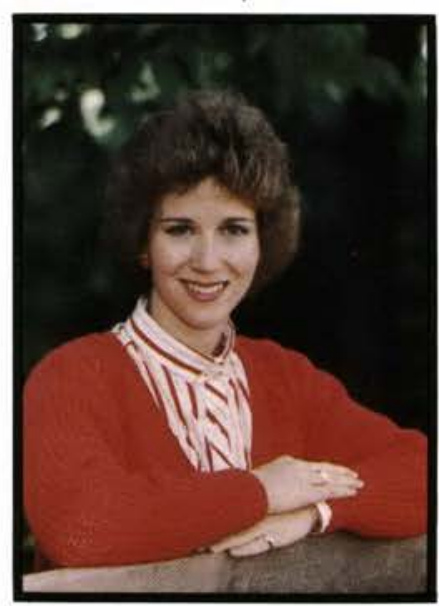

Donna Jane Thomas

Elementary Educarion Elizoberhrown, PA

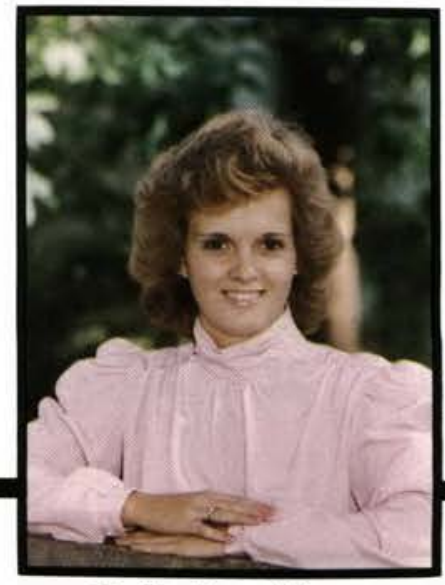

Phyllis Mae Trivett

Elemenrary Educarion

Blanchester, $\mathrm{OH}$ 


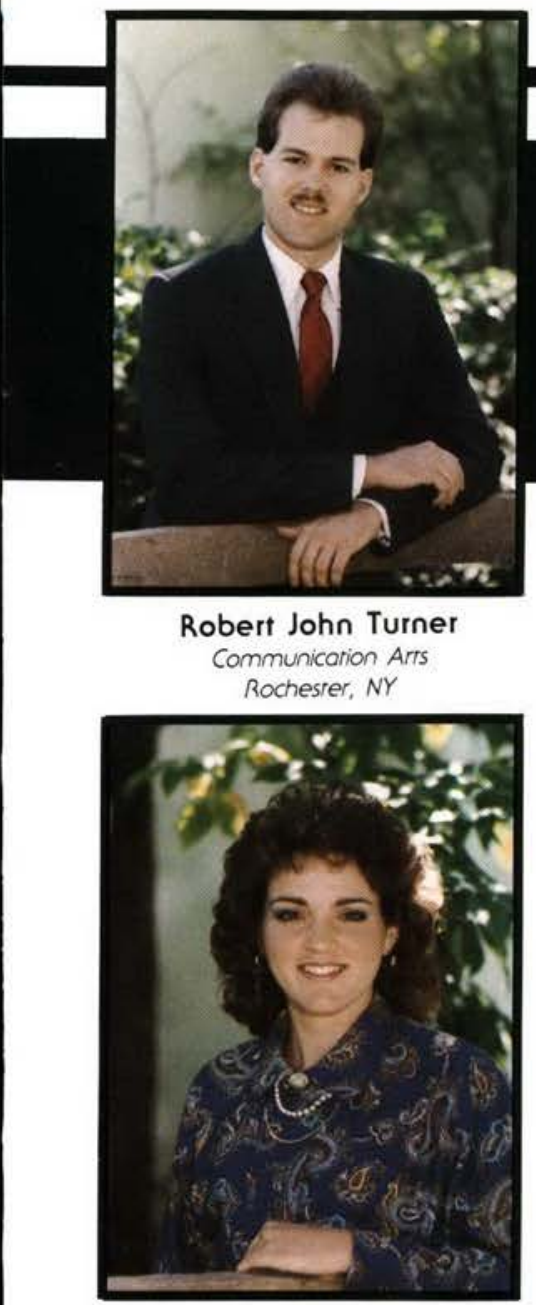

Lisa Lee Tyson English

Elmiro, NY

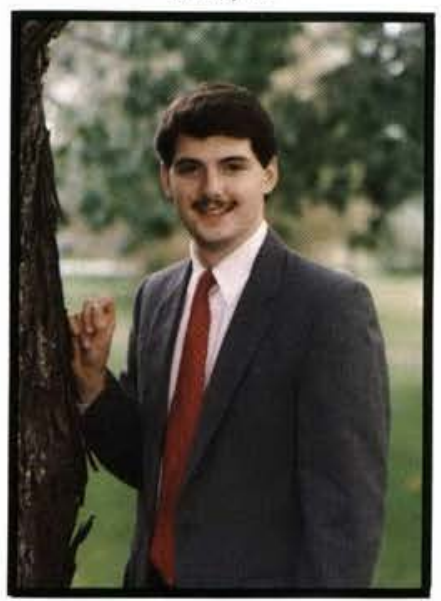

Jeffrey Carl Vander Heide

Broodcosting Coledonia, MI

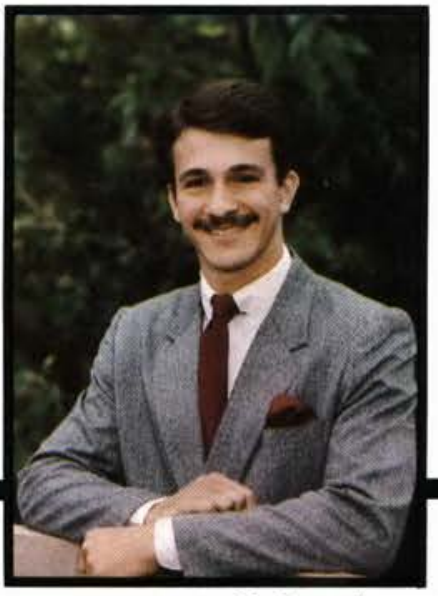

Jeffrey A. VeStrand Behovioral Science Wesffield, NY

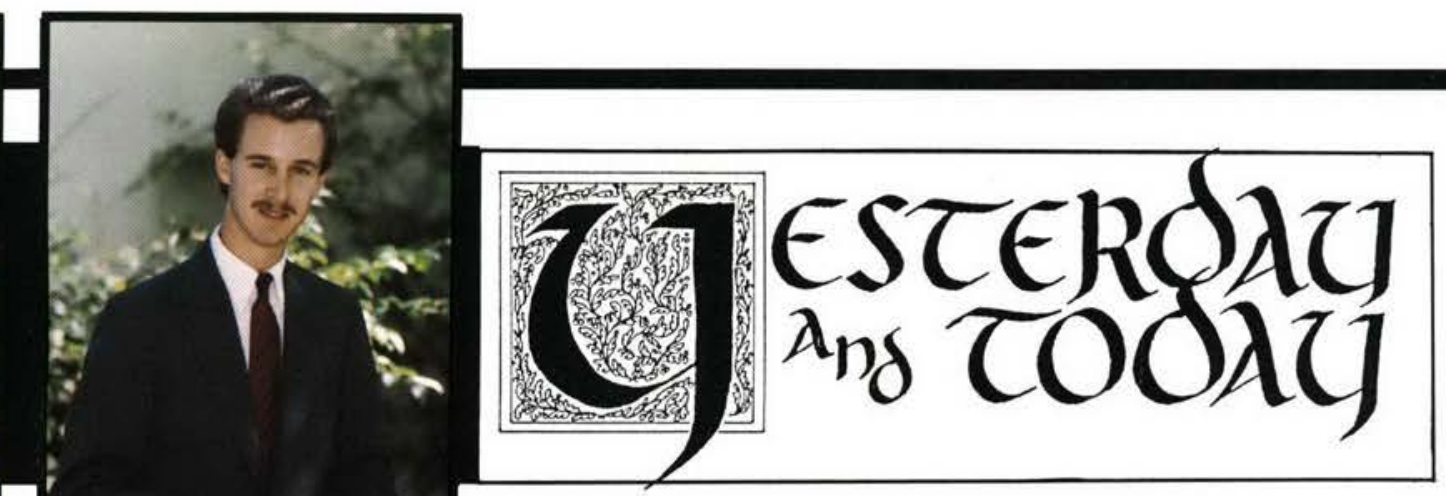

\section{Music Tastes}

College students every. where, no matter what time period, are always concerned with music. Cedarville has never been an exception. Because of its college standard, the school necessarily hold a policy concerning music which is allowed on campus. Within and without - those standards, the taste in music has changed over the years.

Since the days of jazz, big band, and folk music, college students have often listened to music popular at the time. With Elvis and rock and roll and the Beatles, though, the "music policy" began. Even so, Cedarville students in the mid-sixties,

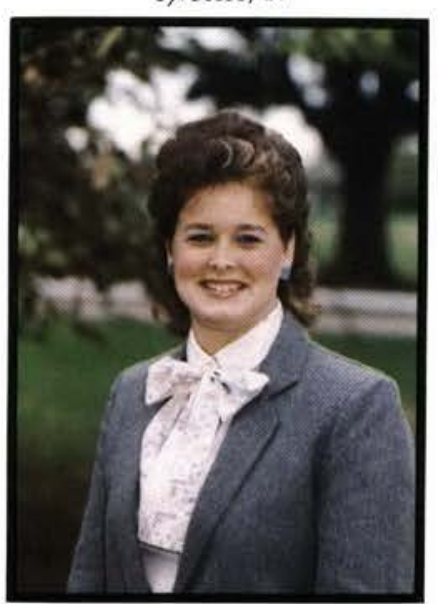

Kimberlee S. Venman Business Administrarion Menror, $\mathrm{OH}$

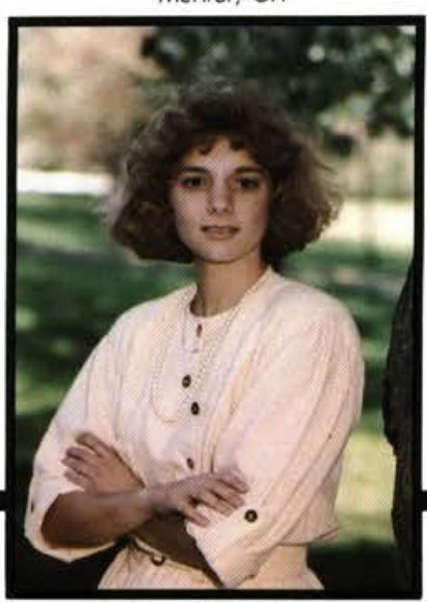

Bridgot K. Vissman

Business Administration Grand Ropids, Ml

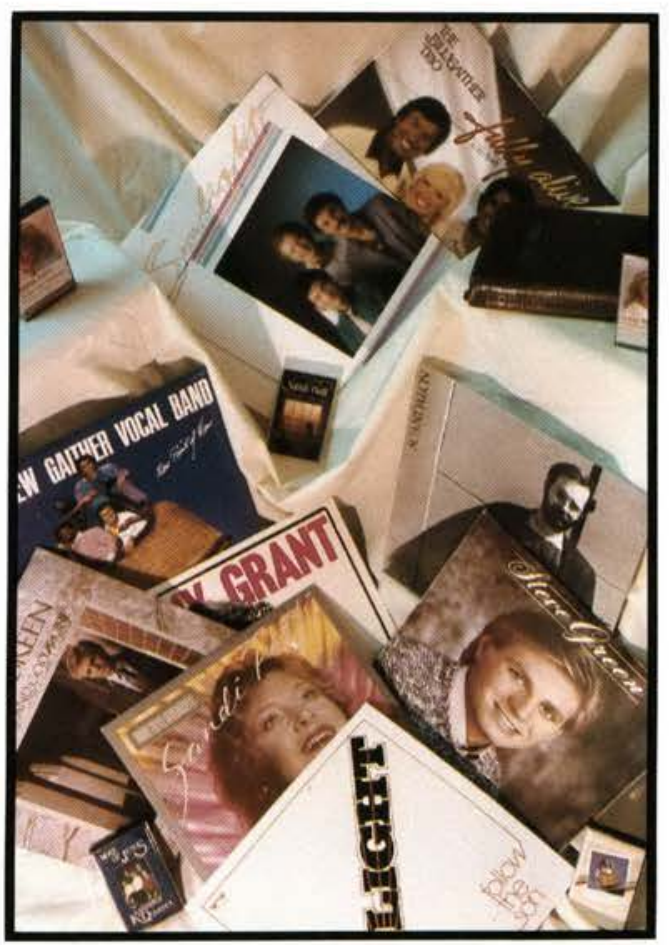

concerned with Viet Nam and politics, listened to people like Joan Baez and Peter, Paul, and Mary, and waited for their draft notices.

Now that Christian contemporary music has grown and become more widely accepted, students listen to every. thing from Christine Wyrtzen, Sandi Patti, and Steve Green to Michael Card, Amy Grant, and Farrell $\&$ Farrell. Cedarville students still struggle with the music policy, and college standards still don't always reflect student tastes; but Dr. Dixon still knows the hit "Christ is All I Need" brings a smile to every. one's face. 


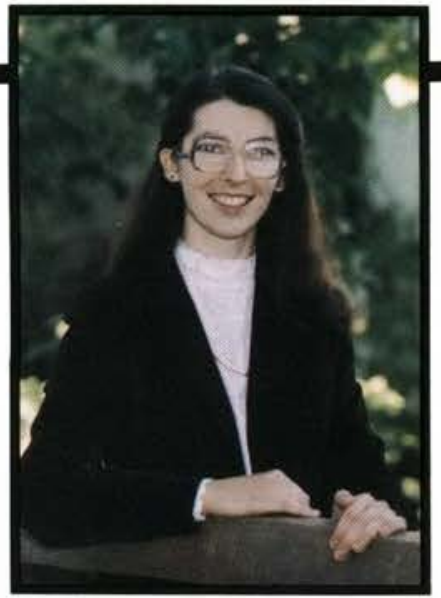

Laurie A. Wagner Elemenrary Educarion

Wellingron, $\mathrm{OH}$

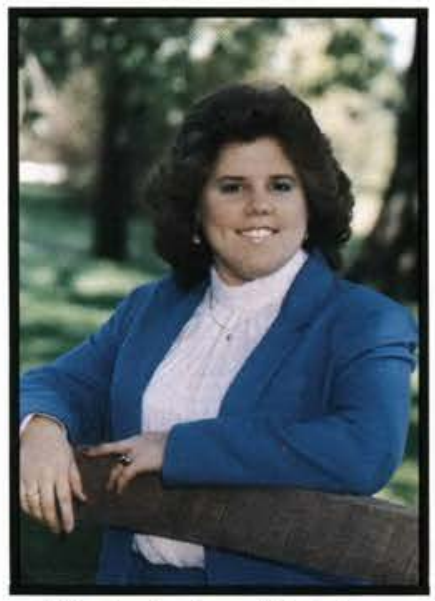

Elizabeth Ann Waltrip Business Administration Strarford, NJ

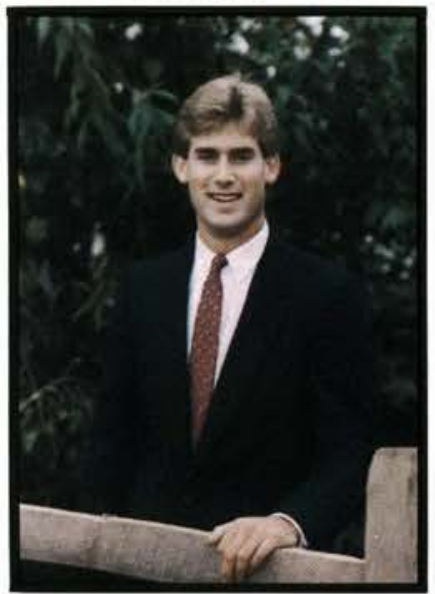

Robert Kent Watson Accounting

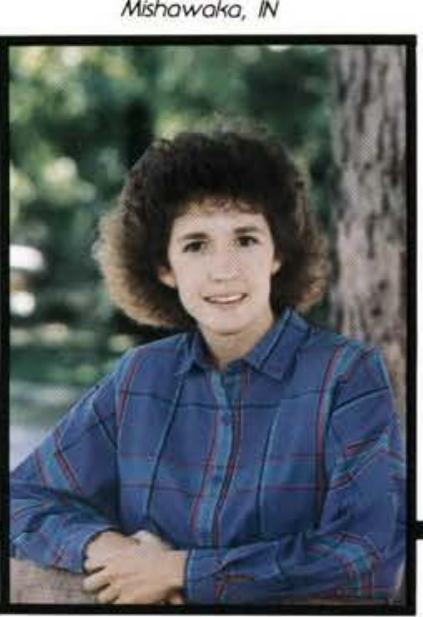

Rujeana Tedford Wenrick

104 Seniors

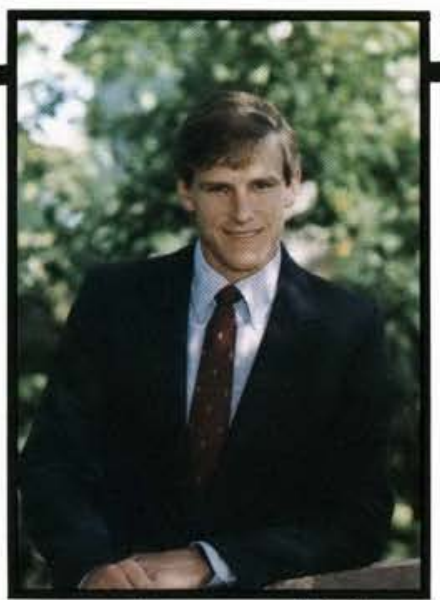

Kevin Lawrence Wallace Physical Educarion

Fosterburg, IL

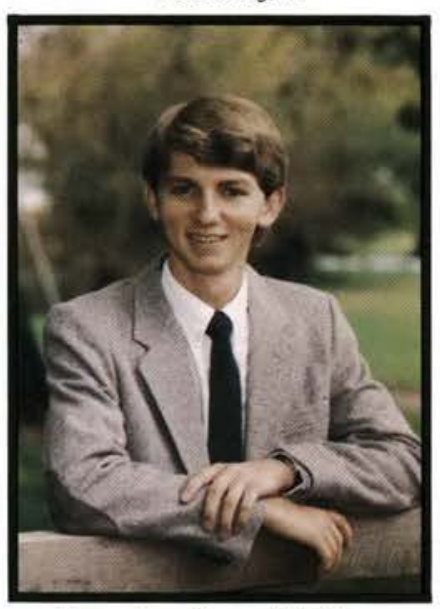

Brandon Troy Waltz

Preseminary Bible Major

Combridge Ciry, $\mathbb{N}$

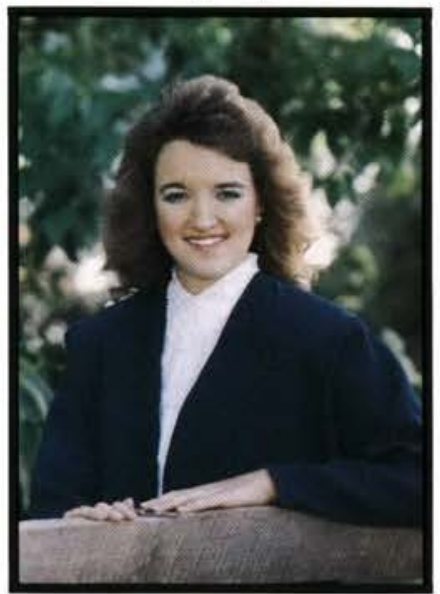

Kristin R. Weber

English

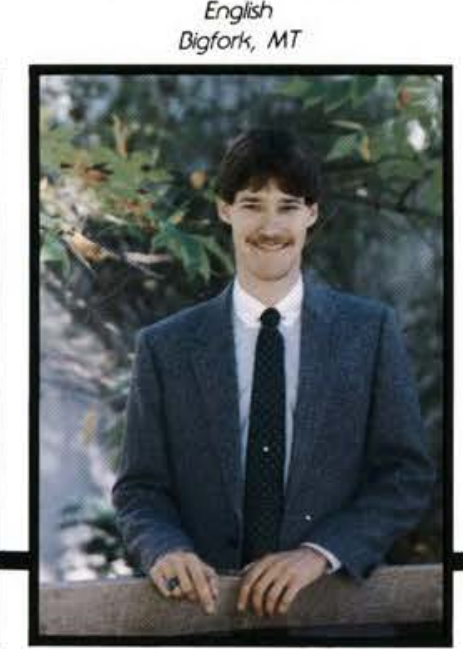

Paul Scott Wenz

Broodcosting

Romulus, MI

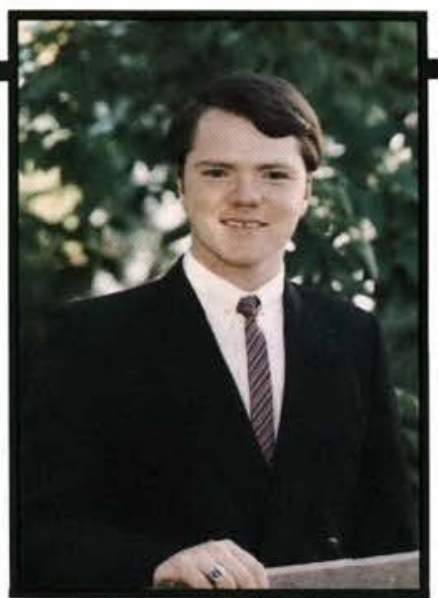

Steven P. Walter

micarion Arts

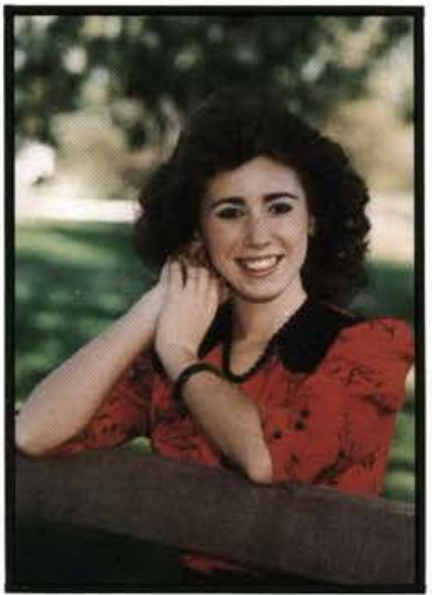

Shari Lynn Washburn Behovioral Science

Lowton, M

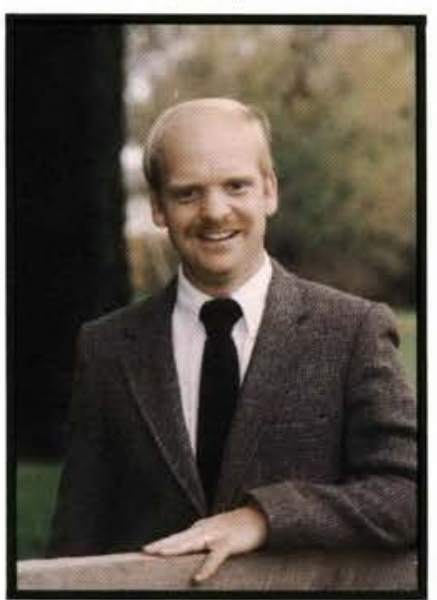

David A. Wehman

Accounring

Son Diego, CA

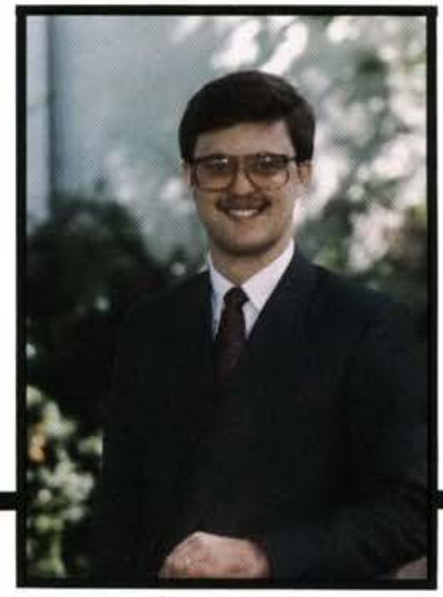

Douglas Dean Whittenburg Accounting

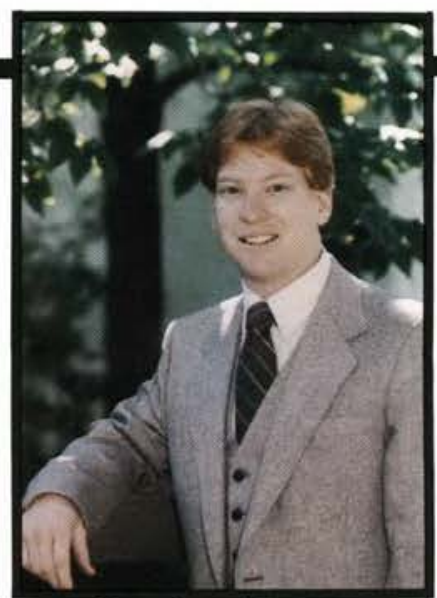

David M. Walters

Preseminary Bible Mojor

Nework, OH

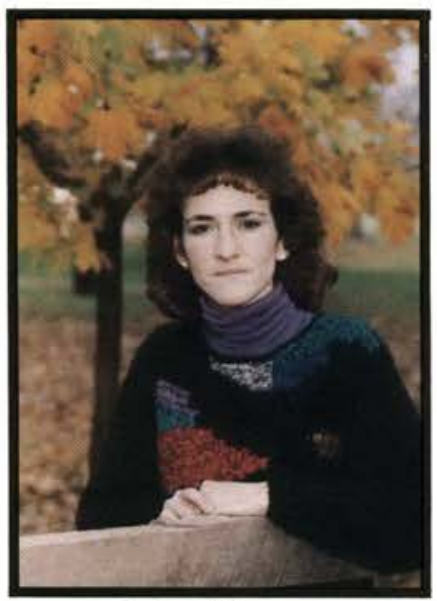

Cathy Watson

Elemenrory Educarion

Roveeno, M

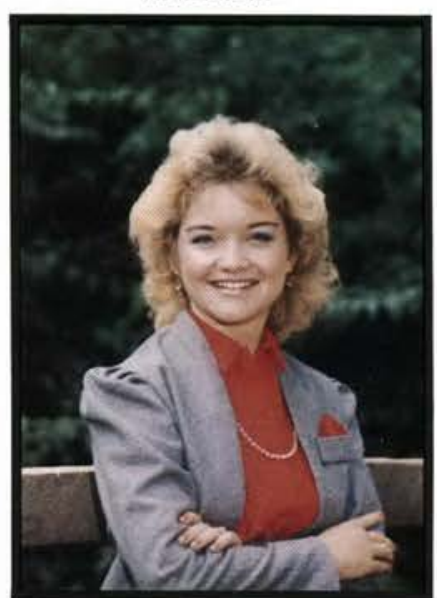

Amy L. Welch

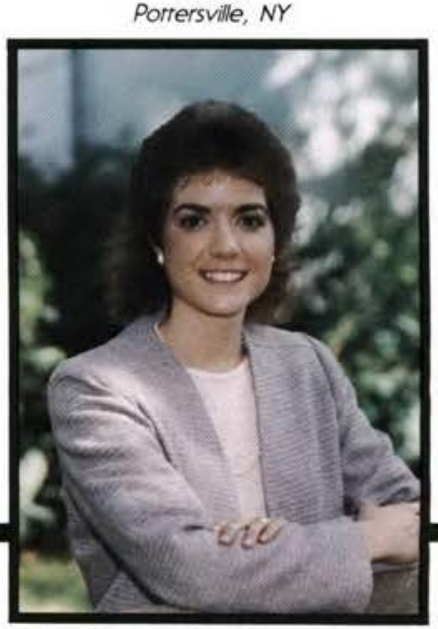

Robin Kimberley

Whittenburg

Spanish 


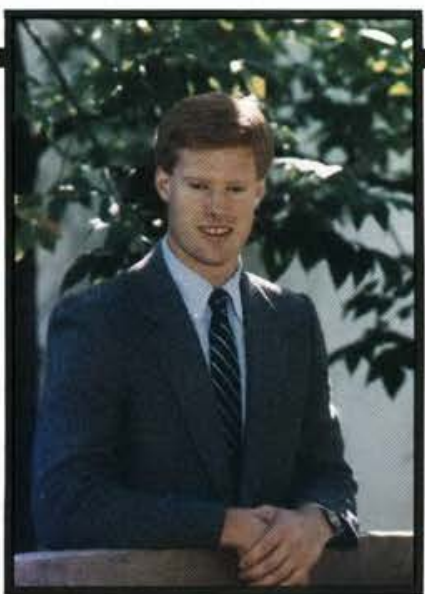

David Alan Wiinamaki

Business Administrotion

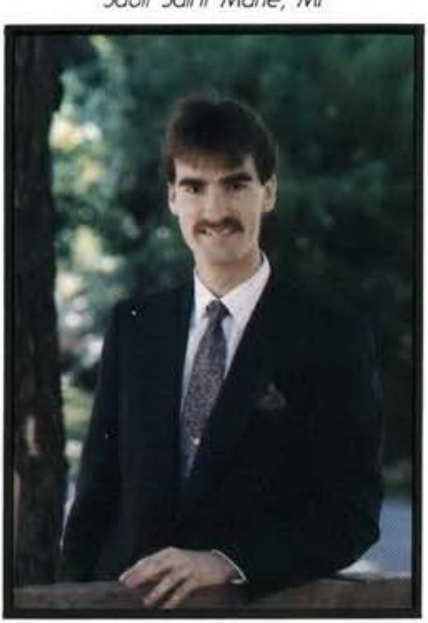

John Andrew Wilson Communicarion Arts

Cincinnari, $\mathrm{OH}$

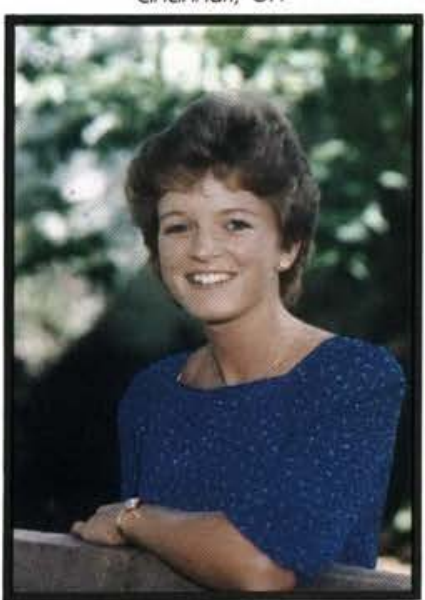

Tara Lee Wise

Business Administrorion

Indianopolis, IN

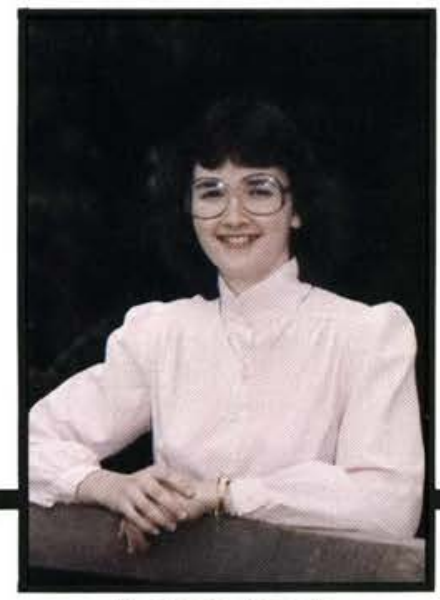

Barbara Yanda

Elementary Educorion

Fillmore, NY

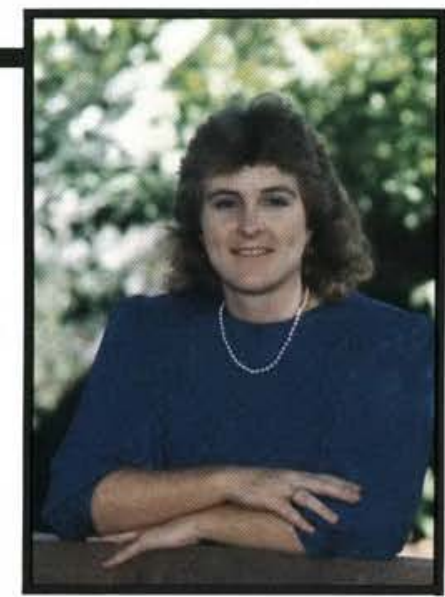

Angela G. Wilcox

Psychology

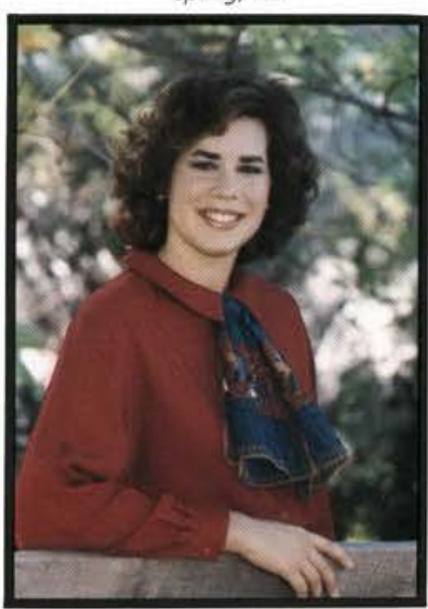

Rachel Lori Wilson

Eincinnoti. $\mathrm{OH}$

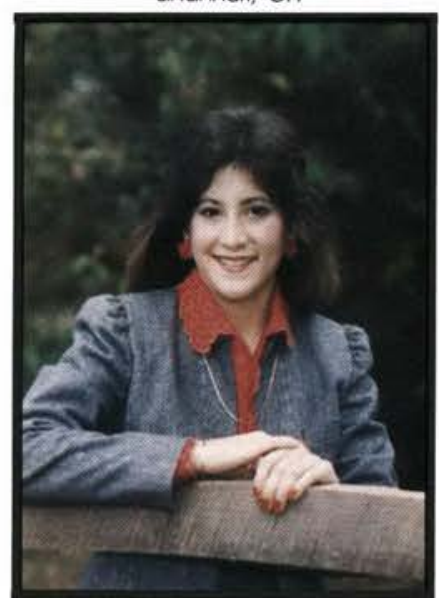

Janet Wittekind

Nursing

Levitrown, NY

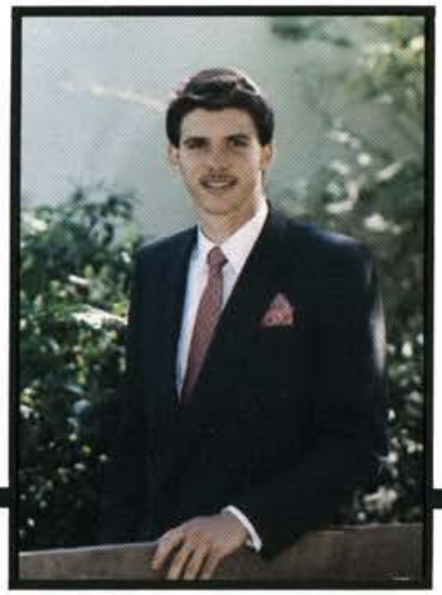

David A. Yeager

Compurer Information Sysrems

North Vernon, IN

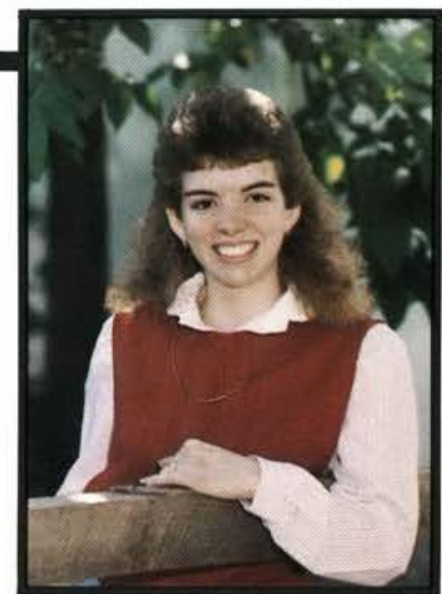

Kathy A. Wilkinson

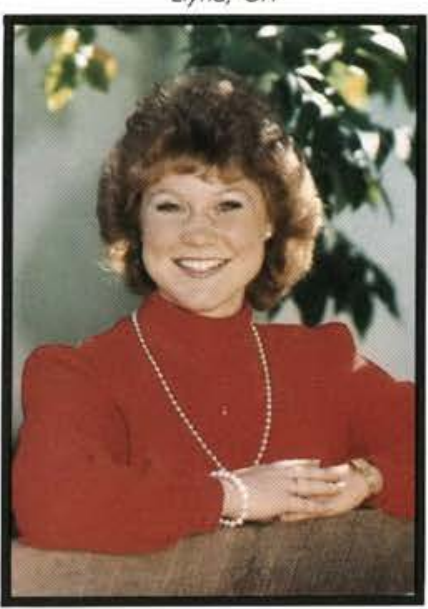

Teresa D. Wilson

Educarion

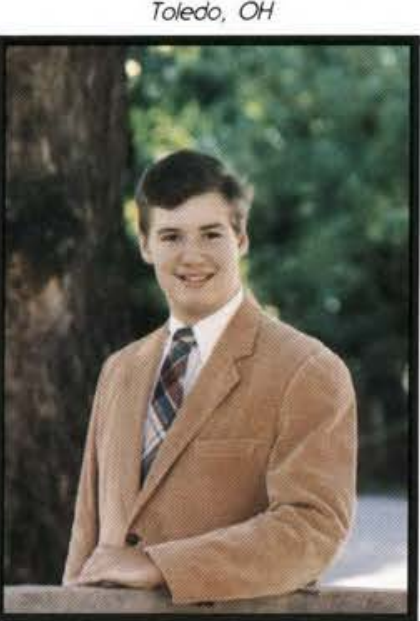

Stephen Edward Wood

Music Educorion

Haddonfield, NJ

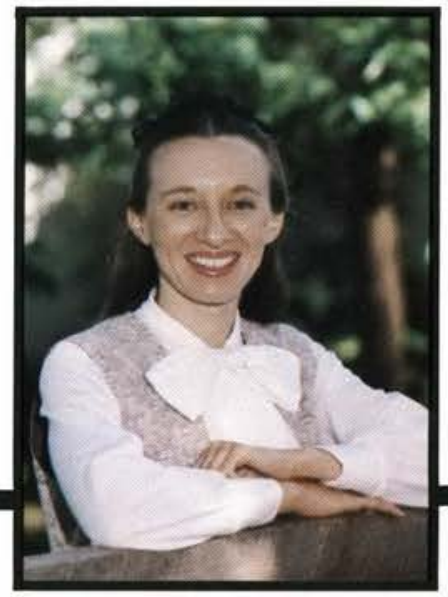

Ethel Paula Yoder

Elementary Educarion

Grantsville, MD

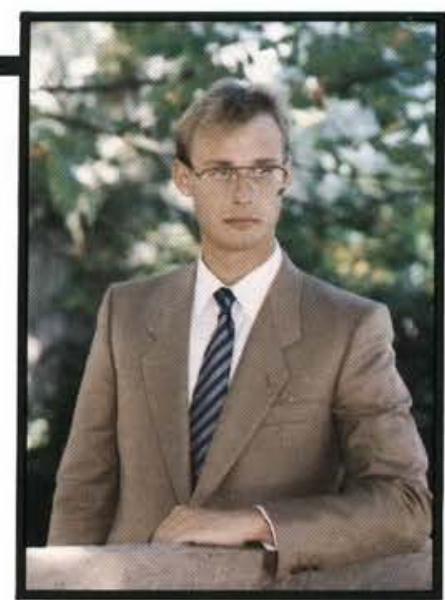

John Timothy Williams

communicarion Arts

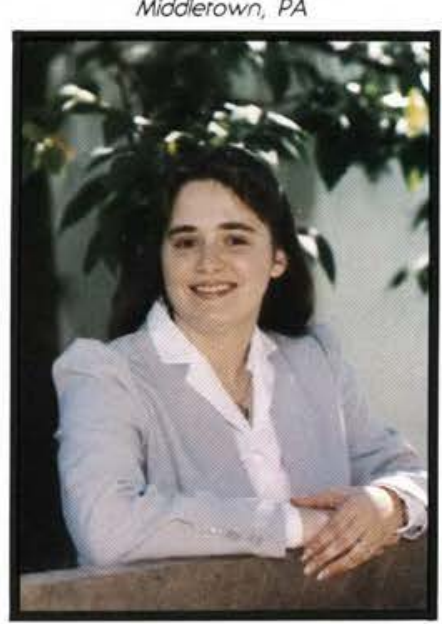

Donna R. Windish Accounting

Middleville, Mi

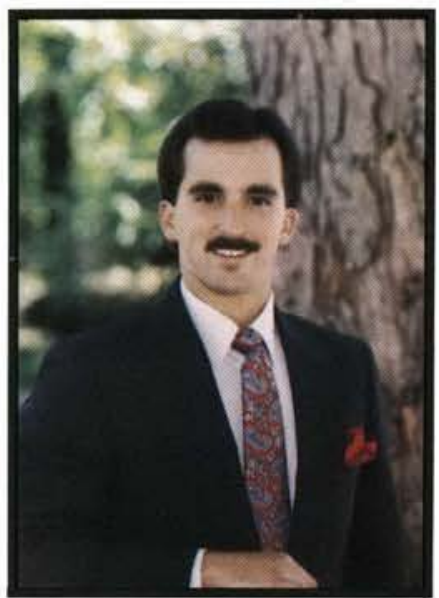

Timothey C. Worosher

Business Administrarion

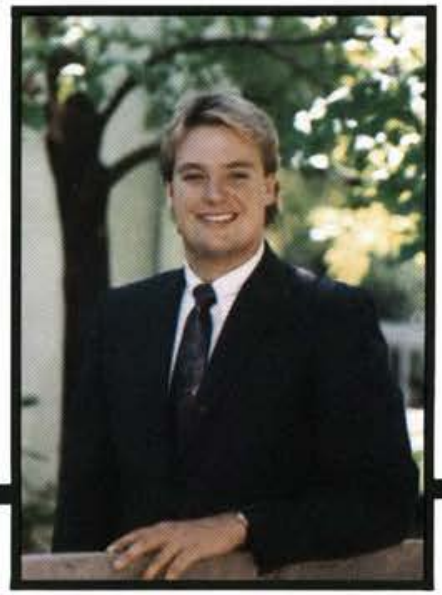

Eric Todd Zehr Business Administrarion

Fr. Wayne, IN 


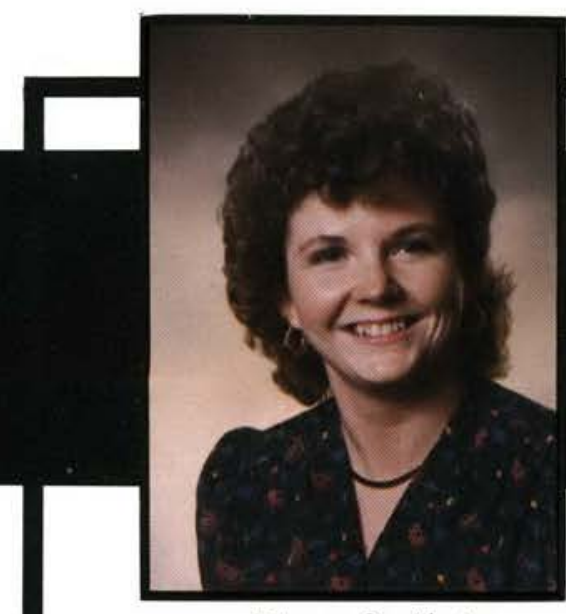

Diane O. Abston

2.Year Secrerarial

Crowfordsvile, IN

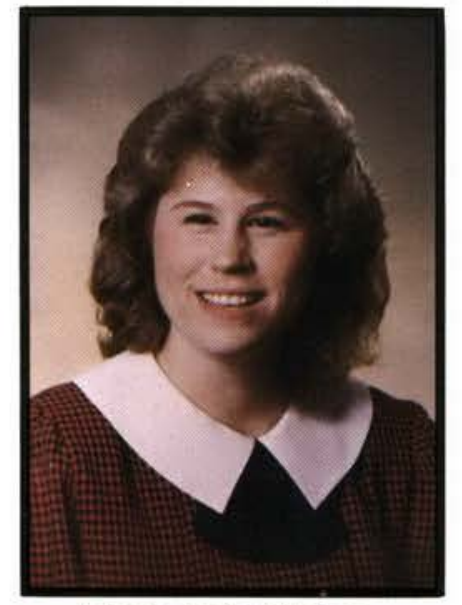

Diane Beth Clymer

2.Year Secretoria

Sellersville, PA

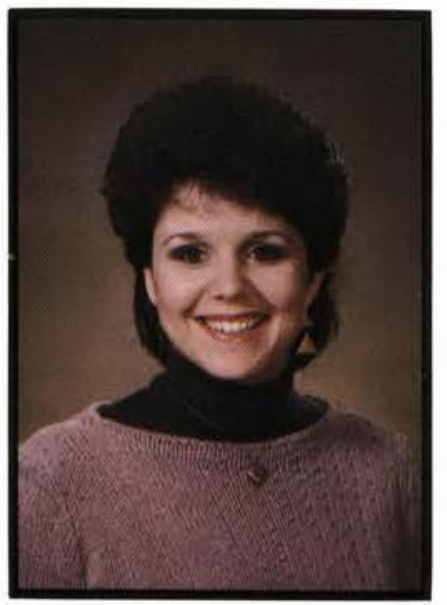

Lisa Grafton

2.Year Secreraria

Cedarville, $\mathrm{OH}$

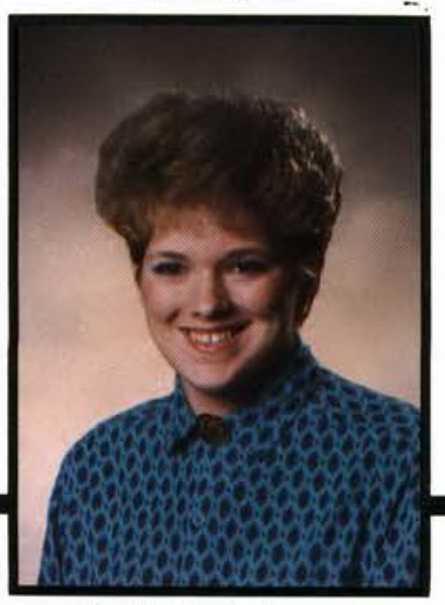

Heidi Marie Ryan

2-Year Secreraria

Almond, NY

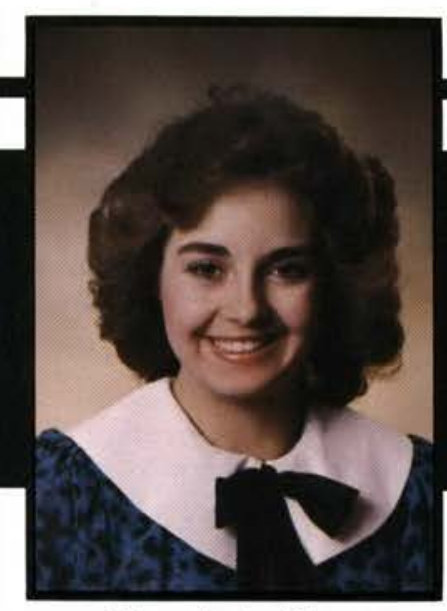

Rhonda L. Altman

2-Year Secrerorial

New Richmond, $\mathrm{OH}$

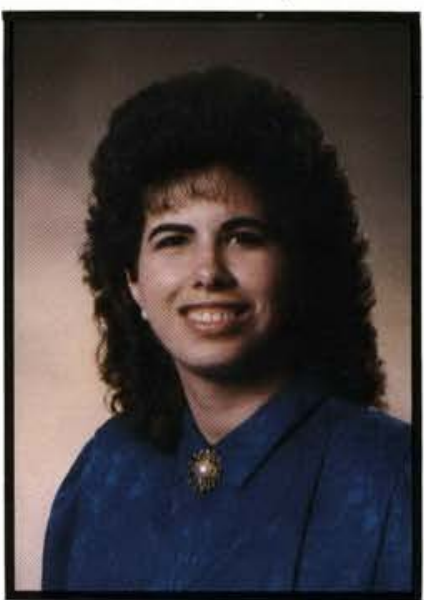

Jennifer Lynn Everage

2.Year Secrerorio

Eminence, IN

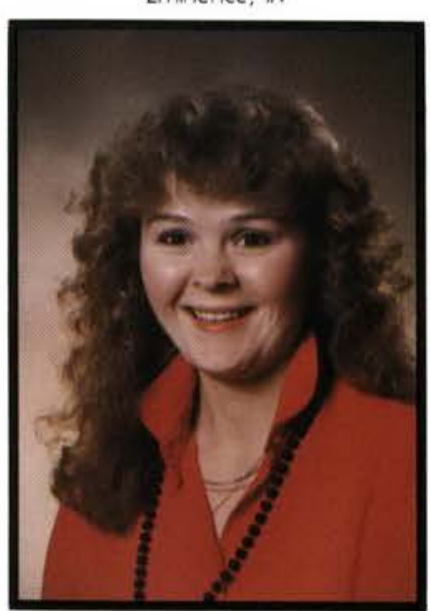

Gina Rae Long

2-Year Secreroriol

Martinsburg, WV

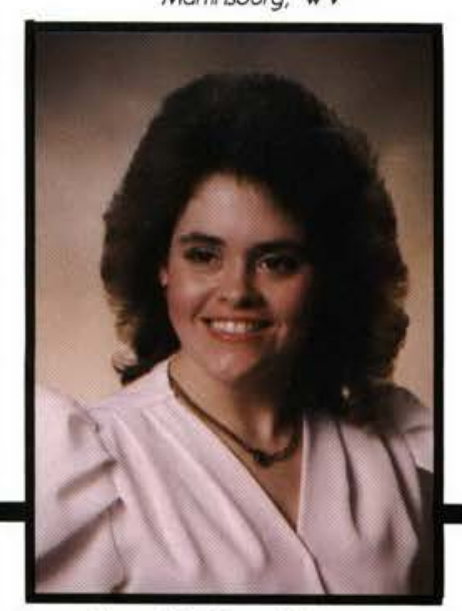

Tina Nadine Skiver

2-Year Secreroria

Continental, $\mathrm{OH}$

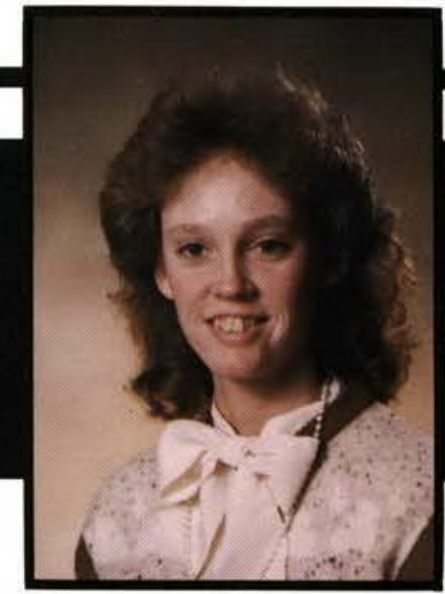

Carol J. Bliss

2.Year Secreraria

Belding, MI

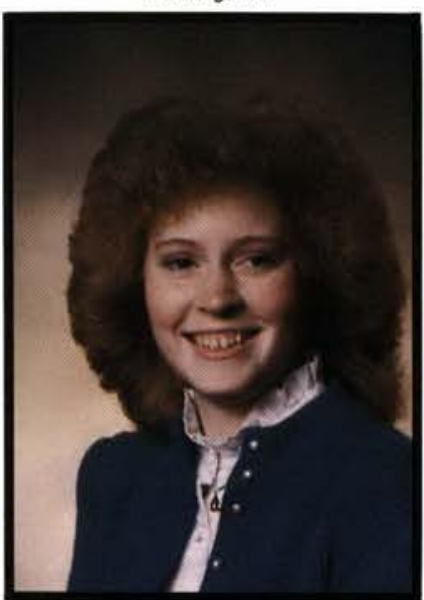

Deborah Lynn Fogle

2-Year Secrerorio

Springfield, VA

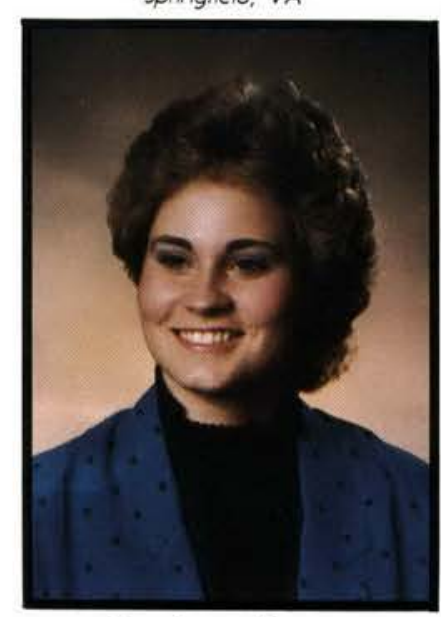

Lisa Lynn Payne

2.Year Secrerorio

Highland, IN

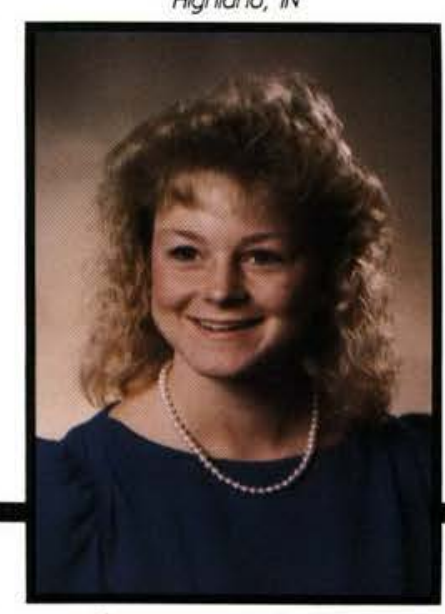

Christine M. Stowell

2-Year Secreroria

Midland, MI

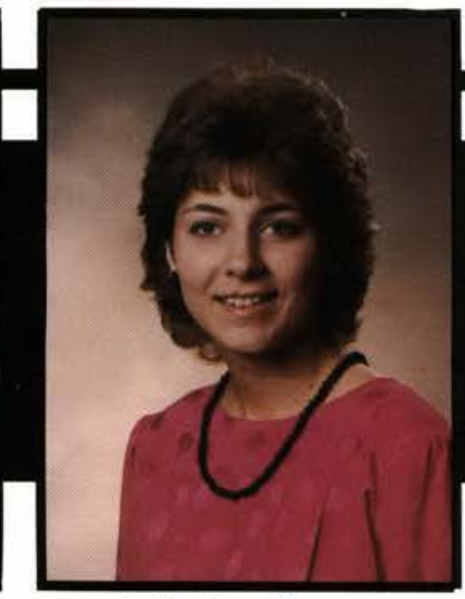

Polly Ann Brewer 2-Yeor Secreroria Salisbury, NC

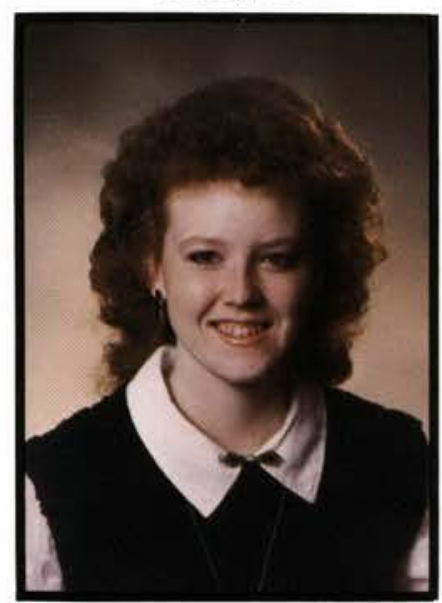

Shannon Rae Fritz

2.Yeor Secrerorio

Mossillon, $\mathrm{OH}$

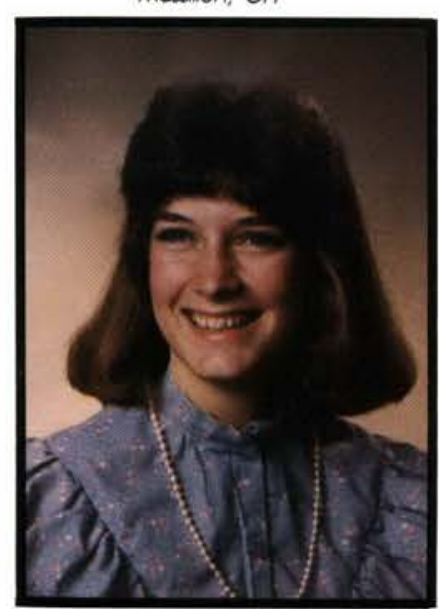

Lisa Beth Raines

2-Year Secreroria

Poroskola, $\mathrm{OH}$

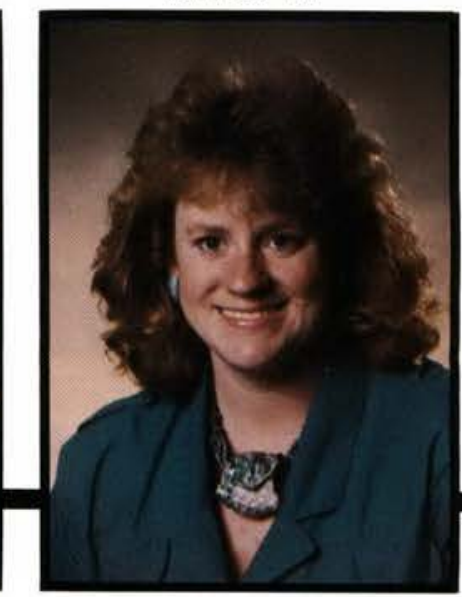

Jennifer $M$. Ward

2.Yeor Secrerorio 


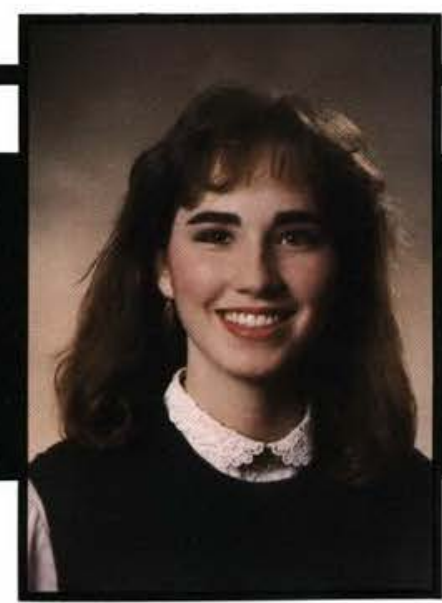

Jodi Lynn Yoder 2.Year Secretarial Alliance, $\mathrm{OH}$
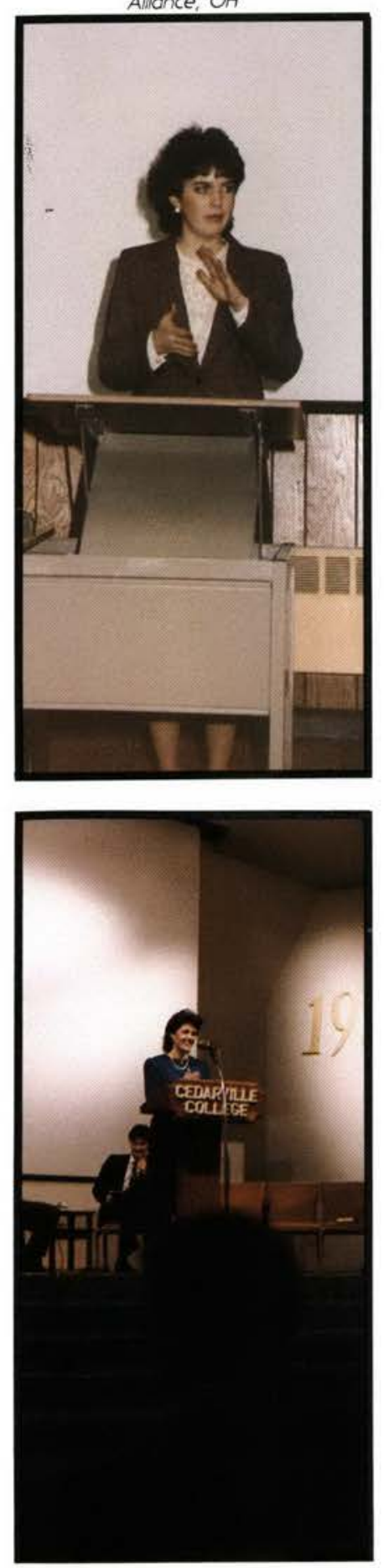

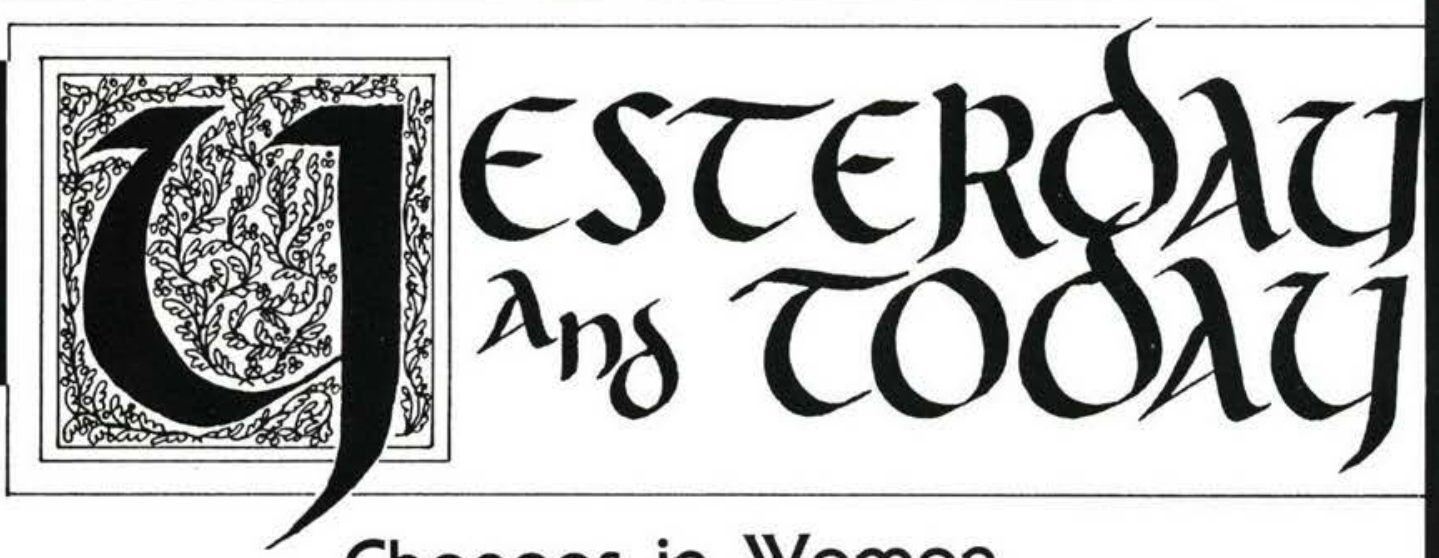

Changes in Women

Along with the changes that have taken place in society concerning women, the women who have attended $\mathrm{Ce}$. darville College in the past 100 years have also changed. In a general sense, the transition has been fairly consistent with the political and social alterations in the woman's status.

Always concerned with political issues, the students of Cedarville were naturally involved in the women's temperance movement. They held marches and meetings and celebrated when the vote was cast.

The independence women gained then has developed over the past sixty years in a number of ways. For example, more women attend Cedarville today than men, and a greater percent-

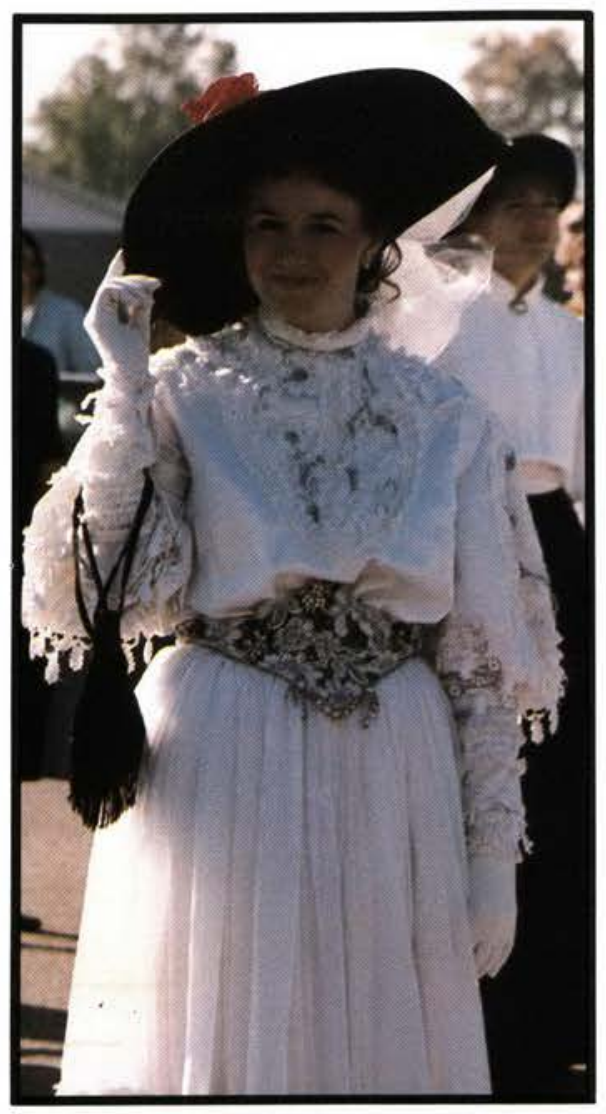

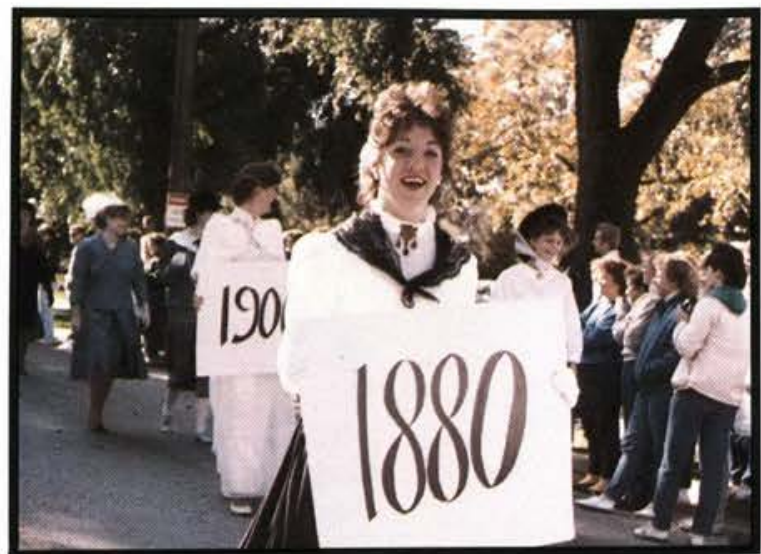

age of those women are choosing career-oriented majors. In some ways, they have come a long way. But in others ... well, it's no longer MotherDaughter teas and the Student Wives' Fellowship; now it's Alpha Delta Omega and Alpha Mu Chi.

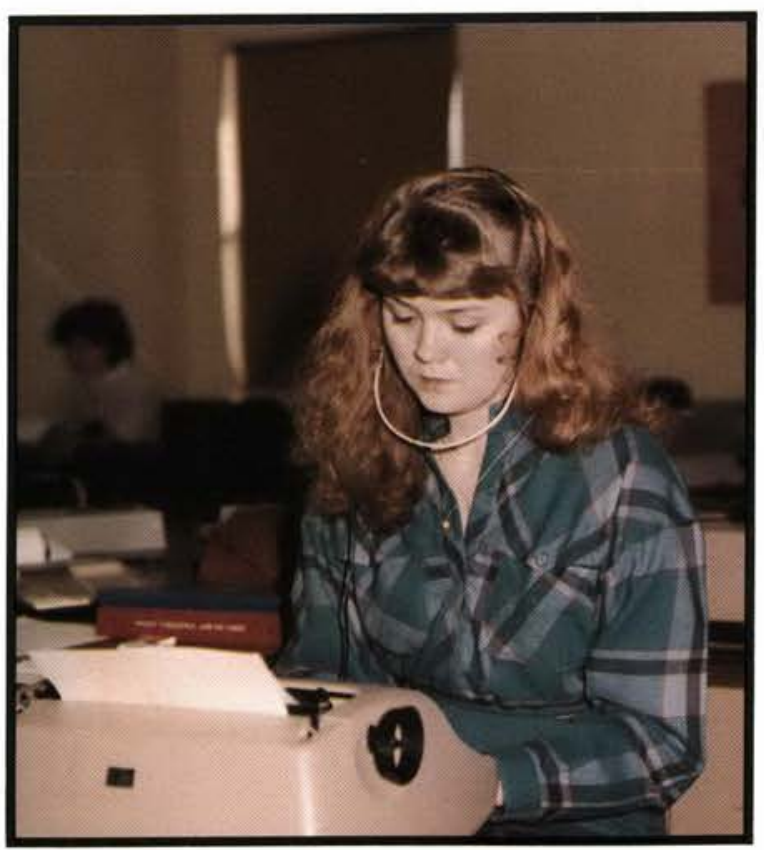




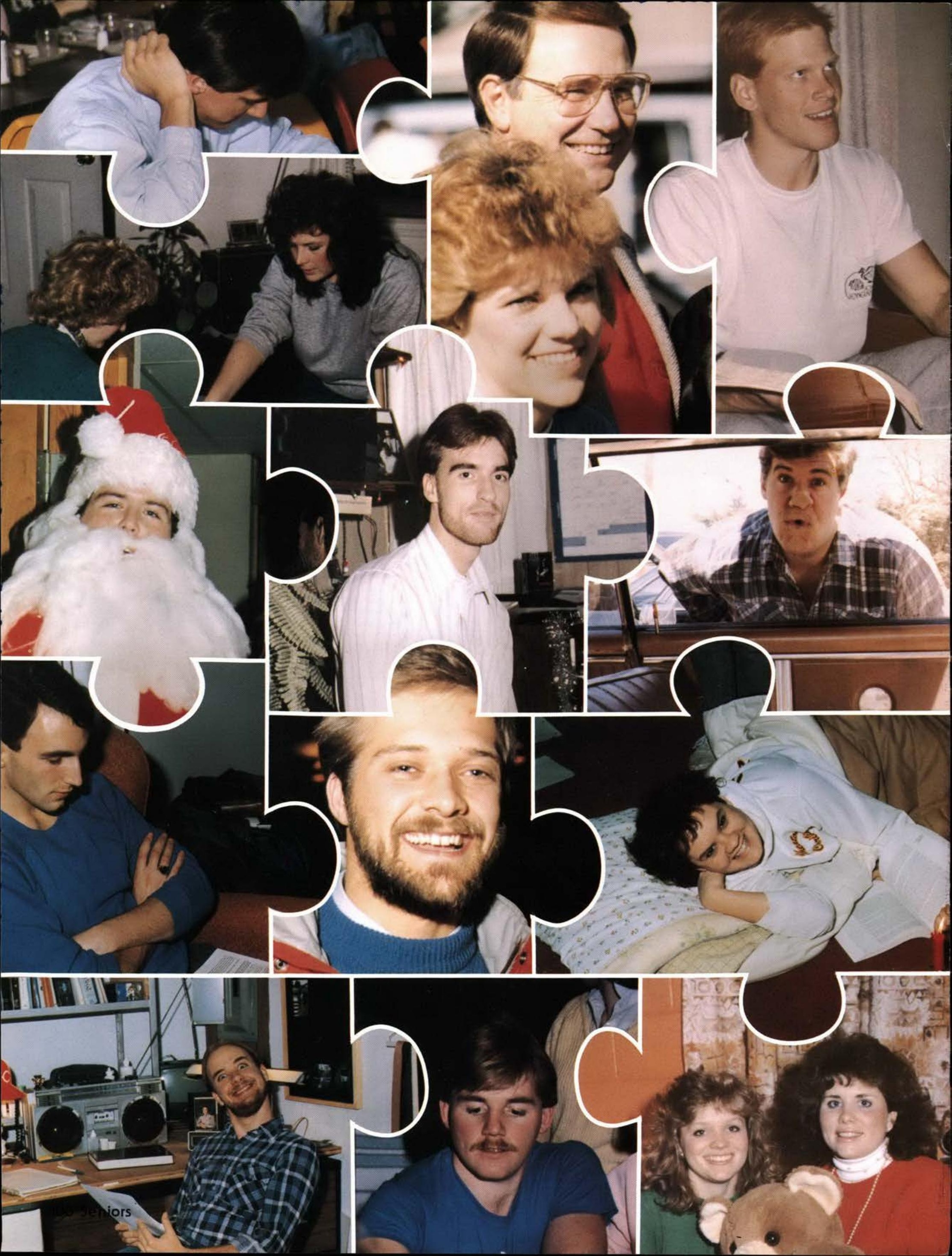




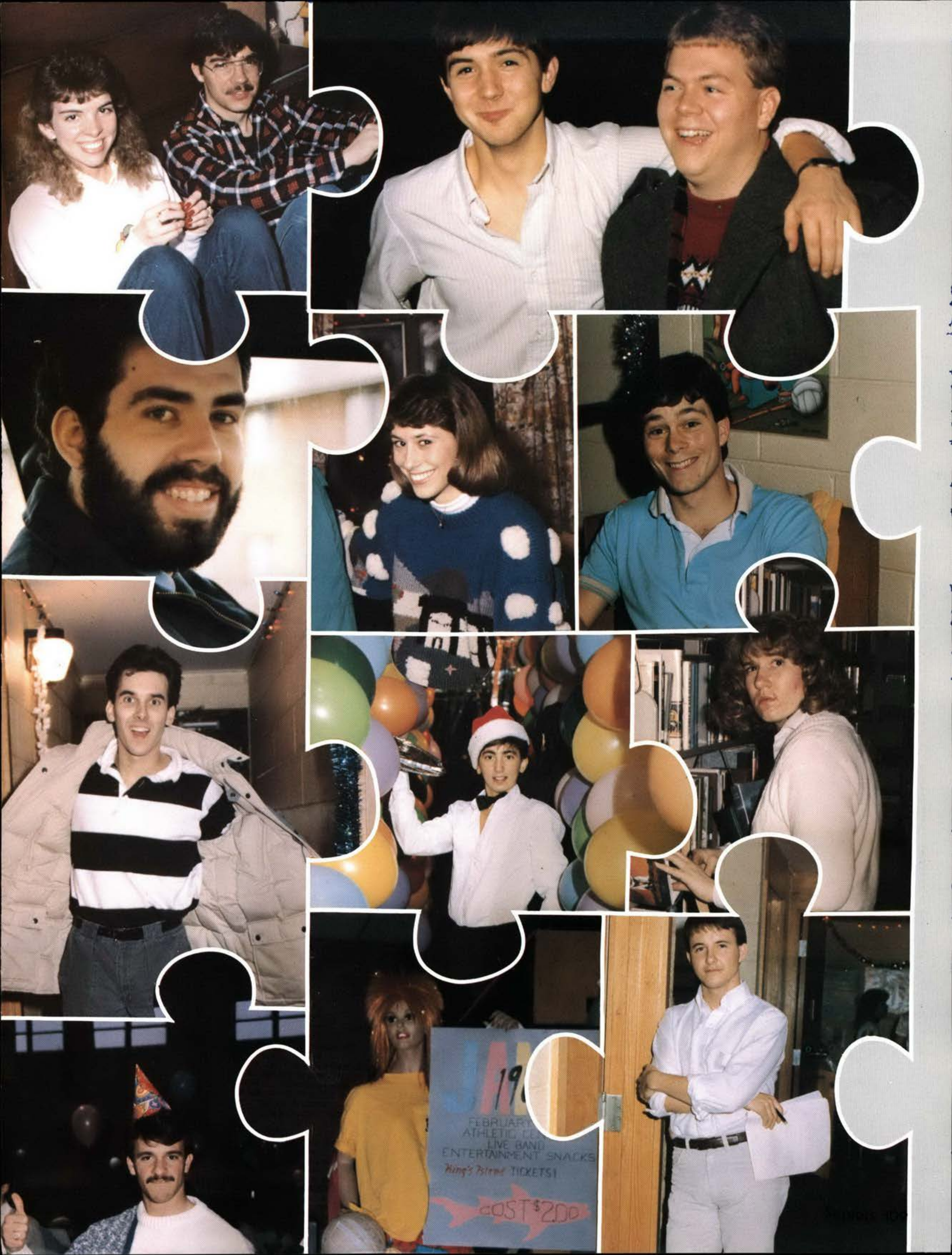




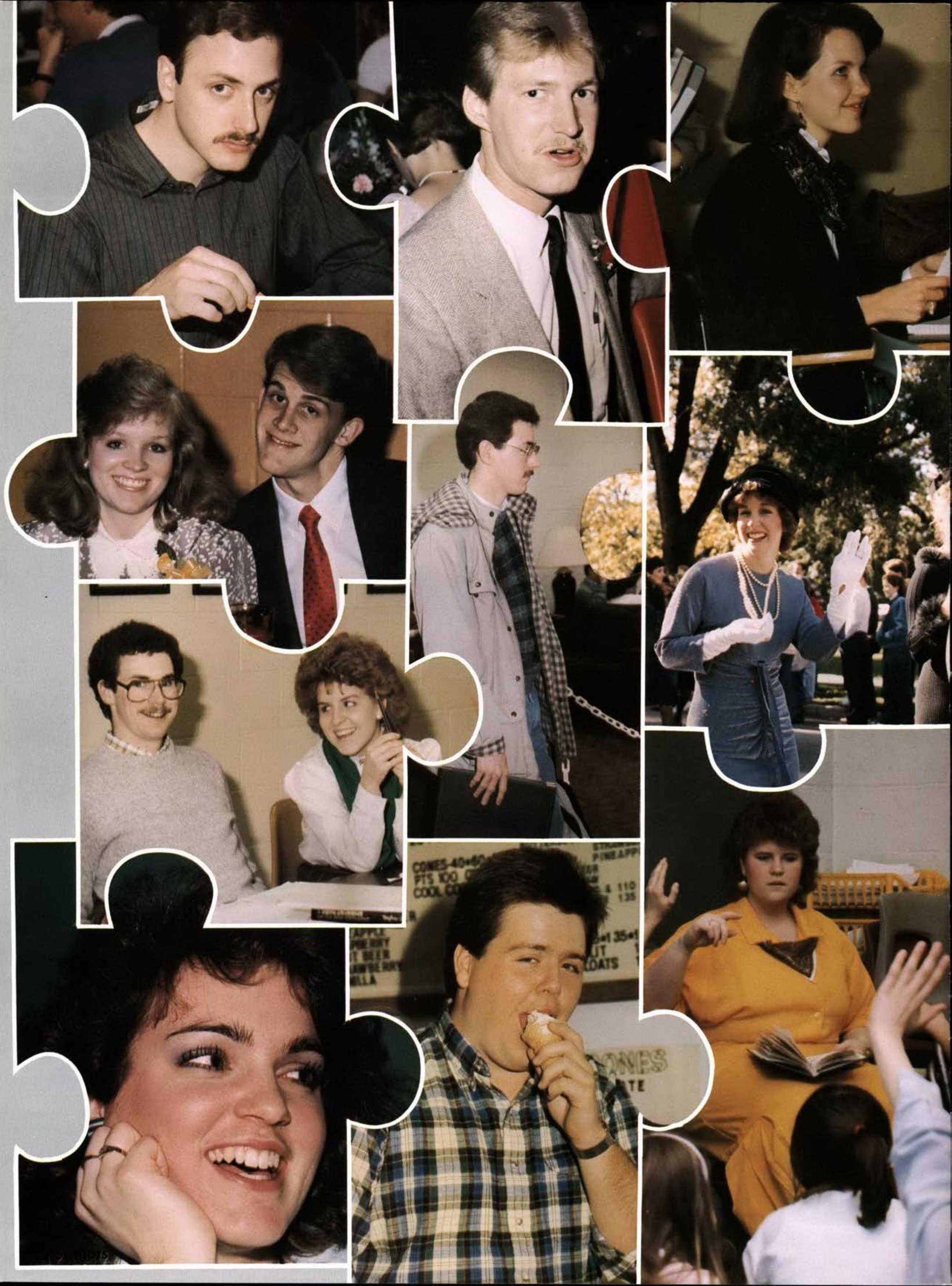




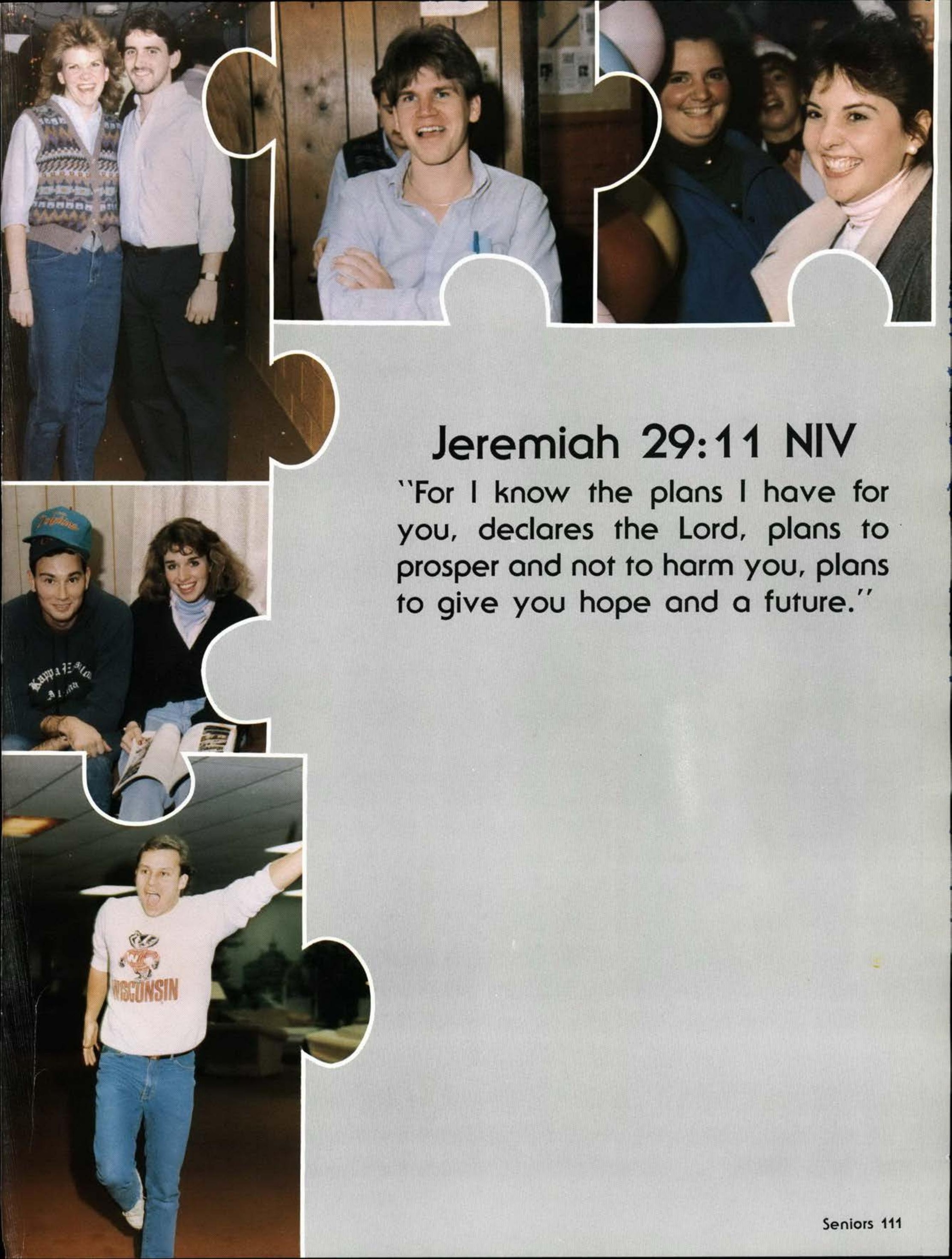



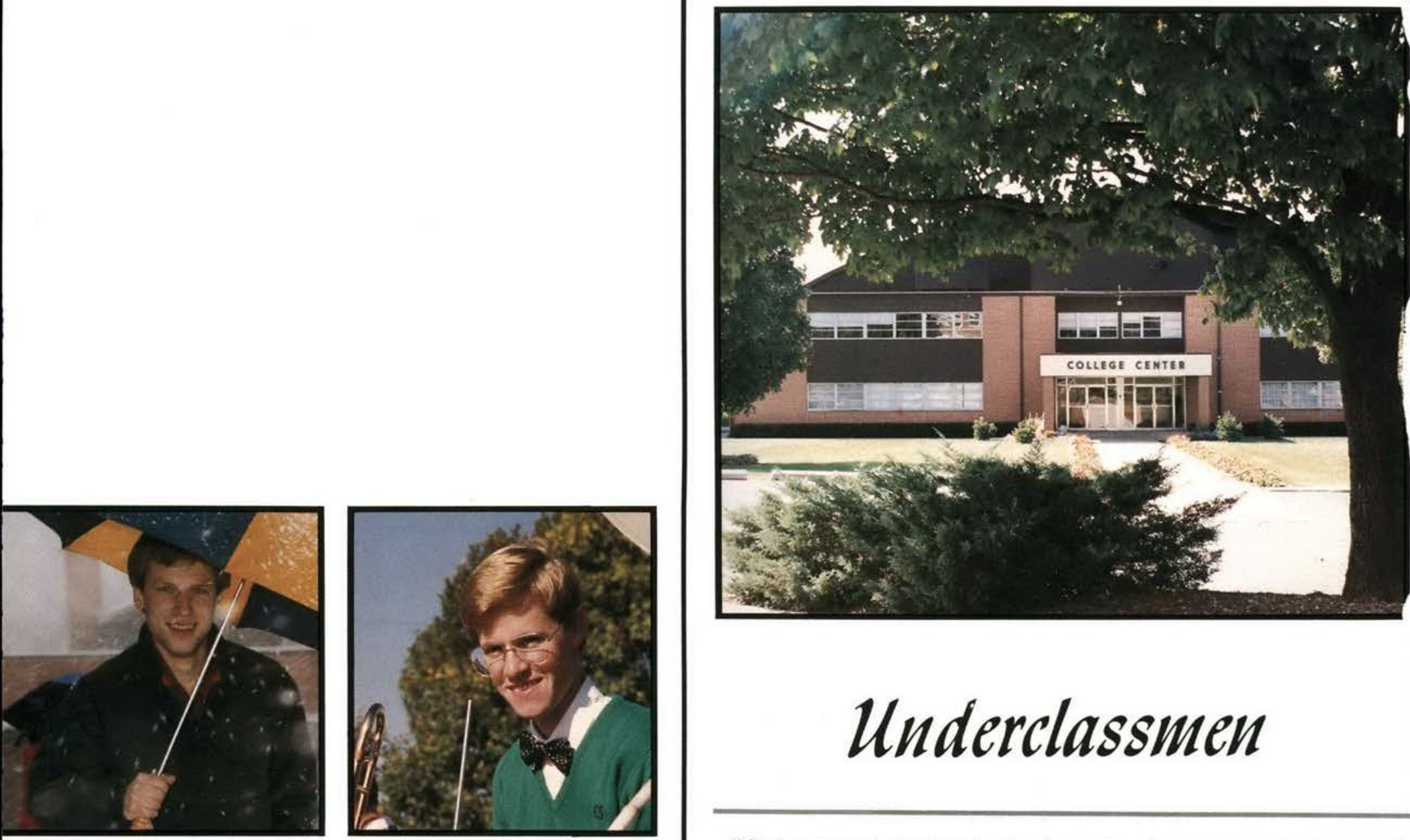

\section{Underclassmen}

When a senior looks back to the rime when he was an underclassman, it seems like yesterday; but when a freshman, sophomore, or junior anticipares "seniority," it seems like forever unil he will don that cap and gown. Some of a college student's fondest memories were formed during his underclass years, especially for those who had the privilege of living in the dormitory. Dorm life has a way of fostering in students an excellent opportunity for learning how to berter live with orhers.

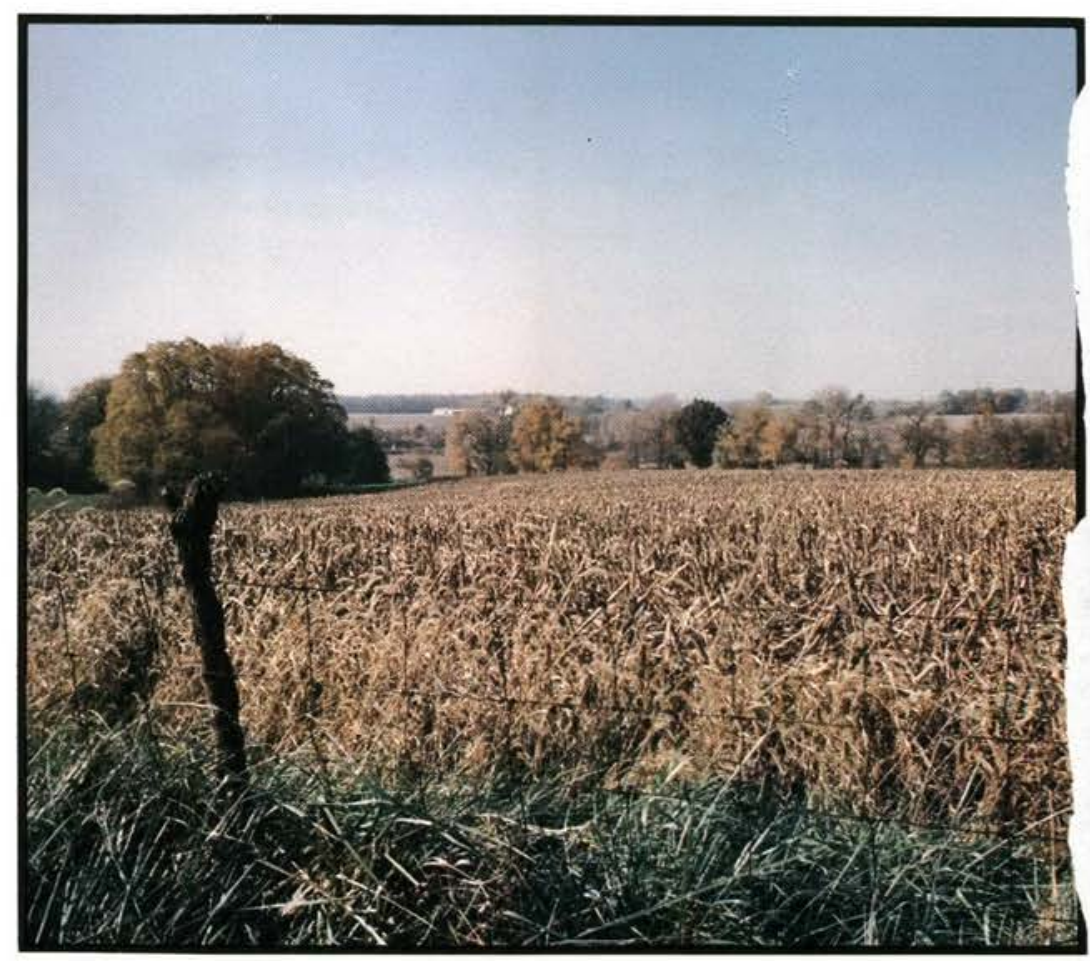




\section{1}
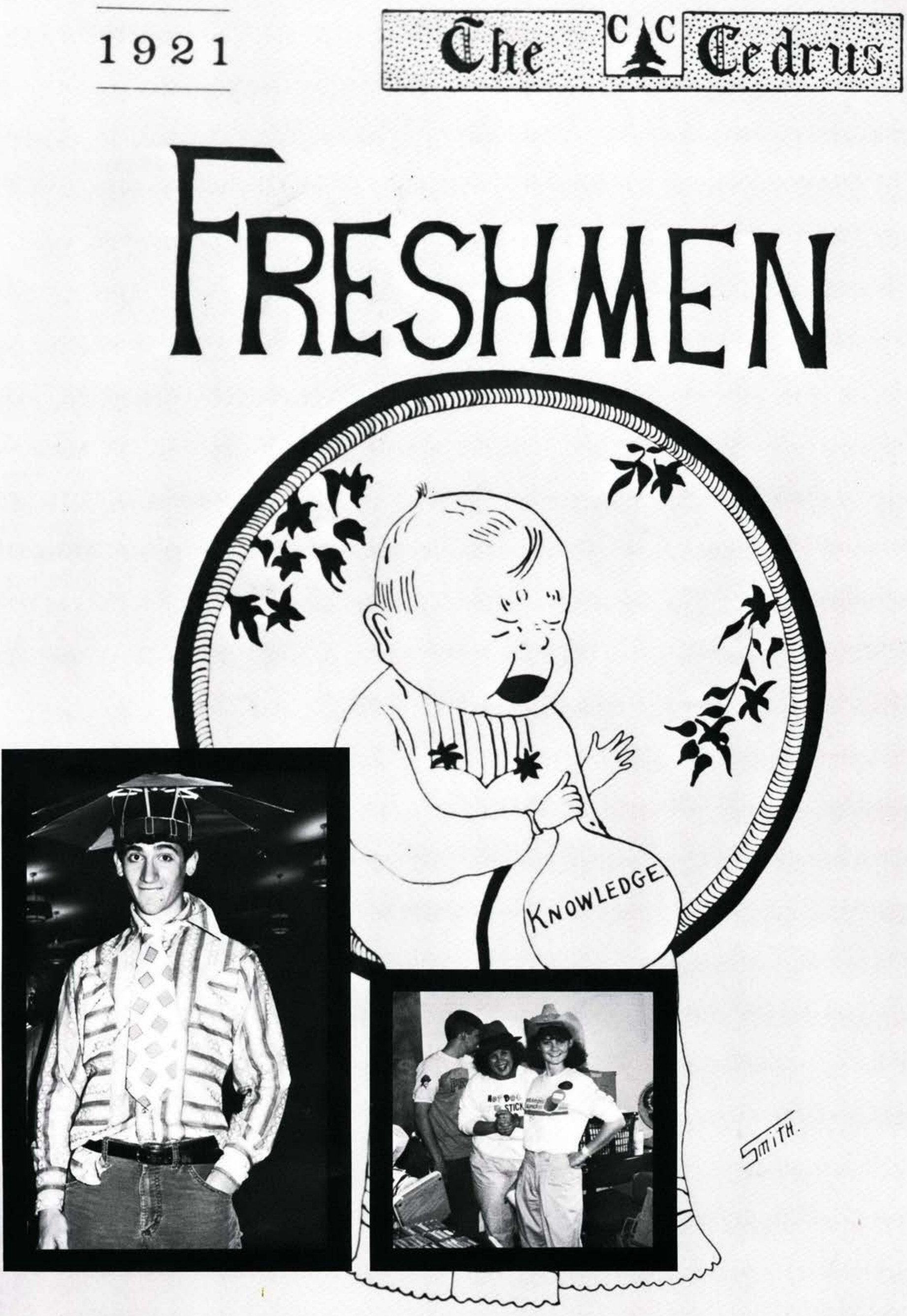


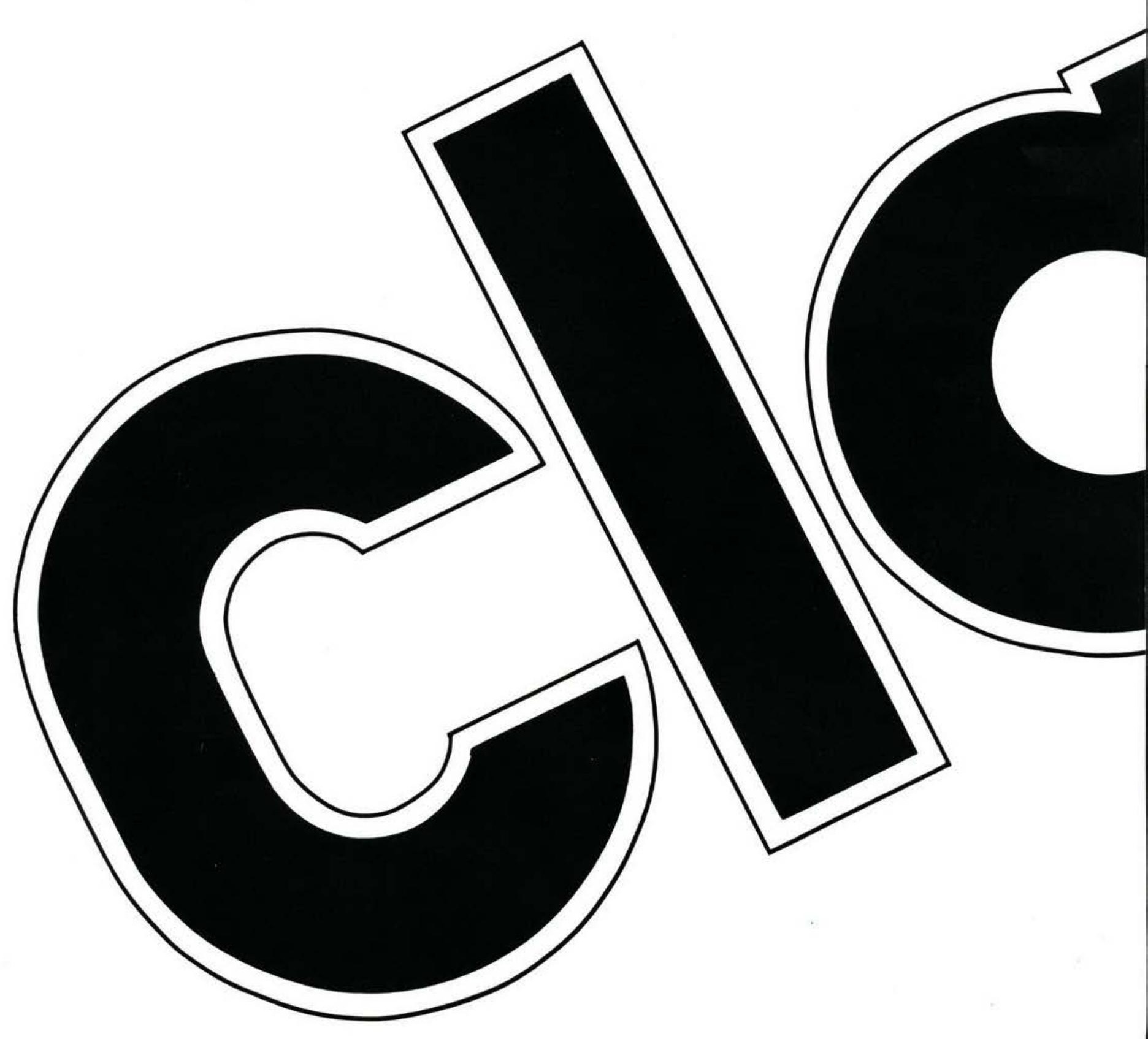




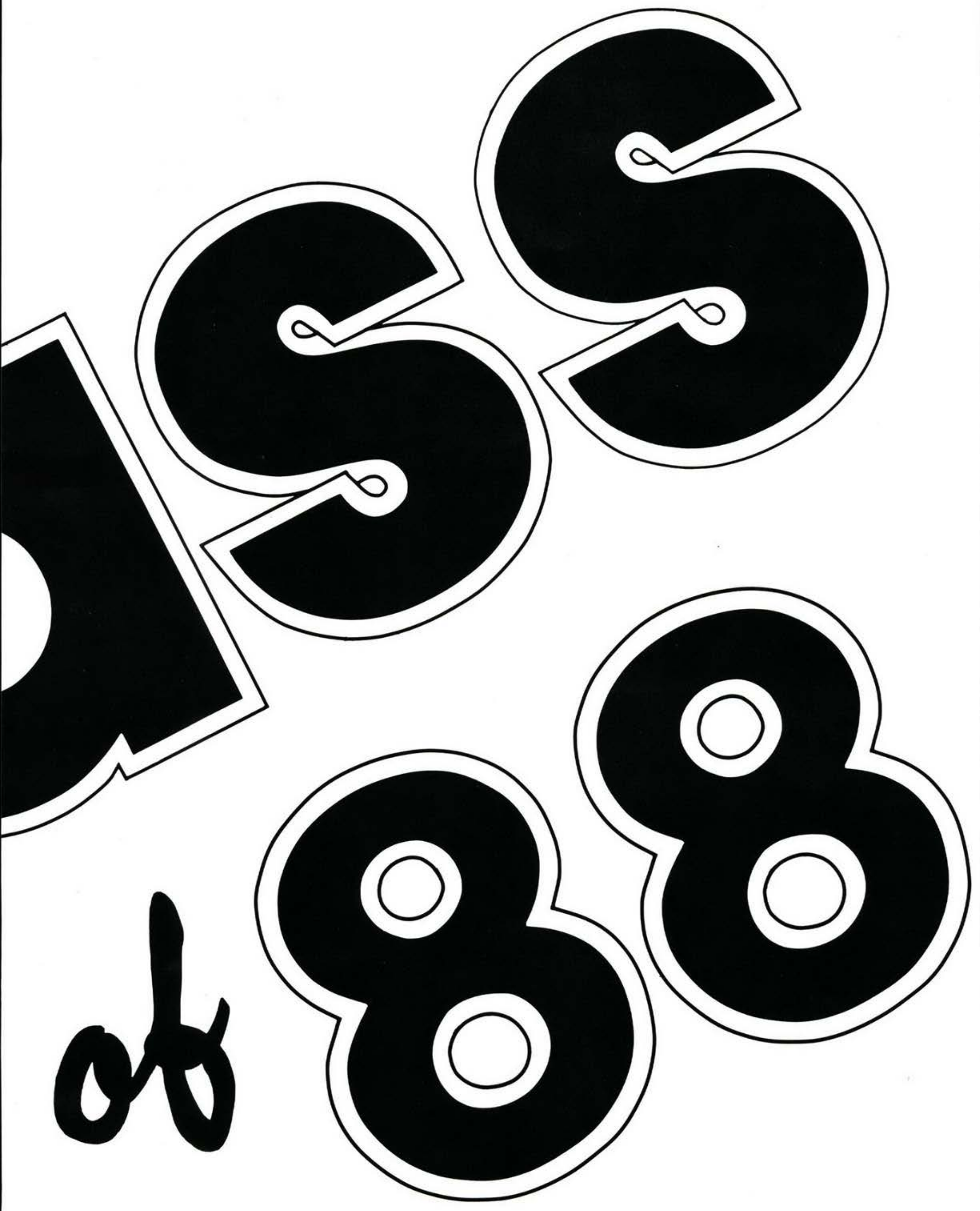




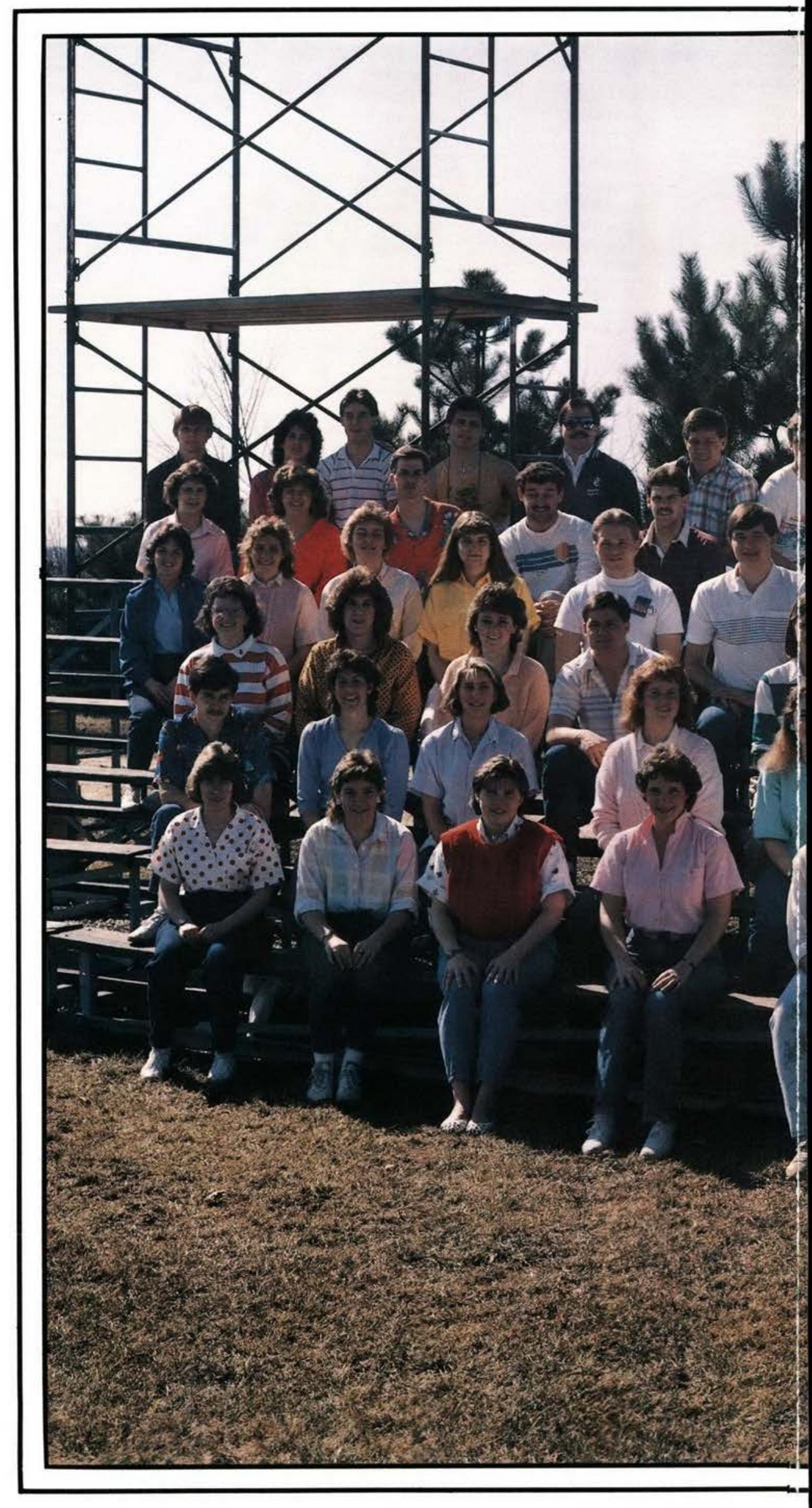



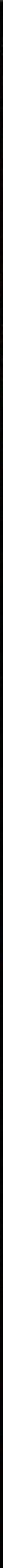


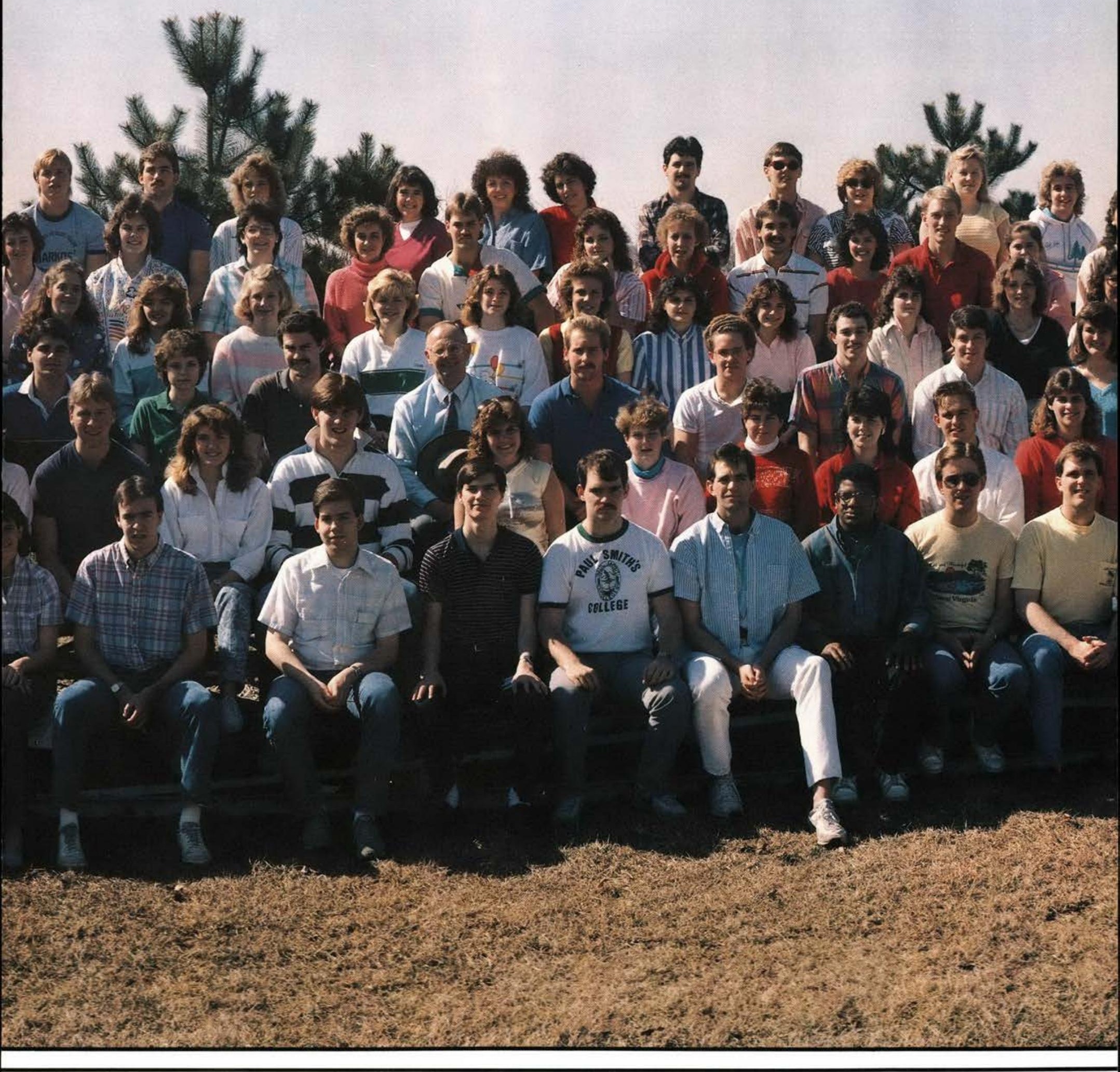




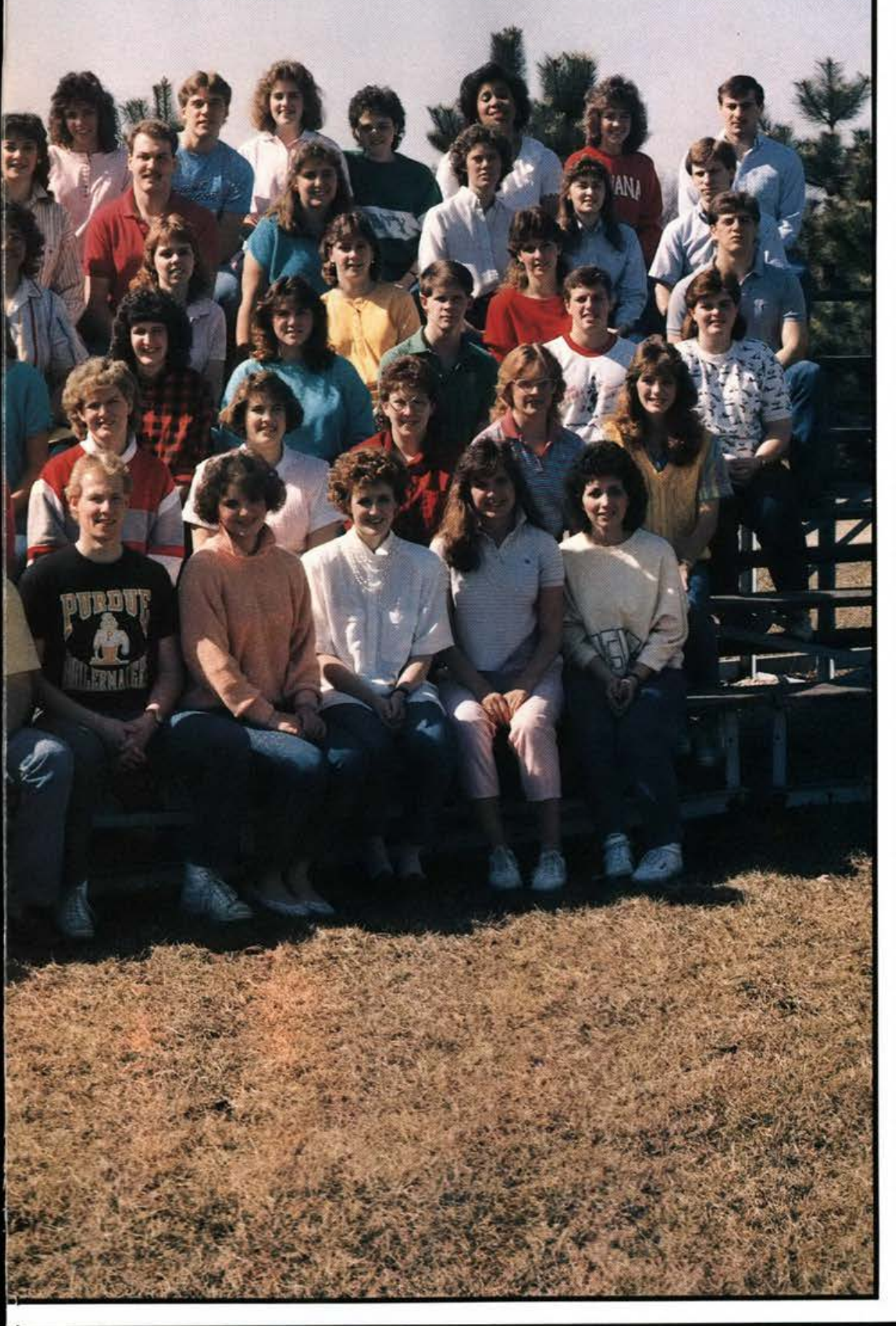




\section{BETHEL HALL}

\section{SOUTH HALL}

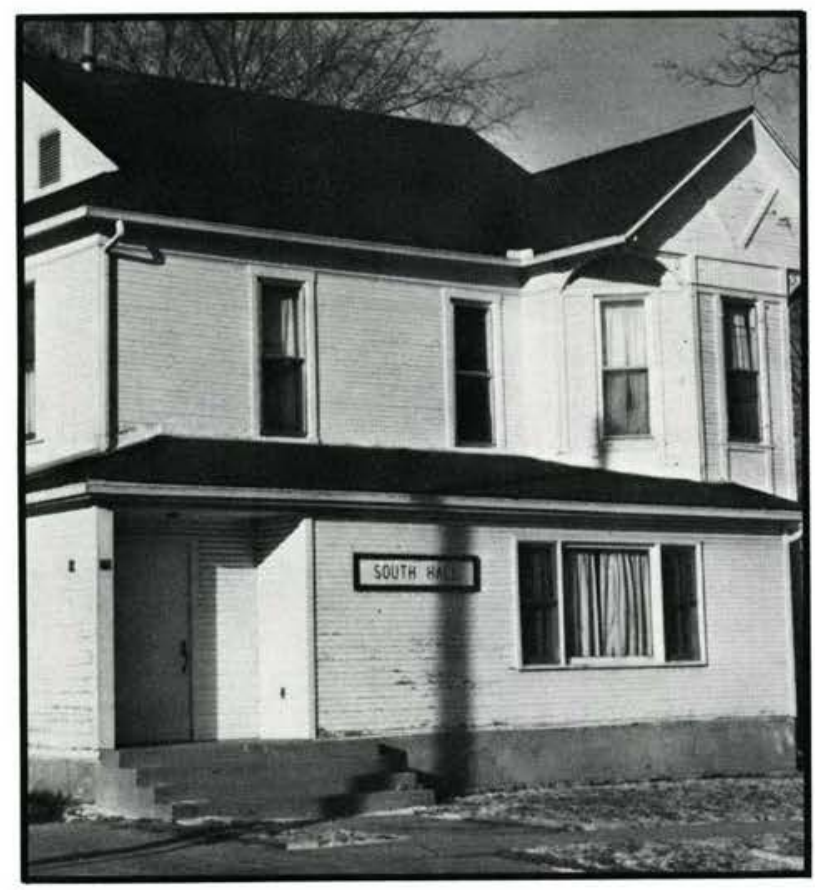

\section{HARRIMAN HALL}
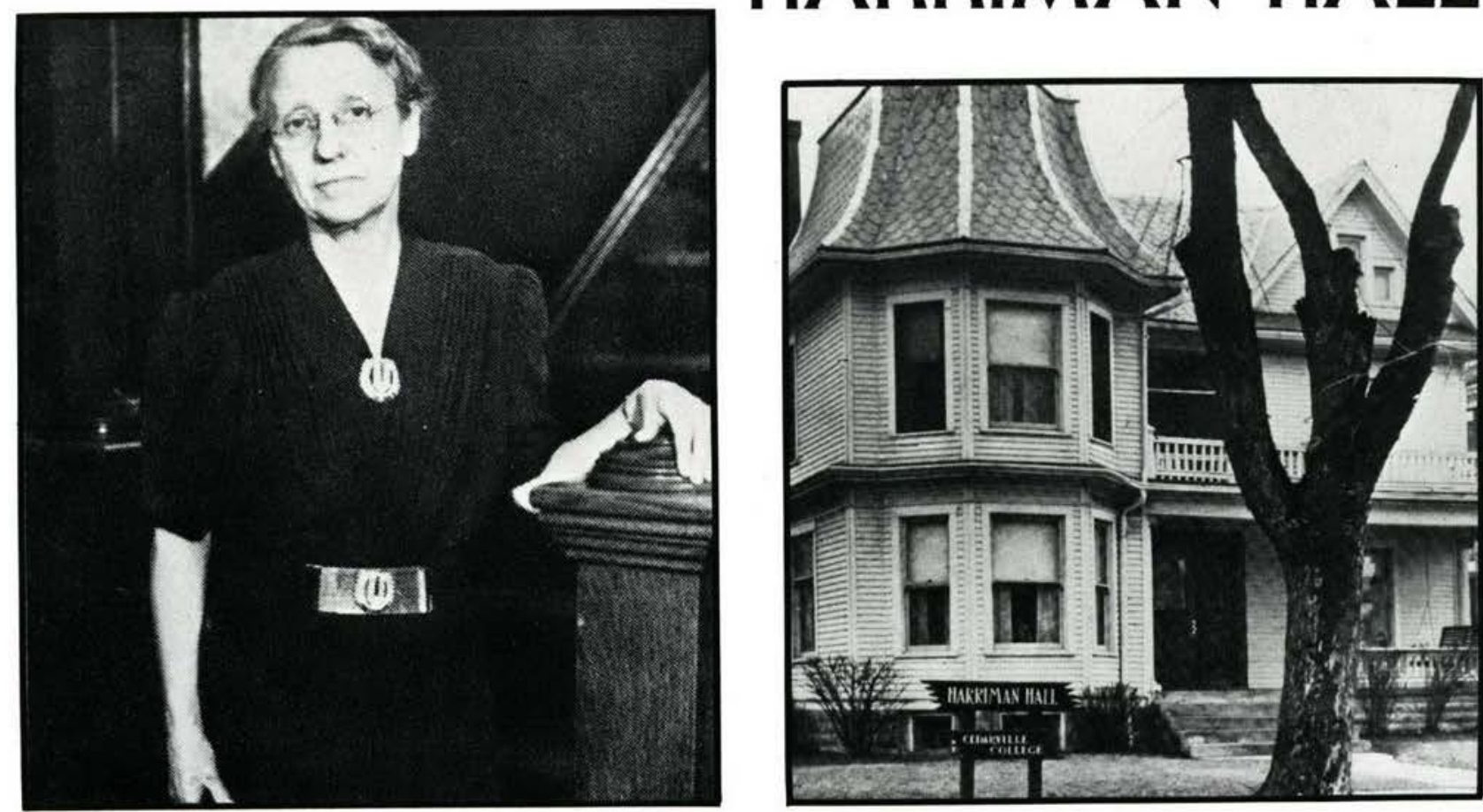

Mrs. W.P. Harriman 

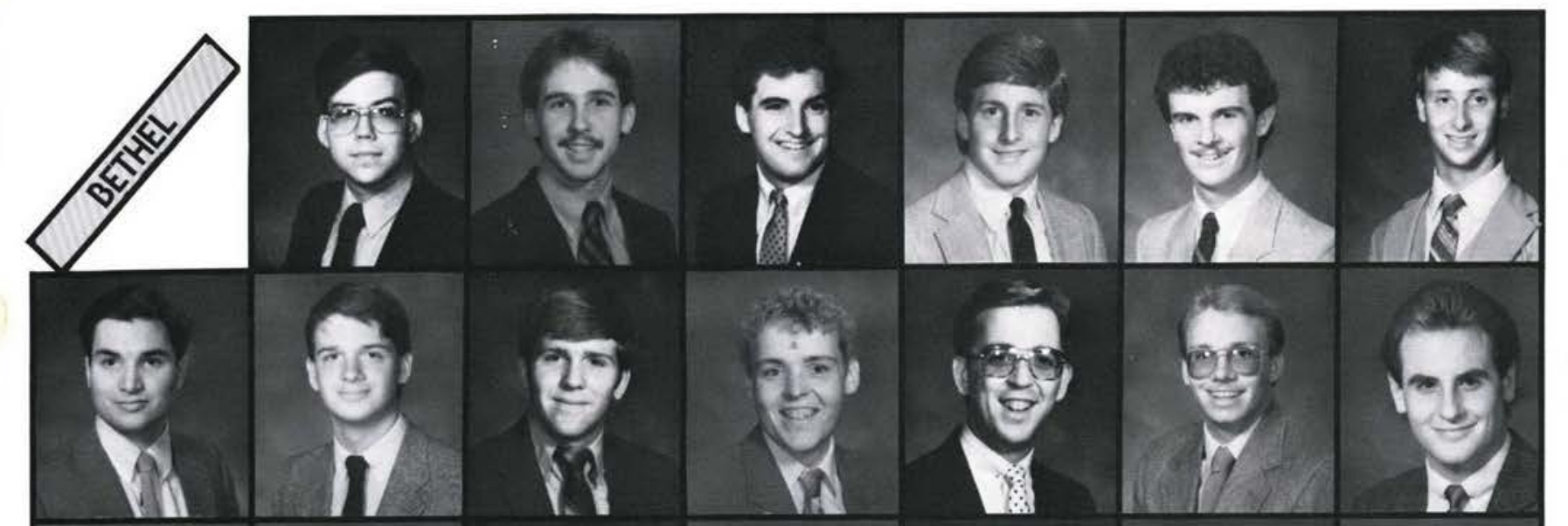

David Beal

Jeffrey Bergandine

Kristion Bradman

Tedd Cossidy

Robert Clemens

Edward Dible

David Gibbs

Thomas Gordon

Philip Grafton

Danny Kloha

Gilbert McClanahan

Timothy Moore

Chod Moyer

David Norris

James Pawelski

Silas Pearson

Jonathan Popo

James Rushe

Cary Underwood

Troy Van Liere

Timothy Willms

Mark Zearfaus

Kelly Belt

Stephen Billingsley

Glen Bowman

Tim Fischer

Benjamin Garrison

Todd Huguenin

Daniel Kain

Timothy Lethbridge

Scott Marsh

Cory Neumann

Gregory O'Dell

Fred Rinehart

Steven Seymour

Andrew Snider

Mark Wolf

Jonathan Wright

David Burk

Wes Cunningham

Robert Donawa

Mark George

Raymond Gesualdo

Kenneth Horton

Todd Hummel

Jay Inman

Mark James

Robert Koch

Patrick Mackenzie

Russell Norman

Christion Pilet 


\section{FAITH HALL}

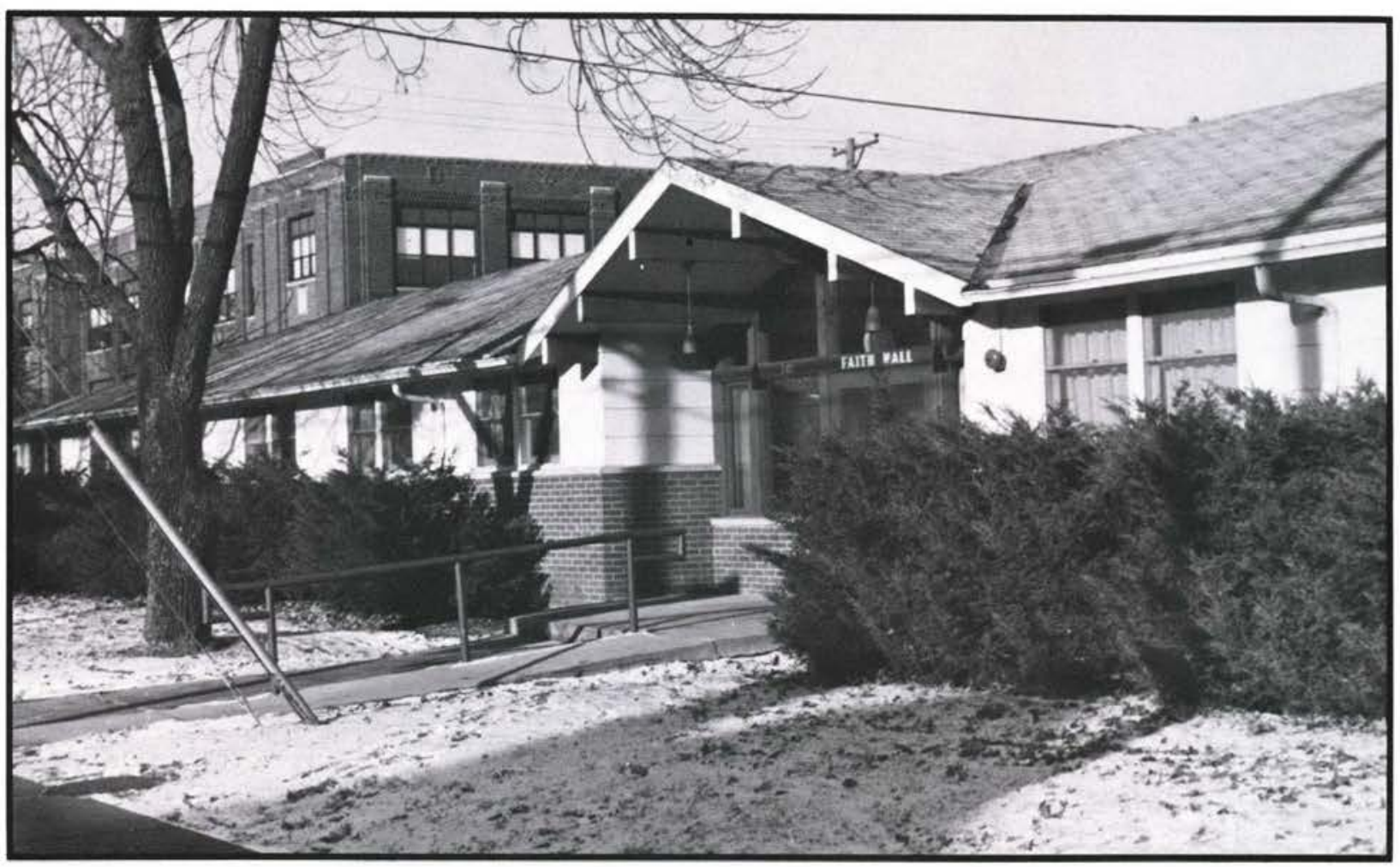

The administration expressed the faith that Cedarville College was built upon by naming this women's dormitory Fairh Hall. The original was purchased from Wright Parterson and moved here in '58. In ' 65 , New Fairh was completed. Faith is the oldest women's dorm and is recognized for having the best pastures for sun bathers and for its lingering atric-like odor. Freshmen usually reside in Faith, bur a few adventure-seekers join up for a second year.

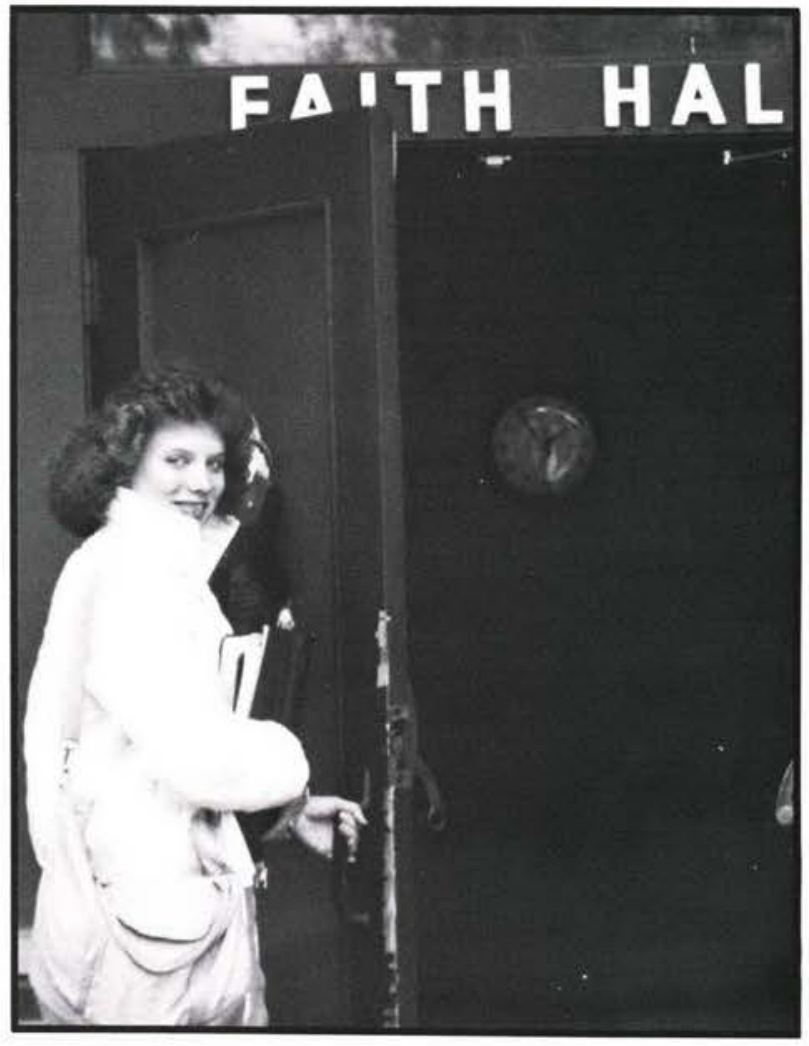




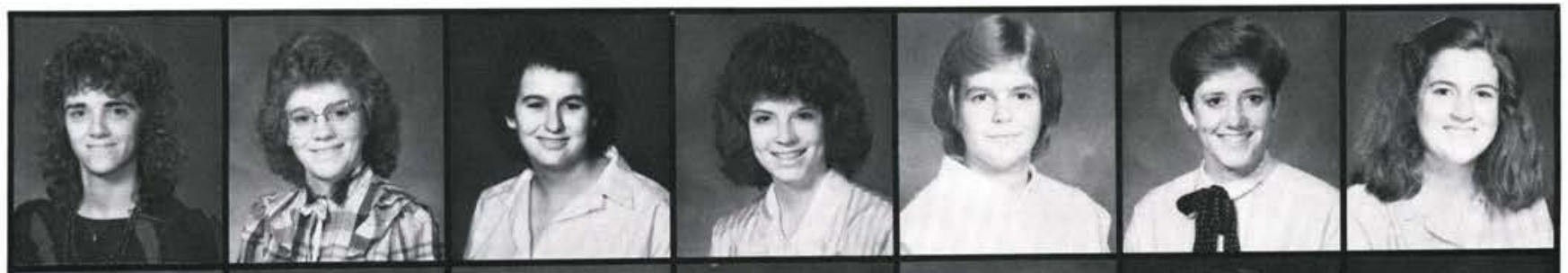

Karen Acker

Wendy Adkins

Rachael Angst

Kim Barch

Denise Bass

Sarah Bassett

Dawn Beach

Jacki Becker

Joan Beltz

Angela Beschler

Mara Blackburn

Linda Blakely

Deborah Borleis

Diane Borleis

\section{Shelia Brown}

Lisa Bryant

Julie Burkholder

Kim Byrom

Janice Caldwell

Michelle Carlyss

Teresa Carter

Cindy Cleaver

Torina Collick

Margaret Collier

Sally Compton

Elizabeth Conger

Pamela Conrad

Tracy Conrad

Terri Cooper

Carol Crowley

Sue Ann Cunningham

Beth Dowson

Carol Delaney

Theresa Dunlap

Kimberly Duzen

Susan Dye

Nancy Eibel

Josie Erwin

Debbie Fenton

Cheryl Fletcher

Darcey Floyd

Deborah Fogle

Christine Friesen

Shannon Fritz

Lisa Garabadian

Sherri Gerdes

Kimberley Gilmour

Melissa Graham

Cynthio Guido

Kristen Harper

Nova Harris

Jenny Havens

Kathleen Hawley

Theresa Henry

Sandra Hitchcock

Sandra Holbrook

Deborah Howard

Cynthia Husband

Kathleen Kenney

Deborah Kettel

Beth Keyes

Valerie Kinsey 
Elicia Kleinpeter

Shelly Lemponen

Kimberly Loekhart

Charlene Lowe

Buffie Mackereth

Julie Maiers

Melisso Martin

Syndy McCafferty

Michelle McGarry

Amanda Mclntyre

Bonnie Miller

Julie Miller

Wendy Miller

Angelia Monnet

Rachel Morgref

Ruth Murtoff

Tammie Nasse

Crystal Patrick

Heidi Petros

Karina Quinn

Lynn Ramsey

Christine Renberg

Chris Rober

Jennifer Rogers

Lisa Rogge

Cathy Rupp

S. Dian Russell

Rebecca Sadler

Sally Jo Sager

Lois Schofield

Deborah Schwartz

Lynette Sebright

Lila Seest

Susan Seibert

Sharon Senay

Beth Ann Smith

Dawn Smith

Fayanne Smith

Ferrol Synder

Amy Springman

Julie Stonehouse

Eliza Stowe

Susan Swann

Jennifer Taft

Linda Tharp

Eileen Thompson

Joanne Thompson

Debra Times

Carol Tinkler

Sharon Tinkler

Julie Titus

Sarah Triehy

Karen Van Dommelen

Elizabeth Vroman

Karen Watkins

Penny Watkins

Melissa Way

Tracy Wemett

Janet Wenger

Julie Williams

Teresa Wilson

Cathie Worthley

Kara Yant
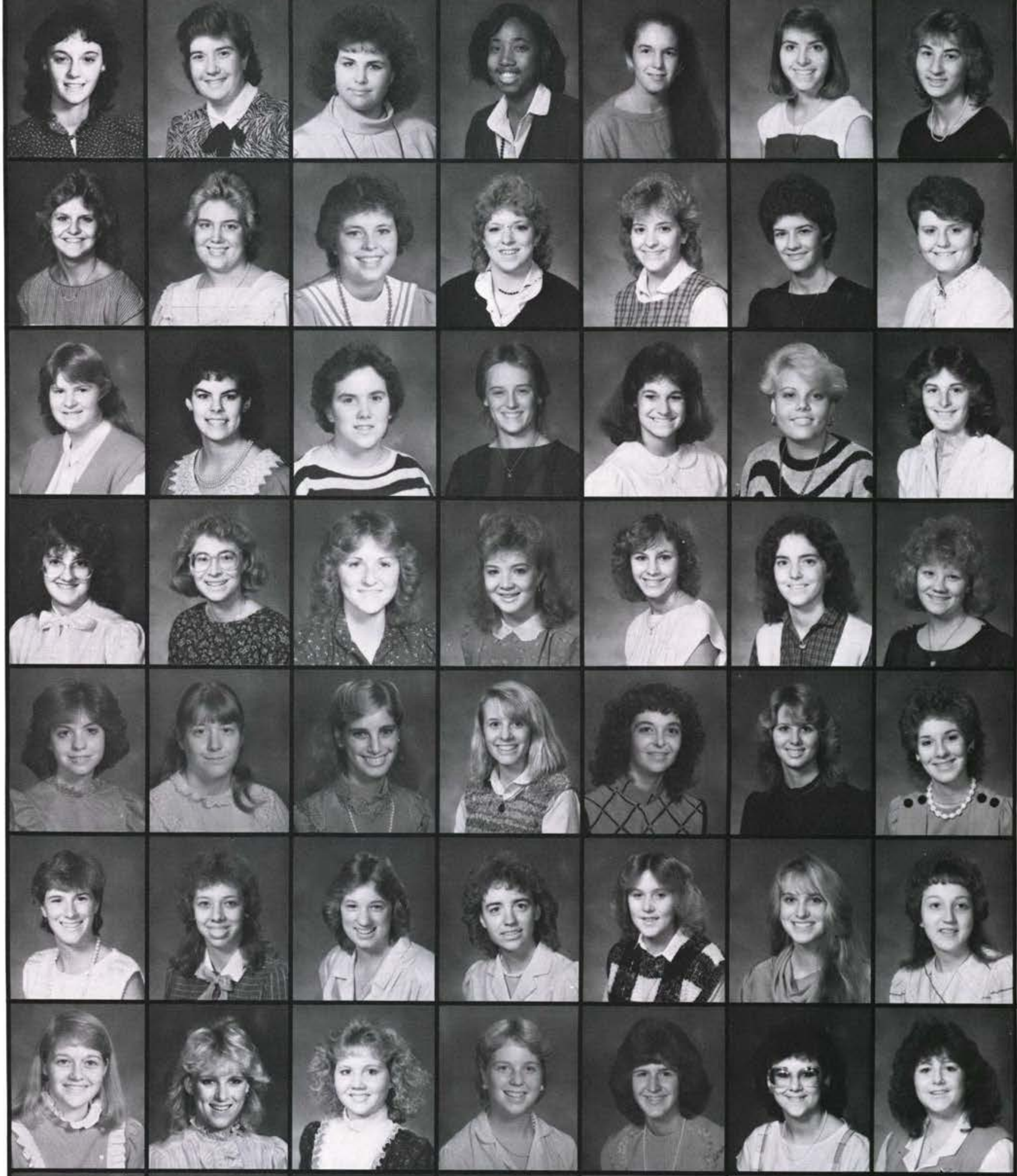

20
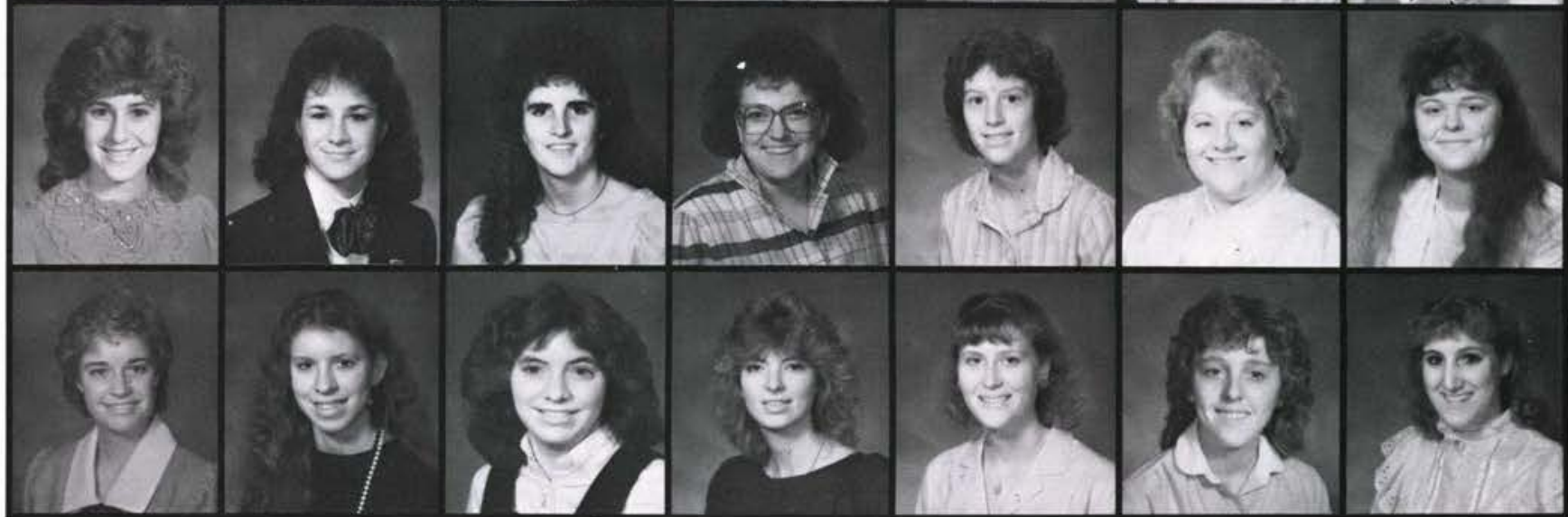


\section{MADDOX HALL}
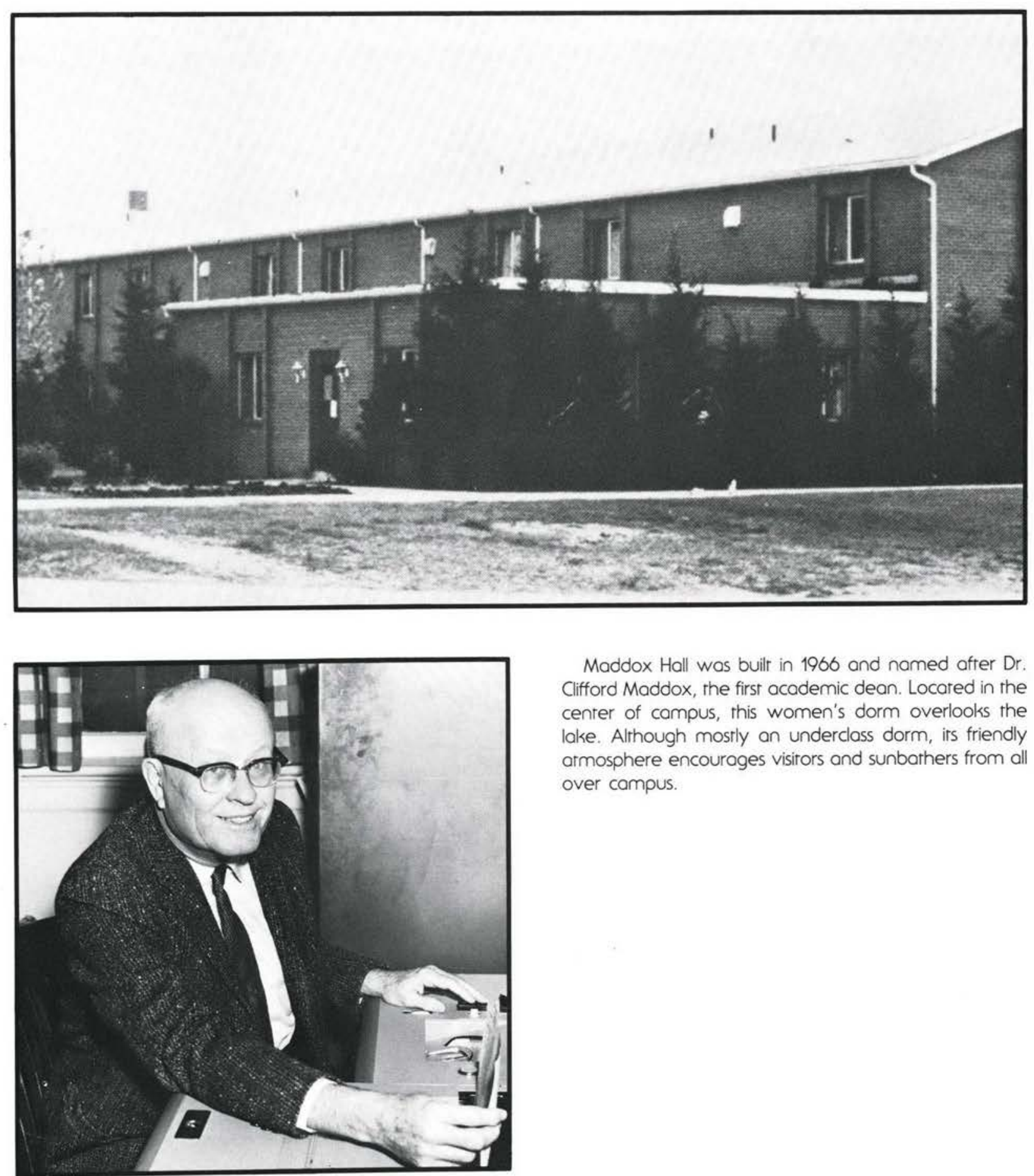

Maddox Hall was built in 1966 and named after Dr. Clifford Maddox, the first academic dean. Locared in the center of campus, this women's dorm overlooks the lake. Although mostly an underclass dorm, irs friendly armosphere encourages visitors and sunbarhers from all over campus.

\section{Dr. Clifford Maddox}

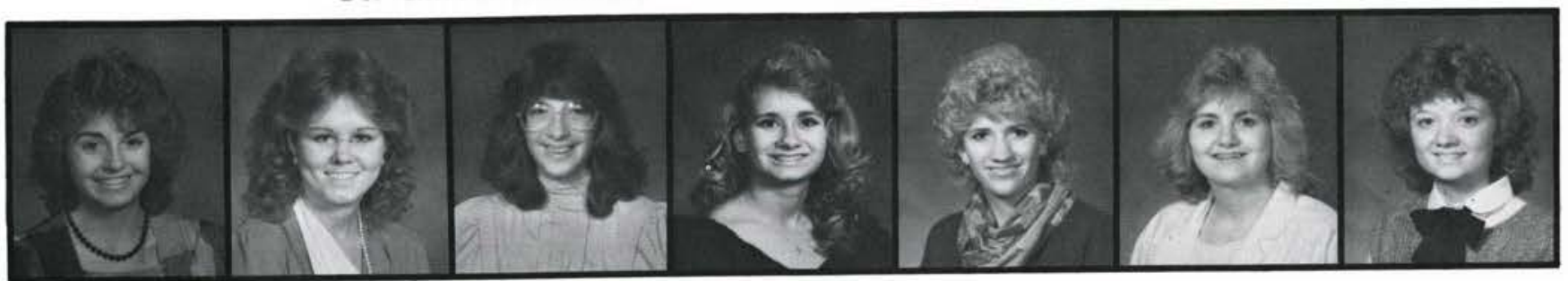

Ronda Altman Amy Armstrong Patricia Armstrong

Teresa Bailey

Beth Baker

Kimberly Baker

LeAnn Barenscheer 
Kathy Barnes Dawn Beachum Katie Beoman

Lori Beasecker

Lisa Bengtson

Lisa Benim

Rachel Benitz

Lisa Bergen Melissa Berner Andrea Berry Melissa Bethel Tolitha Bosworth Mary Bowman Beth Breederland

Mindy Brocious Lori Brown Tonya Bumpus Jody Burtis

Karla Butler Callie Ann Campbell Christine Carpenter

Julie Carter Cari Chamberlin Amy Chandler Samantha Chandler

Patricia Cherry Brenda Clare Crystal Clarke

Karen Clifford Connie Cline Renee Clor Elizabeth Constable Laurie Cooper Brooke Cunningham Carla Curry

Lori Daniels Lisa Davidson Stacey Davis Melisso Denenberg

Angela Dennis

Tamera Detrick Pamela Dillan

Beth Downey Christine Drew Edith Dulost Cathy Dye Ann Dykes Kimberly Ebner Jody Eckert

Michaelle Egel Jeanie Erneston Jennifer Everage

Jennifer Fink

Beverly Fisher Heather Fowler Teresa Francisco

Joann Freyermuth Tami Funderburg Patricia Garcia Brenda Gard Jennifer Geer Carol Geese Sandra Gilbert
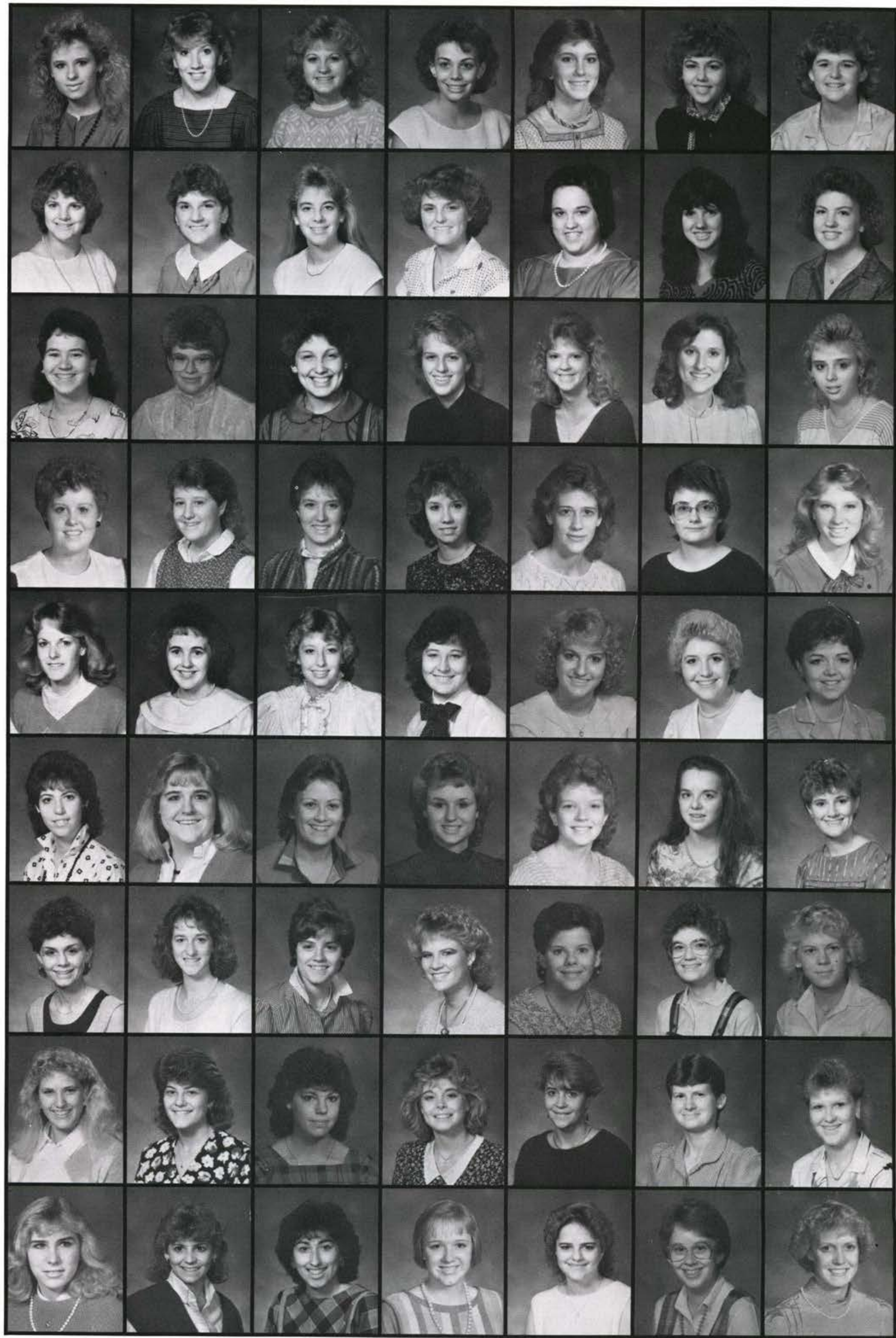

Sandra Gibert 


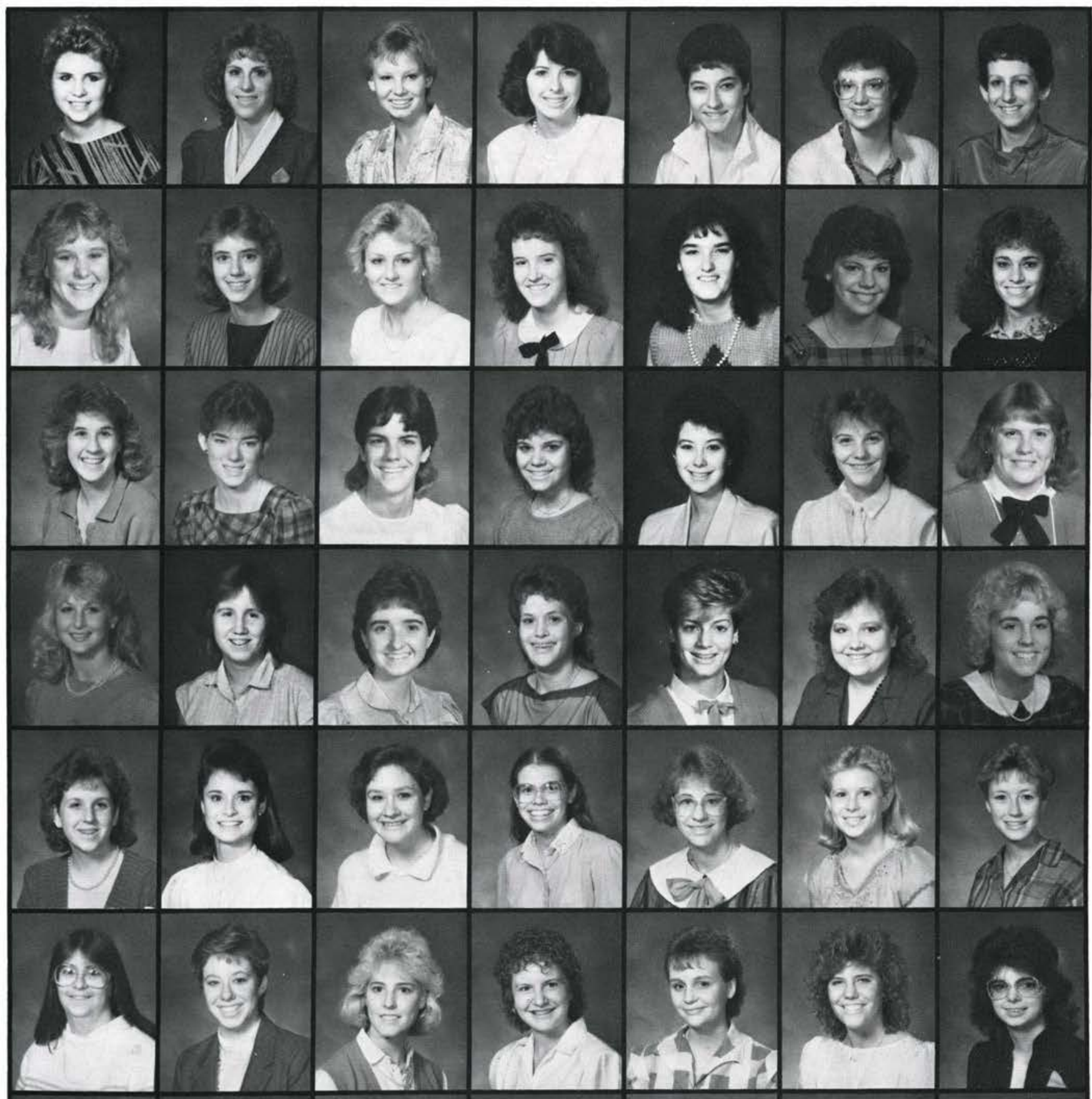

Melissa Glatz Jaime Gonzalez

une Graham

L. Jean Green

Julia Gregory

Sherry Grosch

Beth Grose

Laura Hacker

Sheryh Hambley

Gaye Harden

Nancy Hardin

Jeri Hastman

Karen Haynes

Kathy Haynes

Sarah Hiedorn

Ronda Heise

Kendra Herroid

Paula Hines

Michelle Hinnergard

Derricka Hockensmith

Gretchen Hoffmeyer

Cheryl Holland

Brenda Hollopeter

Cynthia Hoover

Dawn Howard

Paula Howard

Valerie Howard

Rebecca Howell

Angela Huested

Beth Ann Hughes

Kimberlyl Hughes

Esther Hunt

Jody Hutchinson

Sally Inbody

Tami Jeffries

Bonnie Johnson

Kathleen Jones

Nancy Keisacker

Sharon Kerr

Vicky King

Donno Klimkowski

Virginio Knisley

Kristine Kuhns

Christina Lankford

Ramona Larsen

Beth Anne Latoello

Janet Lawrence

Patricio Le Croix

Tina Lethco

Kathleen Low

Laura Maiers

Kristen Marshall

Cynthia Matonti

Janelle Maxwell

Melisso Mee

Karen Meloche

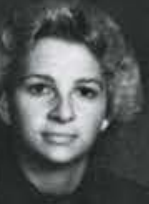

$-2$

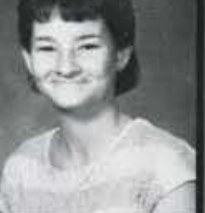

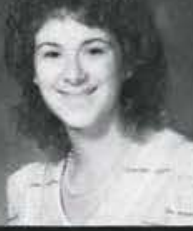
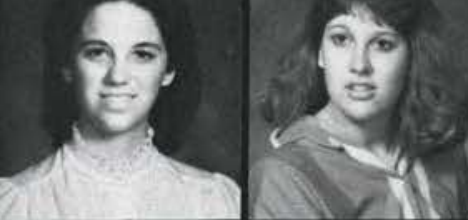

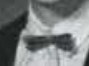

Jolene Merck

Alice Merkh

Nancy Merkh

Poula Merrill

Ann Miller

Annette Mitchell 
Kimberly Mitchell

Heather Moody

Melynda Moody

Michele Moody

Elizabeth Moon

Pomelo Moon

Rebecca Morgan

Bethany Most

Sheila Murdoch

Stephanie Murray

Sue Nosh

Michelle Nester

Ann Nicholson

Leah Nunn

Faith Deloppo

Michele Opitz

Susette Orth

Cheryl Ortloff

Tracy Osborn

Tina Parkinson

Alicia Parnel

Holly Patterson Barbara Paxson Rebecca Pearson

Josette Pena

Christine Penny

Lynne Pitonyak

Katrina Plourde

Gina Poppenhagen Jill Posey

Ann Powers

Michelle Prince

LeiLani Pritts

Lisa Rains

Julie Ream

Vivion Reebel Krista Reed Sharon Reifsnyder

Patricia Rice

Rebecca Richardson

Megan Riley

Tekerro Rinehort

Mary Ritenour Amy Robenolt Deborah Robinson

Teresa Robinson

Susan Rogers

Debra Rotramel

Cheryl Ruble

Melissa Runion

Amy Russel

Deborah Russell

Robin Sanders

Dorothy Schierwage

Jennifer Schritz

Jill Scowcroft
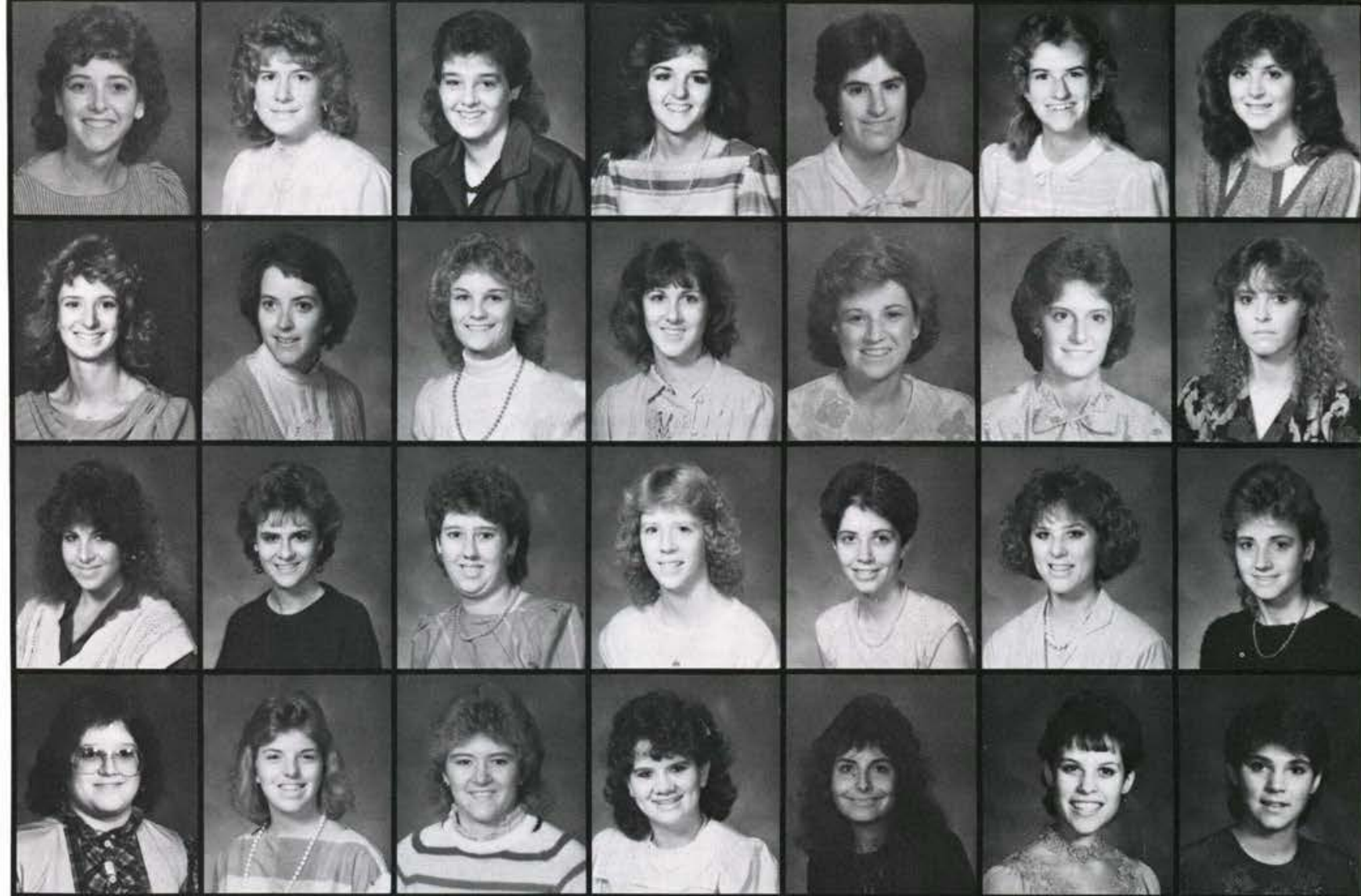

ชู
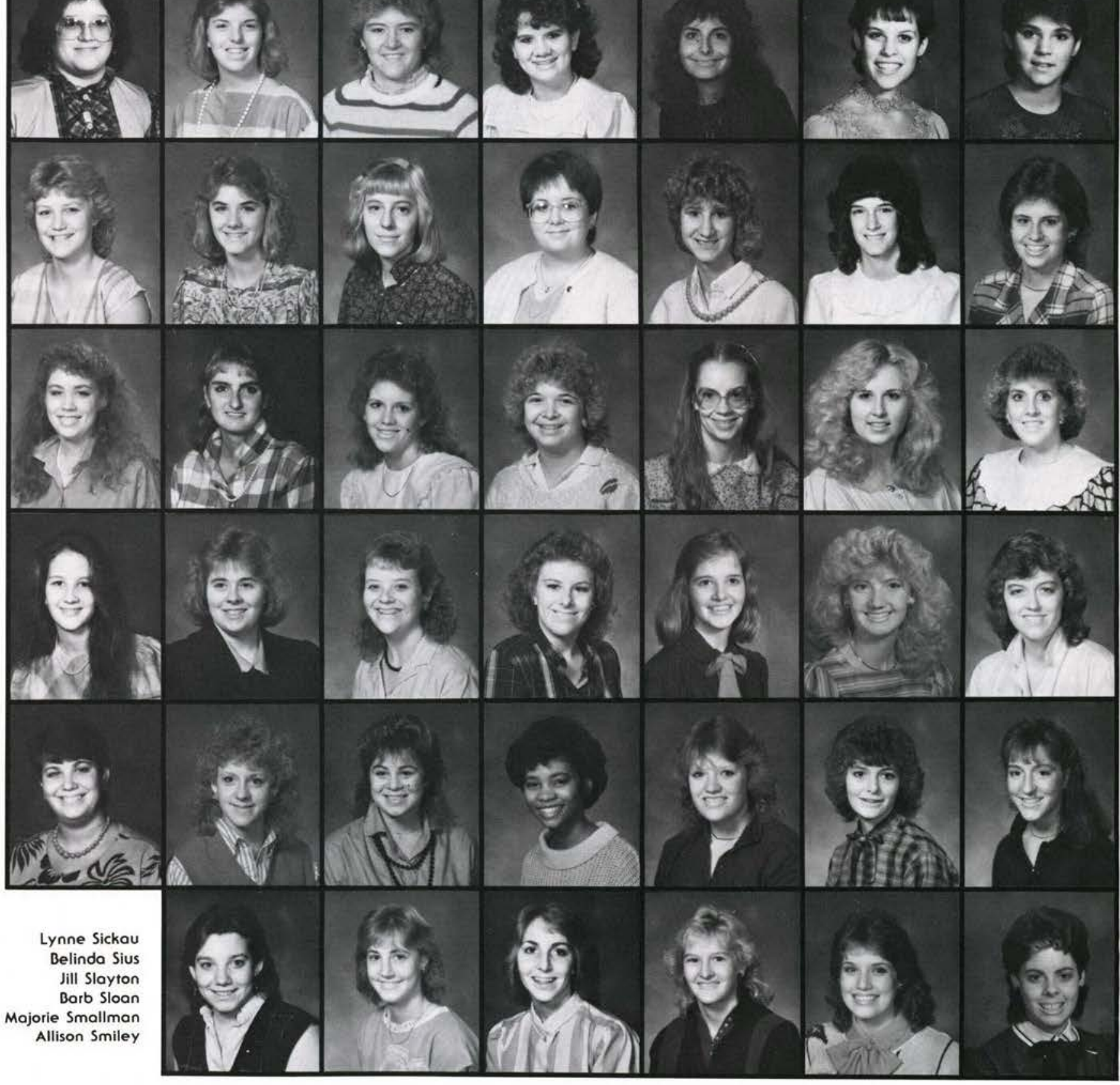


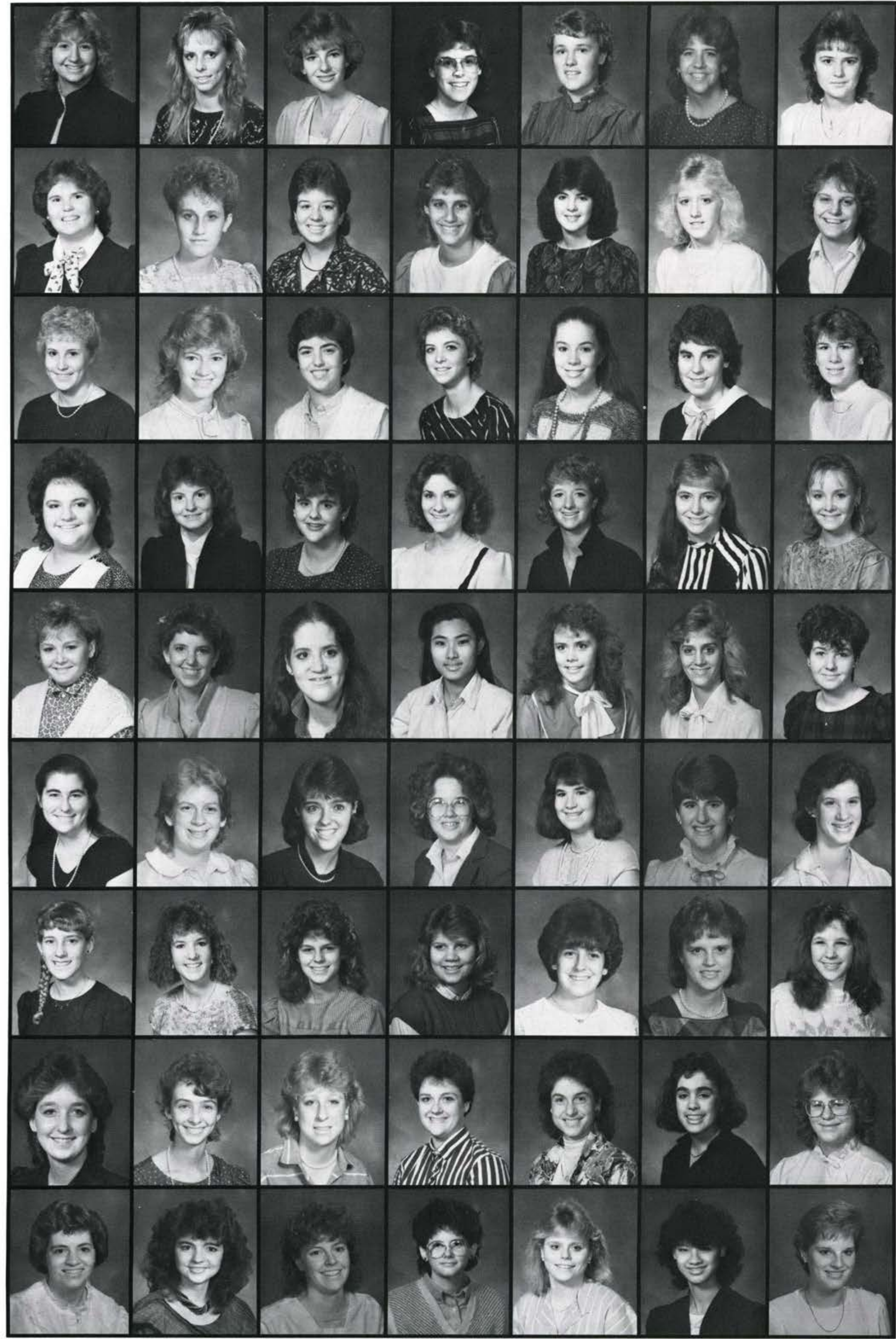

Debbie Smith

Rebecco Lynn Smith

Rebecca Lynn Smith

Ronda Smith

Michelle Snook

Bonnie Synder

Stephanie Solomon

Janet Stauffer

Damaris Stevens

Kimberly Stilwell

Tamara Storm

Jody Stough

Elaine Stroup

Ann Sulek

Joelle Swartz

Kimberly Swee

Stefanie Swift

Lydia Tanner

Greta Tasker

Brenda Taylor

Melinda Taylor

Tami Taylor

Dorothy Thomas

Sora Tilla

Jamelle Toler

Laura Tuttle

Christo Umbough

Karen Valentine

Kelly Valentine

Sharon Van Dommelen Margaret Vanderberg Julie Waddell

Rebecca Wakefield

Christine Walbom

Andrea Wallock

Joy Warman

Deborah Warnshuis

Kristine Watson

Brenda Weber

Kimberly Weidne

Dana Weld

Barbara Wells

Katharine Welner

Janelle Warnli

Brenda West

Wendy Widder

Margaret Wildman

Molly Williams

Susan Williams

Jennifer Williamson

Linda Willis

Jennifer Wilson

Jodi Wilson

Julie Wilson

Melissa Wilson

Laro Wilt

Melisso Windish

Angela Wright

Shawna Wright

Karen Young

Tracy Young

Ruth Yuen

Dawn Zeigler 


\section{ROGERS HALL}

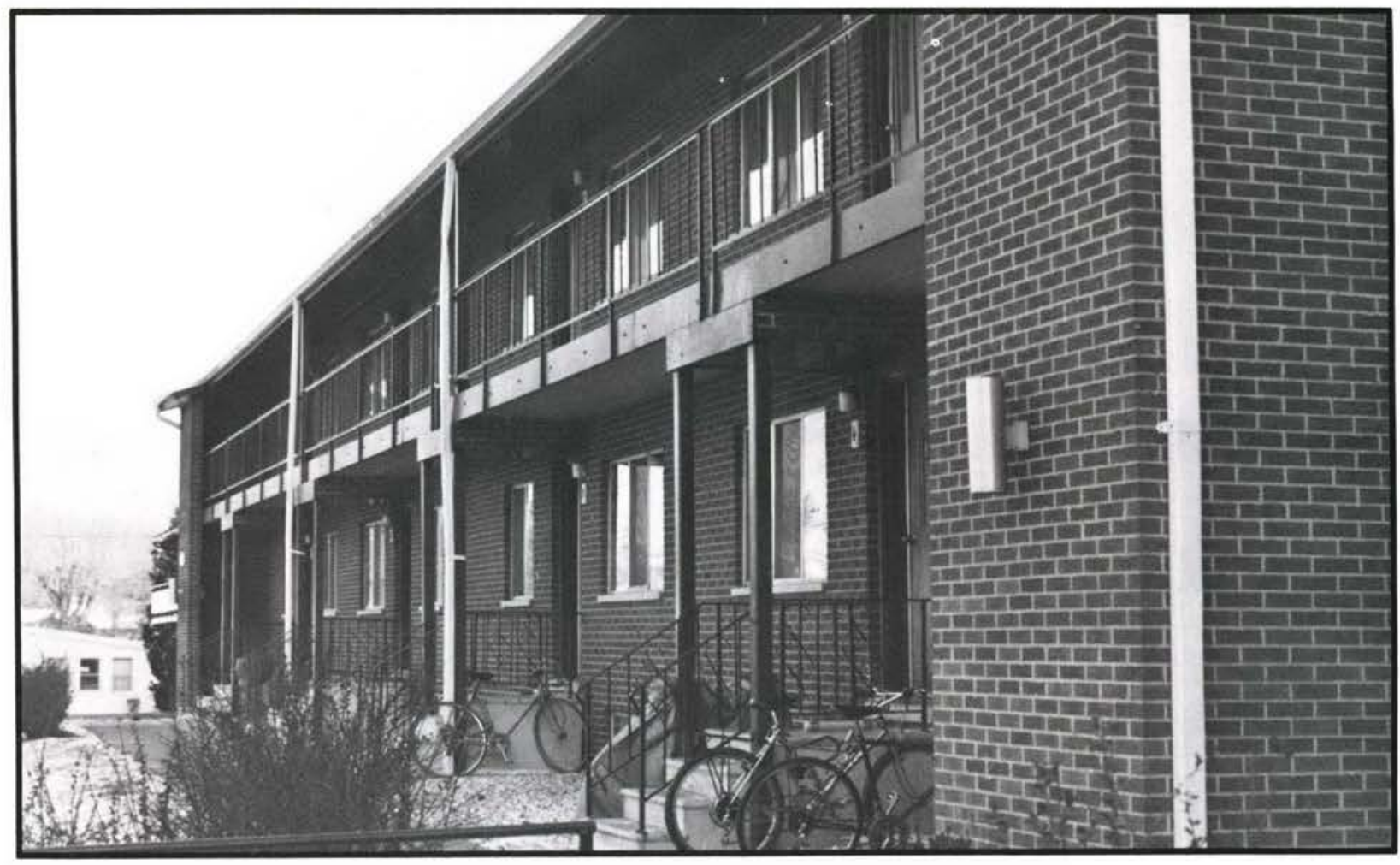

The Hill Home of the "rowdies," the Hill was built in 1974. It is composed of three units; Rogers, named after alumnus Bob Rogers; Marshall, after Gerald "Pop" Marshall in mainrenance; and Carr, after Hugh Carr who was also from Mainrenance.

Someone's always pulling a prank on the Hill, wherher it be warer fights, shaving cream underneath the door, stealing the mourhpiece our of a phone, or purting 500 pounds of snow in front of the RA's door.

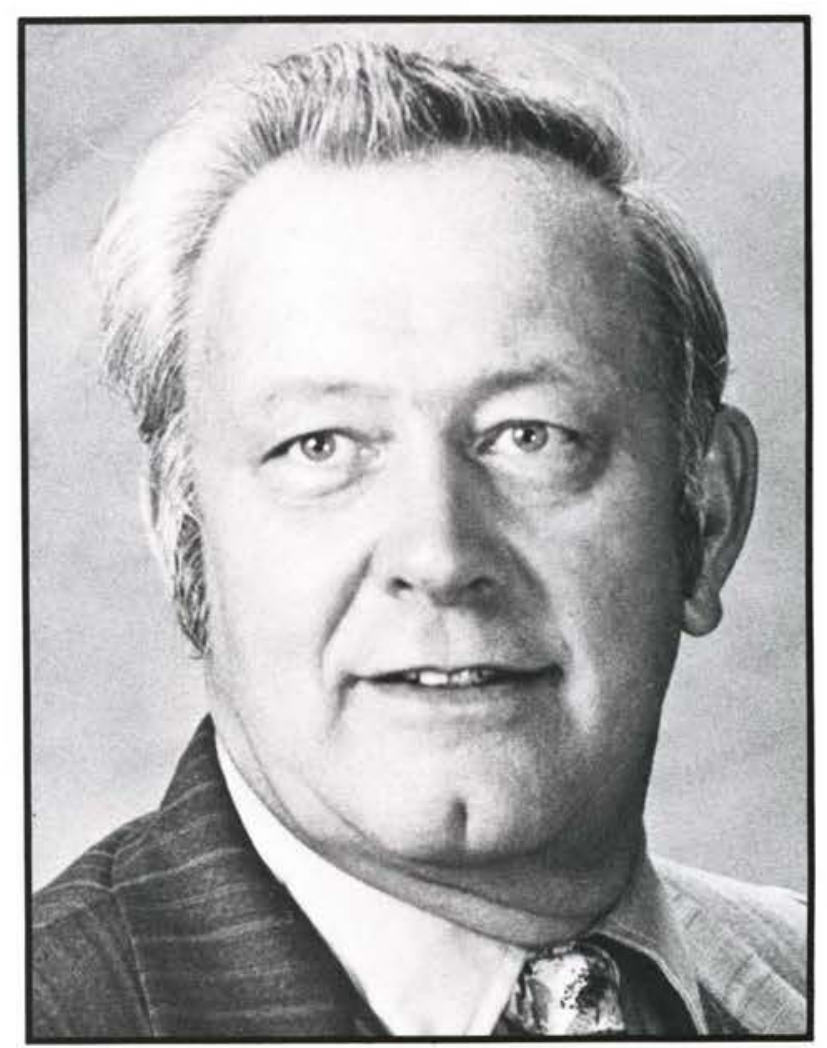

Robert Rogers 


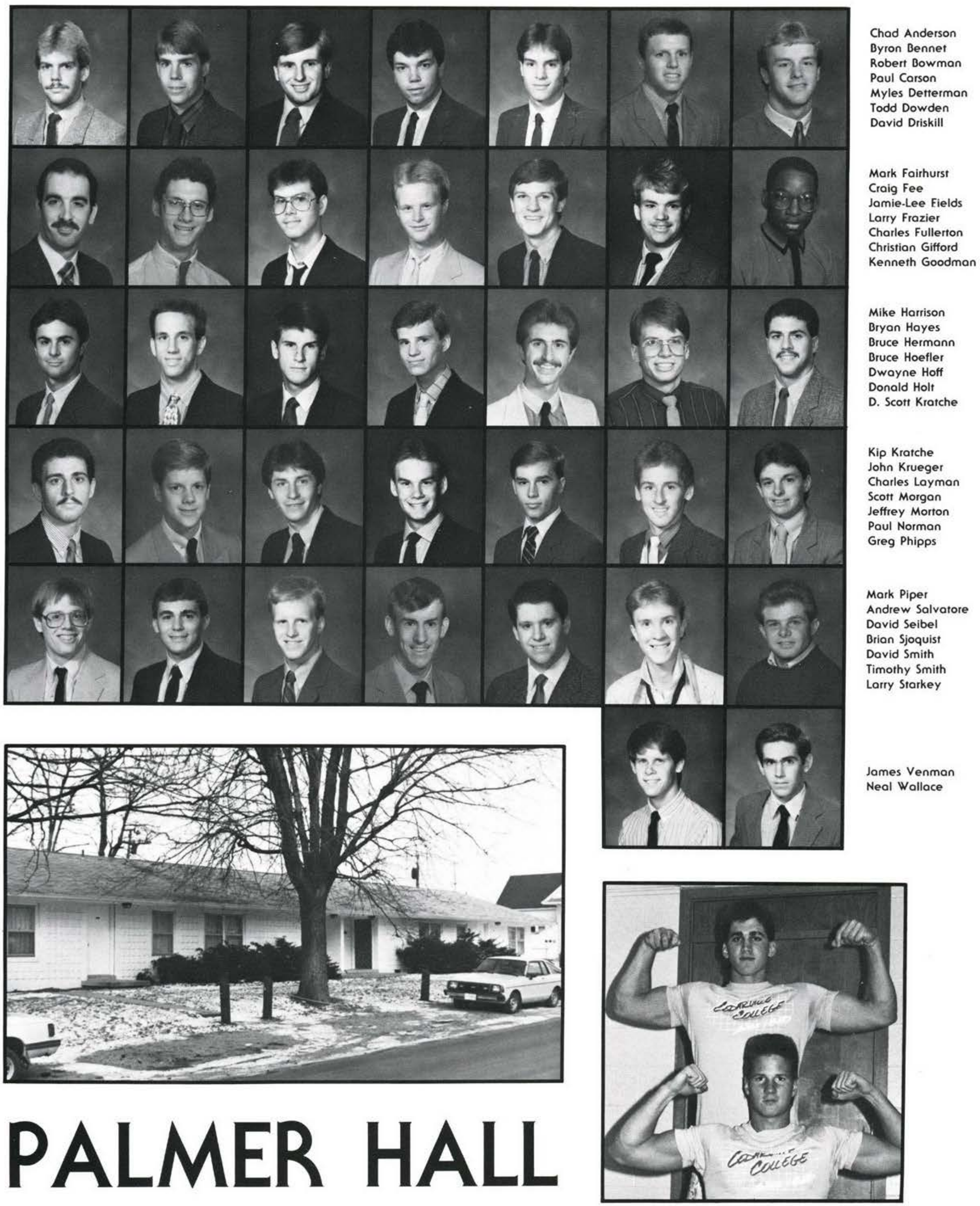




\section{CARR HALL}
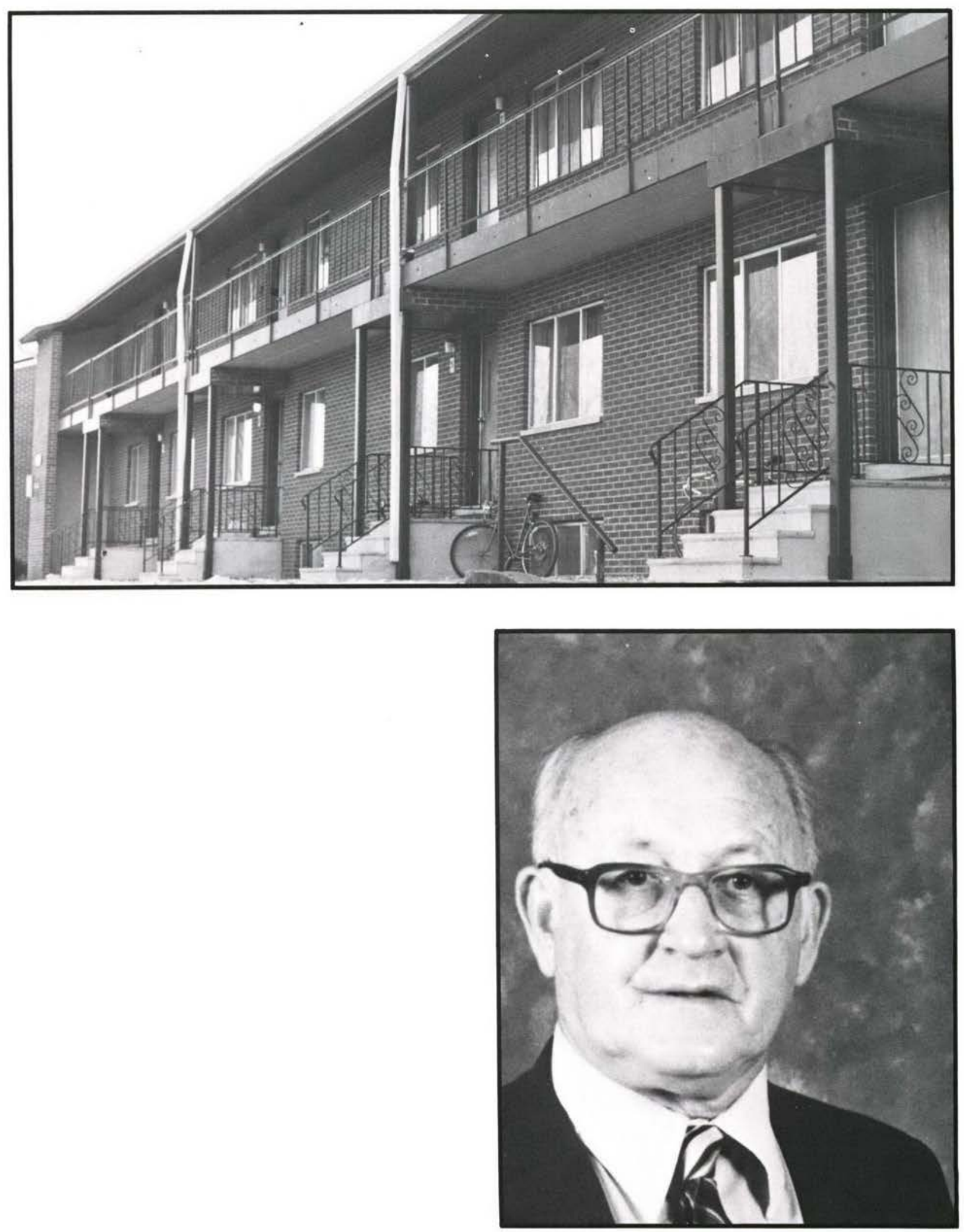

Hugh Carr 


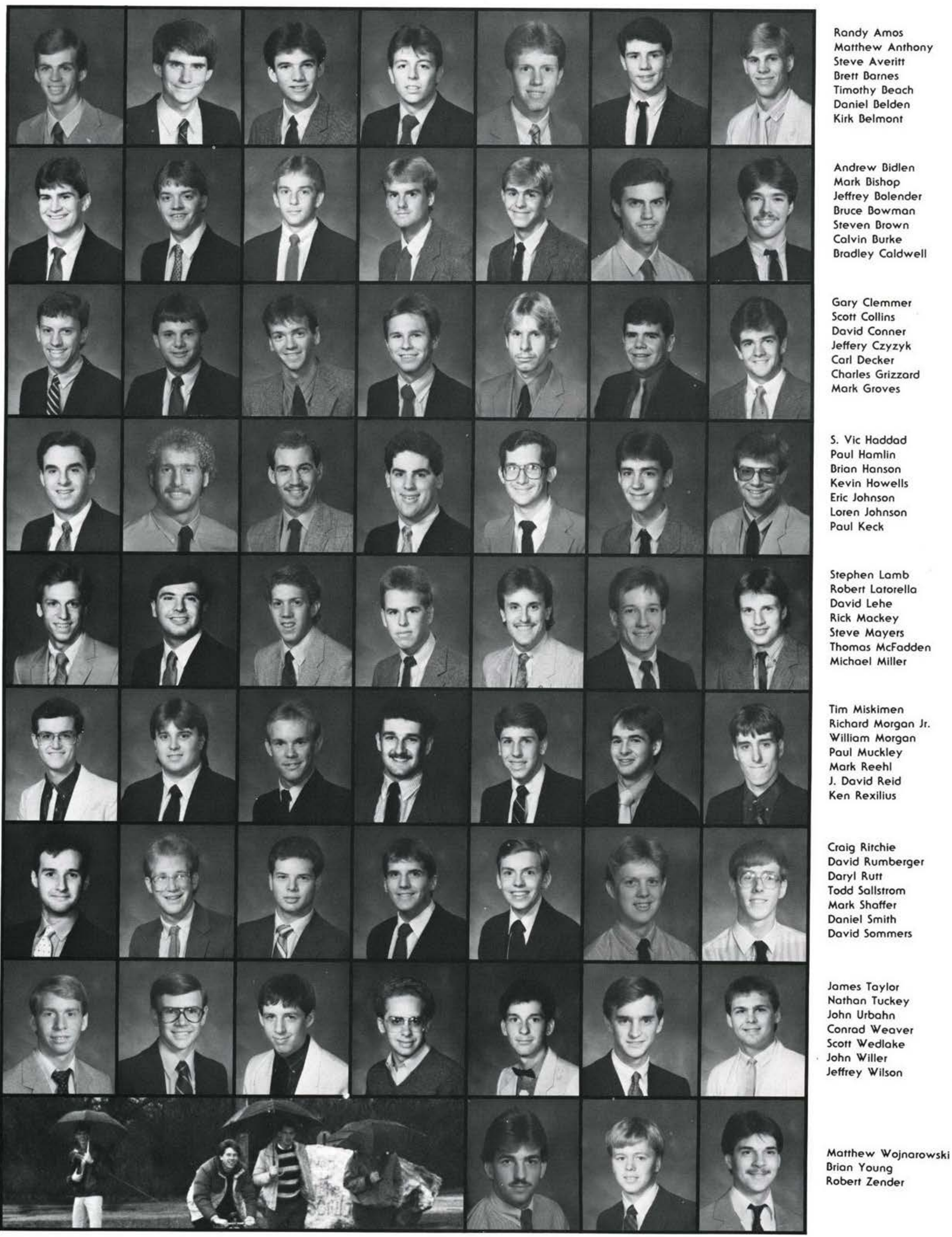




\section{MARSHALL HALL}
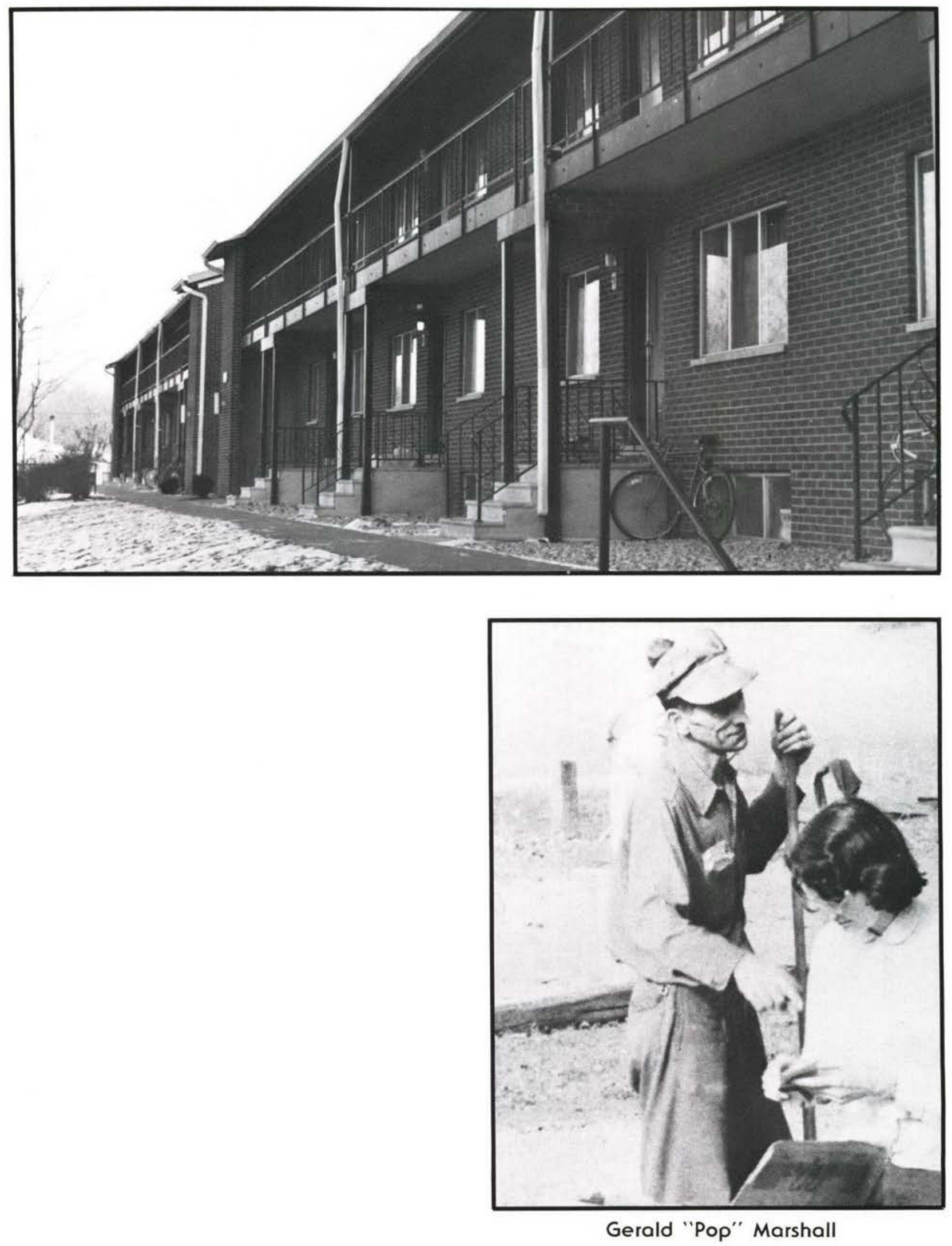


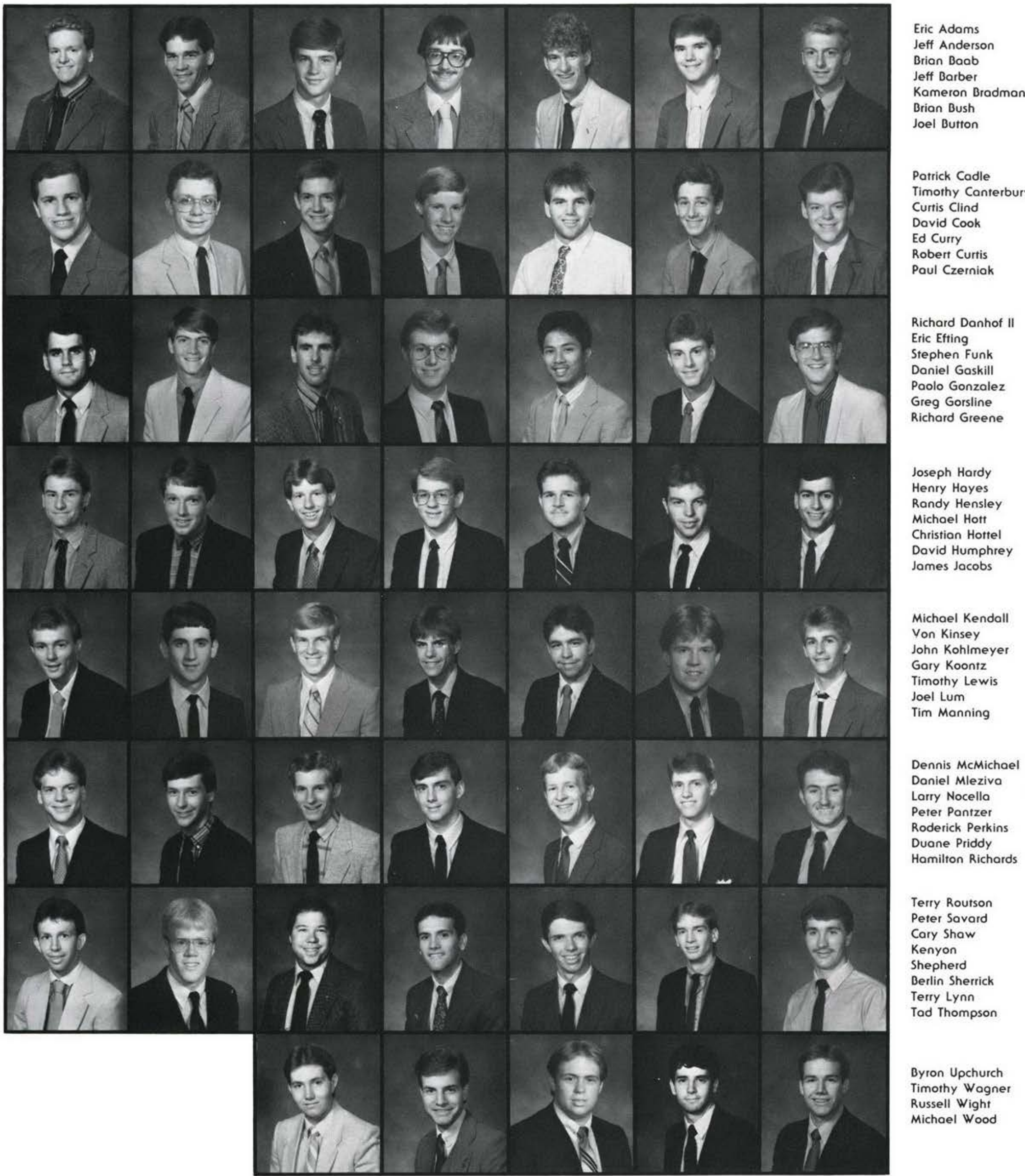




\section{LAWLOR HALL}

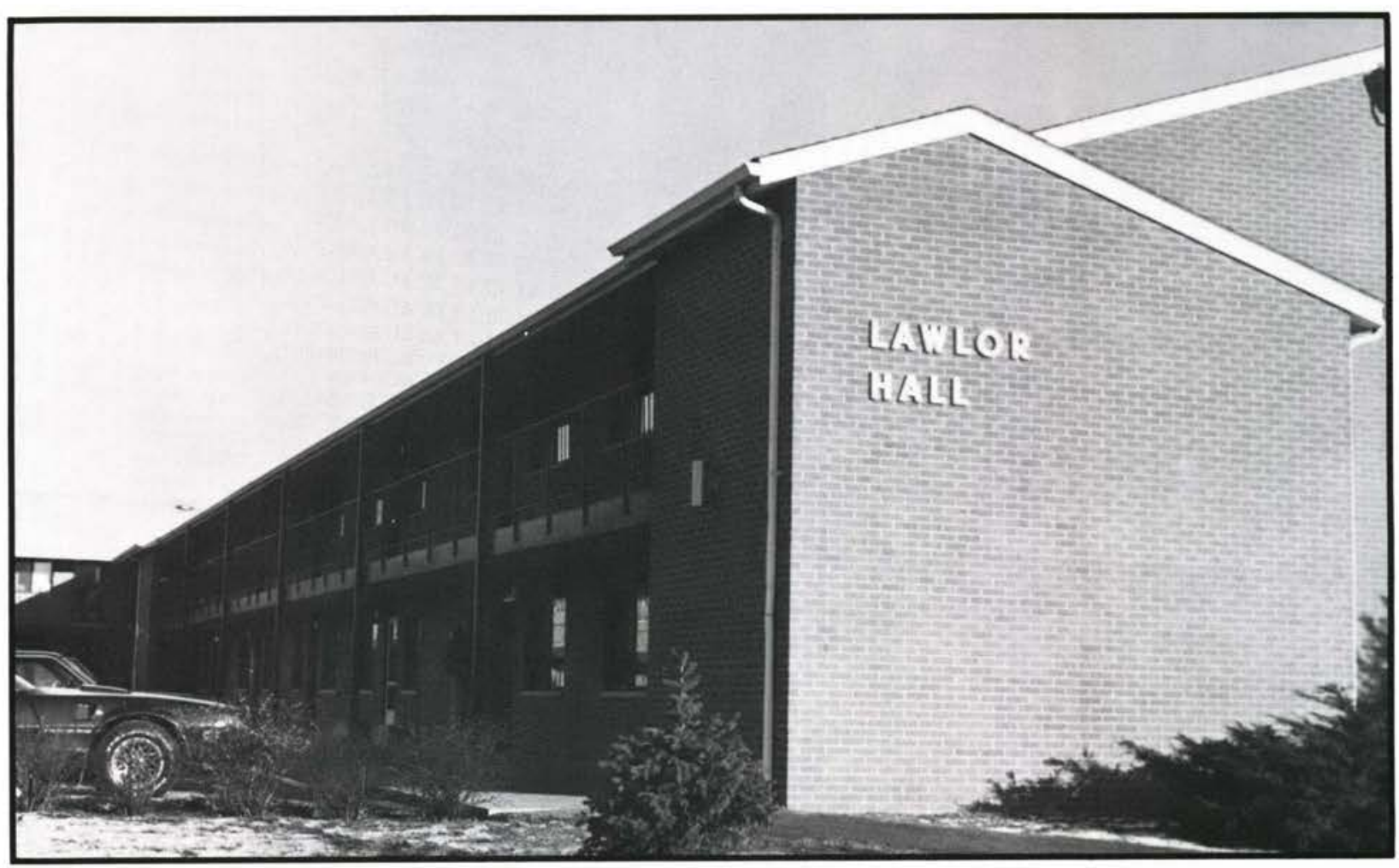

Lawlor Hall, founded in 1978, was named after Dr. George Lawlor, professor of Greek. It is a dangerous dorm, especially for the newly engaged Lawlor man who is thrown into the murky, slime-filled lake, where he is immediarely congratulated. Occasional "buck-bucks," now illegal but secretly practiced underground, and water fights are also traditional practices. One can't forget the midnight snow climbing in December when guys dived into mountains of snow from their windows.

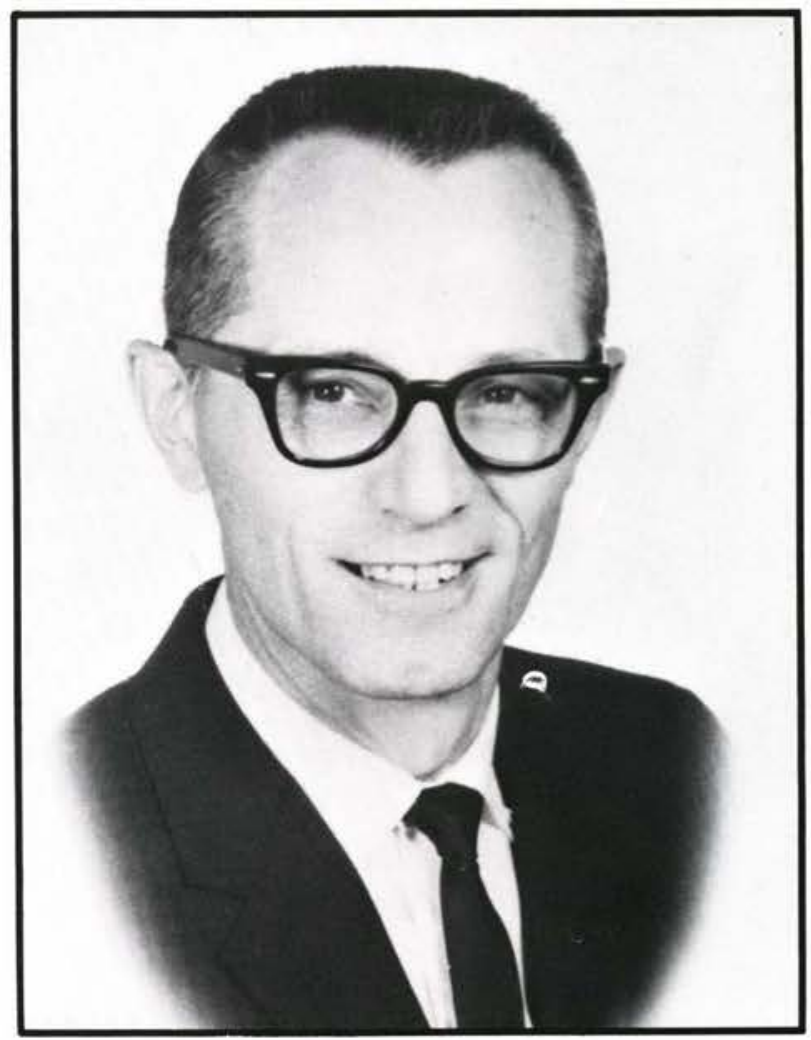

Dr. George Lawlor 
nothy Abramowitz David Allen

Michael Anderson

Stephen Anderson

John Angus

Stephen Apple

Scott Auwarter

Michoel Ayres Joel Barke

Kerry Baumgartner

Scott Beattie

John Bercaw

Chris Bernard

Nathan Bobbett

Scott Boye

Brad Bresson

Jeffery Brown

Mark Brown

Steve Brumbaugh

Brent Budd

Daniel Byrum

Victoir Cahoon

Kelly Callen

Joel Campbell

Glenn Carpenter

Kevin Carpenter

Phillip Chapman

Stanford Charlton

Joel Christensen

Robert Coffman

Timothy Coons

Marc Coope

James Costas

Luis Cruz

Philip Cruz

Doug Cunningham Deron Davis Michael Davis

Nathan Davis

Scott Deetz

Jim Demusz

David Dennis

Wilfred Deshetsky Paul Dillon

David Doud

Donald Drozd

Todd Dunckel

Stephen Elkins

Evan English

Tim Entner

Daniel Erlandson

Richard Ernst

Quentin Eshleman

Brian Ethridge

James Eutsey

Gary Fernandez

Eric Fillinger Douglas Filter Stephen Fisher Kenneth Fleetwood Andrew Ford

Roger Foreman Gregory Frank

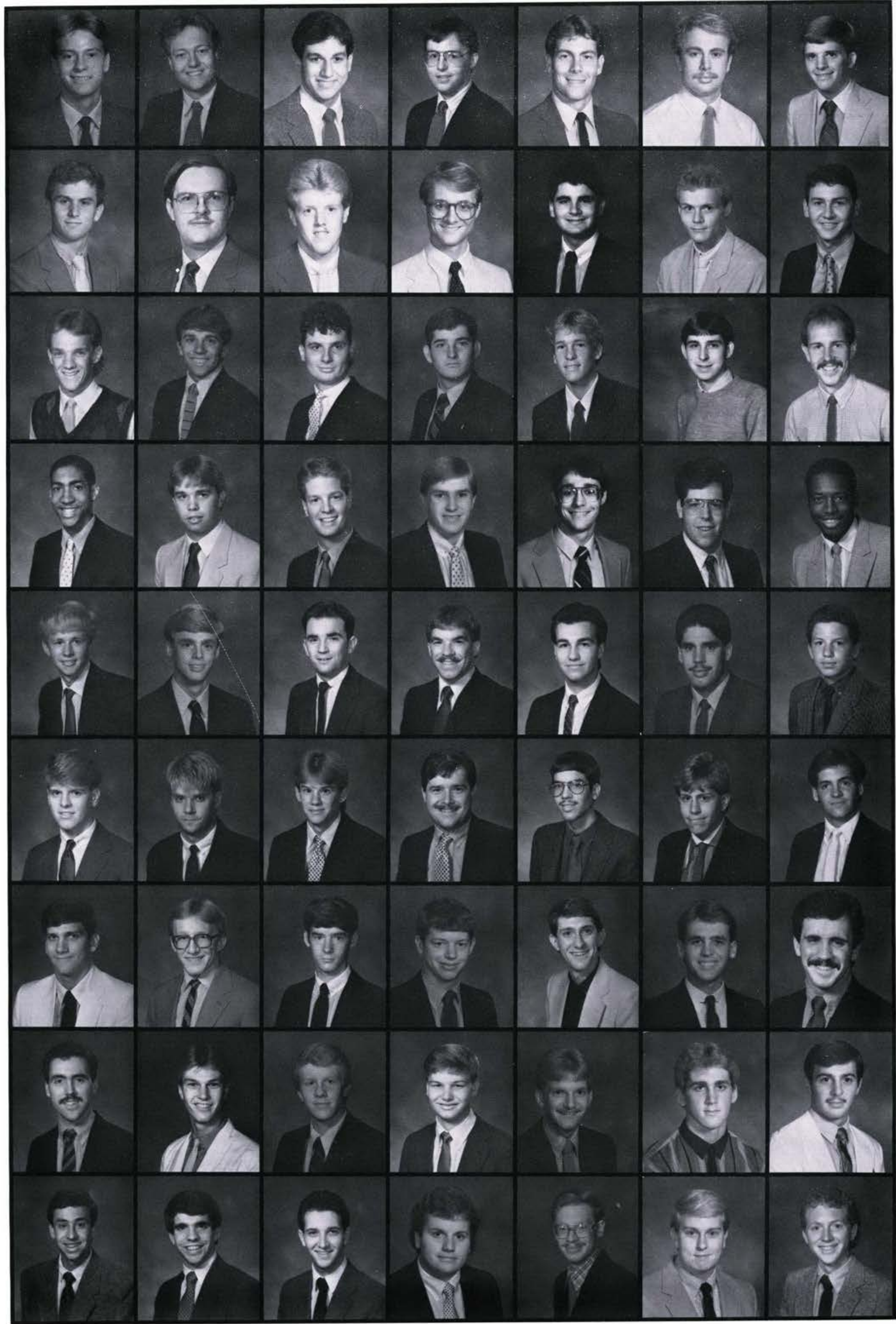




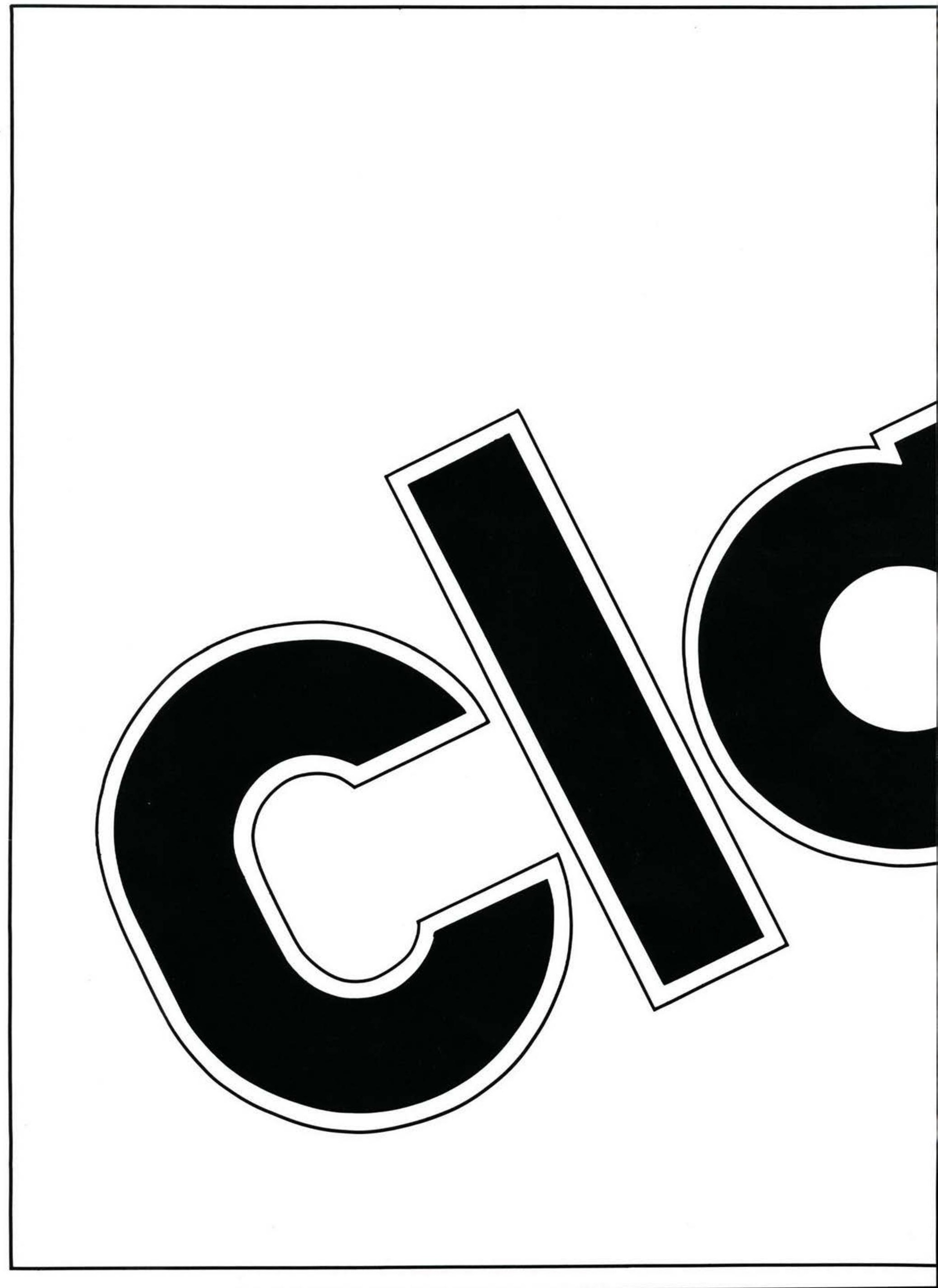




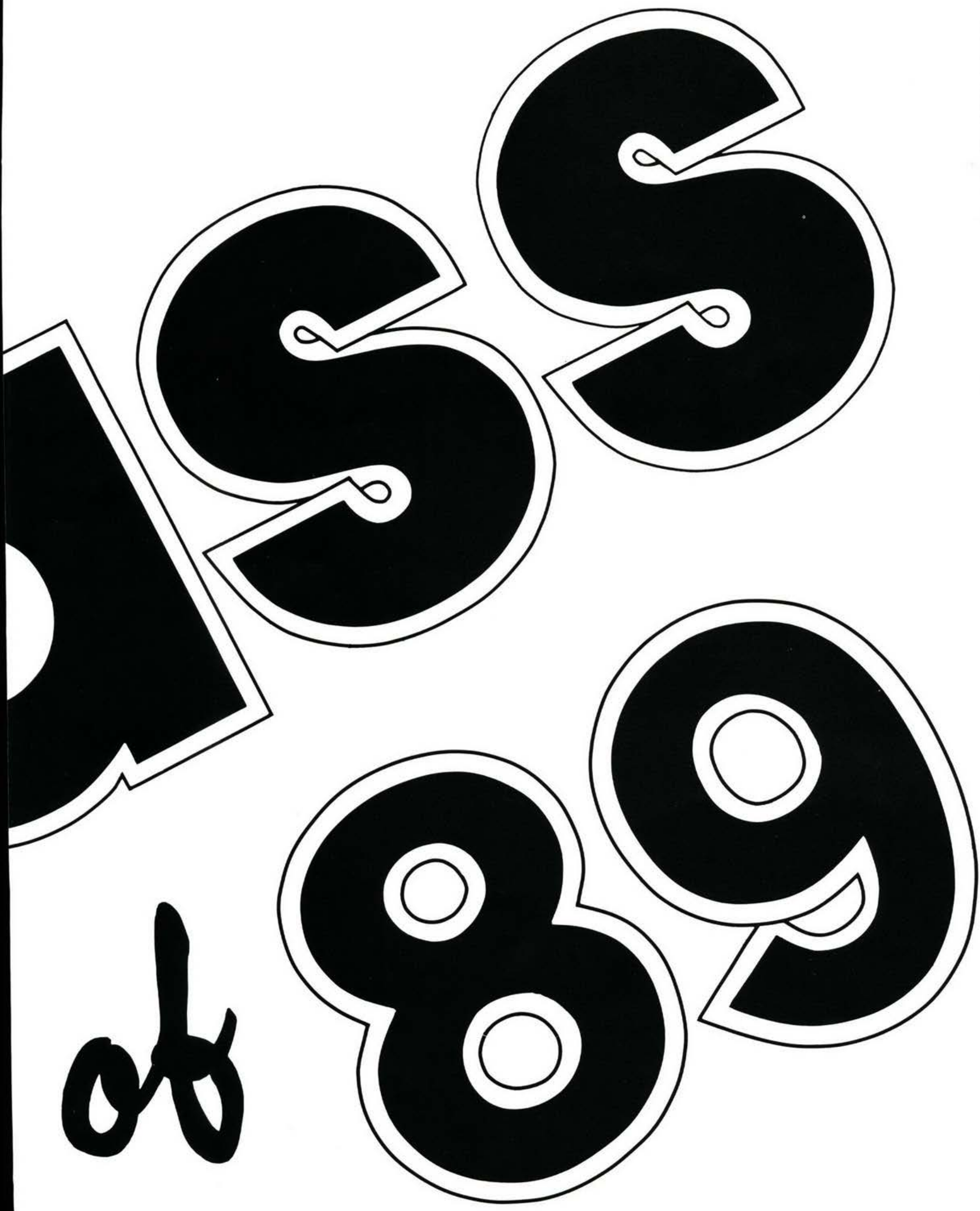




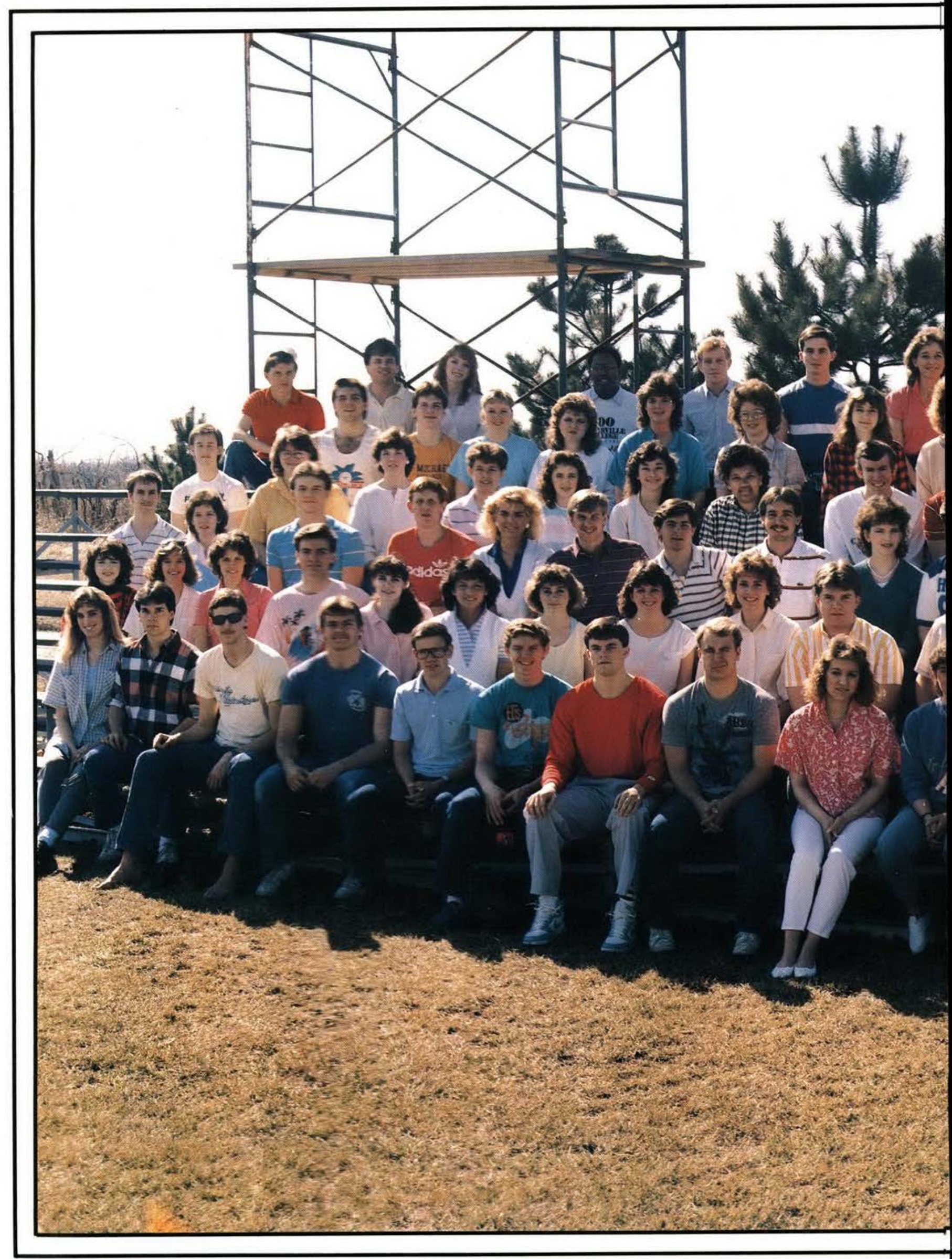




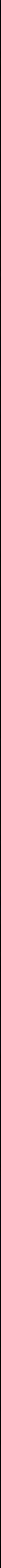


toly

Mrofow, a

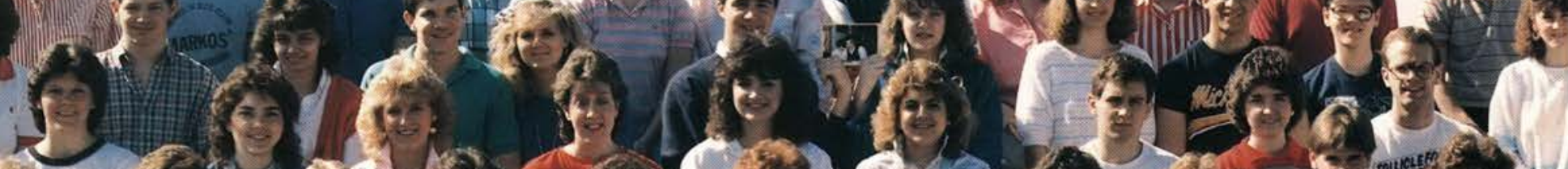

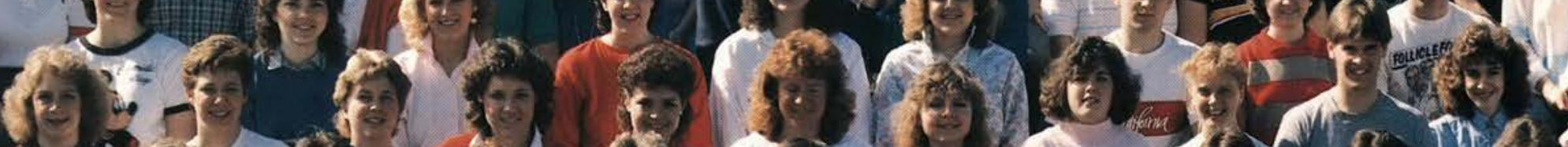

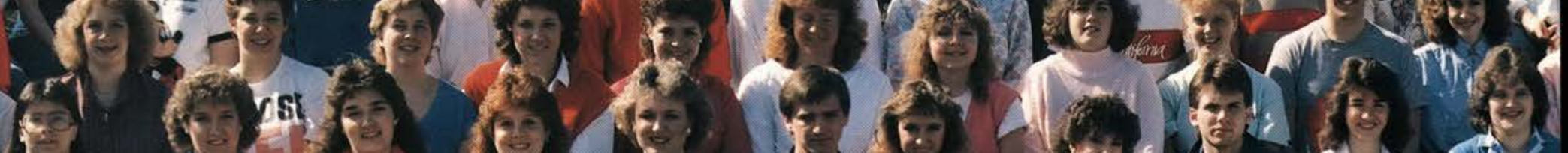
$\int_{-1}^{5}$ 


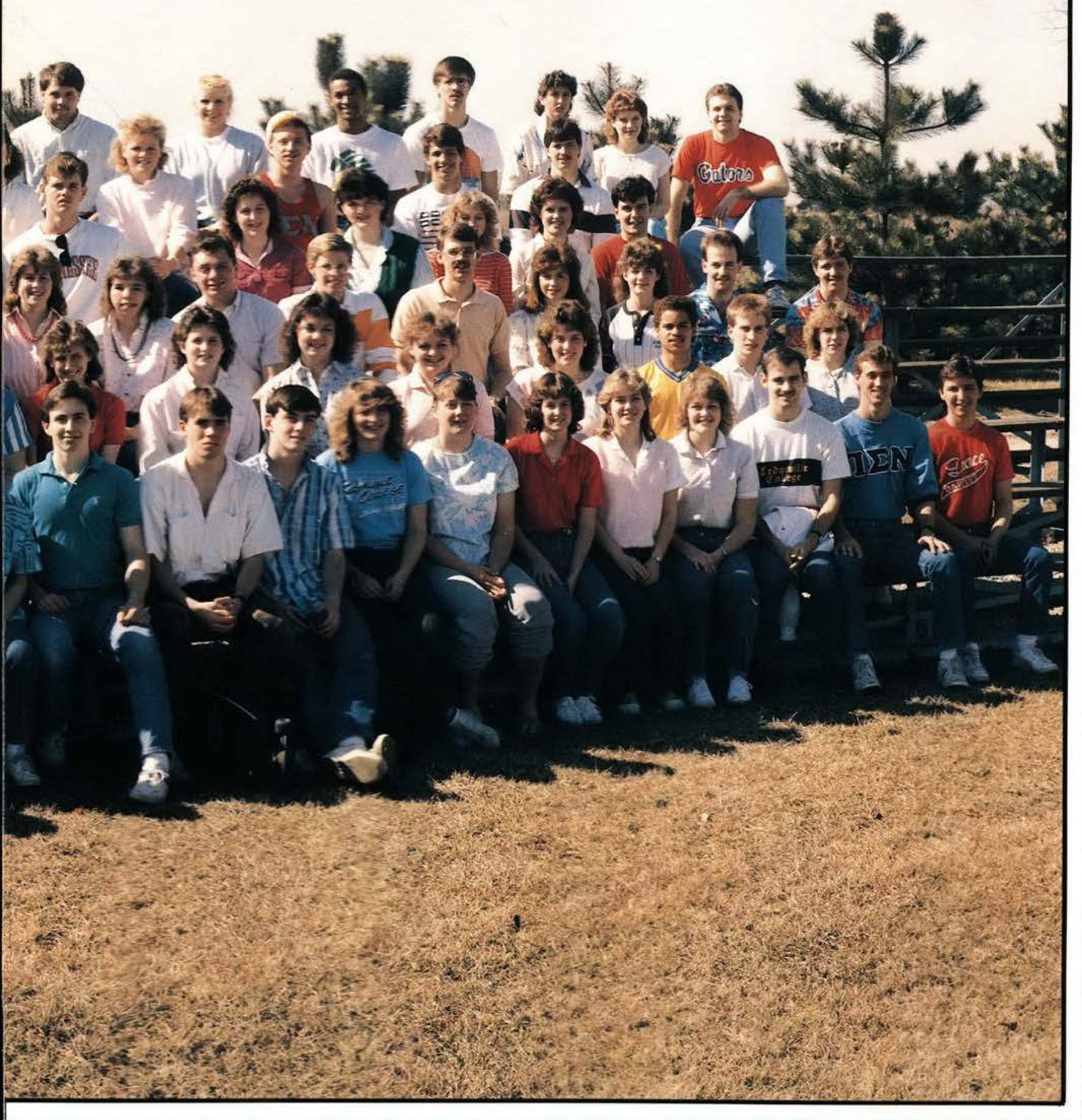


Michael Freeman Dean Frey

Todd Gathany

Stephen Gerhardt

George Gibbs

Gregory Gibbs

Daniel Gonzalez

Brian Gorman

Micirael Green

Bradley Gregory

Robert Gresch

Lowell Halbach

Keith Hammer

Shaun Hannay

Joe Hanssen

Mark Harden

Rodman Haseltine

Jonathan Haskell

Marvin Howk

Raiceon Hawkins

Joe Hayden

Ronald Hayes

Timothy Haynes

David Hays

Eric Hempel

Shawn Hess

Robert Hicks

Robert Hildebrand

Bobby Hile

Michael Hillenburg

Gary Hoog

Greg Hobaugh

Dovid Hoecke

Van Halloway

David Holt

Bradley Hood

Derek Hughes

Brian Hultz

Brian Humphreys

limothy Jeunnette

Dennis Johnson

Robert Joiner

Daniel Jones

Jamie Jordan

Gerald Keller

John Kelley

Kerry Kendrick

Darrin King

Robert Kinney
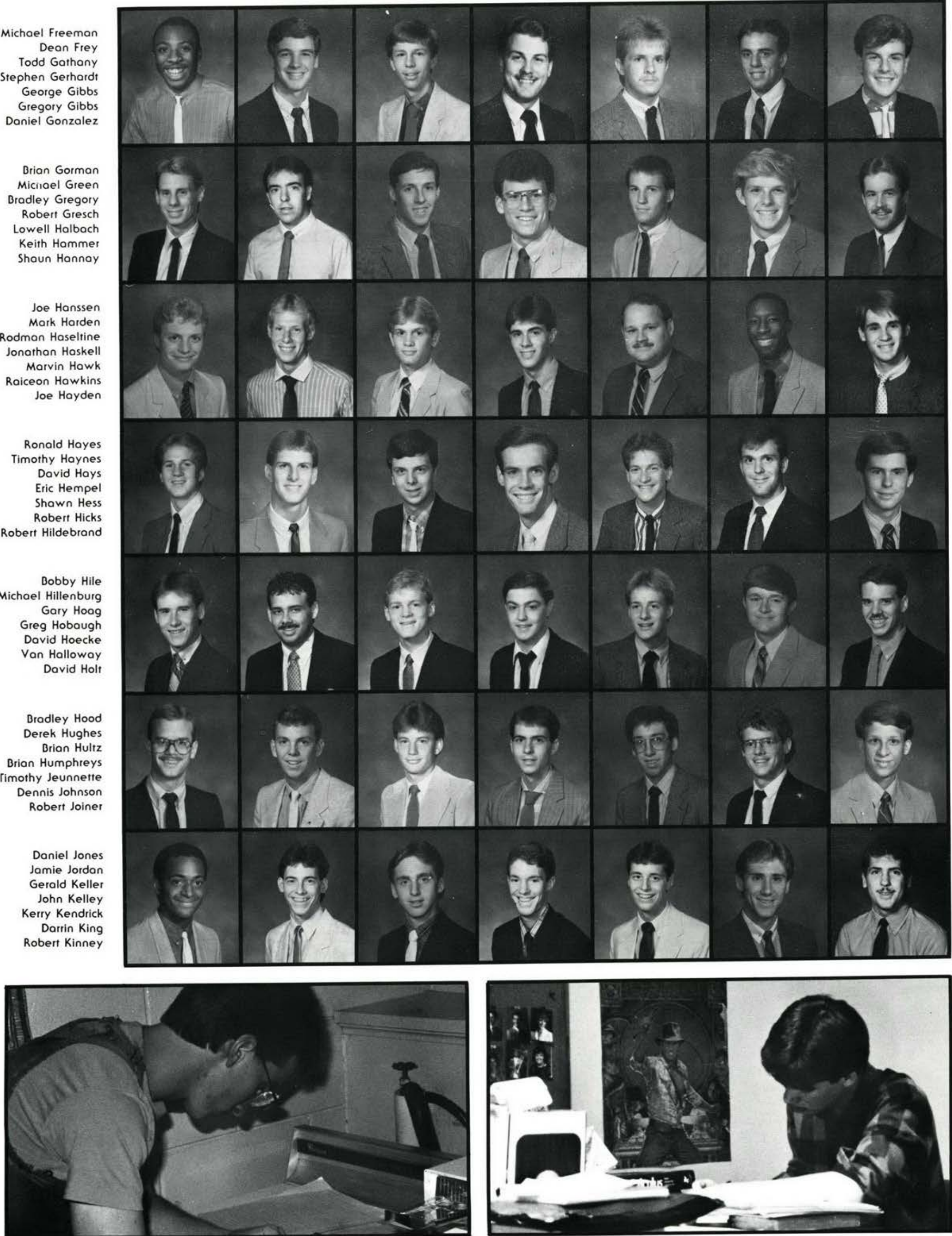

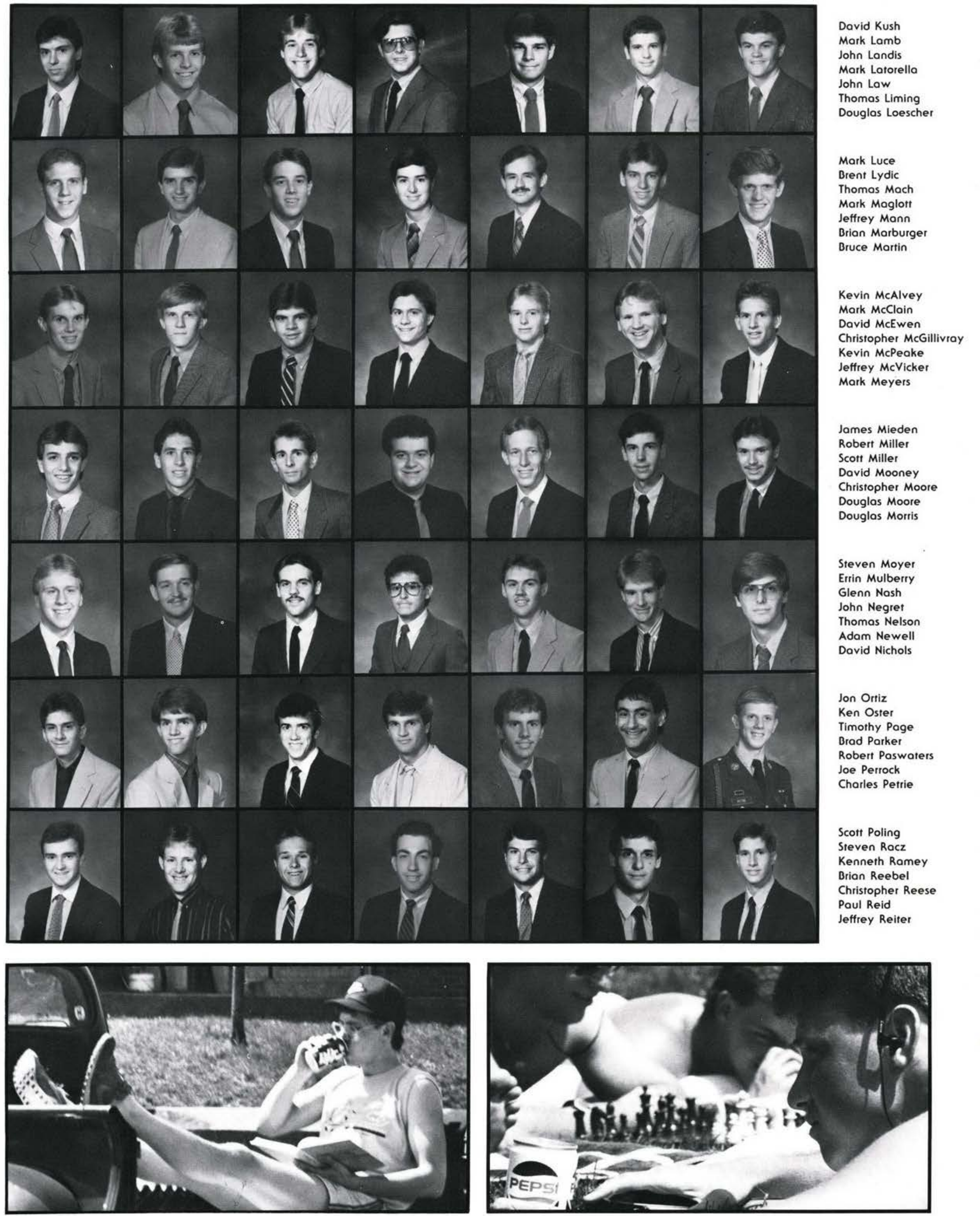


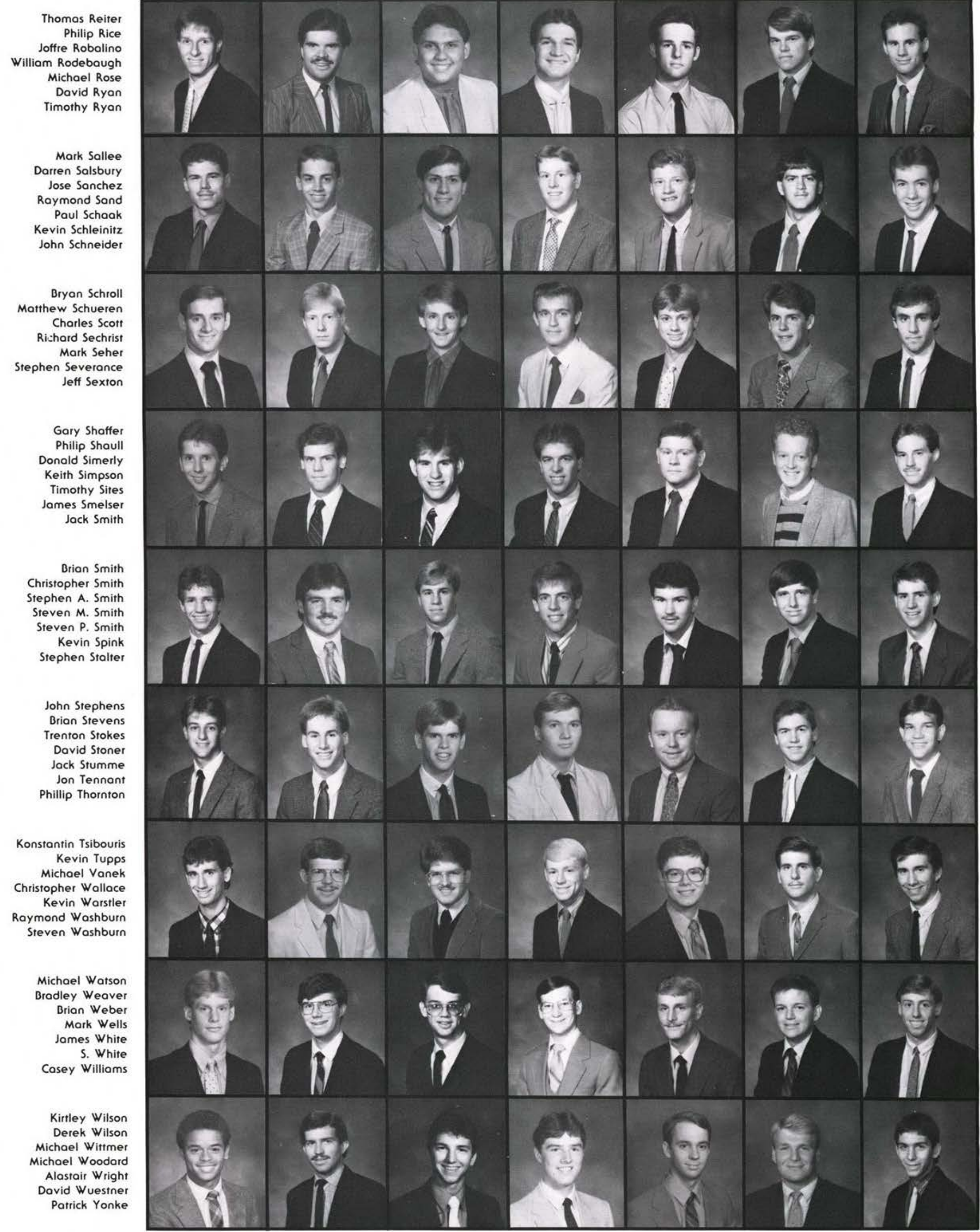




\section{Printy, Hall}

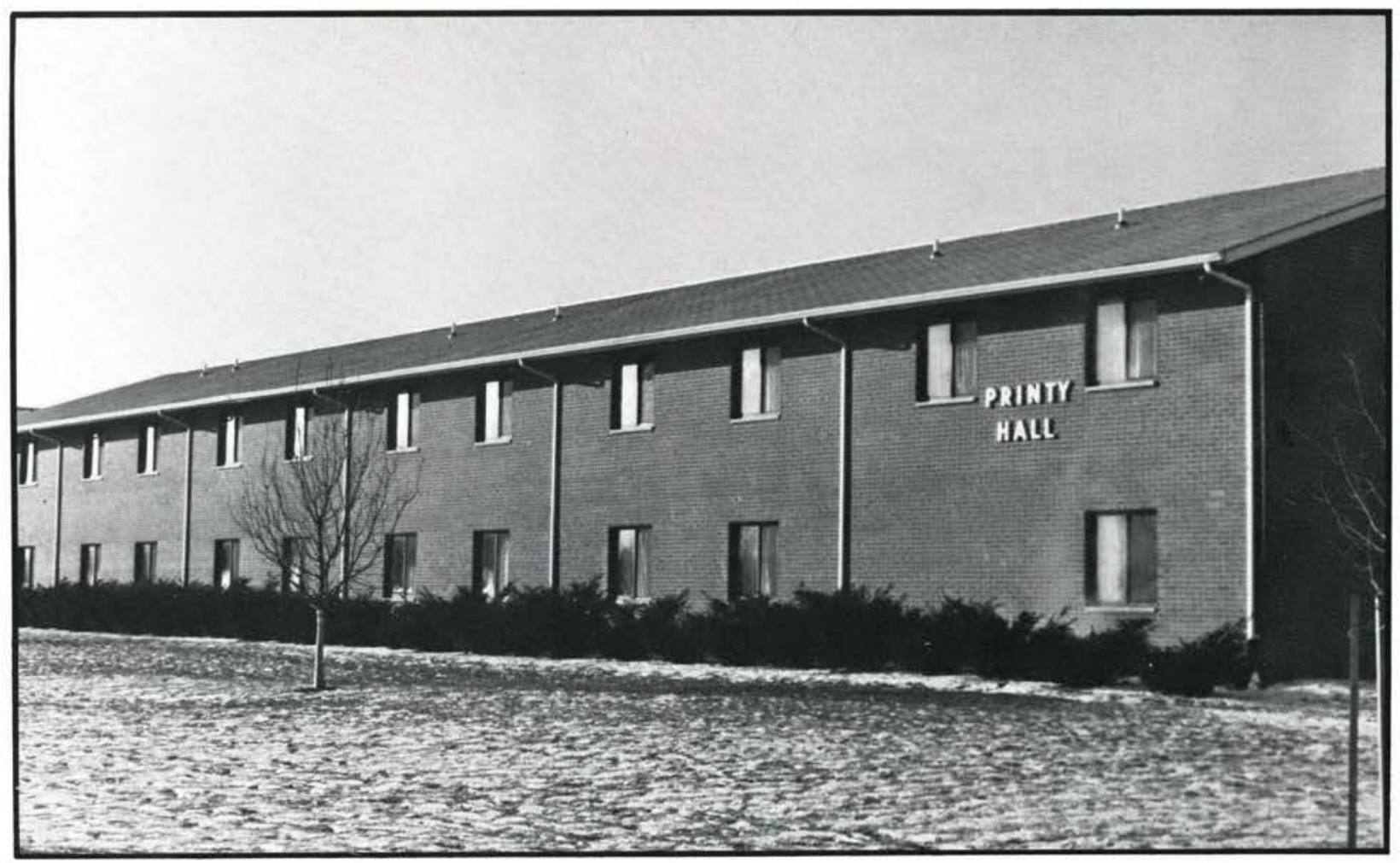

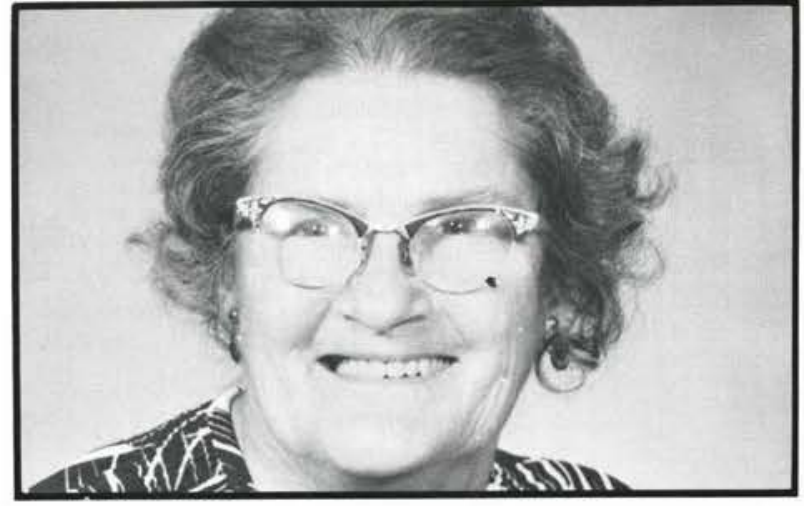

Beatrice Printy

Printy, the well-noted dorm named after the infamous "Ma Printy," was built in 1980. Its enduring love affair with Lawlor and its annual "Printy-Whar" creates jealousy and animosity with the Willerts Women. To the dismay of many Printy women, the dorm is presumed unfortunately to be built on a giant ant hill. Its occupants regularly seize the rooms of many unsuspecring vicrims. But Mrs. Howell, armed with millions of ant traps, always comes to the rescue.

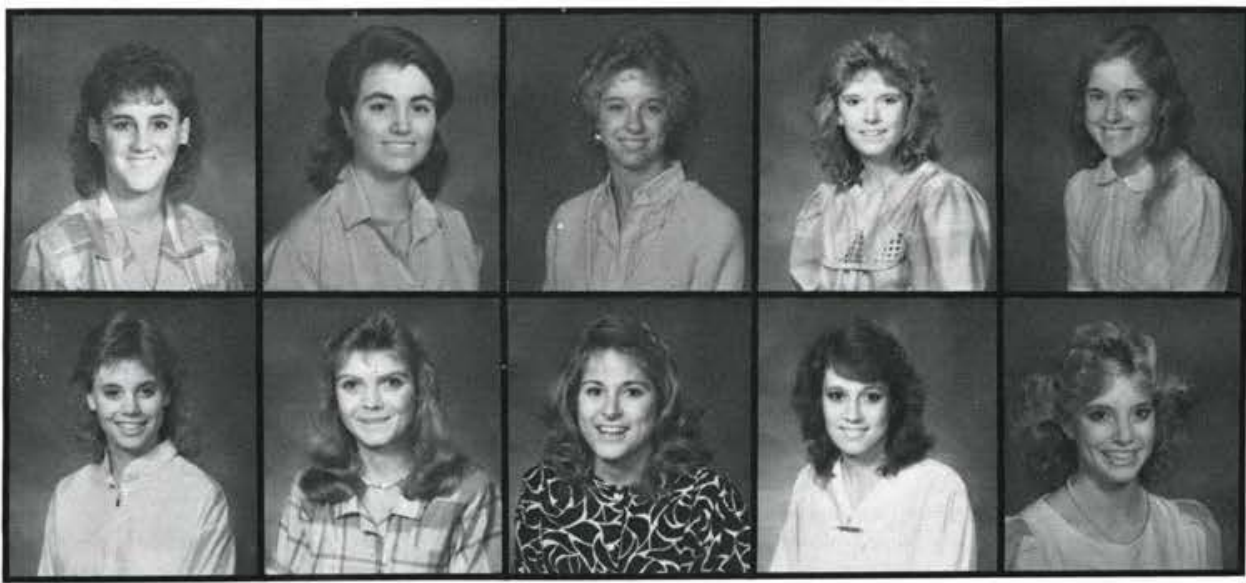

Deborah Ager

Catherine Anderson

Laurly Ann Anderson

Jill Angel

Katherine Augustine

Michelle Babbitt

Kimberly Bailey

Amy Barber

Becky Batey

Sherri Baver 
Kathy Bavec

Kimberly Beaver

Alice BeCraft

Julia Beimly

Amy Belgarde

Marcy Benjamin

Kelli Bensink

Michelle Berkey Kimberly Biddinger

Lynette Bishop

Donna Blumenstock

Lani Sue Borgman

Brenda Bow

Tara Bowling

Toni Bowling Jennifer Bowman Julie Box

Christina Boyle

Kelli Bradds

Judith Bradway

Susan Bradway

Kimberly Bragg Shelley Brandenburg

Jennifer Brand

Janie Bresson

Polly Brewen

Lori Brovont

Renee Brown

Shelley Brown

Shelby Browning Michelle Bumgardne

Kimberly Burrel

Tina Calkins

Dawn Carey

Renee Corr

Jenifer Corrol

Lynn Cose

Susan Chitwood

Jennifer Chon

Elizabeth Church

Rebecca Clifford

Melissa Coffey

Deneen Cole

Monica Coleman

Nancy Columbo

Pamela Commons

Lynn Costly

Kim Coston

Angie Creekmur

Louanne Cruz

Lois Cummings

Jill Cunningham

Cynthia Curry

Tina Daley

Michelle Dalton

Rochelle Daniels

Emily Davenpor

Rebecca Davis

Amy DeCook

Twana Dillard

Melody Dillon

Christine Douglass

Michelle Drummond
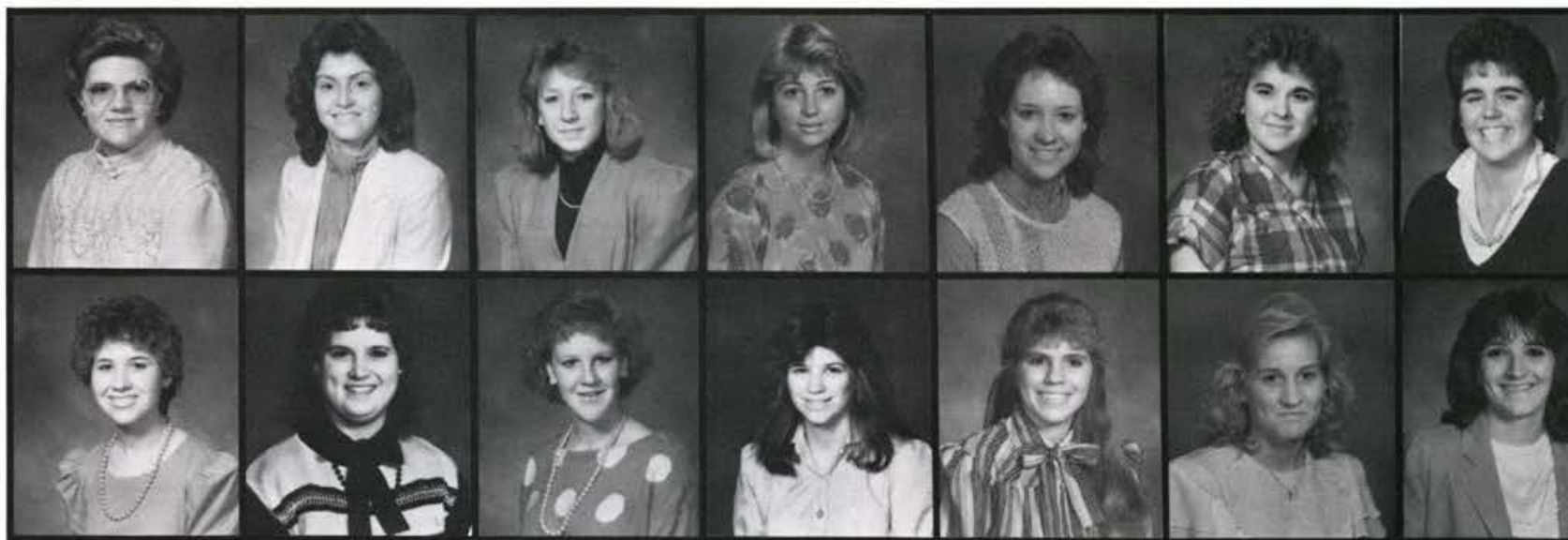

15

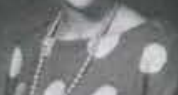

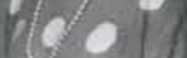
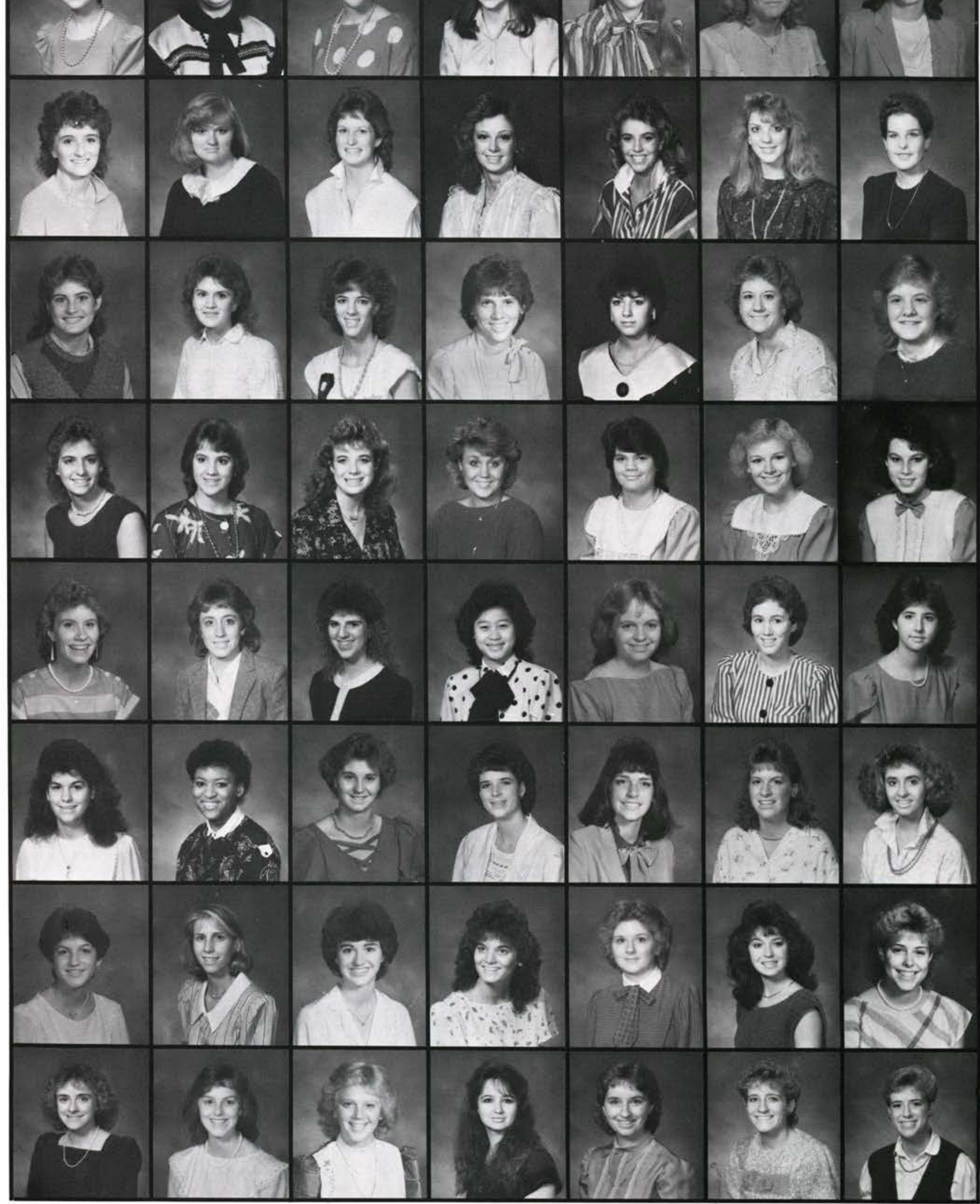


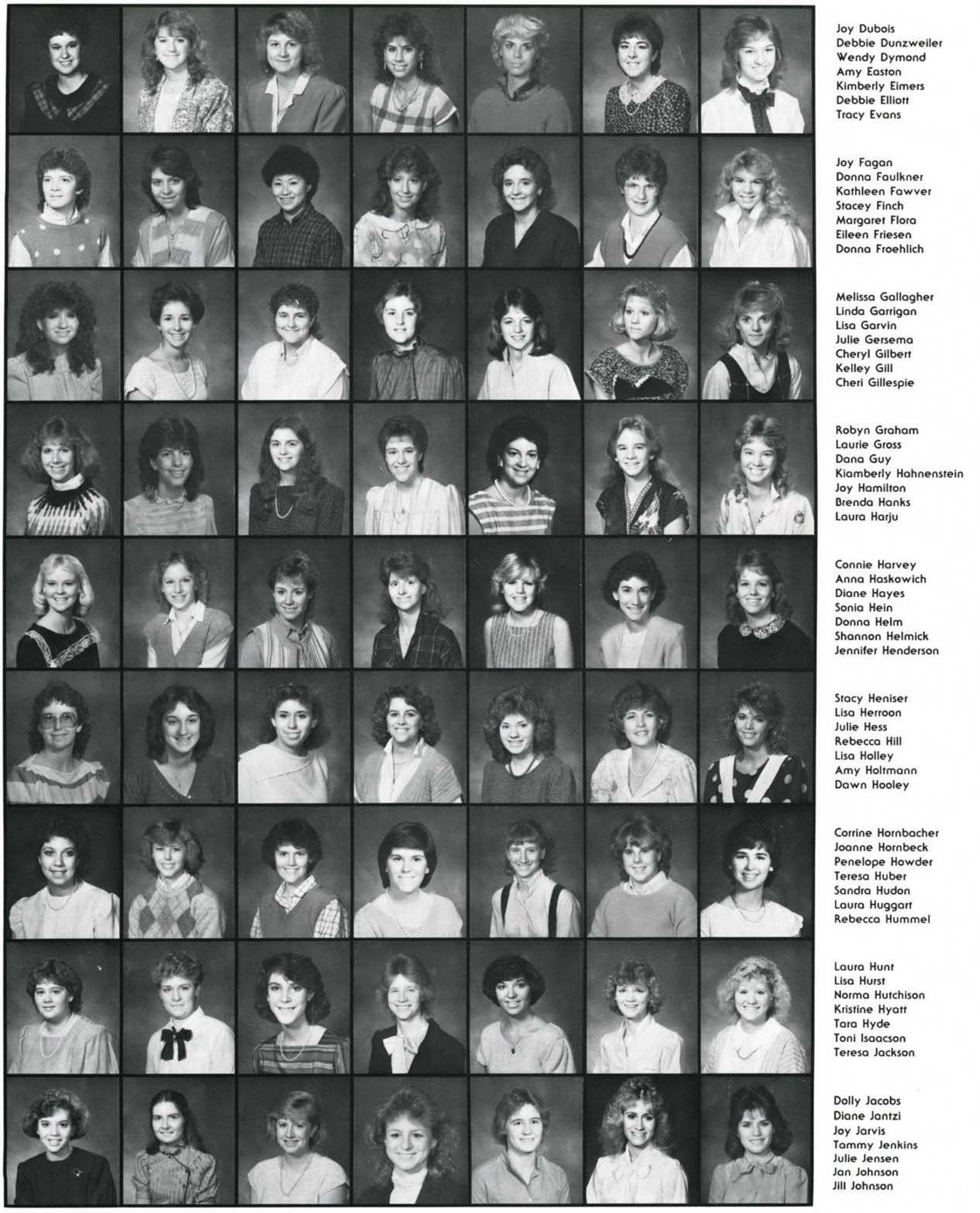


Kristyn Johnson Karen Johnston Karen Jones

Amy Joslin

Susan Kauer

Dionne Keens

Liso Kersten

Kimberly Killian Melissa Kimmel Sheryl King

Danielle Kirkpatrick

Julie Knauff

Kathy Knull

Diane Knuppa

Karen Kuntz

Laura Kuntz

Susan Lacey

Dana Lachmiller

Sheri Larkin

Roge Lash

Heidi Laub

Catherine LeGate Sheri Leach

Michelole Lelah

Jennifer Lenhart

Linda Leshan

Danno Lichty

Heather Lind

Kathleen Lippert

Dana Lott

Tina Love

Heather MacFarlane

Terri MacPherson

Suzanne Mackey

Lori Manley

Kimberly Manz

Tamara Mascari

Jeanne McCarrell

Sharon McClanahan

Christine McCormic

Cherryl McLaughlin

Karen Michene

Lori Miesse

Elizabeth Miller

Darla Mitchell

Kristine Moodie

Linda Mooibroek

Penny Morris

Ruth Morris

Leah Morse

Yolonda Moser

Susan Moyer

Martho Murphy

Ruth Myers

Carol Nauman

Joyce Nazarian

Lana Olson Kristin Ostrum

Pama-Lynn Oswald

Lori Otraway

Lisa Owens

Gloria Par

Alyson Payne
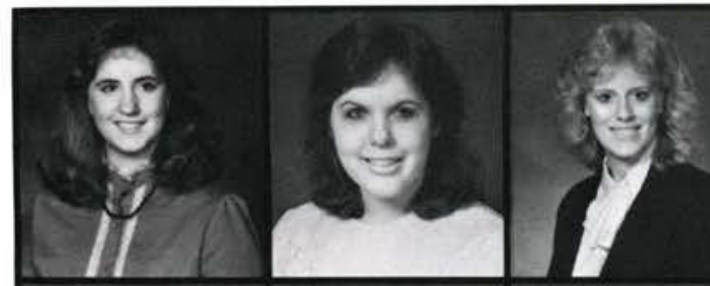

항
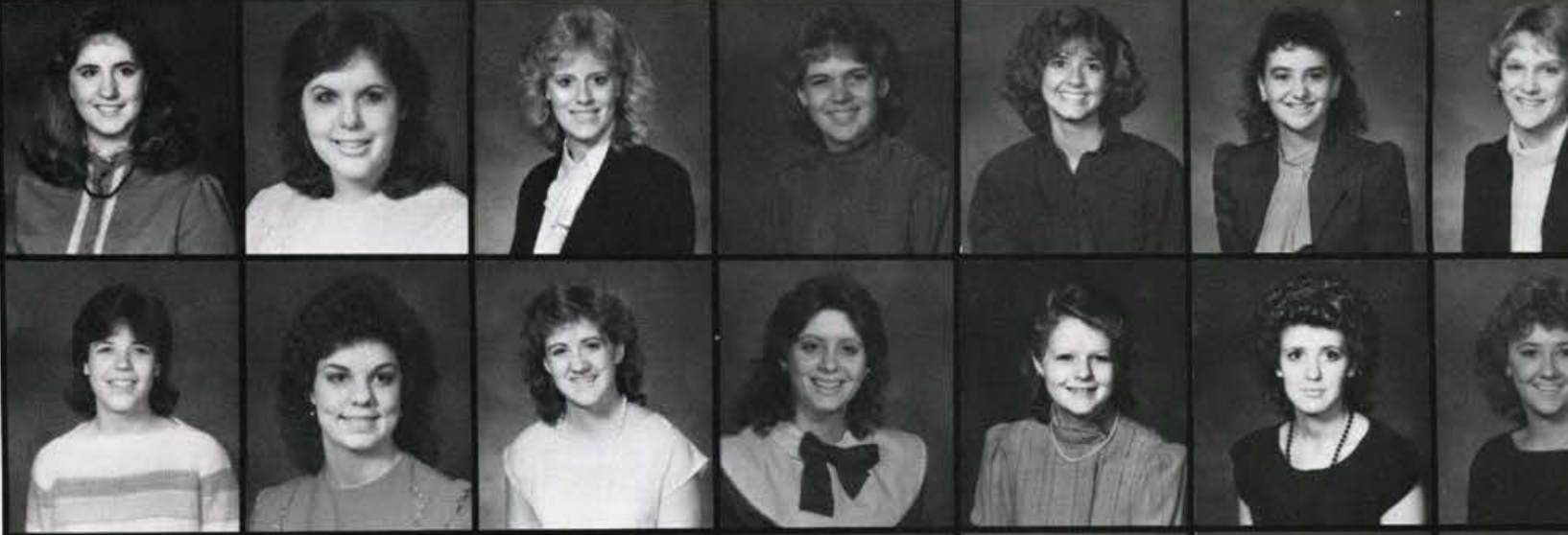

का
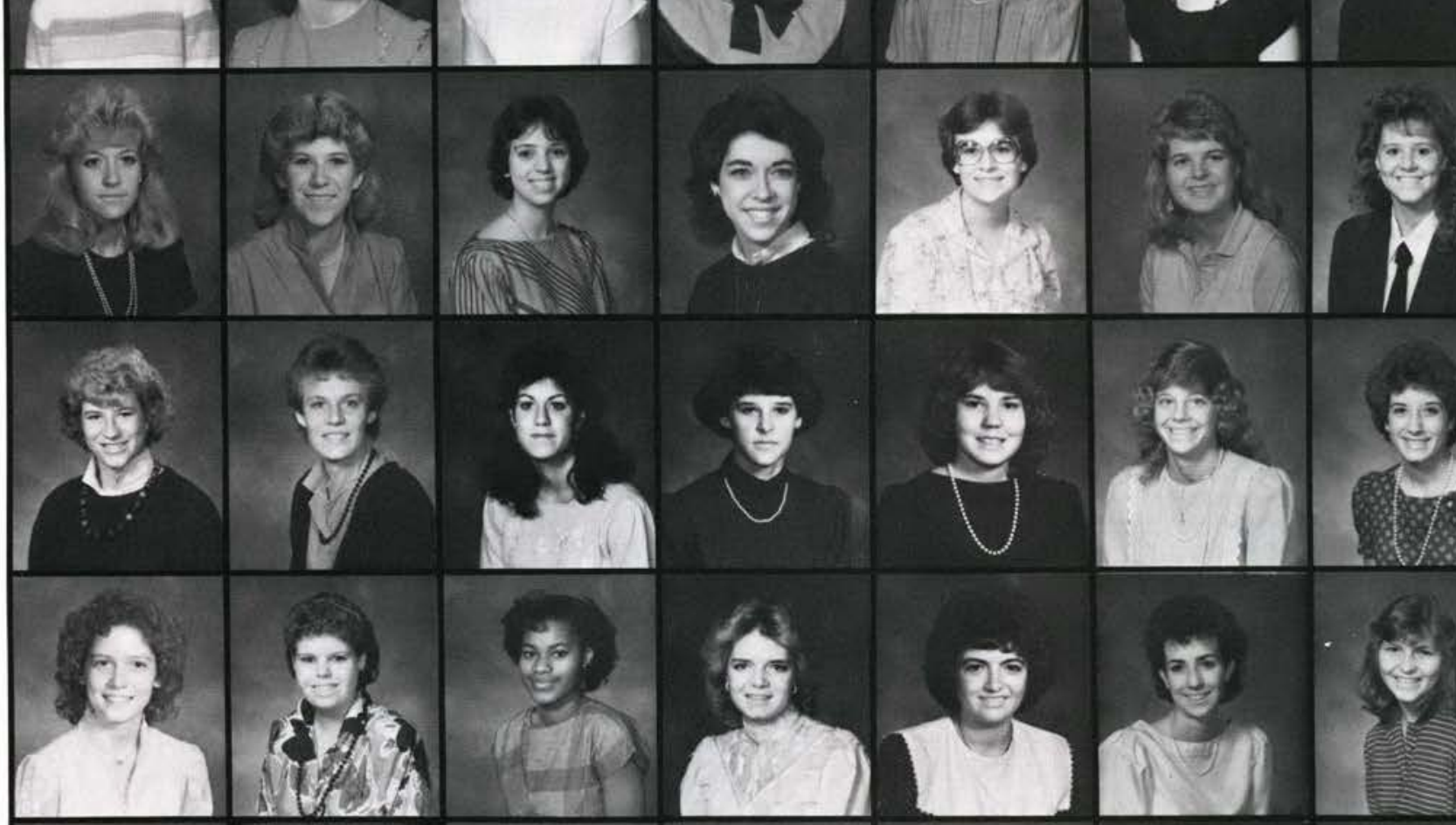

2.
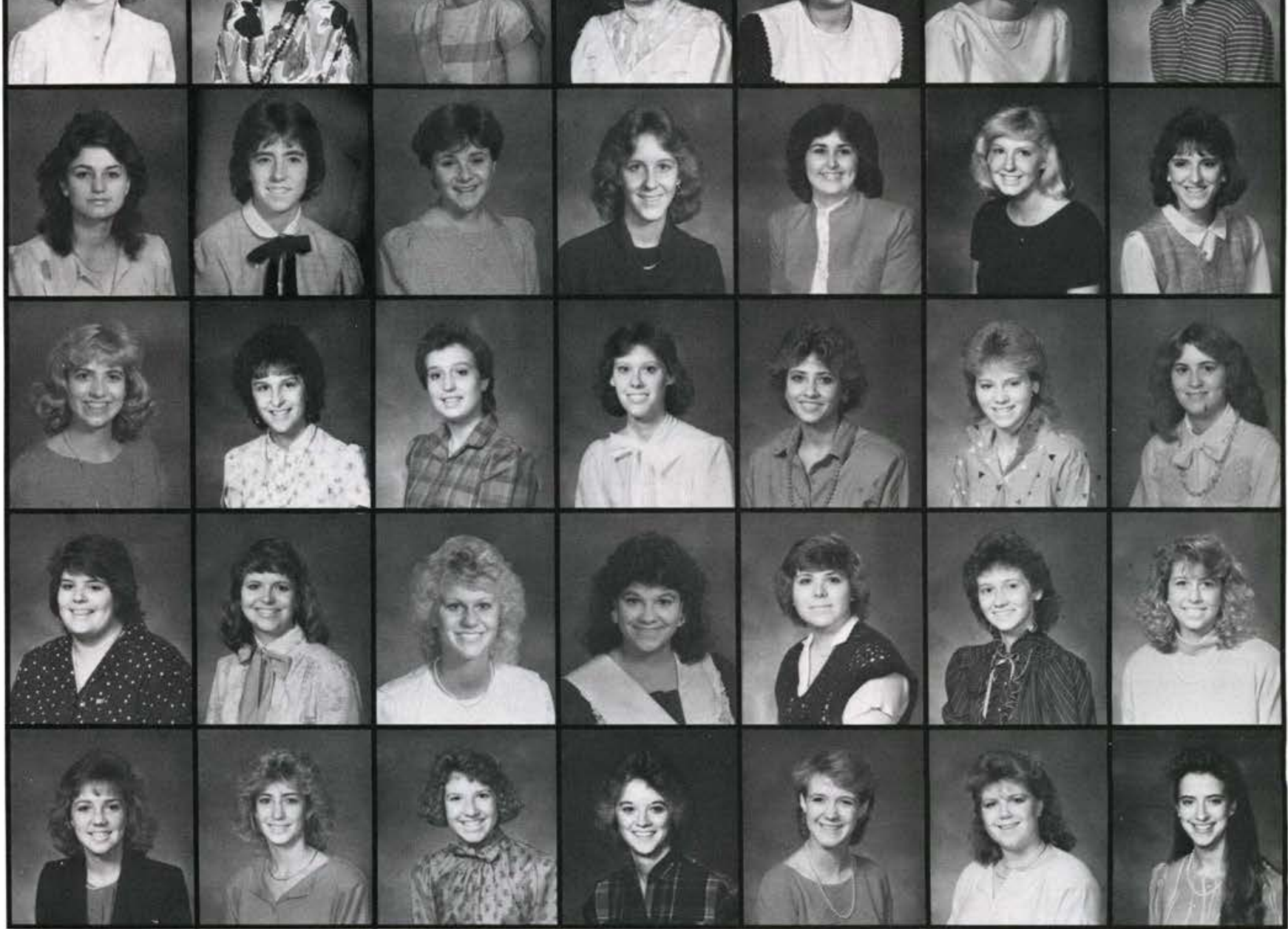


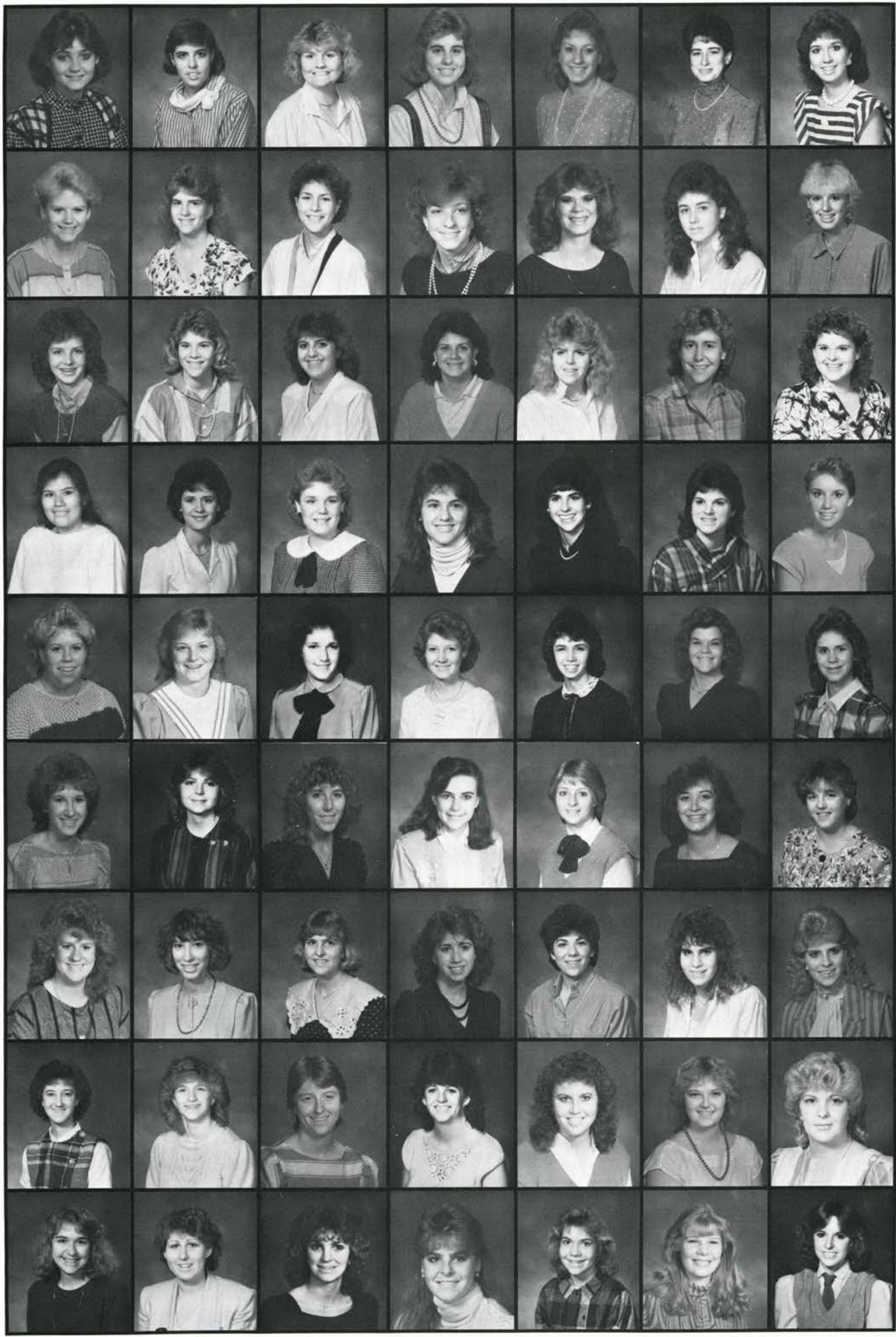

Donna Payne

Kirsten Perkey

Melissa Pope

Laura Potter

Rebecco Pruner

Mary Raisch

Angela Ramsey

Linda Range

Molly Reeves

Kimberly Renshow

Melissa Riggs

Debby Rinehart

Liso Riopell

Sonja Rizor

Leah Robbins

Lori Rogers

Carissa Roig

Wendy Rose

Joanna Rudelitch

Elizabeth Shepherd

Ronda Short

Cheryl Smith

Shawn Spangler

Jill St. John

Penney Stackhouse

Susan Stalter

Leeanne Stevens

Stephanie Stevens

Tina Stinedurf

Christine Stowell

Brenda Sutliff

Sonda Sweet

Alice Sweitzer

Rebecco Tacket

Valerie Teed

Amy Titus

Rebekah Tucker

Heather Tyson

Sharon Van Patten

Tamara Venman

Cynthia Wagner

Jane Walters

Jennifer Ward

Jacquelyn Warden

Cheryl Warren

Sharri Washburn

Sandra Weaver

Kathleen Webber

Carla Weitkamp

Christine Wenge

Loreena Westfall

Wendy Wheeler

Jennifer Whittaker

Suzanne Widder

Carol Wiggins

Rebecco Wingard

Lynn Witt

Patricia Wit

Lynn Wood

Jill Wyse

Laurel Yates

Grace Young

Lisa Zummerele 


\section{WILLETTS HALL}

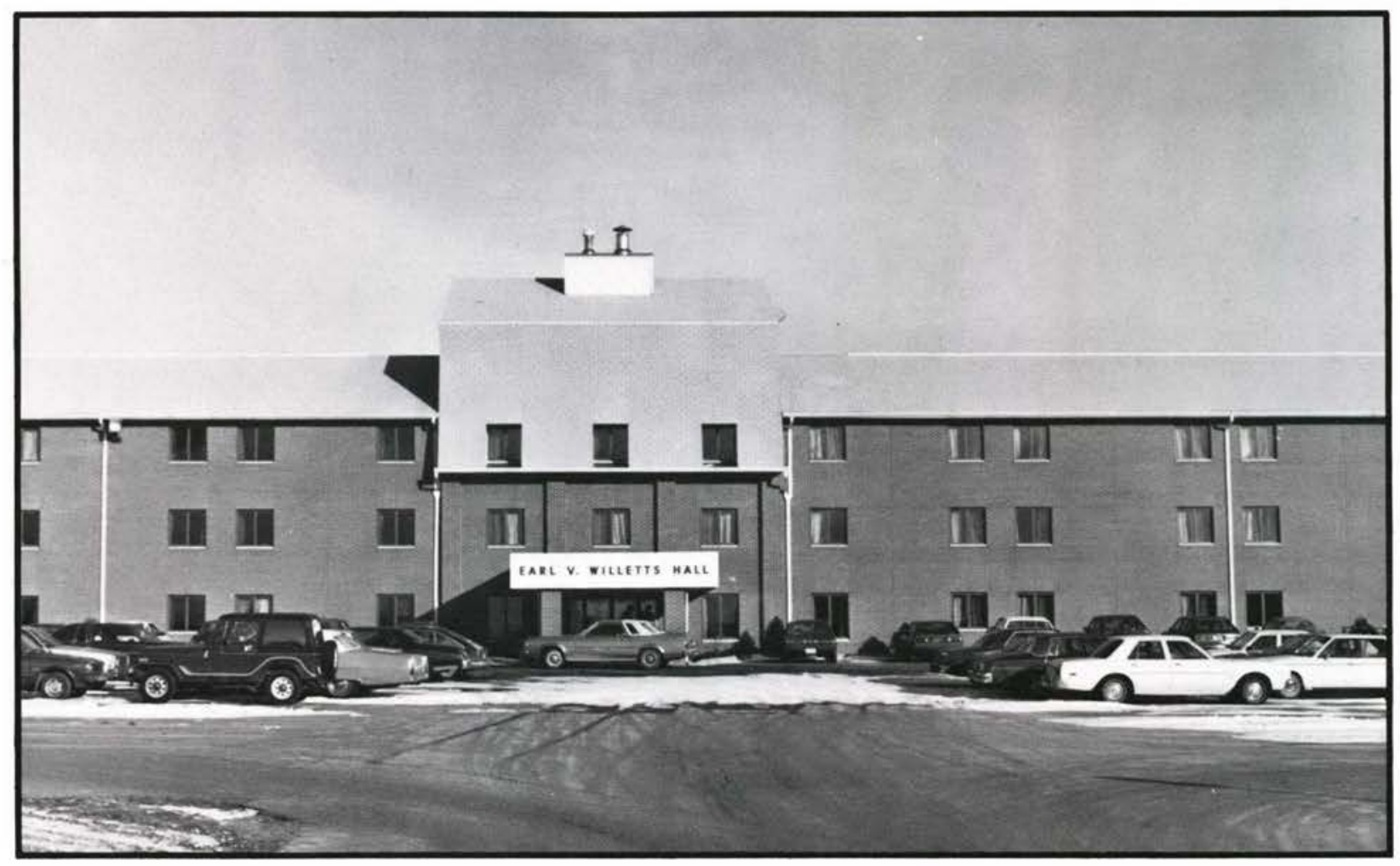

Known affectionately as "the Barn" or the "new dorm," Willerts Hall is the most recent dormitory constructed at Cedarville. Named after trustee Earl Willerts, the structure was built in 1982 to house the increasing number of students.

Often enduring heckling from guys on the north side of Lawlor, these strong women endure not only the brutal winds of an extra long walk but also the brutal words of Lawlor men. Bur the prestige of this dorm, which houses only one freshman, entices most junior and senior women.

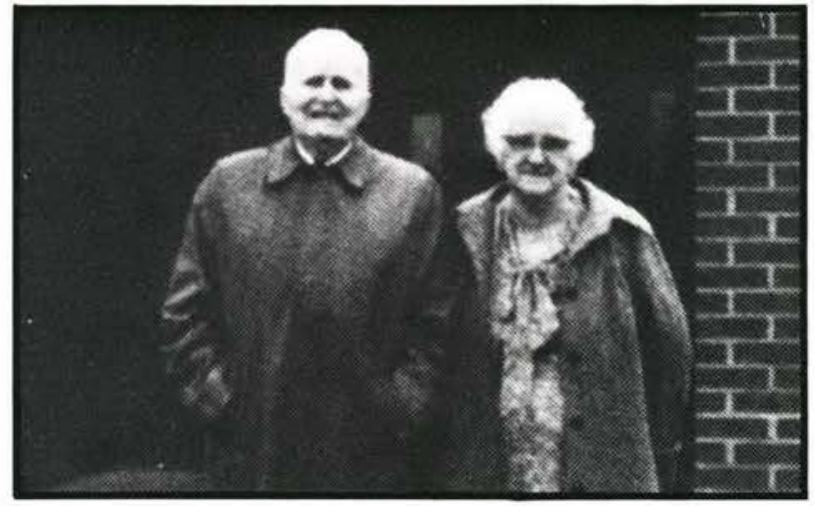

Mr. and Mrs. Earl Willets

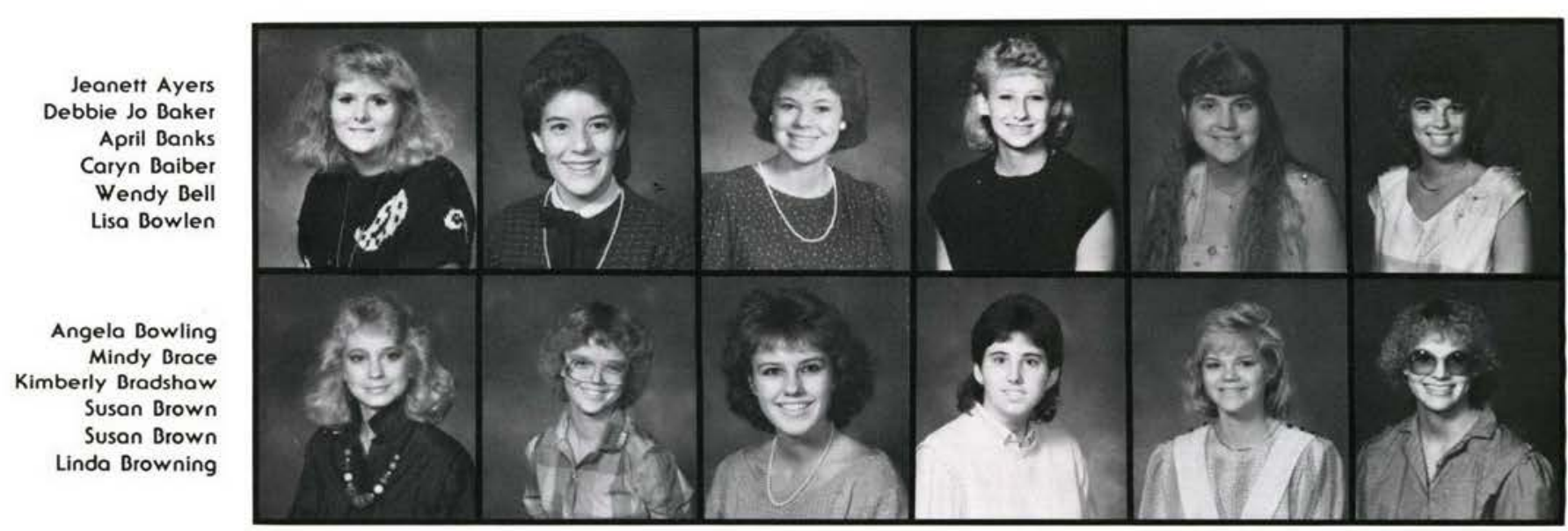




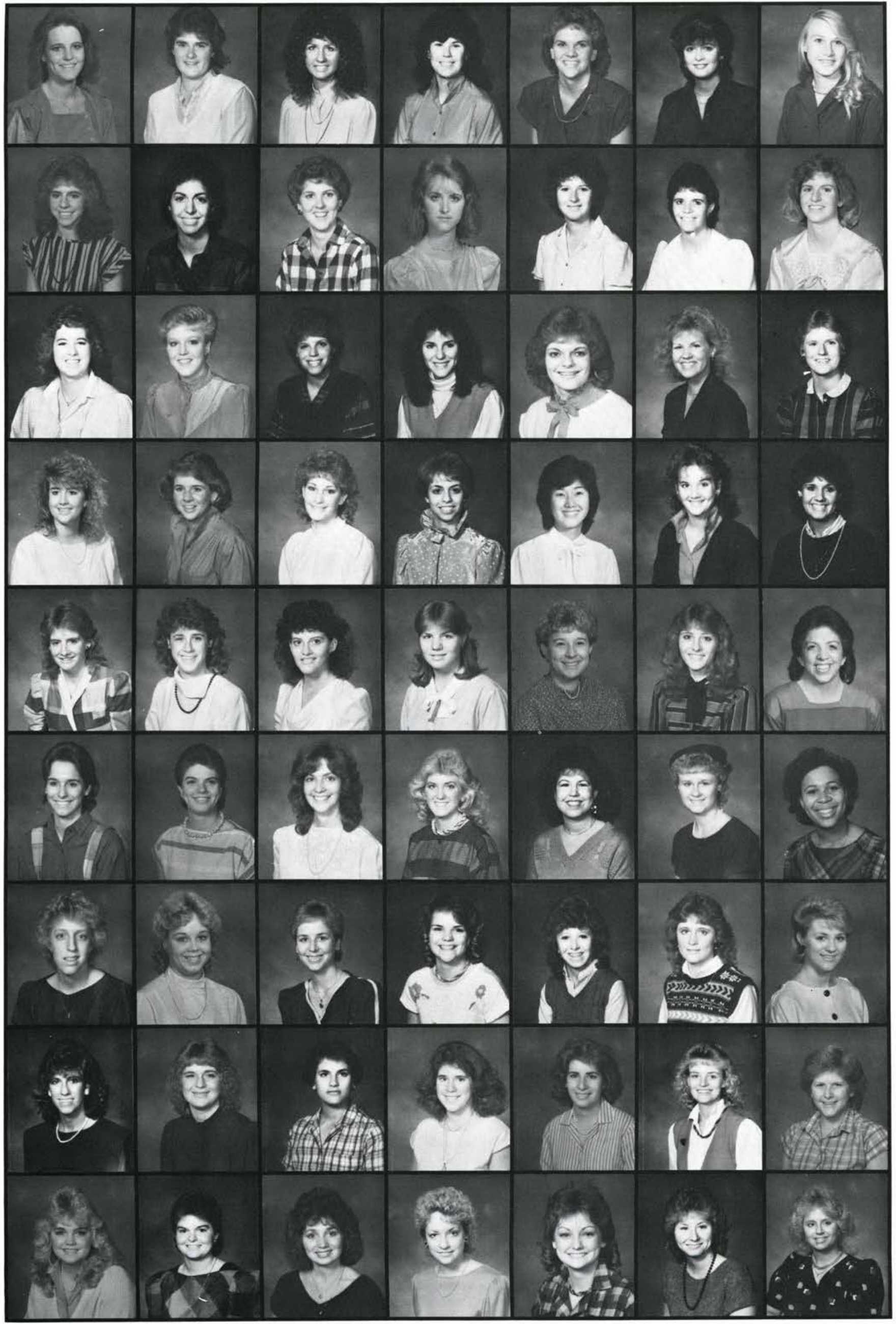

Sherri Cruver

Kristina Culp

Penny Dellinger

Kimberly Eager

Ruth Eissens

Melissa Felt

Melody Ferguson

Laura Fetzer

Claire Fortosis

Christine Friedkin

Rebecca Haga

Heidi Haist

Melanie Hall

Nancy Hanno

Sherri Hannay

Jennifer Haufler

Kirsten Hoddelmann

Kirstina Hoddelmann

Shellie Horton

Cynthia Hunter

Barbara Jacobs

Pamela Johnston

Kathleen Kegel

Pamela Kenyon

Kendra King

Julia Lee

Ruth Margraff

Amy McKibben

Mary McVey

Deborah Merirxether

Lisa Mitchell

Beatrie Moraes

Beth Morse

Gretchen Mountz

Kathy O'Kresik

Jennifer Patry

Cheryl Paxson

Suzanne Perrott

Rebecca Peters

Cheryl Plooy

Gwen Price

Ebbie Radcliff

Debra Reynolds

Renee Rolfe

Nancy Ross

Gayle Ruggles

Kerri Sadle

Sheri Shuler

Joy Shuman

Karla Simpson

Janae Smart

Kristine Smith

Lori Spiegel

Meredith Steiner

Jennifer Stevens

Susan Terkelsen

Deborah VanDorf

Liso Vaughn

Cari Vucish

Bonnie Weber

Charisse Wolf

Elizabeth Zeck

Kimberly Zimmermon 
Robert Aiken
Valerie Baise
Karen Bennett
Judith Bianco
William Bianco
Corol Bliss
Stephen Bovey

William Bovey

Todd Bowen

Roger Bowshier

Penni Bresson

Brian Brock

Phillip Brunner

Holly Burke

Paul Colvert

Cindy Collier Matthew Creamer

Loralee Cruz

Laurie Dalane John Paul Dawes

Sarah Drake

Stephen Dye Debbie Dysert Linda Eberly Layne Etchison

James Evans

Tonya Evans

Susan Everitt

Lisa Ford

Kimberly Fordyce Michelle Fratus

Matthew Grider

Melissa Grimm

Victor Guevaro

Margaret Guido

Fritz Helmuth Lanay Herron

Cheryle Holt

Carolyn Holtz

Ronald Irvin

Kelly Johnson Richard Kaercher

Pamela Kimble Sharon Kresge Michelle Laird

Chester Lehman Christopher Link Jeri Lynne Long

Tim Lowing

Stephanie Manning
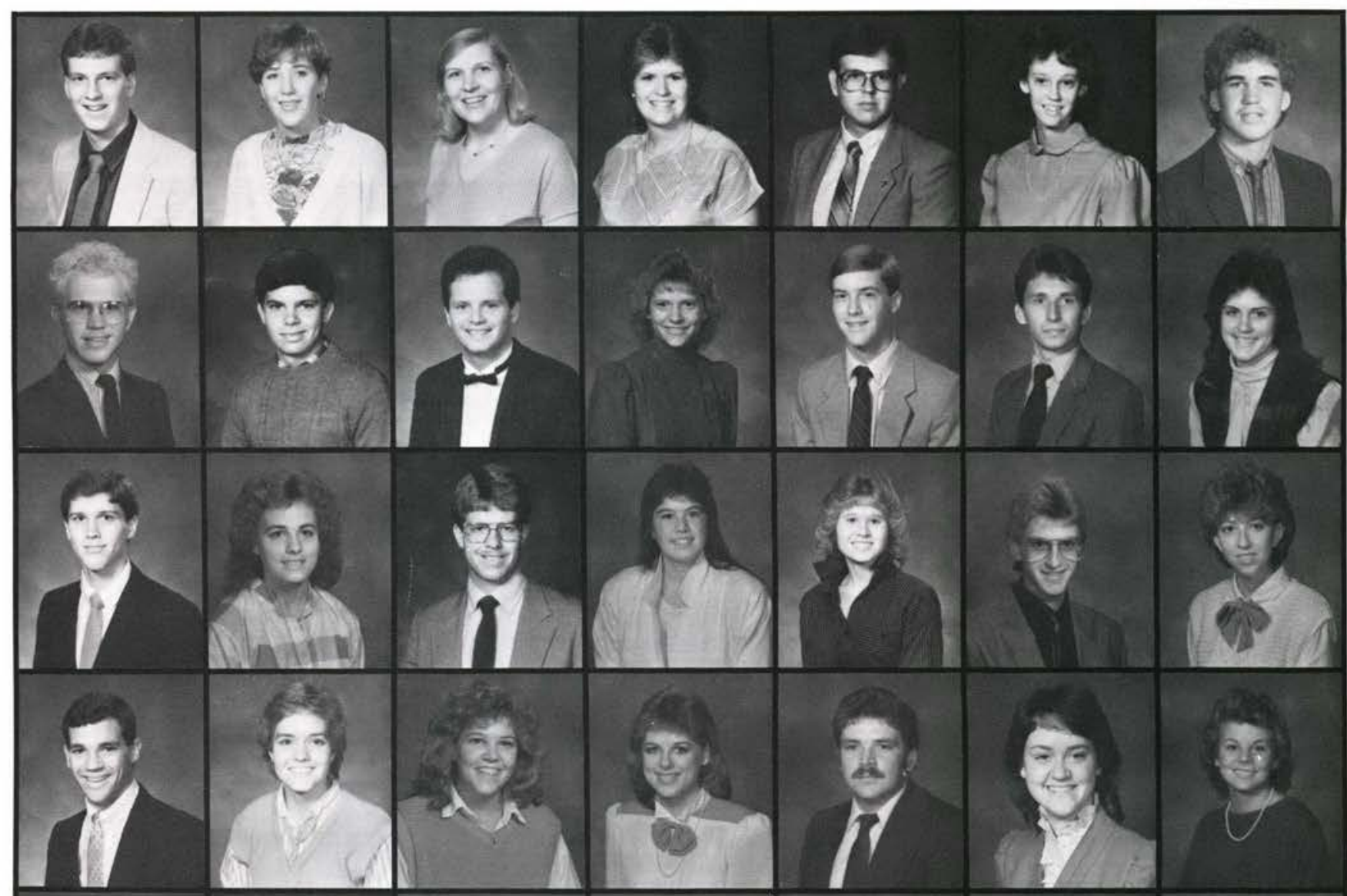

3단
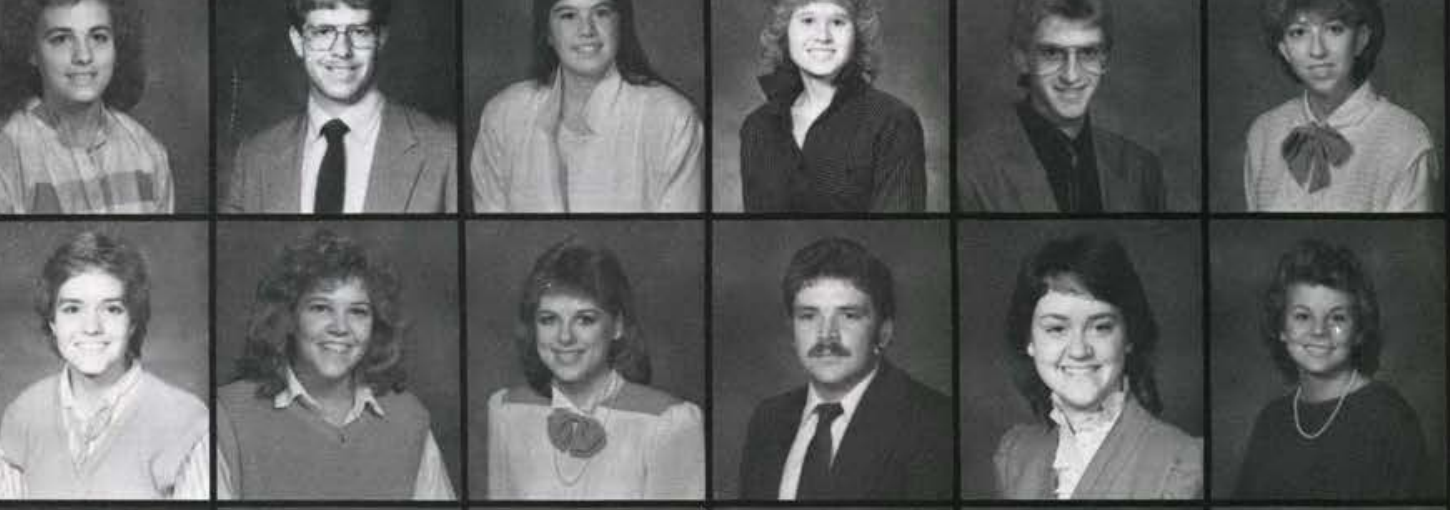
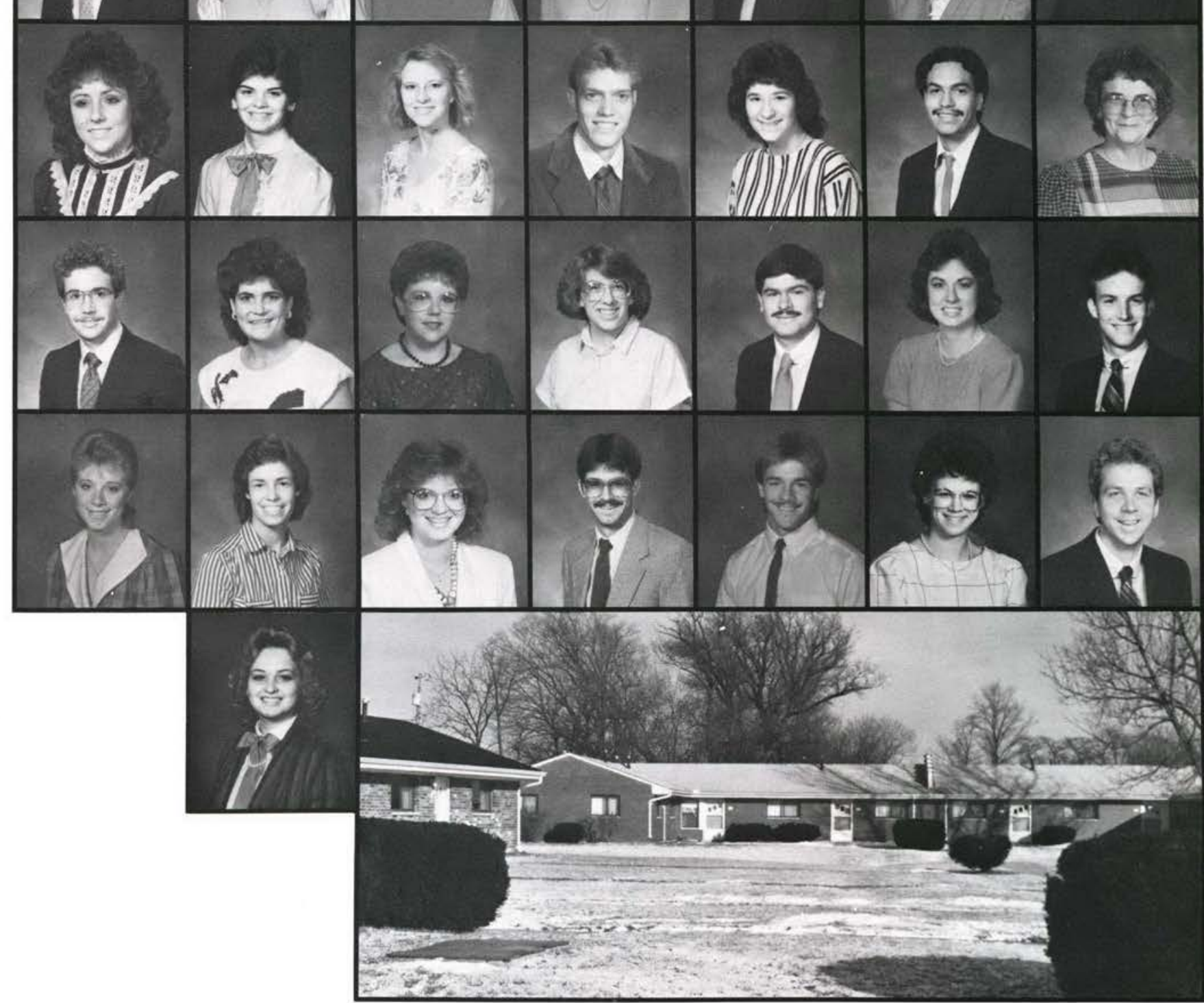


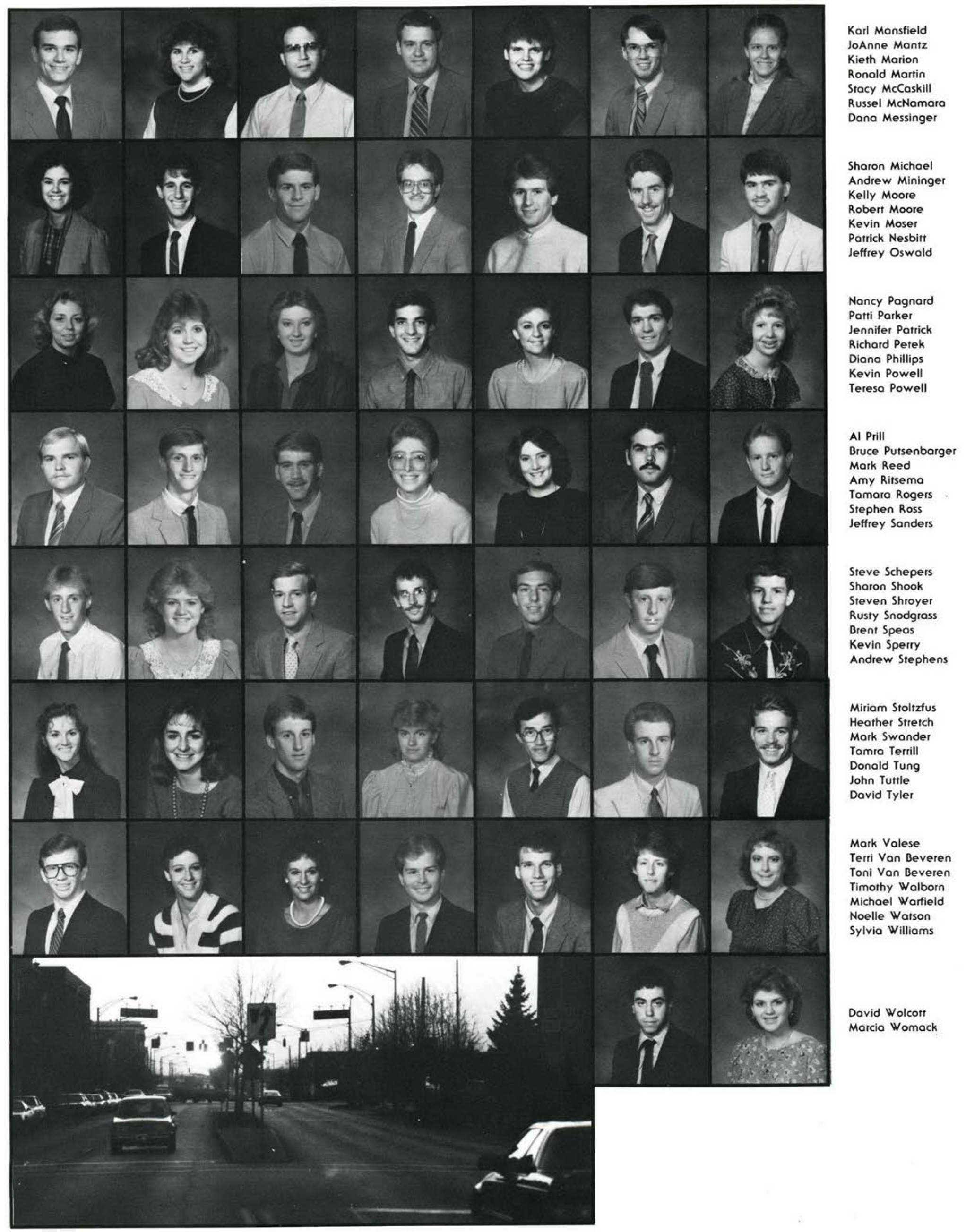




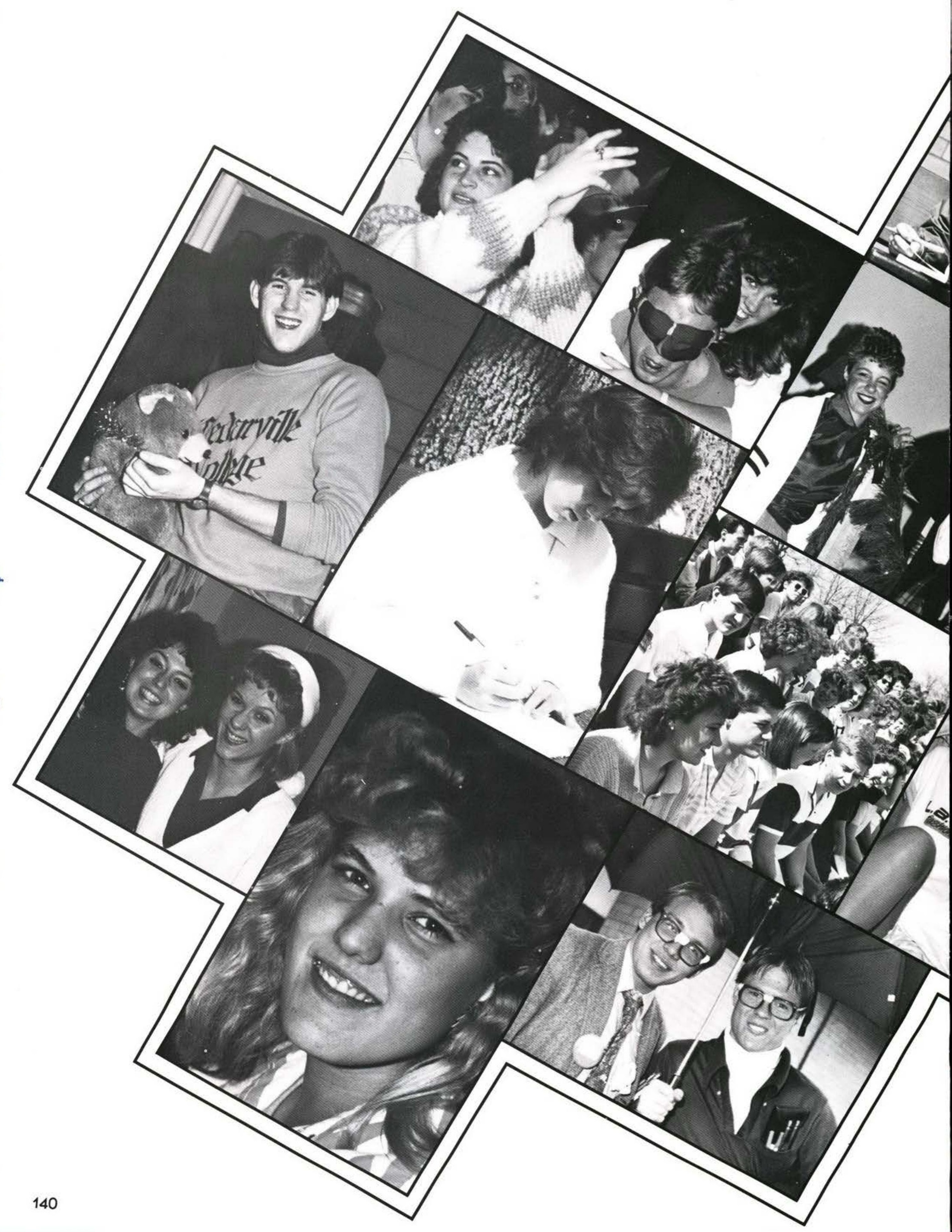




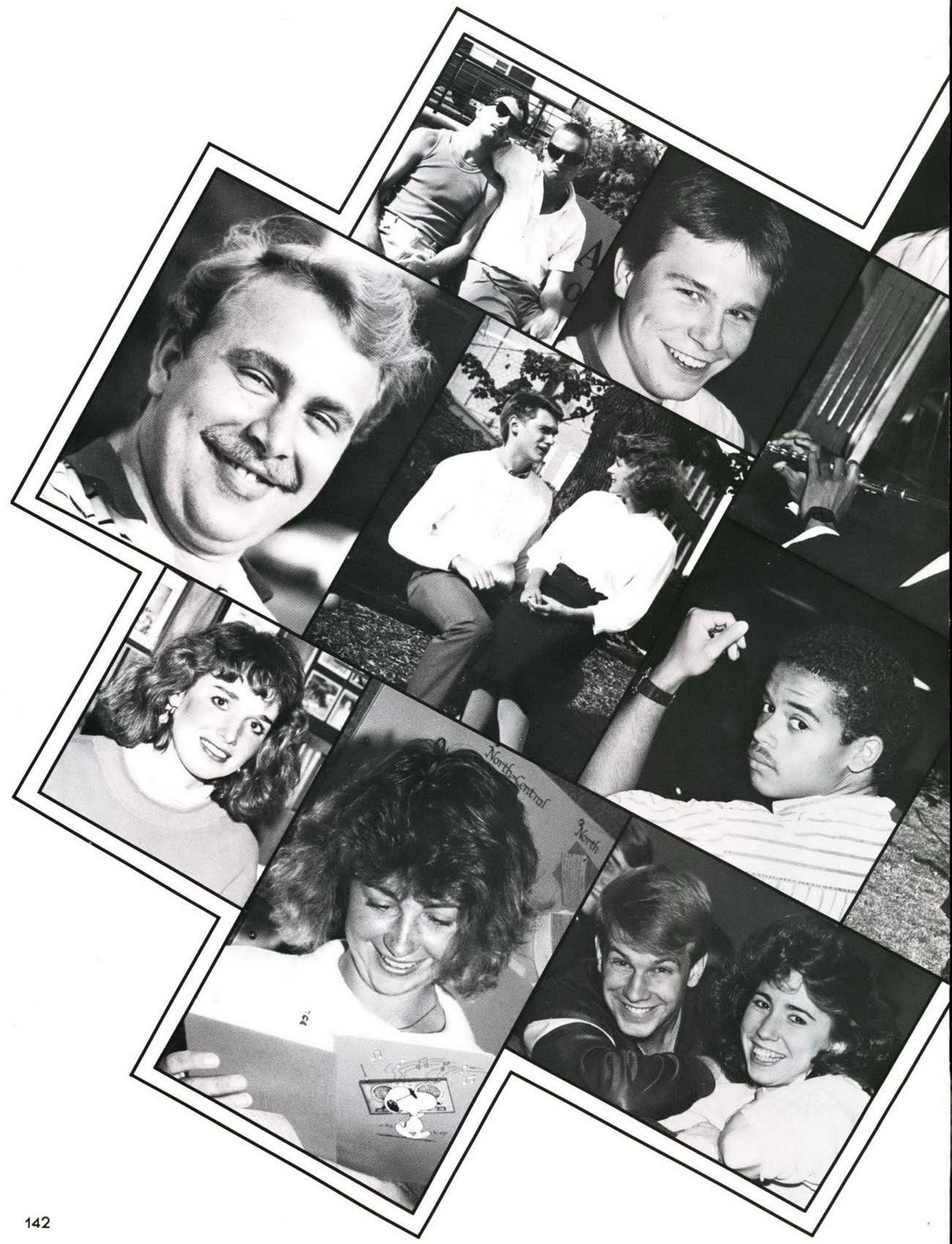




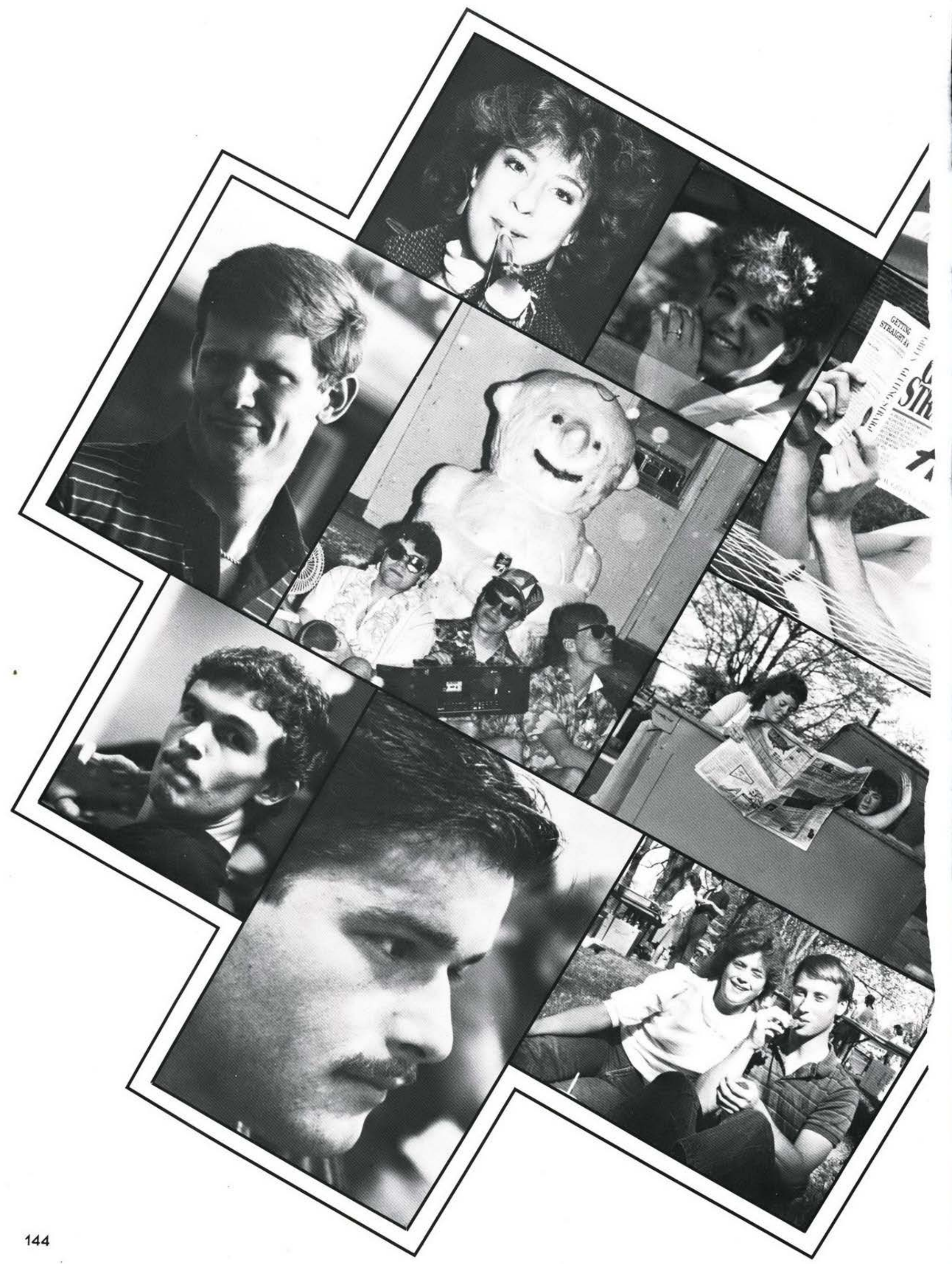




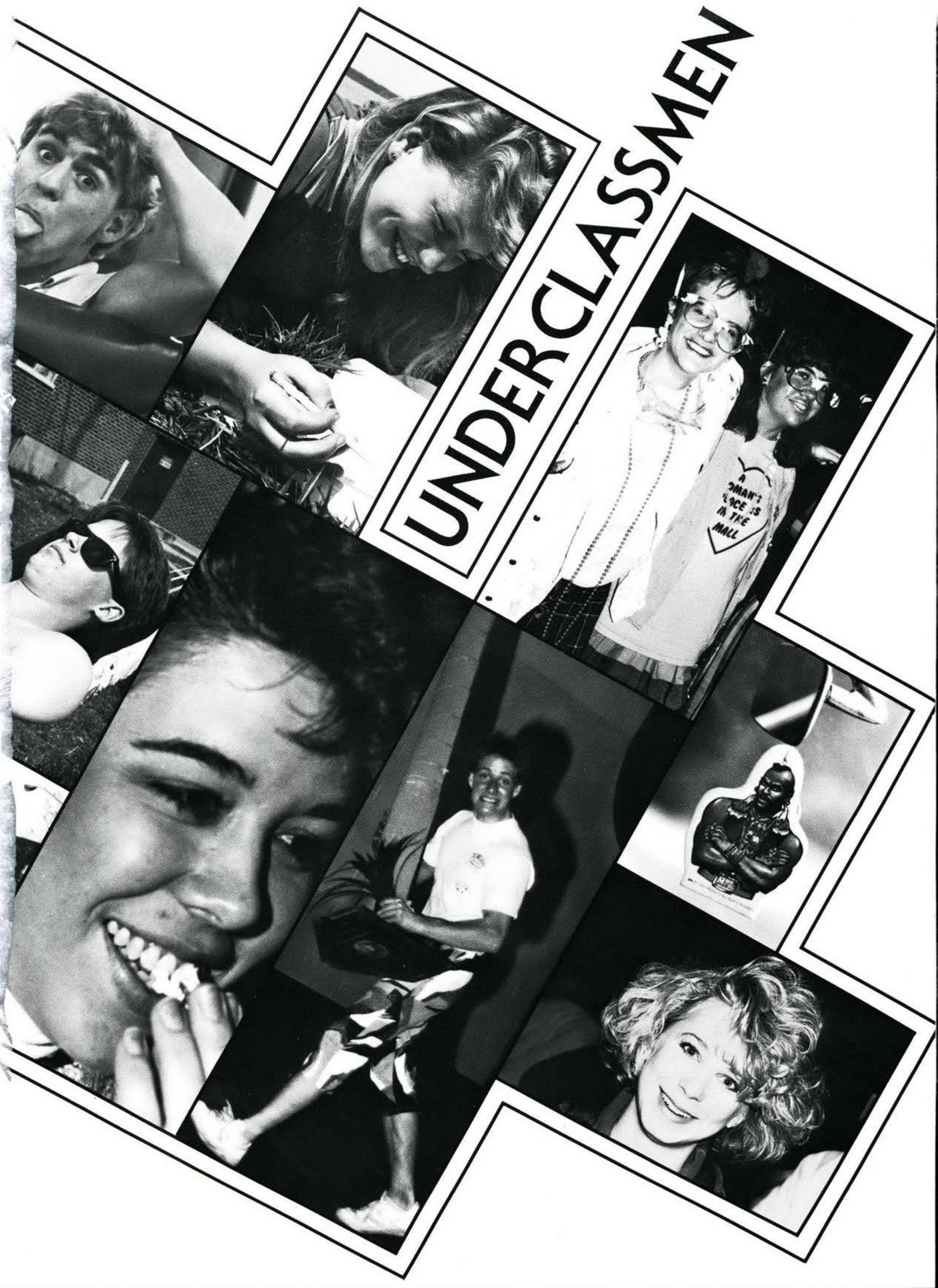




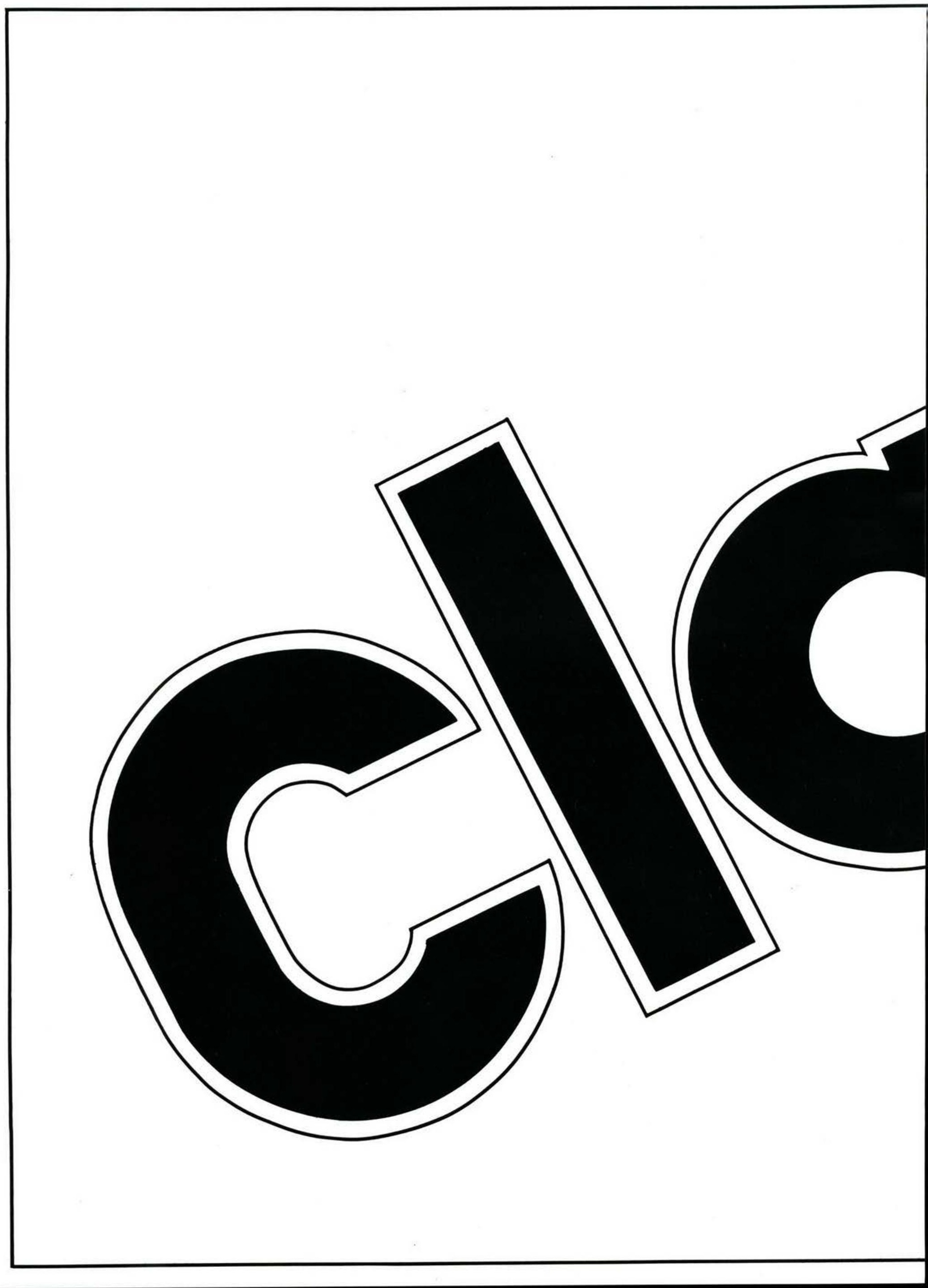




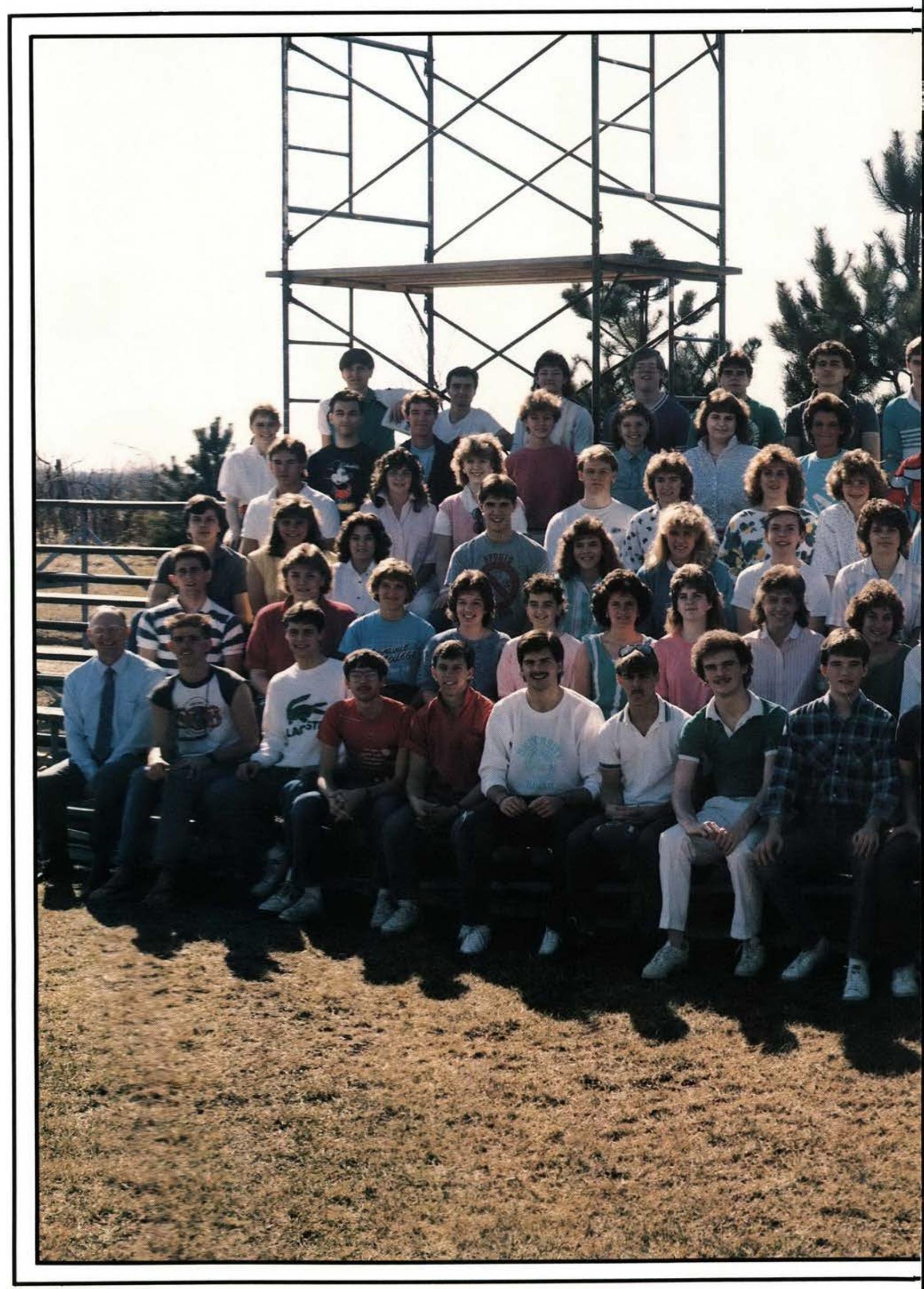



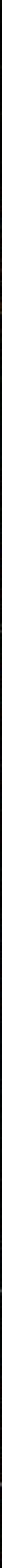


\section{WV \\ 4}

Hot $m^{2}=1$

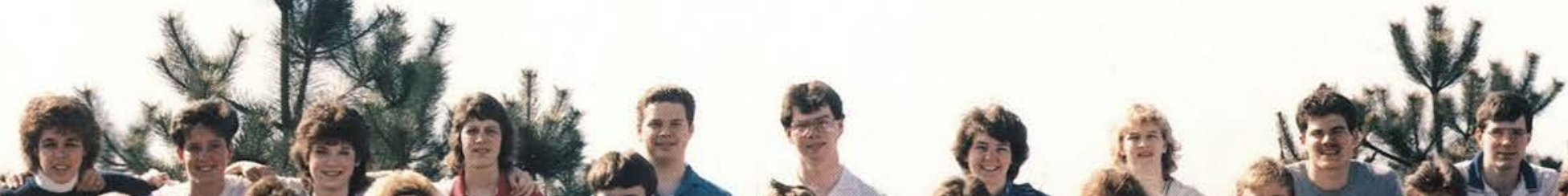

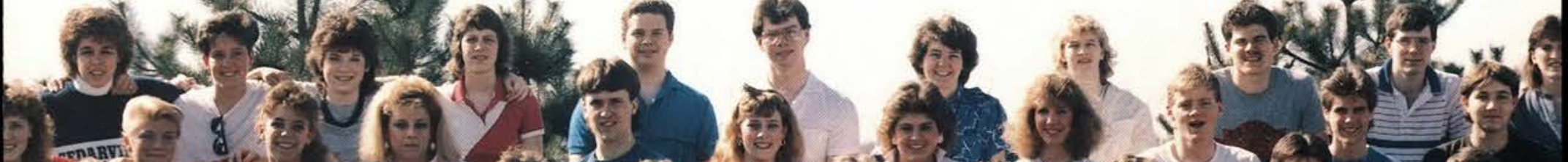

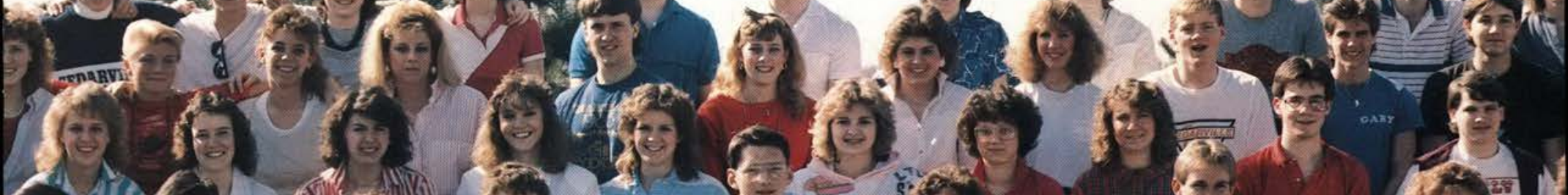
aif 3

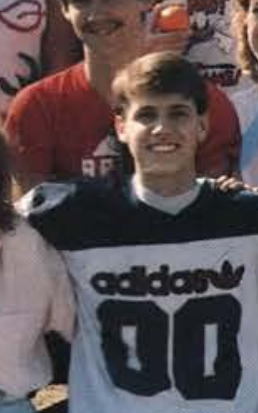

at
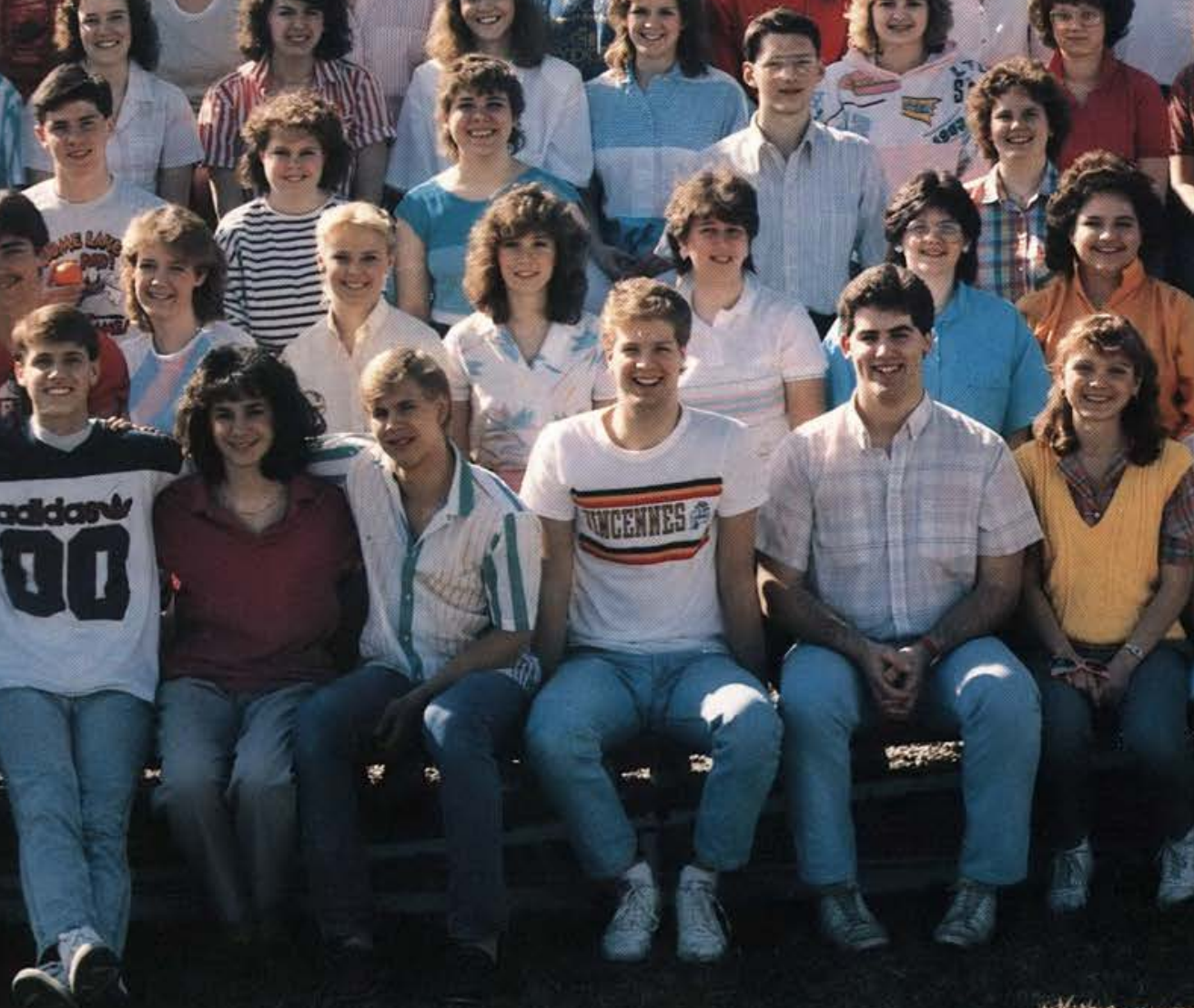

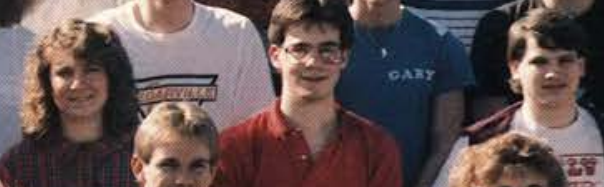

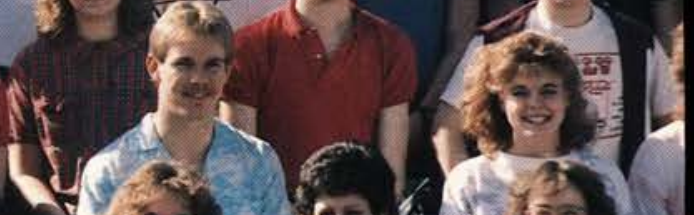

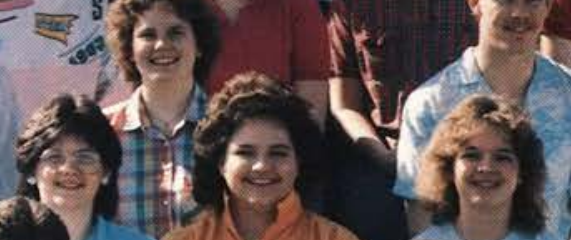

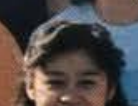

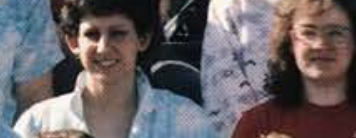
A.

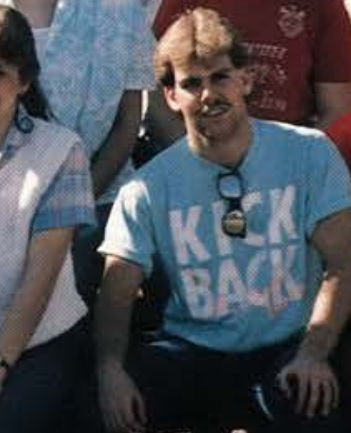

wation

3.t.

Not:

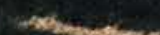

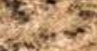

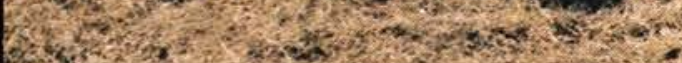

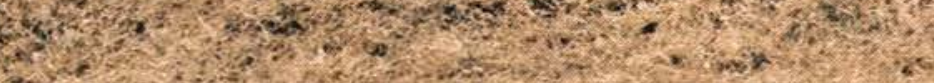




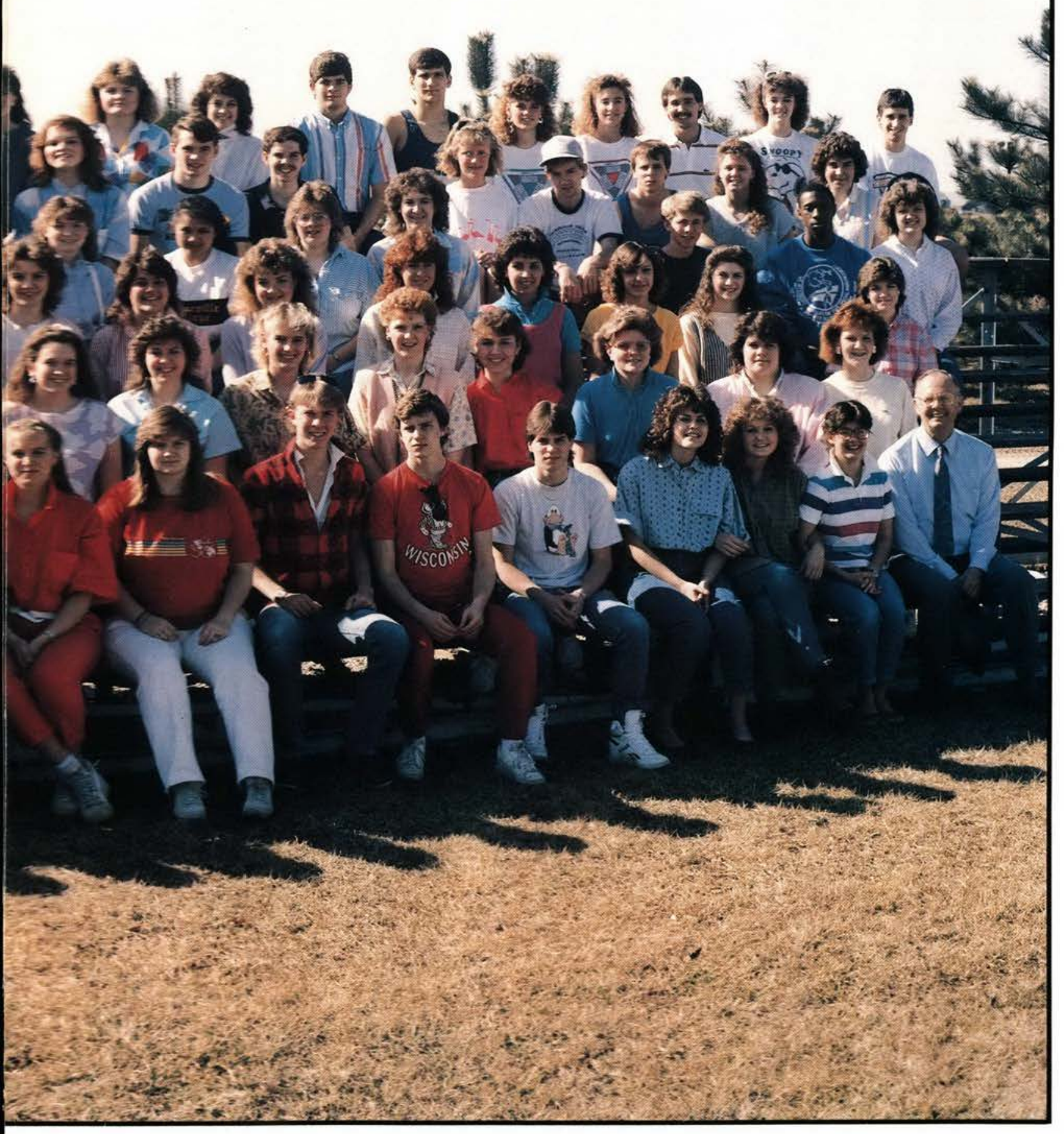




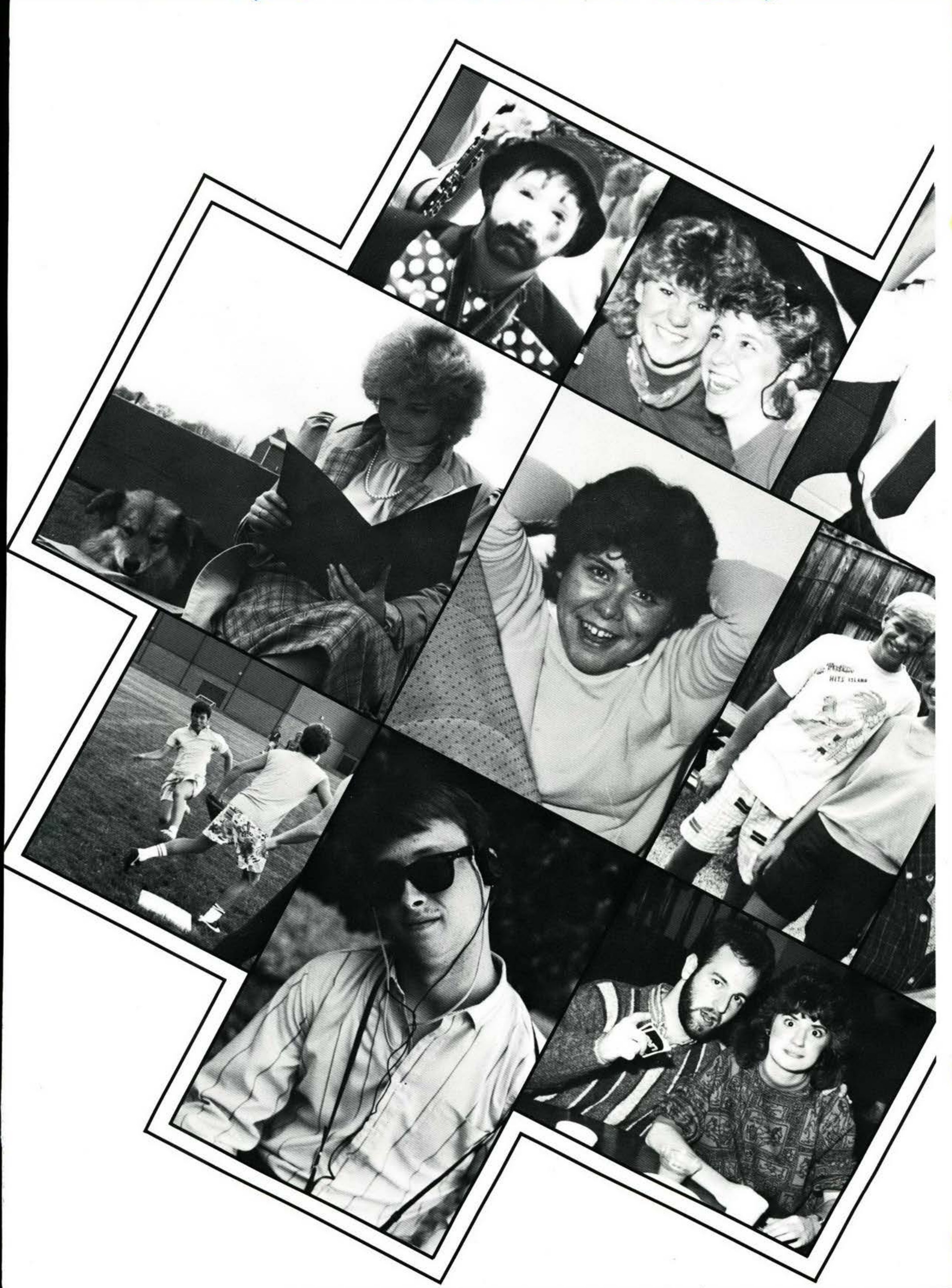



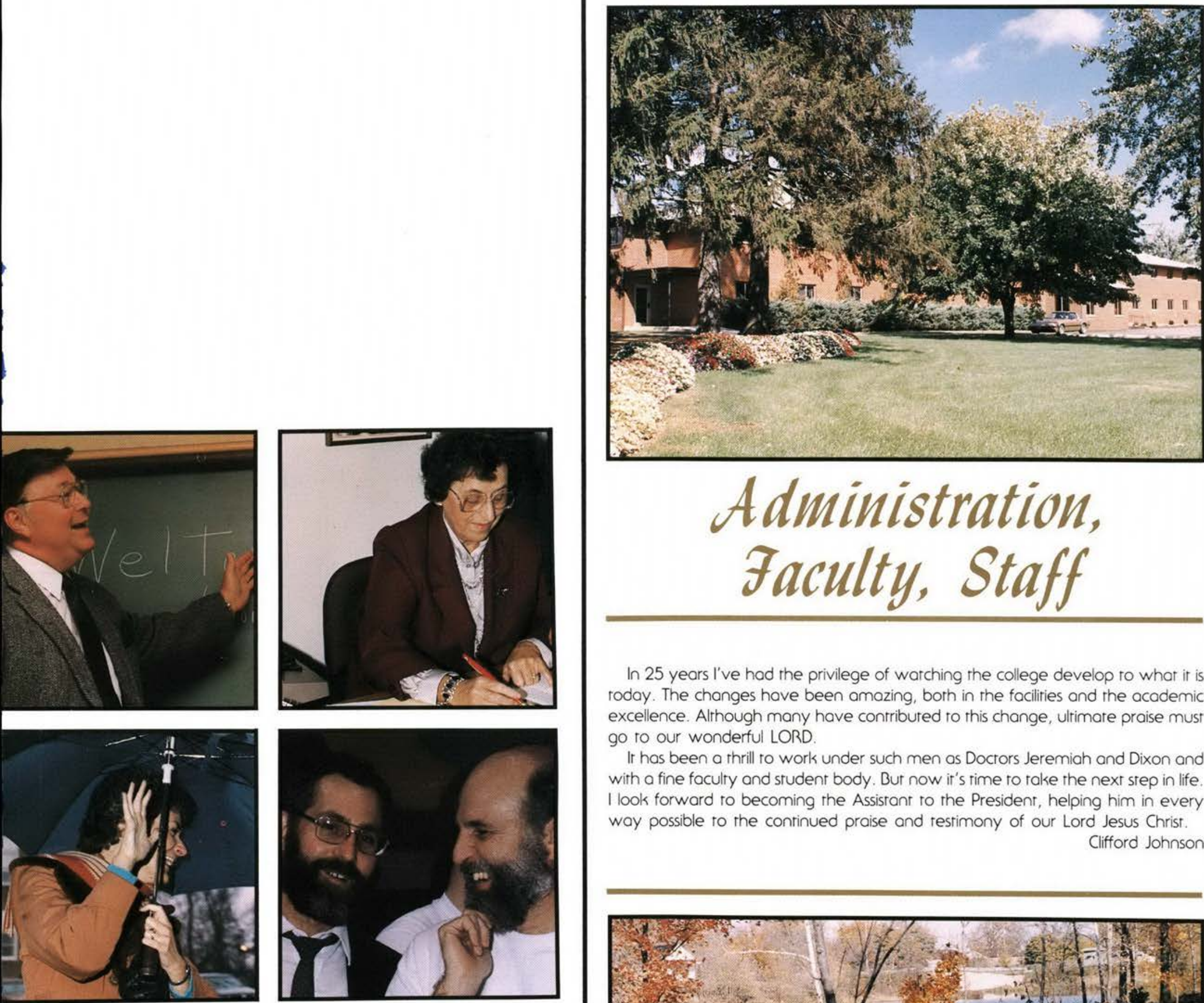

In 25 years I've had the privilege of warching the college develop to what it is roday. The changes have been amazing, borh in the facilities and the academic excellence. Although many have contributed to this change, ultimare praise must go to our wonderful LORD.

Ir has been a thrill to work under such men as Doctors Jeremiah and Dixon and with a fine faculty and student body. Bur now it's time to take the next step in life. I look forward to becoming the Assistant to the President, helping him in every way possible to the continued praise and testimony of our Lord Jesus Christ.

Clifford Johnson

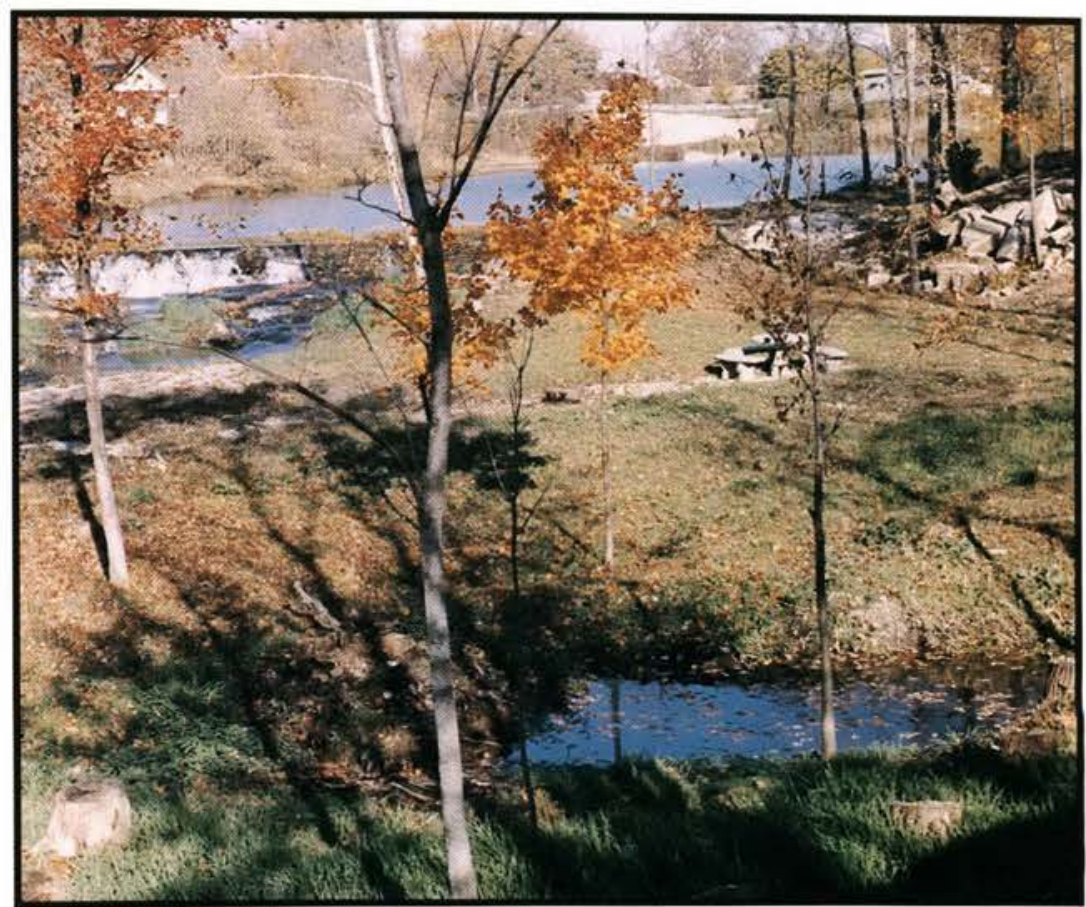


19 ohe acdedrus 19

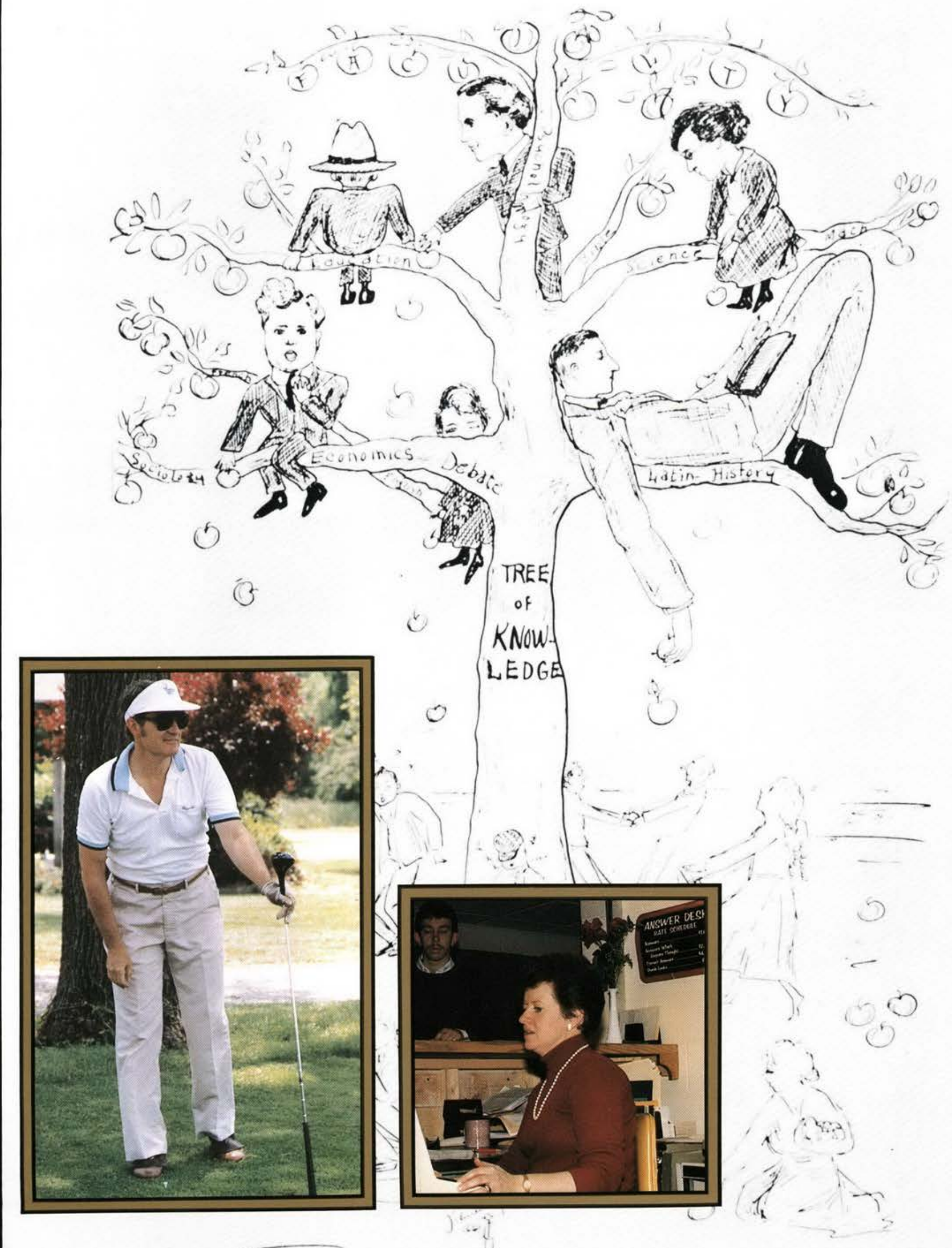




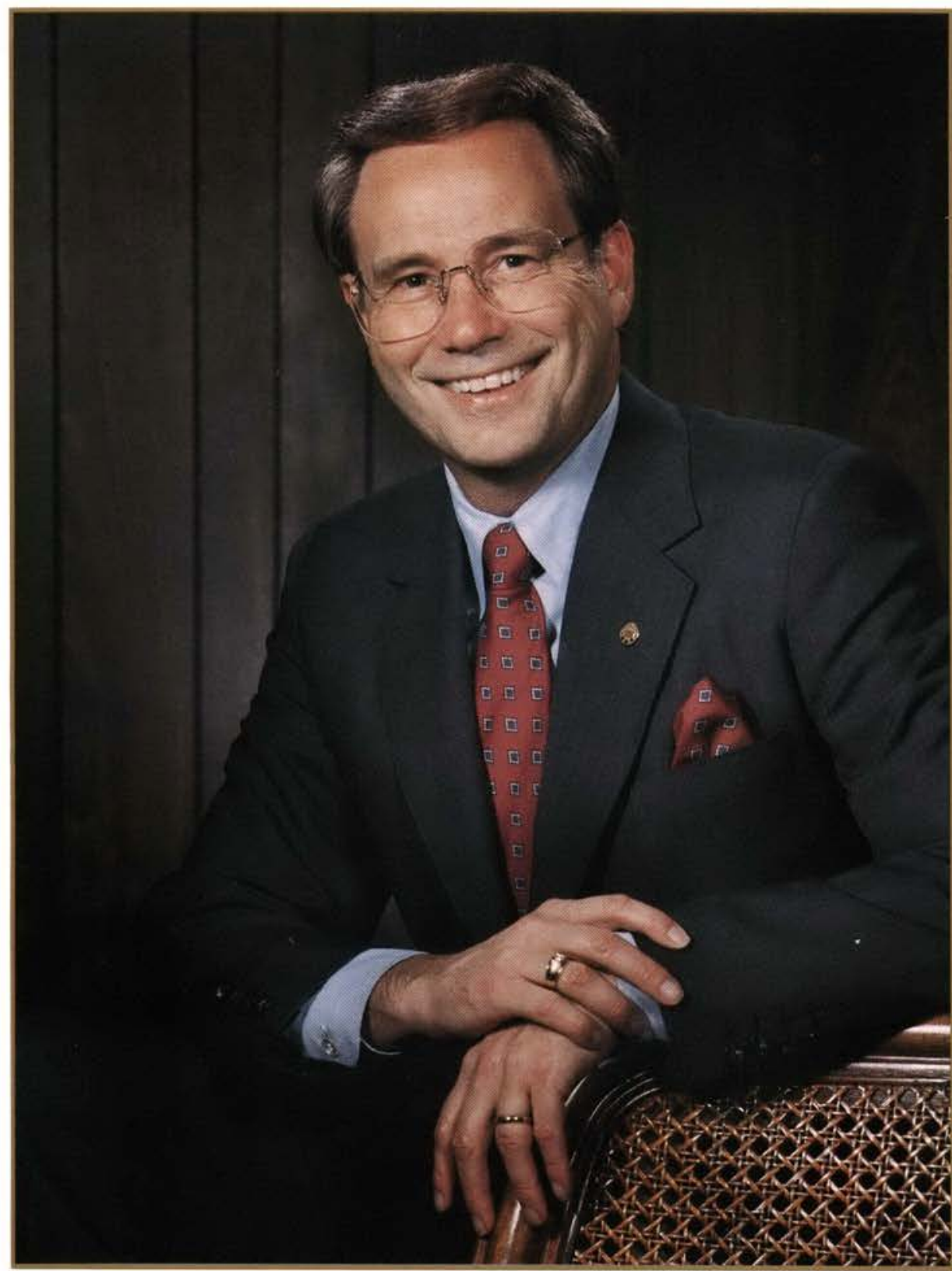

Paul H. Dixon, Ed.D., President

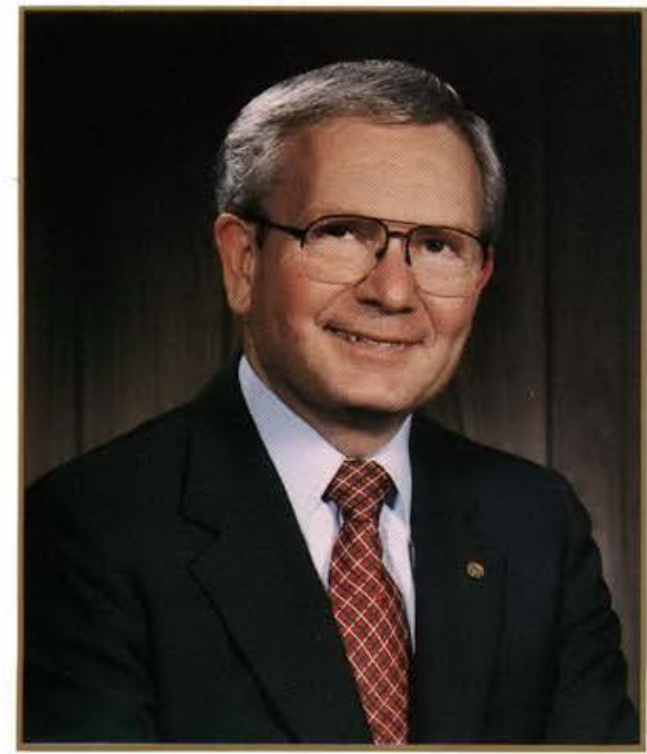

Kenneth St. Clair, M.S., C.P.A. Vice President for Business

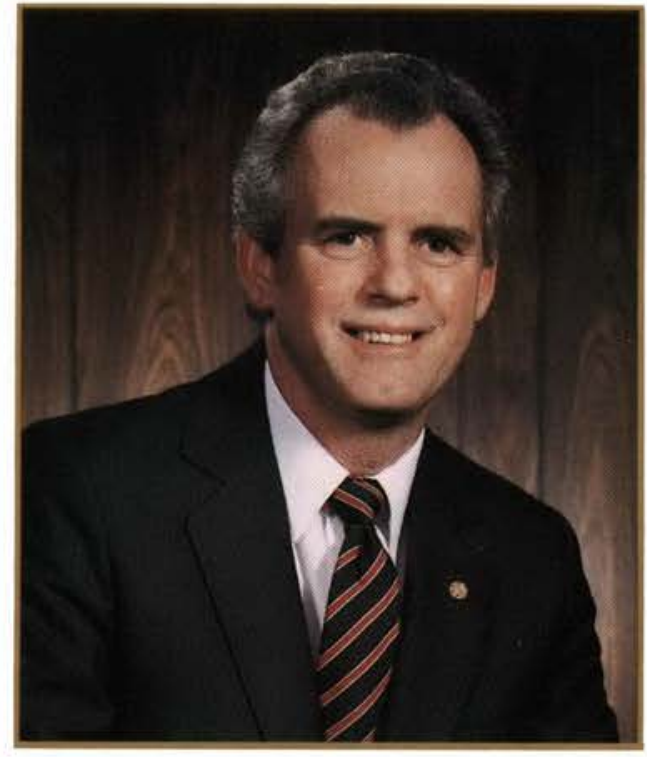

Harold Green, Th.B.

Vice President for Compus Ministries

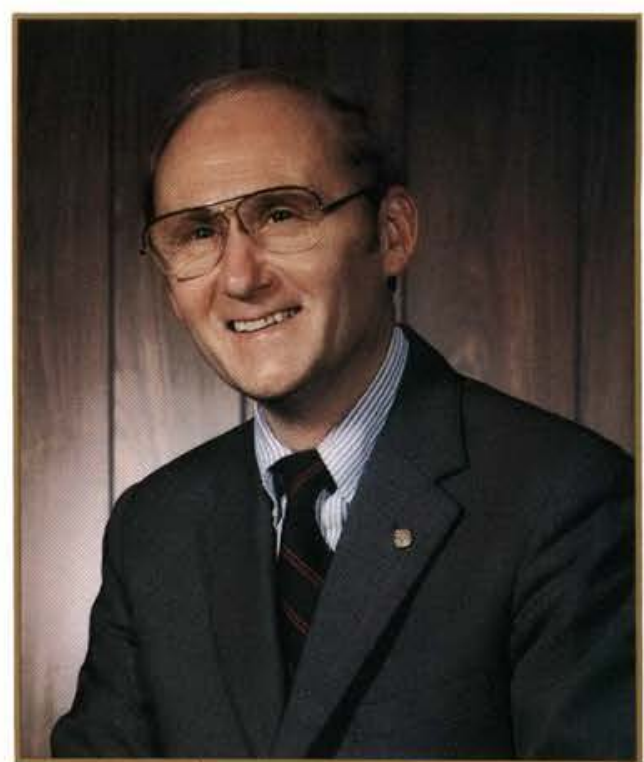

Donald Rickard, M.A.

Vice President for Srudent Services

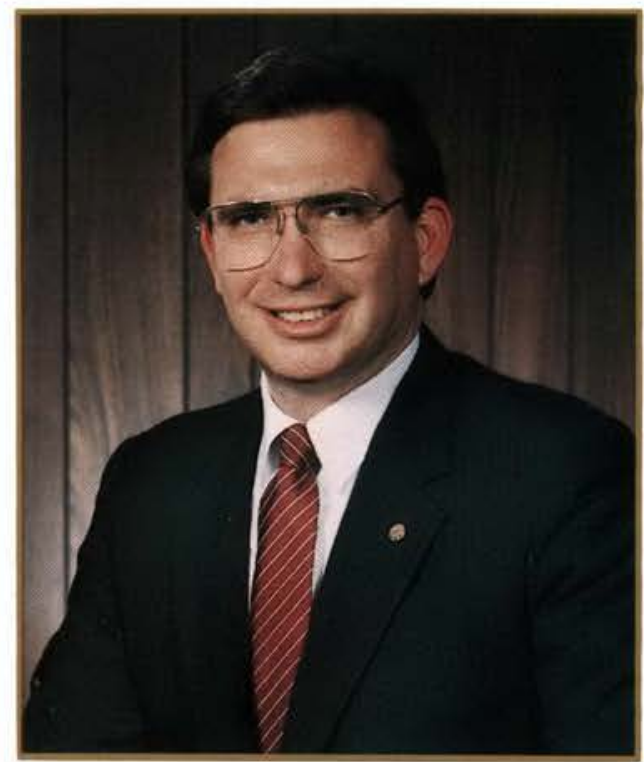

Martin Clark, Ed.D.

Vice President for Develooment

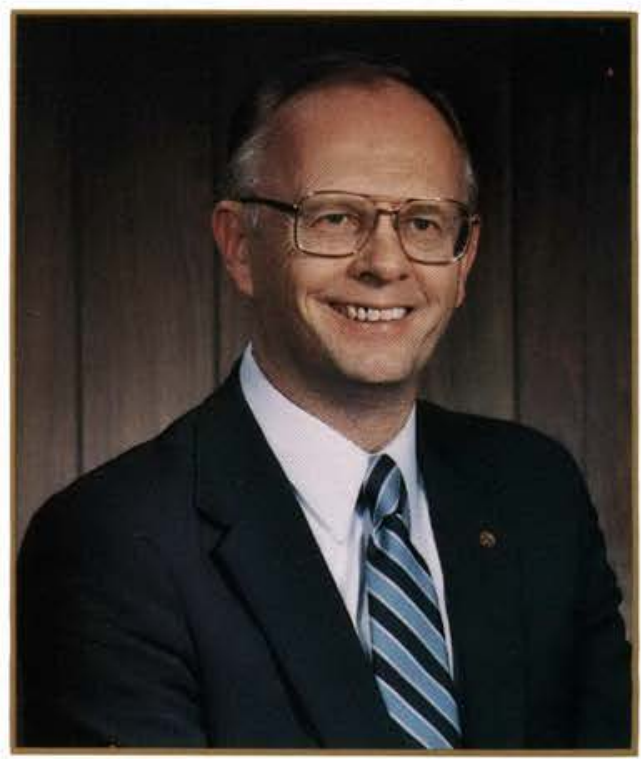

Clifford Johnson, D.Ed.

Academic Vice President 


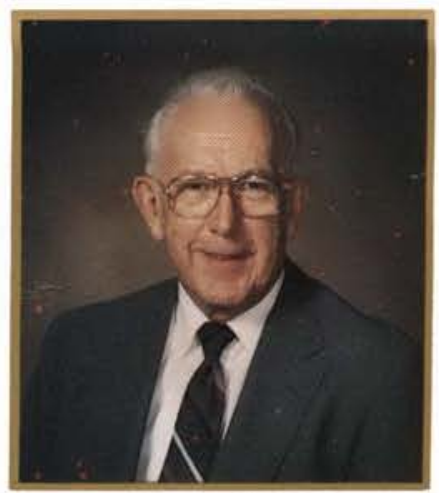

CHANCELLOR

James $T$. Jeremiah

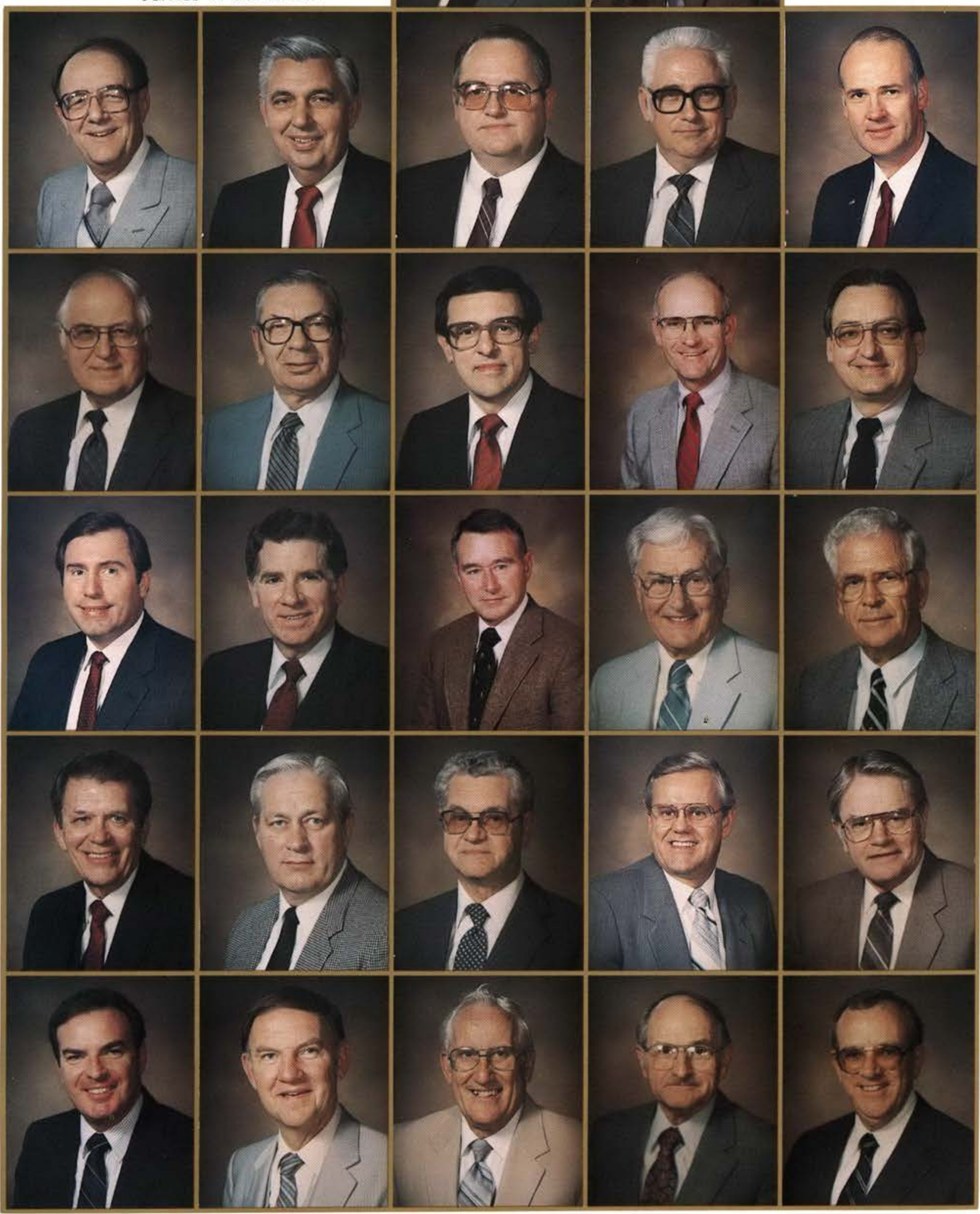

William A. Brock

Gilbert Brueckner

James B. Carraher

Jack Cline

Bill Commons

John Draxler

George Engelmann,

Chairman

Larry Ferzer,

Secretary

Joseph Godwin

Roy Guenin

Jim Henniger

Jack W. Jacobs

C.E. Gene Miller

J. Dale Murphy

George O'Bryon

Irwin Olson

Lynn Rogers

Gerald Smelser

Al Stevens

Robert L. Sumner

Paul Tassel

Donald L. Tyler,

Vice Chairman

Earl Umbaugh

Paul Vernier

W. Thomas Younger 
Jean Fisher, M.A. Assoc. Prof. of Chr. Ed., 30 yrs Harmon Bergen, M.A. Assoc. Prof. of For. Lang., 29 yrs

Donald Callan, Ph.D. Prof. of Phys. Ed.. Chmn., 27 yrs Robert Gromacki, Th.D. Prot. of Bible \& Greek. Chmn., 27

Edward Spencer. M.A. Prof. of English, 25 yrs

Edward Greenwood, D.A. Prof. of English, 24 yrs Daniel Wetzel, Ph.D. Prof. of Physics 6 Math., 24 yrs Donald Baumann, Ph.D. Prof. of Bio. G Chem.. Chmn.. 23

Stanley Ballard, Ph.D. Prof. of Psych., Chmn., 22 yrs

David Matson, Ph.D. Prof. of Music. Chmn., 22 yrs

Murray Murdoch, Ph.D. Prof. of History, Chmn., 22 yrs Merlin Ager, Ph.D. Prof. of Sec. Ed., Chmn., 20 yrs Jack Riggs, Th.D. Prof. of Bible, 20 yrs Ronald Grosh, Ph.D. Assoc. Prof. of English. 19 yrs Larry Helmick, Ph.D. Prof. of Chemistry, 19 yrs

James Phipps, Ph.D. Prof. of Speech, Chmn., 19 yrs Lyle Anderson, Ph.D. Assoc. Prof. of Vocal Music. 17 yrs Joseph Halsey, M.Ed. Assoc. Prof. of Pol. Sci., 17 yrs Elvin King. M.Ed. Assoc. Prof. of Phys. Ed., 17 yrs Robert Abbas, Ph.D. Prof. of Psychology, 16 yrs

Pat Dixon, M.Ed. Assoc. Prof. of English, 16 yrs James McGoldrick, Ph.D.

Prof. of History, 14 yrs

Pamela Diehl, Ph.D. Assoc. Prof. of Phys. Ed., 13 yrs John McGillivray, M.S. Assoc. Prof. of Phys. Ed., 13 yrs Richard Baldwin, M.B.A. Ass't. Prof. of Mgmt., 12 yrs

Charles Ellington, Ph.D. Prof. of Vocal Music. 12 yrs Raymond Bartholomew,

Ph.D.

Prof. of English. Chmn., 11 yrs Edwin Braithwaite, Ph.D.

Ass't. Prof. of Math., 11 yrs

Richard Durham, Th.D. Prof. of Bible and Greek, 11 yrs

Lila Seaman, Ph.D. Assoc. Prof. of Education. 11 yrs
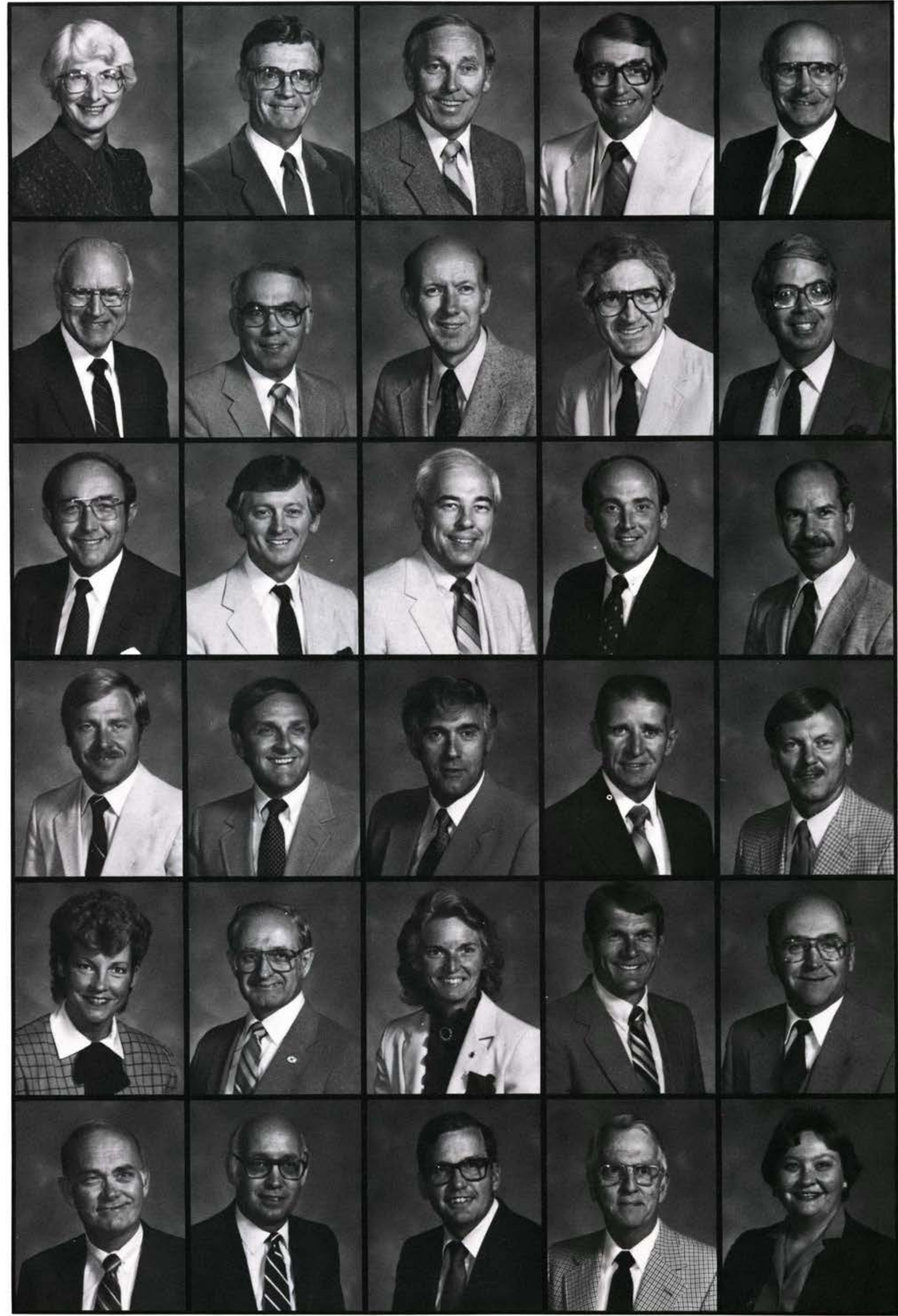


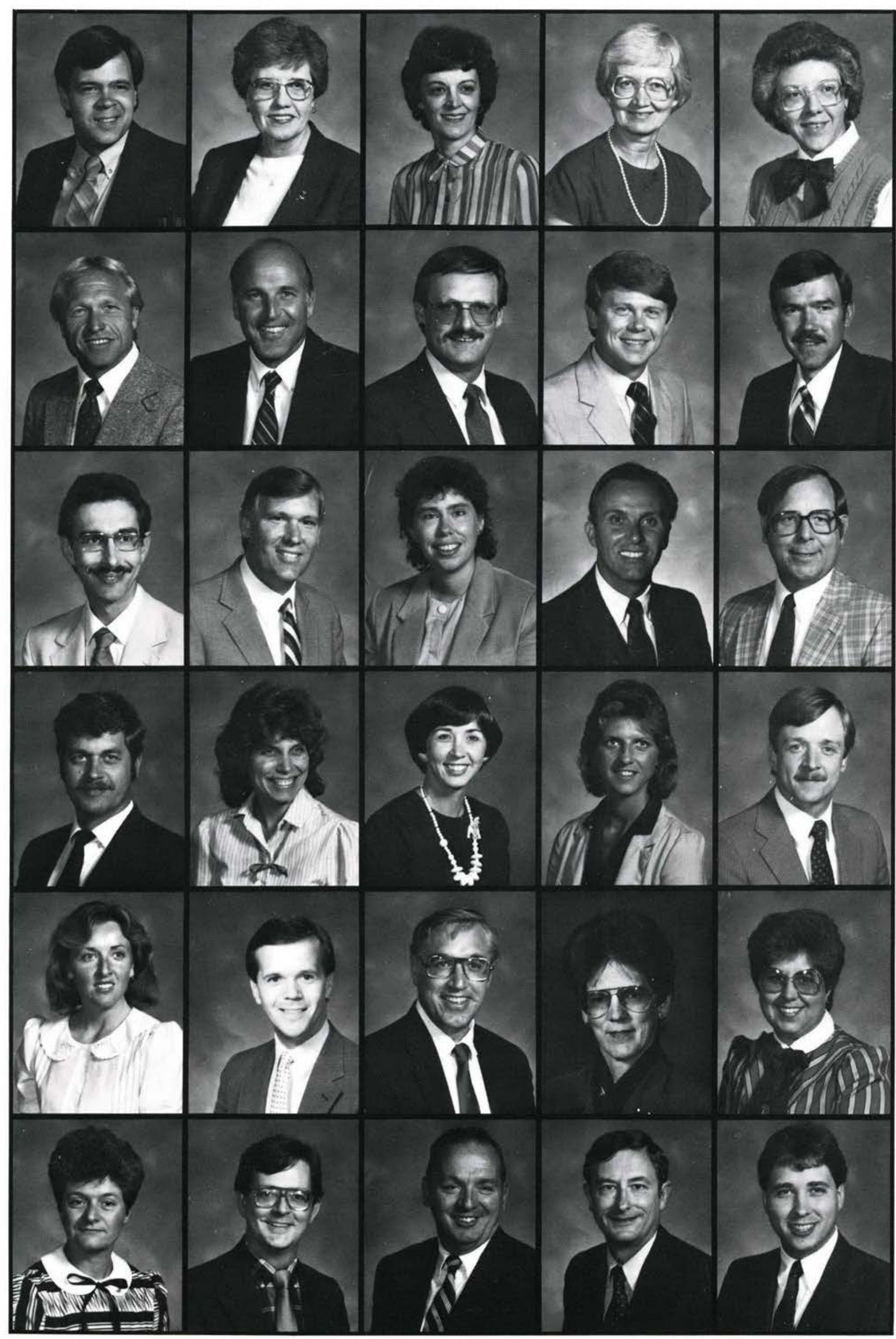

J. Wesley Baker, M.A.

Assoc. Prof. of Speech \& Brdcstg.. 10 yrs Helen Hall, M.A.

Assoc. Prof. of Education, 10 yrs

Beverly Monroe, Ph.D.

Assoc. Prof. of Education, 10 yrs

Sue Baker, M.A.

Assoc. Prof. of Education, 9 yrs

Barbara Loach, M.A.

Ass't. Prof. of For. Lang.. 9 yrs

Terry Phipps, M.S.

Assoc. Prof. of Bio. Sci., 9 yrs

Ronald Walker, D.B.A.

Assoc. Prof. of Mgmt., Chmn.., 9 yrs

Michael DiCuirci, M.M.

Assoc. Prof. of Instr. Music, 8 yrs

Charles Dolph, Ph.D.

Assoc. Prof. of Psych.. 8 yrs

John Silvius, Ph.D.

Prof. of Bio. Science, 8 yrs

Dennis Flentge, Ph.D. Assoc. Prof. of Chemistry, 7 yrs

Robert Parr, M.A.

Ass't. Prof. of Sociology, 7 yrs

Sarah Smith, Ph.D.

Assoc. Prof. of Accounting, 7 yrs

David Warren, Th.M.

Assoc. Prof. of Bible G Greek. 7 yrs

Omer Bonenberger, D.Ed.

Assoc. Prof. of Educotion. 6 yrs

Leroy Eimers, Ph.D.

Assoc. Prof. of Physics 6 Math., 6 yrs

Sharon Eimers, M.Sp.Ed.

Assoc. Prof. of Educotion, 6 yrs

Sandra Harner, M.A.

Ass't. Prof. of English. 6 yrs

Betsy Kempf, M.A.

Ass't. Prof. of Sec. Science, 6 yrs

Kurt Moreland, M.A.

Ass't. Prof. of Comm. Arts. 6 yts

Anne Rich, M.Acct., C.P.A.

Ass't. Prof. of Accrg.. 6 yrs

David Robey, M.A.

Ass't. Prot. of Comm. Arts $G$ Drama. 6

yrs

Galen Smith, M.S.

Ass't. Prof. of Economics, 6 yrs Irene Alyn, Ph.D.

Prof. of Nursing. Chmn., 5 yrs

Janet Baver, M.S.

Ass't. Prof. of Nursing. 5 yrs

Elaine Brown, M.S.Ed.

Ass't. Prof. of Phys. Ed., 5 yrs

Charles Clevenger, D.M.A.

Ass't. Prof. of Piano, 5 yrs

Clifford Fawcett, D.B.A.

Prof. of Monagement, 5 yrs

Marinus Hazen, M.B.A.

Assoc. Prof. of Business, 5 yrs

James Leightenheimer, B.A.

Instr. of Brdestg.. 5 yrs 
Rex Rogers, Ph.D. Ass't. Prof. of Pol. Sci., Dir. of Planning. 5 yrs

Martha Johnson, M.A. Ass't. Prof. of Sec. Sci., 4 yrs

Mark Klimek, M.S.N. Ass't. Prof. of Nursing. 4 yrs

Gary Percesepe, Ph.D. Ass't. Prof. of Philosophy, 4 yrs

Ruth Slocum, M.S. Ass't. Prof. of Nursing, 4 yrs

Stephen Young, B.A. Coach and Instr. of Phys. Ed., 4 yrs

Lois Baker, M.S.N. Ass't. Prof. of Nursing. 3 yrs

Dan Estes, Th.M. Ass't. Prof. of Bible, 3 yrs

Michael Lopez, Ph.D. Ass't. Prof. of Comm. Arts, 3 yrs

Douglas Miller, Ph.D. Ass't. Prot. of Chemistry, 3 yrs

David Rotman, M.A. Ass't. Prof. of Bus., Dir. of Comp. Serv.. 3 yrs

Maureen Salladay, M.P.H.

Ass't. Prof. of Nursing. 3 yrs

Charlotte Crawford, M.S.N.

Ass't. Prof. of Nursing. 2 yrs

Floyd Elmore, Th.M. Ass't. Prof. of Bible. 2 yrs

Deborah Haffey, M.M. Ass't. Prof. of Comm. Arts, 1 yr

NOT PICTURED:

Allen Monroe, Ph.D. Prof. of Social Science, 21 yrs

Dwayne Frank, D.Ed.

Prof. of Education, 19 yrs

Laurence Killian, M.S. Assoc. Prof. of Bio. Sci., 19 yrs

Phillip McClure, M.M Ass't. Prof. of Piano, 13 yrs

Charles Pagnard, M.M

Ass't. Prof. of Inst. Music, 10 yrs

Debra Brown, M.B.A. Ass't. Prof. of Marketing, 5 yrs
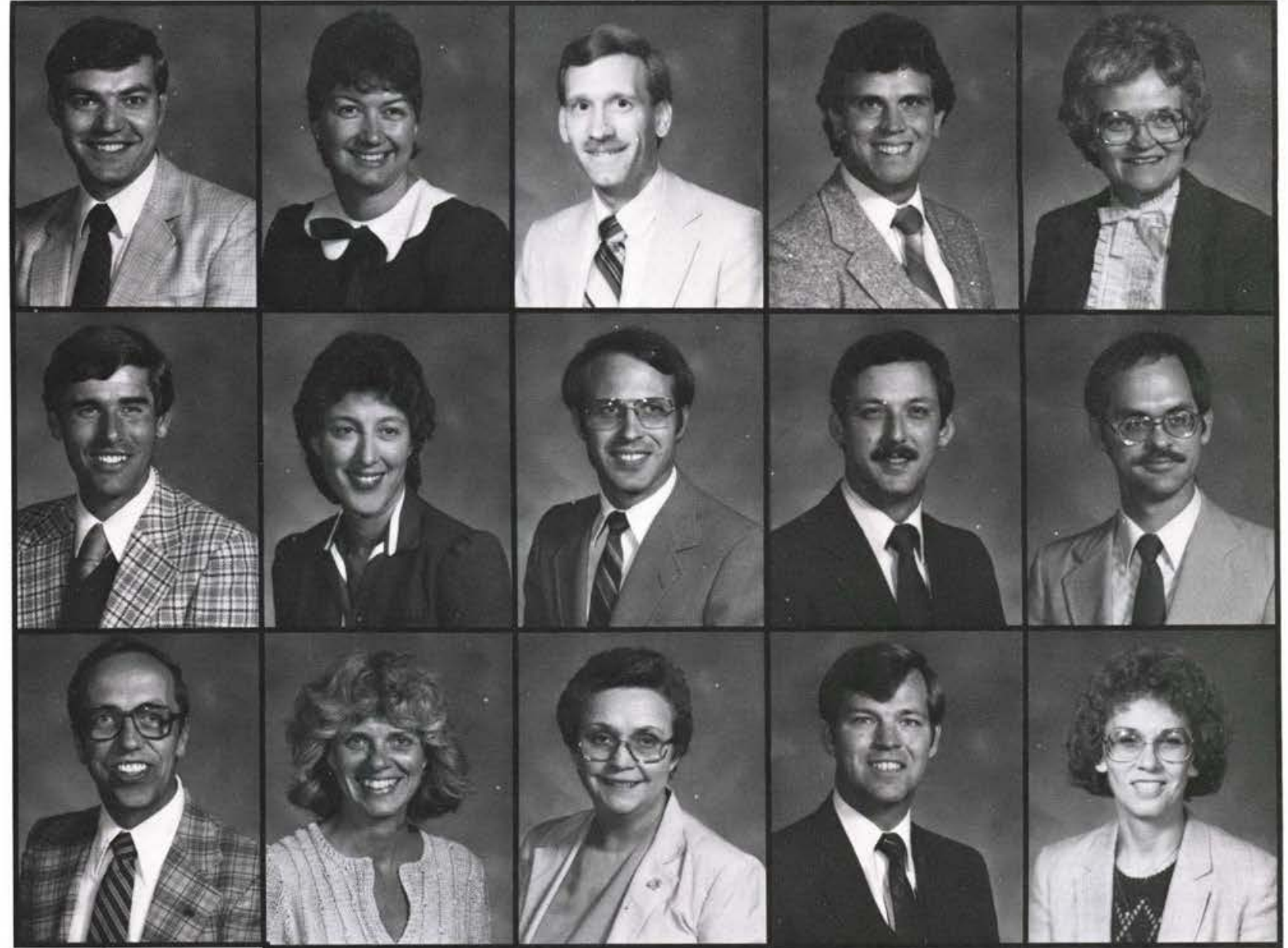

Evan Hellwig, B.S. Ass't. Prof. of Phys. Ed., 1 yr

Lee Kantenwein. Th.D. Assoc. Prof. of Bible, 1 y

David Nicholas, M.A Ass't. Prof. of C.I.S., 1 y

Larry Smith, M.Div. Ass't. Prof. of For. Lang.. 1 yr
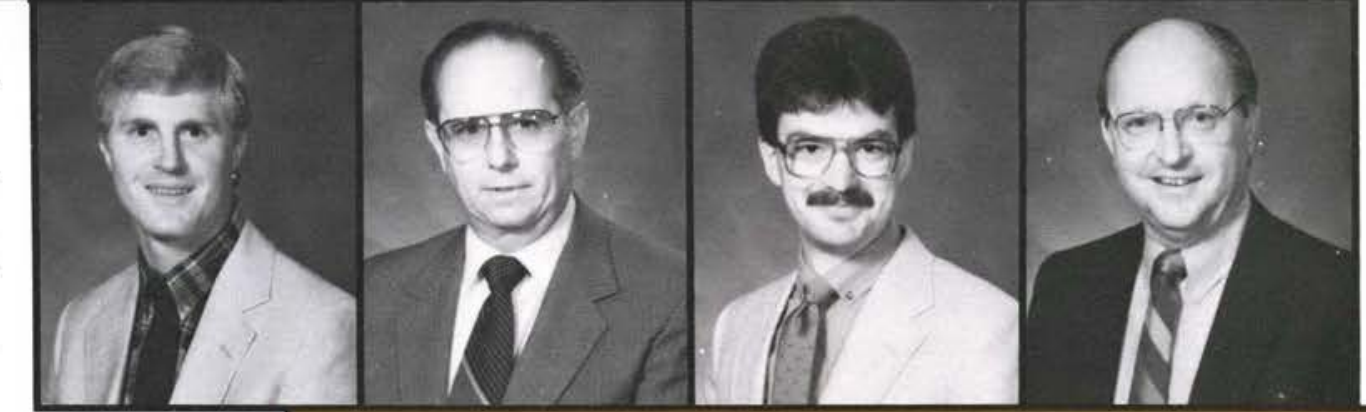
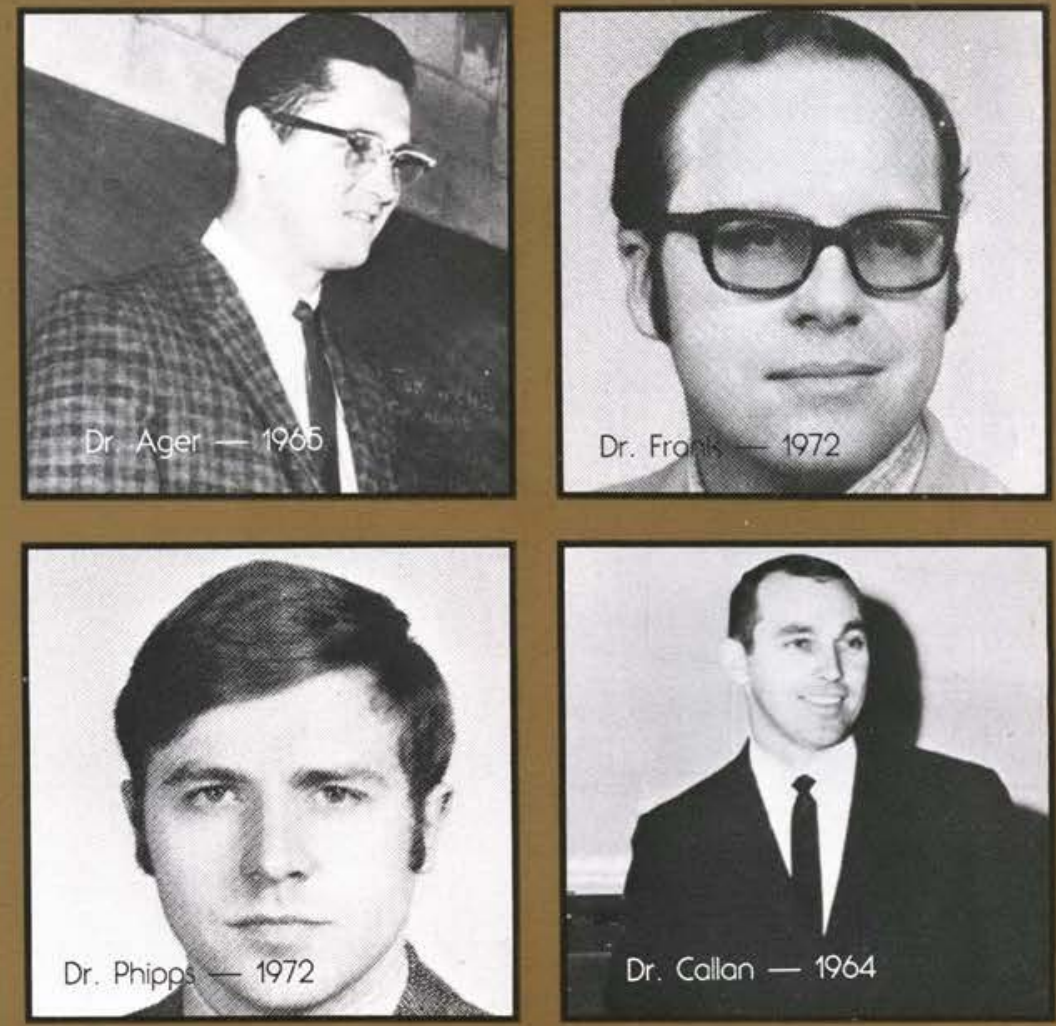

Dr. Callan - 1964 

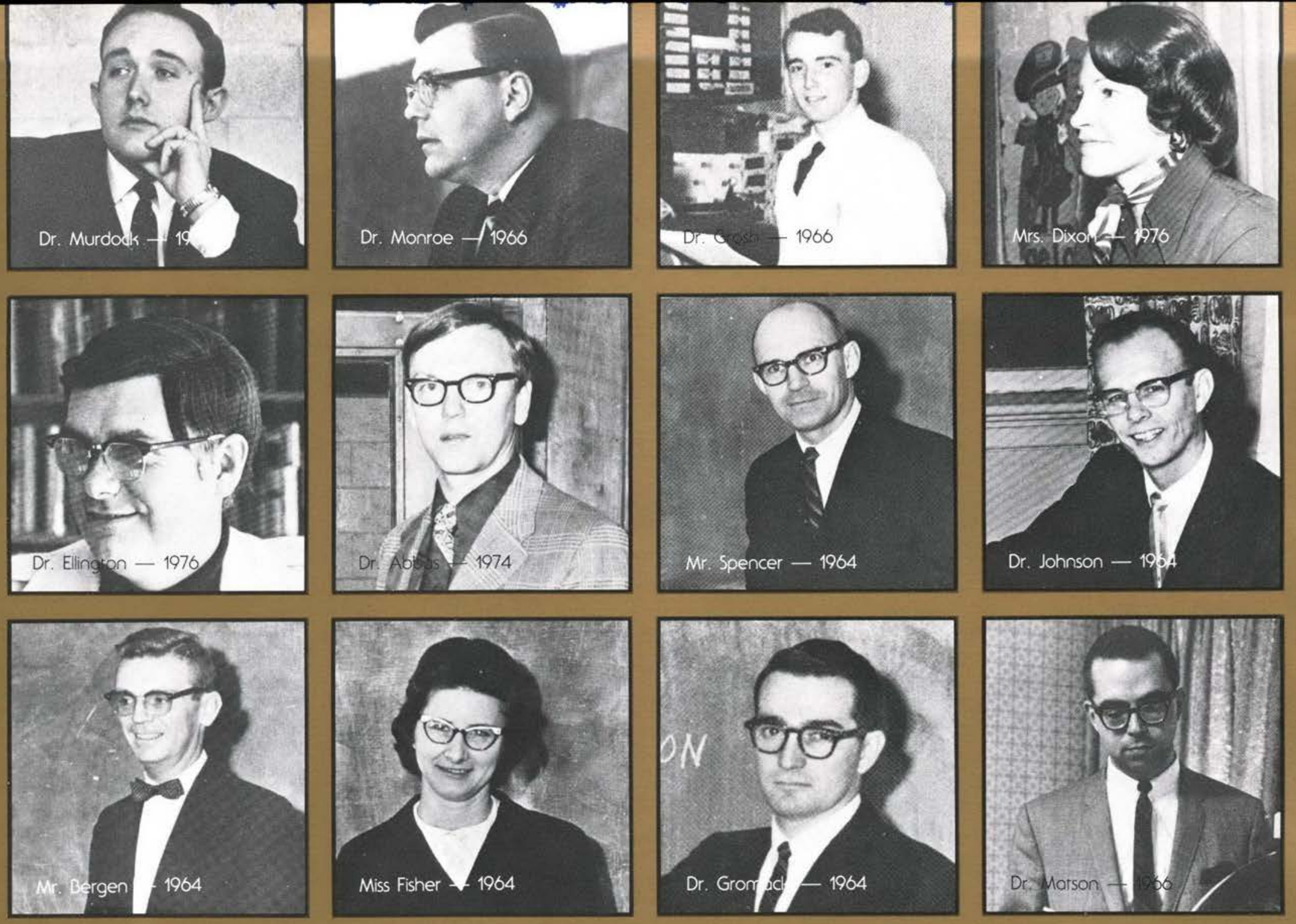

\section{Administrative Personnel}

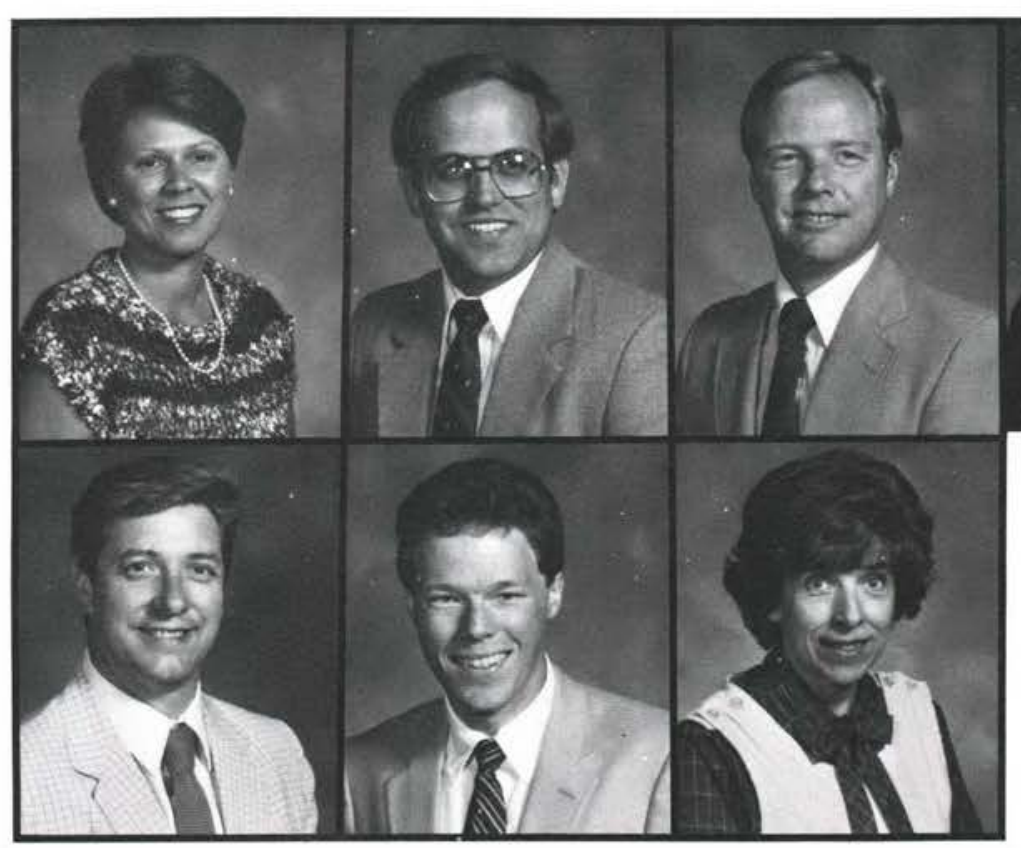

Not Pictured:

Patricia Bates, M.A.

Dean of Women 


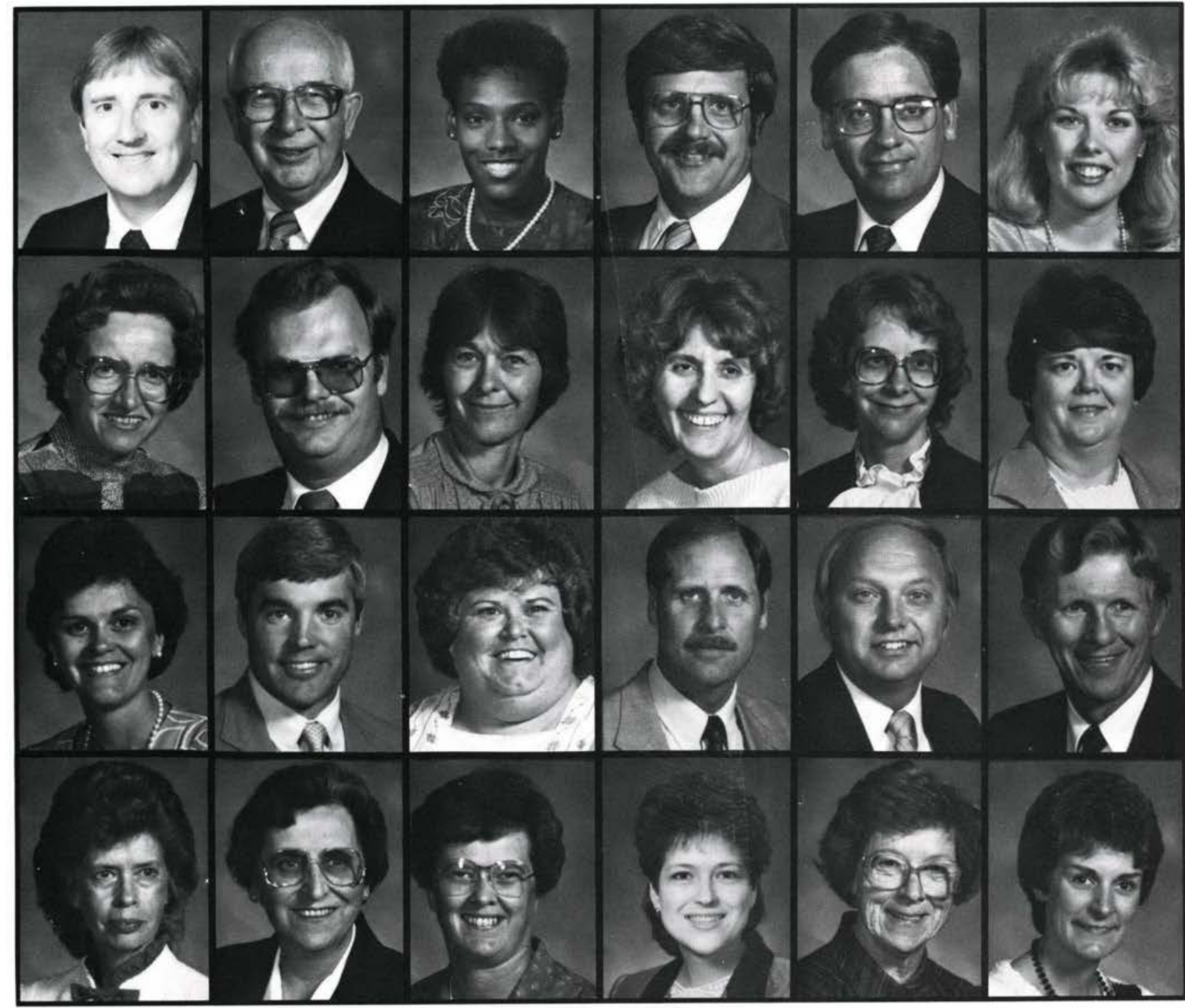

Russell Clark

Harry Cole

Angela Cooke

Paul Cope

Ronald Coriell

Diane Cornish

Sherry Coy

Jeffrey Cunningham

Jeannie Day

Norma Deranek

Linda Divan

Virginia Fields

Judith Fires

Robert Fires

Nancy Fissel

David Gaffne

Paul Gathany

Dovid Gidley

Irene Gidley

May Greenwood

Barbara Henderson

Kelly Herbert

Agnes Howell

Patricia Huber
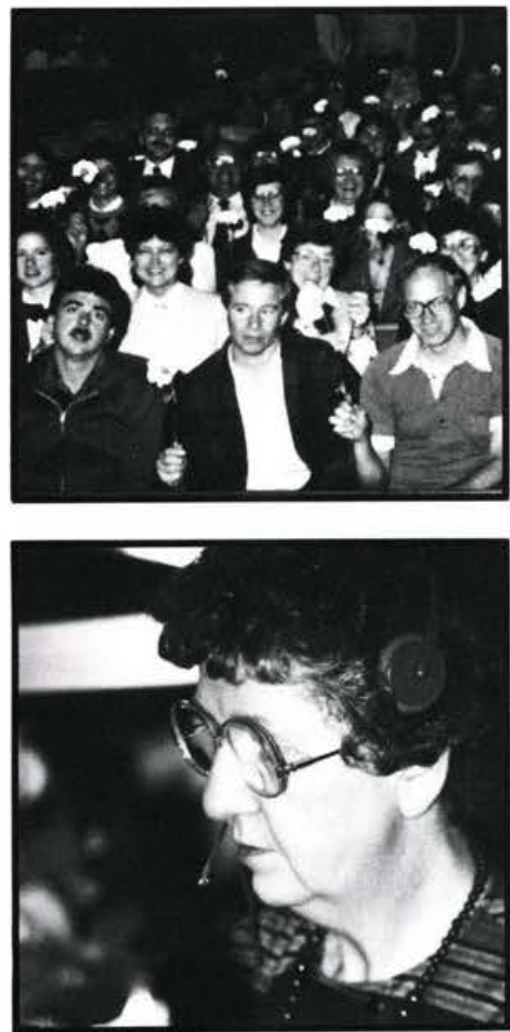
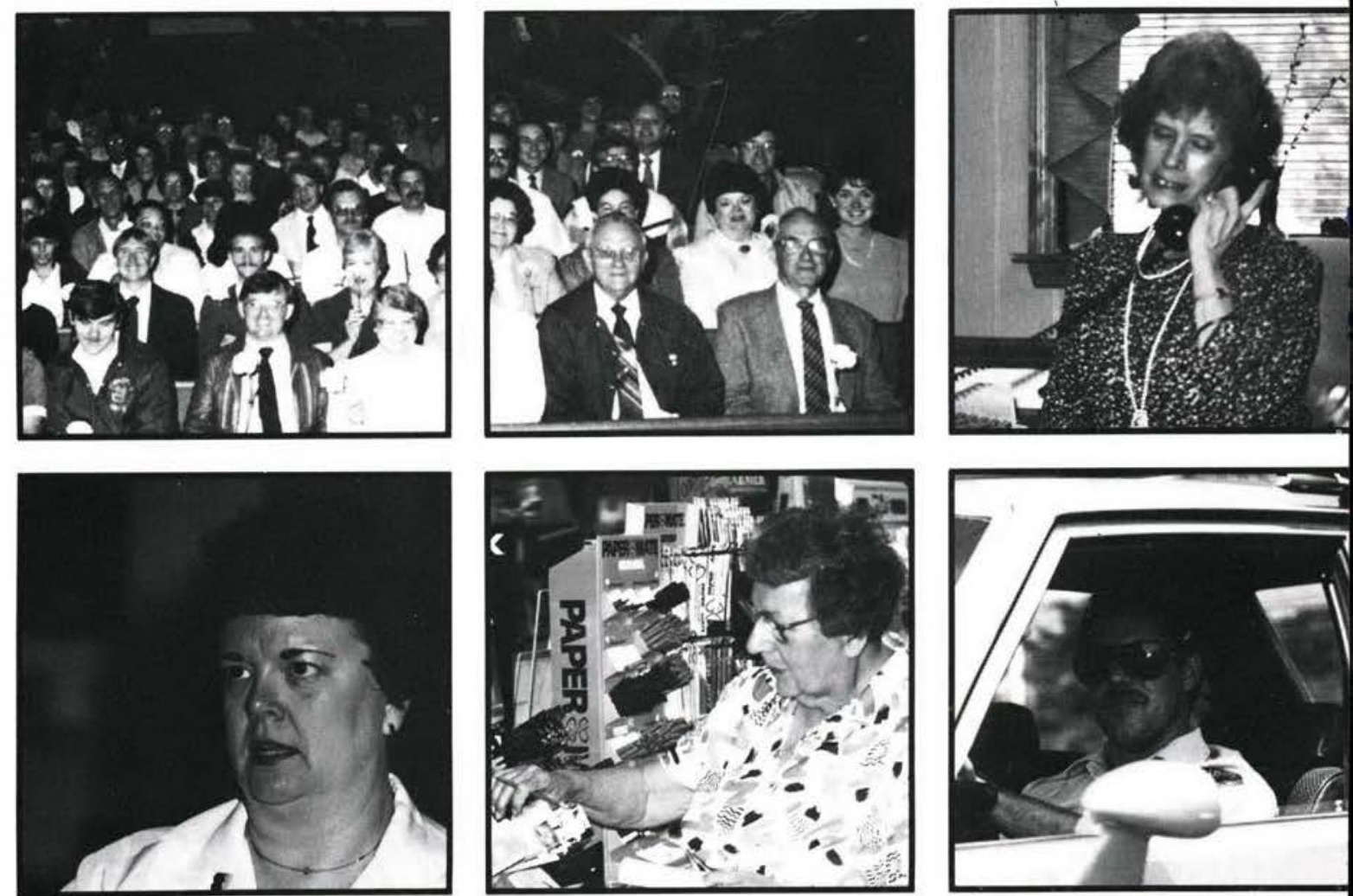

Staff 155 


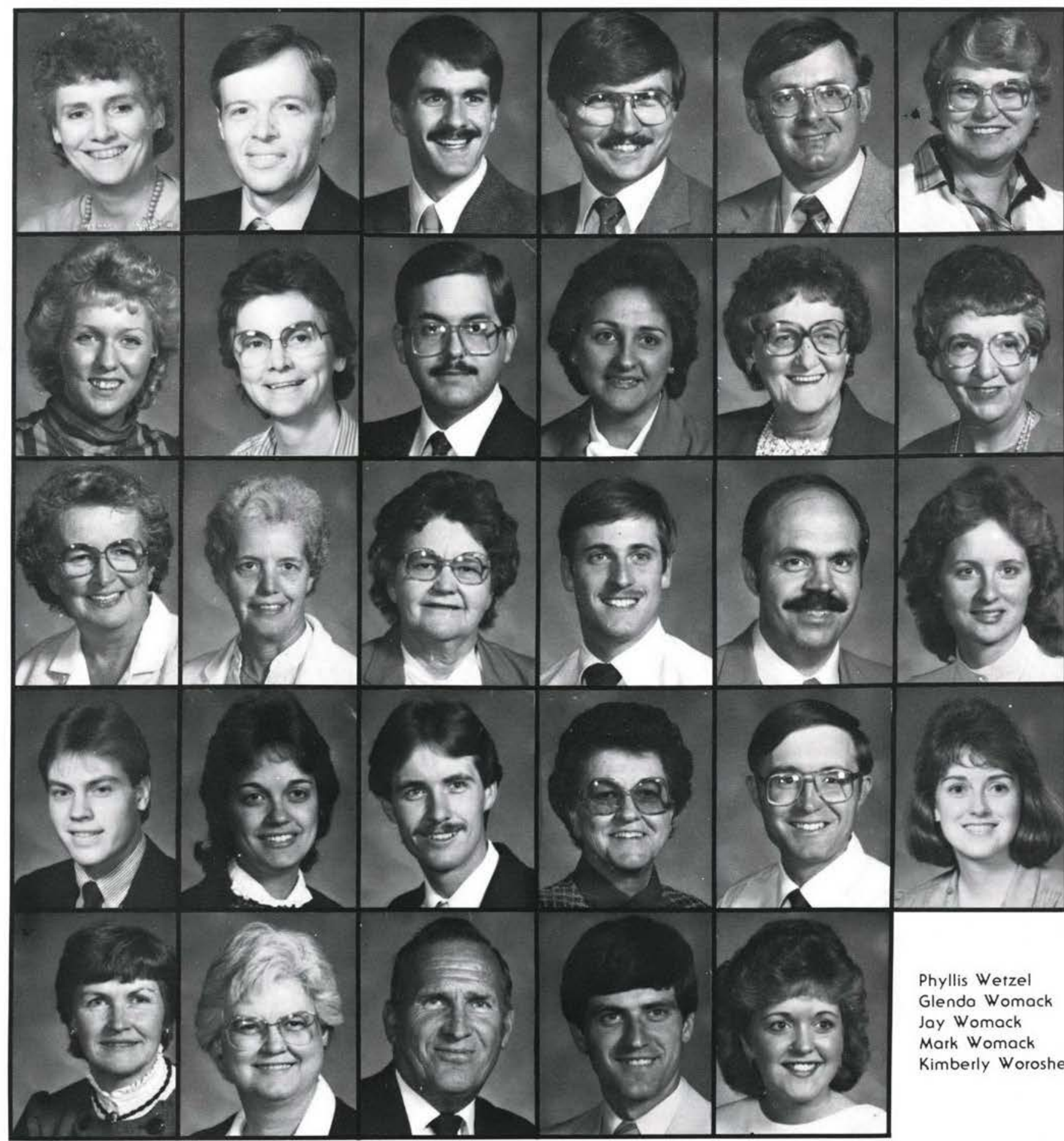

Peg Rickard

Robert Rohm

David Samuel

Douglas Sjoquist

John Skillman

Sylvia Skillman

Valerie Smallman Beverly Smith John R. Smith

Michele Smith

Dorothy Spencer

Ida St.Clair

Joan Street

Eleanor Taylor

Virginia Taylor

Stephen Thompson

John Tocknell

Lori Trautman

Les Troyer

Bonnie Vickman

Jeffrey Vickman

Kitty Vine

Paul Ware

Sherry Watson
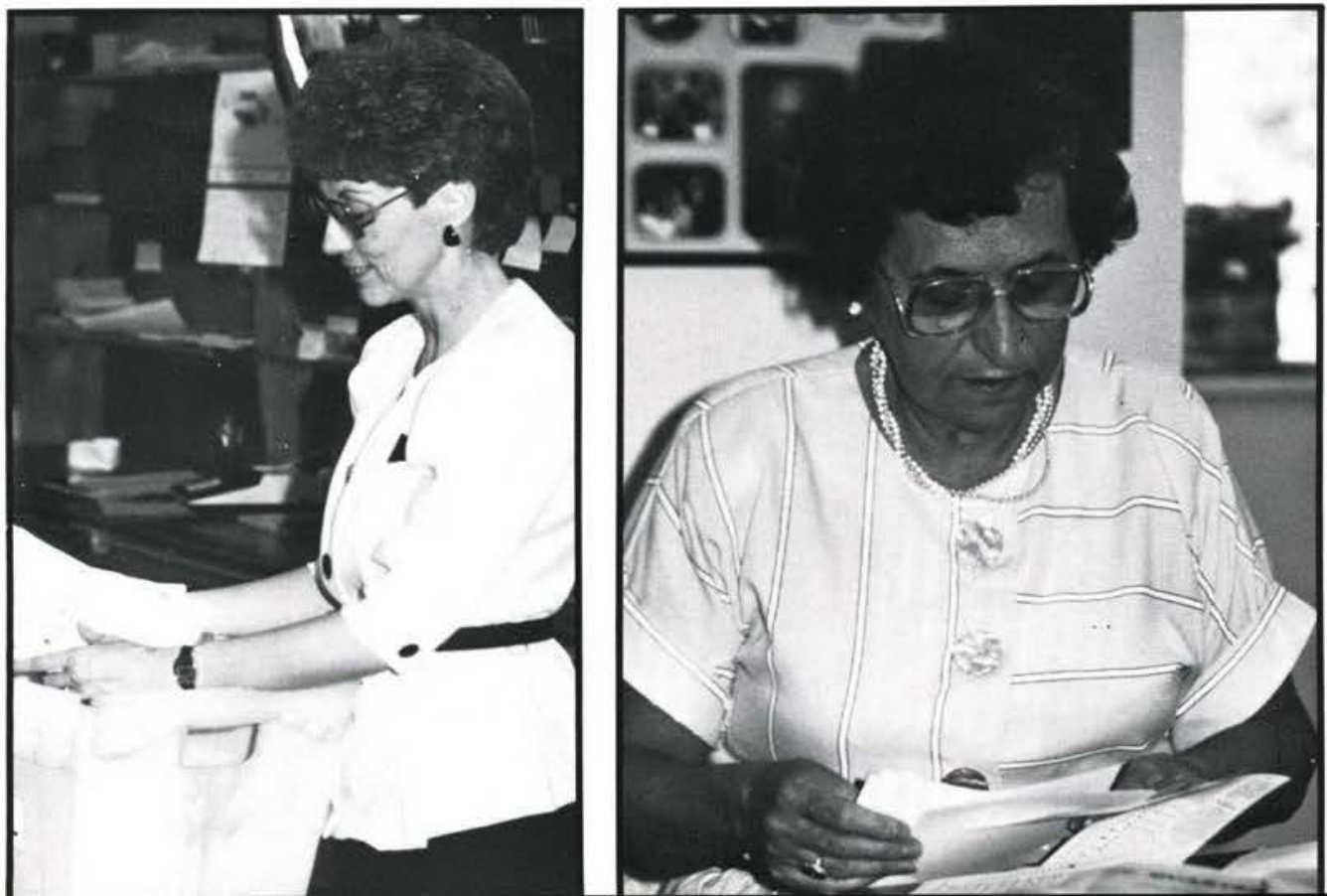


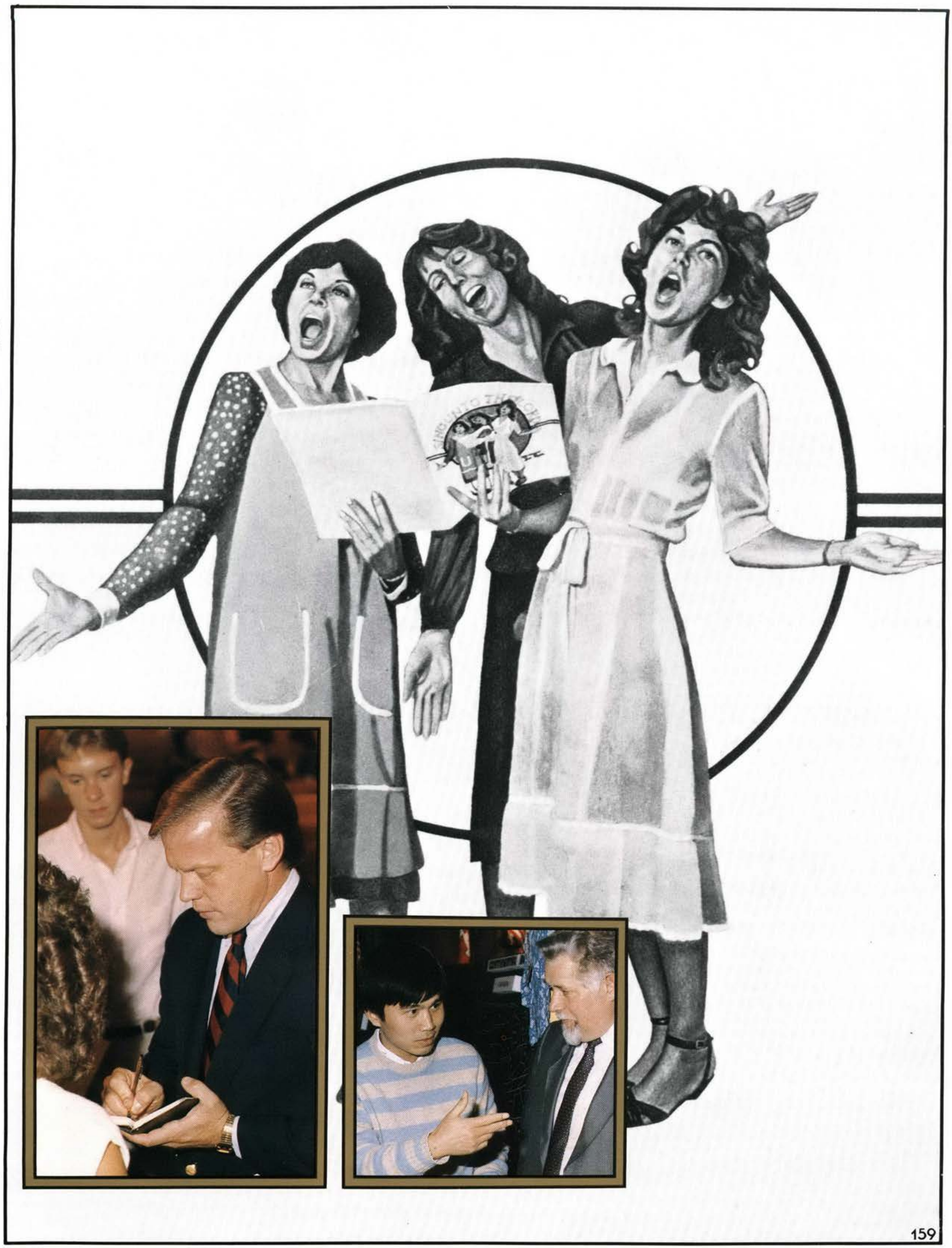




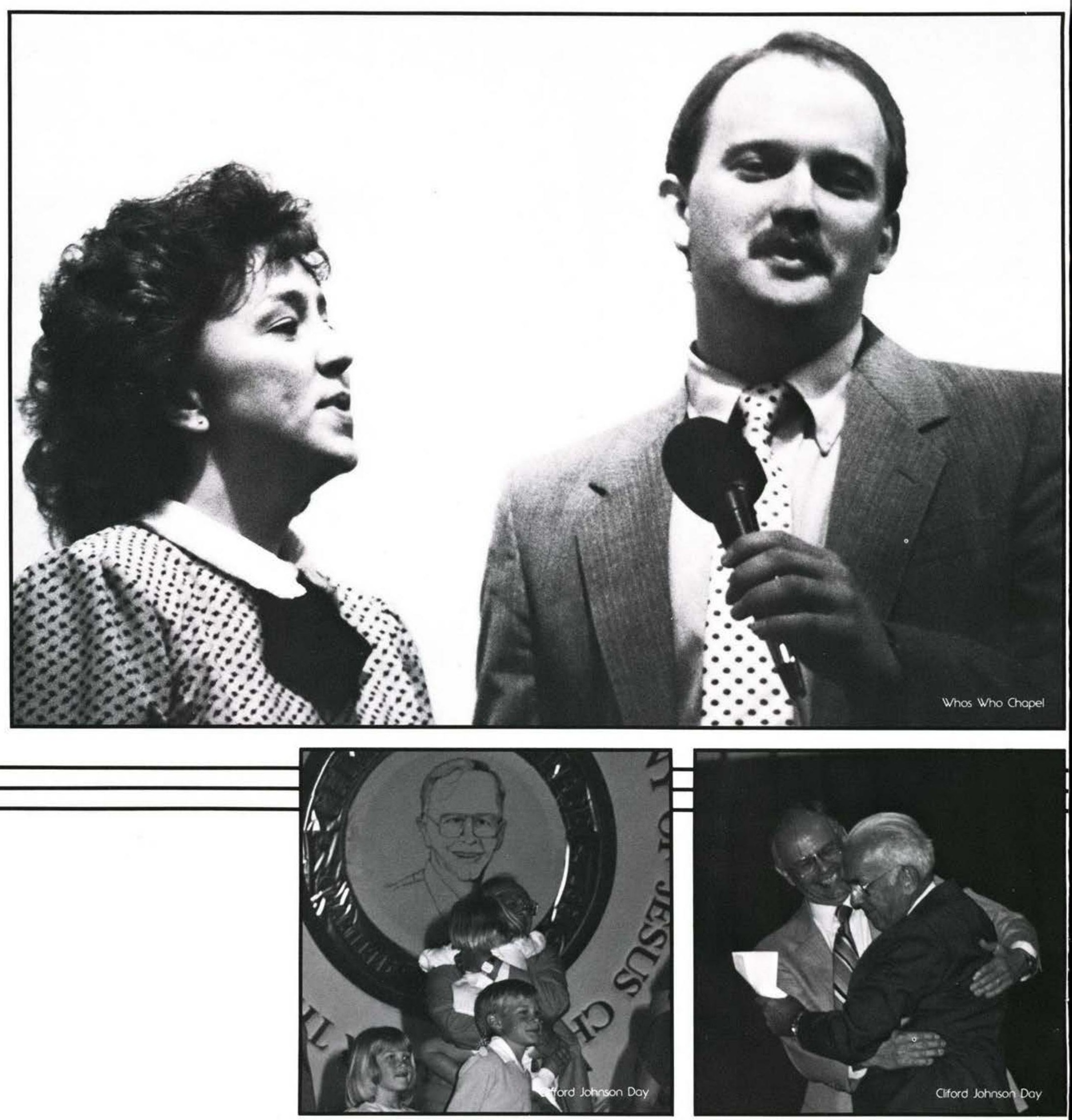

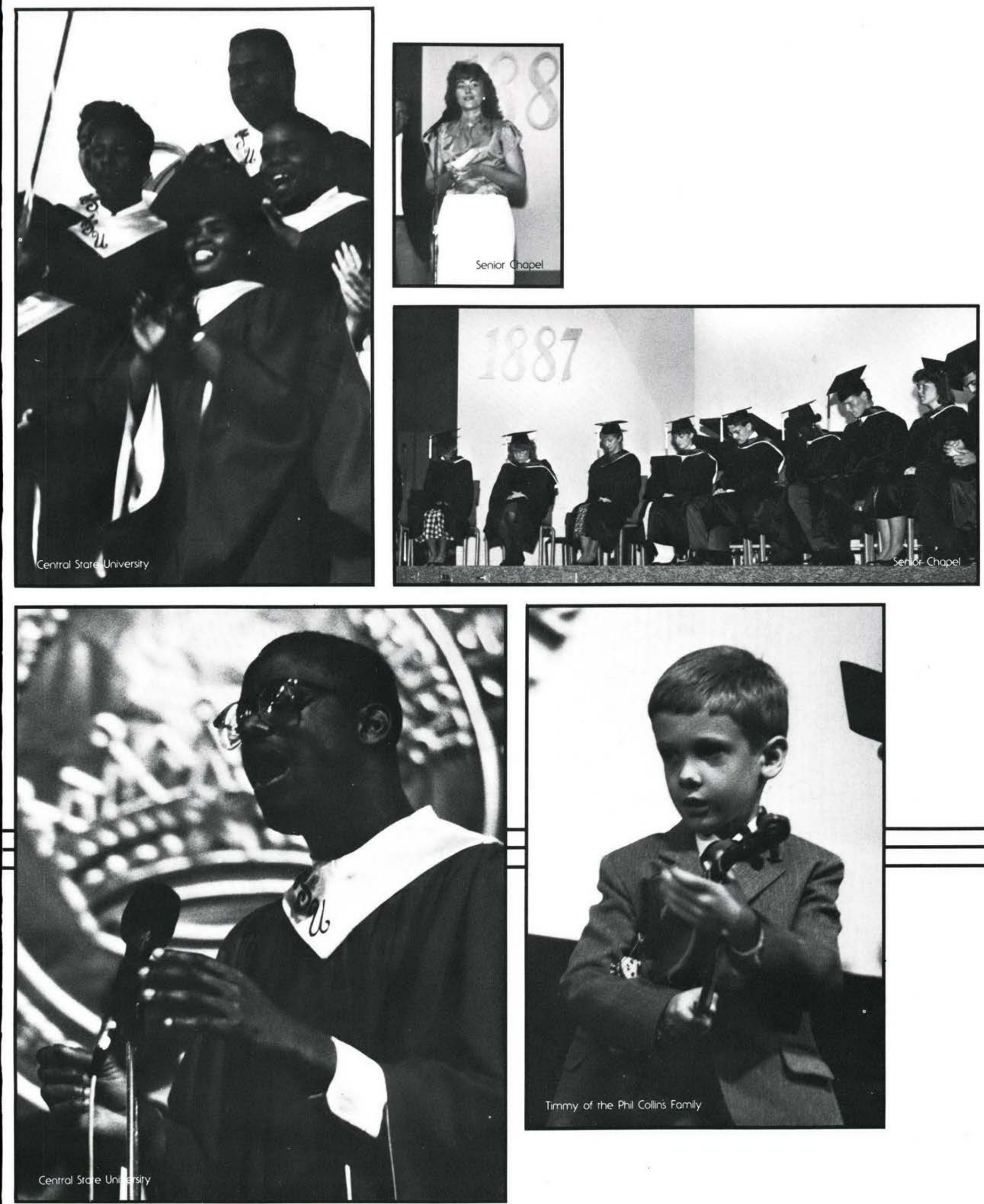

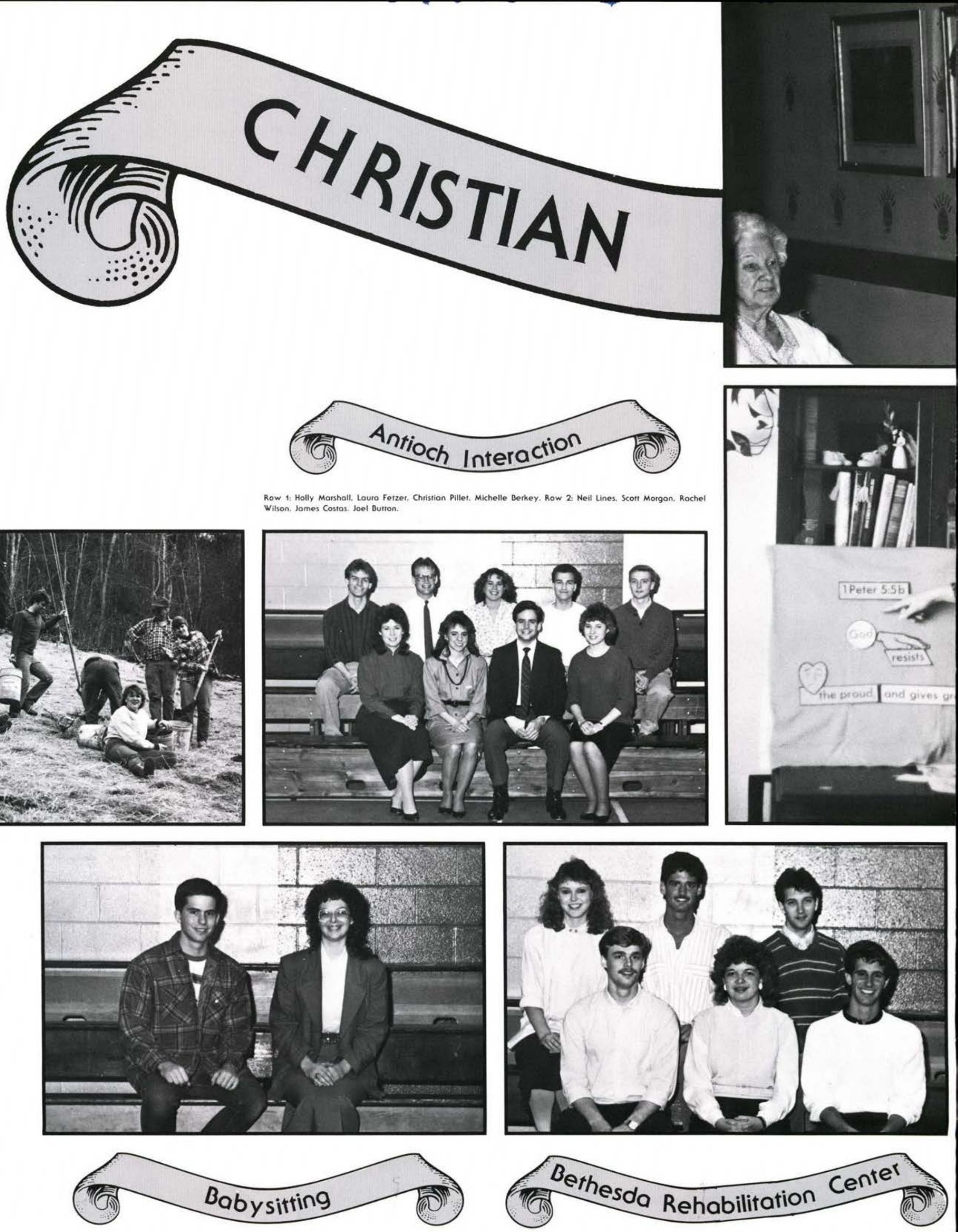

Bob Hildebrand. Lito Seest 


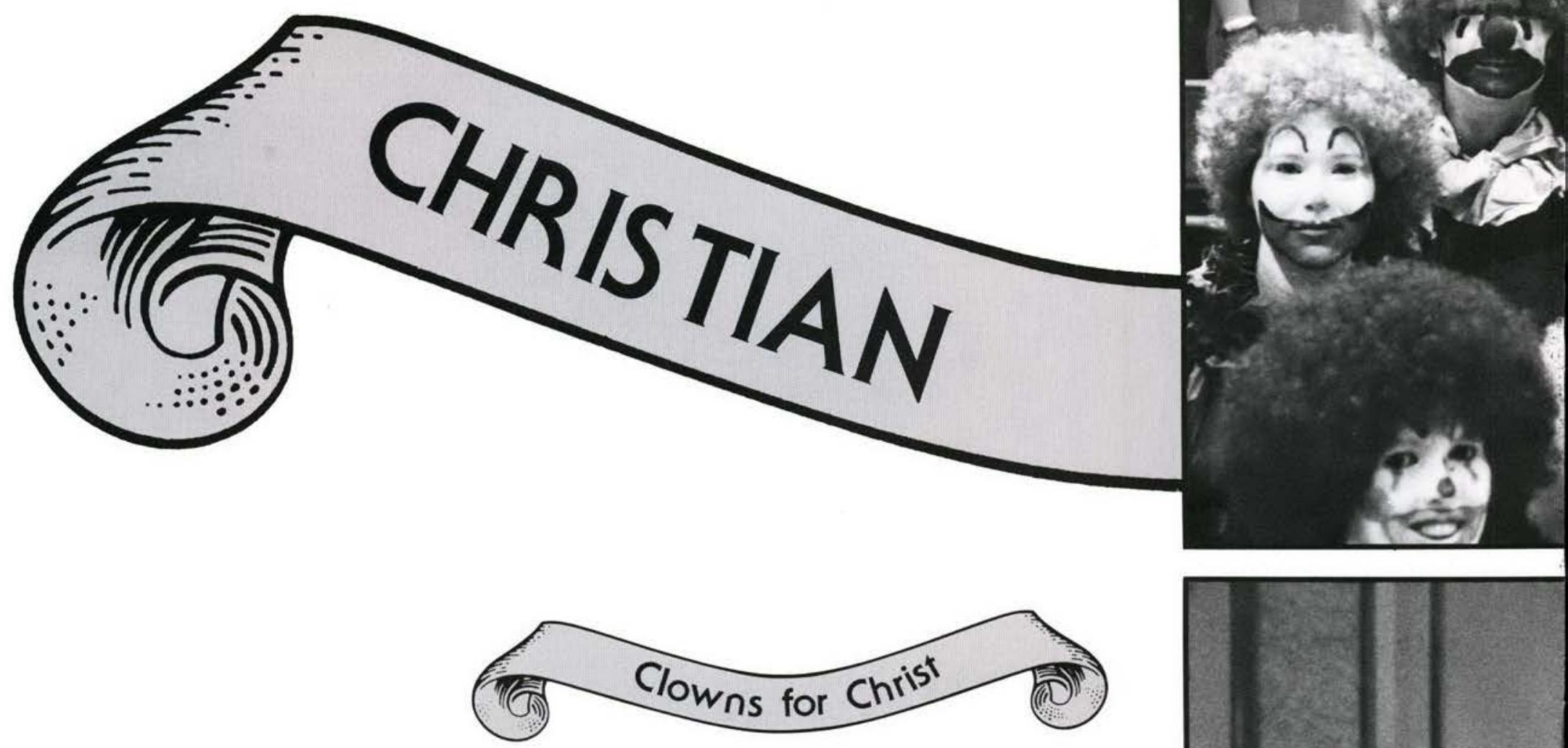

Row 1: Jennifer Houfler. Row 2: Jodi Wilson, Janine Knuutilo. Chris Drew.
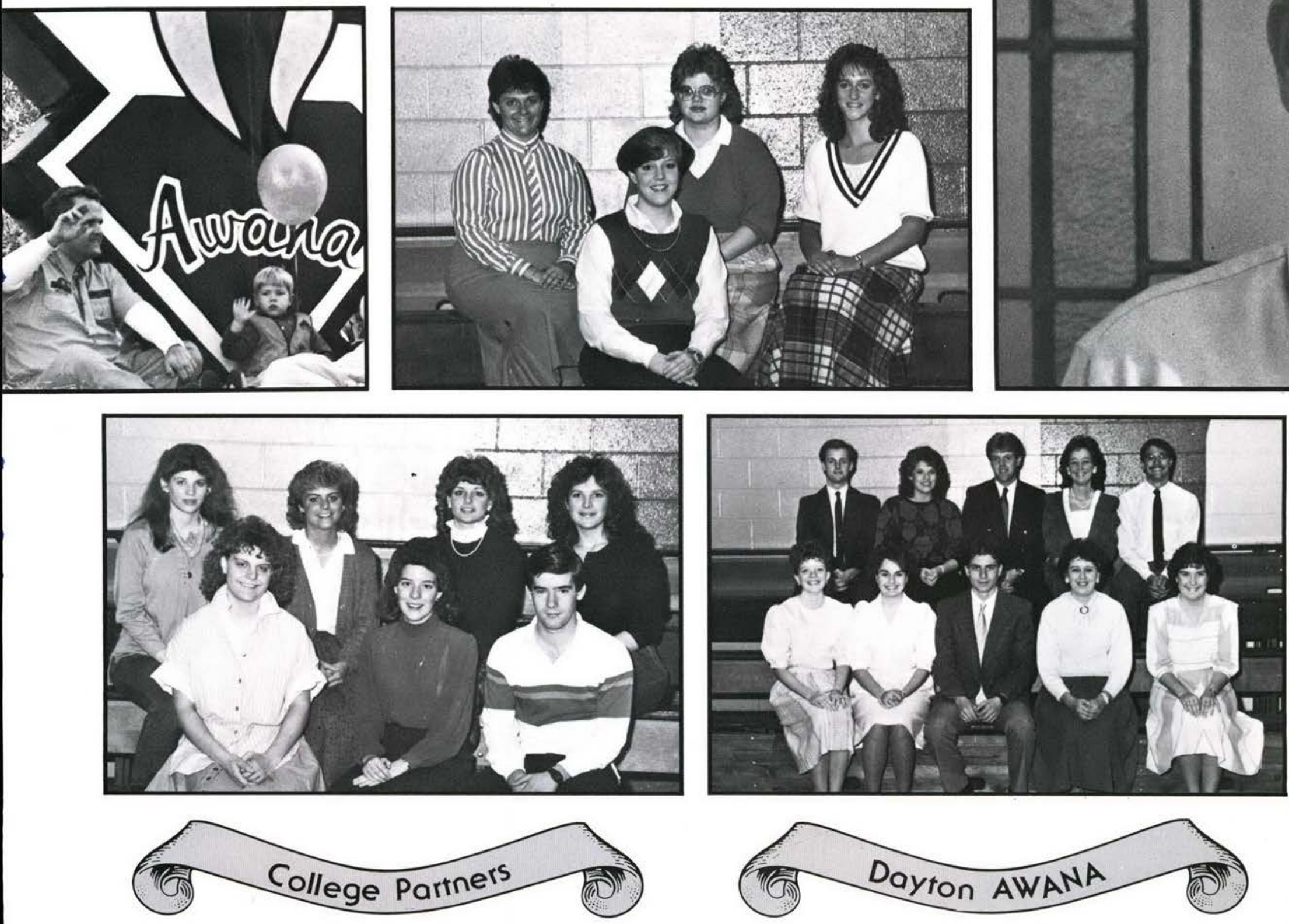

Row 1: Ann Sulek. Debbie Jo Daket, David Doud. Row 2: Donno Dlumenstock. Amy Thomas, Sandy O'Boyle. Linda Row 1: Angie Dennis. Rondo Altman. Brian Humphreys. Sue Otth. Sh 

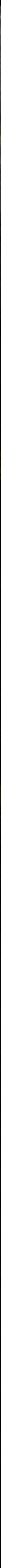

Row 1: Kevin Tupps, Melissa Berner, Dovid Hoecke, Becky Averill. Brian Ethridge, Row 2: Rob Passwaters, Dan Byrum, Vic Hoddod. Scort Poling. Jeff Leoch. Row 3: Tim Abromowitz, Doug Loescher, Dano Guy, Susan Seibert. Alice Becroft.
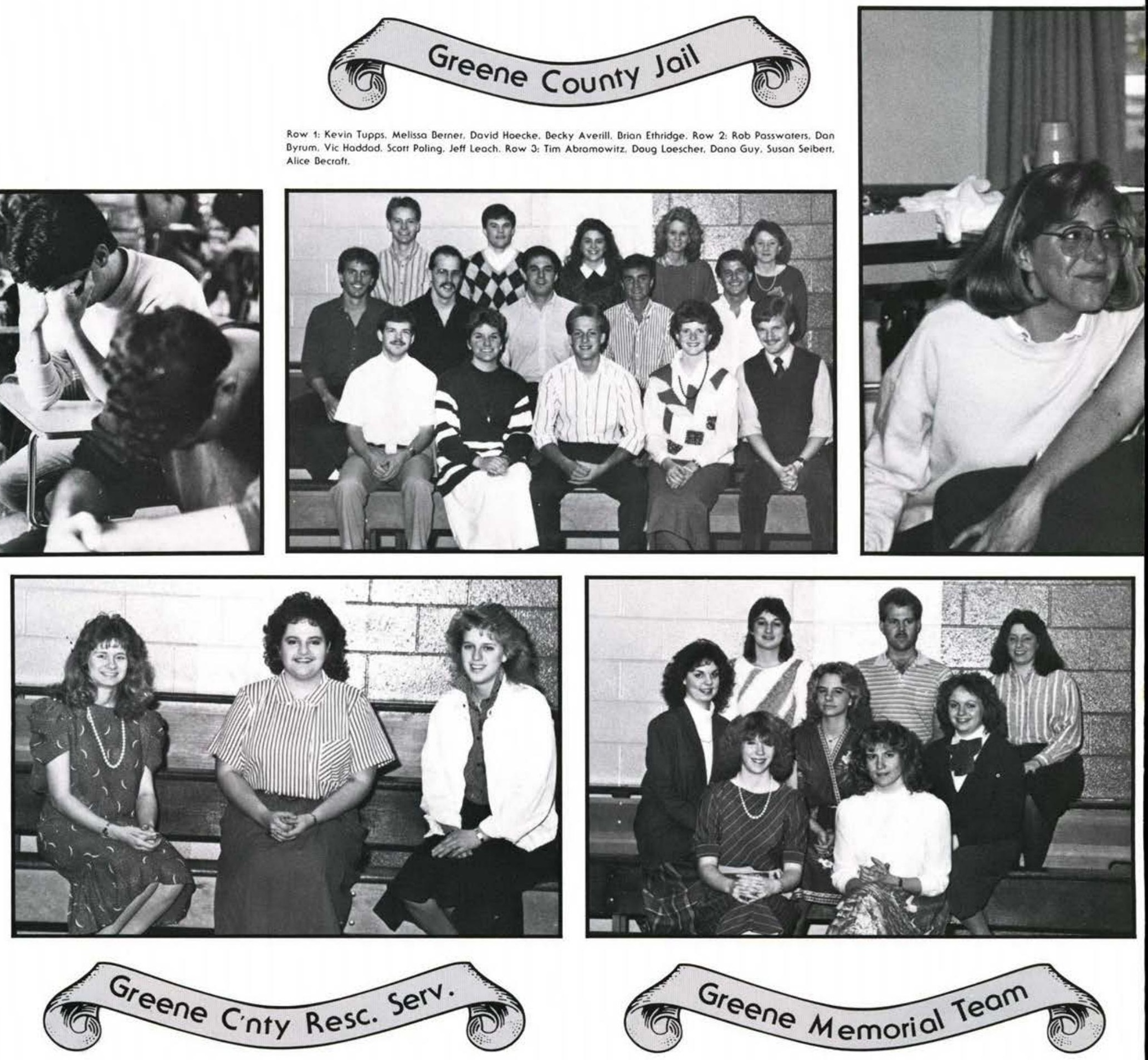

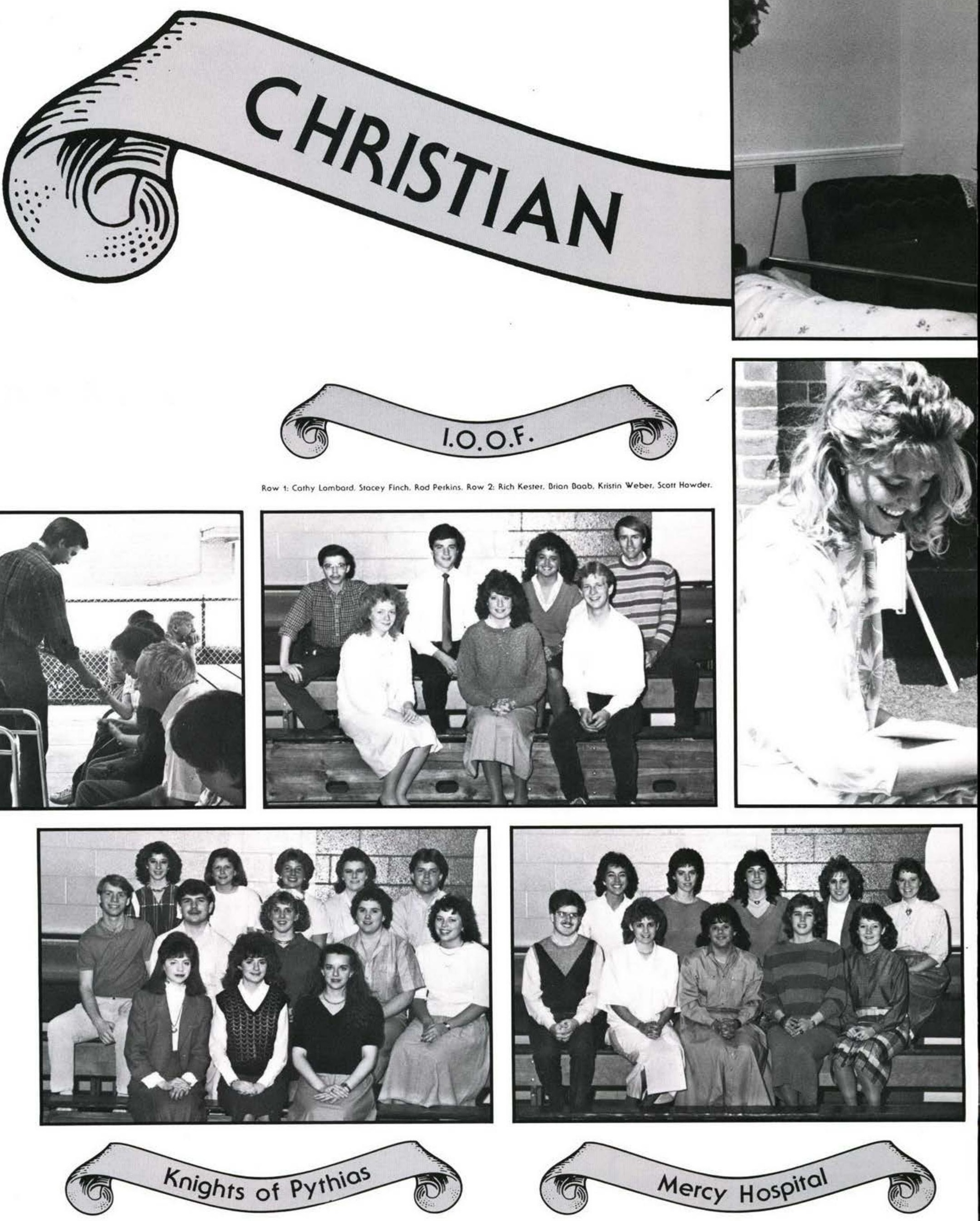


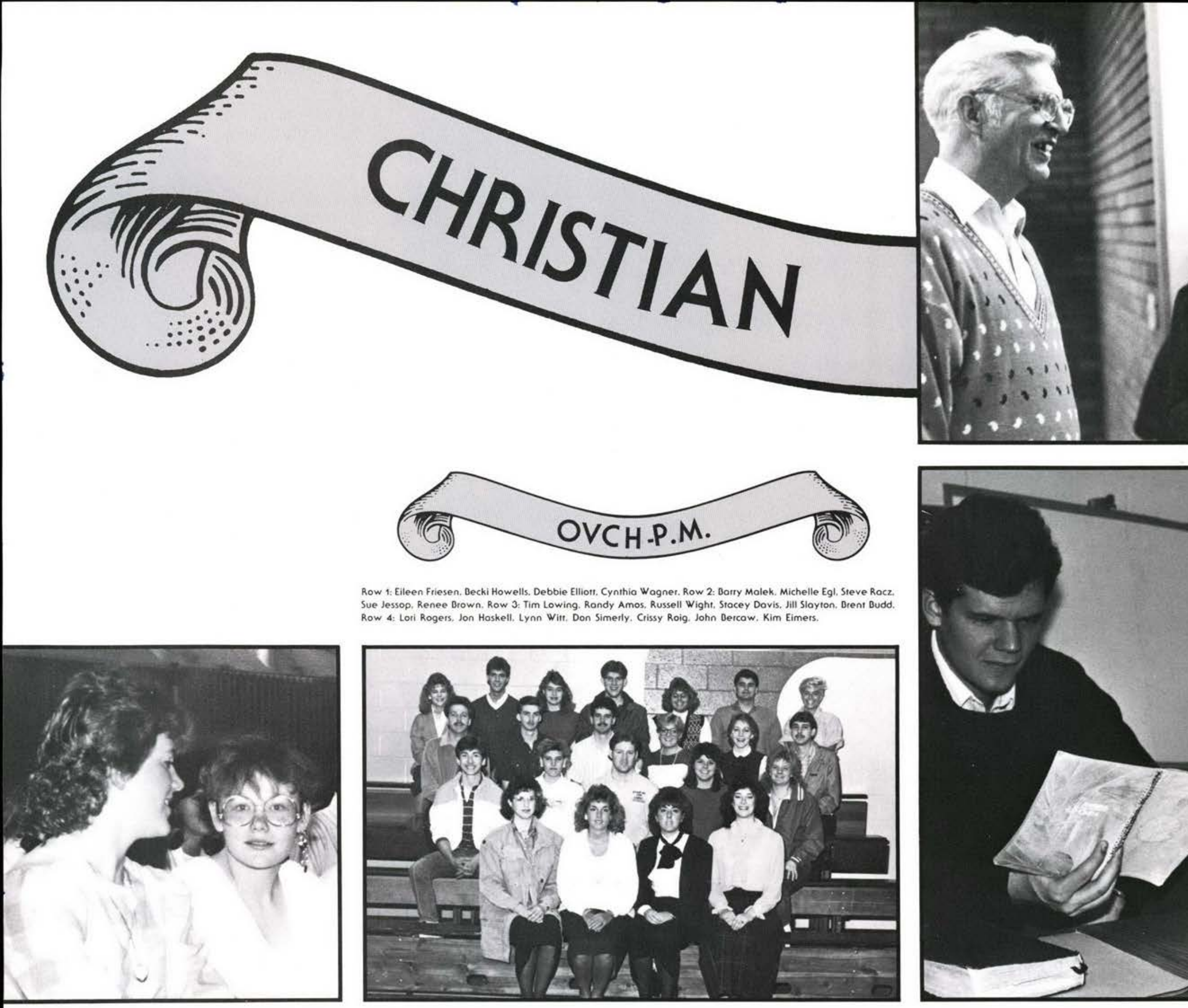

Row 1: Eileen Friesen. Becki Howells, Debbie Elliott. Cynthia Wagnet, Row 2: Barry Malek. Michelle Egl, Steve Rocz Sue Jessop. Renee Brown. Row 3. Tim Lowing. Rondy Amos, Russell Wight, Stocey Dovis. Jill Sloyton. Brent Budd. Row 4: Lori Rogers, Jon Hoskell. Lynn Witt. Don Simerly, Crissy Roig. John Dercaw. Kim Eimers.
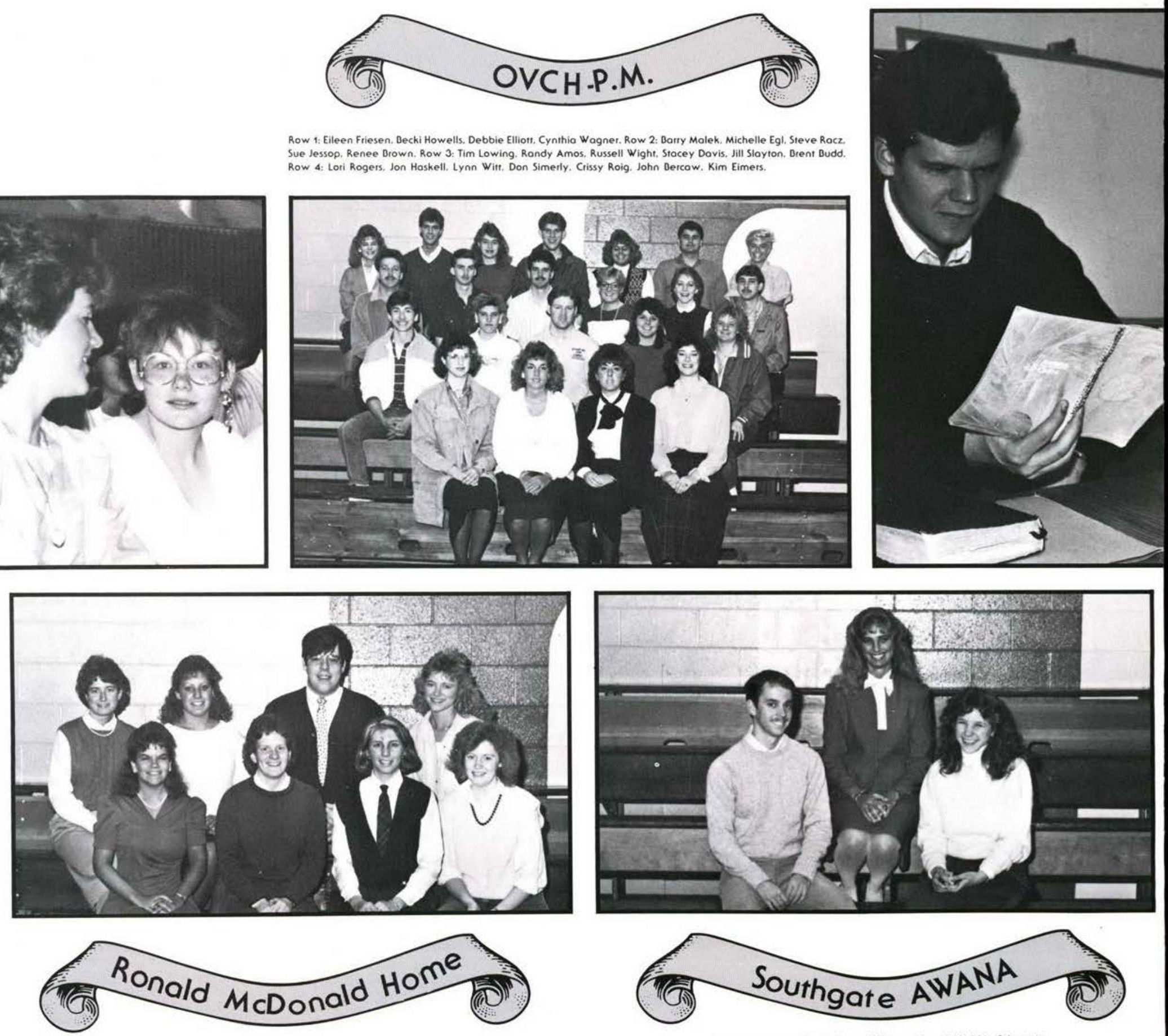

Row 1: Becki Tackett, Dororhy Howdyshell. Lois Cummings, Debbie Fogle, Row 2: Mory Raisch. Decky Pruner, Phil Chapman. Kim Sweet. 

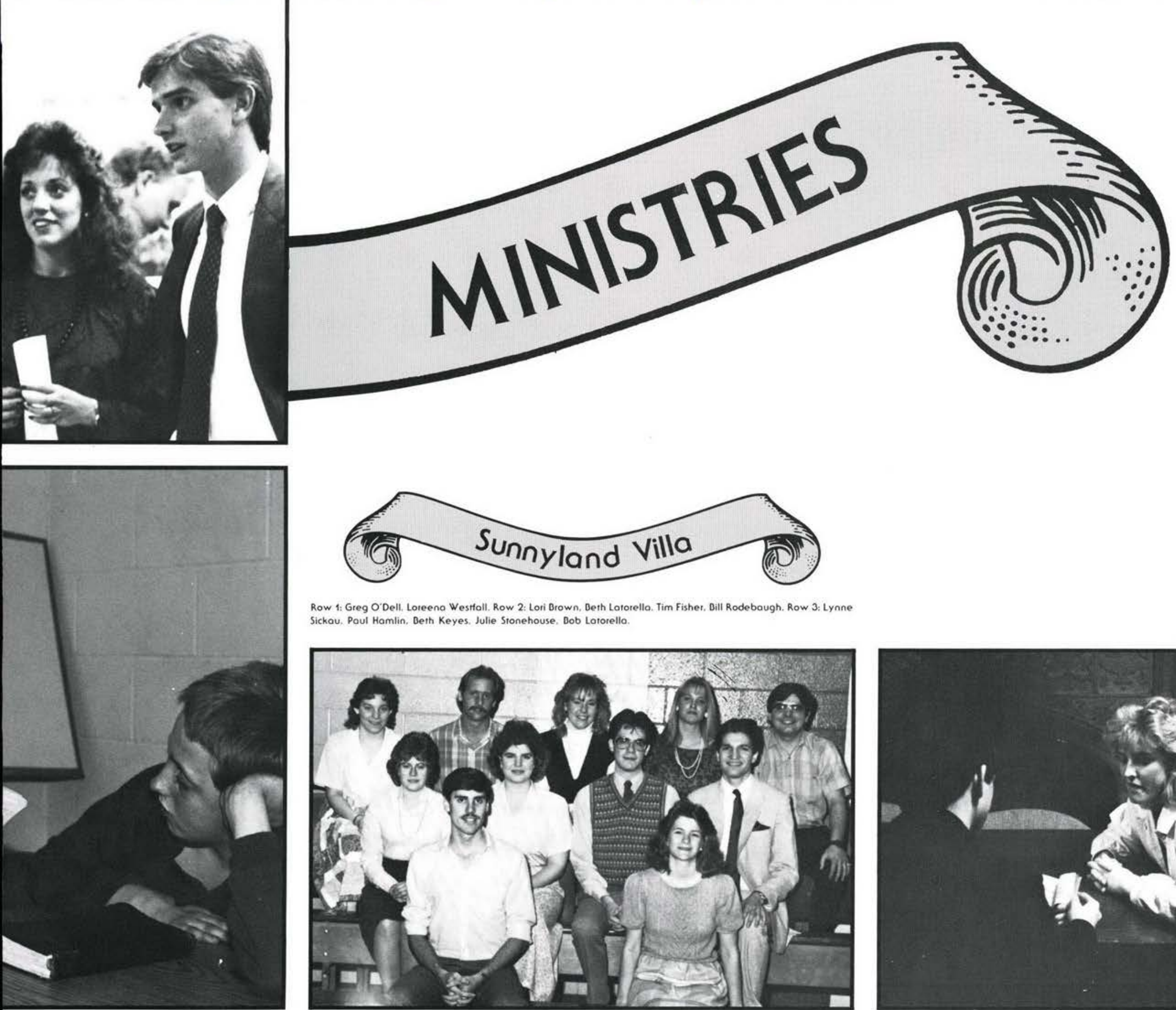

Row 1: Greg O'Dell. Loreeno Westfoll. Row 2: Lori Brown, Deth Lotorello. Tim Fisher, Bill Rodebough. Row 3: Lynne Sickau. Poul Homlin, Beth Keyes. Julie Stonehouse, Bob Lotorello.
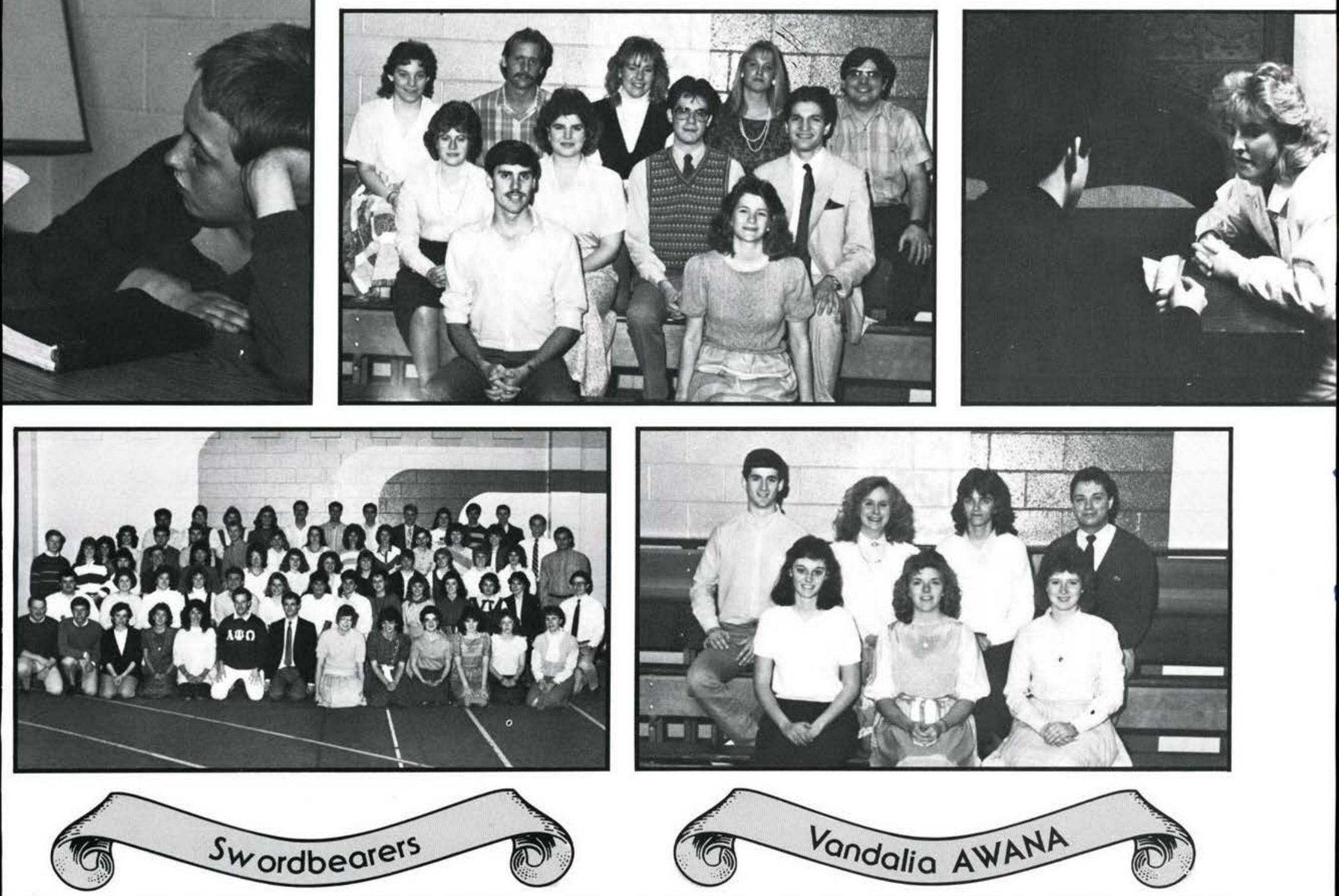

Row 1: Dave Robinette. Tim Deach. Pam Kimble. Mory Jones. Michelle Dolton, Dove Norris. John Kejr. Cheryl Ruble, Sally Compton. Nancy Ebel, Kim Duzen. Ann Hoskowich, Dione Johnson, Mark Volese. Row 2: David Mooney, Julie Corter, Jeri Hostman. Tammy Jenkins. Dave Wuestner. Trocy Young. Poul Merrill, Kathy Hoynes. Angie Huested. Michelle Babbirt, Becky Davis, Jan Caldwell. Joan Rartew, Brian Roget. Row 3: Mark Hinman, Debbie Duzweiler, Schofield, Tevens, Michoel Lne. Pam Comsey, Row 4: Sharon Michoel, Cynthio Wagner, David Beal, Shaun Spongler, David Douglos, Jolene Merck, Buffie MocKereth, Jodi Sofford, Jocki Becker, Jenny Hovens. Tereso Wilson Teresa Carter. Tim Willms. Row 5: Janet Lawrence, Cindy Guido, Susan Swann. Kristyn Johnson. Stephen Gerhordt. David Holt. Brent Lydic, James White, Mindy Brocious. Dwayne Hoff. Andrew Stevens. 

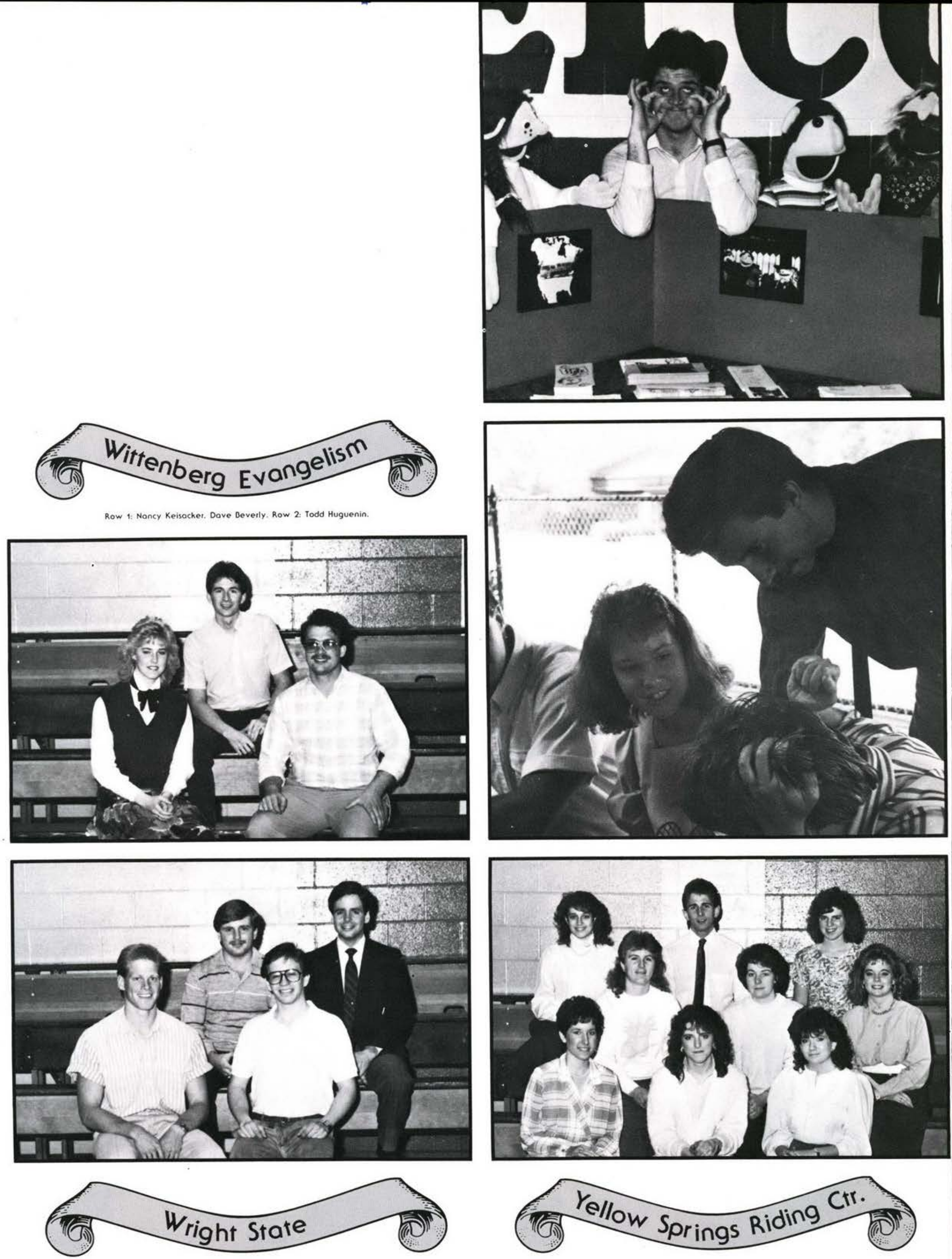


\section{TRAVELING TEAMS}

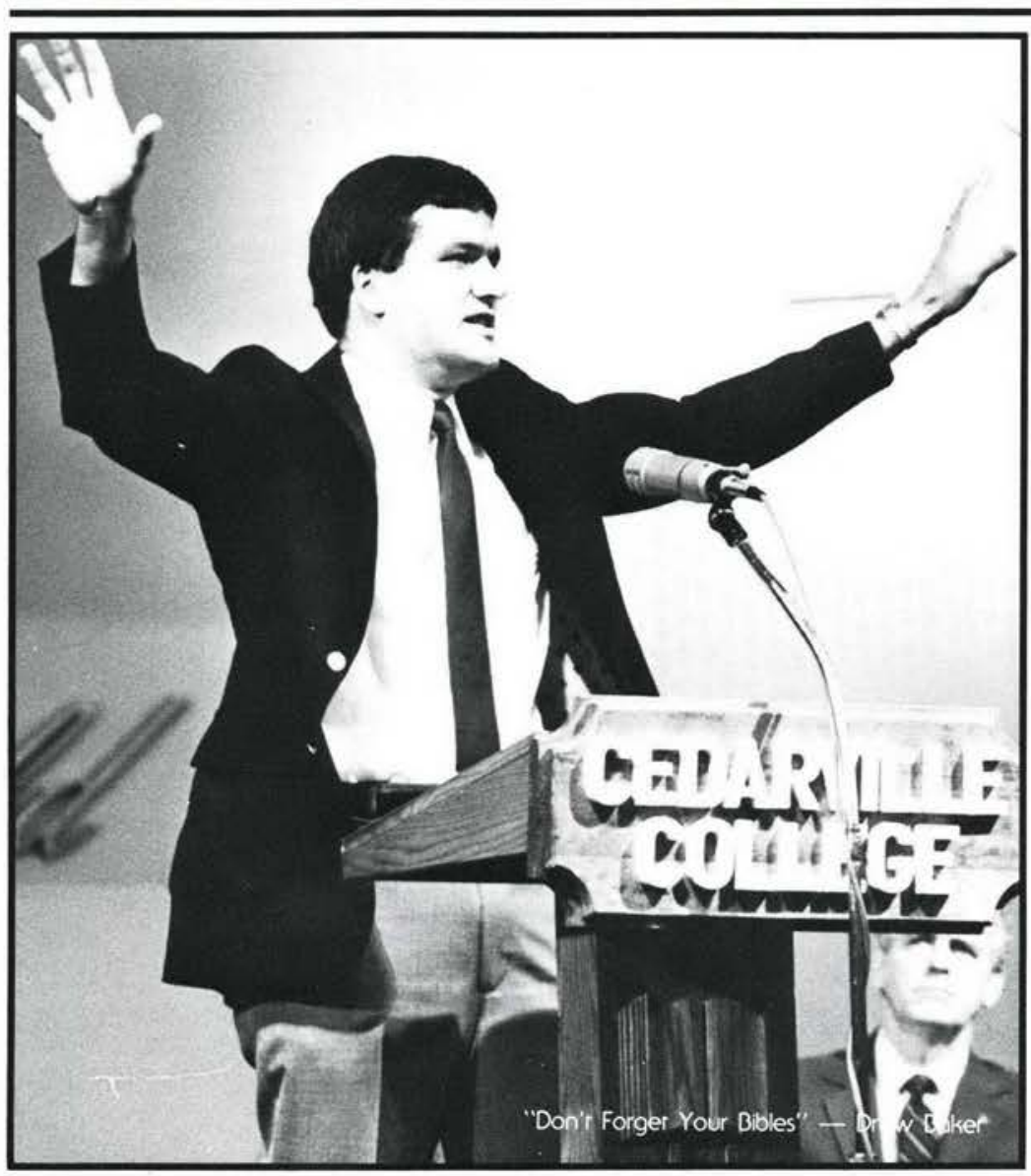

AUSTRALIA MUSIC TEAM: June 24.August 3 Cothy Anderson Jeff Deste, Volerie Dloylock, Dovid Burk, Phil Chopmon. Donielle Kizer, Jennifer Lenhort, Tom Prott, Brion Reebel. Meliso Runion. Jeff Vestrond. Postor Green DRAZIL TEAM: Jufne 10-July 9 Drent Budd. Doug Cherry, Phil Hohulin, Beth Hughes, Loren Johnson. David Smollman. Valerie Smallman, Tim Wagner, Christine Wenger, Dr. and Mrs, Lyle Anderson, Lorie ond Eric Anderson ENGLAND/IRELAND: June 10.July 13 Michelle Dalton. John Kejr. Michoel Lone. Stephonie Manning. David Mooney, David Norris. Karlo Simpson, Leeanne Stevens, David Wuestret, CHINA TEAM. August 5.24 Sorah Bort. Tonyo Dumpus. Blair Carlstrom. Bob Clemens, Jennifer Houflet, Jone Kroner, Brion Roget. Mork Volese. Steve Wolter, Mr. and Mrs. Dovid Rich PHILPPINES BASKETBALL TEAM. June 7July 9 Brod Baird.

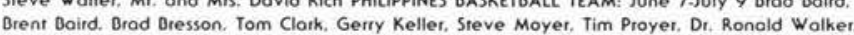
Dr. Don Callon NIGER TEAM: June 10.July 11 Nothan Bobbett. Angelo Huested. Tim Ryan. Loure Yates PERU TEAM: June 23.August 3 Deborah Clem, Sandra Kennedy, Lynell Smith TOGO. AFRICA: June 9.July 20 Beth Honsen INDIVIDUALS TO BRAZIL: John Angus June 30.Sept. 15. Daniel Kain June 8.July 8. Beth McGillivray July 19.Aug. 23 HONG KONG: June-Sept. Bob Poswoters JAPAN: June 23.Aug. 11 Laurie Wogner CORSICA, FRANCE: June 16.Aug. $3 \mathrm{jim}$ Venman EDM OFFICE IN KOKOMO. IN: June 29.Aug. 31 Todd Dowden SUMMER SWORDBEARERS June 13.Aug. 21 Liso Armstrong. David Holt, Steve Gerhardr, Mark Hinmna, Mary Jones, Pionist - Jono Dowsett. Sound Man - Ron Mackey. Brent Lydic, Buffi Mackereth, Jolene Merck. Dove Robinette, Jodi Sofford ABUNDANT LIFE SINGERS: June 13.Aug. 21 Steve Averitt. Roger Foremon. Terti Mockenzie. Thurm Payton, Suzette Ramsey, Sondy Weover, Jomie Beight, Jodi Wilson. Pionist - Kim Borch. Sound man - Don Shepherd KINGSMEN QUARTET. June 13-Aug 21 Steve Dye, Jim Murdoch. Steve Ponther, Jeft Yonder Heide. Tim Wolborn. Sound mon - Scott Deonie SUMMER MASTER'S PUPPETS: June 13-Aug. 21 Janet Gormotter, Dan Gonzoles, Bill Morgon. Andrea Snyder. Brandon Woltz

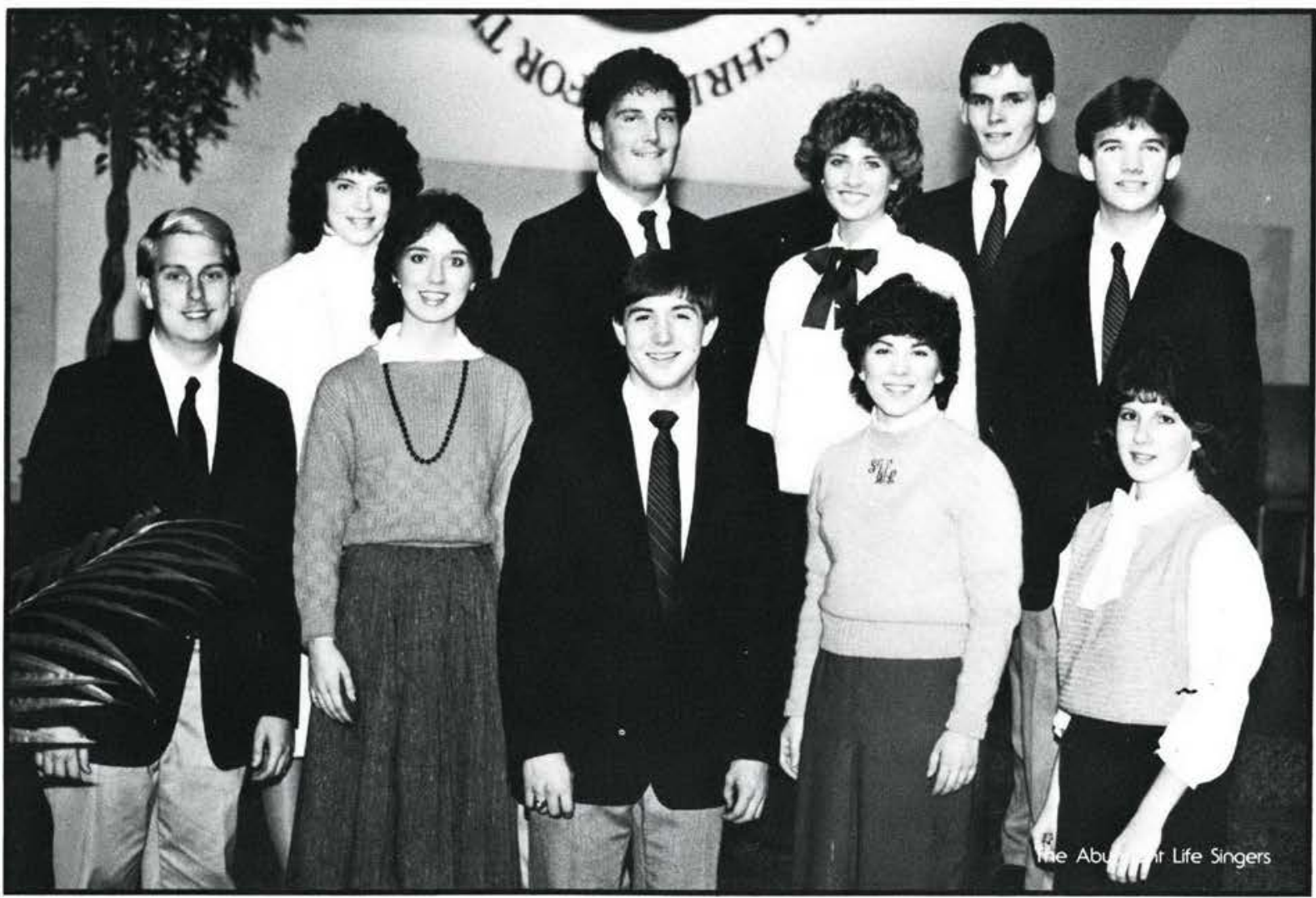



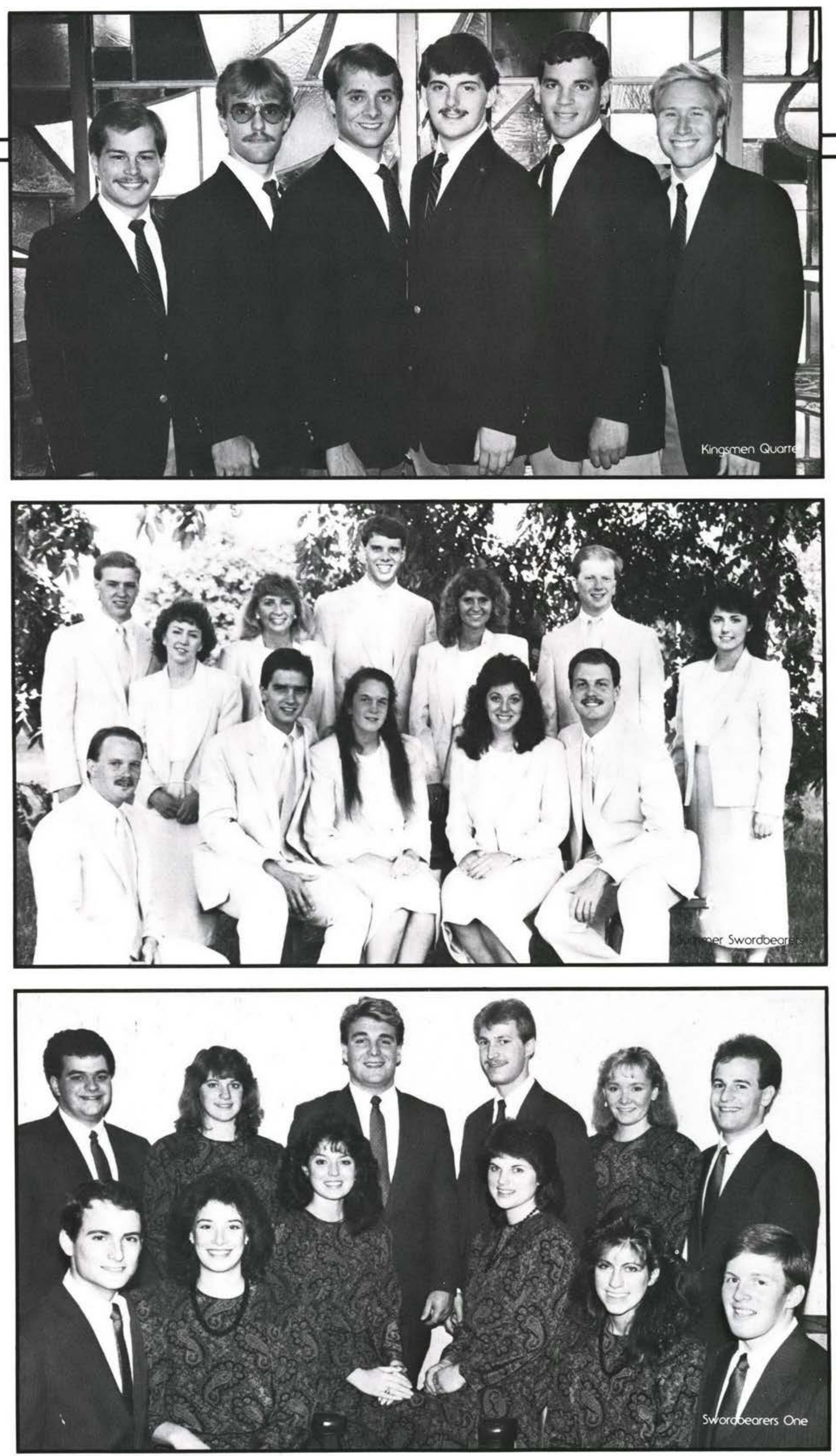

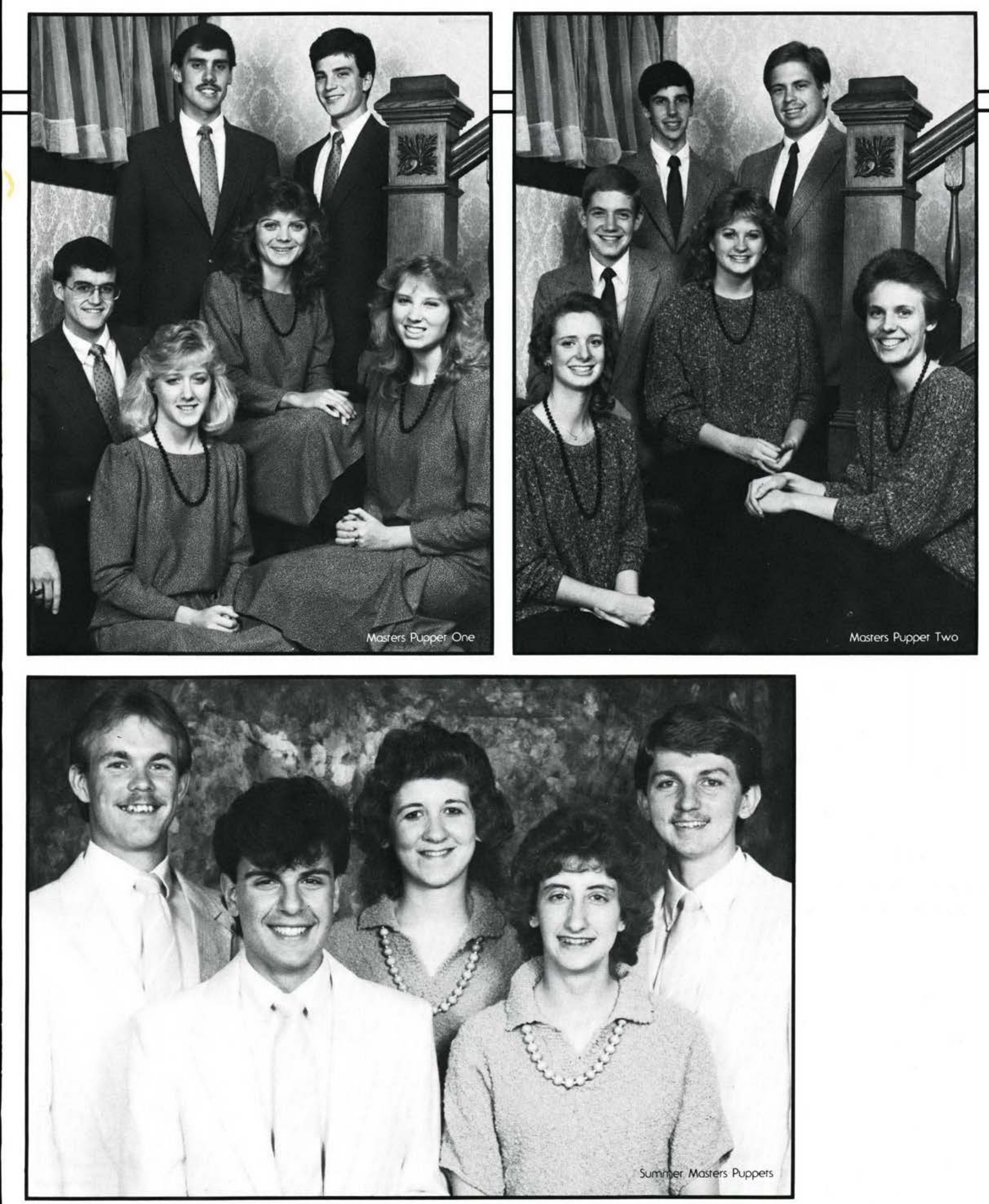

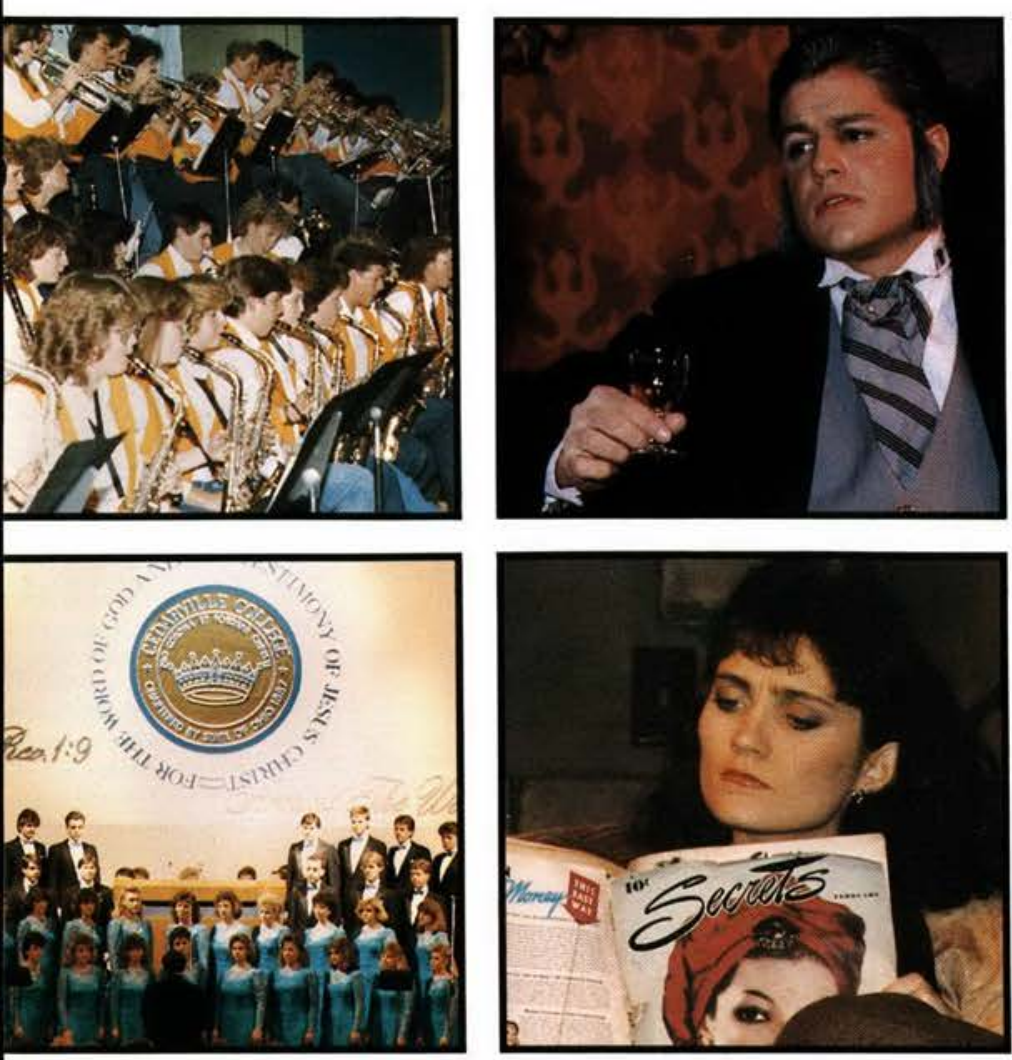
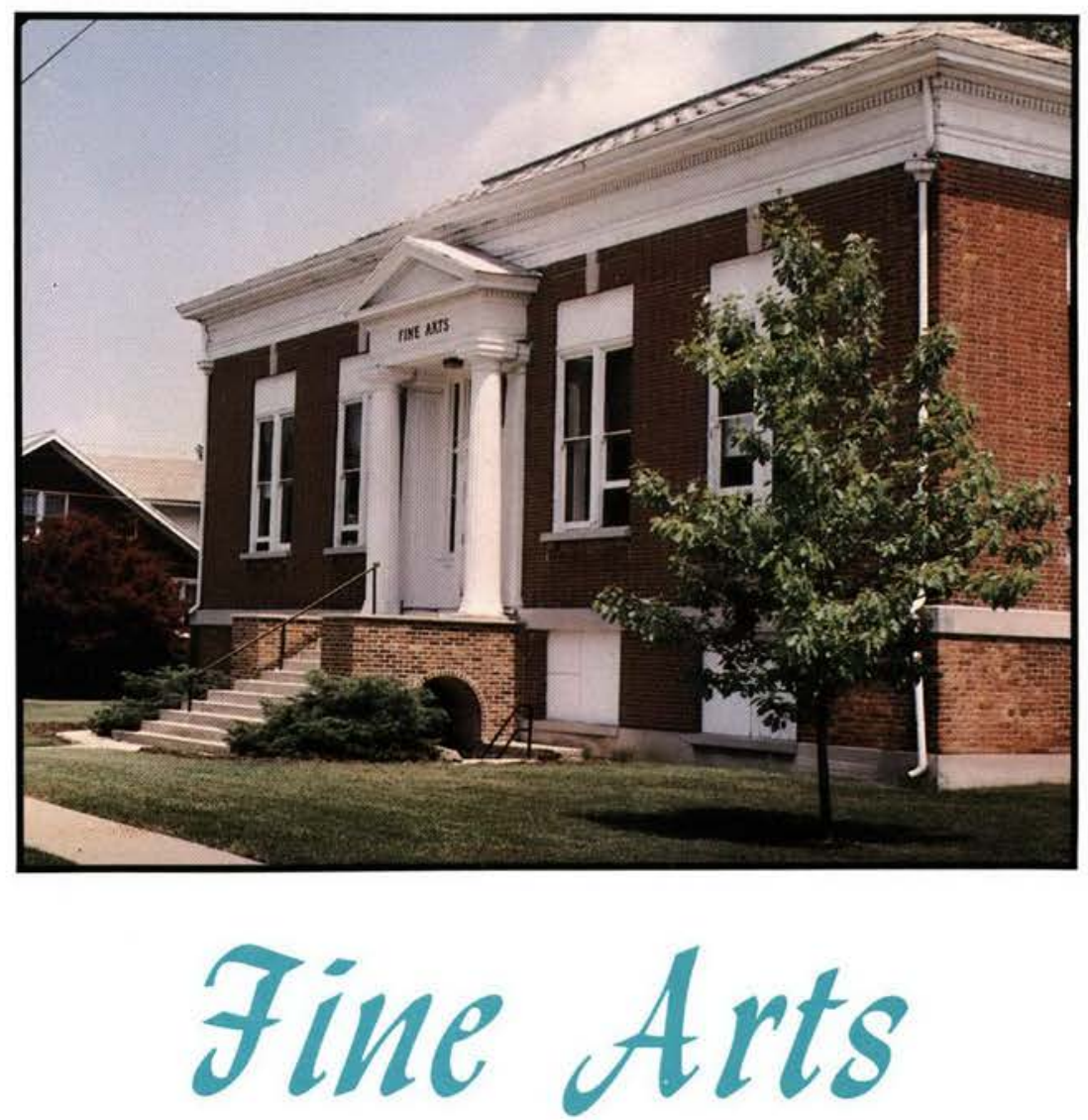

From solo music recitals to full-scale thearrical productions, opportunities abound for students to experience the fine arts. One may develop talents and abilities through active participarion or simply broaden his or her culrural exposure by artending the wide variery of performances given each year. Faculty leadership is commirted to developing student potential to the fullest and to maintaining the highest standards of excellence in performance. For the Christian, cultivaring the fine and performing arts is a magnificent way to glorify the Crearor of all beaury.

Eric Hemurh

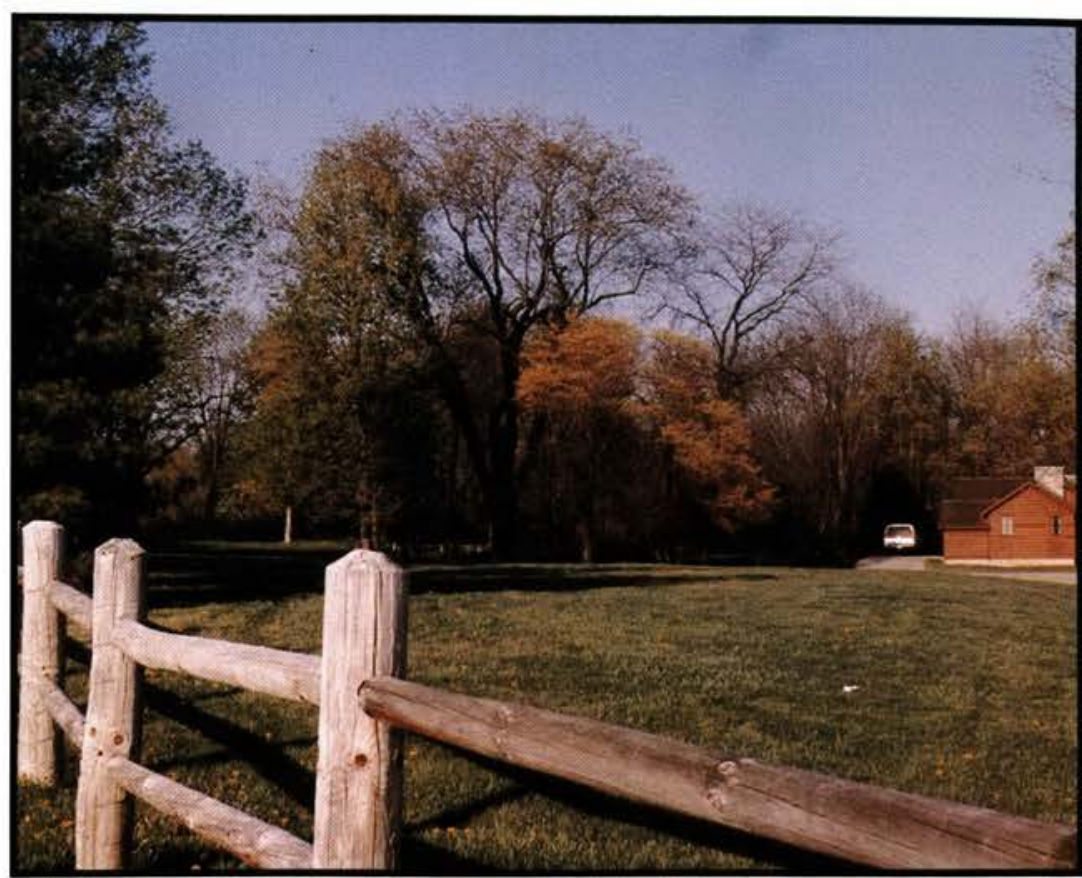




\section{9

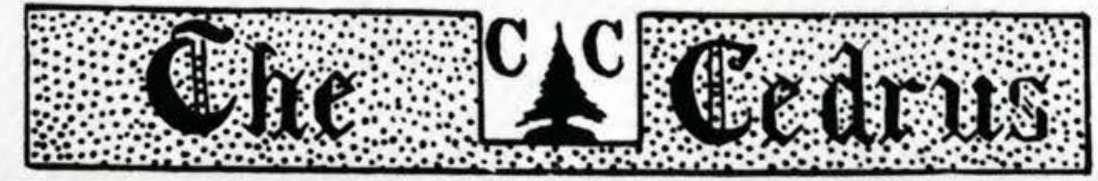 \\ 19}

$6 \sqrt{\left.2+\frac{14}{6}\right)}$

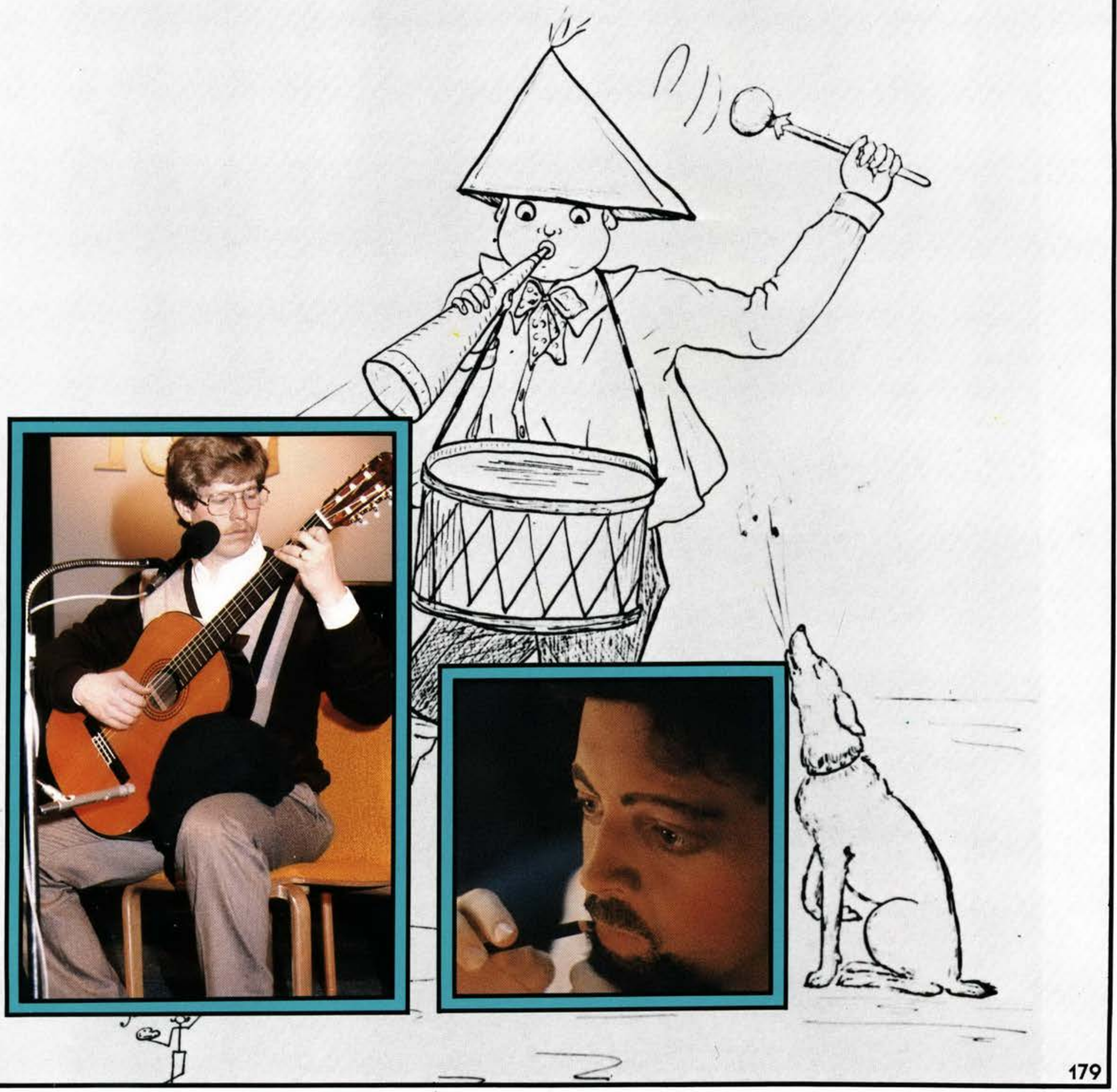




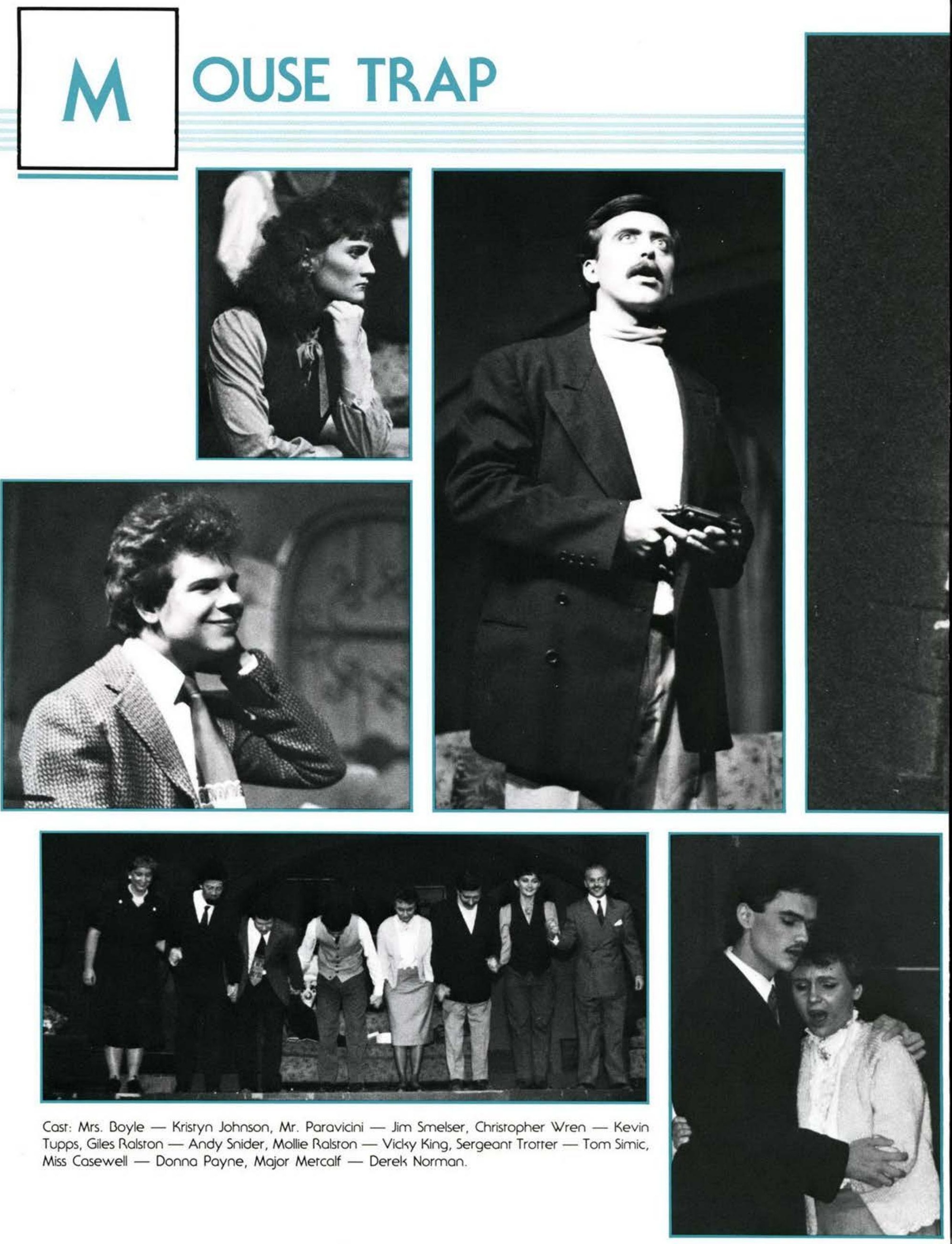




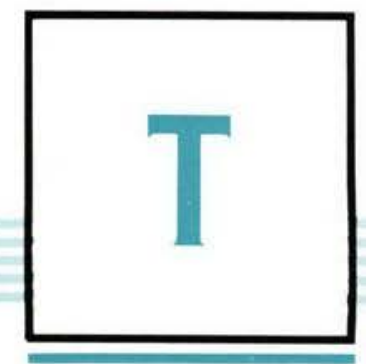

\section{HE IMPORTANCE OF BEING EARNEST}
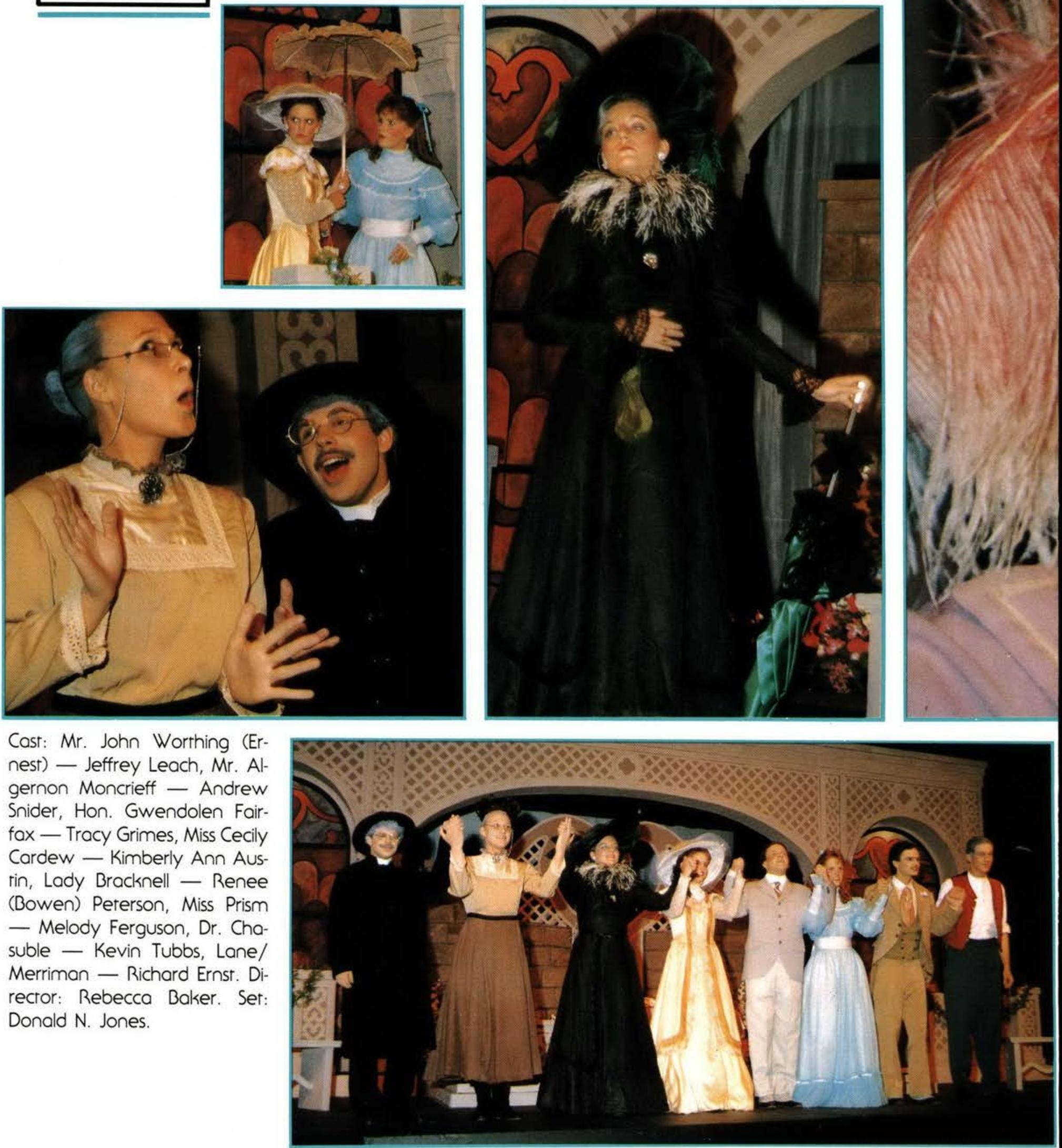

Cast: Mr. John Worthing (Ernest) - Jeffrey Leach, Mr. Algernon Moncrieff - Andrew Snider, Hon. Gwendolen Fairfax - Tracy Grimes, Miss Cecily Cardew - Kimberly Ann Ausrin, Lady Bracknell - Renee (Bowen) Peterson, Miss Prism - Melody Ferguson, Dr. Chasuble - Kevin Tubbs, Lane/ Merriman - Richard Ernst. Director: Rebecca Baker. Ser: Donald N. Jones. 

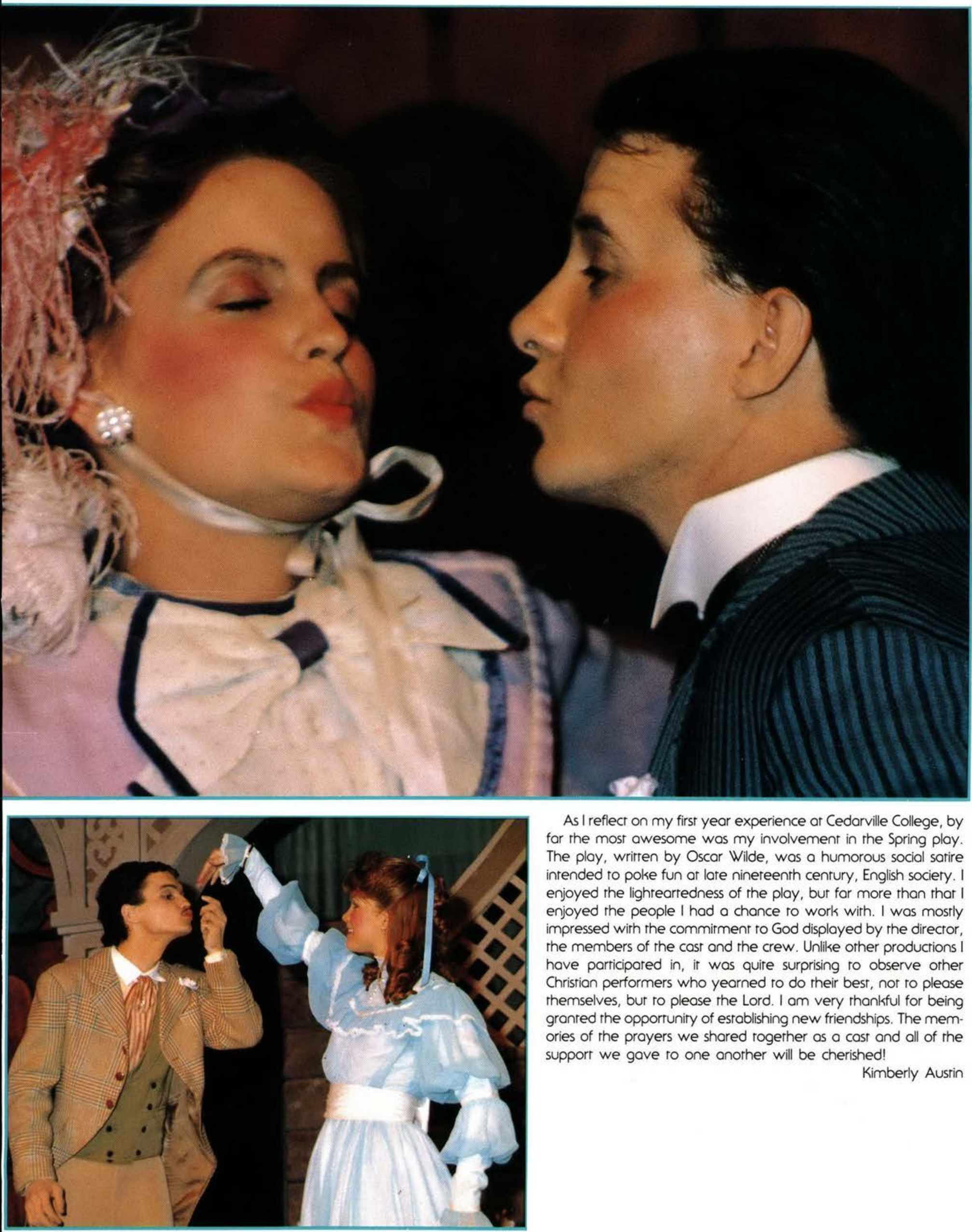

As I reflect on my first year experience at Cedarville College, by far the most awesome was my involvement in the Spring play. The play, written by Oscar Wilde, was a humorous social satire intended to poke fun at late nineteenth century, English sociery. I enjoyed the lighteartedness of the play, but for more than that I enjoyed the people I had a chance to work with. I was mostly impressed with the commitment to God displayed by the director, the members of the cost and the crew. Unlike other productions I have participated in, it was quite surprising to observe other Christian performers who yearned to do their best, not to please themselves, but to please the Lord. I am very thankful for being granted the opportunity of establishing new friendships. The memories of the prayers we shared rogether as a cast and all of the support we gave to one another will be cherished!

Kimberly Austin 


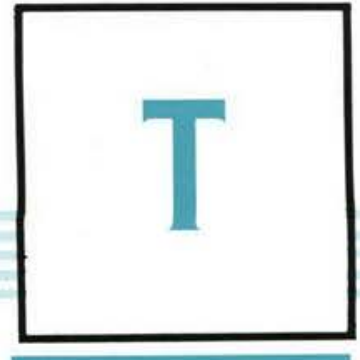

HE LITTLE FOXES
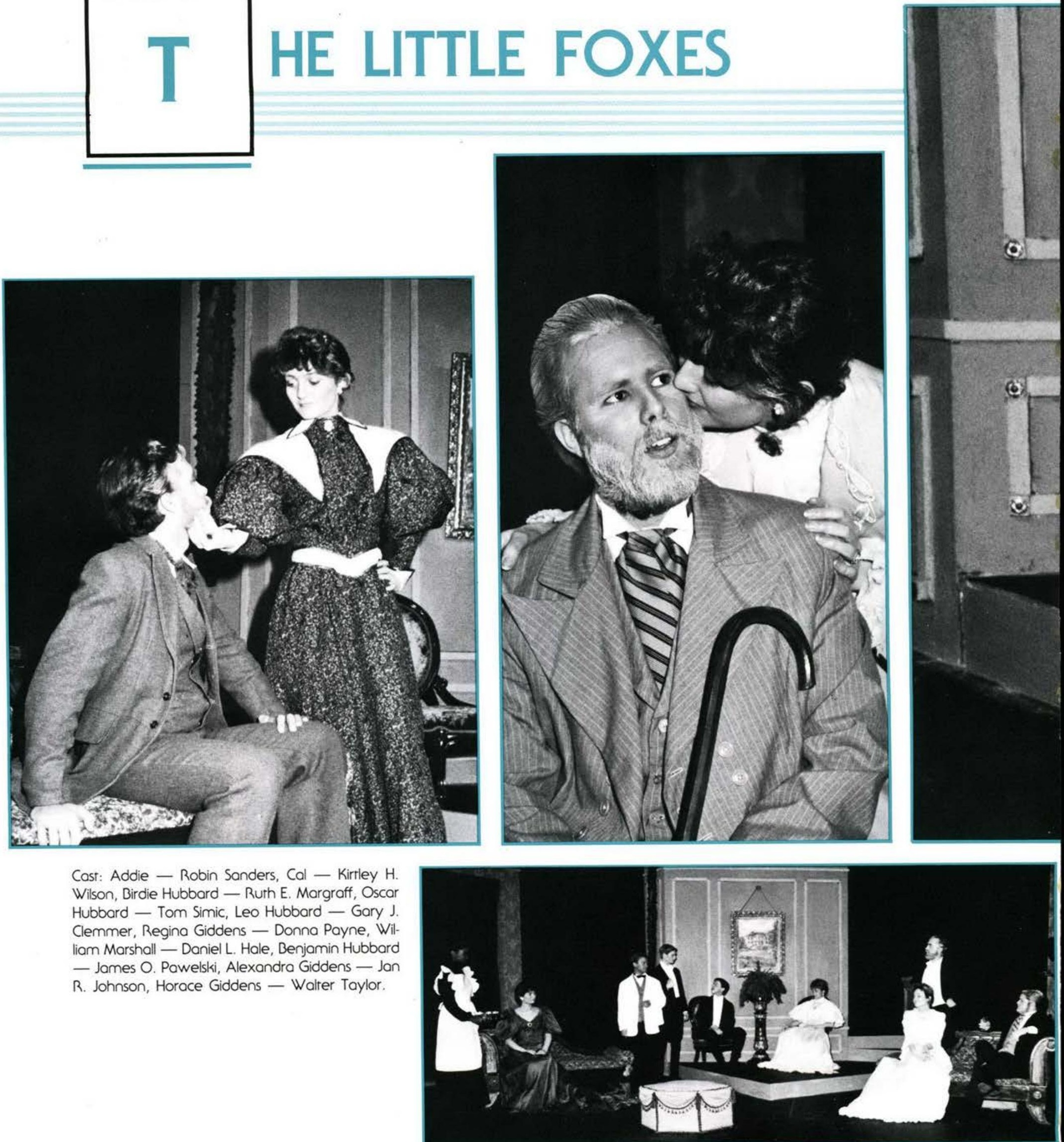

Cost: Addie - Robin Sanders, Cal - Kirtley $\mathrm{H}$. Wilson, Birdie Hubbard - Rurh E. Margraff, Oscar Hubbard - Tom Simic, Leo Hubbard - Gary J. Clemmer, Regina Giddens - Donna Payne, William Marshall - Daniel L. Hale, Benjamin Hubbard - James O. Pawelski, Alexandra Giddens - Jan R. Johnson, Horace Giddens - Walter Taylor.

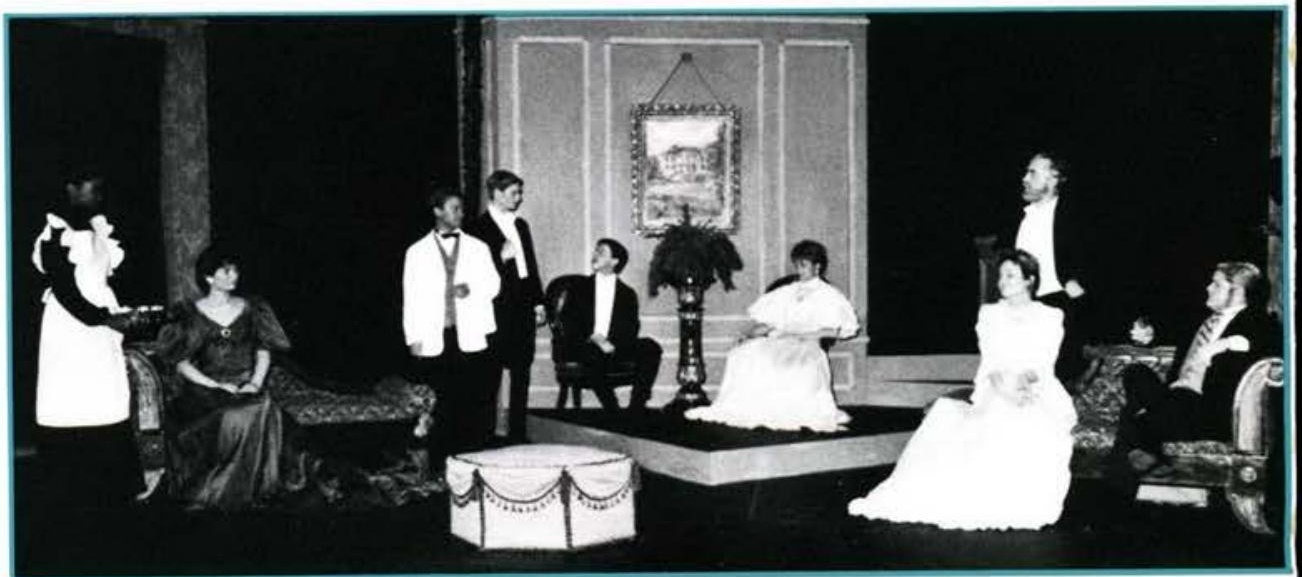



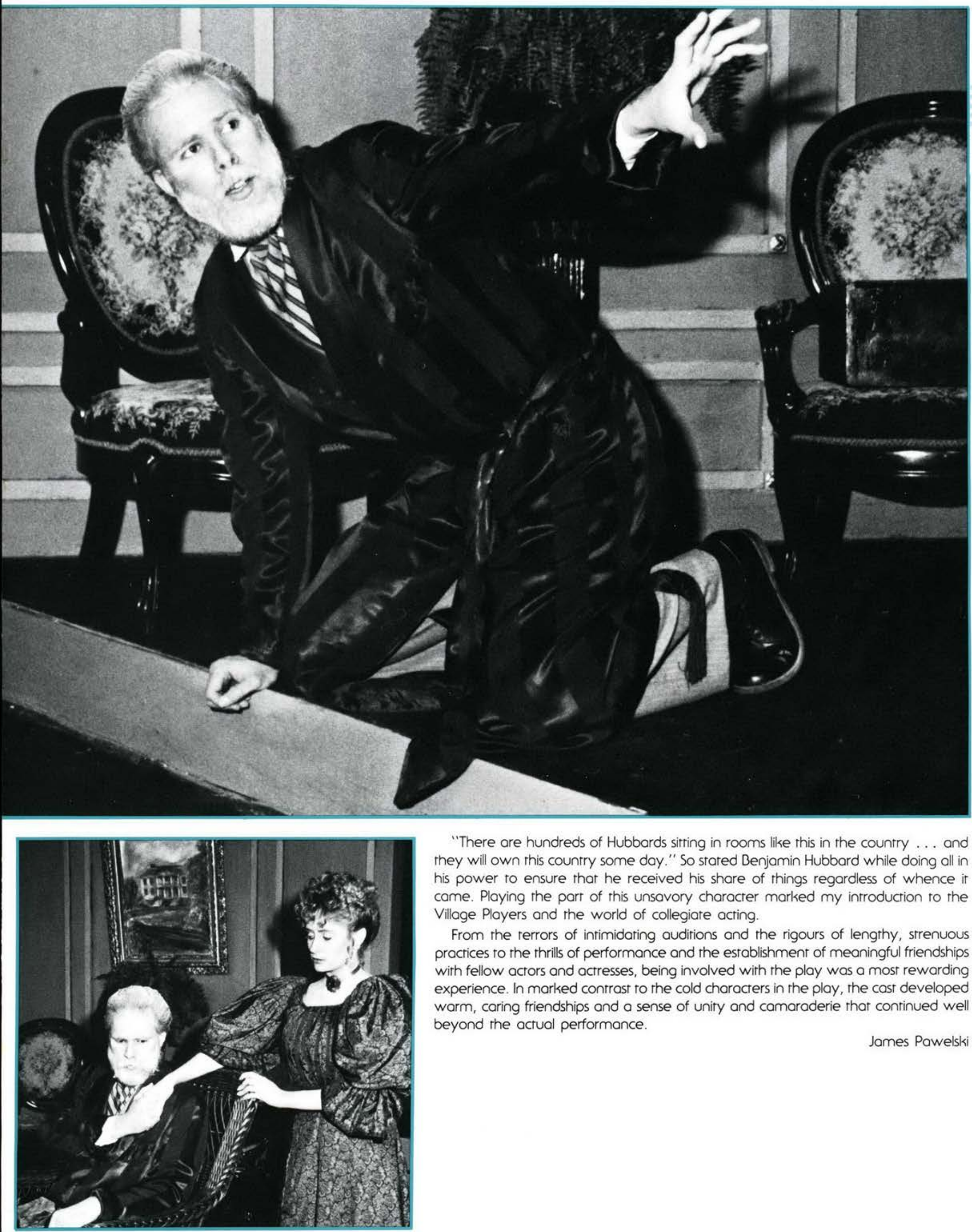

"There are hundreds of Hubbards sitring in rooms like this in the country ... and they will own this country some day." So stared Benjamin Hubbard while doing all in his power to ensure that he received his share of things regardless of whence it came. Playing the part of this unsavory character marked my introduction to the Village Players and the world of collegiate acting.

From the terrors of intimidating auditions and the rigours of lengthy, strenuous practices to the thrills of performance and the establishment of meaningful friendships with fellow actors and actresses, being involved with the play was a most rewarding experience. In marked contrast to the cold characters in the play, the cast developed warm, caring friendships and a sense of unity and camaraderie that continued well beyond the actual performance.

James Pawelski 


\section{Performing Artists}
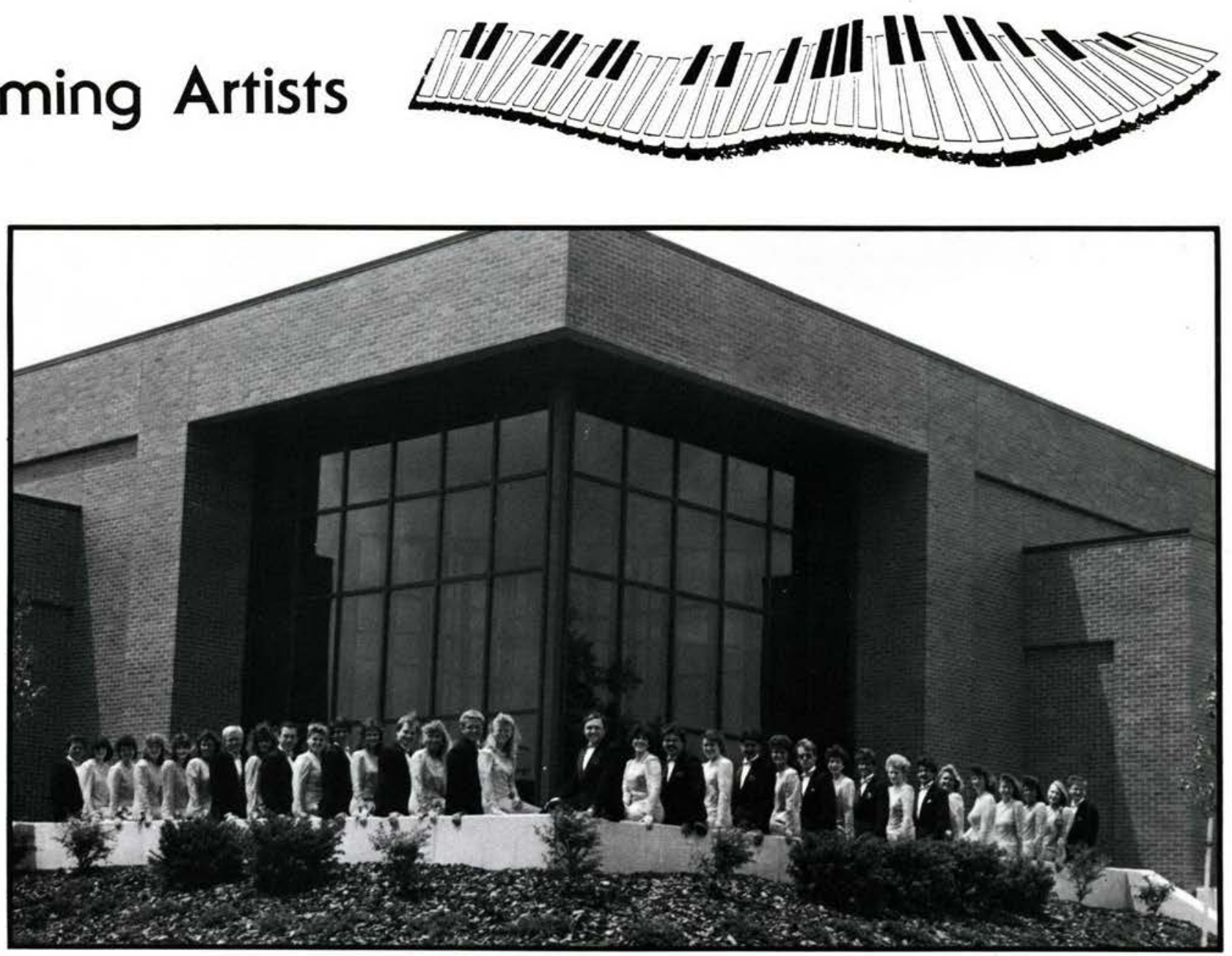

1987 CONCERT CHORALE

Eric Adams, Michael Anderson, Tim Beach, Mara Blackburn, Warren Brown, Mary Coons, Liso Davidson, Berh Dowson, Debbie Dunzweiler, Becky Dye Quentin Eshlemon, Melody Ferguson, Doug Filter, Eileen Friesen, Greg Gibbs, Mark Groves, Karen Hoynes, Michelle Hinnergardr, Dwayne Hoff, Sondro Holbrook, Berh Hughes, Todd Hummel, Jane Inbody, Pam Kenyon, Karen Kuntz, Mike Low, Jennifer Lenhart, Stephanie Manning, Jolene Merck, Jim Murdoch, Steve Panther, Rob Paswarers, Alyson Payne, Suzanne Perrort, Becky Pruner, Lindo Ranger, Brian Reebel, Philip Rice, Jodi Srafford, Jeffrey Vander Heide, Tim

Walborn, Sandro Weaver, Mark Wolf.
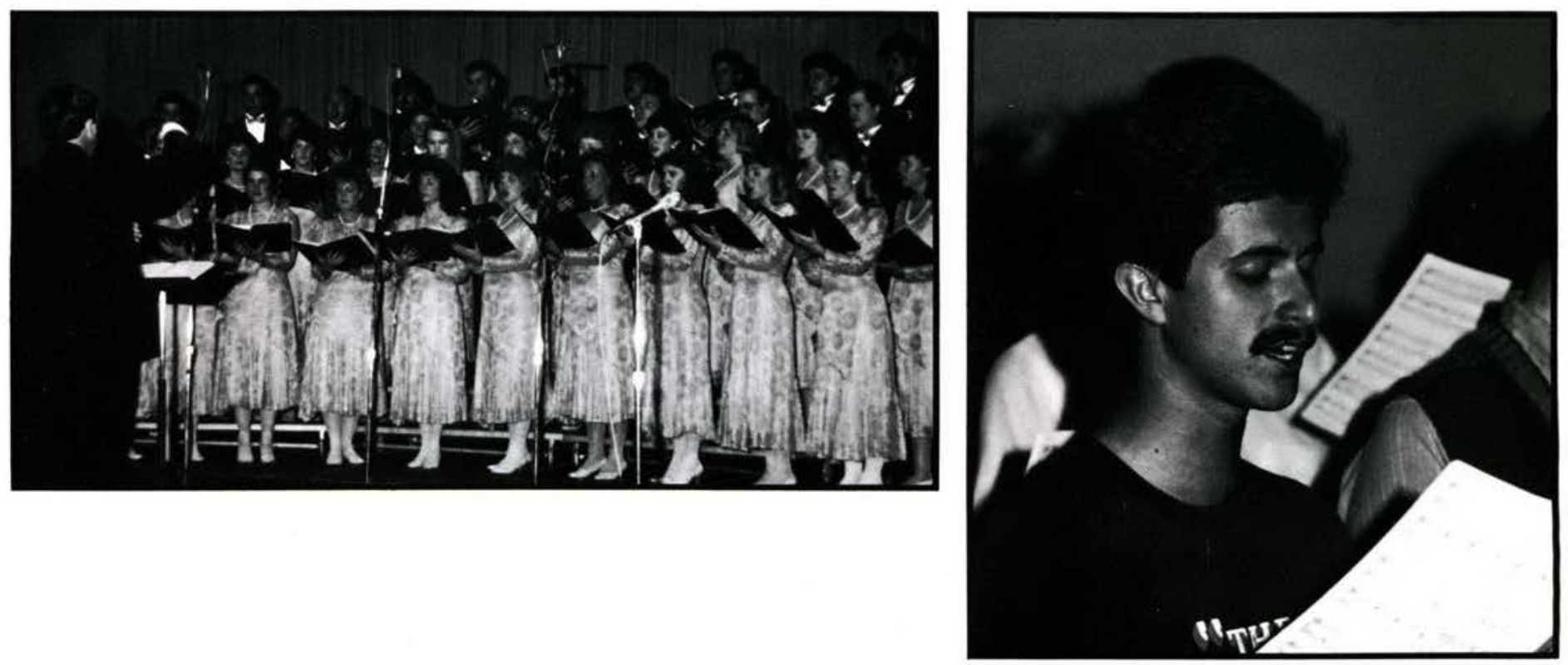


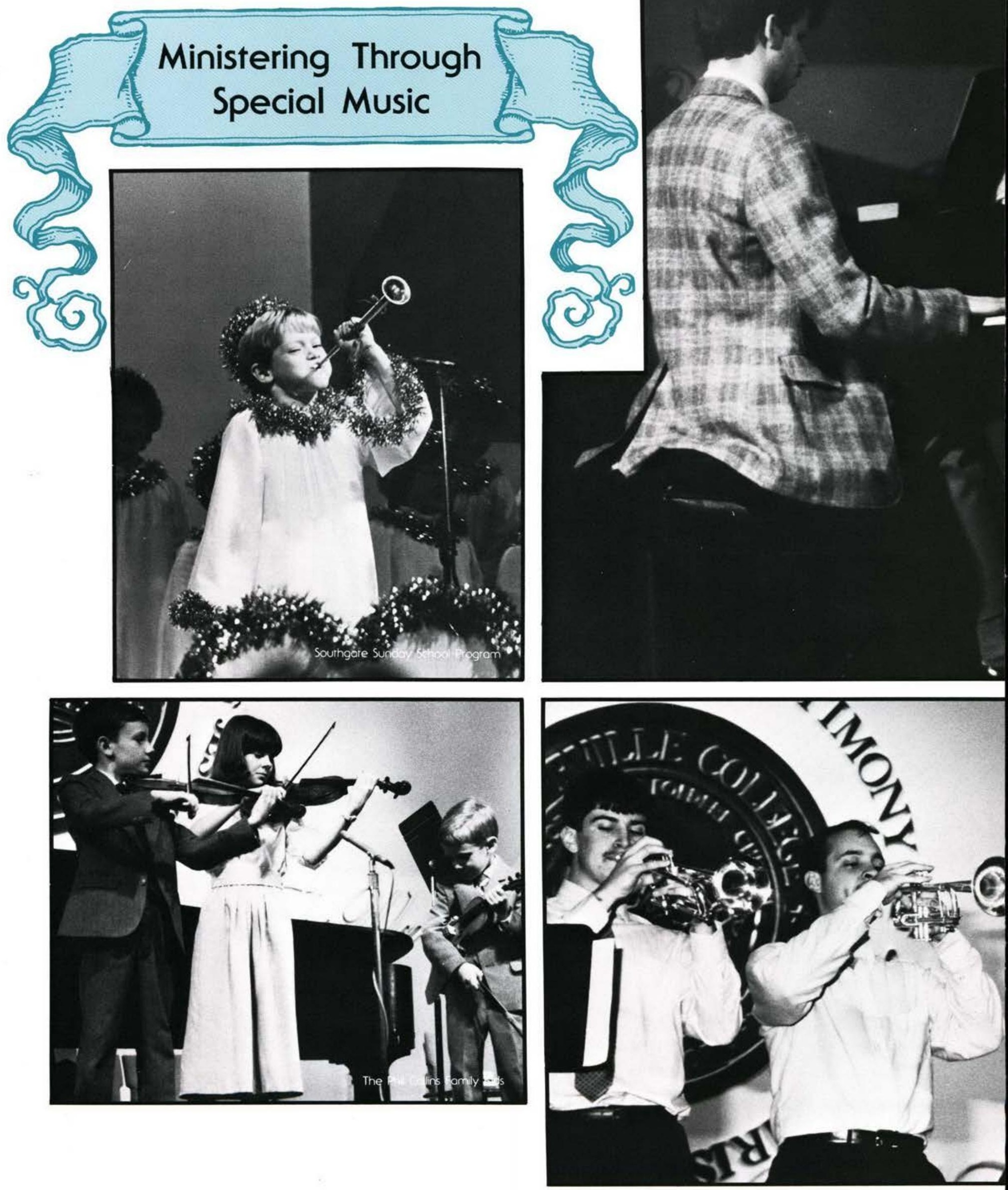



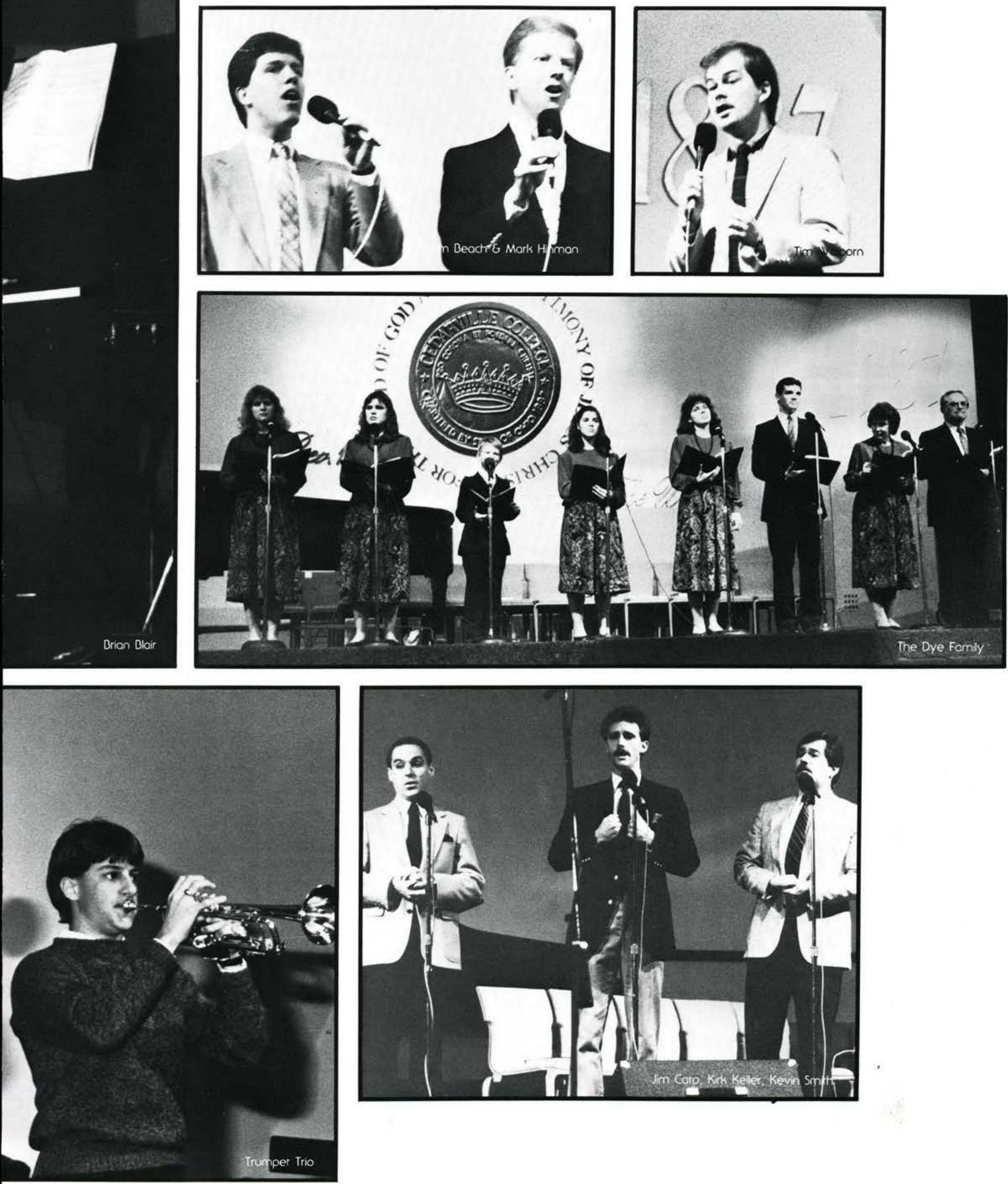

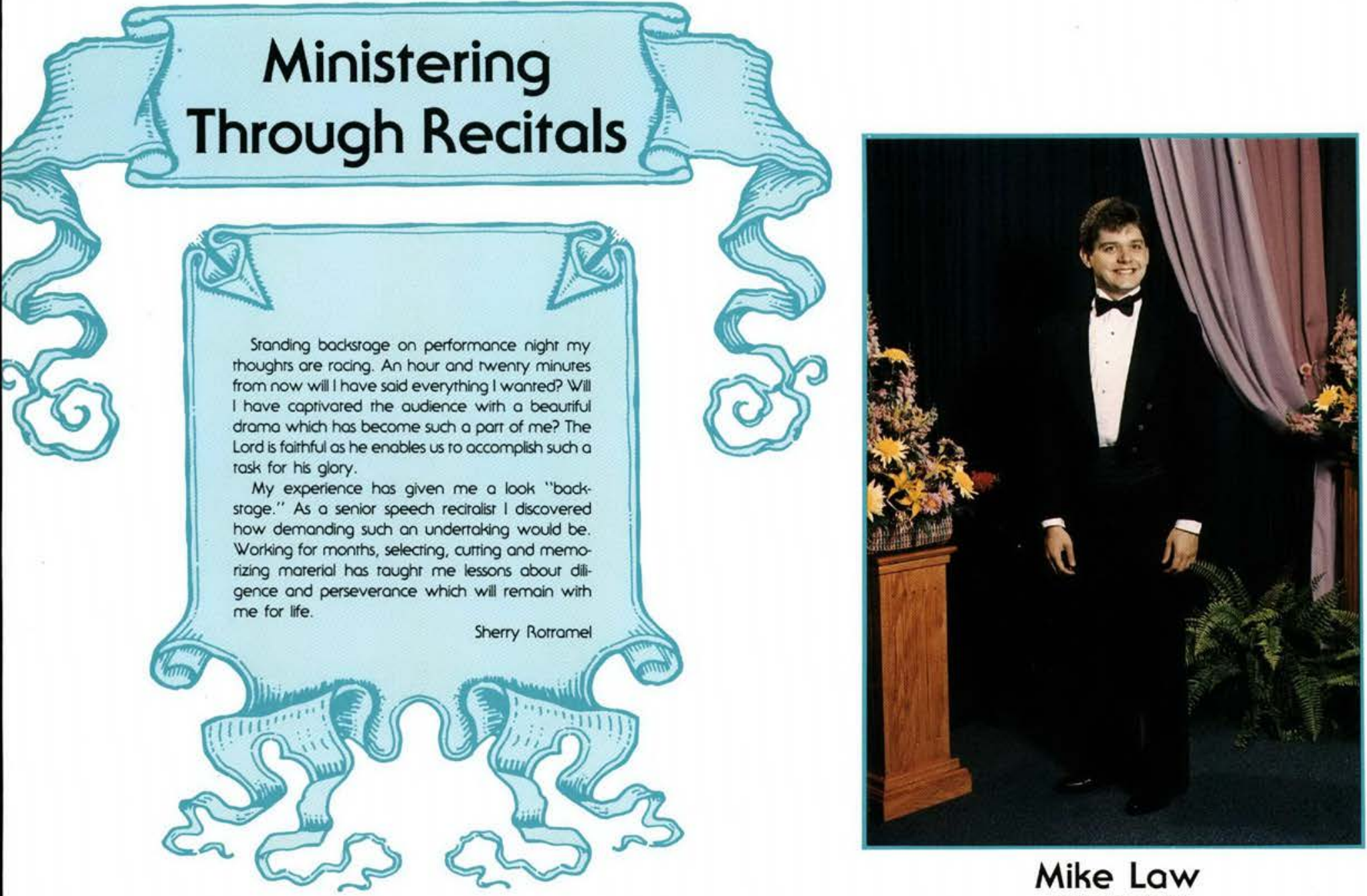

Mike Law

Voice Reciralist

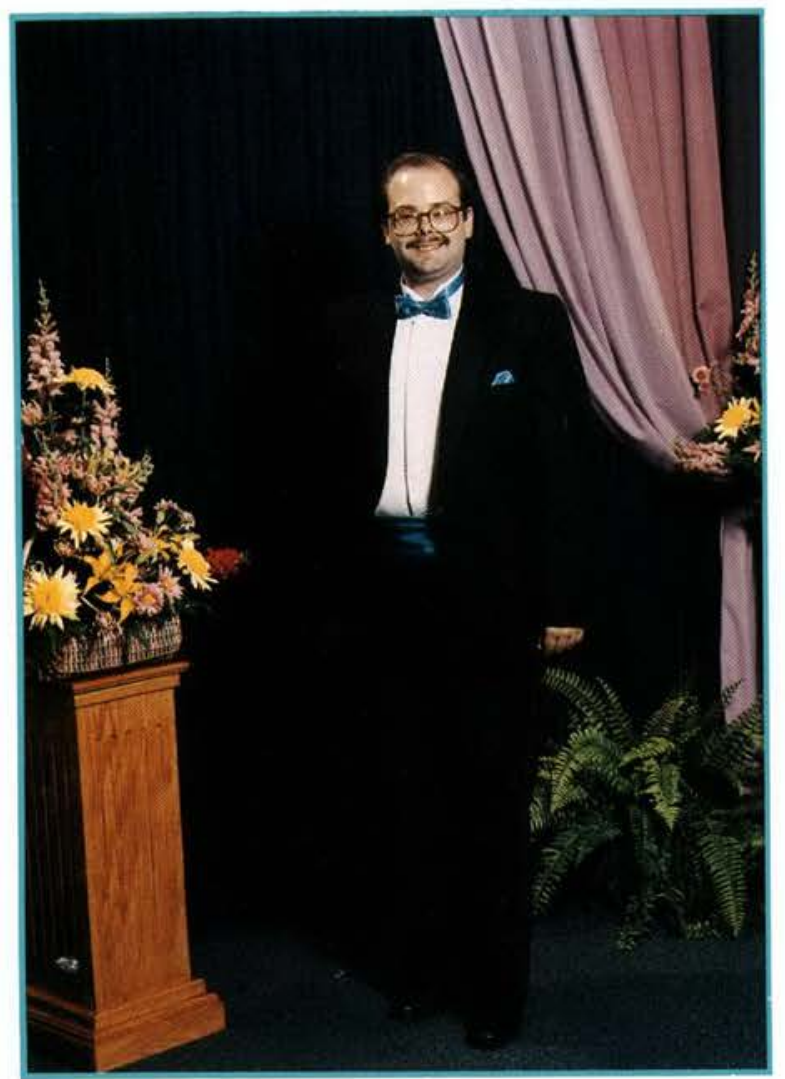

Warren Brown

Organ Recitalist

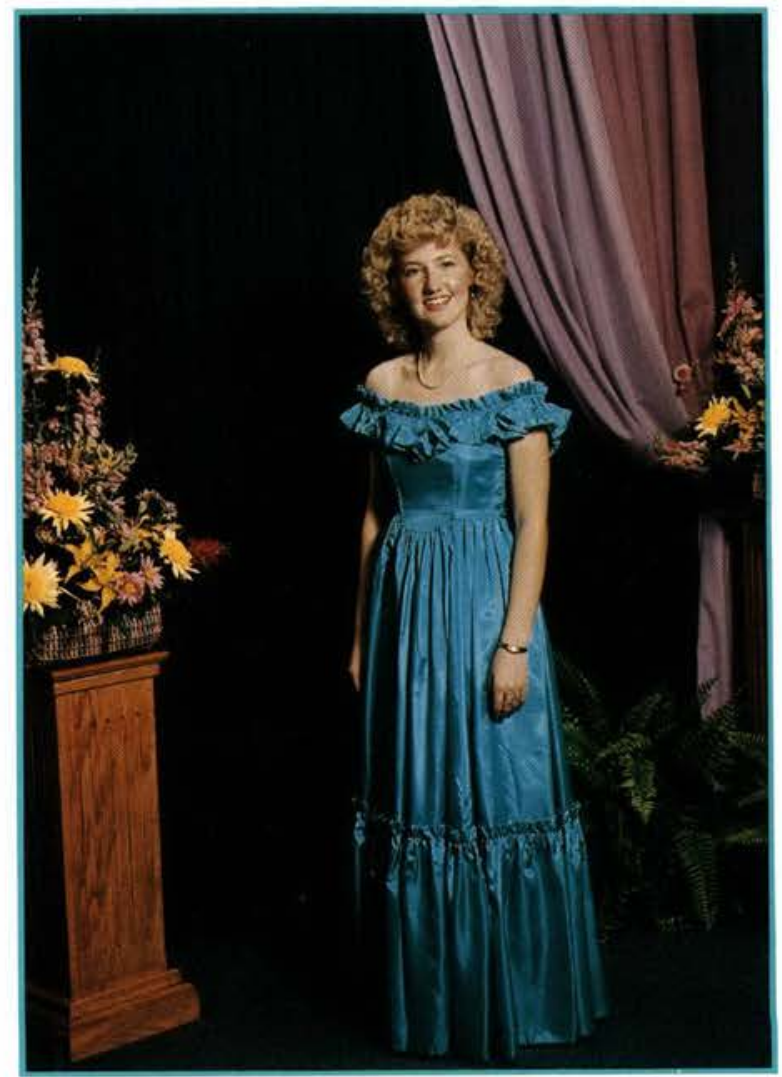

Sherry Rotramel

speech Recitalist 


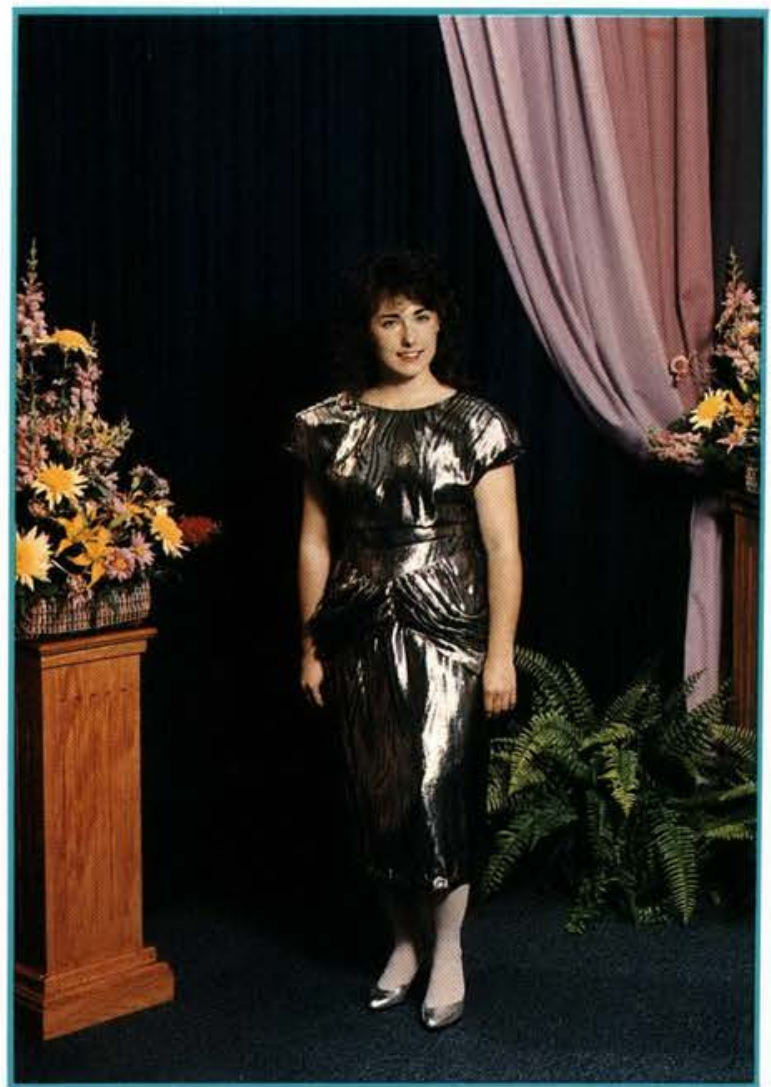

Jana Dowsett

Voice Recitalist

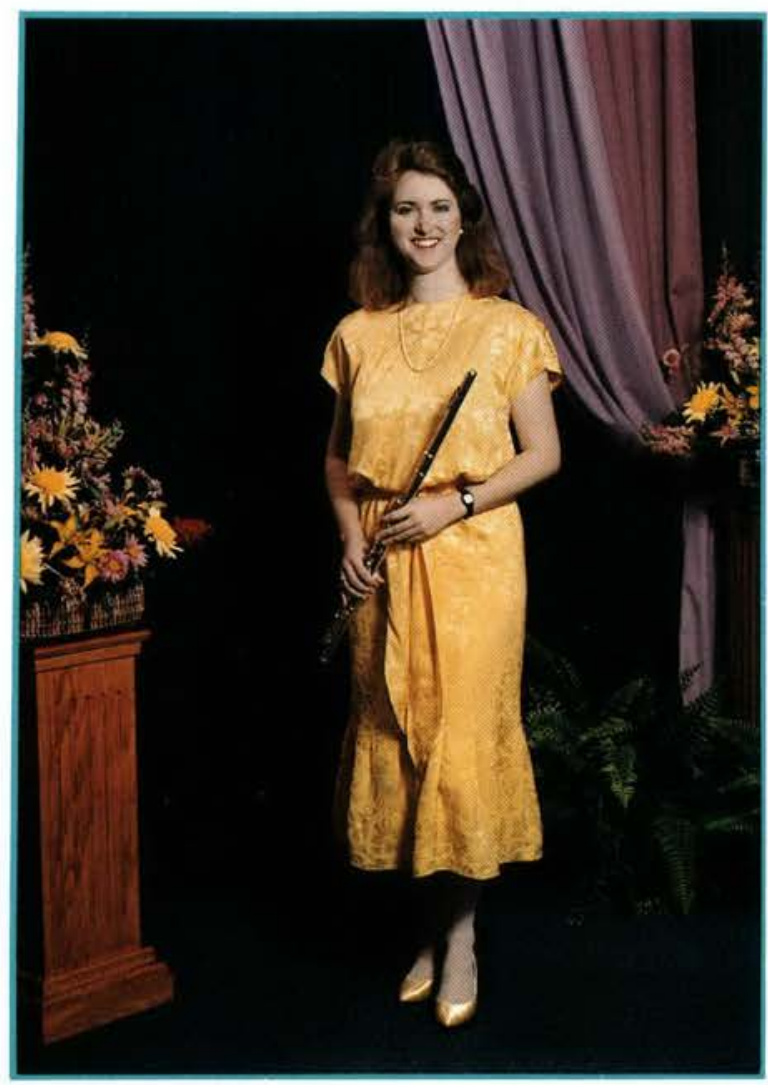

Rebecca Haga

Flute Reciralist

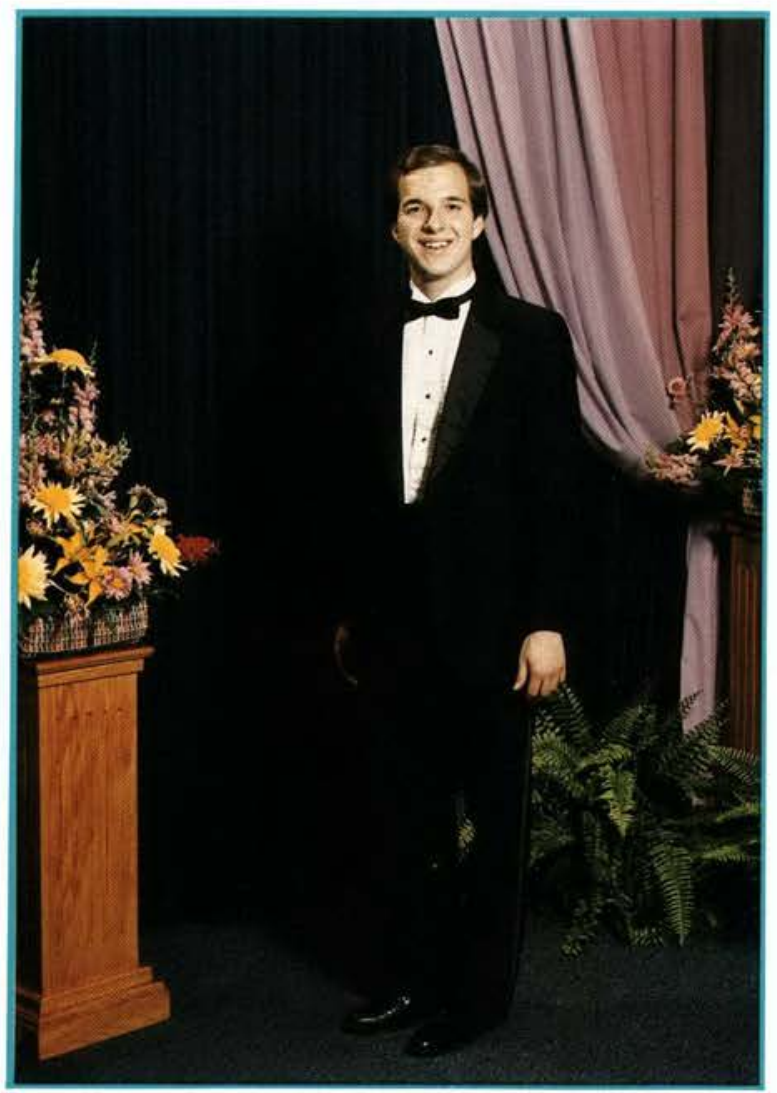

Eric Helmuth

Piono Reciralist

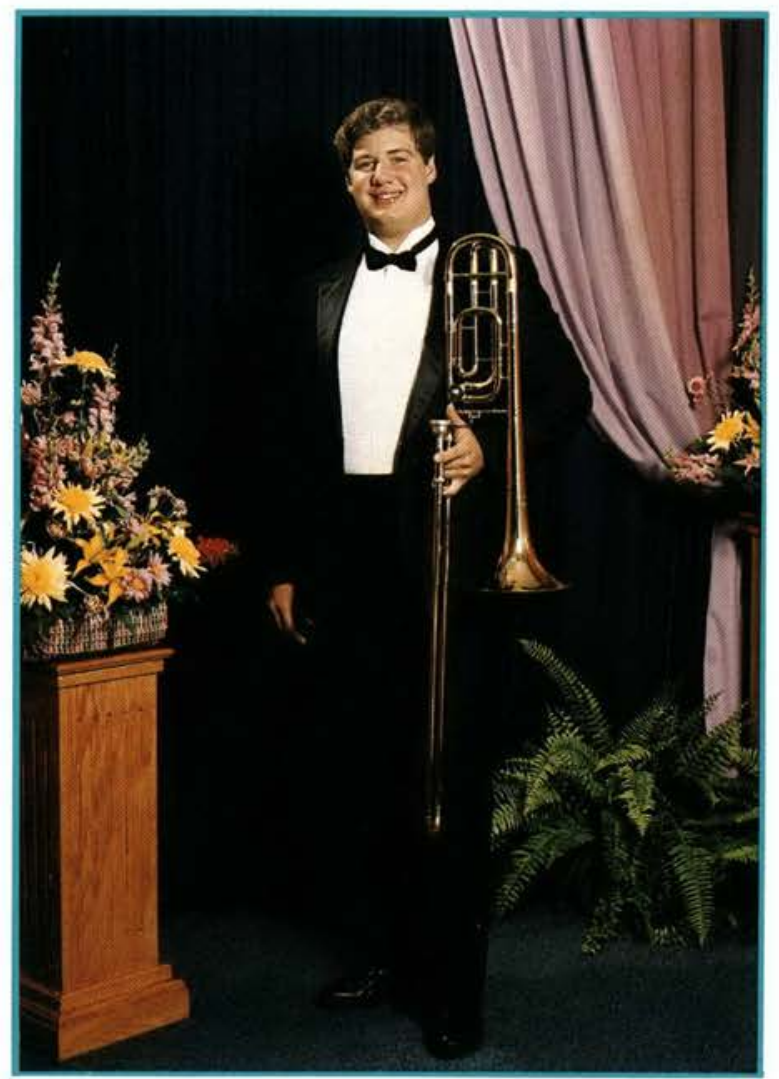

Steve Wood

Trombone Reciralist

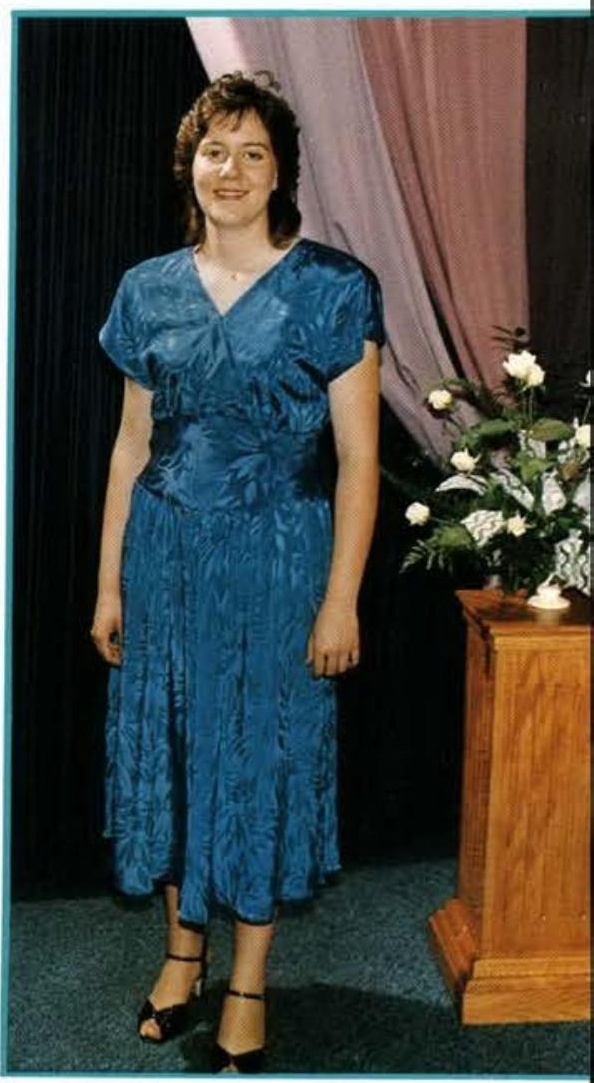

Mary Ruth Cook

Piano Reciralist 


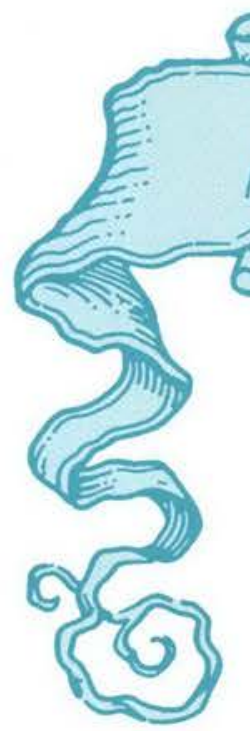

\section{Ministering As Professionals}

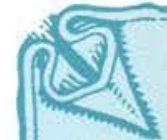

The Compus Acriviries Office sponsored a wide variery of performers this year in the 1986-87 Artist Series. Beginning in October with Andre-Michel Schub, a pianist performing his third rime in the James $T$. Jeremioh Chapel. Orher vererans rerurning for this year's Artist Series were the Ohio Srare Universiry Glee Club, who performed a delighrful concert in March; the Dayton Philharmonic Orchestro; and the Empire Bross, a group of five extremely ralenred gentlemen who once ogain graced our campus in April with their special arrange ments of a variery of music. Borh Christine Wyrtzen and Sreve Green olso rerurned to perform in the James T. Jeremiah Chopel this spring, each reinforcing the already zealous popularity they know here ar Cedarville.

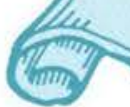

) hininmy
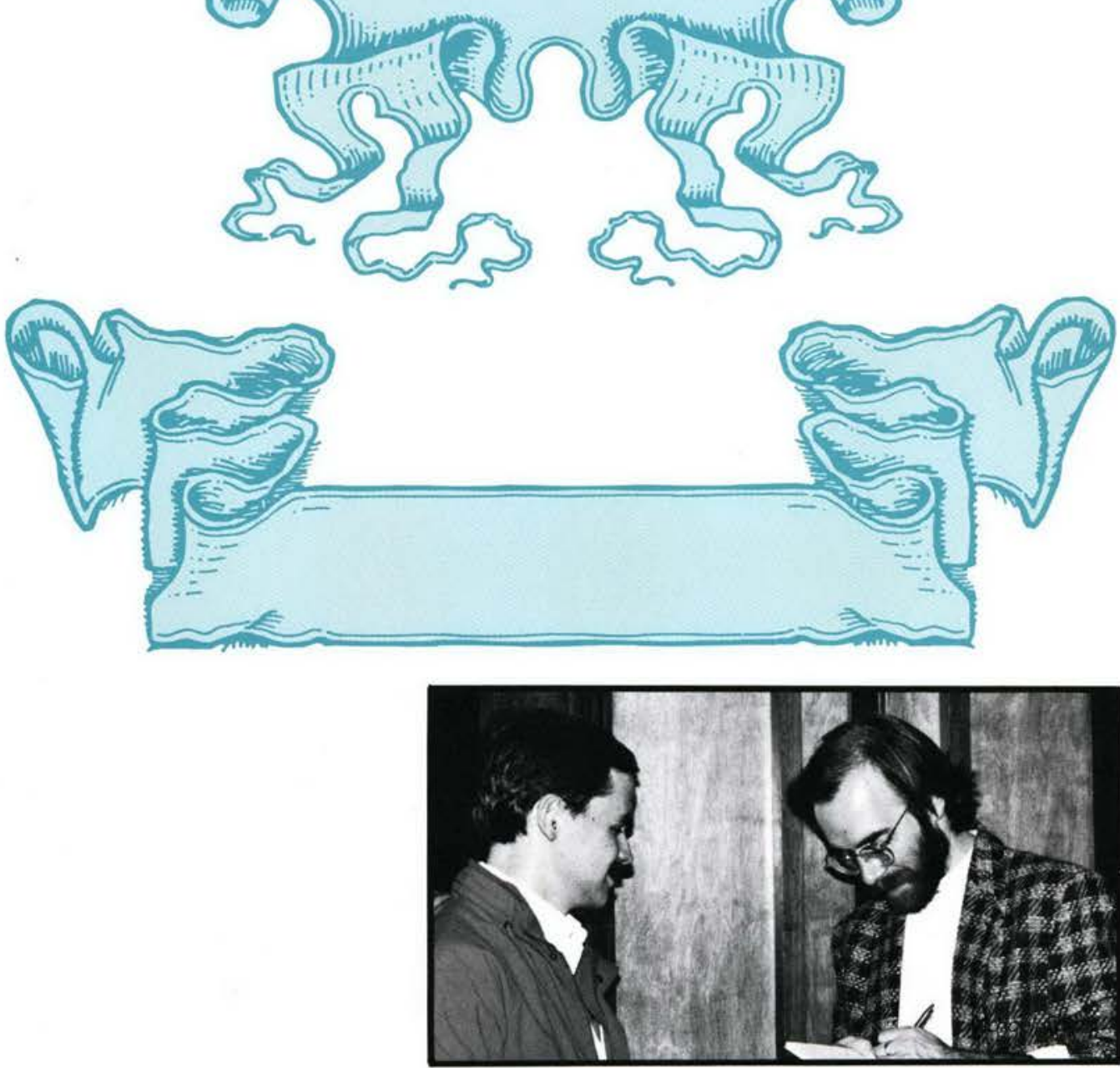

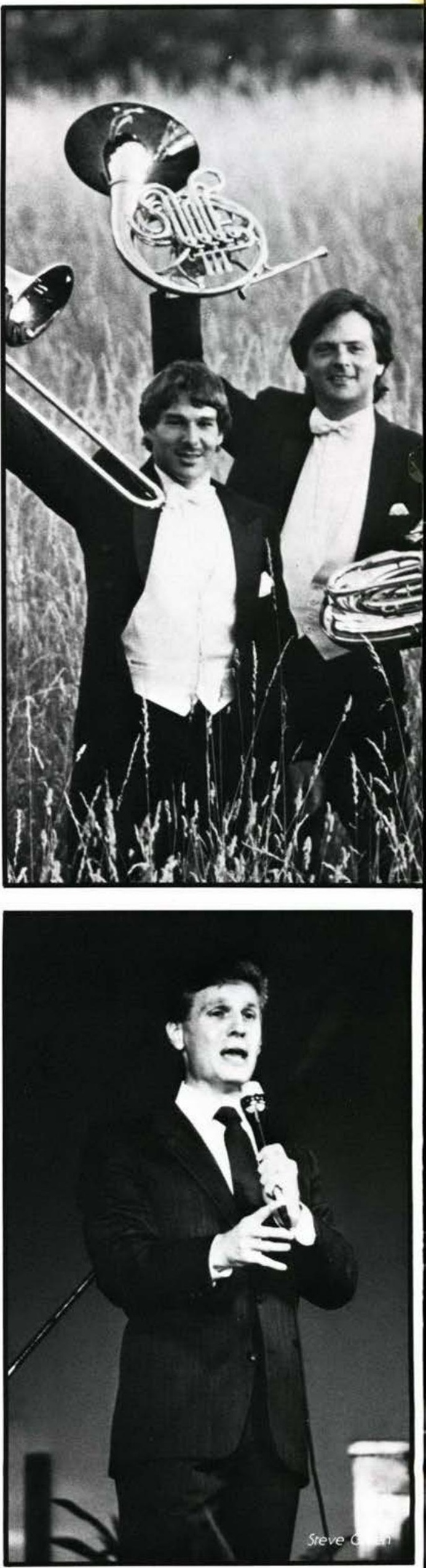



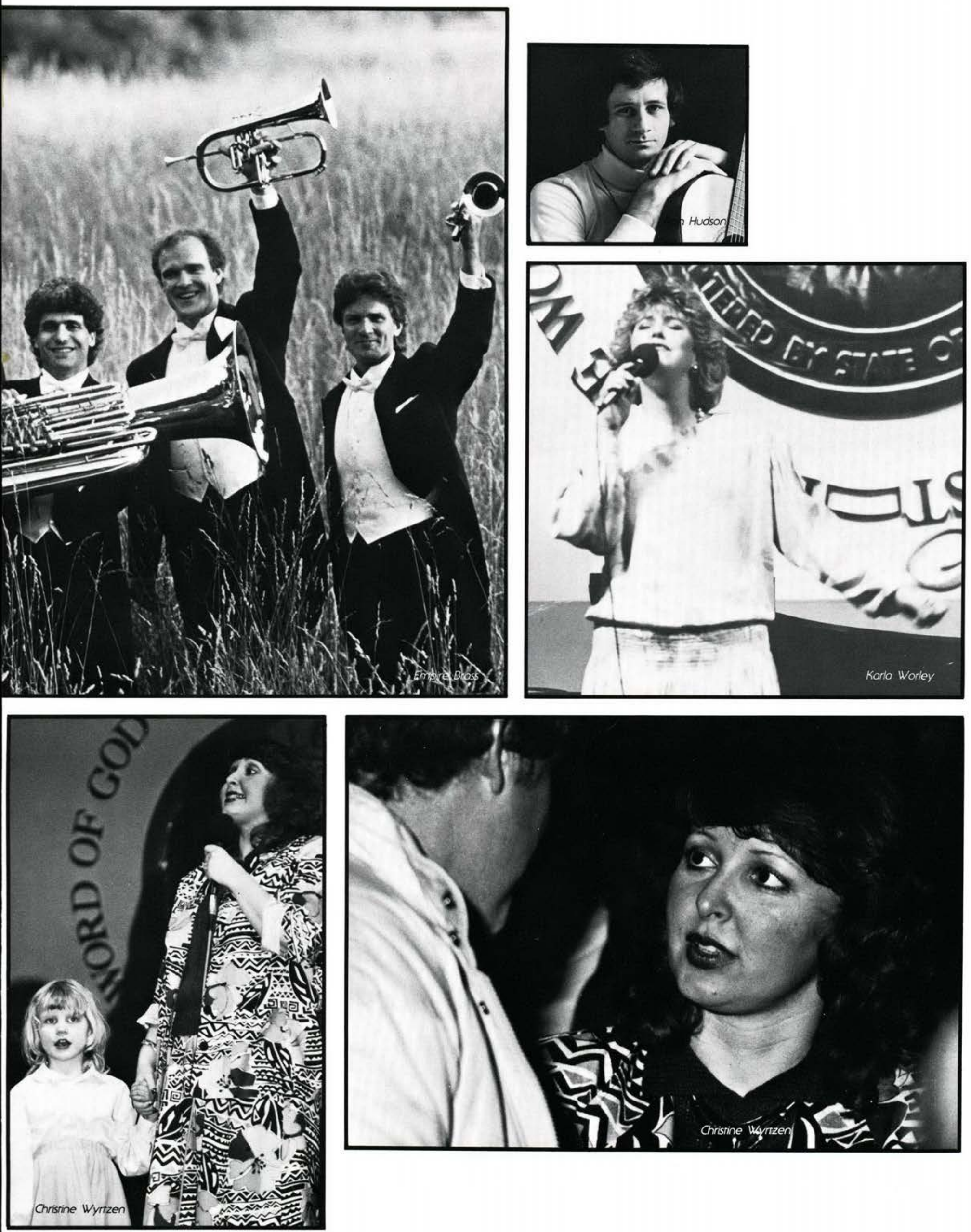


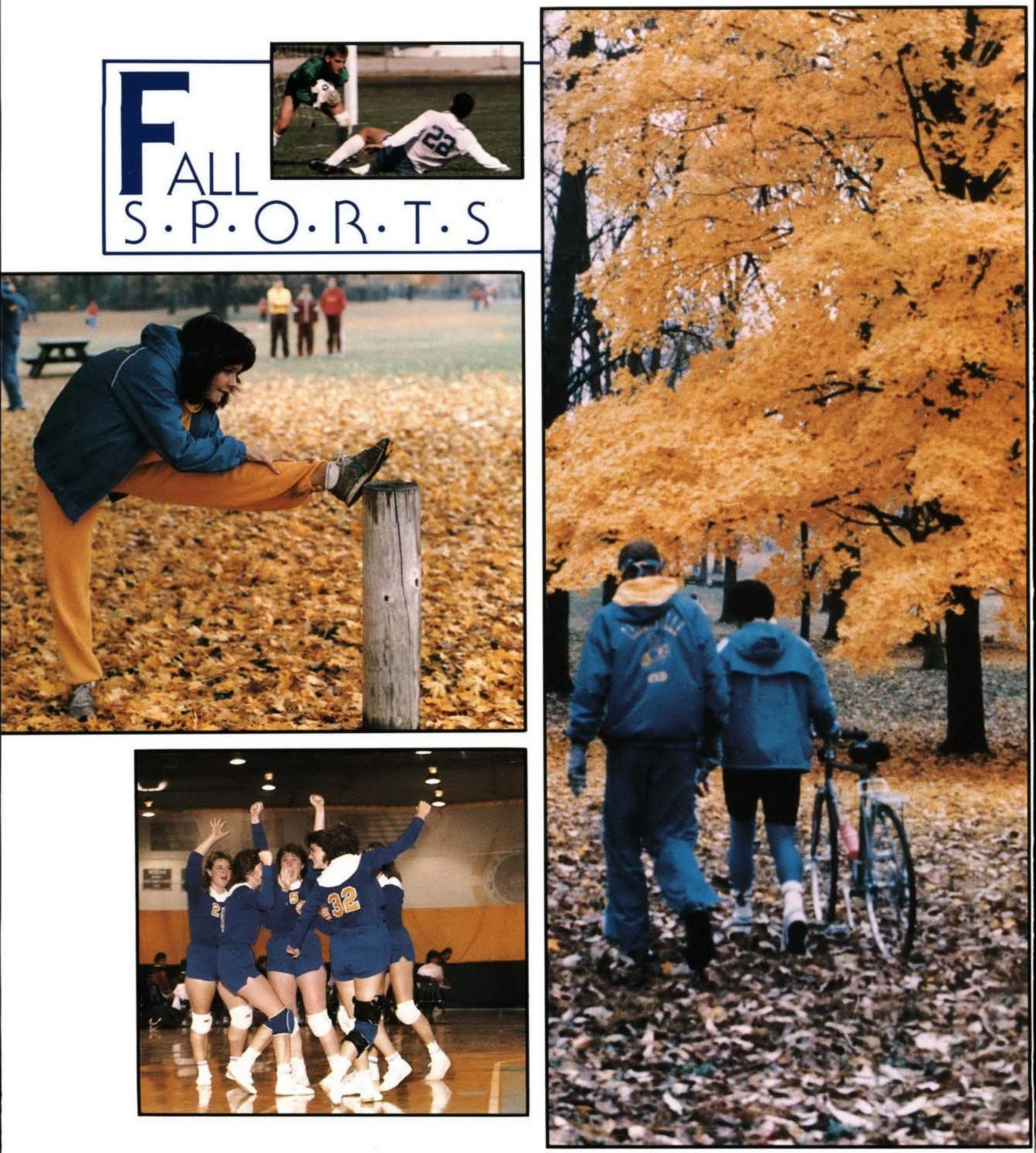




\section{Blighted Hopes}

\section{FINISH LINE}

Cedarville

Denison

Moody Bible

Tenn. Temple

Ohio Dominican

Ohio Wesleyan

N. Kentucky

Each individual on the 1986 soccer team had dreamed of returning to the NCCAA playoffs in Texas for the third consecurive year, but sever al early season victories only created false hopes. Later in the season the ream faltered to opponents of lesser caliber and ended with an 11-7-2 record. Dubbed a "birter-sweet" year, with no chance for the National Championship ritle, it was often difficult to remain commitred ro the team in face of serbacks and defears.

Assessing the season with hindsight, though it is easier to keep it in perspective. Although a third trip to Texas would have been nice, soc cer is not the most important thing in life, and no doubt the memory of the 1986 season will also fade. Coach McGillivray emphasizes the importance of prayer, thanking God for the defears as well as the victories, because both are necessary to grow physically and to foster a healthy relationship with God. As II Corinthians 12:10 srates, "Therefore I rake pleasure in distress for Christ's sake: for when I am weak, then am I strong."

Sreve Hanson

Roger Swiggart, Rod Haseltine, Andy Mininger, Lowel Holbach, Dan Howk, Greg Nordon, Tom Pererson, Kirt Wilson, Sreve Fogle, Phil Fogle, Sreve Rocz, Andy Lew. is, Poul Normon, Tim Davis, Brian Hulrz, Rick Seidel, Sreve Hanson, Mike Dovis, Mike Green, Dan Byrum, Cooch: John McGllivray, Assistont Cooch: Tom Fire, Manoger: Brian Schroll.

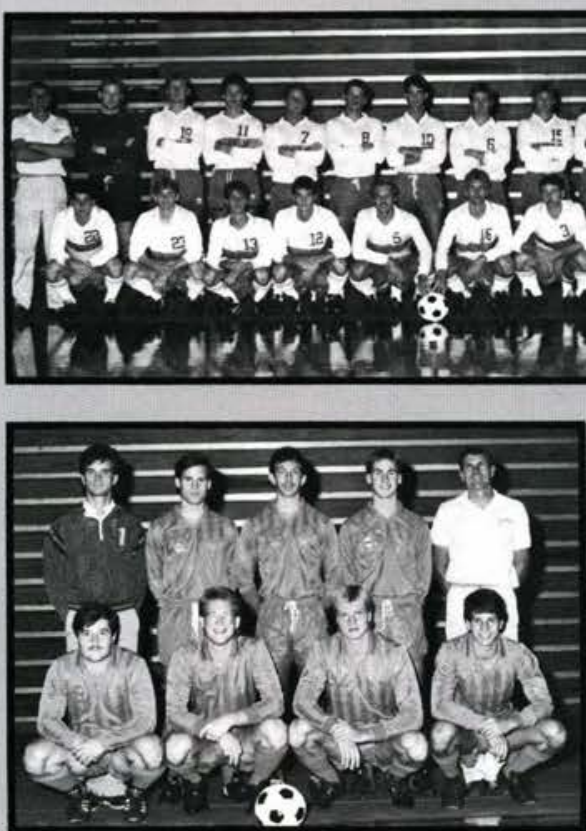

Nathon Davis, Dave Rumberger, Mike Miller, Brian Smirh, Tim Ryan, T.J. Rourson, Brian Stevens, Cooch: John MCGilivray, Assistant Cooch: Cregan Cooke.

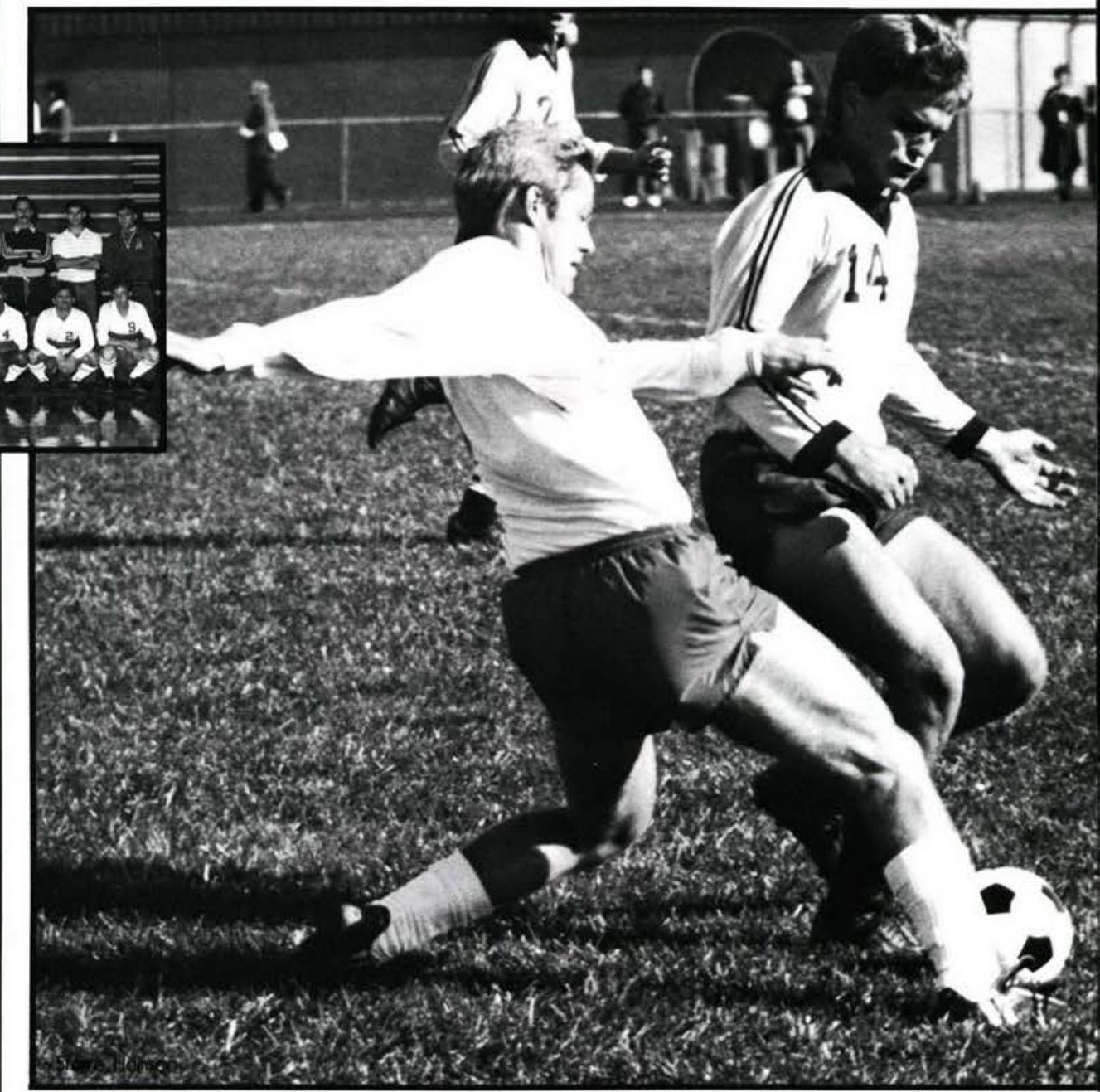



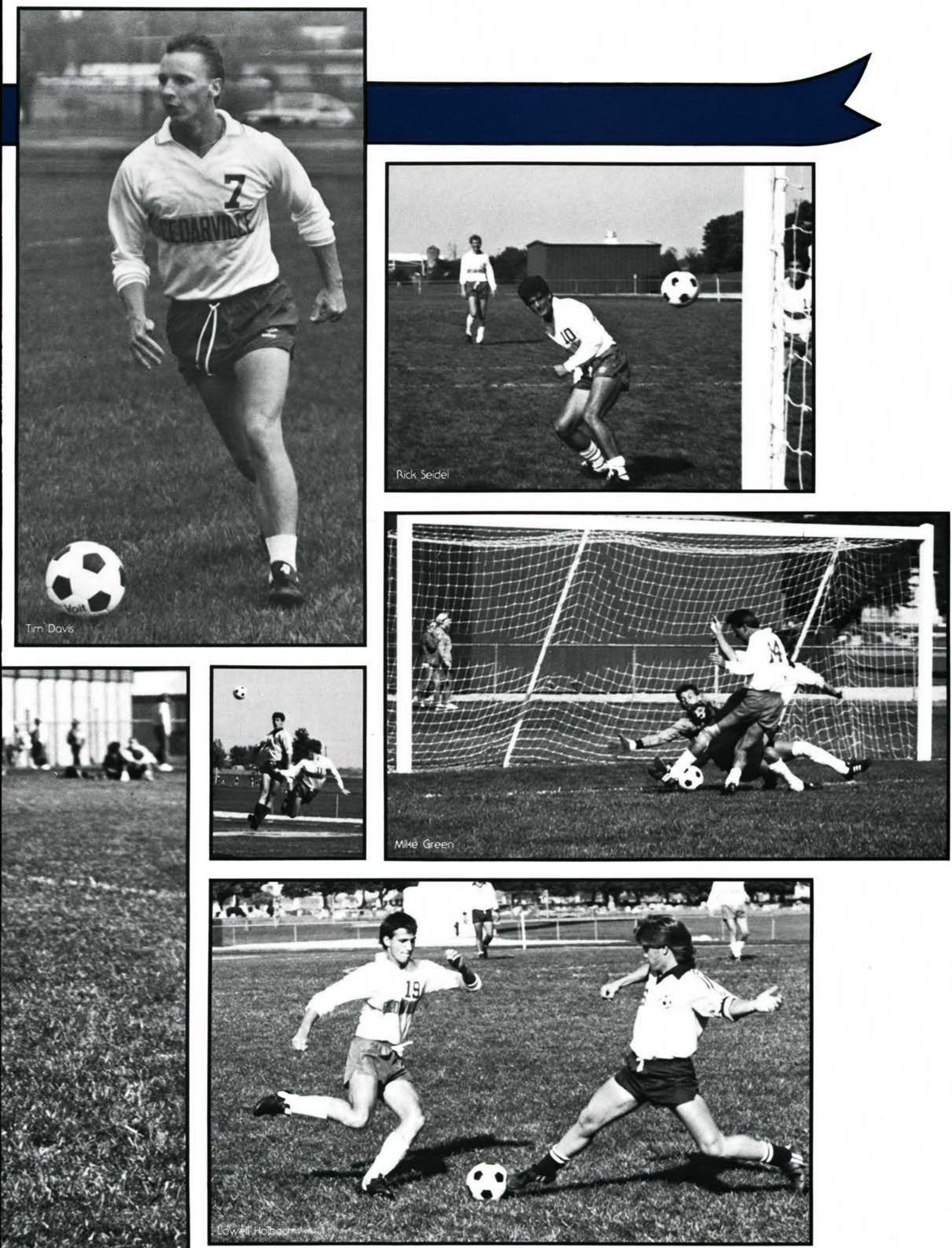


\section{Proven Depth}

\section{FINISH LINE}

Men's Cross Country

Marshall Inv.

Wittenberg Inv.

Wright State Inv.

Rio Grande

Loyola Inv.

All Ohio Meet

MIOC

NAIA District 22

NCCAA Nationals 3rd out of

1st out of

1st out of

1st out of

2nd out of

7 th out of

3rd out of

3rd out of

4 th out of

Women's Cross Country

Wittenberg Inv.

Wright State Inv.

Rio Grande

Loyola Inv.

All Ohio Meet

Cincinnati Inv.

NAIA District 22

NCCAA Nationals

2nd out of
3rd out of
1st out of
8 th out of
16th out of
5 th out of
2nd out of
3rd out of

6

3

12

12

7

3

10

Men's Team: Jeff Bolender, J.P. Dawes, Ben Garrison, Brian Humphreys, Rob Moore, John Oswald, Sreve Schepers, John Urban, Kevin Wolloce, Casey Williams, Sreve Cox, Eric Fillinger, Tom Hill, Chris Heffler, Cooch: Evin King

Women's Team: Becky Averill, Berh McGillivray,

Grerchen Mountz, Belinda Sills, Jennifer Stevens, Carhy Warson, Laurel Yares, Barb Sloan, Deb Pare, Lynn Clark, Bev Fisher, Cooch: Elvin King.

The hard work and commitment of the men on the 1986 cross country team paid off this year when they were rewarded with a strong season from start to finish. Rob Moore led the team almost the entire season, supported by strong performances from John Oswald and Eric Fillinger. At the NCCAA Narionals, Oswald ran first for the team and placed sixth overall.

Although the 1986 women's cross country team had two strong lead runners in Deb Pare and Carhy Warson, the depth of the ream proved to be as much of an asser throughour the season. Pare qualified for All-American Srarus in the NCCAA Narionals as well; and Watson ran a powerful, second position all season, inproving her rime each meet. The remainder of the ream - Becky Averill, Lynn Clark, Beth McGillivray, Gretchen Mounts, Belinda Sills, and
The All-Ohio meer, though, was the site of one of the ream's best performances this season, where the men placed 7th out of 37 teams and second in the college division. The season ended just as strongly with Moore's and Fillinger's positions at the NAIA National meet in Kenosha, Wisconsin.

Berh McGillivroy

Laurel Yares traded positions during the season and provided a solid foundation for the team's overall standing. This paid off at the All-Ohio meer where the women placed 5th our of 17 reams in the college division. Affecred by Malones's entrance in the NCCAA meer, the women placed third overall, but were pleased with a season highlighred by commirment and strong performances from each member.
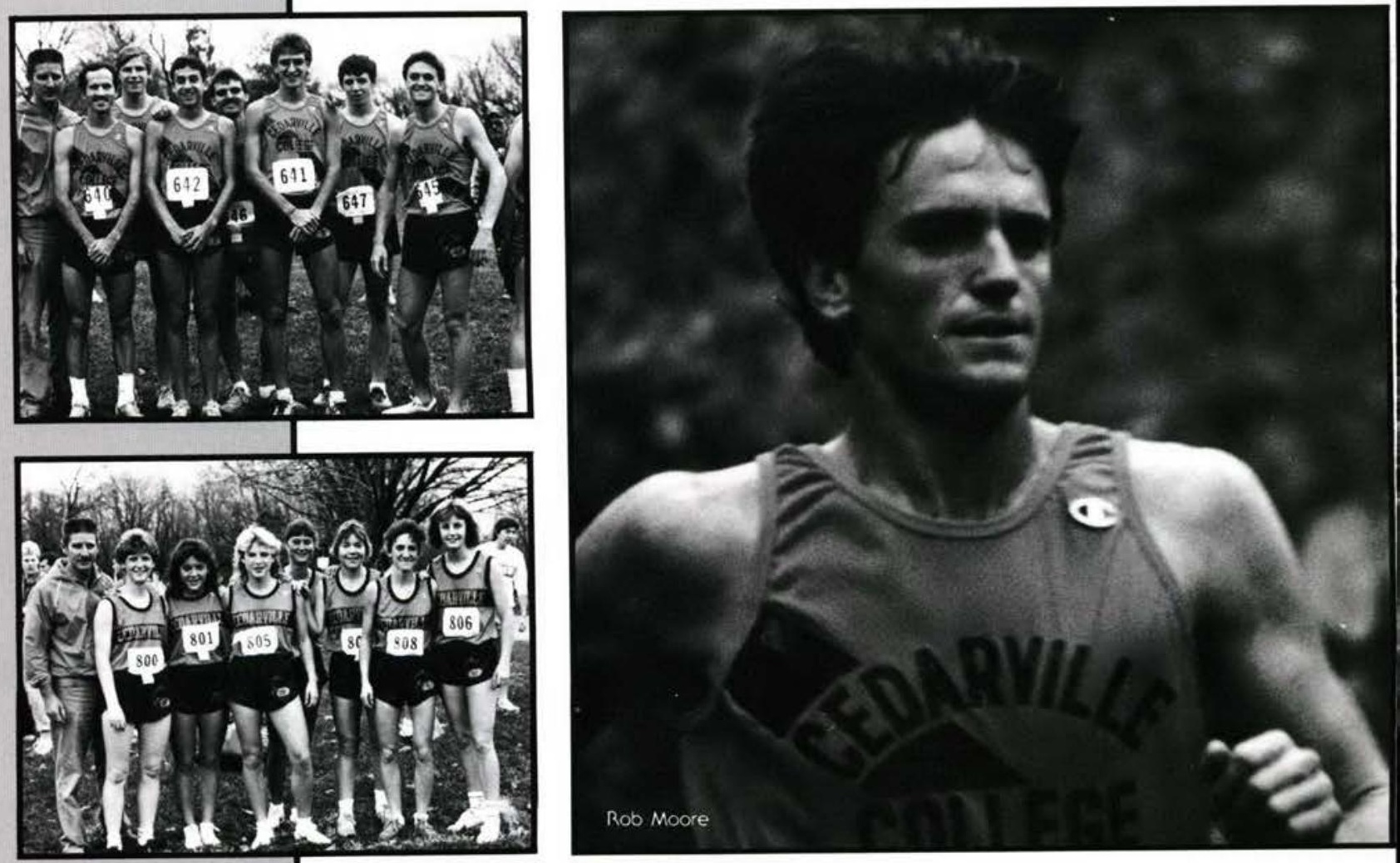

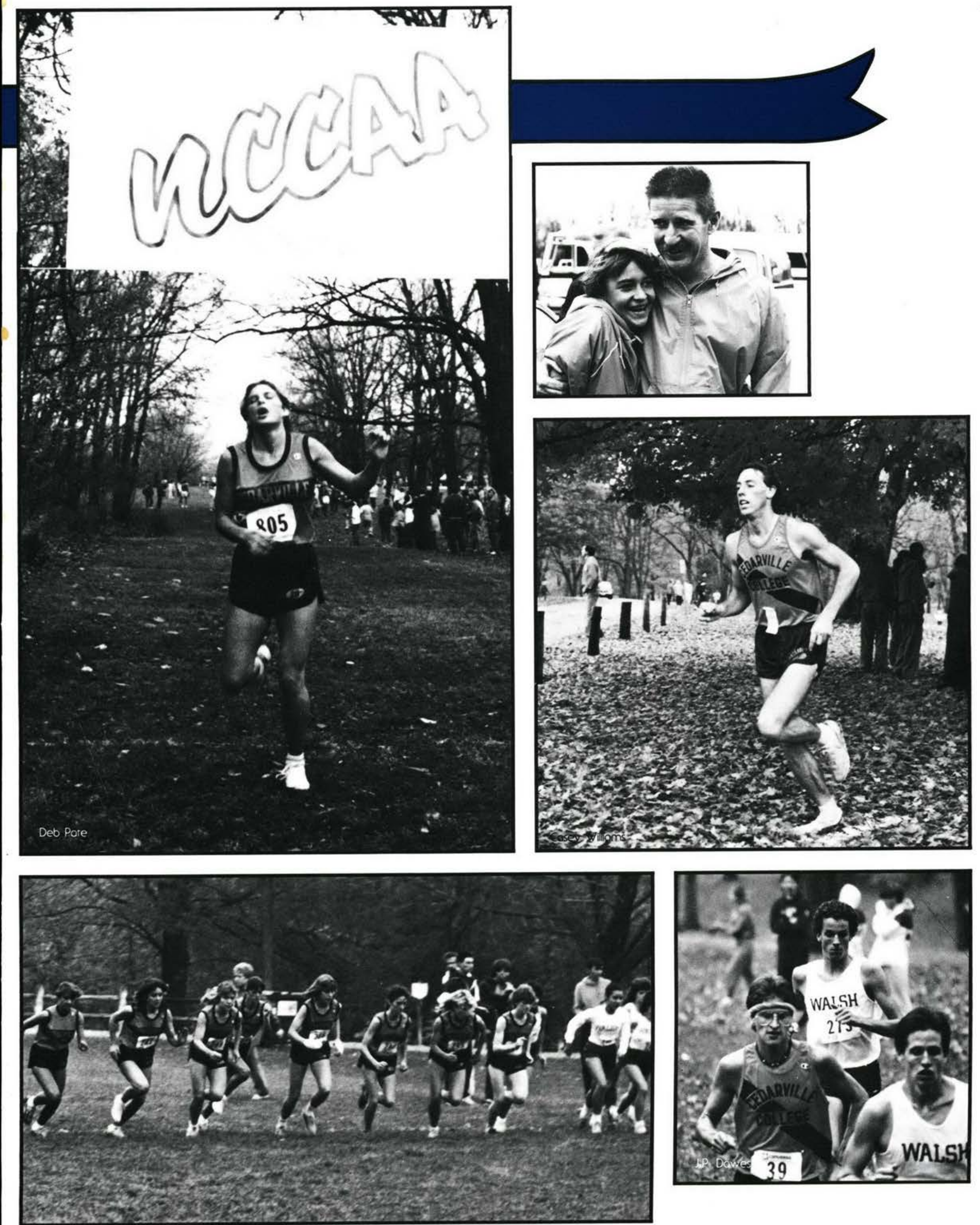


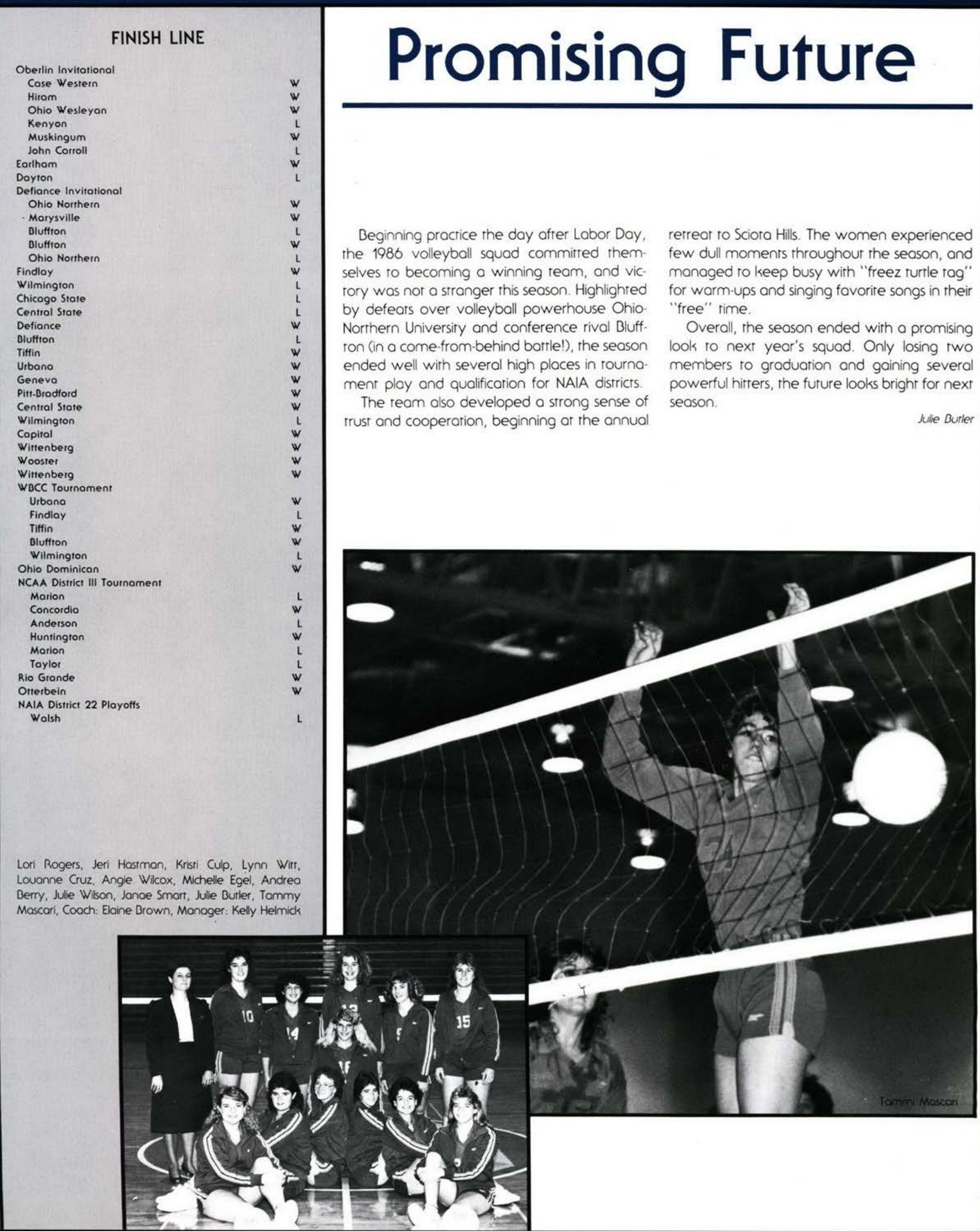



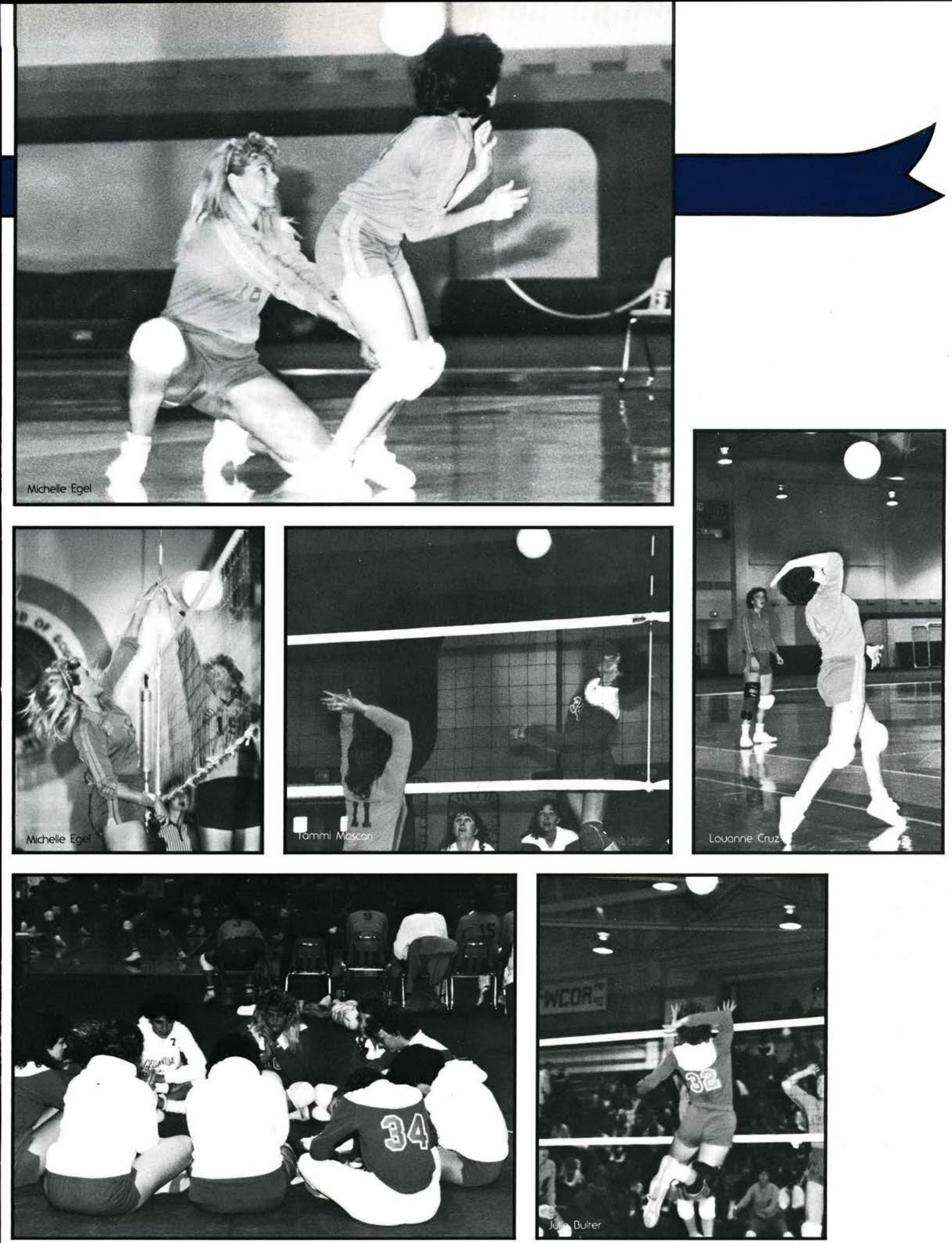

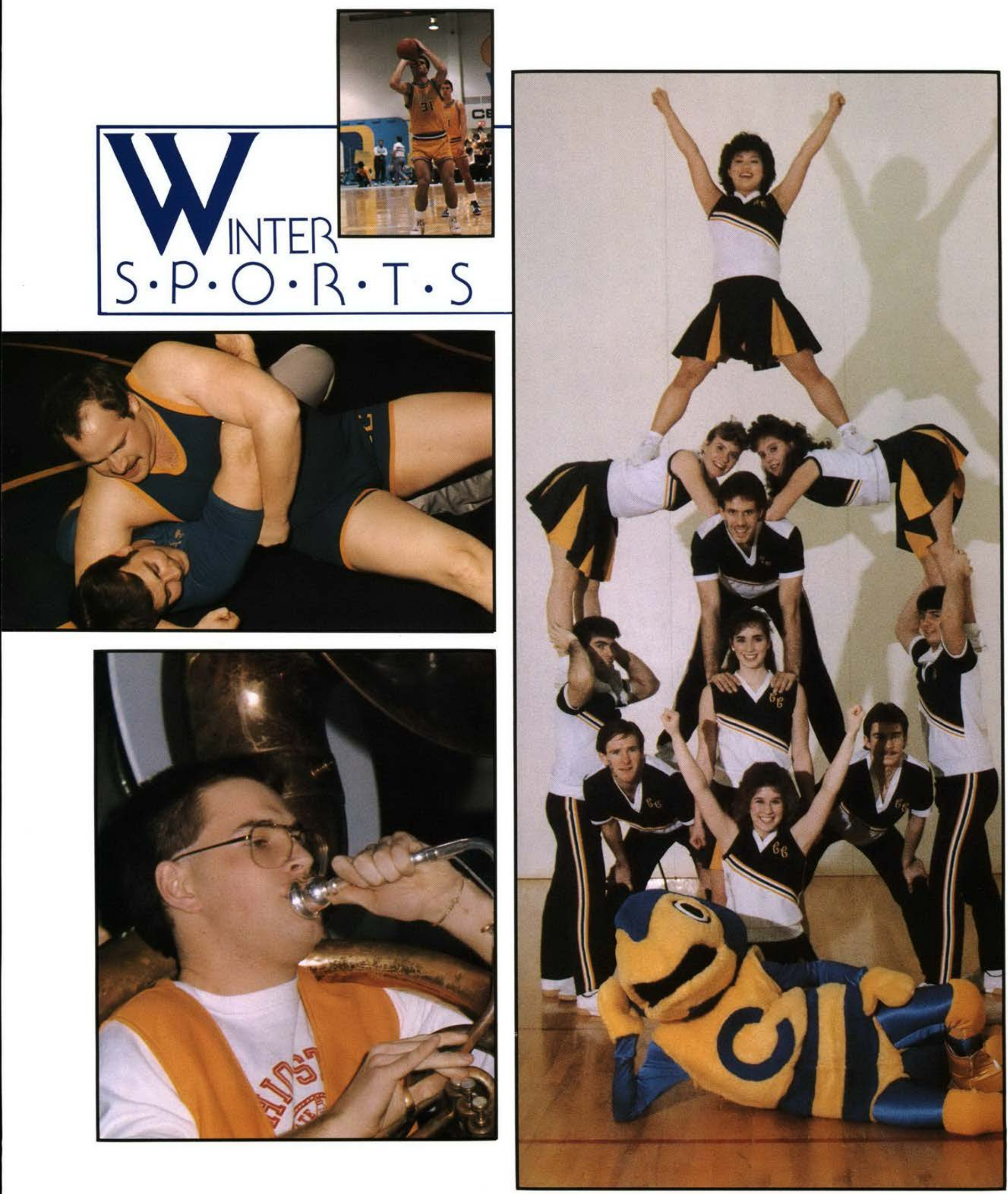

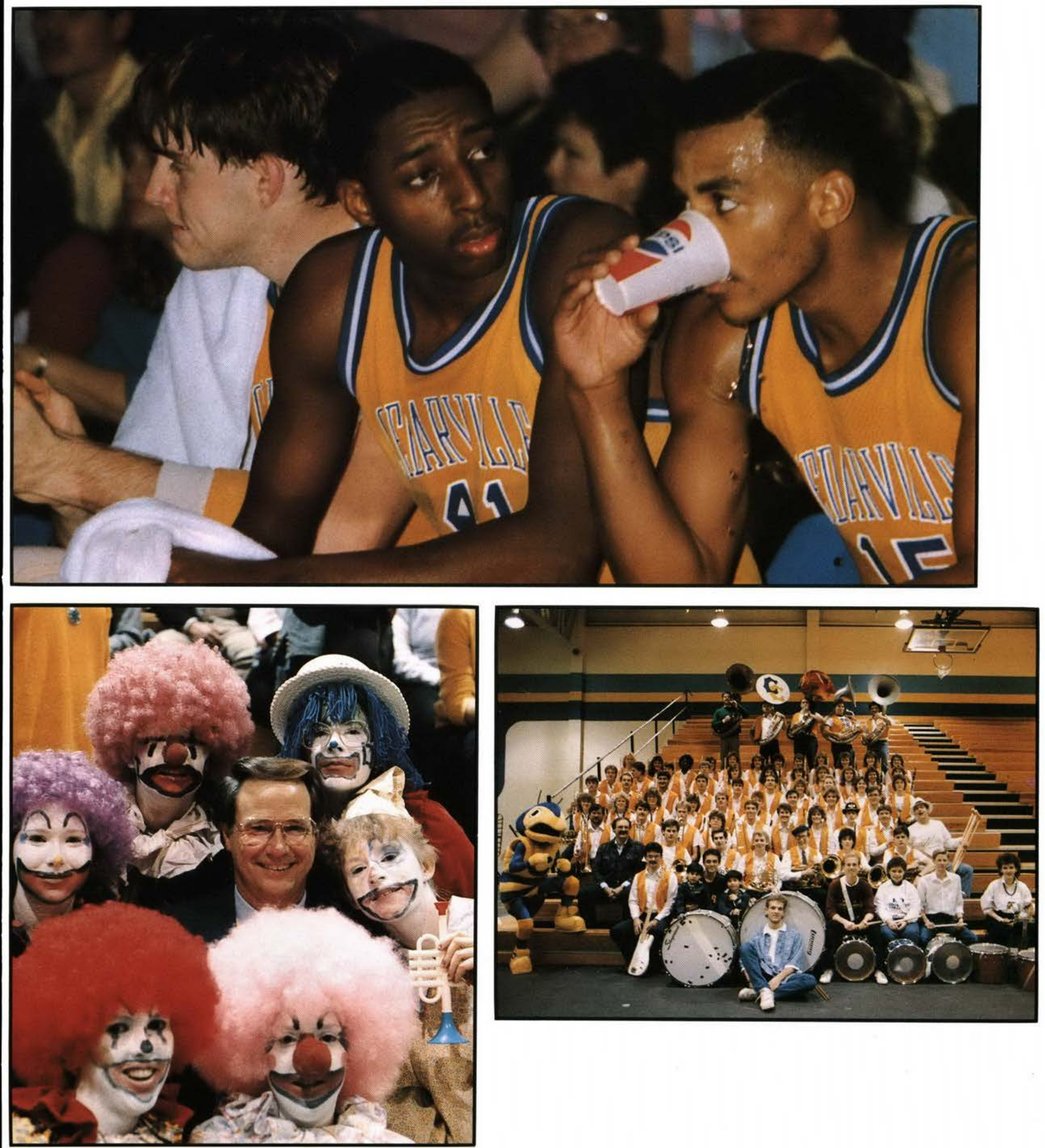


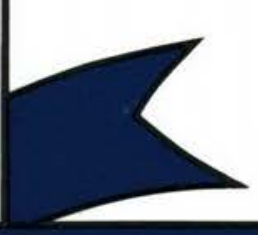

\section{FINISH LINE}

Cedarville

Guelph

Wilfrid Laurie

Concordio

Western Ontario

Indian Tech.

Ohio Wesleyan

Wilmington

Warner Southern

Polm Beach Atlantic

Nova

Urbane

Malone

Mt. Vernon Nazorene

Ohio Dominican

Rio Grande

Walsh

Tiffin

Urbana

Wilmington

Malone

Mt. Vernon Nazarene

Ohio Dominican

Rio Grande

Walsh

Defiance

Findlay

Total Record
Opponent

66

67

80

49

92

114

96

70

88

85

79

74

81

80

68

80

76

72

76

66

75

118

10

Doug Pugh, Derek Hughes, Mike Freeman, Eddie Wokefield, Doug Loescher, Jerry Keller, Chris Reese, David Yeoger, Tony Ewing, Gary Shaffer, Mike Warson, Don Simerly, Head Cooch: Dr. Don Callan, Assistont Cooches: Steve Young, Mark Kempron, Srudent Assistnar: Sreve Terpstra, Manoger: Tim Sites, Trainer: Evan Hellwig

\section{Unwilted Vigor}

The 1986-87 men's boskerball ream was a ream that jumped off ro a $9-0$ make which ried the school record for the most victories at the start of the season. There were dreams of a rrip to the NAIA Narionals in Kansos Ciry. However, problems occurred both on and off the court that forced this group to find a new chemistry. Chris Reese, the rop returning scorer, was lost for the season with a knee injury; and Tony Ewing, this year's leading scorer and rebounder, was declared academically ineligible. Cohesiveness was a key to a strerch run that allowed the Yellow Jackers to perform well in the playoffs.

Cedarville began srumbling through the Mid-Ohio Conference season losing seven games by seven points or less. But the Jack ets refused to wilt and their togetherness paid off in the season's final week. They won back-ro-back games ar Rio Grande and ar Walsh knocking off the MOC's rop rwo reams. That feat earned them a firstround playoff game against Defiance in the NAIA District 22 tournament. Cedarville won their third straight rwo-point decision in a thrilling $77-75$ victory. The Yellow Jackers were then eliminared ar rop-seeded Findlay College, 118-104

Lost because of graduation will be seniors David Yeager (9.3 ppg) and caprain Doug Pugh. "They demonstrared good leadership this season," said Callan. "They were fine examples to the underclassmen." Callan is oprimistic about next year. "We have some very good ball players returning. We are already looking forward to it."

Mark Womack

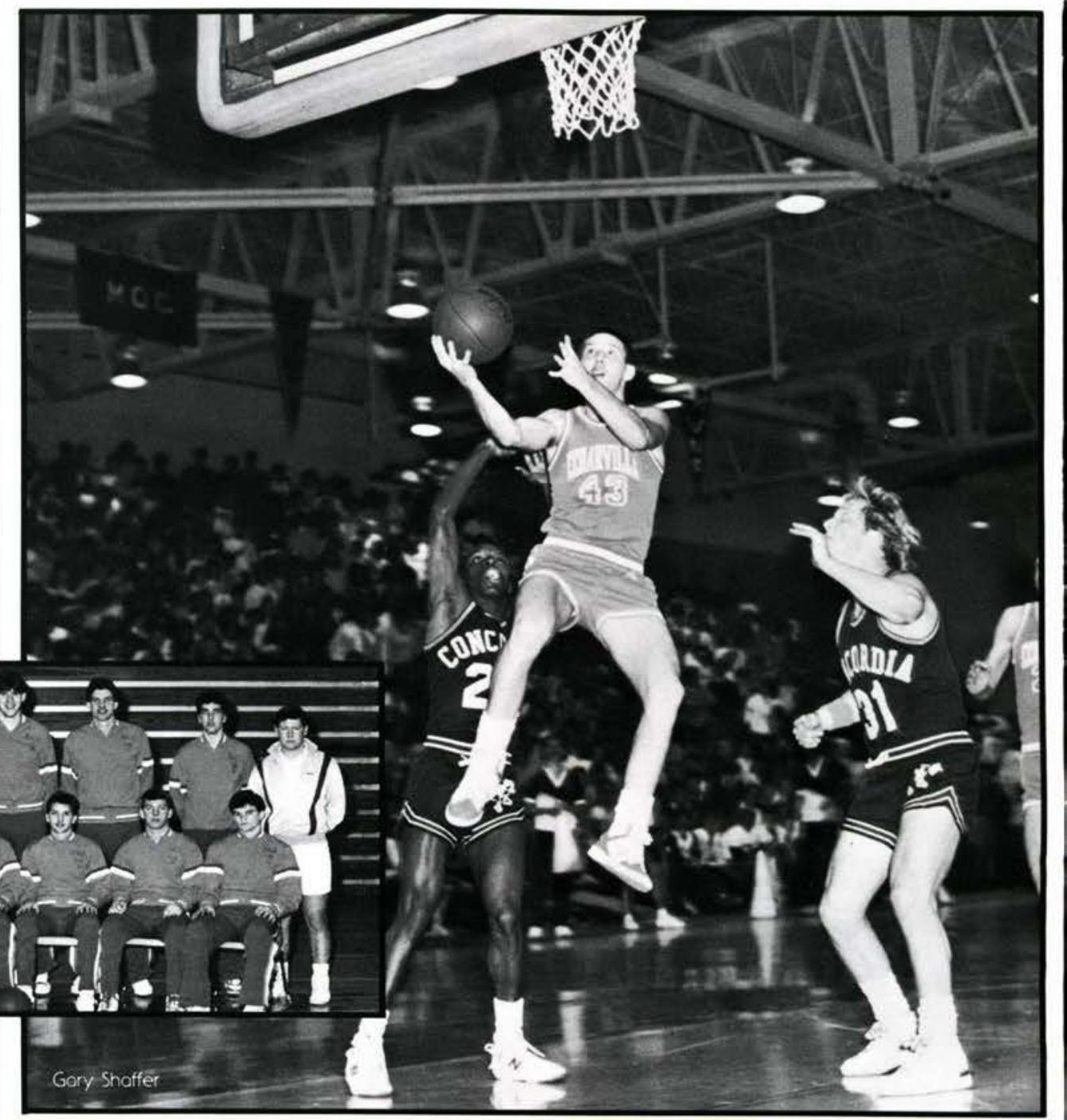



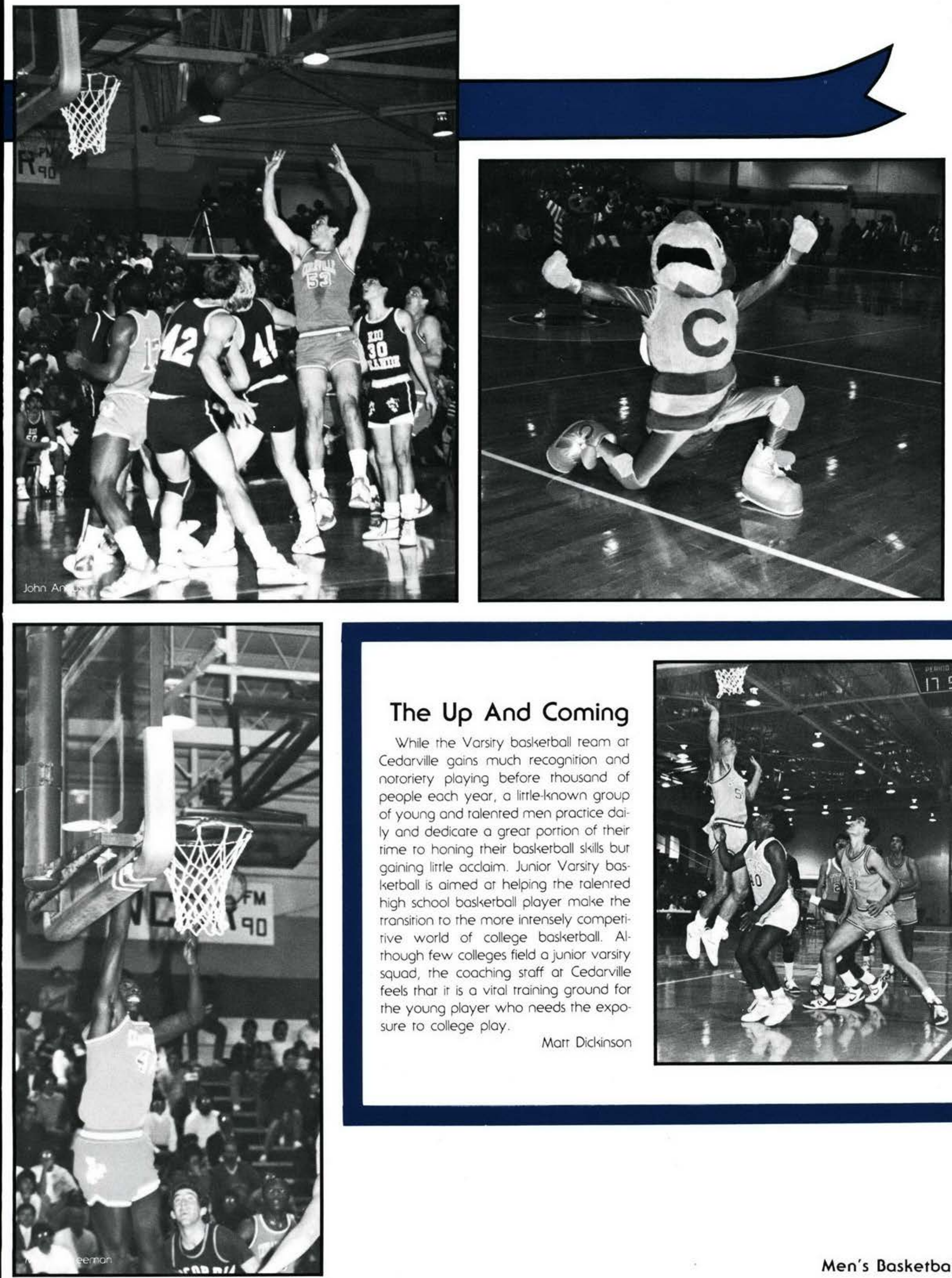

The Up And Coming

While the Varsiry boskerball ream at Cedarville gains much recognition and nororiery playing before thousand of people each year, a lirtle-known group of young and ralented men practice daily and dedicare a grear portion of their rime ro honing their baskerball skills bur gaining lirtle acclaim. Junior Varsity bas kerball is aimed at helping the ralented high school baskerball player make the rransition to the more intensely comperirive world of college baskerball. Although few colleges field a junior varsity squad, the coaching staff at Cedarville feels thar it is a vital training ground for the young player who needs the exposure to college play.

Mart Dickinson

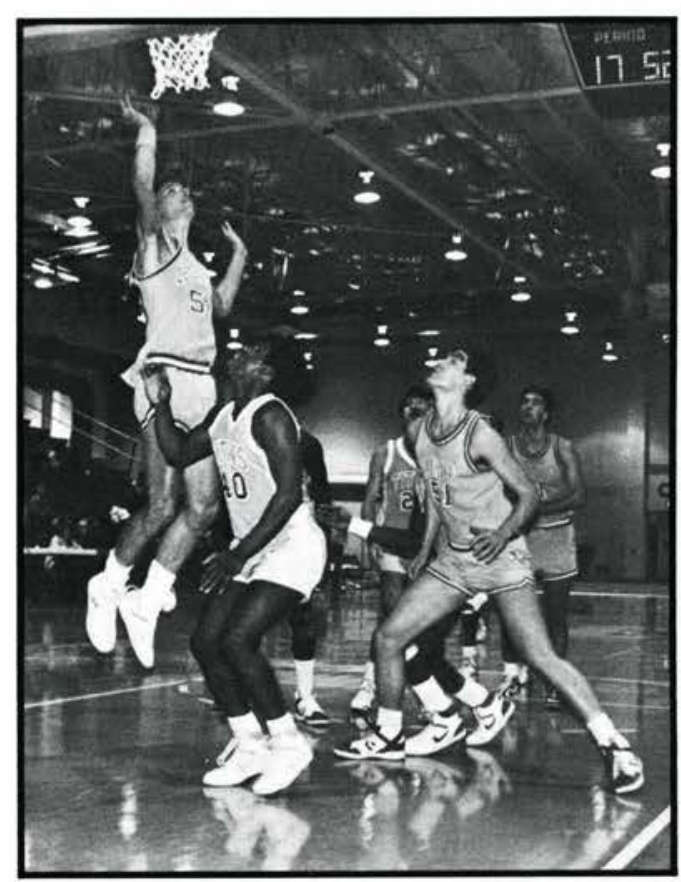




\begin{tabular}{cll}
\multicolumn{3}{c}{ FINISH LINE } \\
Cedarville & & \\
& & \\
9 & Opponent \\
6 & Dalmington & 45 \\
2 & Wittenberg & 42 \\
0 & Wilmington & 50 \\
6 & Wilmington & 51 \\
12 & Cose Western & 49 \\
27 & Urbana & 36 \\
16 & Dayton & 28 \\
19 & Defiance & 39 \\
21 & Defiance & 30 \\
18 & Findlay & 32 \\
15 & Southwest Michigan & 41 \\
6 & Olivet & 42 \\
6 & Heidelberg & 51 \\
0 & Total Record & 50 \\
& & 14
\end{tabular}

\section{Disputed Odds}

The 1986-87 Cedarville College wrestling ream showed a grear deal of dererminarion despire the odds it faced. Beginning with only three rerurning wrestlers, the team fought to overcome the problems of stiffer comperition, injuries, lack of deprh and lack of experience. The ream rrained, condirioned, and wrestled hard. Because of this effort, many first-year wrestlers managed to receive their first college wins by the end of the season.

The climax of the season came when the ream compered in the NCCAA rournament at Baprist Bible College in Clarks Summit, PA. At this tournament, Todd Dowden placed first ar 126 pounds for the third rime; Kelly Moore placed 2 nd ar $150 \mathrm{lbs}$.; Sreve M. Smith placed 5 th ar 142; Jeff Wilson placed 6th ar 158; Sreve A. Smith placed 8th ar 134; and Earl Hawk placed 5 th ar heavyweight

While nor successful enough to be recorded in the official record books, the wrestling team did manage to pull rogerher as a family. They developed a sense of camaraderie that few individuals ever realize. Suffering rhrough pracrices and defears, experiencing Coach Comforr's BaJa 500 van rides, Earl (the Fang) Hawk's war srories, and "Ralph" Richards' encounters with psychoparhic morel cors, the ream had a variery of unique memories of their $1986-87$ season

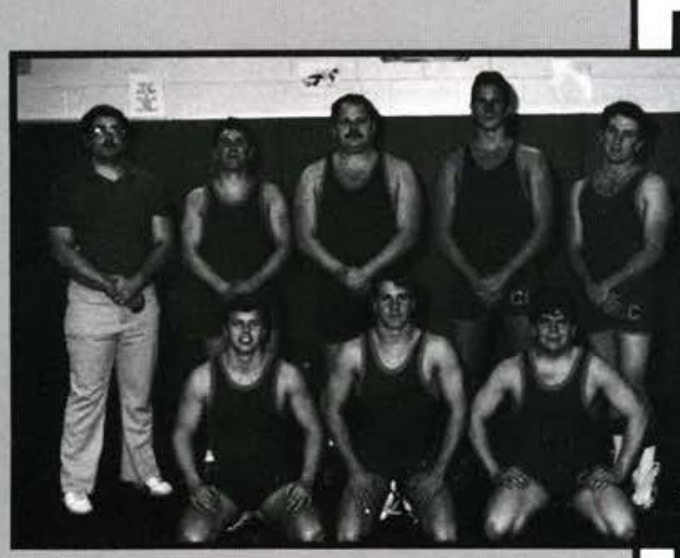

Srephen Smirh, Todd Dowden, Chuck Grizzord, Sieven Smirh, Chris Link, Evan English, Kevin Powell, Andy Salvarore, Jeff Wison, Kelly Moore, Ron Hoyes, Dennis McMichoel, Poul Dilion, Ed Curry, Homilton Richards, Jim Eursey, Head Cooch: Ron Comforr, Assisrant Coach: Jeff Conklin

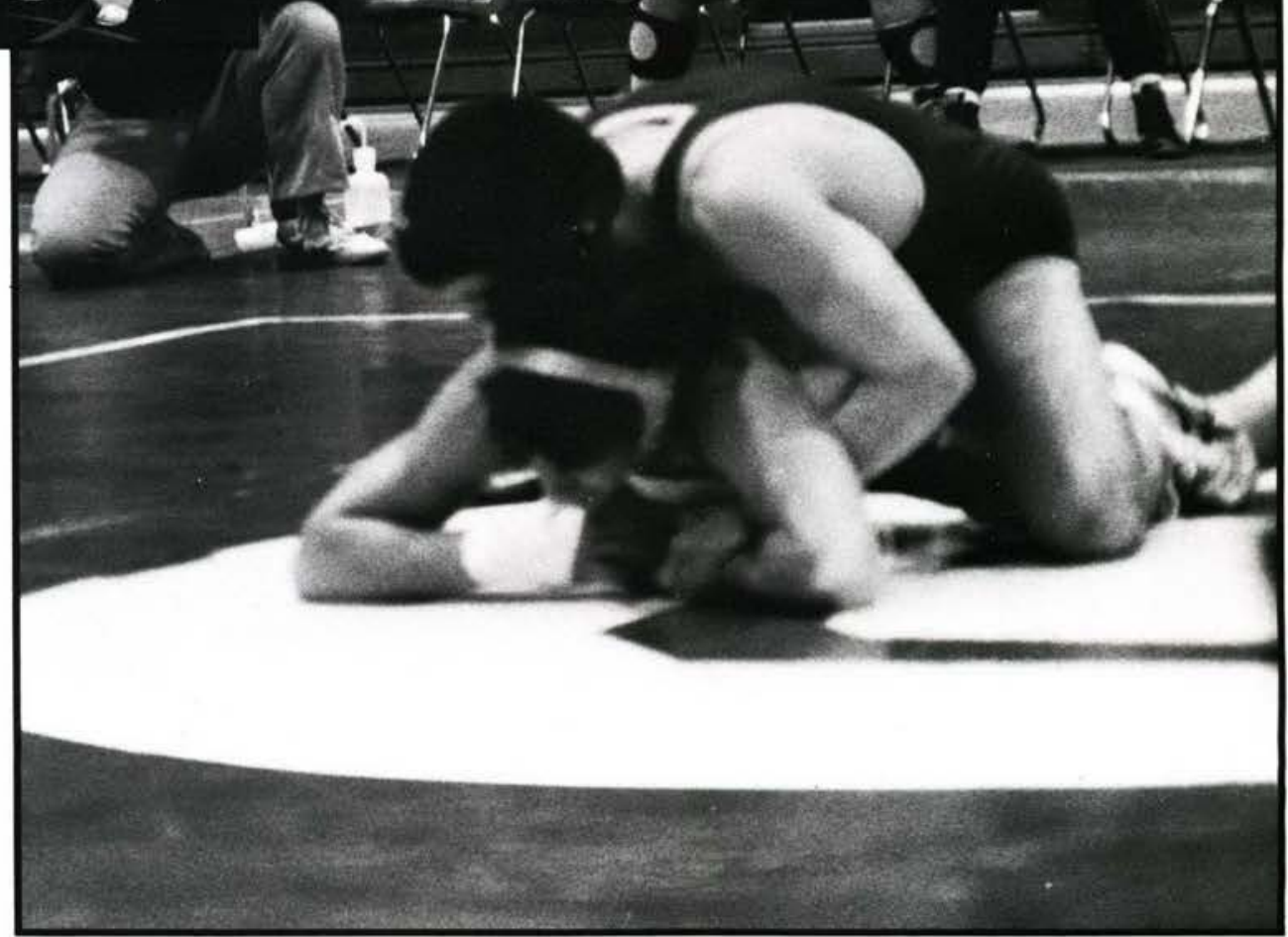




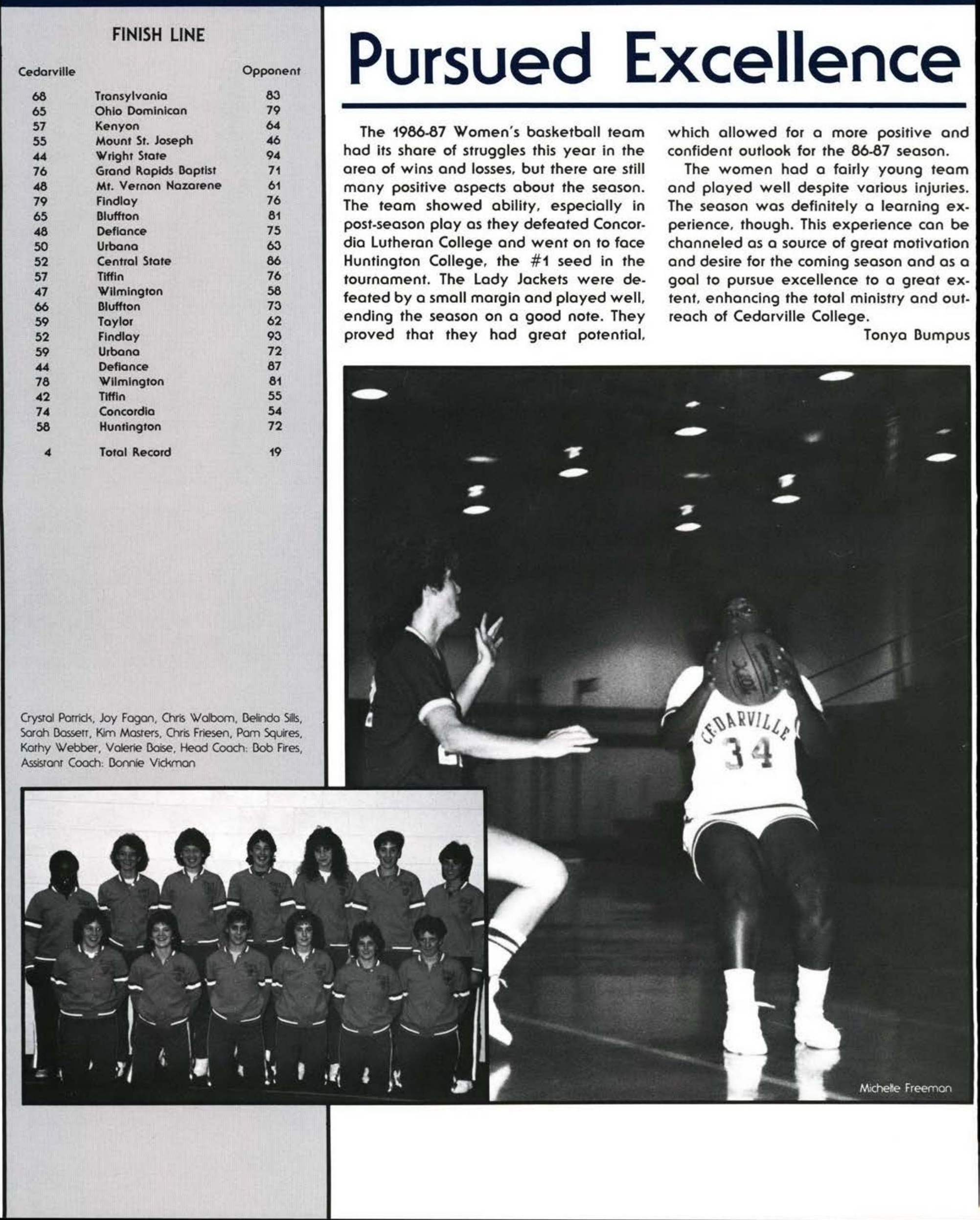




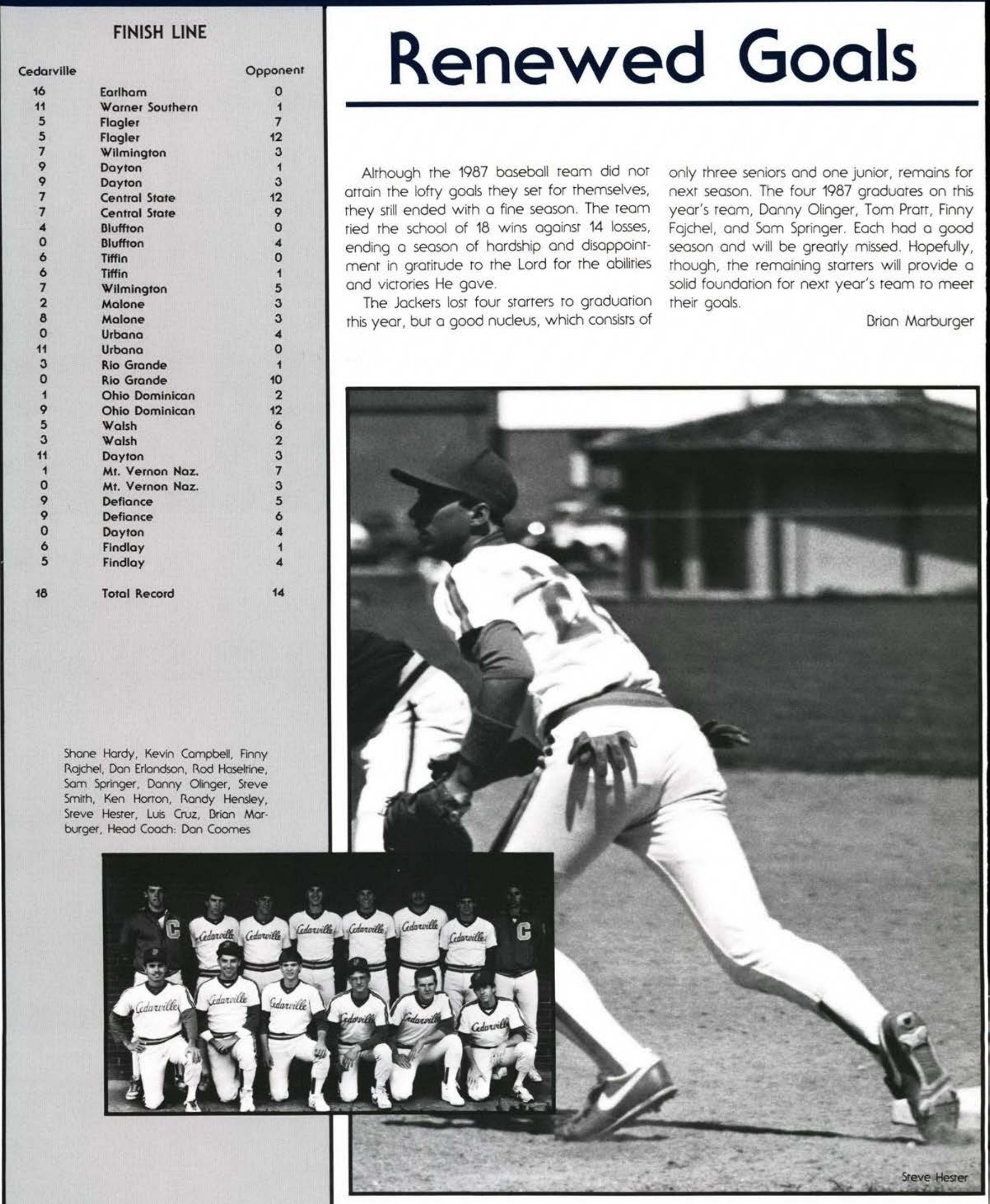




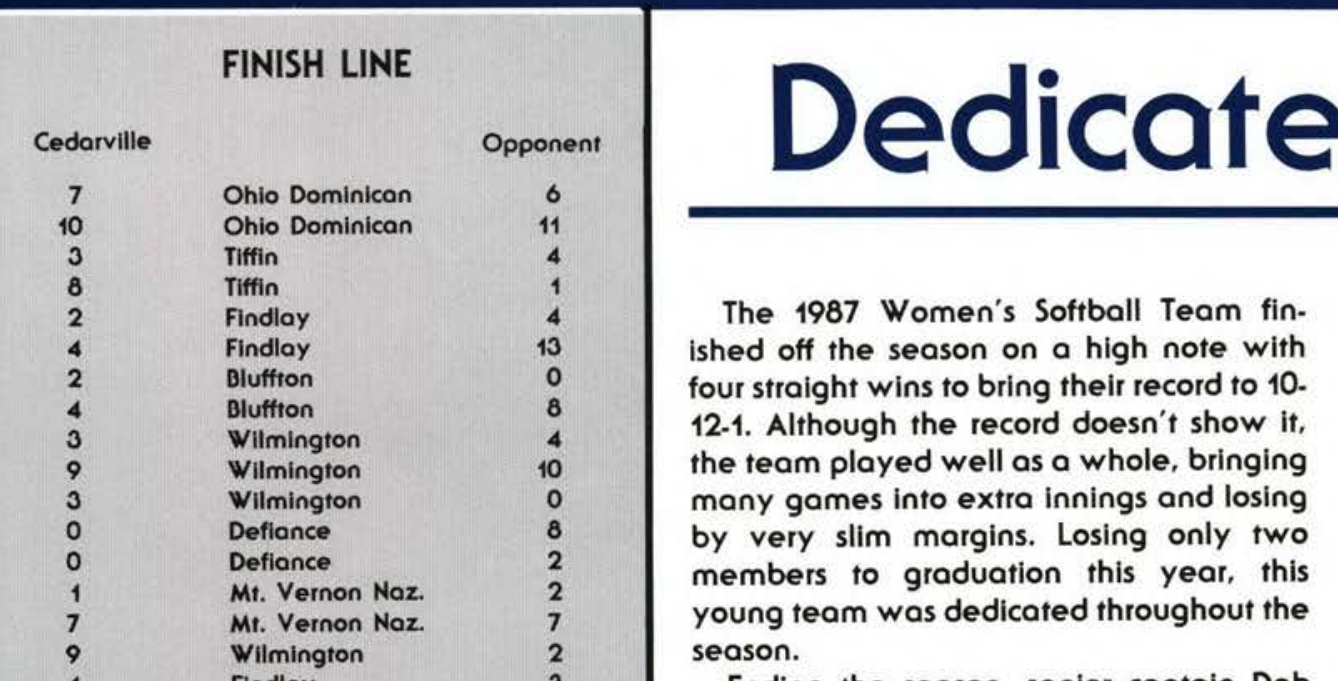

Findloy

Urbana

Wilmington

Taylor

Toylor

Earlhom

Earlham

Total Record

1 Tie
Ending the season, senior captain Deb Berdy was elected for the "mighty in spir- it" award, the award for the player who best displays Christ-like attitudes and leadership on and off the field. Sophomore captain Rebecca Clifford and Senior Nadine Terrill were elected for the "Intensity" award, given to the person(s) who show their dedication through hard work and aggressiveness. Junior pitcher Kristi Culp was elected MVP, and Clifford, Culp, and 3rd baseman Joy Hamilton were elected to the all-conference team.

Sue Baldis

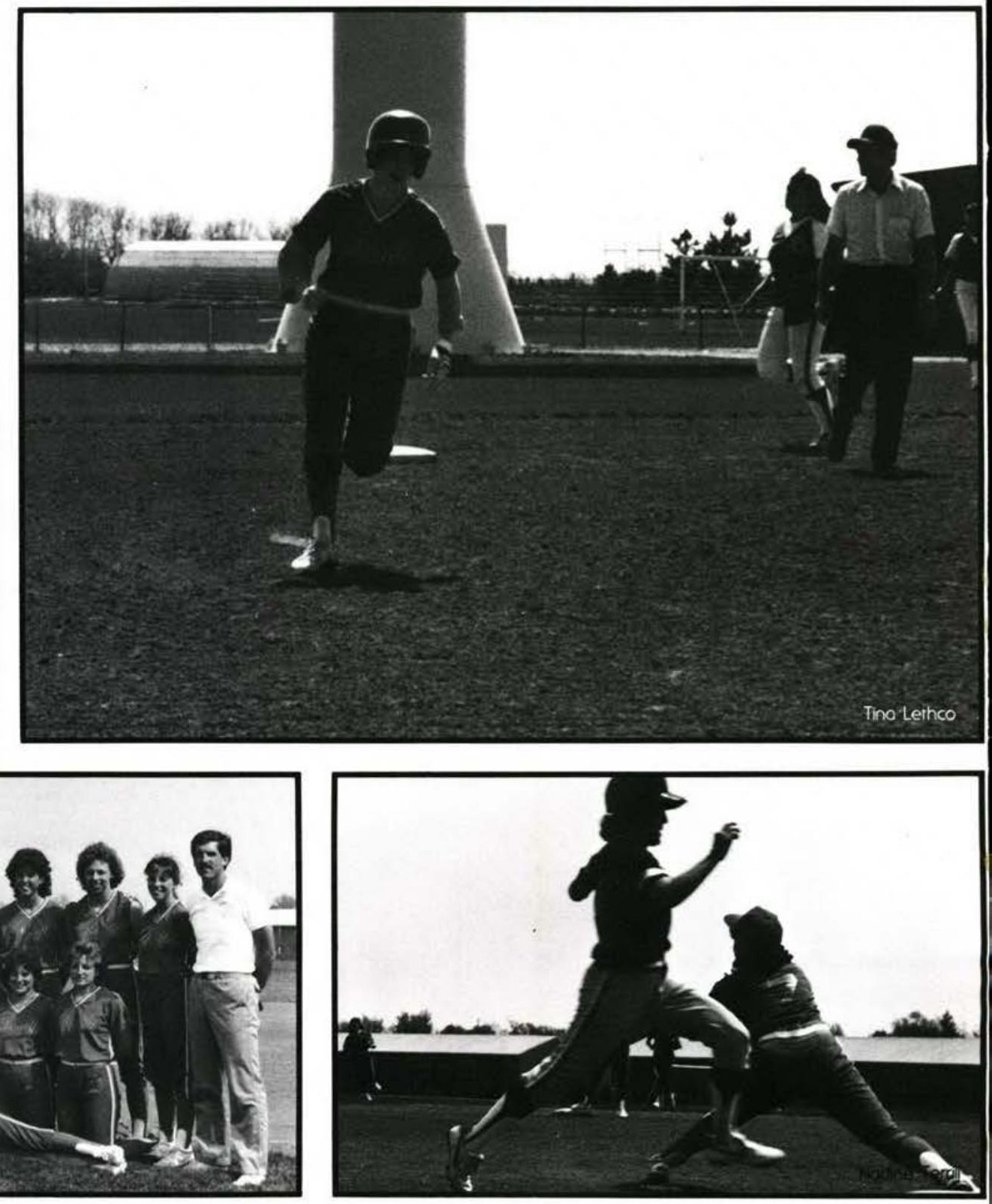

Chris Dougloss, Nadine Terrill, Renee Brown, Dawn Smith, Joy Homilron, Deb Berdy, Berh Smirh, Tamro Terrill, Chris Friesen, Kristy Culp, Rebecca Clifford, Parry Garcia, Kim Fordyce, Andrea Berry. Tina Lerhco, Head Cooch: Mark Marhews, Assistont Cooch: Sue Boldis

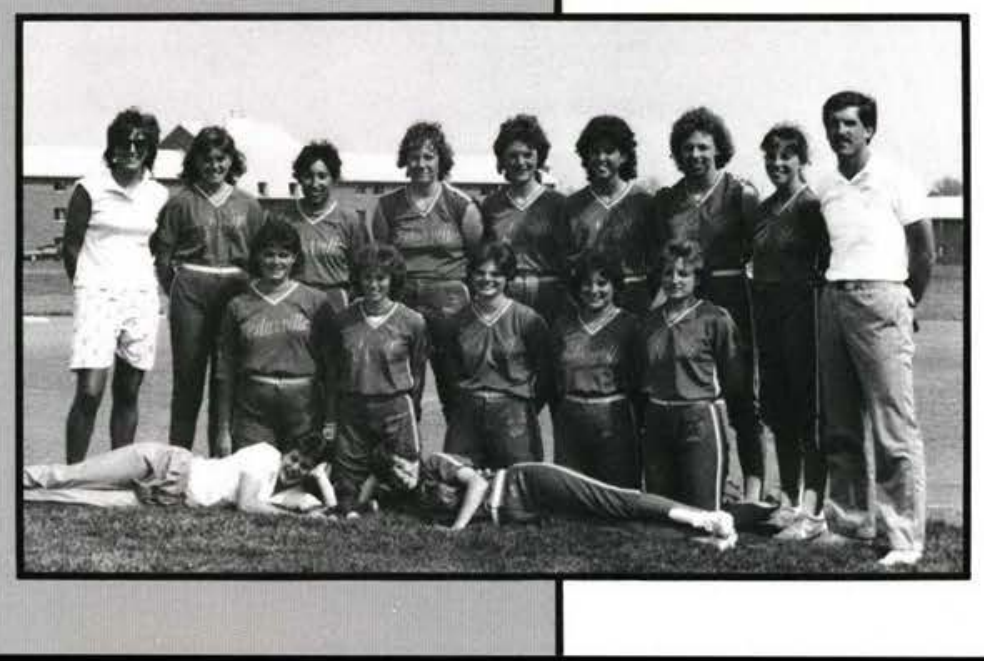




\section{FINISH LINE}

$\begin{array}{lrr}\text { Glenville State Inv. } & \text { 13th out of } & 15 \\ \text { Cedarville Inv. } & \text { 6th out of } & 12 \\ \text { Tiffin Inv. } & \text { 8th out of } & 12 \\ \text { Wright State Inv. } & \text { 11th out of } & 12 \\ \text { Capital Inv. } & \text { 14th out of } & 15 \\ \text { Miami-Hamilton Inv. } & \text { 3rd out of } & 8 \\ \text { NCCAA District III } & \text { 3rd out of } & 6 \\ \text { MOC Match (Walsh) } & 4 \text { th out of } & 5 \\ \text { Malone Inv. } & \text { 13th out of } & 15 \\ \text { NAIA District 22 } & \text { 4th out of } & 8\end{array}$

Mark Reed, John Kohlmeyer, Doug Phillips, Bob Armor, Shawn Hess, Kelly Belt, Willie Deshersky. Head Cooch:

\section{Anticipated Improvement}

In irs second consecurive "rebuilding" year, the 1987 golf ream posed no threat to the school records for ream scores. Mark Reed led the ream for his third straight year, though it was nor his best season. Compering with Mark for the top position was sophomore John Kohlmeyer, who had several brilliant rounds this season. Doug Phillips, the only senior on the ream, managed to play consistently throughour his final season ar Cedarville. Junior Bob Armor rounded off the top four with a fairly good, though often disappointing season. Willie Deshersky, Kelly Bell, and Shawn Hess vied for the fifth position, though Shawn rook over for the final halt of the season. Though the team overall did nor play as well as they hoped they would, Mark Reed finished in the top 10 at the MOC march and placed fourth in the NAIA District 22 rournament, where he was named to the All-District ream.

Doug Phillips

Al Monroe

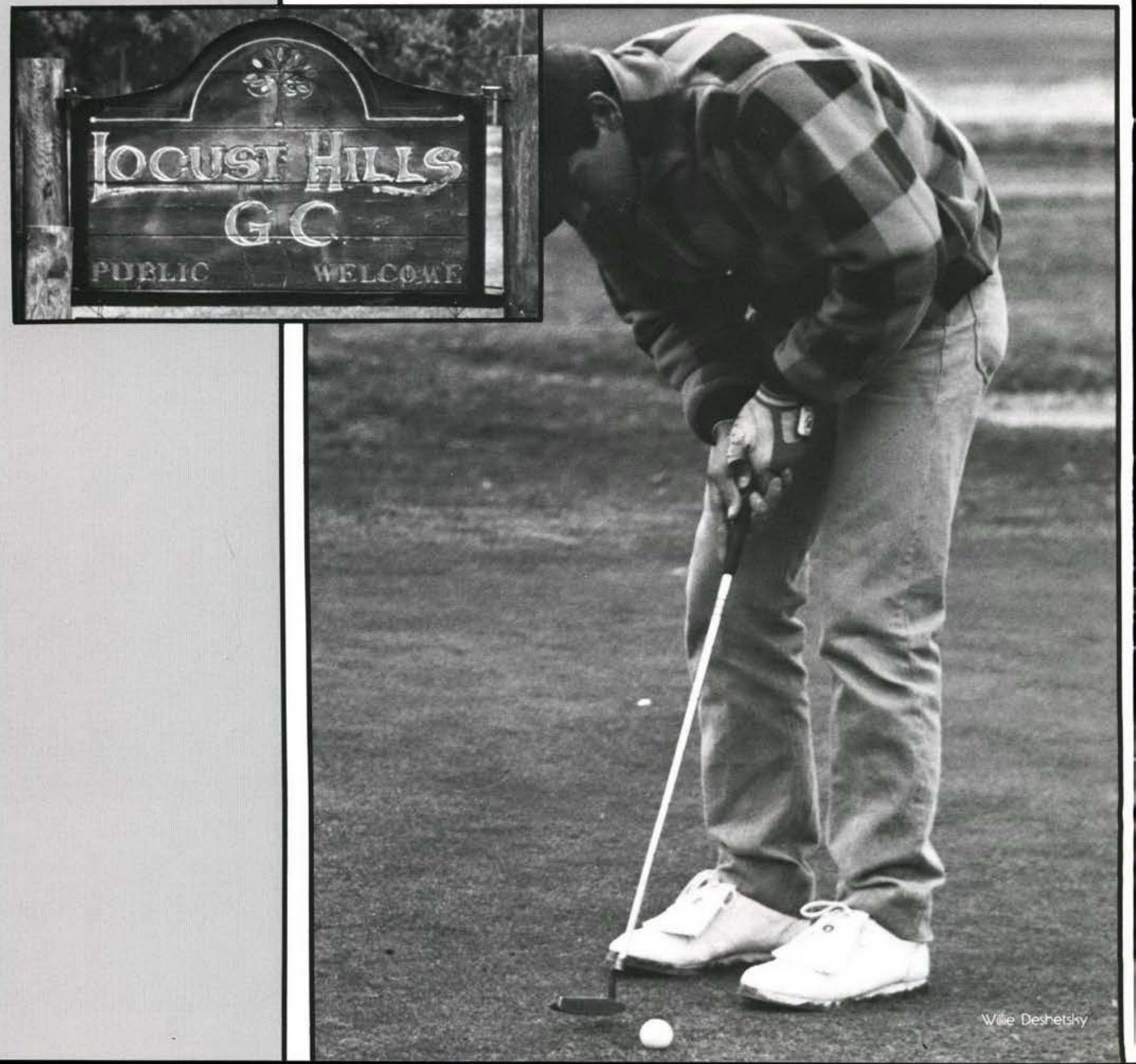




\section{FINISH LINE}

Cedarville

Taylor

IUPA - Ft. Woyne

Grace

Marion

Detroit

Kenyon

Transylvania

Dayton

Fairmont State

Boca Raton

St. Thomas

Florida Atlantic

IUPA - Indianopolis

Findlay

Marion

Charleston, W.Vo.

Georgetown, Ky.

Malone

Sinclair Inv.

1st out of 4

Earlhom

Ashland

Wright Stote

Wilmington Inv.

1st out of 5

Cincinnati

Transylvania Inv.

1st out of 6

Otterbein

Xavier

Wolsh

Mt. Vernon Naz

Wilmington

Wittenberg

Bluttton

NCCAA Not'l Inv.

1st out of 5

Toledo

Wright State

NAIA District 22

1st out of 7

Thomas Moore

NAIA Nationals

12th out of 49

38

Total Record

Opponent

0

Thurman Payton, Greg Frank, Jeff Kohl, Dino Tsibouris, Bruce Toranger, Steve Lesko, Sreve Brumbough, Scorr Poling, John Kelley, Cooch: Murray Murdoch

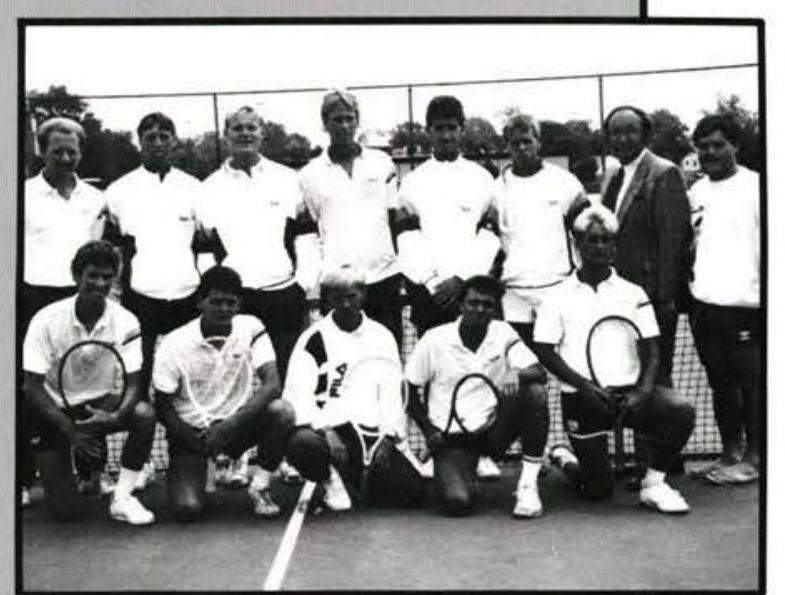

\section{Surpassed Expectations}

The 1986-87 men's rennis ream saw many rhings in our all-year seaon. We saw seven freshmen come in to rake the top seven posirions. We saw that coach Murdoch does nor believe in rennis euthanosia-keeping a lone senior in the line-up at third doubles. We saw the NAIA District 22 Championship title returned ro its righiful owner, and resting more comforrably in its usual home of Cedarville, after a year of absence. We saw an unprecedented eleven points earned at the NAIA Nationals, surpassing the previous record by three points.

The most significant thing the team saw this year, however, was nor in the area of tennis skill, but in growth and marurarion. With such an individual sport, ream cooperarion is most difficult and this year's ream often referred to ourselves as the "Men's Opinion Tennis Team." Yer, through Coach Murdoch's reaching and example of love and unselfishness, we learned the importance of honoring God and loving each orher, building around Philippians 4:13, "I can do all things through Christ who strengthens me."

Thurman Payton 


\begin{tabular}{|c|c|c|}
\hline \multicolumn{3}{|c|}{ FINISH LINE } \\
\hline Cedarville & & Opponent \\
\hline 5 & Mt. Vernon & 4 \\
\hline 6 & Cleveland State & 3 \\
\hline 1 & Wright State & 8 \\
\hline 7 & Miami-Hamilton & 2 \\
\hline 5 & Miami-Hamilton & 4 \\
\hline 1 & Wittenberg & 8 \\
\hline 3 & Walsh & 6 \\
\hline 7 & Malone & 2 \\
\hline 8 & Capitol & 1 \\
\hline 7 & Bluffton & 2 \\
\hline 3 & $\begin{array}{l}\text { Wright State } \\
\text { NAIA District } 22 \\
\text { 3rd out of } 5\end{array}$ & 6 \\
\hline 7 & Total Record & 4 \\
\hline $\begin{array}{l}\text { Cheri Abresc } \\
\text { Michelle Le } \\
\text { Srough, Sref } \\
\text { Corla Weirkc } \\
\text { Delyre Morr } \\
\text { derson }\end{array}$ & $\begin{array}{l}\text { Amy DeCook, Judy } \\
\text { 1, Berh Nesrer, Kir } \\
\text { ie Swift, Becky Tuc } \\
\text { ip, Head Coach: Pom } \\
\text { Eleanor Tayior, Man }\end{array}$ & $\begin{array}{l}\text { Roge Losh, } \\
\text { erkey. Jody } \\
\text { nnifer Word, } \\
\text { hl, Assistants: } \\
\text { Carherine An: }\end{array}$ \\
\hline
\end{tabular}

\section{Unexpected Growth}

The 1987 Women's Tennis Team could summarize their season as an opporrunity to grow through unusual experiences. Of course the first week together in Florida always offers memories as 12 aquaintances board a van and 1 week larer rerurn a ream. From the hor, white sands of Pensacola, we rerurned to the cold whire snow of Cleveland (in April)!

After 2 weeks of cancellations due to wearher, we played our season opener in doors and were promprly caughr in a blizzard unable to rerurn home. From there, our season progressed normally, though filled with many unforgerable hours spent rogether as a ream of friends. Districts closed our year on a rather disappointing nore, for we were unable to pull our talent rogerher for a victory. Although this was a rough farewell to our 4 seniors, it has proved to be a morivaring factor to the rerurning members in their hopes for next year's ream

Our deepest appreciation must go to our godly, caring coach's example, Dr. Pam Diehl, whose leadership challenged us to reach personal porentials on and off the court and to learn from winning as well as losing. Thus, we reflect on a successful ream regardless of its season record.

Amy DeCook

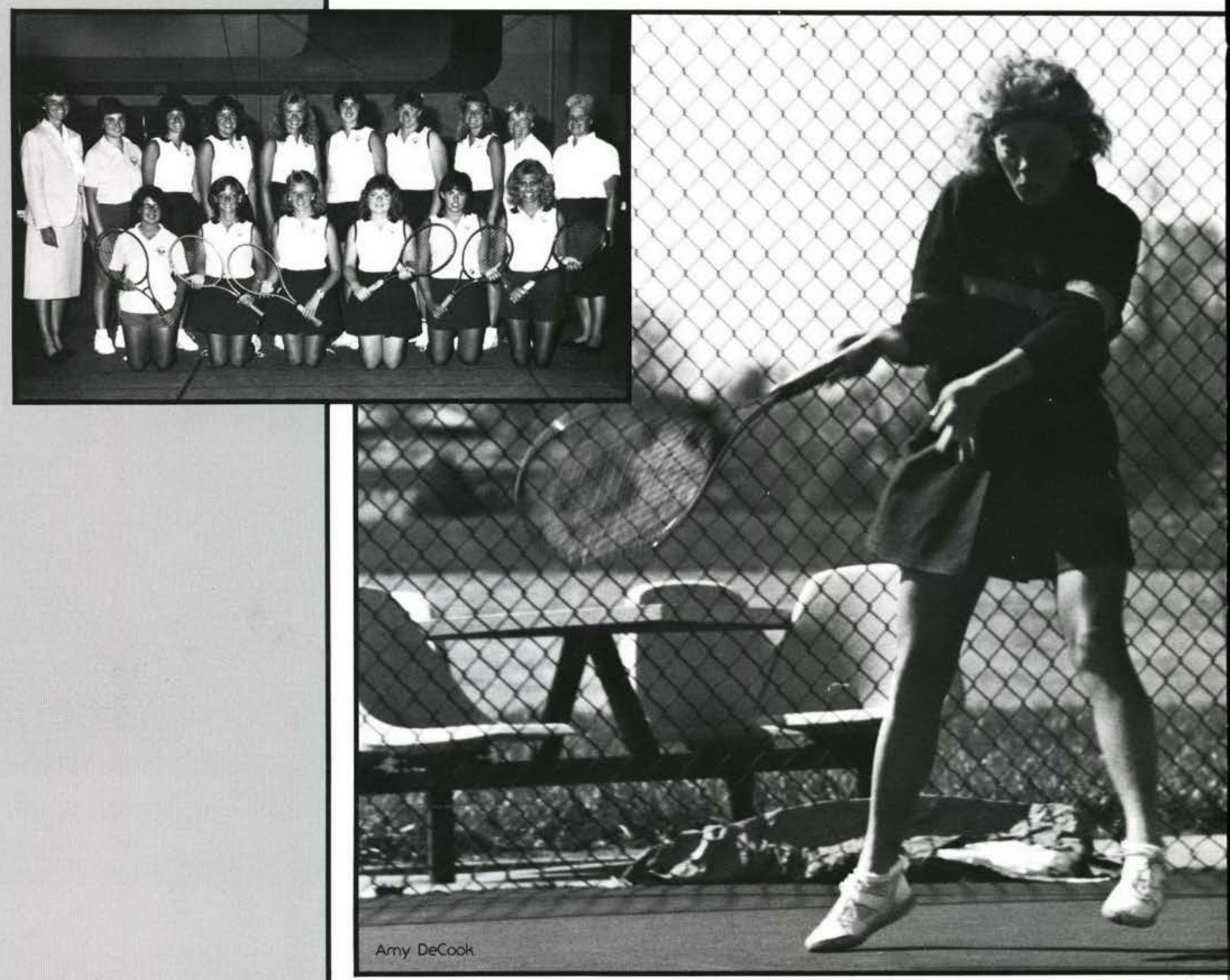




\section{FINISH LINE}

Men's Track
Marshall Invitational Mid.Ohio Conference NCCAA Nationals NAIA District 22 NAIA Nationals

\begin{tabular}{lr} 
3rd out of \\
3rd out of \\
2nd out of \\
tie 1st out of \\
tie 53rd out of & 20 \\
\hline
\end{tabular}

Women's Trock

Marshall Invitational

2nd out of NCCAA Nationals 1st out of 4 th out of NAIA District 22
Men's Track Team: Chris Bernard, Sreve Bilingsley, Jeff Bolender, Stan Charlion, Sreve Cox, Cloncy Cruise, Phil Cruz, J.P. Dawes, Gary Fernandez, Eric Filinger, Shawn Hannay, Tom Hill, Chris Hoefler. Brian Humphreys, Mike Kuykendall, Scort Morsh, Rob Moore, John Oswald Dove Reid, Ken Rexillius, Mike Roberts, Eric Shrum, Dove Seibel, Sreve Seymour, John Urban, Kevin Wal lace, Neil Walloce, Tim Walrers, HEAD COACH: Evin King

Women's Track Team: Becky Averill, Penni Bresson, Lynn Clork, Cindy Colier, Edie Durost, Jody Eckert, Bev Fisher, Shelly Frarus, Wendy Grady, Heidi Loub, Esther Mahr, Berh McGilivray, Sue Nosh, Deb Pare, Cheryl Rendle, Tonyo Schindewolf, Belindo Sills, Pom Squires, Lourel Yores, Rurh Yuen, HEAD COACH: Elvin King

\section{Manifested Discipline}

Abour three years ago, a chapel speaker named Knute Larson shared a message entirled "Running the Race." He suggested in that message a rather radical notion: to actually quit running for yourself and ler your running be a means of worship to the Lord. I learned that can worship Him through the medium of track and field, nor only on a race day bur also as I practice.

One of the great aspects of this notion is that long before the meer, I have been preparing

Individual effort and improvement was the emphasis this season, and personal bests were achieved by several athleres. At the NCCAA National meer, the following women artained All-American starus by placing in the top six in their respective events: Deb Pare $(10,000 \mathrm{~m})$; Berh McGillivray (high jump); Fr. - Edie Durost (javelin); Andy Collier (100m hurdles and 400m hurdles); Esther Mahr (400 m and $800 \mathrm{~m}$ ); Jr. Jody Eckerr (100m and $200 \mathrm{~m})$, and Jr. - Shelley Frarus ( $400 \mathrm{~m}$ hurdles). Narional champions spiritually as well as physically. As I develop the skill of my event, I learn parience; as I practice parience, I learn the principle of progression which is similar to the maruring process I experi ence in the Christion life. As I faithfully submit: myself to the daily rigors of practice, I learn the imporrance of diligence and dedicarion. But? most imporrantly of all, I can be assured that in all of this, I can bring glory to my Lord

Tim Walre

in this group include McGillivray, Frarus and Mahr. Mahr won borh of her events and set os new national record in the $400 \mathrm{~m}$ dash with a time of 57.40 . Freshman Belinda Sills had an impressive first seoson which included the NAIA. District 22 ritle in the $800 \mathrm{~m}$ run. In addition, Sills; along with Mahr, McGillivray and Fratus, tra elled to Russleville, Arkansas, and comper well in the NAIA National Championships. Shelley Fro
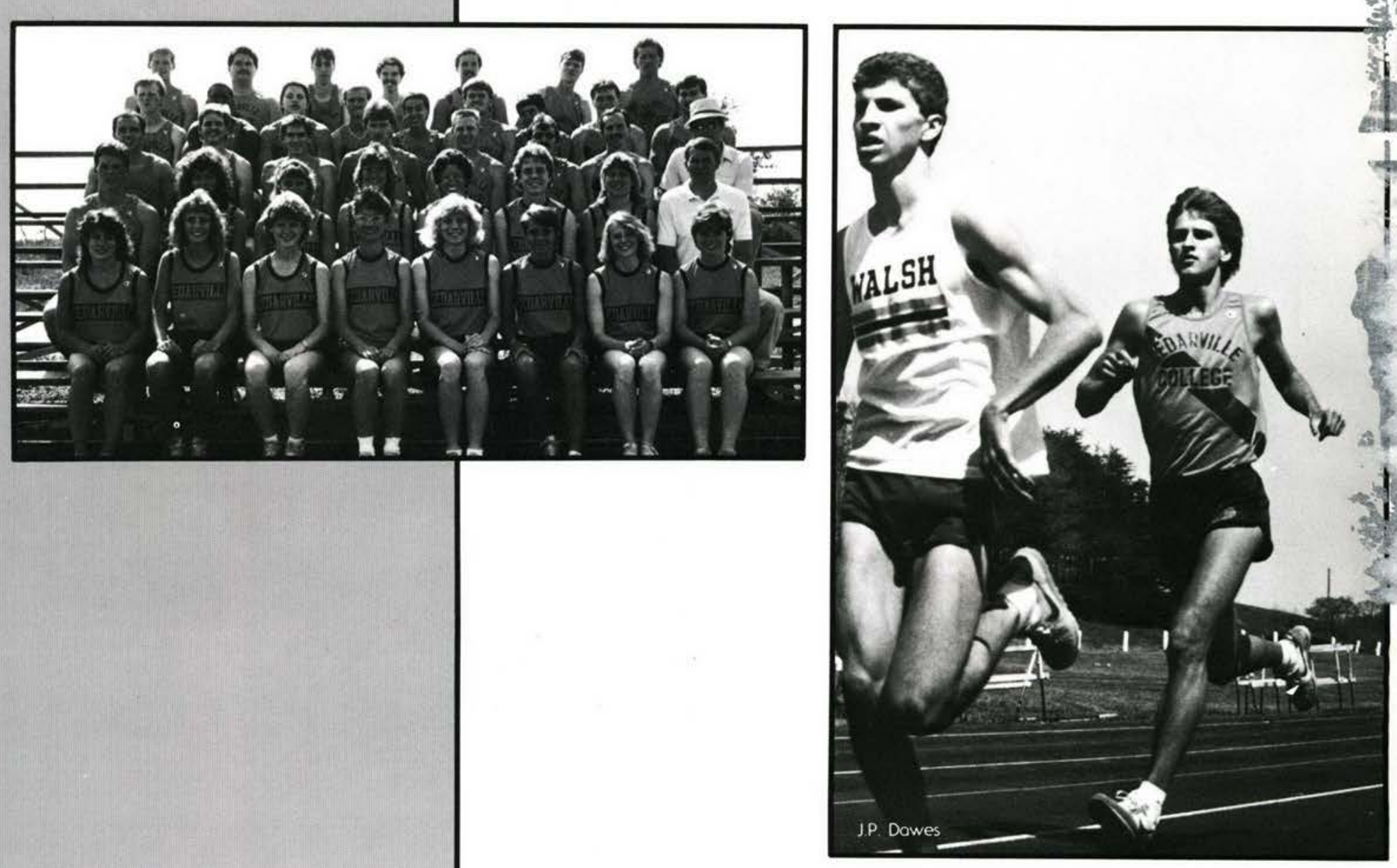


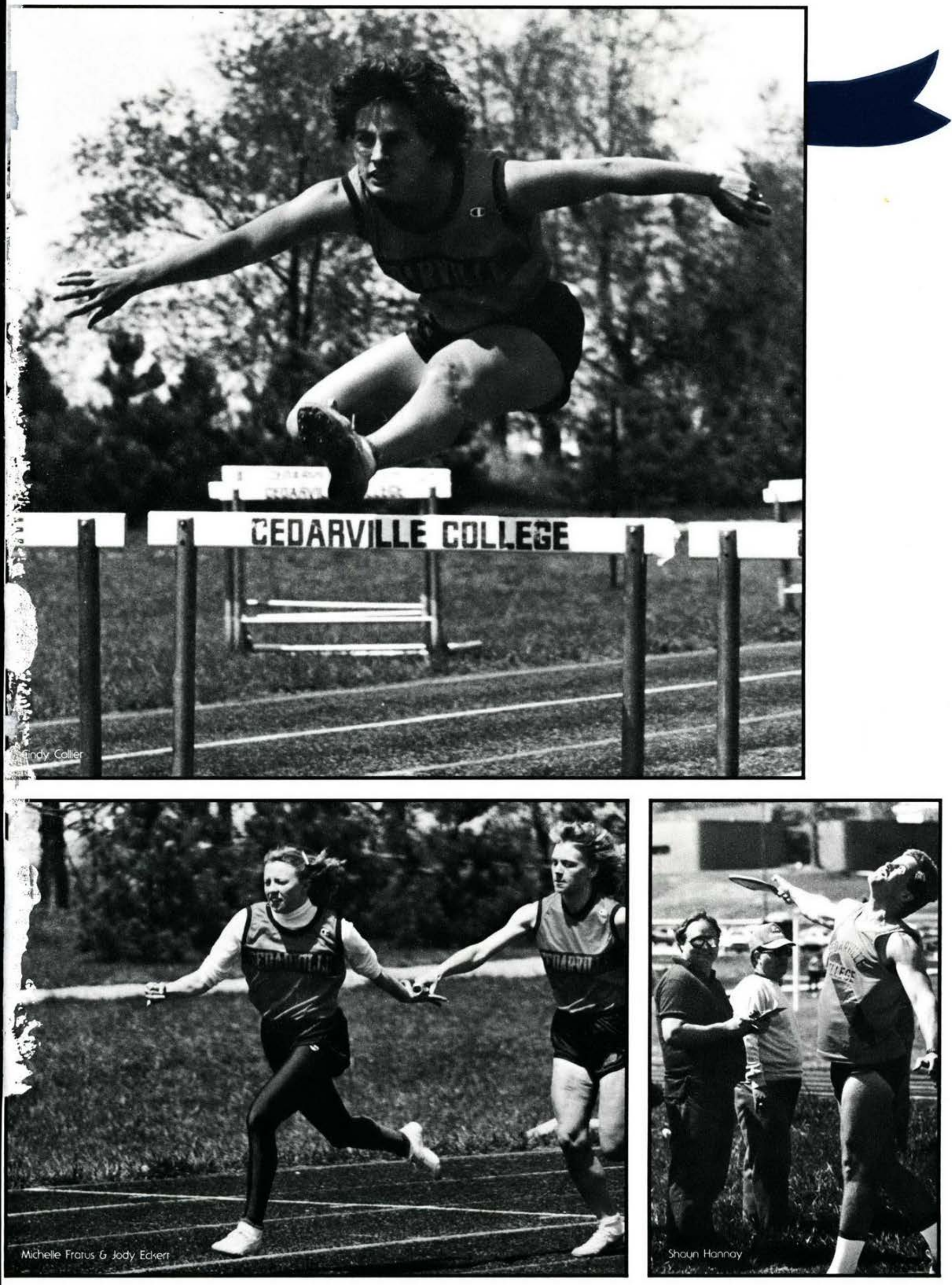




\section{CHURCH PATRONS}

Bailey Road Baprist Church Les Webster, Postor

Berea Baptist Church Kennerh J. Spink, Postor

Big Sewickley Baprist Church David L. Vucish, Pastor

Colvary Baprist Church

Quincy, IL

Cedar Heights Baprist Church Tom Hammond, and Keith A. Kerrenring, Postors

Emmanuel Baprist Church Edward L. Fuller, Posior

First Boprist Church John D. Adams, Postor

First Boprist Church

Kennerh Wilhite, Postor

First Baprist Church

Don Johnston, Jr., Pastor

First Boprist Church

Frederick W. Thomas, Pasror

First Baprist Church

G. Ben Reed, Postor

First Boprist Church

Don Leirch, Pastor

First Baprist Church

Dr. Howard Longcore, Pastor

First Boptsit Church

Robert Wm. Redding, Postor

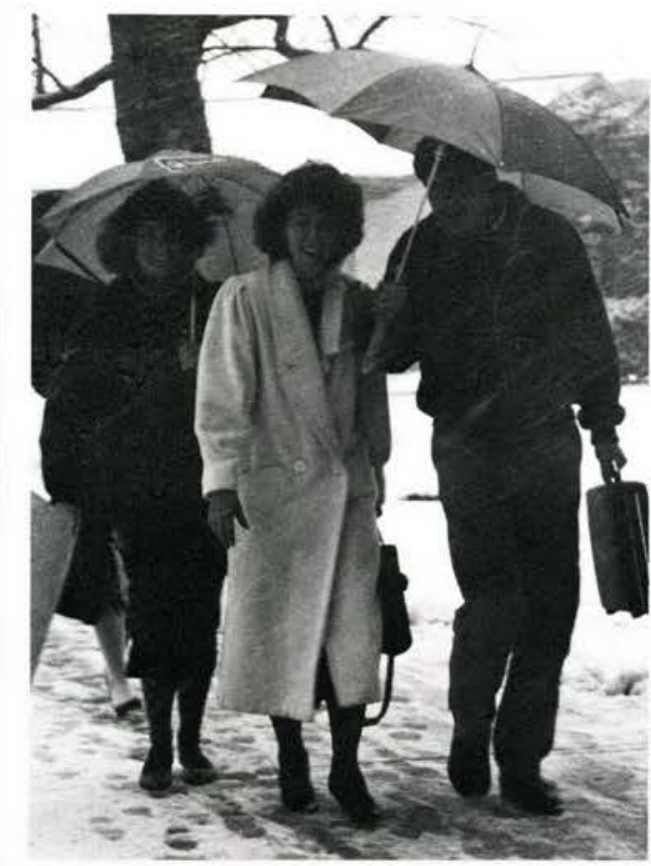

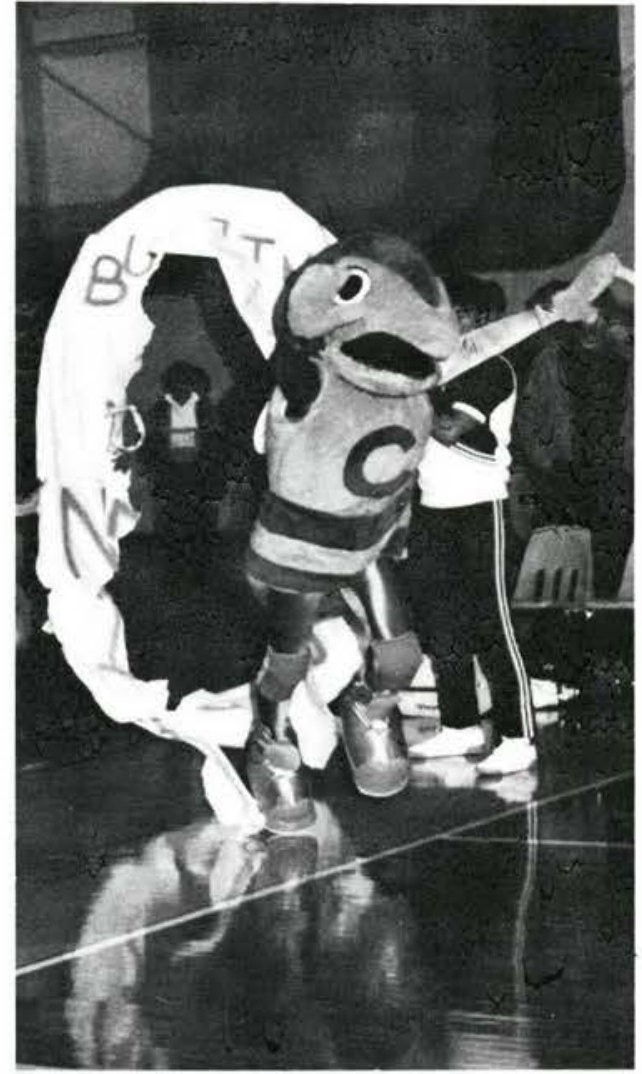

First Reformed

Michael L. Holr, Postor

Foorhills Boprist Church Norvin T. Jones, Pastor

Fox Valley Baprist Church Douglas Compron, Pastor

Garfield Baprist Church

William K. Bernhard, Jr., Postor

Grace Baprist Church

Dr. Ronald L. Schaffer, Postor

Grace Baprist Church

Dr. Jack Jacobs, Pastor

Grace Boprist Church

Stan Knisley, Postor

Heritage Baptist Church

Larry D. Reesor, Pastor

Horsham Bible Church

Paul A. R. Brittain, Pastor

Montrose Boprist Church Richard L. Phillips, Postor

Northfield Baprist Church Lynn E. Rogers, Postor

Nortingham Boprist Church Donald E. McClintick, Postor
Oak Grove Baprist Church James R. Gray, Pastor

Shawnee Hills Baprist Church Dennis L. Henderson, Postor

\section{Sourhgare Baprist Church} John R. Greening, Postor

Sourhside Baprist Church Dr. George A. Starling, Pastor

Tabernacle Baprist Church Herberr Koonce, Postor

Temple Boprist Church Truman Dollar, Pastor

Unired Berhal Mennonite Church Wolrer Beachy, Postor

Wheelersburg Baprist Max K. McCullough, Pastor

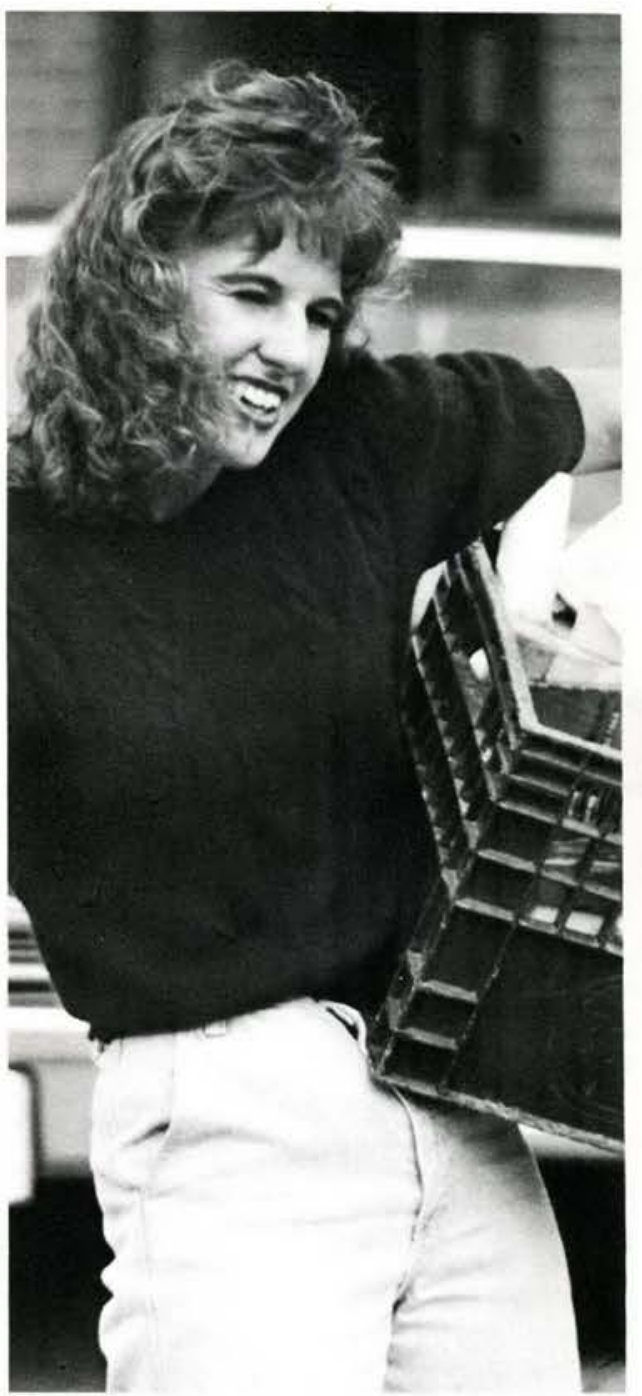




\section{PARENT PATRONS}

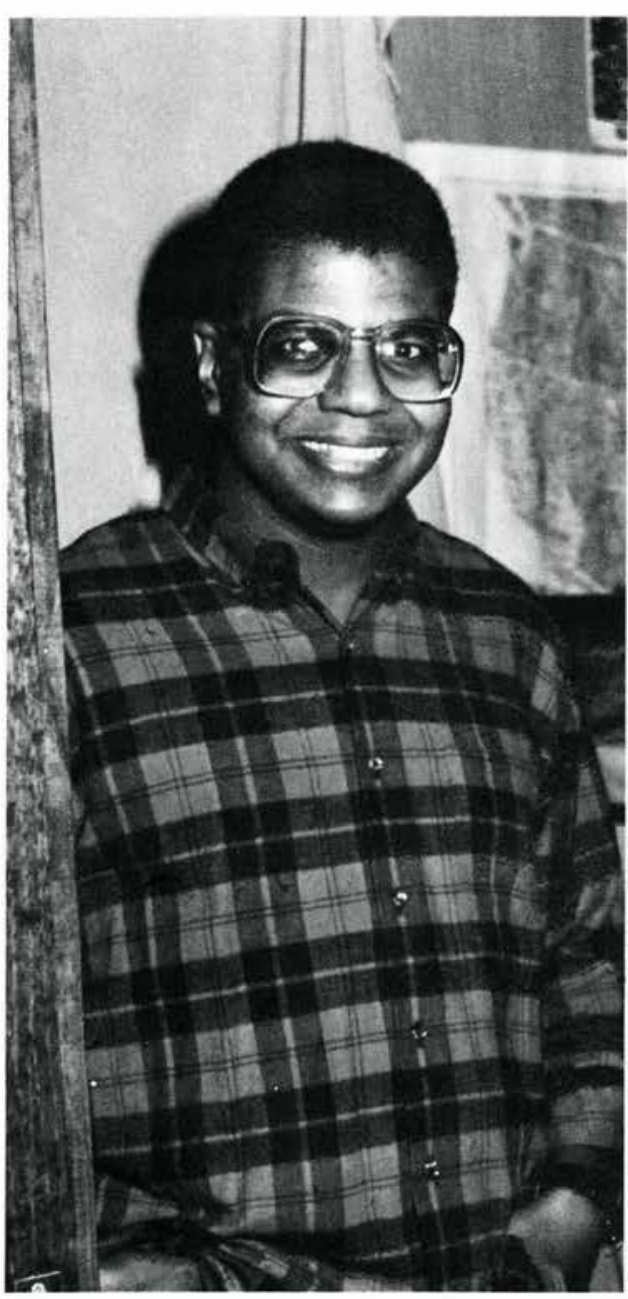

Mr. and Mrs. Al Abramowitz

Freeport, IL.

Ms. Marline Alrman

New Richmond, $\mathrm{OH}$

Dr. and Mrs. Wiliam H. Anderson Jr.

West Springfield, PA

Mr. and Mrs. Fred L. Armbrusrer

Cincinnari, $\mathrm{OH}$

Mr. and Mrs. Robert C. Auckland

Springfield, $\mathrm{OH}$

Edwin and Alice Augenstein

Columbus, $\mathrm{OH}$

Mr. and Mrs. Richard Averill

New Hampron, IA

Karherine L. Boker

Bangor, ME

Mr. and Mrs. Charles Boldis

Simsbury, CT

Mr. and Mrs. Wolrer D. Barker

Stare College, PA

Mr. and Mrs. Richard W. Barey Maple Glen, PA

Mr. and Mrs. Dale R. Bennerr

Lokewood, $\mathrm{OH}$

Mr. and Mrs. Earl E. Bennert

Zanesville, $\mathrm{OH}$

Mr. and Mrs. Robert J. Bercaw

Troy, $\mathrm{OH}$

John and Rosemary Berdy

Tallmadge, $\mathrm{OH}$

Richard and Janer Bergandine

Dundee, IL

Mr. and Mrs. George Berkey
Norristown, PA

Mr. and Mrs. Eugene E. Berhe

Chandler, IN

Mr. and Mrs. Andrew Bidlen

Broodview Hrs, $\mathrm{OH}$

$\mathrm{Mr}$, and Mrs John R. Black

Ontario, NY

Mr. and Mrs. Bob Blaylock

Chartonooga, TN

Mr. and Mrs Merlin B. Bliss

Belding, MI

Mr. and Mrs. Howard C. Bowman Flido $\mathrm{OH}$

Mr. and Mrs. John F. Brodshaw

Mechonicsburg, PA

Larry and Linda Brovont

Lowton, MI

Mr. and Mrs. David H. Brown

Sylvania, $\mathrm{OH}$

Mr. and Mrs. Keirh Brown

Cenrerburg, $\mathrm{OH}$

Mr. and Mrs. Ronald Brown

Lancoster, $\mathrm{OH}$

$\mathrm{Mr}$. and Mrs. Elmer F. Brown Jr.

Mopled Glen, PA

Mr. and Mrs. Ray Burk

Fostoria, $\mathrm{OH}$

Gilbert G. Burziaft

Humacoo, PR

Pastor and Mrs. Dale P. Byrom

Beavercreek, $\mathrm{OH}$

Mr. and Mrs. Roberr Carmack

Bristol, TN

Mr. and Mrs. Srephen E. Corpenter Fonda

Postor and Mrs. Mark R. Chandler

Dacula, GA

Dr. and Mrs. W. E. Cherry

Srerling, IL

The Porenrs of Mr. Perry Ercole Chiarelli

Lincoln Park, MI

Mr. and Mrs. Sranley Christensen

Defiance, IA

Pasror and Mrs. Preble Cobb

Lynnfield, MA

Mr. and Mrs. Parrick V. Cook

Lake Orion, M

John and Corol Coope

New Braintree, MA

Mr. and Mrs. R. Daiker

Sodus, NY

Rich and Martho Davidson

Pork Forest, IL

Mr. and Mrs. Brian Mr. Dovis

Soo Paulo, Brazi

Jim and Lois Day

Belle Center, $\mathrm{OH}$

Dr. and Mrs. Joseph DeCook

Holland, MI

Don and Bonnie Denney

Hebron, KY

Mr. and Mrs. Donald Dickinson

Williamson, NY

Joel Paul Dillon

Woshingron, DC

$\mathrm{Mr}$. and Mrs. Richard E. Durost

Boorhbay Horbor, ME

Dave and Janer Dye

Murual, $\mathrm{OH}$

Mrs. Wonito Dye

Rovenna, $\mathrm{OH}$

Mr. and Mrs. Robert M. Dykes

Urbandale, IA

Mr. and Mrs. Gordon W. Fowcerr

Willowick, $\mathrm{OH}$

Mr. and Mrs. Robert Figary

Norwich, NY

Mr. and Mrs. Gerard C. Fink

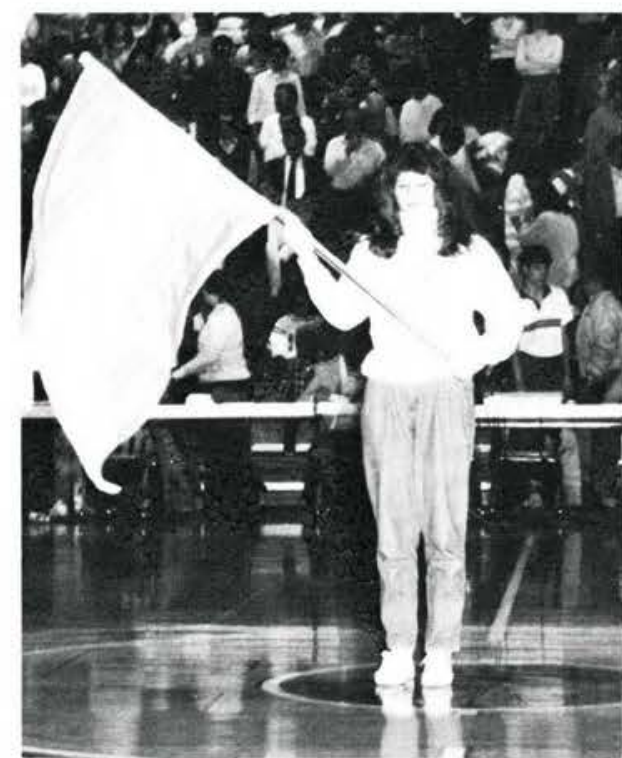

Enfield, CT

Bob and Mary Fowler

Goleron, PA

Wally and Donna Frank

Caldwell, ID

Mr. and Mrs. Robert Fread

Loveland, $\mathrm{OH}$

Mr. and Mrs. Ken Free

Coshocion, $\mathrm{OH}$

Mr. and Mrs. John Frey

Dovison, Ml

Woyne and Linda Gabel

Quincy, IL.

Dr. and Mrs. Charles Garabodian

Pickens, SC

Rurh and Al Garrigan

Town of Poughkeepsie, NY

Rev. and Mrs. Fred Gerhard

Lansing, MI

Mr. and Mrs. David C Gibos Jr

Conneaut, $\mathrm{OH}$

Dr. and Mrs. J. Dean Gifford

Urbano, IN

Mr. and Mrs. Graham Gilbert

Haddon Heighrs, NJ

Mr. and Mrs. Jan C. Gonzolez

Medford, NJ

Mr. and Mrs. Charles Grafton

Troy, MI

Mr. and Mrs. Graham

Ridley Pork, PA

Mr. and Mrs. Cary Grant

N. Canton, $\mathrm{OH}$

Bill and Eva Grimes

Cambridge, $\mathrm{OH}$

Mr and Mrs Leonard A Grosch

Wildwood, II

Bruce and Eileen Gruneisen

Mansfield: $\mathrm{OH}$

Mr. and Mrs. Marvin D. Hocker

Ontario, CA

Hons and Ann Hahnenstein

Conton, NY

Mr. and Mrs. Paul C. Hambley

Cedarville, IL

Donald G. and Barbara Hanno

Champaign, IL.

Rog and Dianne Hansen

Rockford, Ml

Mr. and Mrs. Joe Hanssen

Ankeny, IA 
Mr. and Mrs. Frederick $H$. Houfler Reading, PA

Mr. and Mrs. Rodney E. Hoynes Perry, NY

Robert and Judy $\mathrm{H}$

Fulton, $\mathrm{OH}$

$\mathrm{Dr}$. and Mrs. Larry Hinnergordr

Newrown, CT

Mr. and Mrs. Jack Hoag

Elyria, $\mathrm{OH}$

Mr. and Mrs. James Holland

Eost Auroro, NY

Mr. and Mrs. William T. Holt

Omaha, NE

Mr. and Mrs. Robert E. Horrel Sr.

Advance, NC

Mr. and Mrs. W. J. Howdyshell

Pickeringron, $\mathrm{OH}$

Mr. and Mrs. David Huebscher

Mople Hrs, $\mathrm{OH}$

Leo and Evelyn Huff, Grandparents

Hudson, MI

Mr. and Mrs. David R. Hughes

Pirman, NJ

Mr. and Mrs. Albert A. Hultz

Portsrown, PA

Mr. and Mrs. Paul F. Hunn

New Lebanon, $\mathrm{OH}$

Mr. and Mrs. Joseph Hyde

Cincinnati, $\mathrm{OH}$

Lloyd and Judy Jackson

Bloomfield, IN

Russ and Cloudine Jenkins

McClean, VA

$\mathrm{Mr}$. and Mrs. Robert $\mathrm{E}$. Jones

Wellman, IA

Mr. and Mrs. Francis T. Joslin

Wisconsin Rapids, "W/

Don and Par Kaver

Hudson, $\mathrm{OH}$

Mr. and Mrs. Hugh Kennedy

Cambridge, Onrario

Horold E. and Mary A. Kimmel

Greenville, $\mathrm{OH}$

Mrs. Ruth Klopp

Quebec

Mr. and Mrs. Robert J. Koerber

Colchester, CT

Mr. and Mrs. James W. Krueger

Franklin, WI

Mr. and Mrs. Ralph P. Kuivinen

Foirview Pork, $\mathrm{OH}$

Mr. and Mrs. Glenn C. Kuntz

Grafton, $\mathrm{OH}$

Mr. and Mrs. Charles H. Layman

Philodelphio, PA

David and Virginia Low

Norwich, NY

Mr. and Mrs. Donald D. Leach

Kertering, $\mathrm{OH}$

David and Eleanor Lelah

Sylva, NC

Joseph and Poulerto Lockhorr

Eoston, ME

Ernest and Liliane Low

San Leandro, CA

Mr. and Mrs. Jim McCorrell

Colrs Neck, NJ

Rev. and Mrs. Frederick R. Mackey

Conton, $\mathrm{OH}$

Randy and Lyndo Marriorr

Dayton, $\mathrm{OH}$

Mr. and Mrs. Franklin S. Marshall

Volley Forge, PA

Mr. and Mrs. Woyne A. Meloche

Osseo, MN

Mr. and Mrs. F. R. Michonski

West Simsbury, CT

Mr. and Mrs. Salvarore Mignogna

Greensburg, PA

Mr. and Mrs. Joel Miller

Freeport, IL

Mr. and Mrs. M. Luke Miller

Auroro, CO
Mr. and Mrs. Earl Moniford

Mr. Hope, KS

Walter and Fredo Moon

Haymarker, VA

W. Eugene Morris

Marietro, $\mathrm{OH}$

Walter and Lois Moser

Brook Park, $\mathrm{OH}$

Mr. and Mrs. Arthur Morzer

Hamilon, $\mathrm{OH}$

Wayne and Bonnie Nosh

Midland, MI

Mr. and Mrs. Carl Nasse Jr.

Conojoharie, NY

$\mathrm{Mr}$. and Mrs. Larry Nocella Sr.

and M. Elizaberh Gale-Hall

Columbus, $\mathrm{OH}$

Mr. and Mrs. Webster Orton

Algono, IA

Mr. and Mrs. Rolph Polmer

Hibbing, MN

Jock and Ann Porsons

Columbus, $\mathrm{OH}$

Mr. and Mrs. Silos Pearson

Springfield, MA

Rev. and Mrs. Douglas E. Perers

Troy, $\mathrm{OH}$

Mr. and Mrs. Gary W. Phillips

Columbus, $\mathrm{OH}$

Mr. and Mrs. Samuel L. Pierce

Dublin, $\mathrm{OH}$

Mr. and Mrs. Ronald Plooy

Waukesho, WI

Mr. and Mrs. Delbert W. Ports

Foresr Lake, PA

Edwin and Jean Prart

Ypilanri, MI

Mr. and Mrs. Victor Priester

Grand Ledge, MI

Mr. and Mrs. John Racz

Hoffman Estares, IL

Mr. and Mrs. Wm. Howard Rase

Portsmourh, $\mathrm{OH}$

Mr. and Mrs. Eugene Ream

Woterloo, IA

Mr. and Mrs. George E. Reede

Birdsboro, PA

Mr. and Mrs. Pere Reese

Saugus, CA

Mr. and Mrs. Leonard R. Reeves

Derry, PA

Mr. and Mrs. Larry L. Rice

Lordstown, $\mathrm{OH}$

Mr. and Mrs. Vincent Rice

Ironton, $\mathrm{OH}$

Mr. and Mrs. Charles W. Rizor $\mathrm{Cols}, \mathrm{OH}$

Mr. and Mrs. William K. Rogge

Toledo, $\mathrm{OH}$

Mr. and Mrs. Wendell Rose

Norwich, NY

Mr. and Mrs. Jomes F. Rushe

Fulton, MD

Mr. and Mrs. Robert D. Sager

Urbano, $\mathrm{OH}$

Mr. and Mrs. Frank Schrirz

Spencer, $\mathrm{OH}$

Mr. and Mrs. James Senay

Kirtland, $\mathrm{OH}$

Donald and Florence Shaffer

Corbondale, PA

Mr. and Mrs. Horry Shriver

Dover, PA

Mrs. Herbert L. Shroyer

Dunkirk, $\mathbb{N}$

Mr. and Mrs. Don E. Simmen

Morton, IL

Duane and Shirley Smirh

Dunkirk, $\mathrm{OH}$

Herb and Judy Smirh

Hudsonville, MI

Norris and Judy Smith

Amherst, $\mathrm{OH}$
Mr. and Mrs. Larry Snyder

Elizoberhrown, PA

Mr. and Mrs. Paul W. Srackhouse

Moorestown, NJ

Rev, and Mrs. Eldon Srirn

Lima, $\mathrm{OH}$

Ronald L. and Bonnie C. Srokes

Roonoke, VA

Mr. and Mrs. Reuben Srolizfus

Souderton, PA

Yvonne and Ted Srough Jr.

Honover, PA

Mr. and Mrs. Wm. V. Strebig

Troy, $\mathrm{OH}$

Paul and Joan Srurgis

Hoddon Heighrs, NJ

$\mathrm{Jim}$ and Marcia Swanson

Cuyahoga Falls, $\mathrm{OH}$

Mr. and Mrs. C. Earldean Taylor

Edgewood, KY

Joel G. Toylor

Indianola, IL

Mr. and Mrs. Gerald Terrell

Dayton, $\mathrm{OH}$

Mr. and Mrs. Fredrick J. Tinkler Jr.

Norristown, PA

Ralph and Jessie Todd

Ploinview, MN

Dove and Vicki VanLiere

Wolcort, NY

Mr. and Mrs. Gilbert Vanderberg

Grand Hoven, MI

Perer and Card Venman

Mentor, $\mathrm{OH}$

Mr. and Mrs. David E. Wagner

Wellingron, $\mathrm{OH}$

Mr. and Mrs. William Warfield

Indianapolis, $\mathbb{I N}$

Mr. and Mrs, John Weber

Bigfork, MT

Mr. and Mrs. C. Robert Weidner

Beovercreek, $\mathrm{OH}$

Mr. and Mrs. William Wenger

Mossillon, $\mathrm{OH}$

Lucille Wighr

Ravenswood, WV

Dovid and Judy Williams

Mouldin, SC

Gene Williamson

Midlorhion, VA

Mr. and Mrs. Elmer Wilims

Dike, IA

Mr. and Mrs. Lowrence Wilson

Toledo, $\mathrm{OH}$

Mr. and Mrs. Charles Wise

Indionopolis, IN

Mr. and Mrs. H. Edward Wood

Audubon, NJ

Donno and Tom Young

Mairland, FL

Duane and Karhy Young

Columbus Grove, $\mathrm{OH}$

James and Joan Zimmerle

Medinah, IL 


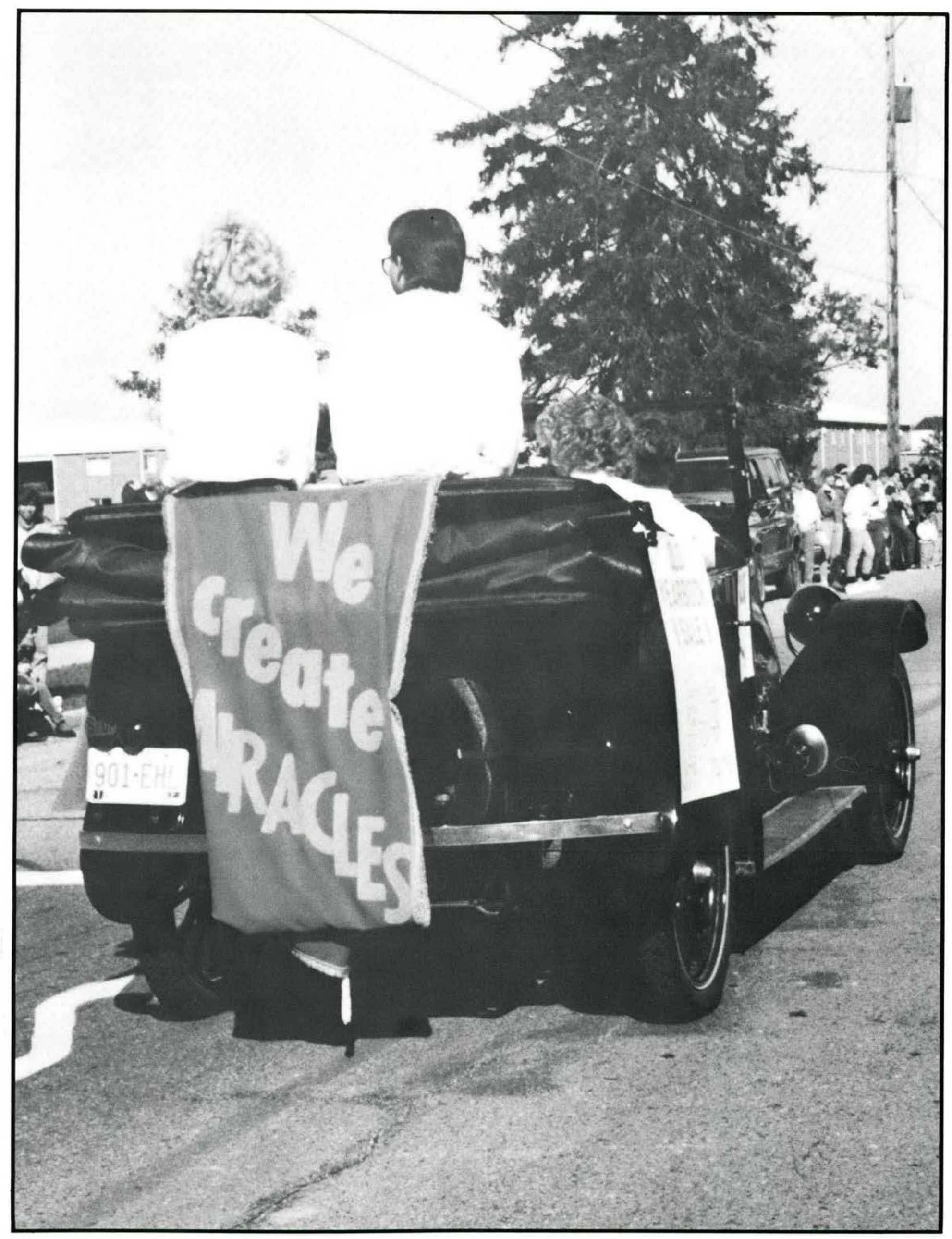




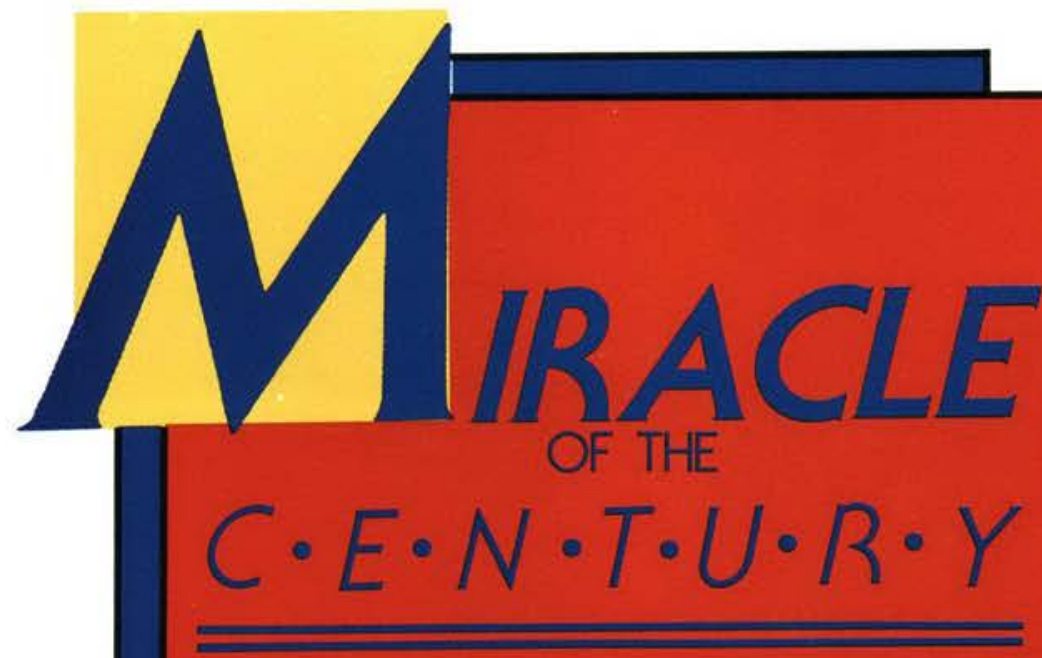

\section{FROM THE EDITOR}

I cannor keep from thanking the Lord for bringing me through 1986 - the most painful year of my life. He accomplished this through reaching me many life-changing lessons and through sending many dear people inro the sphere of my friendships. God has certainly blessed my life with people! God has also blessed the life of Cedarville College with people godly people like Dr. Mc Kinney, Dr. Mc Chesney, Dr. Jeremiah, Dr. Dixon and hundreds of orhers. God has used these people ro bring Cedarville College through many hardships and ro bless the lives of thousands.

As I reflect upon my own life and thank God for the many dear friends (borh old and new) He has given me, I also thank Him for Cedarville College and for the scores of people who have labored to make it whar it is roday. May God continue to bless the college so that it may continue to glorify Him!

Acknowledgements. To Mis. D for your support and loving concern through. out this trying year, ro Donnoh Barker for your hard work and dear friendship (GOOD LUCK NEXT YEARI), 10 all of my stoff for the gallons of "midnight oil" you burned; ro all studenis, foculiry, and staff who wrore copy and showed up for picrures, to Tina Daley, foll quarrer secretary, to Margarer Richards and Elayne Howard for your anistic coniributions, to Dr Dixon for your understanding when had to call Mrs. D or home (and always internupred "Cosby and Reds games); ro Mom and Dod for your just being there!

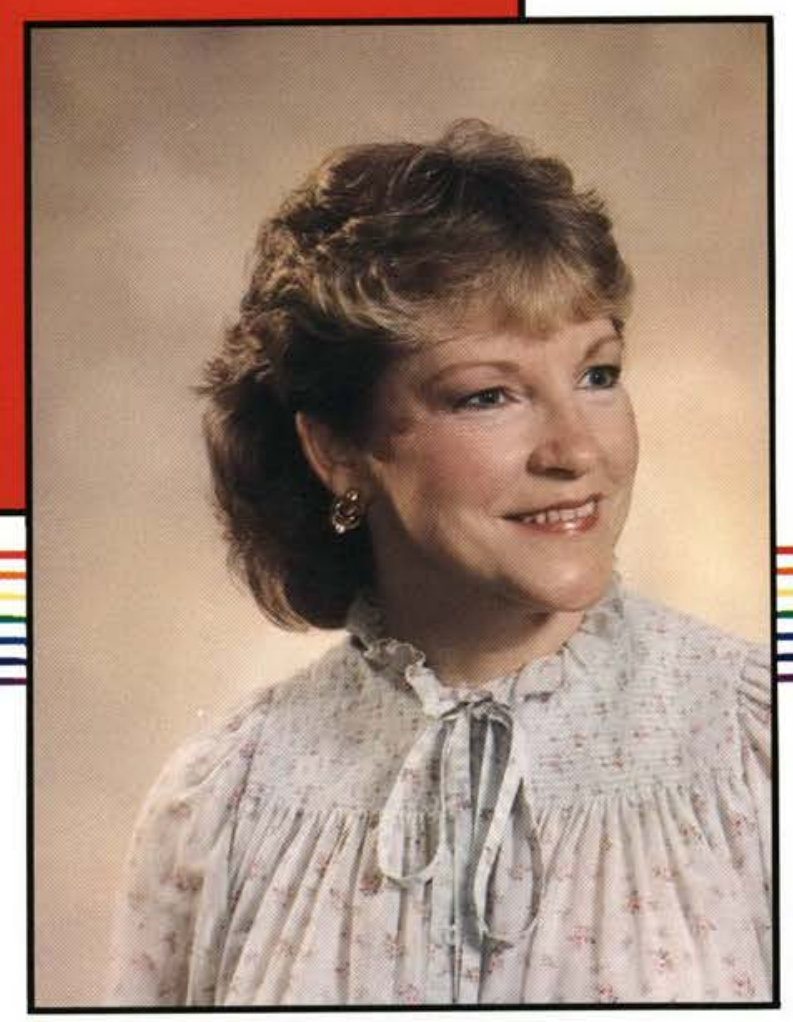

MRS. PAT DIXON

Advisor

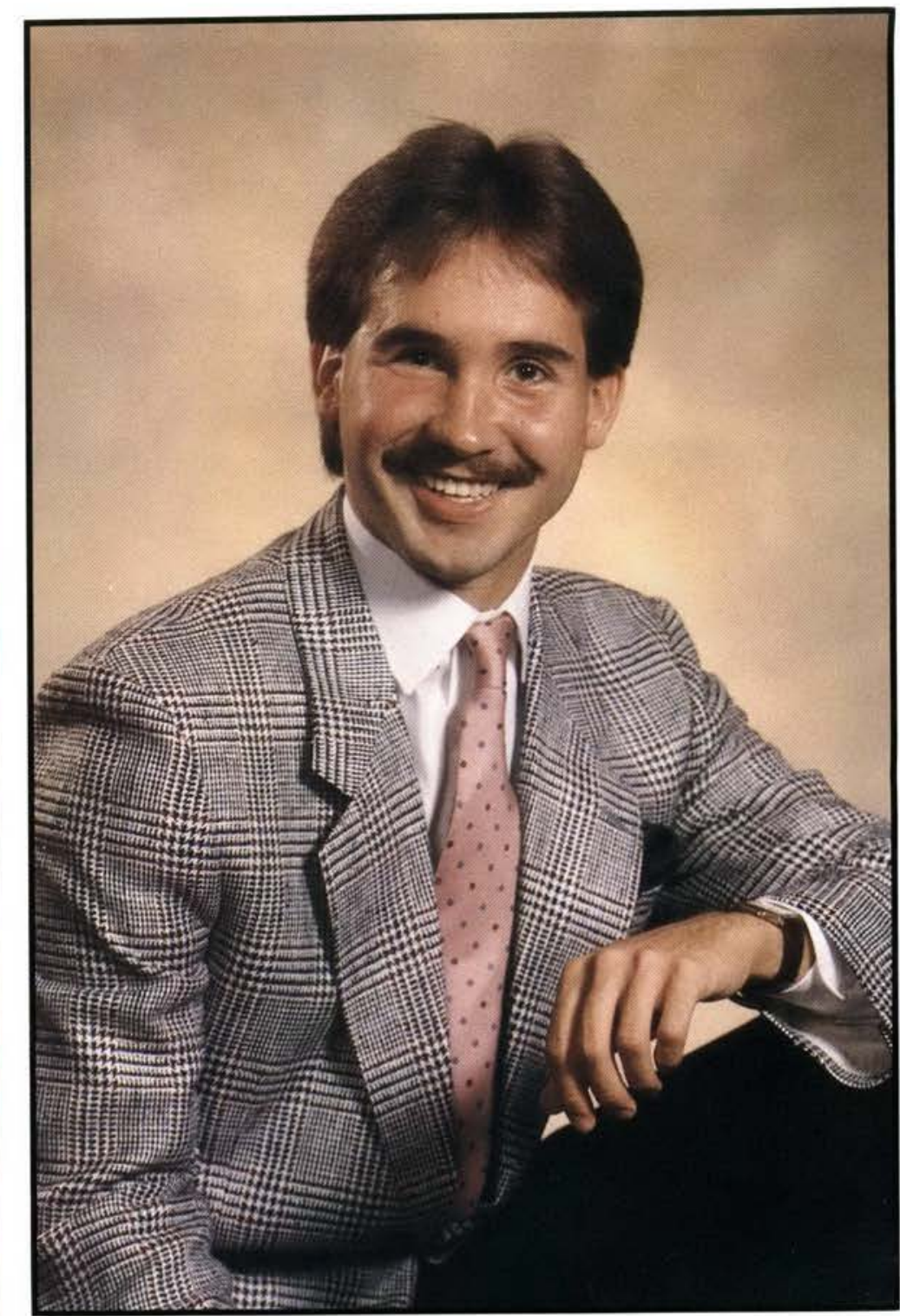

\section{JEFFREY BERGANDINE}

Editor-In-Chief

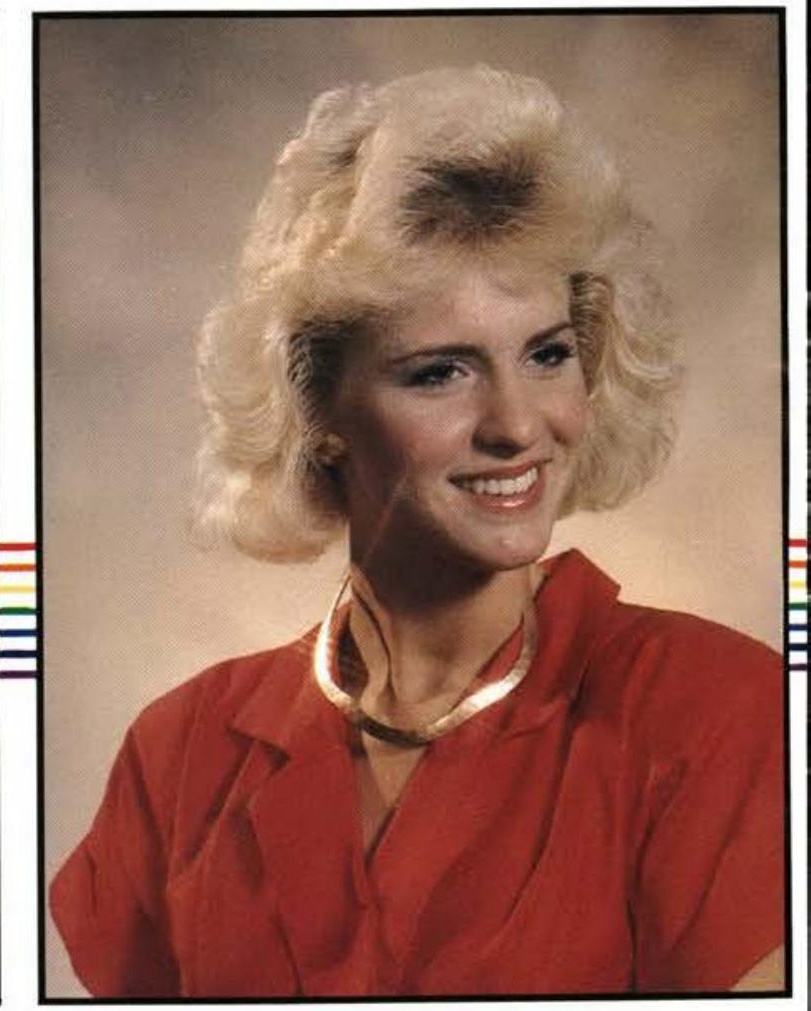

DANNAH BARKER

Assistant-ro-the-Editor 


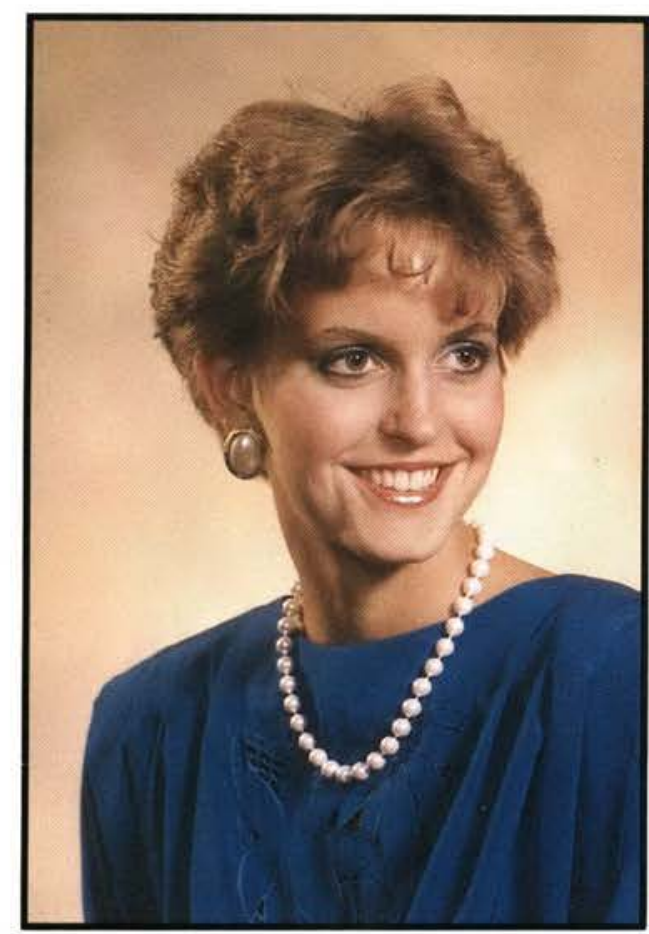

TRACY GRIMES

Copy Subediror

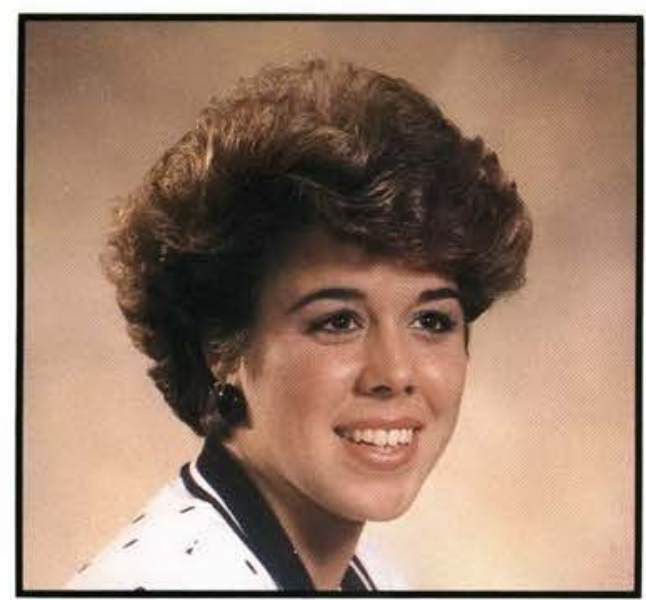

JOSIE ERWIN

Sraff Member

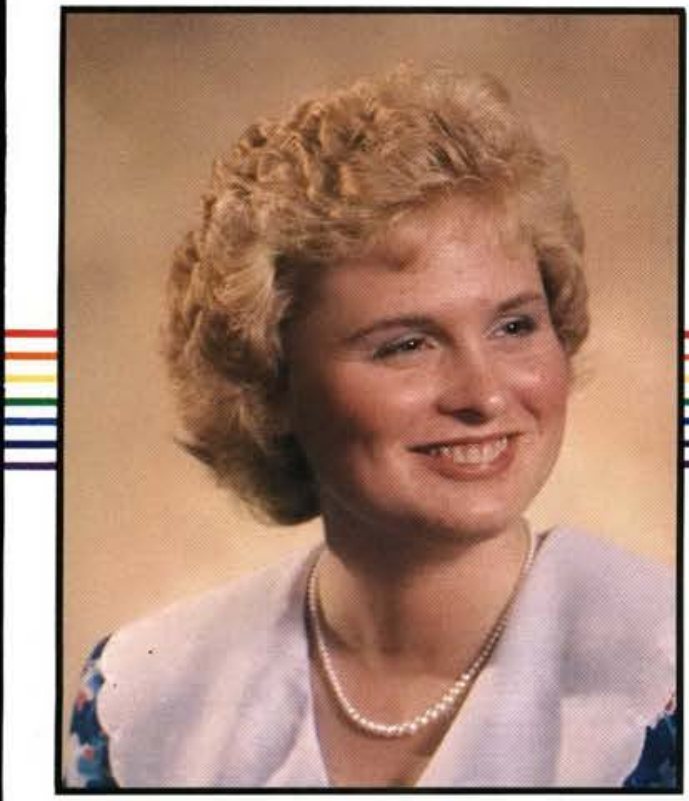

SANDRA POTTER

Secretary

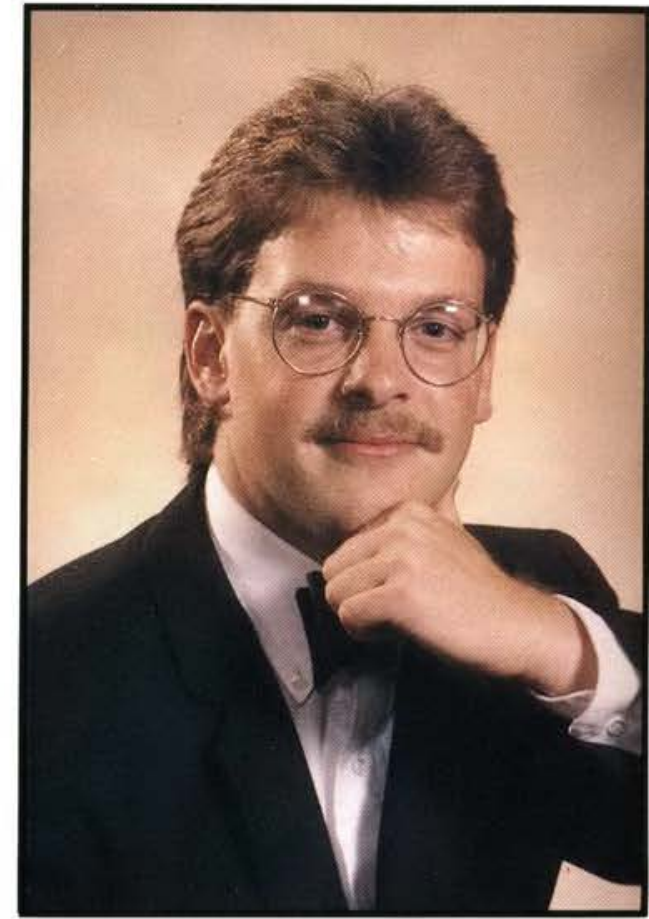

MATT DICKINSON

Sports Sub-ediror

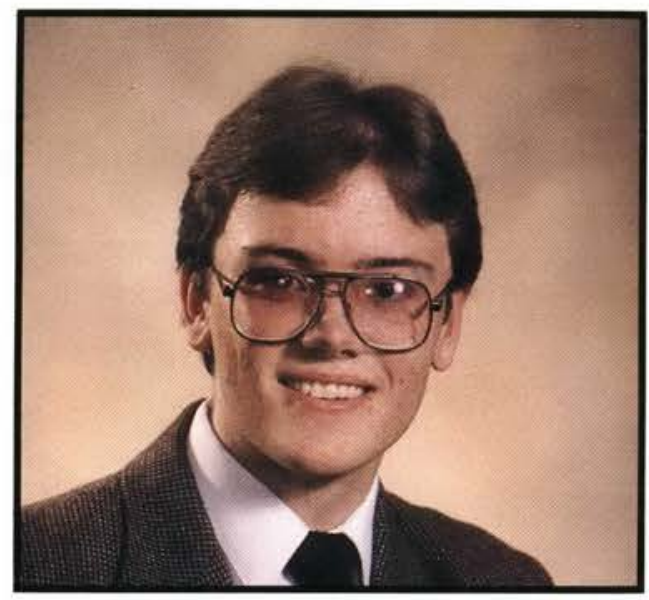

CARY UNDERWOOD

Staff Member

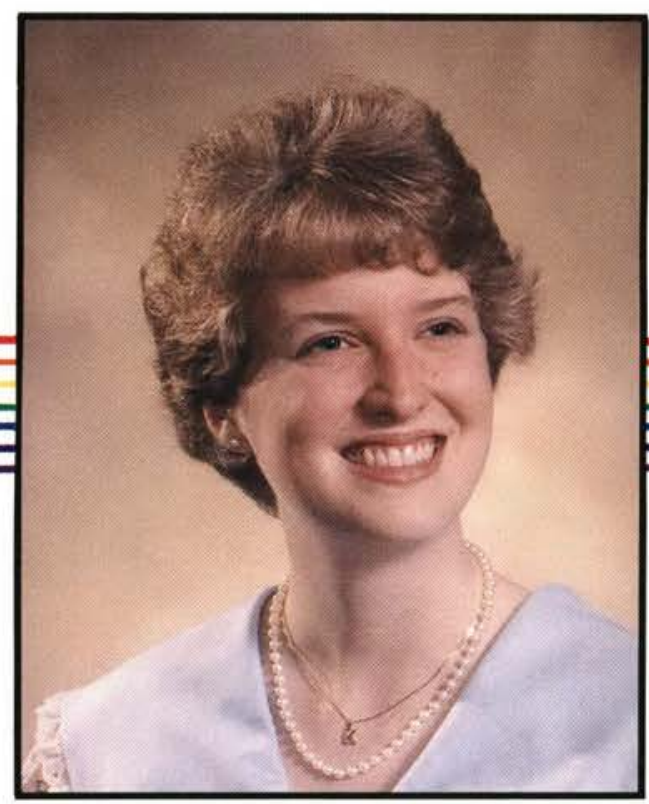

KIM BYROM

Classes Sub-edito

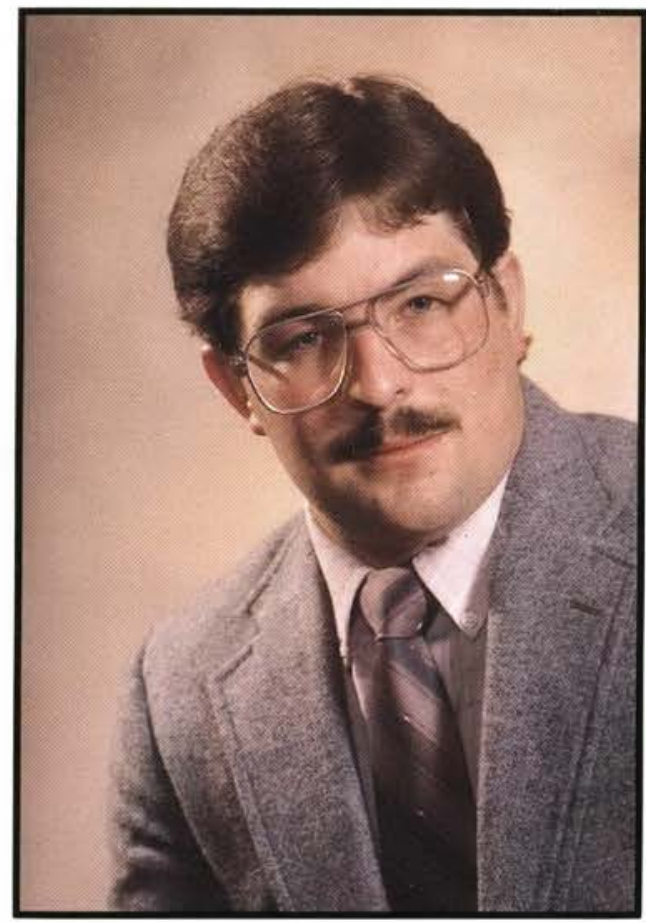

PERRY CHIARELLI

Photography Sub-editor

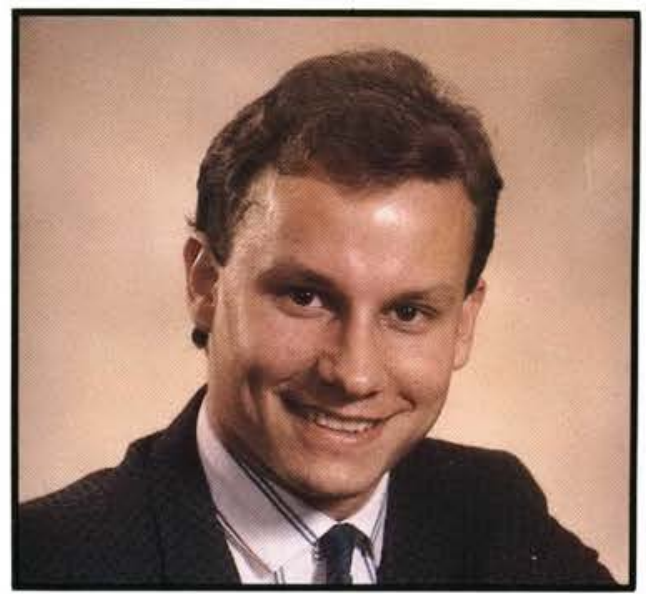

BRIAN ROGET

Darkroom Sub-ediror

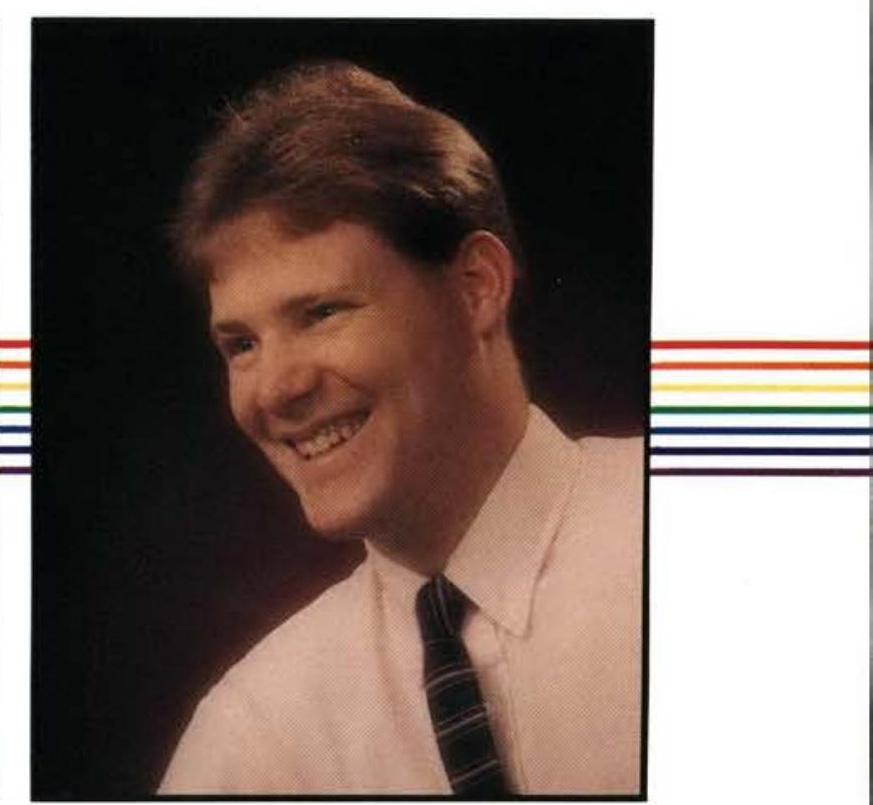

STEVE RACZ

Compurer Sub-editor 

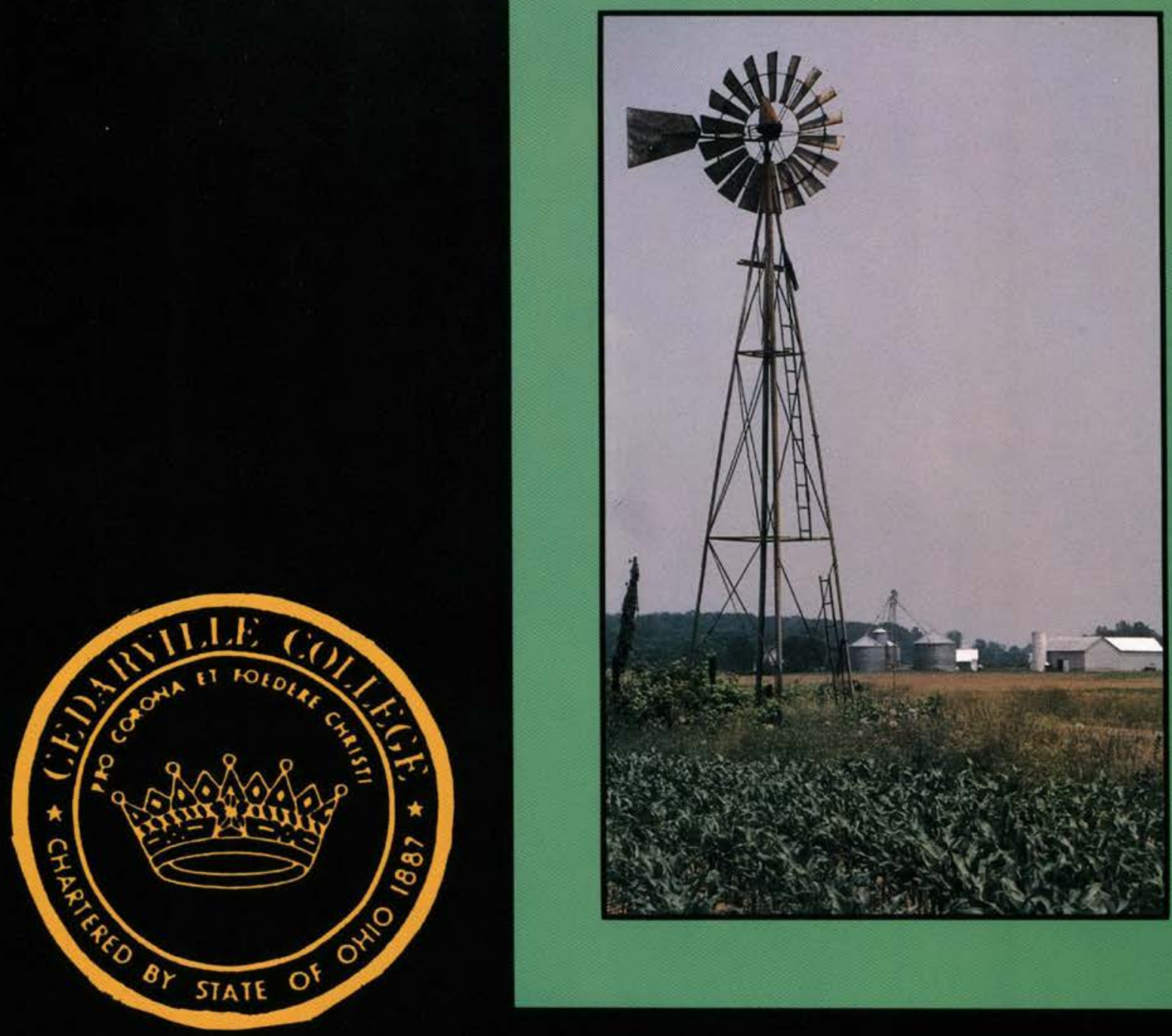

\section{CEDARVILLE COLLEGE}




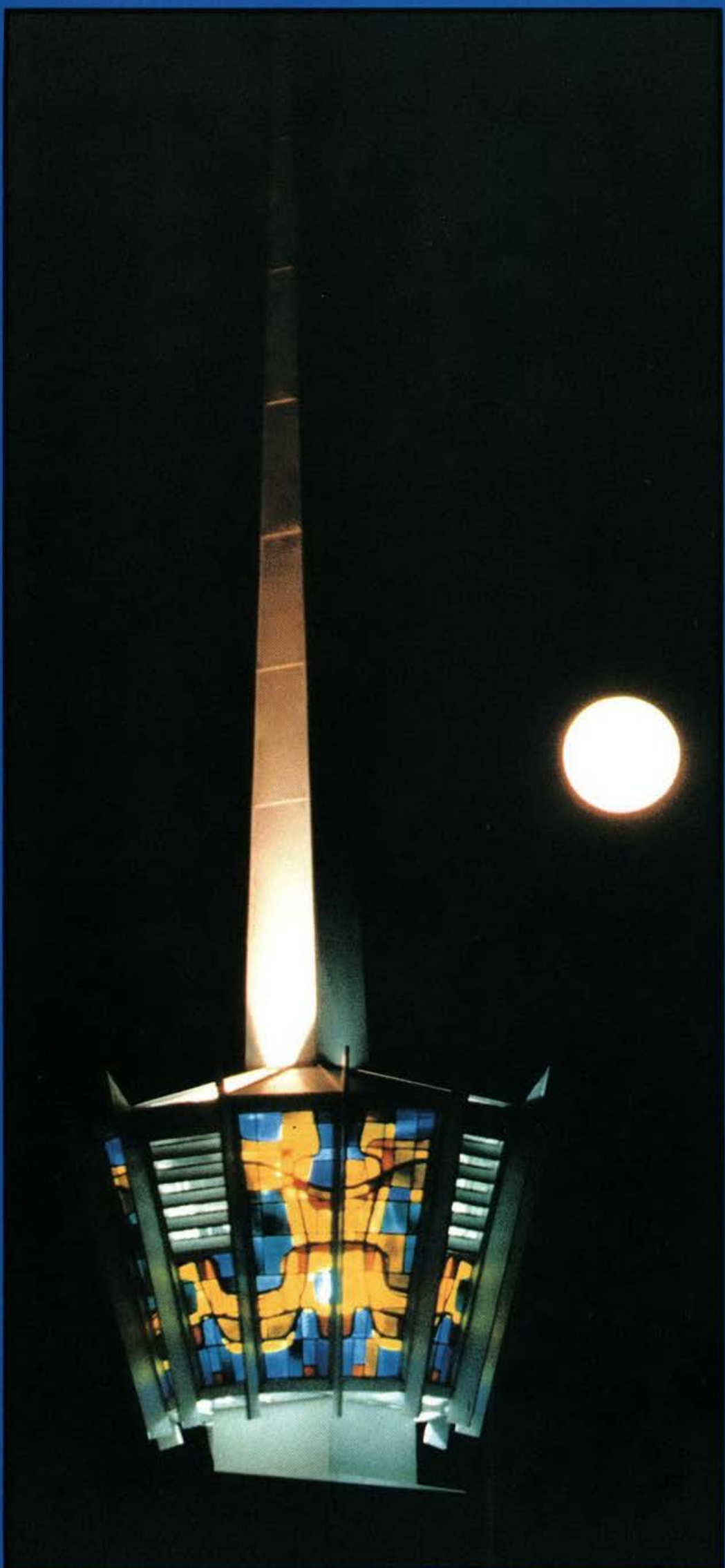

". . . the heartbeat of every Christian College

- Dr. Paul Dixon 


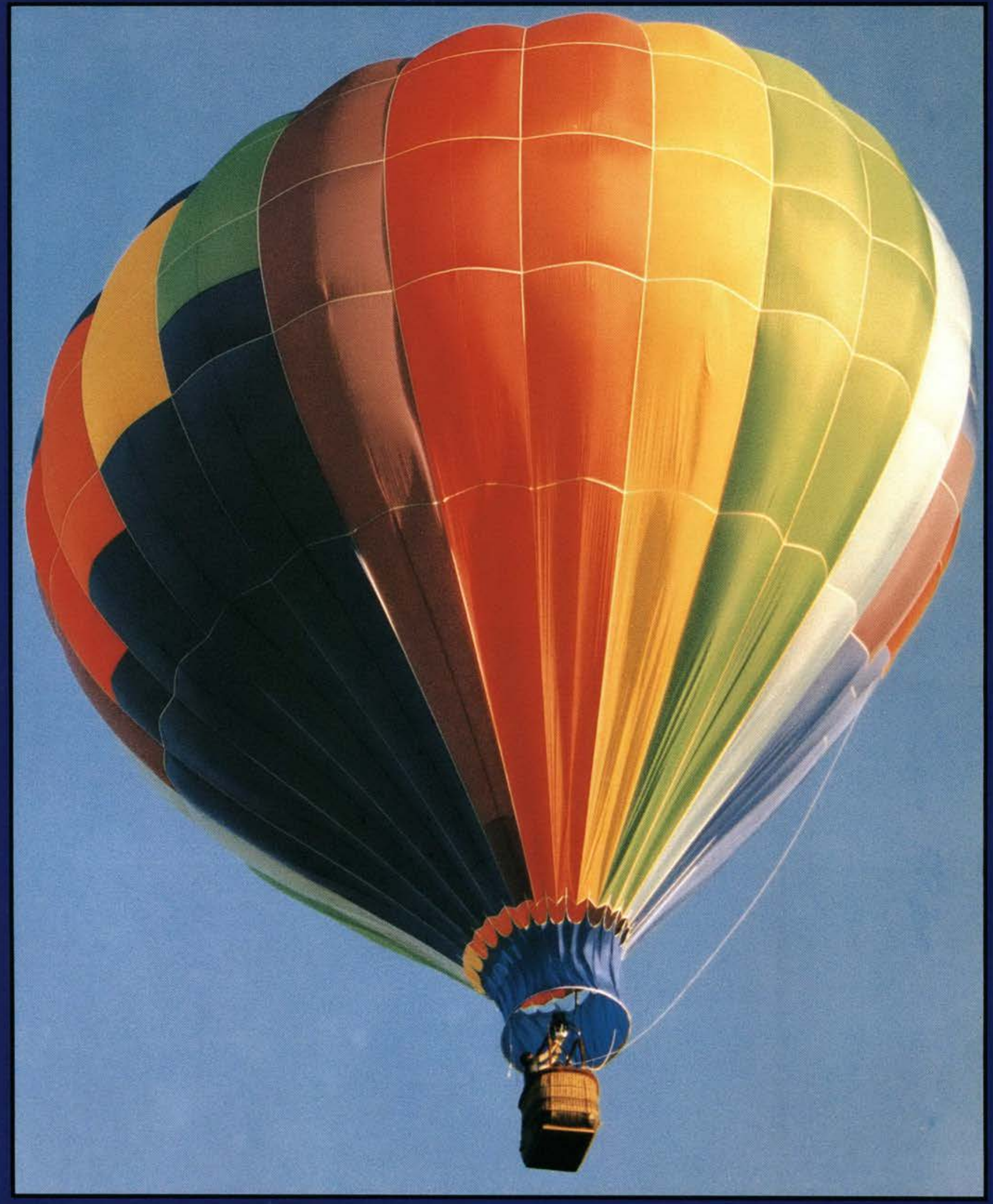




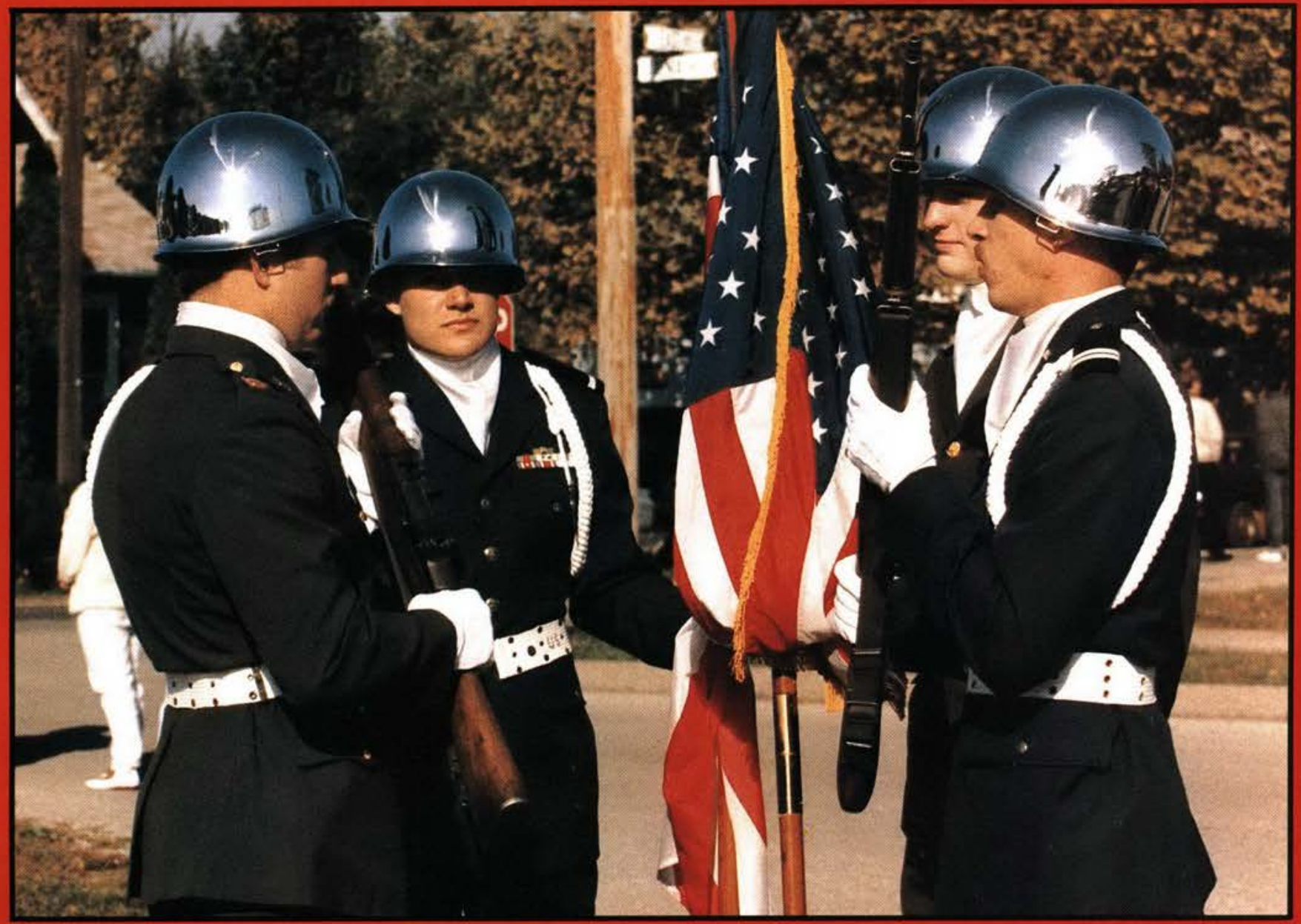

For 100 years Cedarville College has stood strong, unwavering in its commitment to the cause of Christ. God has blessed this faithfulness by carrying our school through the very core of hardships and trials. When to the world it seemed that there was no hope for Cedarville College, faithful men persevered, and the Lord blessed them with what seemed to have been an impossibility - a miracle. Because the Lord's power has worked through Cedarville, our anumal is proudly maned "che Miracle."

Cedarville College can proudly reflect upon its first 100 years and can look forward to diligently protecting "the word of God and testimony of Jesus Christ."

"Be sober, be vigilant; because your adversary the devil, as a roaring lion, walketh about, seeking whom he may devour." 


\section{THE CENTENNIAL MIRACLE IS}

JAMES T JEREMIAH, D D

CEDARVILLLE, OHIO 45314

Through the years it has been my ptivilge to observe the grouth and influence of Cedaralls Collex and d rejocice. With many atterim d pray Hod vill cextemus to strengthen the collex and increse its outread, ton the glong of the hold Fon thes to hapen thes must nexax bo a

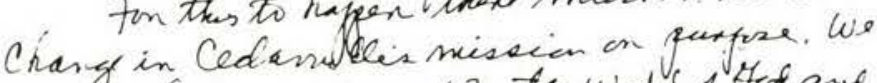
must alwarn serve "Zentle Wort 1 tod and the testimiony o fous Cerest."

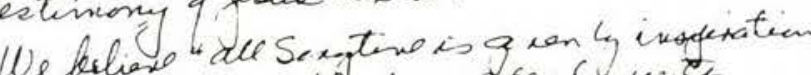

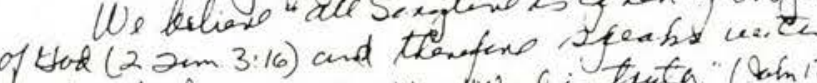

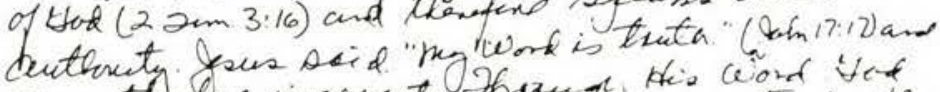

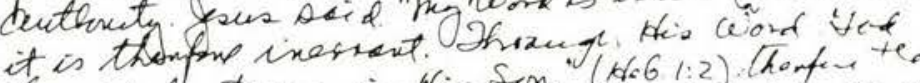

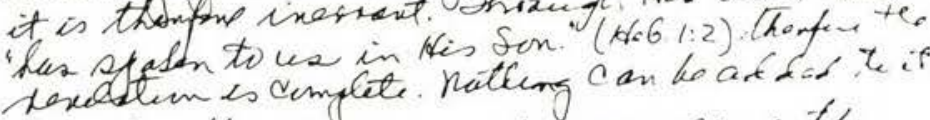
Fon these xeasona and many snore te Butle, Fods wort, munt ke th fourriation aid

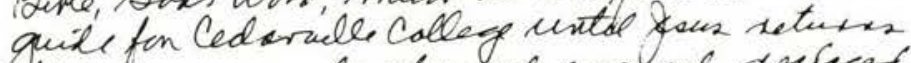
dt must nexen be changed, e gnoxed, saglaced or reglected.

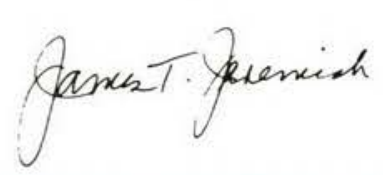

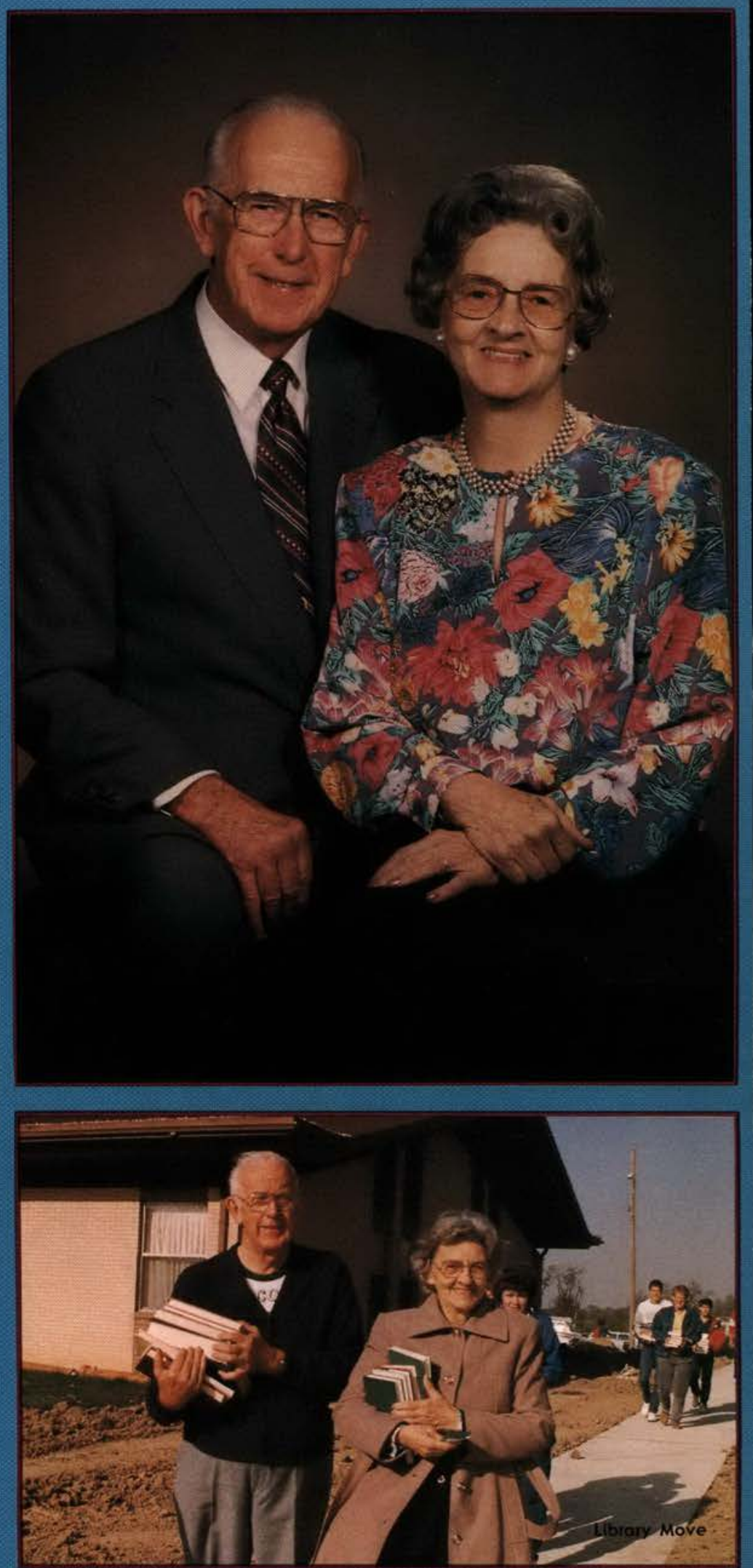




\section{PROUDLY DEDICATED TO DR. AND MRS. JAMES T. JEREMIAH}

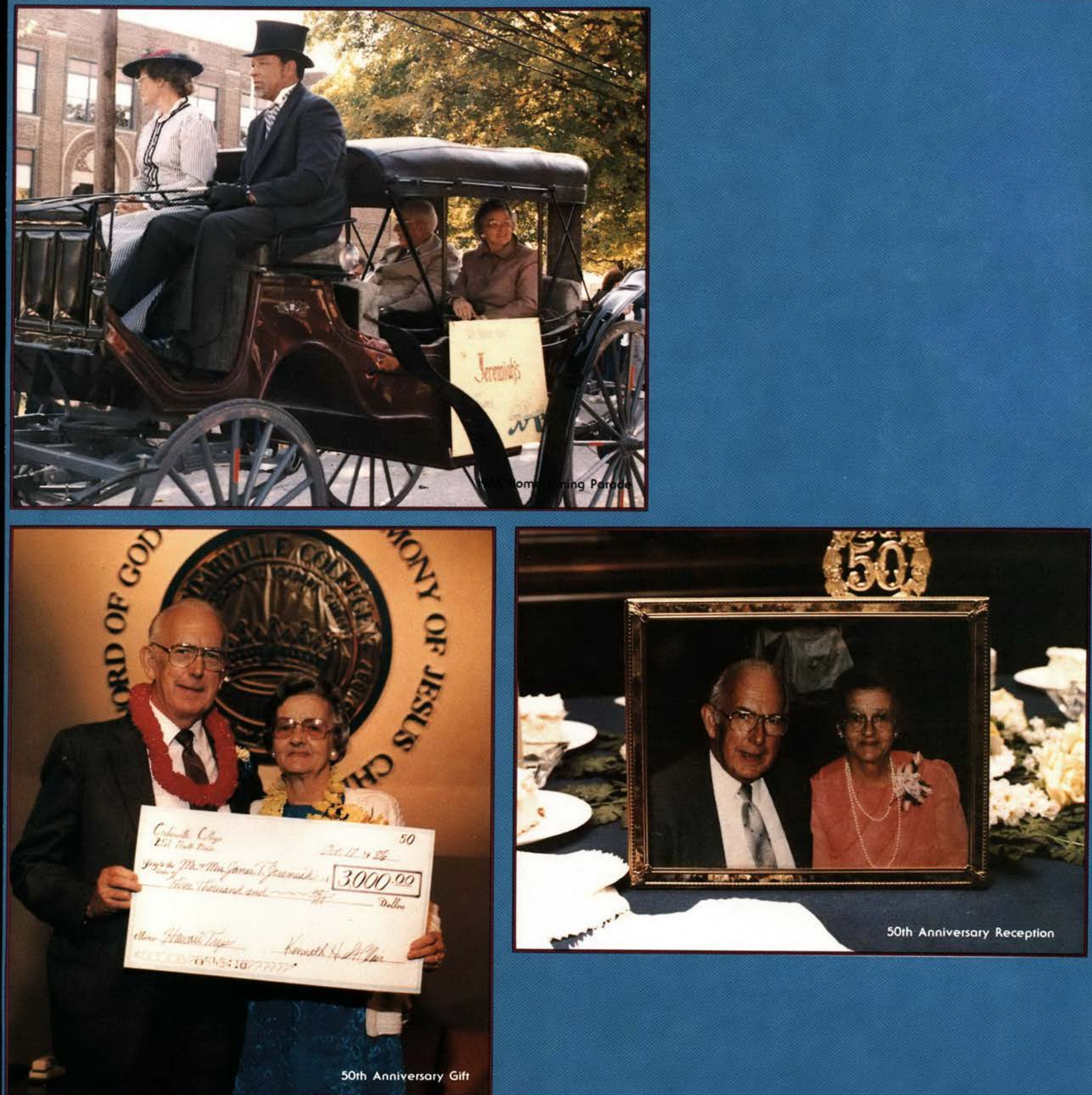




\begin{tabular}{|c|c|c|c|c|}
\hline 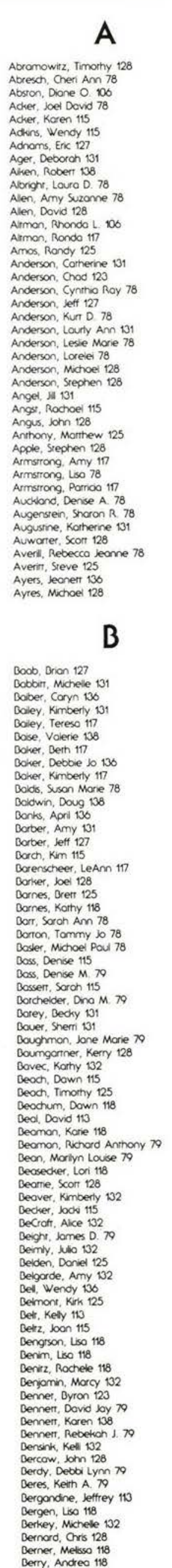 & 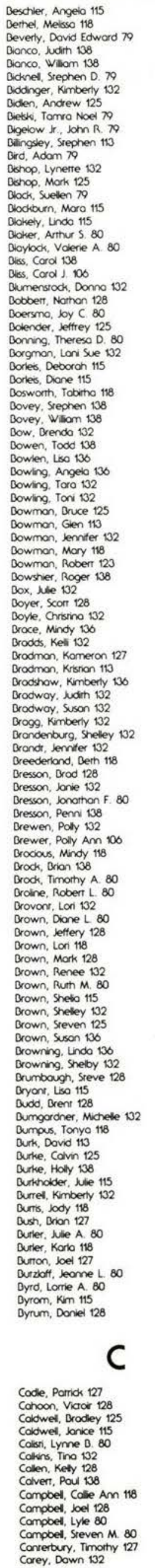 & 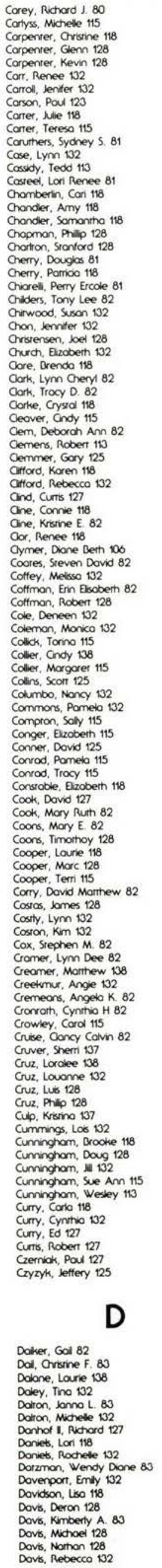 & 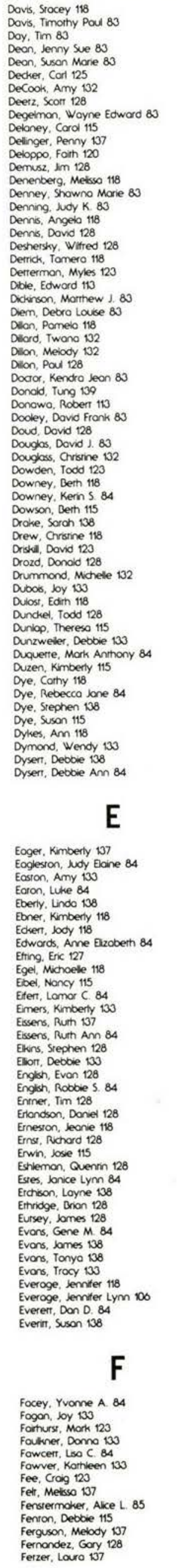 & 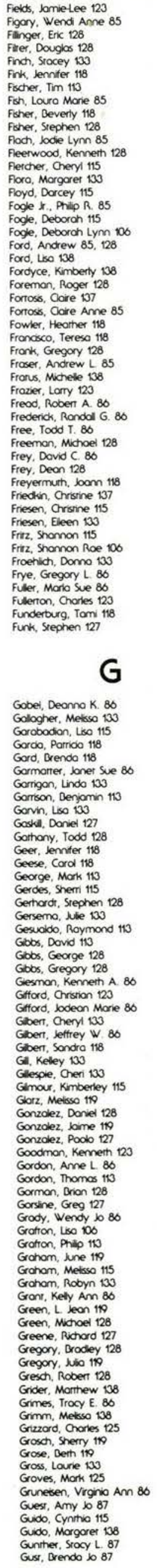 \\
\hline
\end{tabular}


$\mathrm{H}$

Hoberli, Lori R 87

Hockenberry, Lyn M 87

Hodker, Louro 119
Hoddod's Vic 125

Hoddod, S Vic 125
Hogo, Rebecco 137

Hogo, Rebecco 137
Hogo, Rebecco Jo 87

Hoga, Rebecco Jo 87
Hohnensten, Kimberly 130

Host, Heid 137

Hoboch. Lowel 128

Hol, Mesone 137

Holloway, Von 128

Hombley, Sheryh 10

Hamizon, Joy 133

Hamin, Poul 125

Hommer, Keirh 128

Hancock, Loure 5.87

Hanks, Brenda 133

Honno, Noncy 137

Hannay, Shoun 128

Hansen, Elizobert loy 87

Hanson, Brion 125

Honson, Steve J. 87

Honssen, doe 128

Hoppe, Judit E. 87

Horden, Goye 119

Horden, Mork 128

Hordin, Nancy 119

Hordy, loseph 127

Horju, Loura 133

Horper, Kristen 115
Horper, Srephen J 87

Harris, Karth 87

Harris, Novo 115

Horrson, Mke 123

Horrsough, Louro A. 87

Horvey, Conne 15

Hosket, Jonorhan 128

Hoskes, Jonarhan 128

Hostmon, Jeri 199

Houfler, Jennfer 137

Hovers, Jenny 115

Howk, Morvn 128

Howkins, Roiceon 128

Howley, Karhleen 115

Hoyden, Joel 128

Hoyes, Dione 133

Hoyes, Henry 127

Hoyes, Ronold 128

Hoynes, Koren 19

Hoynes, Karhy 119

Hoynes, Timothy 1

Hoys, Dovid 128

Hoywood, Nichard $G 87$

Heocox I, Lary Leox 87

Heoley. Hearher A. 88
Heffefinger, Jeftrey Lee 88

Hedorn, Sarch 19

Hein, Sonia 133

Hese, Rondo 199

Helm, Dorno 133

Heimick, Cynthio Joan 88

Hermick, Kelie L 88

Heimick, Shannon 13

Hemor, Eric 128

Henderson, Jemnter 133

Henderson, Nodeen
Heniser, Srocy 133

Henry, Liso Derh 88

Henry, Thereso 115

Henry. Theresa Rondy 127

Henthorn, Rebecco L. 88

Hermom, Bruce 123

Herrick, Kendall Lym 88

Herroid, Kendro 119

Herron, Lanay 138

Herroon, Lso 133

Hess, Jute 133

Hess, Shown 128

Hicks, Robert 128

Hidebrand, Robert 128

Hle, Dobby 128

Hili, Reberco 133

Hilenburg, Michoel 128

Hines, Korio Marie

Hines, Poulo 119

Himmon, Mork Joseph 88

imergardr, Michele 119

Hirchcock, Sandro

Hoog. Gary 128

tobough, Greg 128

Horter Virginio Morie 88

Hochsrenter, Virginia Morie

Hockensminth, Derricko 119

Hoddelmam, Krsten 1 TI

Hoecke; Dert I. 88

Hoecke, David 128

Hoecke, Dovid 128

Hoefler. Orristopher Jomes 89

Hoff, Dwayne 123

Hotfmeyer, Greschen 119

Hohuen, Philip I. 89

Holbrook, Sondro 11

Holland, Chery! 10

Holley, Liso 133
Holoperer, Brendo 19
Hoit, Cherye 138

Hor, Dovid 128

Hotr, Donold 123

Holrmam, Amy 133

Hotrz, Corolyn 138

Hood, Drodey 128

Hooley, Down 133

Hoover, Cynthio 19

Hornbocher, Corrine 130

Hornbeck, loanne 130

Horton, Kennert 113
Horron, Shelle 89,137

Horton, Shelle 89,137
Hoskinson, Joet M 90

Hort, Mchoel 127

Horrel, Christion 127

Howard, Down 119

Howard, Deboroh 115

Howard, Poula 119

Howard, Volene 190

Howder, Penelope 133

Howdyshet, Dororhy Eloine 90

Howell, Rebecco 19

Howets, Kevin 125

Huber, Tereso 130

Hudon, Sondro 133

Huebscher, Mark Dovid 90

Huested, Angeto 110

Huggar,, Louro 133

Hughes, Derek 128

Hughes, Kimberlyl 110

Hughes, Poul R. 90

Huguenin, Todd 113

Hul, banie Marie 90

Hutzz, Brion 128

Hummel, Rebecco 133

Hummel, Todd 113

Humphrey, Dovid 127

Humphreys, Brian 12

Hum, Poula $M$

Hunt, Esther 119
Hunt, Judy $M 90$

Hunt, Judy M 90

Hunter, Cynrinia 137

Hunfer, Cynrhios
Hurs, Liso 133

Hussoand, Cynthio 115

Hurchinson, Jody 119

Hurchison, Norma Kay 90

Hyarr, Krisine 133

Hyde, Toro 133

\begin{tabular}{|c|}
\hline $\begin{array}{l}\text { Imhoff, Jeffrey A. } 90 \\
\text { Inbody, Solly } 119 \\
\text { Inman, Joy } 113 \\
\text { Invin, Ronold } 138 \\
\text { Irving } \mathrm{k} \text {. John T, } 90 \\
\text { koocson, Toni } 133 \\
\text { Iverson, Douglas Scort }\end{array}$ \\
\hline
\end{tabular}

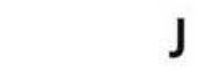

Jackson Jr., L. Charles 90

Jackson, Teresa 133

Jocobs, Dolly 133

Jocobs, Dolly 133

Jocobs, lames 127

James, Mork 11,3

James, Richord D

Jantzi, Done 13
Janvs, Joy 133

Jarvis, Joy 133

Jenkins, Kimberty Down 90

Jenkins, Kimberty Down

Jensen, Jule 130

Jessop. Suson L 90

Jeunnerte. Timothy 128

Johnson. Domie 10

Johnson, Denns 128

Johnson, Dione Renoe 91

Johnson, Enic 125

Johnson, Jan 133

Johnson, jill 133

Johnson, Kely 138

Johnson, Kely Lym 9

Johnson, Kristyn 13

Johnson, Loren 125

Johnson, Virginio L. 91

lohnston, Koren 134

lohnston, Pometo 137

tohnston, Robin L.

oiner, Robert 128

lones, Doniel 128

Jos, Kartion

Jones, Karhleen 119

Jones, Kelly K o1

lones, Mary $C$. 91

ones, Suzonne Eve or

ones, suzanne Eve 91

Jordon, Jame 128

bosin, Amy 134

$\begin{array}{ll}\text { K } & \text { Loethorr, Kimberty } 110 \\ \text { Loescher, Douglos } 129 \\ \text { Lombord, Corherine Mory } 94 \\ \text { Long, Gina Roe 106 }\end{array}$

Kline Jeffrey W 92
Love, Tino 134

Low, Karhieen 119

Lowe, Chorlene 110

Luce, Mork 129
Lum, Joel 127

Lumrel, Roger Dole 94

Lydic, Brent 129
Lym, Terry 127

Kelley, Jom 128

Kendoll, Michoel 127

Kennedy, Sondro Frances 91

Kerney, Karhleen 115

Kenyon, Pometa 137

Kerr, Shoron 119

Kester, Richard T or

Kercham, David Willom 91

Kerting, Jomes 91

Keyes, Derh 115

Kiener, Susan 1.91

Kimble, Pamelo 138

Kimble, Pamela Jane 82

Kimmel, Metssa 134

King, Dorrin 128

King. Kendra 137

King, Sheryl 134

Kimey, Robert 128

Kinsey. Volene 115
Kinsey, Von 127

Kirkporrick. Daniele 134

Kirtiand $\mathrm{K} r$. James L $\mathrm{L}$.

Kiser, Kimberley sue 92

Kizer, Doniele Kay 92

Klempeter, Eliod 110

Kinho, Danny 113

Klopo, Rebecca Am की

Knoutt, Jube 13

Knicely, Mory Derh 92

Knisley, Virginio 169

Knull, Karhy 134

Knuppo, Dione 134
Koch, Robert 113

Koerber, Jomes R. 92

Kohimeyer, John 127

Kooniz, Gory 127 123

Kreidler, Sonyo R Q2

Kresge, Sharon 138

Krikke, Lorin Kennerh 92

Krveger, John 123

Kuthrs, Kristine 119

Kuntz, Koren 134

Kunrz, Louro 134

Kyser, Rhondo L 92

Locey, Suson 134

Lochmiler, Dano 134

Lofferty, Suson lone 92

Lahoie, Daniet A.

Lamb, Stephen 125

Lands, John 129

Lone, Michoet C. 92

Lankiord, Crrstino is

Larsen, Ramono 10

Latcelo, Derh Anne 19

Laroreto, Mark 129

Larorelo, Robert

Loub. Hed 124

Low, John 129

Low, Michoel Dovid 93

Loymon, Charles 123

Le Croix, Pornio 19

Leoch, Jeftrey A 93

Leoch, Sheri 134

Lee, Juso 137

Legore, Cortherine 134

Lehe, Dovid 125

Letmon, Chester 138

Letoh, Michetole 134

Lemponen, Shely 110

Lenharr, Jennter 134

Lerhbridge, Tmorhy 113

Lertico. Tino 19

Lewis, Tmothy 127

ichry, Danno 134

Liming. Thomas 129

Ind, Heorher 124

Lindley, Dorr AMn 93

Link, Orristopher 138

ippert, Kortheen 134

getshor, Kimberty

ombord, Cortherine Mory

Long. Jem wa

Lough $\mathrm{K}$., Donald H. 94

Mooibroek, Lindo 134

Moon, Elzoberh 120

Moon, Pomela 120

Moore, Onrisopher 129

Moore, Douglas 129

Moore, John Andrew 95 
Peorson, Rebecca 120 Pearson, Silos 110

Peno, loserte 120

Penny, Crristine 120

Perkins, Roderick 127

Perkins, Shele 97

Perrock, be 129

Perek, Doriel Chorles 97

Perek, Richard 109

Perers, Rebecco 137

Pererson, Renee' A. 97
Pererson, Thomos L. 97

Perne, Charles 129

Perros, Heid 110
Philips, Amy Louise 98

Phillips, Amy Louse
Philps, Diano 139

Phipps, Greg 123

Prikerton, Jean E.98

Pinkerton, Jeon E. 98
Piper, Jeftrey S. 98

Piper, Jeffrey S. 90
Plper, Mork 123

Pronyok, Lynne 120

Plooy, Cheryl 137

Plourde, Korrina 120
Poing, Scont 129

Poing. Scorr 129

Popo, Jonarhon 113

Pope, Metsso 105

Poppenhogen, Gina 120

Posey, III 120

Porrs, Kim E. 98

powel, Tereso 139

Powers, Am 120

Priddy, Duone 127

Prince, Michelle 120

Prins, Lelloni 120

Pruner, Rebecco 135

Pursenborger, Bruce 139

\section{Q}

Quim, Karino 116

\section{R}

Rocz, Steven 129

Roddiff, Ebbie 137

Raines, Liso 120

Ranes, Lso Berh

Rajchel, Ferdinand Henry 98

Ramey, Kennerh 129

Rarnsey, Angeto 135

Ramsey, Lym 11:

Ramsier, Kimberly Jean 98

Randol, Roger Lee 98
Ranger, Lindo 105

Rose, Daniet Howard 98

Roy, Kara Janele 9

Reom, Jule 120

Reebel, Orian 129

Reebel, Vivion 120

Reed, Kristo 120

Reed, Mark 139

Reed, Porrica J. 98

Reede It. George Edwin 98
Reeder, Lowrence G. 98

Reeder, Lowrencis 125

Reehl, Mork 125

Reeves, Jennifer Eleen 98

Reeves, Moly 135

Reid, J. David 125

Reid, Rebecco D. 98

Reifsnyder, Shoron 120

Retrer, Jeffrey 129

Rerer, leftrey 129

Renberg. Orrisine 116

Rendle, Oneryl A. 99

Renshow. Kimberty 135

Rexilus, Ken 125

Reynolds, Debro 137

Rice, Porricio tro

Rice, Philip 130

Richord Dye 84

Richords, Homiton 127

Richardson, Rebecco 120

Rickard, Pameta to 9

Riggs, Metsso 135

Rivey, Megan 120

Rinehor, Deboy 135

Rinehor, Fred 113

Rinehor, Tekerso 120

Riopel, Liso 125

Rirche, Groig 125

Rirsemo, Amy iso

Rizor, Sonjo 135

Rizor, Sonjo 135

Roboino, loftre 130

Roboris, Lech 135

Rober, Oris 116

Robinerte, Dovid Lee $\phi 9$

Robinson, Deborch 120

Robirson, Kimberty is

Robinson, Tereso 120

Rodebough, willom

Rogers, Jennifer 116
Rogers, Lori 135

Rogers, Suson 120

Rogge, Liso 116

Roig. Corissa 135

Rolfe, Renee 137

Rolfe, Renee leorne $\phi$

Rose, Michoel 130

Rose, Wendy 135

Ross, Nancy 137

Ross, Srephen 109

Rorromet, Debro 120

Rorromel, Sherry M. $\phi$

Rourson, Terry 127

Ruble, Oreny 120

Ruby, Dorren L 99
Rudeirch, Joamo 135

Rudelirch, bama 135
Ruggles, Gayle 137

Ruggles, Goyle 137

Runion, Metsso 120

Rupp, Carty 116

Rushe, James 113

Russel, Deborch 120

Russell, kAmy 120

Russell, Suson 116

Rutr, Daryl 125

Ryon, Heid Morie 00

Ryan, Timorthy 130

\section{S}

Soder, Kerri 137

Sodler, Rebecca 116

Soger, Solly to 110

Soliee, Glen W. 99

Solee, Mark 130

Solstrom, Todd 125

Solsbury, Darren 130

Solvarore, Andrew 123

Sonchez, lose 130

Sand, Raymond 130

Sonder, Mark 139

Sanders, Roobn 120

Sovord, Perer 127

Shook, Poul 130

Schoker, Timorthy Mork $\varphi$

Scrierwogen, Dororhy
Schleinitz, Kevin 130

Scheider, Jom 130

Schofield, Los 116

Sctritz, ennter 120

striz, enrifer 120

Schueren, Morthew 130

Schuirz, Elizobert A. $\phi 9$

Sthuiz, Metody Am $\phi 9$

conver, Mesoy Am $\phi$

sciwarz, Deborch tho

scorr, Charles 130

Scowcot, 1il 120

Secrles, Daniet R. 99

Sebright, Lynerte 110

Sectrist, Nichard 130

Seest, 1 ito 116

Seher, Mork 130
Sebel, Dovid 123

Setber, Suson 110

Seidel, Enick I. 9

Selers, Forrest Randd 100

Semmerorh, Judin 100

Senoy, Sharon 116

Severance, Siephen 130

sexion, Jeft 130

Seymour, Sreven 113

Shatfer, Gary 130

Shatfer, Lindo L. 100

Shatfer, Mark 125

Sharp. Terry D. 100

showi, Philp 130

Show, Kevin Andrew 100

Show, Woyne 113

shepherd, Kenyon 127

sherrick, Berin 127

shook, shown 139

shook, Shown 139
shorr, Rondo 135

Shriver, Deena Renee' 100

Shrum, Enic L. 100
Shuier, Sheri 137

Shuler, Sheri Lym 100
Shuman, by 137

Stbert. Karhryn Mone 100

Sickou, Lyme 120

Simerty. Donold 130

Simk, Thomos D. 100

Simmen, Deboroh Lee 100

Simpson, Karlo 137

Simpson, Keth 100
Sindar, Rebecco Elen 100

Sindor, Rebecco Elen

Sus, Delindo 120

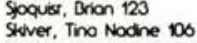

Sloyton, jall 120

Stoan, Dart 120

Sloan, Tommy W. 100

smolman, Dovid A. 100

smalman, Mojorie

smar, Mhomas James 100

smeter, James 130

Smiley. Allson 120

Smith, Allison 120

Sman, Derh Ann 110

Smint, Drian 100

Smith, Cheryl 103

Smirh, Orristopher 130

Smirh, Daniel 125

Smith, Dovid 123

Smirt, Debole 121

Smith, Foyame 110

smich, krebecco Lym 121

Smirh, Krisine 137

Smith, Pomelo Mriam 101

Smith, Rebecco Lym 121

Smith, Rhondo 121

Smith, Robert 1. 101

Smath, Ronold M. 101

smith, Srephen A. 130

Smath, Sreven M. 130

Sminh, Sreven P. 100

Smith, Tmorhy 123

snider. Andrew 113

snodgrass, Rusty wo

Snyder, Andrea 0.01

snyder, Domo lean 101

Snyder, Domo lean 101

Solomon, Srephanie

sommers, Dovid 125

Sourhwel, Sheto Am 101

Spangler, Shown 135

spes, kern 139

Sperry, Kevin 139
Solegel, Lori 137

Spink, Kevin 130

Springer, Somvel T. 101

Springmon, Amy

Srodthouse, the Lym 101

Srodhouse, Penney 135

Srarer, Siephen 130

Stroder, Suson 135

Storkey, Lomy 123

Srouffer, Joner 121

Srener, Meredirh 137

Srephens, John 130

Stevers, Drion 130

Srevers, Domor's 121

Srevers, ternifer 137

Srevens, Leecrne 105

Srevers, Srephanie 135

Sollwet, Kimberty 121

Srinedurt, Tho

Sirn, Philp 110

Srokes, Trenton 130

Srotrztus, Minom 139

Sronehouse, tive 116

Sroner, Dovid 130

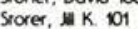

Srom, Tamaro 121

Srough, body 121

Srowe, dazo 110

Srowed, Orisine 135

Srowel, Orisine M. 100

Srowel, Rondid Lee

Srretch, Hearther 139

Srroup, Elone 121

Srumme, lock 130

Srurgs, Dian Poul 101

Sulet, Amn 121

Suritf, Drenda 135

Swander, Mark Alan 101

Swam, Suson 110

Swanson, Pamelo M. 101

Swornz, bete 121

Swoyzee, ill A. 102

Sweer, Kimberty 121

Sweer, Sondra 135

Swerzer, Alce 155

Swetzer, Mark Fredert

Swope, Kennerh E. 102

Synder, Domie 121

Synder, Ferrol 116

Synmort, Deboroh Am 102

\section{$T$}

Tocker, Rebecco 105

Toth, Jennifer 116

Tonner, Lyolio 121

Torke, Shetey Ame 102

Tosker, Greto 121

Toylor. Alson Koy 102

Toylor, Drendo 12

Toylor, lomes 125 




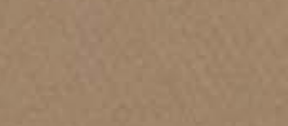

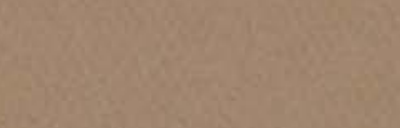

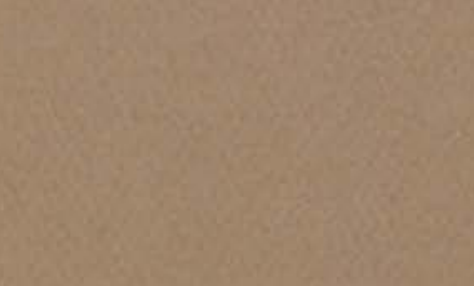

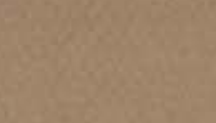

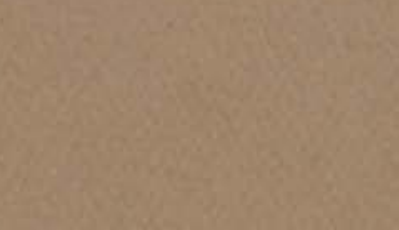

isip Pot

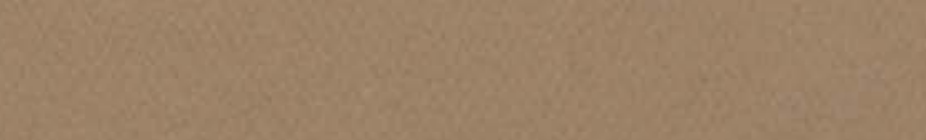

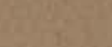

(a)

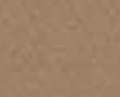

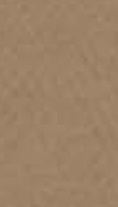

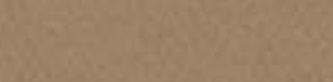

F.

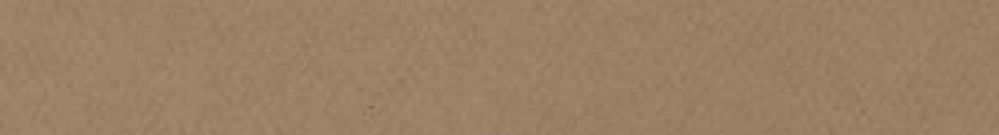

ing

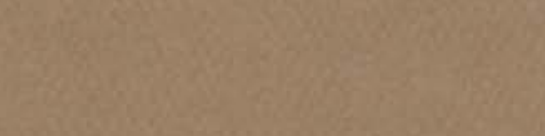

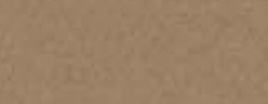 \\ Whe}

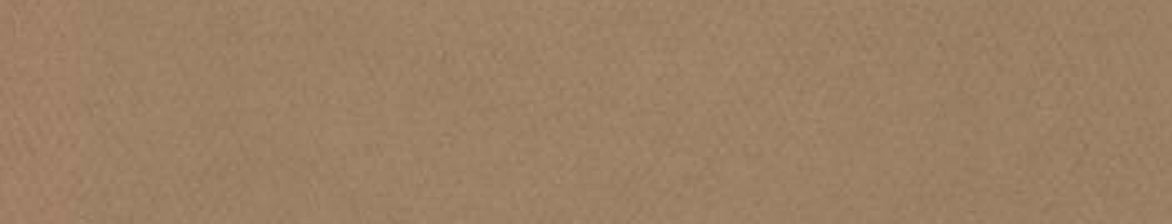

Whete

and

\section{ahe 30}
W.

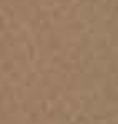

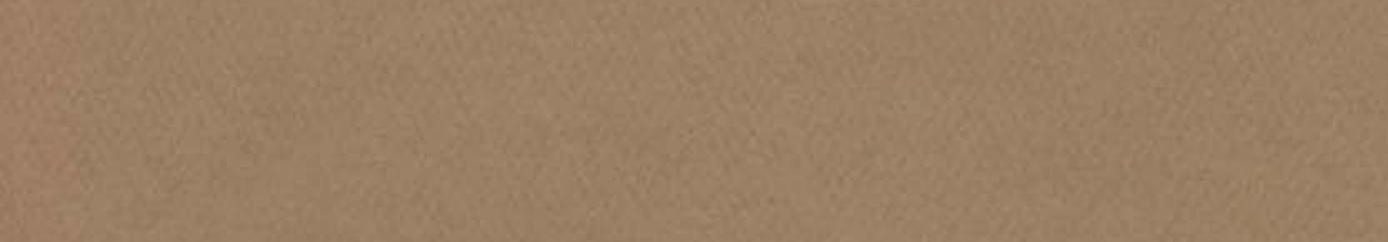

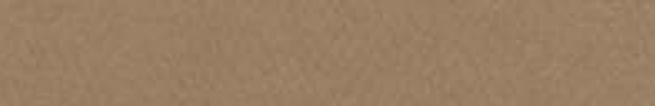

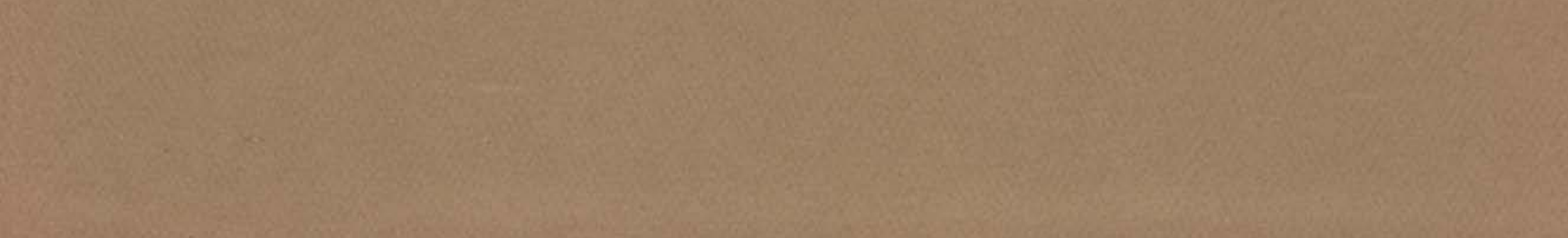
- 4 ia

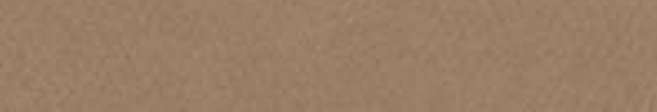

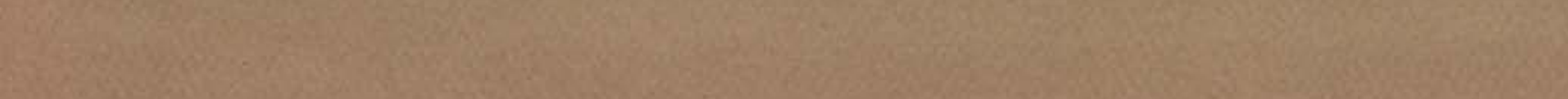

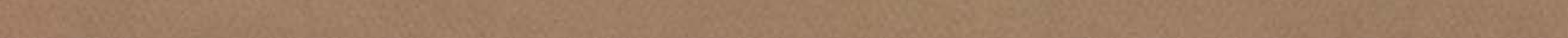


